\title{
Origami Cylinders
}

\author{
Dissertation \\ for the award of the degree \\ Doctor rerum naturalium (Dr.rer.nat.) \\ of the Georg-August-Universität Göttingen \\ within the doctoral program Mathematical Sciences \\ of the Georg-August University School of Science (GAUSS)
}

submitted by

Friedrich Bös

from Kassel

Göttingen, 2017 


\section{Thesis Committee}

Max Wardetzky,

Institut für Numerische und angewandte Mathematik, Universität Göttingen

Gert Lube,

Institut für Numerische und angewandte Mathematik, Universität Göttingen

Etienne Vouga,

Department of Computer Science, The University of Texas at Austin

\section{Members of the Examination Board}

Reviewer:

Max Wardetzky,

Institut für Numerische und angewandte Mathematik, Universität Göttingen

Second Reviewer: Gert Lube,

Institut für Numerische und angewandte Mathematik, Universität Göttingen

Additional Reviewer: Etienne Vouga,

Department of Computer Science, The University of Texas at Austin

Further members of the Examination Board:

Anja Fischer,

Institut für Numerische und Angewandte Mathematik, Universität Göttingen

Stephan Huckemann,

Institut für Mathematische Stochastik, Universität Göttingen

Preda Mihăilescu,

Mathematisches Institut, Universität Göttingen

Gerlind Plonka-Hoch,

Institut für Numerische und Angewandte Mathematik, Universität Göttingen

Date of oral examination: July 6th 2017 


\begin{abstract}
Origami, the age-old art of folding intricate three-dimensional structures from flat material, has found numerous applications in e.g. the design of deployable structures and mechanical metamaterials. This thesis investigates the axial compressibility of cylindrical origami, i.e., cylindrical structures folded from a single rectangular sheet of paper. It is shown via purely geometric arguments that a general fold pattern has only finitely many strain-free cylindrical embeddings. Therefore, continuous deformations must either induce elastic strain or deform the preexisting folds. A counterexample shows that the obtained necessary flexibility conditions are sharp. The results restrict the space of possible constructions for designing rigid-foldable deployable structures and metamaterials.

Despite this rigidity result, origami cylinders are nevertheless observed to compress apparently isometrically. In a second step, this apparent flexibility is modeled by replacing hard rigidity constraints with simple soft constraints in a way inspired by physical experiments, numerical simulations, and theoretical arguments. The resulting energy minimization problem is solved in two different ways: numerically using the particular geometry of the feasible set and qualitatively using topological arguments about the set of critical points. The results exhibit marked buckling phenomena reproducible in experiments, indicating a geometric as opposed to a physical origin. The model can be used for rapid prototyping in the design of foldable cylindrical structures with a prescribed strain response to axial compression.
\end{abstract}




\section{Contents}

1 Introduction 9

$1.1 \quad$ Background . . . . . . . . . . . . . . . . . . . 10

$1.1 .1 \quad$ Rigid polyhedra . . . . . . . . . . . . . 10

1.1 .2 Elasticity of thin shells . . . . . . . . . . . . . 12

1.1 .3 Targeted design of origami $\ldots \ldots \ldots \ldots \ldots$

1.1 .4 Elastic origami . . . . . . . . . . . . . . . 14

1.2 Overview and contribution $\ldots \ldots \ldots \ldots \ldots \ldots$

1.2 .1 Rigid origami cylinders . . . . . . . . . . . . . . . . . . . . . . .

$1.2 .2 \quad$ Nearly rigid origami cylinders . . . . . . . . . . . . 17

$\begin{array}{lll}2 \text { Rigid Origami } & 19\end{array}$

$2.1 \quad$ Basics of developable surfaces $\ldots \ldots \ldots$. . . . . . . . . . 19

2.2 Rigid origami cylinders . . . . . . . . . . . . . . . . . 22

$2.2 .1 \quad$ Flatness of faces $\ldots \ldots \ldots \ldots \ldots \ldots$

2.3 Deformations of origami cylinders . . . . . . . . . . . . . . . 27

$2.3 .1 \quad$ Embedding a single strip . . . . . . . . . . . . . . . . 29

2.3 .2 Turning angles at interior vertices $\ldots \ldots \ldots \ldots$

2.3 .3 Turning angles at exterior vertices and apices . . . . . . . . 32

2.3 .4 The gap function $\ldots \ldots \ldots \ldots \ldots \ldots$

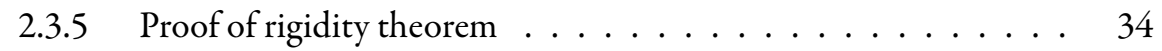

2.3 .6 Proof of vertical fold theorem . . . . . . . . . . . . 35

2.4 Designing folded structures of given height $\ldots \ldots \ldots \ldots$

2.5 Construction of multistable origami $\ldots \ldots \ldots \ldots$

3 Nearly rigid origami 43

3.1 Setup . . . . . . . . . . . . . . . . . 44

3.2 Analytic solution in special cases $\ldots \ldots \ldots \ldots \ldots \ldots$

3.2 .1 Single strips . . . . . . . . . . . . . . . 46

3.2 .2 Multiple strips . . . . . . . . . . . . . . . . . . 48

3.3 Parametrizing the constraint manifold $\ldots \ldots \ldots \ldots \ldots$

$3.3 .1 \quad$ Planar vertex layers $\ldots \ldots \ldots \ldots \ldots$

3.3 .2 Nonplanar layers. . . . . . . . . . . . . . . . . . 52

3.3 .3 Basics of Lie groups $\ldots \ldots \ldots \ldots \ldots \ldots$

3.3 .4 Incorporating the inequality constraints . . . . . . . 57

3.4 The simple energy model $\ldots \ldots \ldots \ldots \ldots \ldots$

3.4.1 The case $m=2 \ldots \ldots \ldots \ldots \ldots$ 
\begin{tabular}{|lll}
\hline & Implementation details & 69
\end{tabular}

$4.1 \quad$ Projection onto tangent cones . . . . . . . . . . . . . . . . . . . 69

4.1 .1 Projection onto tangent spaces . . . . . . . . . . 70

4.1 .2 Tangent cone projection: straightforward method . . . . . . 70

4.1 .3 Tangent cone projection: Lemke's algorithm . . . . . . . . 72

4.2 The line search method . . . . . . . . . . . . . . . . . 75

4.3 Coordinate conversion . . . . . . . . . . . . . 77

$\begin{array}{lll}5 & \text { Results and discussion } & 81\end{array}$

5.1 Code validation. . . . . . . . . . . . . . . . . . 82

$5.1 .1 \quad$ Comparison to analytic results $\ldots \ldots \ldots \ldots \ldots 2$

5.1 .2 Comparison to soft constraint minimization . . . . . . . . 82

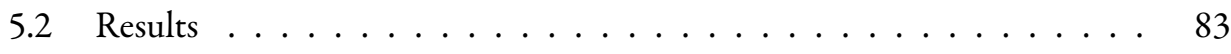

$5.2 .1 \quad$ The simple energy model . . . . . . . . . . . . . . . . . 87

5.2 .2 Verifying the planarity assumption $\ldots \ldots \ldots \ldots$. . . . 91

5.2 .3 Further observations. . . . . . . . . . . . . . . . . . . . 92

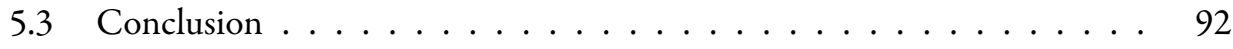

$\begin{array}{lll}6 \text { Conclusion } & 95\end{array}$

\begin{tabular}{lll}
\hline A Further results & 105
\end{tabular}

\begin{tabular}{lll}
\hline B Fold patterns & 177
\end{tabular}

B.1 Acknowledgments . . . . . . . . . . . . . . . . . 228 


\section{List of Figures}

1.1 An origami cylinder of ordinary paper compresses. But is it because the material stretches? . . . . . . . . . . . . . 9

1.2 The Tachi-Miura fold pattern (TMP) and two sample embeddings. The purely vertical fold lines marked in red are perfectly sharp in all embeddings, so that the adjacent orange faces lie back to back. . . . . . . . . . 12

1.3 A cylinder of Guest and Pellegrino type. . . . . . . . . . . . . . 15

1.4 The bellows theorem does not apply to this origami cylinder, but it nevertheless deforms. This apparent flexibility is shown to be a phenomenon of elasticity . . . . . . . . . . . . . . . . 16

2.1 Top row: a fold pattern is cut horizontally along its vertices into strips (orange line). Bottom row: the lower strip fold pattern is embedded, once as a closed cylinder and once as an open cylinder, with the gap length marked in dashed red. . . . . . . . . . . . . . . . . . 28

$2.2 \quad$ A fan with three end vertices is embedded. . . . . . . . . . . . . . . . 29

2.3 Left: $P_{i+1}$ lies on one of two definite rays. Right: the corresponding section of the bold pattern. Note that the value of $u_{i}$ is negative. . . . . . 30

2.4 Overview of the different possibilities of embedding the neighbors of the apex $A$ with $n=3$ end vertices. The end vertices $P_{1}$ through $P_{3}$ are fixed. 32

2.5 Illustration of various angles $\ldots \ldots \ldots \ldots \ldots \ldots \ldots$

2.6 Example for a periodic repetition fold pattern that embeds to a rotationally symmetric strip. Marked in red and blue are the initial vertices, the black vertices are added by the refinement process. . . . . . . . . . . 35

$2.7 \quad$ Illustration of the notation used in the proof of Theorem 2.4.2 . . . . 36

2.8 Two steps of the fan construction process $\ldots \ldots \ldots \ldots \ldots \ldots$

$2.9 \quad$ A short embedding is completed to an isometric embedding . . . . . . 38

2.10 Plot of the two right-hand sides of Equation (2.5.2) for specific parameters $u_{1}=-1, u_{3}=0.4, h=2, \ldots \ldots \ldots \ldots \ldots \ldots$

2.11 A bistable origami cylinder where a bistable strip has been glued to its mirror image. The two orange folds joined to the orange vertex are completely Hat in the left embedding. Theorem 2.3.2 shows that there exists no continuous isometric deformation between the two states. . . . . . . . . 41

3.1 A single fold is cut and rejoined by an elastic rubber band of zero rest width. 43

3.2 Illustration of the construction process for rotationally symmetric fold pat-

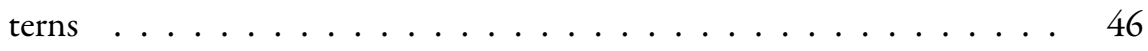

3.3 Exploded fold pattern of a single Guest and Pellegrino strip. . . . . . . 47 
3.4 Two energy functions for different Guest and Pellegrino strips. Left: $r=$ $1, n=8$. Right: $r=1.1, n=7$. In both cases, the initial height is $H=1$. 48

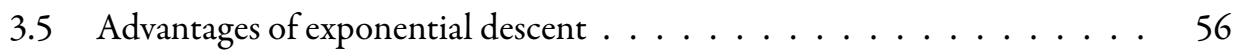

3.6 As $x$ decreases during the compression, the critical point changes its type. The label "-" indicates that the critical point is a minimum, the label " + " indicates a maximum. Horizontal lines correspond to constant ratio $t / x$. .62

3.7 A local minimum splits into two local minima enclosing a local maximum. Horizontal lines correspond to constant ratio $t / x \ldots \ldots$. . . . . 62

3.8 Two different pair events may occur. Left: a minimum and a maximum annihilate into a saddle which then disappears. Right: a pair of minimum and maximum is created from a saddle point. . . . . . . . . . . . 63

3.9 The sign of $\partial_{t}^{3} g$ determines both the type of branch and whether they curve towards or away from $\nabla \partial_{t} g . \ldots \ldots \ldots \ldots \ldots$

$4.1 \quad$ Illustration of the tilt angle $\psi \ldots \ldots \ldots \ldots \ldots \ldots$

$5.1 \quad$ Two examples of input fold patterns. . . . . . . . . . . . . 81

5.2 Comparison to soft-constrained minimization $\ldots \ldots \ldots \ldots . \ldots 83$

5.3 Heights and energies of two example fold patterns . . . . . . . . 84

5.4 The energy landscapes for different cylinder heights $\ldots \ldots \ldots$. . . . 85

5.5 Snapshots of the compression process. . . . . . . . . . . 86

5.6 Comparing simple energy against numerical minimizer . . . . . . . 87

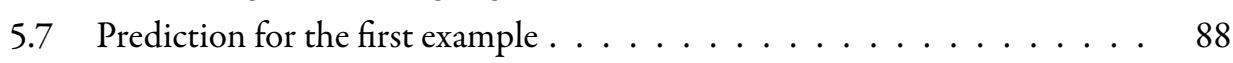

5.8 Prediction for the second example . . . . . . . . . . . . . . . . . 89

5.9 Energy predictions for $r=1, n=8$ deviate significantly in two places. . $\quad 90$

5.10 Two landscape functions showing different shape of simple and true energy. Note that the true energy has three minima while the simple energy has only one local minimum. . . . . . . . . . . . . . . . 90 90

5.11 At a later time, the landscape prediction is again correct. . . . . . . . 90

5.12 Comparing planar and nonplanar minimizers . . . . . . . . . . 91

5.15 Comparison of nonplanar and planar minimizers. . . . . . . . . . 92

5.16 Plot of the elastic yield for $r=0.6$ and $n=10 . \ldots \ldots \ldots 93$

5.17 Plot of the elastic yield for $r=1.2$ and $n=10 . \ldots \ldots \ldots 3$

6.1 Another compressible origami cylinder. Left: uncompressed state. Right: compressed state . . . . . . . . . . . . . . . . 96 


\section{Introduction}

If a thin-walled cylinder — such as the side of a soda can —is subjected to axial compression, a strange phenomenon occurs: the material does not deform uniformly, but instead buckles to form a number of creases. Moreover, these creases appear in a completely regular pattern of diamonds [63, 18] and encompass most of the in-plane stress that is unavoidably generated by the compression $[39,59]$.

According to the classical elasticity theory for thin surfaces (e.g. [13]), the elastic energies associated to different deformations of such thin-walled structures scale differently with the shell's thickness: while stretching and in-plane shearing causes strain proportional to the thickness, pure bending of the shell results in strain scaling with the cube of the thickness. Thus, idealized infinitely thin materials are perfectly incompressible and can only deform via bending of infinitely sharp folds.

These infinitely thin shells are thus located in the domain of origami, the age-old art of paper folding. Its obvious aesthetic aspects notwithstanding, the mathematics of folding as an independent subject has sparked considerable research interest due to a great number of possible applications in various engineering disciplines.

One major problem of mathematical origami is that of flexibility: given a folded structure made from perfectly rigid material, bendable only at the existing folds, is that structure deformable or is it locked into its original shape? As evidenced by Figure 1.1, there exist origami cylinders that are indeed flexible if folded from ordinary paper. But are they rigidly flexible or only because they are made from slightly flexible material? And what aspects of a fold pattern determine flexibility of the origami cylinder?
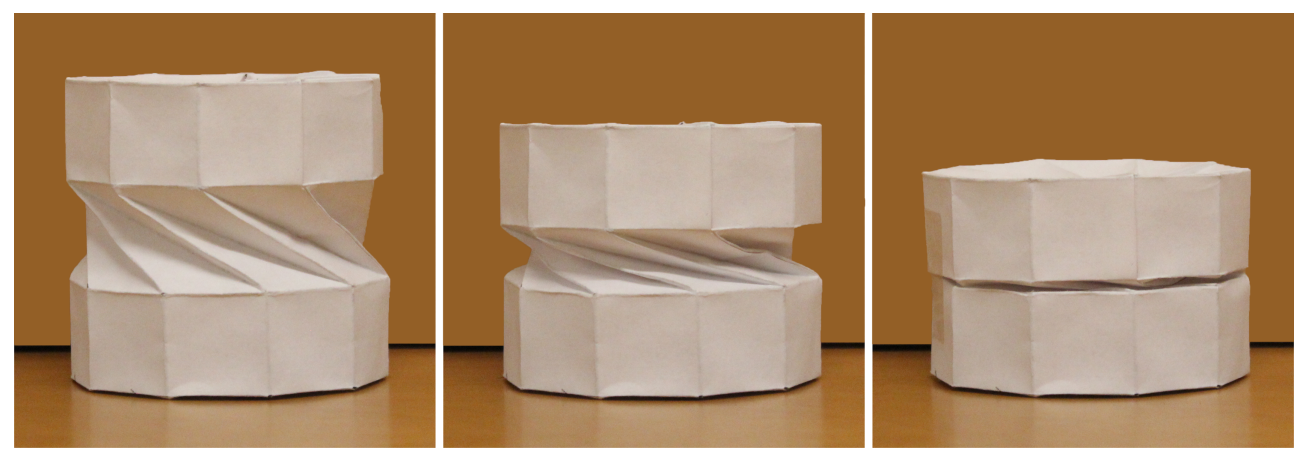

FIGURE 1.1: An origami cylinder of ordinary paper compresses. But is it because the material stretches? 


\subsection{Background}

The obvious representation of straight-fold origami as polyhedra leads to the problem of rigidity of polyhedral surfaces, a question that already occupied mathematicians' minds at the time of Euler and is still not perfectly solved. Supporting the validity of this representation, the properties of perfect rigidity and perfectly sharp folds also emerge as direct consequences of the theory of elasticity if one seeks approximation relations between threedimensional elasticity of thin objects and the treatment of their middle surface as a twodimensional elastic object.

Besides asking about the flexibility of a given folded structure, the other great branch of origami studies focuses on systematic design. To achieve a variety of ends, folded structures with a predefined set of properties and the associated fold patterns need to be found. Such properties include rigid flexibility, defined elastic responses to axial compression, or bistability between a compact storage state and a vastly different, expanded deployed state.

\subsubsection{Rigid polyhedra}

The study of polyhedral rigidity for closed surfaces, without holes or boundaries, has a very long mathematical history. The first formulated result dates back to 1766, when Leonard Euler first conjectured (but was unable to prove) that all polyhedral surfaces are rigid [21]. However, the first proven mathematical result only appeared in 1813, when Cauchy was able to prove that convex polyhedral surfaces (such as e.g. the surfaces of Platonic solids) are infinitesimally rigid [12, 15], that is, the only infinitesimal isometric deformations are the infinitesimal rigid body motions. (Cauchy himself actually proved non-infinitesimal rigidity, a slightly weaker statement, but it was later discovered by Alexandrov [2] that his arguments are equally applicable to show infinitesimal rigidity, which is why this result bears Cauchy's name.)

The case of non-convex polyhedra proved to be much harder to treat: the first example of a flexible mechanical linkage, that is, a polyhedron without its solid faces (a network of rigid rods connected by pin joints), was only found in 1896 by Bricard [10, 11]. Unfortunately, his counterexamples cannot be extended to polyhedra since filling in the faces of Bricard's linkages would create self-intersections. Rigidity of true polyhedra thus remained an open question.

Another 80 years later, in 1975, Herman Gluck proved that "almost all" polyhedra are rigid, in the sense that the set of rigid polyhedra is open in the space of all polyhedra [25]. Finally, in 1977, Connelly found the first true (read: not self-intersecting) counterexample of a flexible polyhedron [14], which has since been simplified into the now canonical example of Steffen's polyhedron. A final noteworthy result, the famous Bellows theorem, was again proven by Connelly, Sabitov, and Walz in 1997 [16] and states that any flexible closed polyhedral surface must maintain its enclosed volume. Its name stems from the equivalent 
formulation that every bellows (which must change its volume in order to expel air) must incur material strain during operation, to the effect that no "perfect" bellows exists.

In contrast to these global results, there are numerous results about general local flexibility, applicable to folded surfaces of arbitrary topology, detailing whether a single vertex and the collection of faces around it is flexible. It is clear that if the single-vertex origami is rigidly flexible, then it must flex solely by changing the dihedral angles associated to each edge. These angles are subject to a natural closing condition: any closed curve that travels around the central vertex must remain closed during the flexing operation. This closing condition results in algebraic flexibility conditions which have been proposed in a variety of formulations such as rotation matrices [6], quaternions [60], or in the language of spherical geometry [52]. Common to all of these is that they essentially express the notion, first introduced by Huffman [32], that the discrete Gauß curvature at the vertex must remain constant during any isometric deformation, in analogy to Gauß' famous Theorema Egregium [19] for smooth surfaces. Any solution to the algebraic conditions is a valid configuration of the origami and flexibility is then a consequence of the algebraic equations possessing a connected family of solutions.

One way to extend this analysis to multi-vertex origami is to simply periodically repeat a suitable flexible single-vertex origami to obtain a flexible polyhedral surface resembling a wrinkled sheet of paper. The classical example of such a multi-vertex flexible origami is the doubly-corrugated Miura-ori [43], composed of quadrilateral faces, which possesses a single degree of freedom. Variations of this quadrilateral fold pattern have been analyzed extensively, culminating in the classification of all flexible periodic quadrilateral patterns [53]. Moreover, it has been shown that, given careful selection of parameters, these fold patterns can be folded up into cylinders [58]. Such cylindrical Miura-ori represent isolated points in the family of solutions, though, as they will unfold into a planar surface if the origami is evolved along its degree of freedom.

This thesis is concerned with a slight modification of the original question of polyhedral flexibility: does removing two edge-disjoint faces from a polyhedron (thereby turning it into a cylinder) increase its flexibility? Since the two absent faces are now essentially made flexible, it is clear that the classical results about global flexibility can no longer be applied.

The question was answered in the affirmative by Tachi [54], who created the first rigidly flexible origami cylinders. His example is made of quadrilateral faces, but is not developable, that is, it cannot be folded from a single flat piece of paper without tearing or stretching. The first nontrivial developable rigidly flexible origami cylinder, the Tachi-Miura pattern (TMP) depicted in Figure 1.2 was discovered only in 2010 [54, 45].

Thus, the existence of the TMP answers the question about existence of rigidly flexible origami cylinders. But not only is it the only known example of its kind, there are also no structural statements about necessary or sufficient conditions for flexibility of general origami cylinders: while much work has been done to explore the variety and flexibility of specific fold patterns, or simple families of regular fold patterns, no efforts towards the anal- 


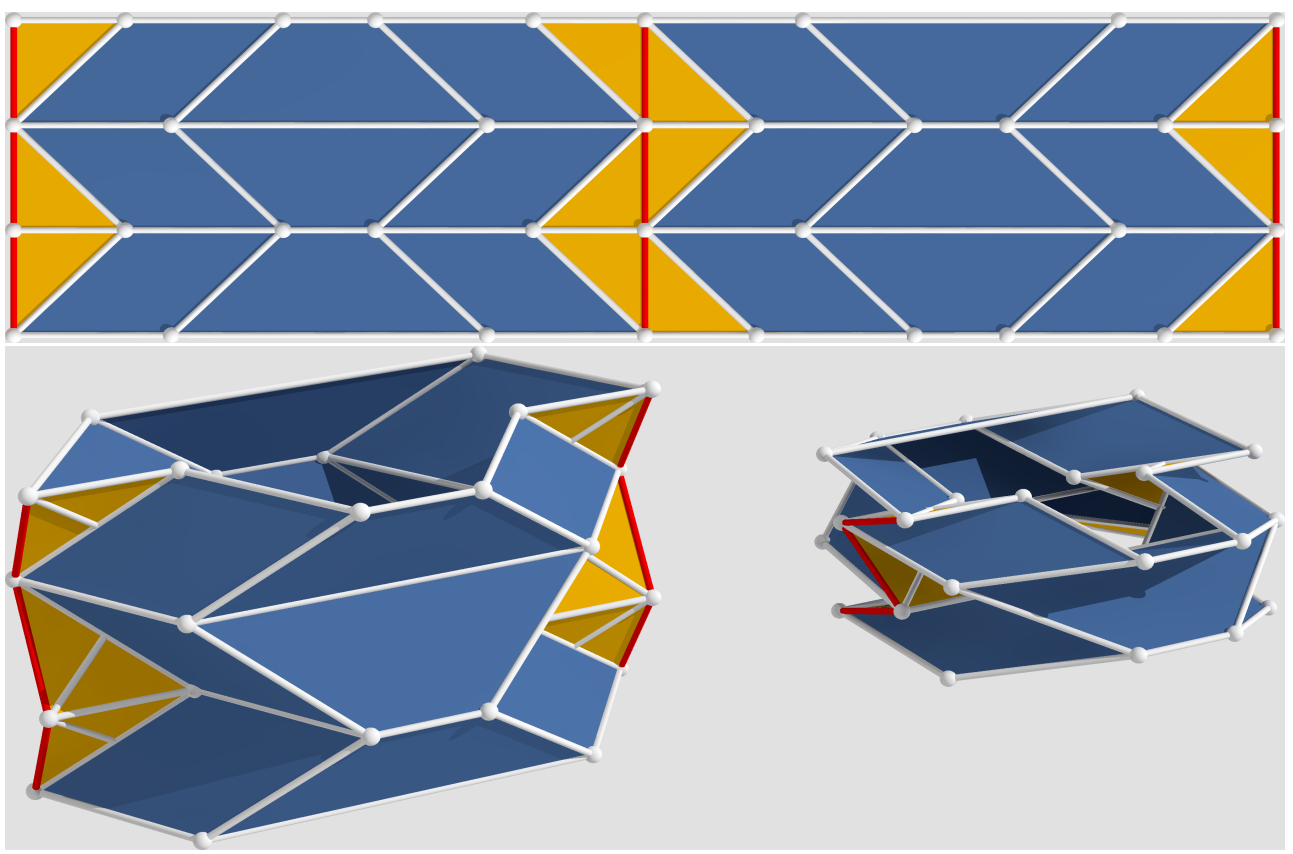

FIGURE 1.2: The Tachi-Miura fold pattern (TMP) and two sample embeddings. The purely vertical fold lines marked in red are perfectly sharp in all embeddings, so that the adjacent orange faces lie back to back.

ysis of arbitrary fold patterns have been undertaken. Analyzing the structure and flexibility of general, permanently cylindrical origami will be a main subject of this thesis.

\subsubsection{Elasticity of thin shells}

The notion of rigid origami also arises quite naturally in a vastly different area of mathematics: the study of thin elastic surfaces. In the most general terms, the classical elasticity theory describes the relation between forces and elastic energies on one hand and deformations of three-dimensional bodies on the other hand. A very important special case is that of three-dimensional bodies which are very thin, i.e., two of their dimensions greatly exceed the third. Such thin bodies are called sheets (if their rest state is flat, such as a sheet of paper) or, more general, shells (if their rest state is curved, such as a hollow pipe).

One of the most familiar instances of deformation of thin plates and the one that is relevant to this work is the crumpling of paper. Here, the challenge is to derive the associated complicated phenomena-the generation of creases, the pattern that these creases form, their number and sizes, et cetera-from the relatively simple laws of elasticity.

If we consider the shell to be the extrusion of a two-dimensional middle surface, an intuitive question to ask is whether there exist any "limit theories", i.e., two-dimensional elasticity theories that when applied to the middle surface yield approximations for the threedimensional elastic theory of the full body if the shell thickness $h$ approaches zero. Although various models for elastic thin shells have been proposed throughout history, most promi- 
nently the Kirchhoffmodel [34], these models are derived from heuristic assumptions on the nature of thin shell deformations and have not been established as mathematically rigorous limits.

Indeed, this ad hoc notion of "limit theory" was given a mathematically precise meaning only in recent years first by Le Dret and Raoult [36] and then by Friesecke, James, and Müller [23] with the notion of $\Gamma$-convergence or, closely related but maybe more familiar, convergence of minimizers. Loosely speaking, a sequence of functionals $F_{h}$ (in this case: the three-dimensional elastic energy per unit thickness $E:=h^{-1} \mathcal{E}$ over the space of embeddings of thickness $h) \Gamma$-converges to a limit $F$ if a) every minimizer of $F$ is the limit point of a sequence $\left(x_{h}\right)$ where each $x_{h}$ minimizes the functional $F_{h}$ and b) conversely, the limit of every convergent sequence $\left(x_{h}\right)$ of minimizers is itself a minimum of $F$.

Such $\Gamma$-limits have been found not only for the elastic energy itself, but also for various rescaled functionals of the form $h^{-\alpha} E$, roughly analogous to different terms in the expansion of $\mathcal{E}$ into a power series in $h$ :

The membrane model The simplest case is that of no additional rescaling, $\alpha=0$. The classical theory of elasticity dictates that the leading order contributor to the unscaled en$\operatorname{ergy} \mathcal{E}$ is the membrane energy arising from stretching or shearing in the shell's tangent plane, scaling linearly in $h$. The rescaled elastic energy $E$ is thus expected to have a finite limit.

Experiments and numerical simulations paint an even stronger picture: if a strip of paper is confined to form a single ridge, the normalized energy $E$ not only has a finite limit, but scales with $h^{5 / 3}[38,39,59,37]$. This empirical observation was given rigorous mathematical support by Venkataramani [56, 57] under some simplifying assumptions, and in full generality by Conti and Maggi [17].

They showed, building upon Le Dret and Raoult [36] (who completed the case $\alpha=$ $0)$, that the $\Gamma$-limit of $h^{-\alpha} E$ for $\alpha \in[0,5 / 3)$ of an embedding $u$ of the center surface is given by

$$
\left(\Gamma-\lim _{h \rightarrow 0} h^{-\alpha} E\right)(u)= \begin{cases}0 & , u \text { is a short map } \\ \infty & , \text { else }\end{cases}
$$

where $u$ is typically taken from the function space $W^{1, \infty}\left(\Omega, \mathbb{R}^{3}\right)$ for a suitable parameter domain $\Omega \subset \mathbb{R}^{2}$. Such a map $u$ is called short map if it is nowhere extending, that is, the matrix $\operatorname{Id}_{2}-\left(\nabla u^{T} \nabla u\right)$ is positive semidefinite almost everywhere. Intuitively, the matrix $\nabla u^{T} \nabla u$ measures the change in metric tensor and thus the deformation of infinitesimal volumes by the deformation $u$. It is also called the Cauchy-Green strain tensor in elasticity theory.

The Kirchhoff model Friesecke, James, and Müller [24] covered the limits of functionals $h^{-\alpha} E$ for values of $\alpha \geqslant 2$. For $\alpha=2$, the $\Gamma$-limit gives the Kirchhoff plate energy when 
applied to isometric maps $u$ (where $\nabla u^{T} \nabla u=\mathrm{Id}_{2}$ almost everywhere) and is infinite on all non-isometric $u$. The Kirchhoff energy, first proposed by Kirchhoff [34], is a simplified 2D elastic energy derived from the full 3D elasticity theory using the additional assumption that the thin plate is not sheared along its offset direction during the deformation, that is, points on a given normal to the middle surface remain on a common normal to the middle surface throughout the deformation. For even higher values of $\alpha$, the limit functionals are only ever finite if $u$ is an affine map. The remaining range of $\frac{5}{3} \leqslant \alpha<2$ remains open.

\subsubsection{Targeted design of origami}

The earliest application of rigidly foldable origami sheets (not necessarily glued into a cylinder) is the Miura-ori from 1969 [43, 44], which was originally proposed to store satellite solar panels in flat-folded compact form during launch.

Since then, techniques and insights from the study of rigidly foldable origami have been applied to diverse areas, such as the design of mechanical metamaterials [49, 50] and shock absorbers [64, 51], the construction of self-inflating stent grafts in medical engineering [35], or flat-folding of packaging material [1, 3, 61]. Consequently, the targeted design of rigid origami structures [20, 48, 33, 22] is a very active field of research. Nevertheless, despite such a wide range of interests and considerable research effort, various aspects of the global rigid flexibility of origami cylinders and the scope of possible rigidly flexible origami structures still remain poorly understood as current research into the design of origami cylinders focuses on exploring only a very narrow set of fold patterns.

\subsubsection{Elastic origami}

Analogous to the study of elastic thin shells, the field of elastic origami is concerned with the influence of folds on the elastic behavior of thin shells, as it has long been known that a material's mechanical properties can change when it is folded. A very simple experiment illustrates this fact: try to hold a flat sheet of paper horizontally by one edge. The opposite edge will flap down due to gravity. Now fold the paper perpendicular to the held edge into a wedge-like shape and hold the same edge. The paper will no longer bend downwards 1 .

The elastic flexibility of cylindrical origami under axial compression in particular were examined in detail by Guest and Pellegrino [26, 27, 28]. They focused on a specific class of fold patterns, exemplified in Figure 1.3, that they showed are all strain-free in both their fully extended and fully compressed configuration. Furthermore, they found that the maximum strains during the compression can be made arbitrarily small by refining the fold pattern to a suitable amount.

Another field of interest in the study of elastic origami is the relation between the predicted behavior of idealized polyhedral origami and their real-world counterparts. We only

\footnotetext{
${ }^{1}$ This is more commonly applied to pizza wedges.
} 


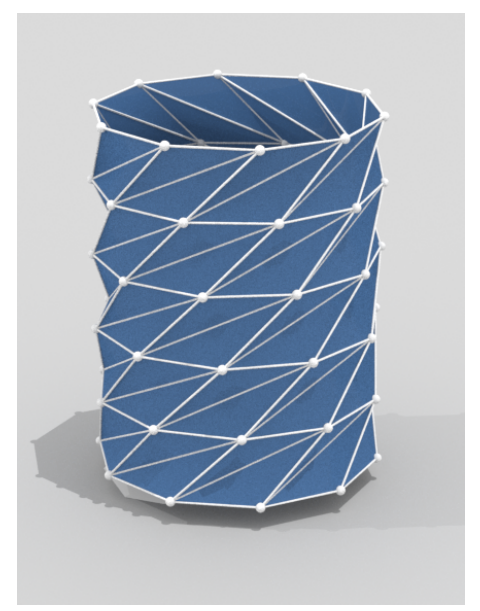

Figure 1.3: A cylinder of Guest and Pellegrino type.

highlight the Tachi-Miura pattern here: it is rigidly flexible as an infinitely thin polyhedral surface, but still requires compressive force when actually folded from paper [62].

\subsection{Overview and contribution}

The main focus of this work is to analyze origami cylinders with respect to two different notions of flexibility. Within the classical setting of perfectly isometric deformations, we obtain strict conditions on the rigidity of such cylindrical origami, but these are unsuited to explain why some paper cylinders deform nevertheless. We therefore employ a relaxed notion of nearly perfect rigidity as the basis for finding numerically a cylinder's dynamic response to axial compression.

\subsubsection{Rigid origami cylinders}

As evidenced by the existence of the Tachi-Miura pattern, (perfectly) rigid origami cylinders can exhibit flexibility despite the fact that all their faces are rigid polygons. In order to describe the nature of this flexibility, Chapter2/introduces a novel and very general framework for describing rigid origami and shows that even under more general regularity assumptions, all origami cylinders must necessarily be polyhedral surfaces. This model describes origami cylinders by the intuitive folding process: on a rectangular domain, fold lines are drawn. This fold pattern is then folded rigidly along its fold lines such that the domain's left and right boundaries align perfectly, to the effect that the entire folded rectangle forms a cylindrical polyhedral surface.

Furthermore, it is shown that the TMP possesses a striking feature, being constructed of two mirror-symmetric pieces glued together along folds whose fold lines are vertical, that is, 

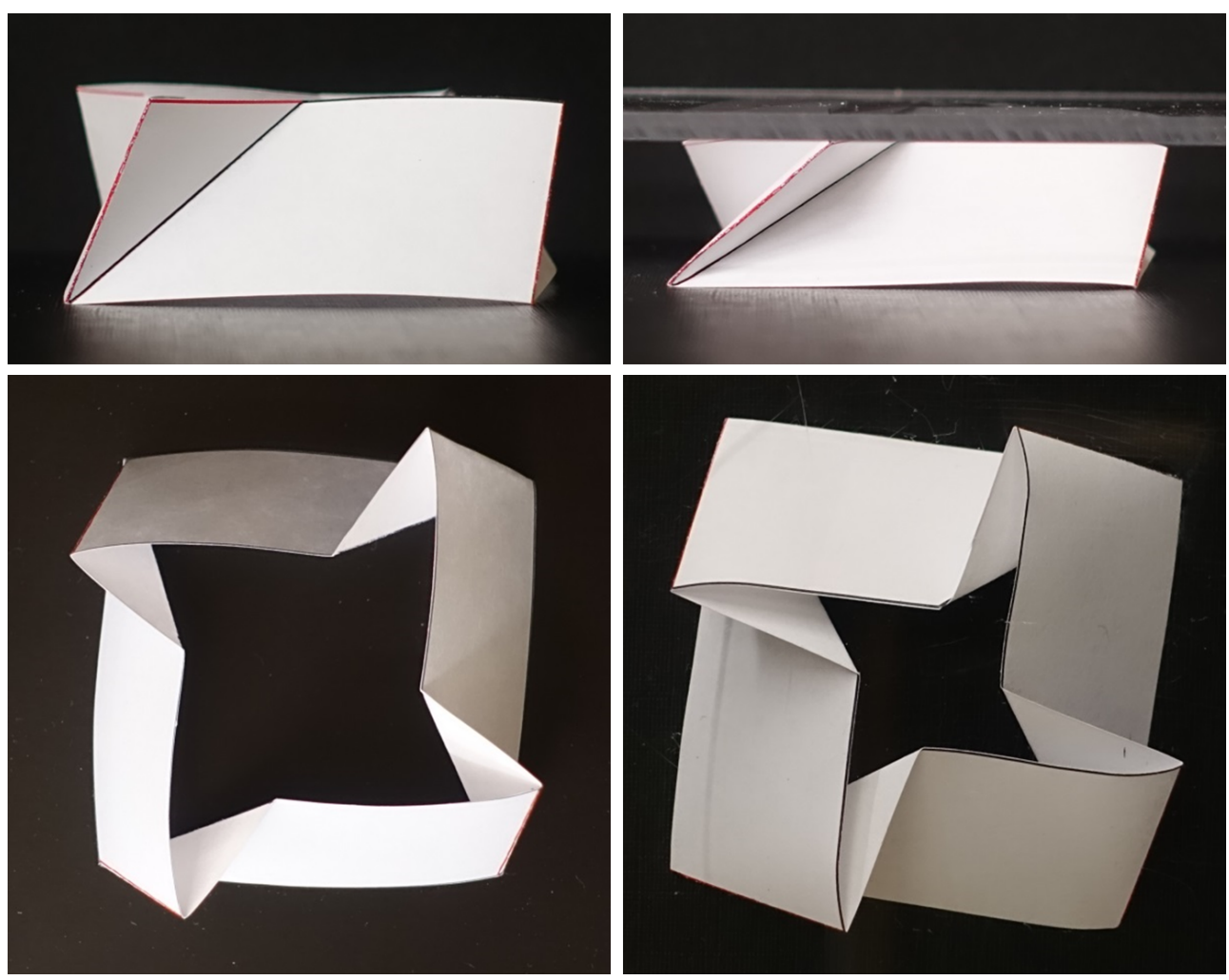

FIGURE 1.4: The bellows theorem does not apply to this origami cylinder, but it nevertheless deforms. This apparent flexibility is shown to be a phenomenon of elasticity.

parallel to the domain's left and right boundaries. To be precise, it is shown that fold patterns that do not include at least two vertical fold lines are incompressible and that, moreover, at least two of the vertical fold lines must be folded sharp, that is, the adjacent faces must lie directly on top of each other. This analysis not only covers the realm of regular, rotationally symmetric fold patterns, but extends to all fold patterns with finitely many folds.

The cornerstone to our argument is to represent the problem of rigid foldability as a root finding problem for a certain real analytic function. We show that this function has at least one nonzero value, implying that it can only have isolated zeroes, which rules out continuous isometric deformations of origami cylinders. Our result extends the bellows theorem by proving that it is impossible to crush a large class of origami cylinders (e.g., those shown in Figure 1.4) without the restricting assumption that the cylinder's top and bottom maintain their shape during deformation. These results have been published in the Journal of Mechanical Engineering [8], and parts of this article are reproduced in Chapter2 without continued citation.

Additionally, two different design applications of this origami model are presented. Section 2.4 sketches a possible design process for origami cylinders of arbitrary height while Section 2.5 highlights the construction of origami cylinders that are bistable: a fold pattern that can be folded in two different ways to origami cylinders of different height. 


\subsubsection{Nearly rigid origami cylinders}

Having established that rigid origami are in fact mostly inflexible, we relax the rigidity condition to analyze nearly rigid origami cylinders. As described in Chapter 3, these are initially polyhedral surfaces whose faces are separated along the edges and then rejoined via elastic rubber bands. All faces can move freely via individual rigid body motions and are only constrained by the elastic energy incurred by the rubber bands.

This model is then used to simulate the response of real folded structures to axial compression. To this end, the boundary edges are clamped to specific heights and the energy is minimized over the space of all face configurations subject to clamping and rigidity constraints. The minimization process exploits the geometric fact that the constraint manifold can be expressed as a simple Lie group to enable the use of efficient numerical algorithms, a fact that is laid out in greater detail in Chapter 4 .

Finally, we show that a suitable approximation to the elastic energy allows to predict with great ease the general qualitative behavior of the minimizer as the compression progresses. Both the approximate prediction and the numerically obtained minimizer show several striking phenomena: not only do they restrict to a very narrow set of possible states with only short intermediary transitions, they furthermore exhibit discontinuous jumps, which we associate with buckling phenomena observed in crushing experiments. Our model thus shows that buckling is by no means an intricate consequence of the elasticity theory of thin shells but arises instead very naturally from geometric conditions. 


\section{Rigid Origami}

We now present the framework that is used to describe rigid origami cylinders. Within this framework, we will then prove this chapter's main result, Theorem 2.3.2 about continuous flexibility of rigid origami cylinders.

At its heart, our model is a description of polyhedral surfaces in the language of differential geometry. We therefore begin by introducing the requisite notions of this mathematical area. Following that, we establish in Section 2.2 the central concepts and assumptions of our model for rigid origami cylinders. In Section 2.3, we introduce the notion of deformations into our model and proceed to prove this chapter's main result, which is that rigid origami cylinders are, in fact, quite rigid. We reiterate at this point that the attribute "rigid" is used with two different meanings: the term "rigid origami" refers to the condition that each individual face cannot be deformed, while "rigid" rigid origami means that the origami cylinder as a whole does not allow for deformations in our sense.

This chapter closes with sketching out how design problems can be handled in the context of the rigid origami model. Section 2.4 provides a design process to imprint on a given rectangle a fold pattern that can be folded into an origami cylinder of given arbitrary height. Section 2.5 shows how to construct fold patterns that are multistable: they can be folded to different origami cylinders with different heights.

Note that the results presented in this chapter have been published in the ASME Journal of Mechanical Design [8]. For the sake of legibility, they will be presented here without explicit citations.

\subsection{Basics of developable surfaces}

We begin by introducing the necessary mathematical framework for our model of rigid origami. In this, we follow loosely do Carmo's book on differential geometry of curves and surfaces [19].

As we will see, the central notion is that of curves and surfaces that are given as subsets of the ambient space $\mathbb{R}^{3}$. Such surfaces are called embedded:

Definition 2.1.1. An embedded surface is a two-dimensional real Riemannian manifold $M$ together with an injective proper immersion $f: M \rightarrow \mathbb{R}^{3}$.

We will only consider embedded surfaces and therefore omit the attribute "embedded" and only talk about the image $S=f(M)$. Unless explicitly stated otherwise, our surfaces will be assumed smooth. We will also only consider orientable surfaces, to the effect that a consistent choice of unit normal $N$ exists. 
On embedded surfaces, we consider the Riemannian metric that is generated from the Euclidean metric of the ambient space. This leads to the notion of fundamental forms:

Definition 2.1.2. Let $S$ be an embedded surface. The first fundamental form $\mathrm{I}$ is the metric tensor of the metric on $S$ induced by $\mathbb{R}^{3}$. If $S$ is parametrized by local coordinates $u$ and $v$ and parameter map $f$, the metric tensor takes on the form

$$
\mathrm{I}:=\left(\begin{array}{cc}
\left\langle f_{u}, f_{u}\right\rangle & \left\langle f_{u}, f_{v}\right\rangle \\
\left\langle f_{v}, f_{u}\right\rangle & \left\langle f_{v}, f_{v}\right\rangle
\end{array}\right) .
$$

Here, $f_{u}$ and $f_{v}$ are the first partial derivatives of $f$.

Definition 2.1.3. A smooth map $f: S \rightarrow \bar{S}$ between two embedded surfaces $S$ and $\bar{S}$ is called isometric if the metrics on $S$ and $\bar{S}$ are related via the differential $d f$, that is, one is the pullback of the other:

$$
\mathrm{I}_{S}=f^{*} \mathrm{I}_{\bar{S}}
$$

In particular, we also call the embedding $f: M \rightarrow S$ isometric if the Riemannian metric on the parameter manifold $M$ coincides with the pull-back of the metric on $S$ induced by the standard Euclidean metric on $\mathbb{R}^{3}$.

Definition 2.1.4. The second fundamental form II measures the change of the unit surface normal $N$ along tangent directions. Expressed in coordinates, it takes on the form

$$
\mathrm{II}:=\left(\begin{array}{cc}
\left\langle f_{u u}, N\right\rangle & \left\langle f_{u v}, N\right\rangle \\
\left\langle f_{v u}, N\right\rangle & \left\langle f_{v v}, N\right\rangle
\end{array}\right) .
$$

For isometric embeddings, where the first fundamental form is the identity matrix, it is equal to the shape operator as matrices. Hence, the Gauß $\beta$ curvature of $S$ is given by the function $K=\operatorname{det} I$ II. The principal curvatures are the eigenvalues of II, the mean curvature is the mean of the principal curvatures or half the trace of II.

Points where exactly one principal curvature is zero are called parabolic points. If both principal curvatures are zero, they are called flat points.

The Gauß curvature is particularly important because it is invariant under isometric deformations, that is, it can be expressed purely in terms of the first fundamental form, even though the second fundamental form itself may well change.

Theorem 2.1.5 (Theorema Egregium). Let $f$ be an isometric map between embedded surfaces $S$ and $\bar{S}$. Then the respective Gauß curvatures $K$ and $\bar{K}$ satisfy

$$
K=\bar{K} \circ f
$$


A consequence of the Theorema Egregium is that any smooth object that can be created from a flat piece of paper without cutting or stretching must have Gauß curvature zero everywhere. Surfaces with this property are called developable:

Definition 2.1.6. An embedded surface $S$ is called developable if its Gauß curvature is zero everywhere.

Another important class of surfaces are ruled surfaces. The classical definition of a ruled surface is that it can be generated by attaching to each point of a smooth curve a line such that the union of these lines forms a smooth surface.

Definition 2.1.7. A surface $S$ is called a ruled surface if through each point $x \in S$ there exists a straight line that is wholly contained in $S$. The surface can thus be parametrized by asymptotic lines via a function $f(s, t)=\gamma(t)+s \cdot v(t)$, where $s, t$ are real variables, $\gamma$ is a smooth curve in $\mathbb{R}^{3}$ and $v$ is a smooth curve on the unit sphere $S^{2}$. The curve $\gamma$ is called directrix or base curve and the curve $v$ is called director curve. The straight lines $l_{t}: s \mapsto f(s, t)$ are called rulings of the surface $S$.

We extend this definition to bounded surfaces by requiring that the parameters $s, t$ are suitably bounded.

Classical examples of smooth ruled surfaces are cylinders and hyperboloids. The relation between ruled and developable surfaces is the following:

Theorem 2.1.8. Every ruled surface has nonpositive Gauß curvature everywhere.

We point out that there is some ambiguity about the relation between developable and ruled surfaces in the mathematical literature. Some authors, for instance do Carmo, define developable surfaces to be those ruled surfaces where each ruling has constant tangent plane (the Gauß curvature is zero as a consequence), while some authors, such as Hartman and Wintner [30], use our definition purely in terms of Gauß curvature and denote surfaces of the former kind by torses. The relation between torses and developable surfaces was again characterized by Hartman and Wintner [30, Theorem 5]:

Theorem 2.1.9. Let $S$ be a $C^{2}$ surface parametrized by $f(u, v)$. If $S$ is a torse, then its Gau $\beta$ curvature is identically zero; conversely, if the Gau $\beta$ curvature of $S$ is identically zero and if either every point of $S$ is a flat point or every point of $S$ is a non-flat point, then $S$ is a torse.

Thus, every torse is developable (in our sense) and the converse holds only for surfaces of Gauß curvature zero that consist only of flat points or only of non-flat points. This leaves open the case of surfaces with both types of points. It is clear that such surfaces must be piecewise torsal and that the individual torsal pieces must correspond to the connected components of the sets $F$ of flat points and $P$ of non-flat points. Moreover, as Massey shows [41, Theorem 3], the individual pieces are joined together by straight lines: 
Theorem 2.1.10. Let $S$ be a $C^{4}$ surface with Gauß curvature zero. Let $F$ be the set of flat points of $S$ ( $a$ closed set in $S$ ), and let $P$ be the set of all non-flat points (an open set in $S$ ). Then through each point of the topological boundary $\partial P$ there passes a straight line on $S$ and this straight line is entirely contained in $\partial P$. Thus $\partial P$ is the union of straight line segments.

In order to transport the crucial notion of Gauß curvature to the case of origami cylinders, which will only be piecewise regular surfaces, we need to adapt the corresponding notions, in particular that of Gauß curvature, to the surface's irregularities. In principle, polyhedral surfaces have two types of nonregularities, edges and vertices, that require a redefinition. In order to preserve the spirit of the Theorema Egregium 2.1.5, edges are assigned zero Gauß curvature (as it is always possible to flatten two faces joined at a single edge) and vertices are assigned their angle defect $2 \pi-\sum_{i} \theta_{i}$, where the $\theta_{i}$ are the interior angles of all faces joined at that vertex. Under this discrete Gauß curvature, all notions and theorems introduced for the smooth setting remain true for origami surfaces.

Finally, we also need to consider embedded curves in $\mathbb{R}^{3}$. An important concept is the notion of a frame along a curve, in particular the Frenet frame:

Definition 2.1.11. Let $I \subset \mathbb{R}$ be a compact interval and $\gamma: I \rightarrow \mathbb{R}^{3}$ be a $C^{2}$ curve such that $\ddot{\gamma}(t) \neq 0$ and $|\dot{\gamma}(t)|=1$ for all $t \in I$. Define $T(t):=\dot{\gamma}(t), N(t):=(\ddot{\gamma}(t) /|\ddot{\gamma}(t)|)$ and $B(t):=T(t) \times N(t)$. Then the triple $(T, N, B)$ is an orthonormal frame for all $t$ and is called the Frenet frame of $\gamma$.

Theorem 2.1.12. Let $\gamma: I \rightarrow \mathbb{R}^{3}$ be a $C^{2}$ curve such that $\ddot{\gamma}(t) \neq 0$ and $|\dot{\gamma}(t)|=1$ for all $t \in I$, and let $(T, N, B)$ be its Frenet frame. Then $T, N, B$ satisfy the so-called Frenet equations: there exist functions $\kappa, \tau: I \rightarrow \mathbb{R}$ such that

$$
\left(\begin{array}{c}
\dot{T} \\
\dot{N} \\
\dot{B}
\end{array}\right)=\left(\begin{array}{ccc}
0 & \kappa & 0 \\
-\kappa & 0 & \tau \\
0 & -\tau & 0
\end{array}\right) \cdot\left(\begin{array}{l}
T \\
N \\
B
\end{array}\right)
$$

The function $\kappa$ is called curvature of $\gamma$, the function $\tau$ is called torsion of $\gamma$.

\subsection{Rigid origami cylinders}

We now lay out the framework in which we conduct our analysis. The central notion is that of "folding instructions:"

Definition 2.2.1. A fold pattern is a finite set of straight lines (fold lines) imposed on a rectangle of size $w \times h$. We denote the points where fold lines intersect each other or meet the boundary by vertices. 
If the rectangle domain is cut along the fold lines, one obtains a collection of disjoint components. We call (the closure of) these components faces.

Intuitively, the rectangle is then folded along the fold lines into a three-dimensional object such that the left and right boundaries come to match and the upper and lower boundaries lie in parallel planes: by

Definition 2.2.2. A rigid origami cylinder is a fold pattern together with an embedding of the rectangular domain into $\mathbb{R}^{3}$ that satisfies the following conditions:

1. it is continuous,

2. it is $C^{4}$ and isometric on each face of the fold pattern including the face boundaries,

3. fold lines are mapped to straight lines, and

4. horizontal sections of the domain are mapped to closed curves contained in horizontal planes each. Note that this condition implies the closing of the fold pattern into a cylinder.

This last assumption is motivated by the prevalence of vertically layered fold patterns among those fold patterns that appear to be flexible, such as the one in Figure 1.1.

The distance between the two boundary planes is called the (embedded) height of the cylinder. Note that folds can be flat, i.e., the union of its two adjacent faces forms a single $C^{4}$ surface.

The embedding map can be visualized as follows: the fold pattern is folded along its fold lines to the prescribed angles, thereby lining up the left and right rectangle boundaries, where corresponding points are identified ("glued together") to form the embedded cylinder. It is clear that different fold patterns can be folded to the same cylinder: simply cut the embedded cylinder open along a different seam to obtain a new fold pattern, which is a periodic horizontal translation of the original pattern.

On the other hand, a fold pattern could be folded and glued into different embedded cylinders, but it is less obvious if that is a general property or conditional on certain characteristics of the fold pattern. We will answer this question over the course of Section 2.3, where we will show that general fold patterns have at most finitely many different embeddings (if any).

At this point, our notion of origami cylinder leaves room for the possibility of curved faces. Indeed, the usual smooth cylinder is a rigid origami cylinder in our sense: its fold pattern has no fold lines. Contrary to that, the origami community usually defines rigid origami to be a polyhedral surface whose faces are rigid. The next section will reconcile these two notions and show that the definition given here is actually only a slight generalization of the usual definition. 


\subsubsection{Flatness of faces}

This section is devoted to proving the following theorem:

Theorem 2.2.3. Arigid origami cylinder whose embedded height is less than its material height $h$ is made up entirely of flat faces that meet at the embedded fold lines. In particular, there are no creases in the embedded cylinder apart from those given by the fold lines.

The proof of this theorem is split into two parts. First, we consider in Lemma 2.2.4 the faces that are completely enclosed by fold lines and do not touch the cylinder's boundaries except possibly in isolated vertices. The leftover boundary faces will be treated using an auxiliary result about developable ruled surfaces.

Lemma 2.2.4. Let $P$ be a closed non-intersecting polygon with finitely many corners in the plane, which is embedded isometrically via a $C^{4}$ map $f$ into $\mathbb{R}^{3}$ such that each boundary edge of $P$ is mapped to a straight line segment in $\mathbb{R}^{3}$. Then the resulting surface $S=f(P)$ is contained in an affine plane.

Proof. Since $f$ is an isometric map, the surface $S$ is developable and thus its Gauß curvature vanishes everywhere. We divide the points of $S$ into parabolic (where not both principal curvatures are zero) and flat (where both principal curvatures are zero). Furthermore, we call a curve in $S$ asymptotic if its tangent vectors coincide with directions of vanishing directional curvature at every point. (Intuitively, asymptotic curves correspond to rulings.)

We then require the following two facts about $C^{4}$ developable surfaces [41]:

(1) Every asymptotic curve that contains a single parabolic point must consist entirely of parabolic points.

(2) If an asymptotic curve contains a parabolic point, then it must be a straight line segment.

The claim is that $S$ does not contain parabolic points. Suppose for contradiction that $S$ contained a parabolic point. By continuity of the mean curvature function, the set of parabolic points (as the preimage of $\mathbb{R} \backslash\{0\}$ ) is an open subset of $S$. Hence there exists a curve $\Gamma$ of nonzero arc length that consists entirely of parabolic points and that intersects asymptotic curves transversally. (For instance, an integral curve of the nonzero principal direction vector field.) Without loss of generality, $\Gamma$ is assumed to be parametrized by its arc length $t$.

This means that $S$ contains a family of straight line segments, all consisting entirely of parabolic points, that originate from each point of $\Gamma$. Because $S$ is a bounded surface, each of these straight lines intersects the boundary of $S$ in (at least) two different points. If these intersections did not coincide with vertices, then they have two linearly independent directions of vanishing directional curvature: along the edge and along the asymptotic line. Thus, the asymptotic lines must either only intersect the boundary $\partial S$ along an entire edge, 
or at most on the vertices. For this there are only finitely many possibilities, which, together with continuity of the family of asymptotic lines, means that the family is constant. But then $\Gamma$ cannot have positive length, contradicting the initial assumption that there exist parabolic points.

It cannot be that both $P_{1}$ and $P_{2}$ are independent of $t$ : if that were true, all straight lines would in fact be the same (there is only one straight line connecting any two points). But this cannot be the case as $\Gamma$ was chosen to be perpendicular to the straight lines. Therefore, at least one of the intersections must vary with $t$. Hence, there must exist some point on $\Gamma$ whose asymptotic line meets the boundary of $S$ in a point $P$ that is not a vertex. But $P$ is a flat point as there are two linearly independent directions with zero directional curvature: one is the asymptotic curve that meets $P$ and one is the boundary line segment of $S$ that contains $P$. So there exists an asymptotic curve containing both parabolic points and the flat point $P$. This is a contradiction to condition (1). We conclude that $S$ cannot contain parabolic points.

This proves that all faces whose sides are all fold lines must be flat. We now turn to those faces that meet the cylinder's boundary. We first prove a result about the reconstruction of developable ruled surfaces from transversal geodesics.

Theorem 2.2.5. Let $S, \tilde{S}$ be two $C^{3}$ developable ruled surfaces that are generated by the same directrix curve $\Gamma$ (parametrized such that $|\dot{\Gamma}(t)|=1$ for all $t$ ) and two director curves $v, \tilde{v}$. If $\ddot{\Gamma}(t) \neq 0$ for all $t$ and $\Gamma$ is a geodesic in both $S$ and $\tilde{S}$, then $v$ and $\tilde{v}$ must agree up to sign.

Proof. The surface $S$ can be parametrized via a map $f(s, t)=\Gamma(t)+s v(t)$ for suitable $s, t$. Since $\Gamma$ is a geodesic, its geodesic curvature in $S$ must be zero and hence its curvature vector $\ddot{\Gamma}$ must be normal to $S$. Let $N:=\ddot{\Gamma} /|\ddot{\Gamma}|$ be the corresponding unit normal. Note that since $S$ is developable, $N(t)$ is normal to $S$ along the entire ruling $\{\Gamma(t)+s v(t)\} \subset S$. The ruling direction is obviously a tangent direction to $S$, so that it can be parametrized by

$$
v(t)=\cos \phi(t) \cdot \dot{\Gamma}(t)+\sin \phi(t) \cdot(N(t) \times \dot{\Gamma}(t)),
$$

where $\phi(t)$ is a suitable angle function.

Furthermore, the second fundamental form of $S$ is given by

$$
\mathrm{II}=\left(\begin{array}{cc}
\left\langle f_{t t}, N\right\rangle & \left\langle f_{s t}, N\right\rangle \\
\left\langle f_{t s}, N\right\rangle & \left\langle f_{s s}, N\right\rangle
\end{array}\right) .
$$

Developability of $S$ implies that the determinant of II vanishes on an open and dense subset of $S$. Since $f_{s s} \equiv 0$, we conclude that the off-diagonal entries of II must also be zero. Hence, the vector $f_{s t}=\dot{v}$ is perpendicular to the surface normal $N$, that is, it is a tangent vector. 
If we differentiate Equation (2.2.1) with respect to $t$, we obtain

$$
\dot{v}=-\dot{\phi} \sin \phi \cdot \dot{\Gamma}+\cos \phi \cdot \ddot{\Gamma}+\dot{\phi} \cos \phi \cdot(N \times \dot{\Gamma})+\sin \phi \cdot(\dot{N} \times \dot{\Gamma}+N \times \ddot{\Gamma}) \text {. }
$$

We have $N \times \ddot{\Gamma}=0$ since they are parallel. Furthermore, we have established that $\dot{v}$ is tangential, to the effect that

$$
0=\langle\dot{v}, N\rangle=\cos \phi\langle\ddot{\Gamma}, N\rangle+\sin \phi\langle\dot{N} \times \dot{\Gamma}, N\rangle
$$

We now use the fact that $\langle\dot{\Gamma}, N\rangle \equiv 0$, which implies that $\langle\ddot{\Gamma}, N\rangle+\langle\dot{\Gamma}, \dot{N}\rangle \equiv 0$. This turns the equation above into

$$
\begin{aligned}
0 & =-\cos \phi\langle\dot{\Gamma}, \dot{N}\rangle+\sin \phi\langle\dot{N} \times \dot{\Gamma}, N\rangle \\
& =\cos \phi\langle\dot{\Gamma}, \dot{N}\rangle+\sin \phi\langle\dot{\Gamma} \times \dot{N}, N\rangle \\
& =\cos \phi\langle\dot{\Gamma}, \dot{N}\rangle+\sin \phi\langle N \times \dot{\Gamma}, \dot{N}\rangle .
\end{aligned}
$$

Since $N$ has unit length, $\dot{N}$ is again tangential to $S$. We can thus introduce a second angle function $\psi$ to write $\dot{N}=(|\dot{N}| \cos \psi) \cdot \dot{\Gamma}+(|\dot{N}| \sin \psi) \cdot(N \times \dot{\Gamma})$. We obtain

$$
\begin{aligned}
0 & =|\dot{N}|(\cos \phi \cos \psi+\sin \phi \sin \psi) \\
& =|\dot{N}| \cos (\phi-\psi)
\end{aligned}
$$

Finally, note that $|\dot{N}|$ can never be zero: if it were zero for some $t$, then the Frenet equations (Theorem 2.1.12) imply $\ddot{\Gamma}(t)=0$, contradicting our assumption that $\ddot{\Gamma}$ is nowhere zero. Therefore we can divide by $|\dot{N}|$ and obtain $0=\cos (\phi(t)-\psi(t))$.

The angle $\psi(t)=\angle(\dot{N}(t), \dot{\Gamma}(t))$ only depends on $\Gamma$ alone. But if $\psi$ is known, one can infer $\phi$ up to integer multiples of $\pi$, which, when combined with Equation (2.2.1), uniquely determines $v$ up to sign.

We have shown that, under the assumptions of the theorem, any developable surface $S$ that contains the given $\Gamma$ both as geodesic and as directrix has its associated director curve defined up to sign. Since $S, \tilde{S}$ are two such surfaces, their director curves $v, \tilde{v}$ must agree up to sign.

Having established this auxiliary result, we can now proceed to prove Theorem 2.2.3.

Proof of Flatness Theorem. For a given origami cylinder whose embedded height is strictly less than the material height, consider a segment $\Gamma$ of either of its boundaries between two vertices that is thus a $C^{4}$ curve. We now show that $\Gamma$ must be a straight line segment. Since all folds are straight lines and the boundaries are straight lines between vertices, flatness of all faces is then a consequence of Lemma 2.2.4.

Assume, by contradiction, that $\Gamma$ was curved, i.e. that $\ddot{\Gamma}(t) \neq 0$ for some $t$. Then by restricting the domain of $\Gamma$ if necessary, we can assume that $\ddot{\Gamma}(t) \neq 0$ for all $t$. Since now 
$\Gamma$ consists entirely of points which are parabolic points of the attendant face, each point carries a unique asymptotic line, whose collection is a ruled developable surface (coinciding with the face that is bounded by $\Gamma$ ), to which we apply Theorem 2.2.5.

Note that by our definition of origami cylinders, $\Gamma$ is a horizontal curve. This means that all derivatives of $\Gamma$ are horizontal vectors as well, in particular $N$ and $\dot{N}$. Now both $\dot{N}$ and $\dot{\Gamma}$ are horizontal and perpendicular to $N$, implying that they must be parallel. Therefore, $\psi=k \pi, k \in \mathbb{Z}$, and thus $\phi=\pi / 2+k \pi, k \in \mathbb{Z}$, meaning that the ruling direction must be purely vertical along the entire curve $\Gamma$.

Since $\Gamma$ does not contain vertices, the vertical rulings cannot coincide with vertical fold lines. As a consequence, they cannot meet other folds except at isolated points since the folds are straight lines whereas $\Gamma$ is not.

Therefore, almost all rulings (i.e., those which do not originate from a set of measure zero) must pass all the way to the other cylinder boundary, implying that the cylinder's height is equal to its material height. This contradicts the assumption that the cylinder height is strictly less than the material height. Therefore, the initial assumption that the boundary curve $\Gamma$ was actually curved must be false.

A simple example shows that the statement of Theorem 2.2.3 is strict: an ordinary round cylinder is an origami cylinder in our sense whose fold pattern has zero folds.

It is perhaps surprising that this result has only been proven for such high regularity of the embedding. One would expect to require less regularity, given that all relevant notions such as curvature and lengths are already defined for $C^{2}$ surfaces (and can even be extended to even less regular embeddings in a weak sense much akin to weak derivatives in the study of partial differential equations). However, the given proof crucially relies on the two cited facts about developable surfaces, whose proof in turn requires second derivatives of curvature.To the best of our knowledge, there do not exist counterexamples for surfaces of less regularity, so that it remains an open question whether the statement is still true for $C^{2}$ or $C^{3}$ surfaces.

\subsection{Deformations of origami cylinders}

This section introduces deformations of origami cylinders and proves that in general continuous deformations are impossible.

Definition 2.3.1. A deformation of an origami cylinder is a continuous one-parameter family of embeddings of the same fold pattern each satisfying the conditions of Definition 2.2.2 such that the heights of this family of cylinders vary continuously with the family parameter. Accordingly, a compression is a deformation where the height is (strictly) monotonically 

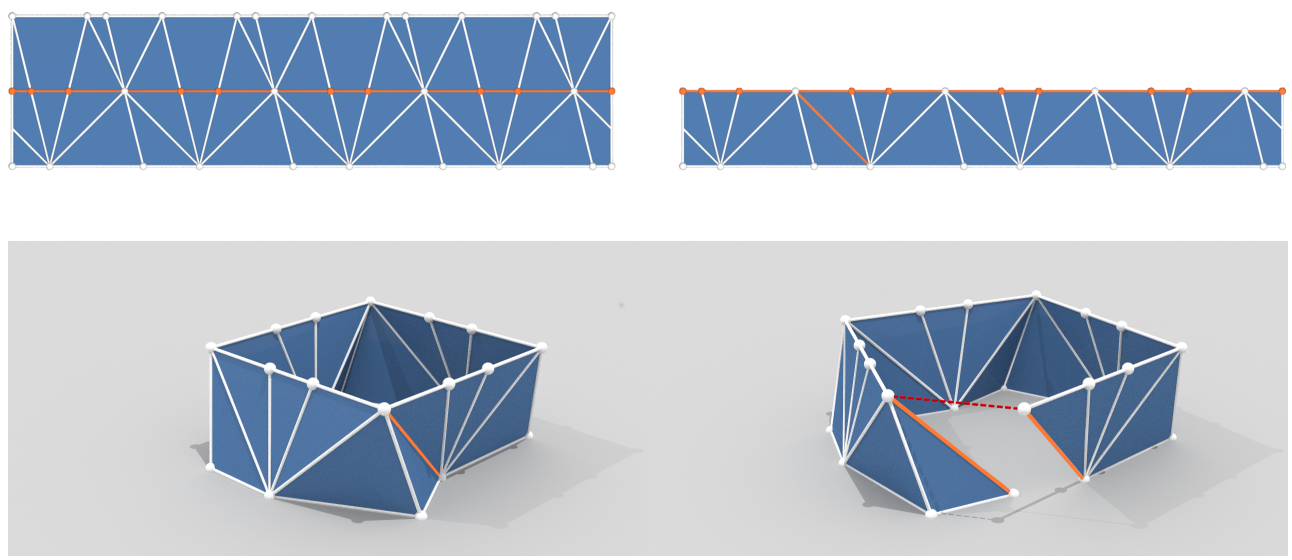

FIGURE 2.1: Top row: a foldpattern is cut horizontally along its vertices into strips (orange line). Bottom row: the lower strip fold pattern is embedded, once as a closed cylinder and once as an open cylinder, with the gap length marked in dashed red.

decreasing. A fold pattern/origami cylinder is called rigid or incompressible if no such continuous family exists.

The main theorem of this section states that most fold patterns are incompressible:

Theorem 2.3.2. An origami cylinder whose fold pattern does not contain vertical fold lines has at most a finite number of heights at which it can be isometrically embedded. Hence, the absence of vertical folds probibits isometric deformations with continuously varying heights. Conversely, any continuously deformable origami cylinder must have at least two vertical fold lines.

Furthermore, there exist additional conditions on the vertical fold lines.

Theorem 2.3.3. If an origami cylinder whose embedded height is less than the material height contains vertical fold lines, the corresponding folds must be either flat or sharp, that is, the associated dihedral angle (the angle between the adjacent faces' unit normals) is either 0 (for flat folds) or $\pi$ (for sharp folds). This is independent of the compressibility of the origami cylinder.

Note that the single known family of examples of flexible origami cylinders, the TachiMiura pattern, follows exactly along these two statements: it has two vertical fold lines whose dihedral angle is equal to $\pi$ at all times.

The proof of these two theorems is composed of three steps: first, a candidate fold pattern is split into a stack of horizontal strips by cutting horizontally through each vertex, see the top row of Figure 2.1. Strips are a special class of fold pattern whose fold lines may only meet on the pattern's boundary. Ruling out compressibility of the entire cylinder then reduces to ruling out compressibility of each of the fold pattern's strips.

Secondly, Section 2.3.1 analyzes the space of all possible embeddings of single strips as origami cylinders with prescribed height. While such an embedding might not be possible for all values of $H$, it is possible if one relaxes the condition that the strip closes into a cylinder, while still maintaining the rest of the assumptions. Intuitively, this corresponds to cutting the cylinder along any of its seams and then changing its height. 
Finally, for each open embedding we measure the distance between matching points on opposite sides of the cut. Embeddings that close into a cylinder correspond to zeroes of this so-called gap function. The proof is completed by showing that, given an initial embedding into a closed cylinder, this gap function is an analytic function of the embedding's height alone and that the gap function must have isolated zeroes.

\subsubsection{Embedding a single strip}

Having split the given fold pattern into strips, we begin analyzing all possible ways to embed a given strip fold pattern to varying heights. As the first step, we further decompose strip fold patterns into fans, using the observation that adding new folds will only increase the cylinder's flexibility. We can thus assume that the fold pattern is triangulated by adding arbitrary diagonals to all non-triangular faces. Then since folds can only meet on the strip's boundary, we can assign to each vertex the collection of all fold lines emanating from that vertex, see Figure 2.2. This collection will be called a fan:

Definition 2.3.4. A fan is a maximal subset of fold lines with at least two elements that all emanate from a common vertex. This common vertex is called the apex of the fan, the other vertices are called end vertices. The outermost end vertices are furthermore called exterior, all others are called interior. We will label the fold lines connecting apex and end vertices accordingly.

Since we assume that the strip fold pattern has been triangulated, the entire fold pattern decomposes into fans. In particular, exterior end vertices are apices of neighboring fans themselves.
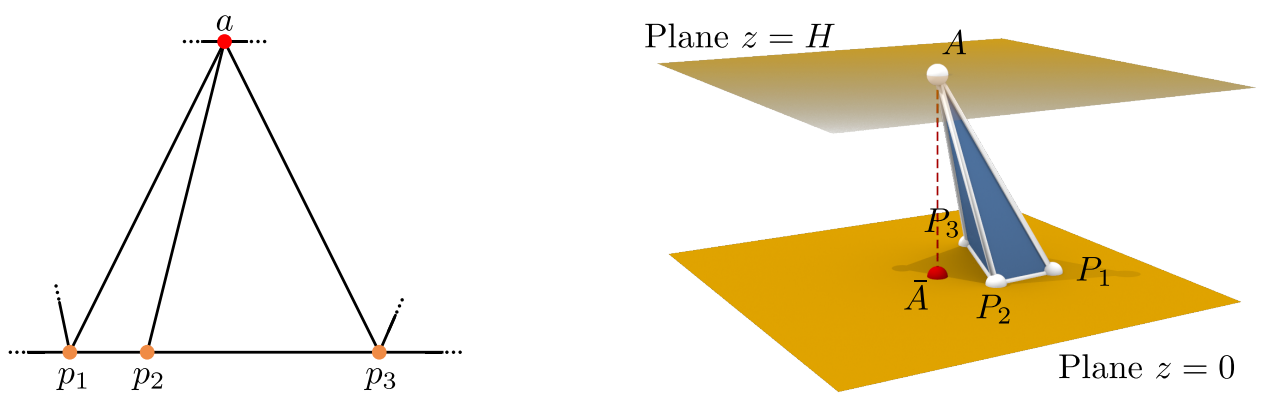

FIGURE 2.2: A fan with three end vertices is embedded.

Embedding a given fan to a height $H$ strictly less than its material height $h$ is now easily done via iteratively embedding all triangles. Each new triangle is joined to an already embedded triangle by one fold. Because of rigidity, the only freedom left in embedding the new triangle is to rotate it about the joint edge, to the effect that the yet unembedded vertex traverses a circle around this edge. Since the third vertex must belong to one of the strip's boundaries, it must lie on a specific horizontal plane, which intersects the circle of possible locations in precisely two points. Thus, each successive triangle can be embedded in 
only two different positions, limiting the total number of embeddings of the entire fan to $2^{\text {number of triangles- } 1}$.

\subsubsection{Turning angles at interior vertices}

We now phrase the restrictions on the embeddings of triangles in terms of the dihedral angles of the folds or, equivalently, of the turning angles of the fan's boundary segments. To this end, first note that the strip's two boundaries are polygonal lines. This is because all faces of the original cylinder are flat and the strip boundaries are obtained by cutting the flat faces with a horizontal plane. Since the position of the vertices in the strip's fold pattern determines the lengths of all line segments of the two polygonal lines independent of the strip's embedded height, the only variables are the turning angles of the boundaries. We will analyze these turning angles in this section.

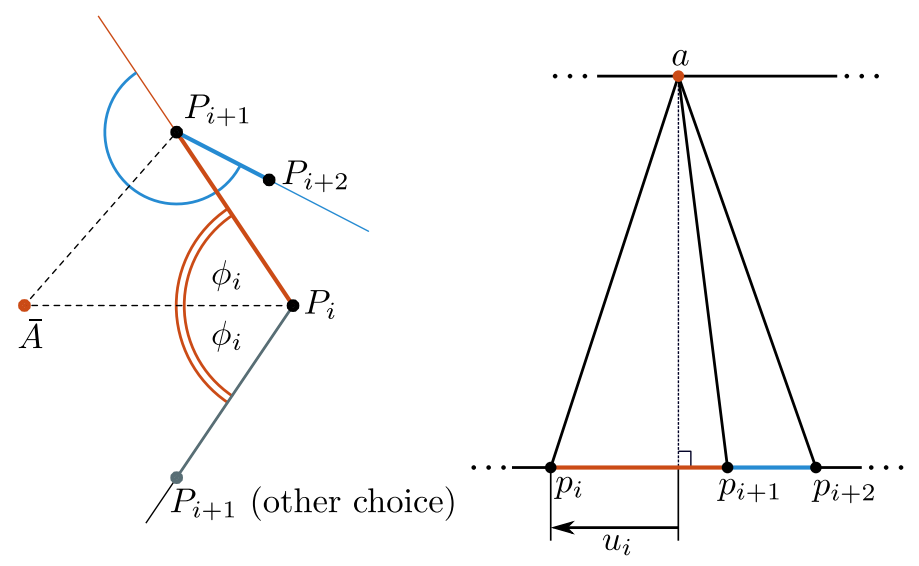

FiguRE 2.3: Left: $P_{i+1}$ lies on one of two definite rays. Right: the corresponding section of the bold pattern. Note that the value of $u_{i}$ is negative.

We first turn to turning angles at vertices that lie in the interior of a fan. In this section, we will show that the turning angles can only attain finitely many values which each depend analytically on the strip's embedded height $H$.

A key observation is the following lemma:

Lemma 2.3.5. For a given fan with apex $a$ and end vertices $p_{1}, p_{2}, \ldots, p_{n}$, denote by uppercase letters the embedded positions of each vertex. Denote by $\bar{A}$ the orthogonal projection of $A$ onto the plane containing the $P_{i}$, see Figure 2.2. For $i=1,2, \ldots, n-1$ denote by $\phi_{i}$ the angle $\angle \bar{A} P_{i} P_{i+1}$. Then $\phi_{i}$ satisfies:

$$
\cos \phi_{i}=\frac{-u_{i}}{\sqrt{h^{2}-H^{2}+u_{i}^{2}}},
$$

where $u_{i}$ is the signed horizontal distance from $p_{i}$ to $a$ and $h$ is the material height of the fold pattern. See Figure 2.3 for an illustration. 
Proof. The triangle $\bar{A} P_{i} P_{i+1}$ has side lengths $\left|\bar{A} P_{i}\right|=\sqrt{h^{2}-H^{2}+u_{i}^{2}},\left|P_{i} P_{i+1}\right|=$ $\left(u_{i+1}-u_{i}\right)$, and $\left|\bar{A} P_{i+1}\right|=\sqrt{h^{2}-H^{2}+u_{i+1}^{2}}$. From here, the claim is equivalent to the law of cosines.

This lemma opens a way to embed the vertices of a fan given only the embedding of one of the boundary folds, that is, the positions of $A$ and $P_{1}$.

1. Project the embedded apex $A$ orthogonally onto the horizontal plane containing $P_{1}$.

2. Draw two rays emanating from $P_{1}$ which each enclose an angle of $\phi_{1}$ (defined by Equation (2.3.1)) with the line $P_{1} \bar{A}$. The second vertex $P_{2}$ must lie on either ray at a distance of $u_{2}-u_{1}$ from $P_{1}$.

3. Repeat this process: at $P_{i}$, draw rays under an angle of $\phi_{i}$ to the line $P_{i} \bar{A}$ and mark off $P_{i+1}$ at the correct distance.

Another easy calculation shows that one can also perform this ray construction process backwards: given the apex and $P_{n}$, draw rays at an angle of $\psi_{n}=\pi-\phi_{n}$, mark off $P_{n-1}$ and so on.

Finally, we summarize the effects on the possible turning angles.

Lemma 2.3.6. The turning angle of the strip boundary at an interior vertex $P_{i}$ is either 0 or equal to $\pm 2 \phi_{i}$, where $\phi_{i}$ is defined by Equation (2.3.1). In particular, if the fan's embedded height $H$ is prescribed, there exist only finitely many possible embeddings of the fan up to rigid body motions.

Proof. This lemma is a direct consequence of Lemma 2.3.5 and the observation that the straight embedding (where all folds have zero dihedral angle) is always possible. But the straight embedding of a fan must be constructible using the ray method detailed above. Therefore, one of the two rays emanating from $P_{i}$ that could contain $P_{i+1}$ must be the parallel continuation of the line segment $P_{i-1} P_{i}$. This implies that the line $P_{i-1} P_{i}$ and the second ray enclose an angle of $\pm 2 \phi_{i}$.

Note also that this provides the possibility to continue the successive embedding process in the next fan. Having embedded all vertices $A, P_{1}, P_{2}, \ldots, P_{n}$ of the current fan, using the fact that $P_{1}$ and $P_{n}$ are the neighboring fans' apices and $A$ is an exterior end vertex in both neighboring fans, one can apply either forward or backward ray drawing to both neighboring fans independently of each other.

This section showed how to construct all possible embeddings of single fans to a given embedded height $H$ and fixed the turning angles of the strip's boundaries at interior vertices. All that remains is to do a similar discussion for exterior vertices. 


\subsubsection{Turning angles at exterior vertices and apices}

In this section, we will prove an analogue of Lemma 2.3.6 for exterior vertices. As the preceding section showed, exterior vertices are themselves apices of the neighboring fans. The result corresponding to Lemma 2.3.6 is the following:
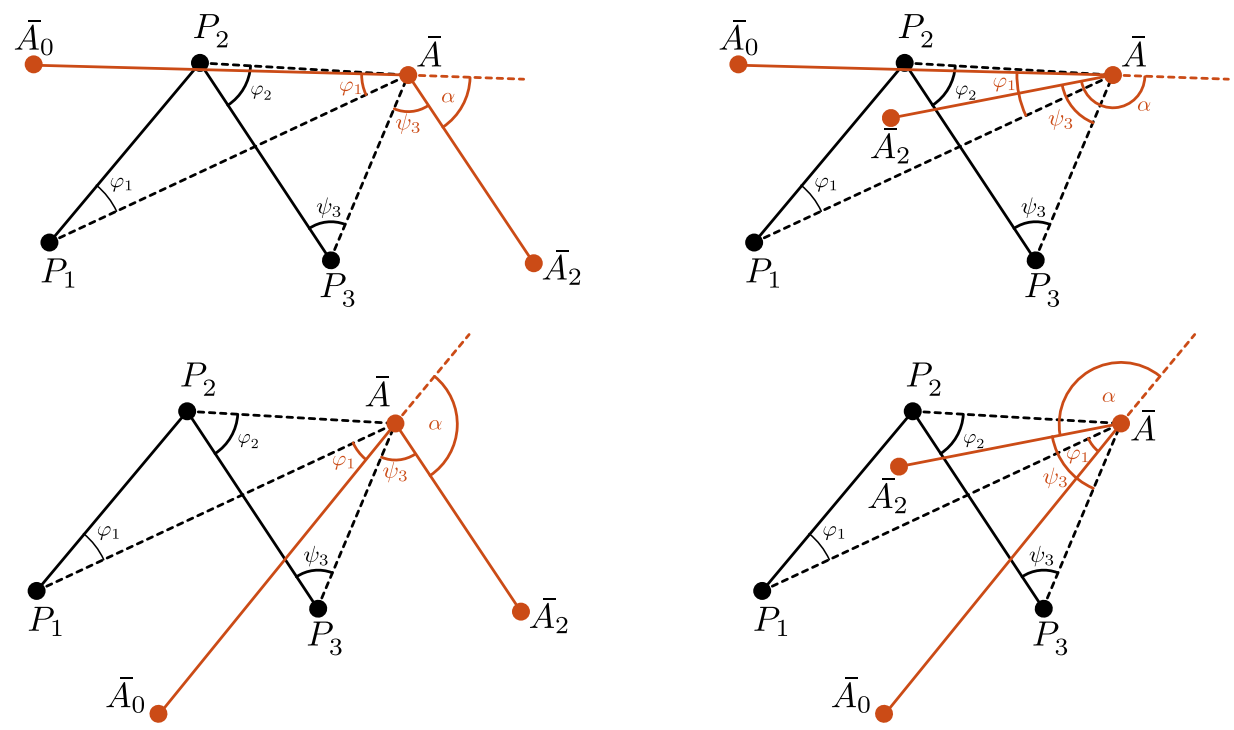

FIGURE 2.4: Overview of the differentpossibilities of embedding the neighbors of the apex A withn $=3$ end vertices. The end vertices $P_{1}$ through $P_{3}$ are fixed.

Lemma 2.3.7. For a given fan with apex $a$ and end vertices $p_{1}, p_{2}, \ldots, p_{n}$, denote by uppercase letters the embedded positions of each vertex. Denote by $\bar{A}$ the orthogonal projection of $A$ onto the plane containing the $P_{i}$. Let $a_{0}, a_{2}$ be the neighboring vertices of $a$ on the same strip boundary. Let $\beta$ be the angle between the lines $P_{1} P_{2}$ and $P_{n-1} P_{n}$ and let $\psi_{n}:=\pi-\phi_{n}$, where $\phi_{n}$ is defined by Equation (2.3.1). Then the turning angle $\alpha=\pi-\angle A_{0} A A_{2}$ satisfies $(\alpha+\beta) \in\left\{0 \pm 2 \phi_{1}, \pm 2 \psi_{n}, \pm 2 \phi_{1} \pm 2 \psi_{n}\right\} \bmod 2 \pi$.

Proof. From the right-hand side of Figure 2.5, observe that $a$ is the leftmost boundary vertex of the fan with apex $p_{1}$. Also note that the horizontal distance is the same $u_{1}$ used to compute the angle $\phi_{1}$ in Lemma 2.3.5. Therefore, the rays from $\bar{A}$ containing $\bar{A}_{0}$ must enclose the same angle $\phi_{1}$ with the line $P_{1} \bar{A}$. Likewise, the rays from $\bar{A}$ containing $\bar{A}_{2}$ must enclose the angle $\psi_{n}$ with the line $P_{n} \bar{A}$. Since $\alpha=\pi-\angle A_{0} A A_{2}=\pi-\angle \overline{A_{0}} \bar{A} \overline{A_{2}}$, proving the claim is then a matter of simple addition of angles depending on the actual positions of $A_{0}, A_{2}, P_{2}$, and $P_{n-1}$. Figure 2.4 provides an overview of the different possibilities of embedding the points $A_{0}$ and $A_{2}$.

The auxiliary angle $\beta$ can be obtained by repeated application of the interior turning angle lemma 2.3.6. As a consequence, all possible turning angles of either boundary are only dependent on the embedded height $H$. 

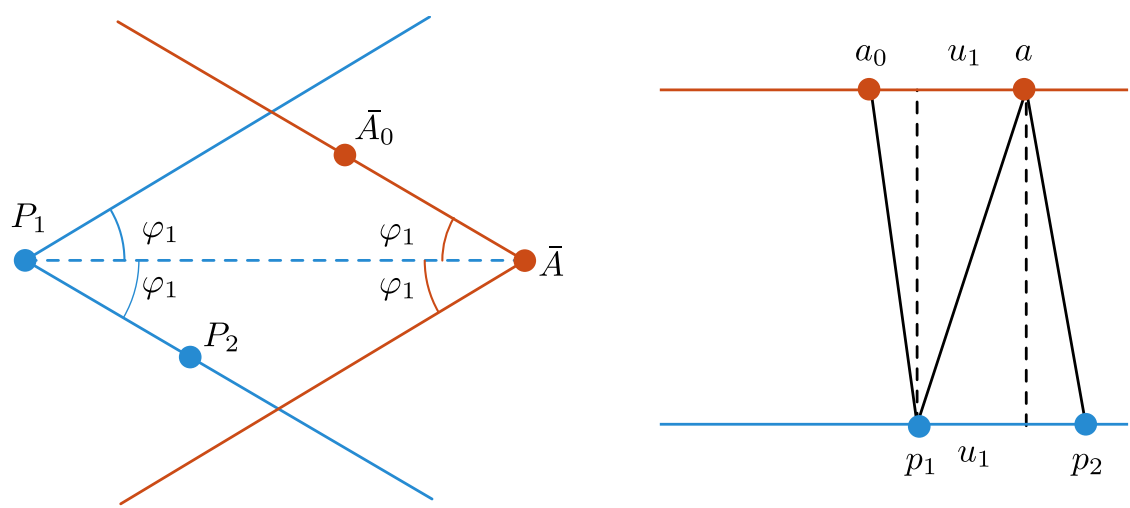

FIGURE 2.5: Left: the line $\overline{A_{0}} \bar{A}$ encloses the same angle with the line $P_{1} \bar{A}$ as the line $P_{1} P_{2}$. Right: the reason is that the horizontal distance $u_{1}$ is the same for $a$ as an end vertex of the apex $p_{1}$ and for $p_{1}$ as an end vertex of the apex $a$.

\subsubsection{The gap function}

We now introduce the main tool used in the proof of Theorem 2.3.2. The key change is that in this section, the strips no longer have to close up into cylinders but can instead remain open.

Definition 2.3.8. An open cylinder is a fold pattern together with an isometric embedding into $\mathbb{R}^{3}$ satisfying the same conditions as origami cylinders except that the left and right boundaries do not need to coincide.

Note in particular that we still require that horizontal lines in the material embed to horizontal curves. As it turns out, leaving the cylinder cut open makes it flexible and thatat least for individual strips—-this flexibility is uniquely determined:

Theorem 2.3.9. The evolution of an open embedded strip with $H<h$ under continuous compressions is uniquely determined by its initial embedding up to global rigid body motions. In particular, every strip fold pattern can be embedded to an open strip to all values of $H$.

Proof. Prescribing the initial embedding fixes the turning angles to one of the different possible values established in Lemmas 2.3.6 and 2.3.7. We now show that these possible values are pairwise either distinct for all values of $H<h$ or identical for all values of $H$, to the effect that changing from one distinct value to another is a discontinuous event.

Consider first the turning angles at interior end vertices. According to Lemma 2.3.6, this turning angle is either 0 or $\pm 2 \phi_{i}$. If $\phi_{i}$ were equal to an integer multiple of $\pi$ for some $H$, then Equation (2.3.1) would imply $H=h$. Because the turning angle can only change continuously with $H$, it is either equal to zero for all $H<h$ or equal to $\pm 2 \phi_{i}$ for all values of $H<h$ and is thus uniquely determined from the initial cylinder.

As the angle $\beta$ is composed only of a number of turning angles of interior end vertices, its evolution with $H$ is also uniquely prescribed. Furthermore, it also holds that either $\phi_{1}= \pm \psi_{n}$ for all $H<h$ or $\phi_{1} \neq \pm \psi_{n}$ for all $H<h$ : if $\left|\phi_{1}\right|=\mid \psi_{n} \|$ for some $H_{0}$, 
Equation 2.3.1 implies $\left(u_{1}^{2}-u_{n}^{2}\right)\left(h^{2}-H_{0}^{2}\right)=0$, to the effect that $\left|\phi_{1}\right|=\left|\psi_{n}\right|$ for all $H$. Thus, all possible values of $\alpha+\beta$ are either identical for all $H$ or different for all $H$, so that the evolution of $\alpha+\beta$ with $H$ is uniquely prescribed. As a consequence, the apex turning angle $\alpha$ itself is also uniquely prescribed for all $H$.

Thus, all turning angles of folds and thus the entire embedding is uniquely determined from the initial embedding for all values of $H<h$.

This means that, given an initial embedded strip, the motion of its four corners (the intersections of upper and lower boundaries with the seam) is a unique function of the strip height alone. We measure the distance between corresponding points on both sides of the seam with the gap function:

Definition 2.3.10. Let $C_{1}$ and $C_{2}$ be corresponding points on both sides of the cut of an open strip cylinder. The gap function $g$ assigned to $C_{1}$ and $C_{2}$ is defined to be

$$
g(H):=\left|C_{1}(H)-C_{2}(H)\right|^{2},
$$

where the argument $H$ is the strip's embedded height. See Figure 2.1 for an example.

Because we cut along a straight line, it is sufficient to examine the gap functions for the two pairs of corner vertices, as all intermediate gaps can be obtained from there as linear combinations.

\subsubsection{Proof of rigidity theorem}

The ingredients for the proof of Theorem 2.3.2 have now been introduced. To prove this theorem, consider a strip origami cylinder without vertical fold lines and cut it open along one of the fold lines. Suppose for contradiction that this origami cylinder were compressible. Compressibility implies that the gap function for this embedding is zero for all values of $H$ in an entire interval. On the other hand, the gap function $g$ is univariate real analytic in the variable $H$ : it is the difference between the two end points of a polygonal line where the line segments have constant length and whose turning angles depend on $H$ analytically according to Lemmas 2.3.6 and 2.3.7 as long as $H<h$. Since $g$ is zero on an entire interval, it must be zero for all values of $H$ (as every univariate real analytic function is either the zero function or has isolated zeroes). On the other hand, consider the limit as $H$ approaches $h$ : explicitly taking limits in Equation 2.3.1 gives that $\cos \phi_{i}=-1$ unless $u_{i}=0$. As all fold lines are assumed to be non-vertical and thus all $u_{i}$ are indeed nonzero, this implies that all turning angles $\pm 2 \phi_{i}$ become integer multiples of $2 \pi$. But then the embedding becomes the flat embedding: all folds are flat folds. Clearly, this embedding's gap length is equal to the squared domain width $w^{2}$ and thus nonzero. We have arrived at a contradiction. Therefore, the strip origami cylinder is incompressible unless it contains vertical fold lines. 


\subsubsection{Proof of vertical fold theorem}

Finally, the proof of Theorem 2.3.3 is a consequence of Lemma 2.3.5: for vertical fold lines, the value $u_{i}$ is zero and therefore $\phi_{i}=\pi / 2$, independent of $H$. If the vertical fold is an interior fold, then the turning angle of the corresponding end vertex of that fold is either zero or $\pm \pi$ by Lemma 2.3.6. If the vertical fold line is an exterior fold line, that is $i=1$ or $i=n$, then the lines $A_{0} A$ and $P_{1} P_{2}$ (if $i=1$ ) are parallel, as Figure 2.5 illustrates. In all cases, the dihedral angle of the vertical fold is either zero or $\pm \pi$.

This finalizes the rigidity analysis of rigid origami cylinders. The remainder of this section will be dedicated to additional applications of the rigid origami framework.

\subsection{Designing folded structures of given height}

Another useful application of the rigid origami model established in this chapter is the design of strip fold patterns that embed to an origami cylinder of prescribed height, a construction of which will be outlined in this section. Even though this discussion is restricted to rotationally symmetric fold patterns for the sake of simplicity, the underlying principles and resulting design method can be applied to arbitrarily asymmetric fold patterns as well.

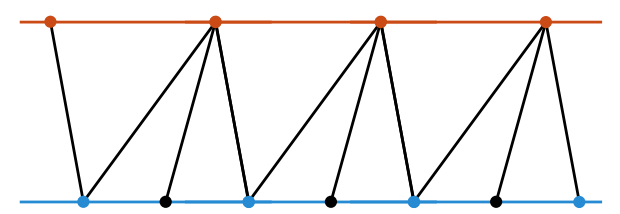

FIGURE 2.6: Example for a periodic repetition fold pattern that embeds to a rotationally symmetric strip. Marked in red and blue are the initial vertices, the black vertices are added by the refinement process.

The simplest class of fold patterns with $k$-fold rotational symmetry is a periodic repetition of a single fan as in Figure 2.6. To find embeddable fold patterns, begin with $k$ equidistant vertices each on top and bottom boundary, with fold lines connecting them in a trusslike configuration. This "skeleton" fold pattern will now be refined to one that embeds as a rigid origami cylinder to the given height in such a way that the cylinder's top boundary is a regular $k$-gon.

The following theorem will ensure that this process is always feasible. As it turns out, the central premise required for this existence result is that the given vertices are embedded short:

Definition 2.4.1. A short embedding of a strip fold pattern is a (not necessarily isometric) embedding of the pattern's vertices into $\mathbb{R}^{3}$ where

1. all vertices of each boundary lie in a common horizontal plane, 
2. the length of each fold is equal to the length of the fold line in the fold pattern and

3. the distance between adjacent vertices on the same boundary is less than or equal to their material distance. In order to achieve "cylindrical adjacency", the leftmost and rightmost vertex on each boundary also count as adjacent.

In other words, a short embedding is almost a rigid origami cylinder embedding, except that neighboring vertices on either boundary may be closer than their material distance.

As it turns out, any shortly embedded fan can be completed to an isometrically embedded fan if $H<h$ by adding a single fold between each pair of adjacent vertices. This is due to the following geometric fact:

Theorem 2.4.2. Consider a triangle abc in the plane and a triangle $A B C$ in $\mathbb{R}^{3}$. Let $\mathcal{E}$ be any plane containing $B$ and $C$. If the following conditions are satisfied:

1. $|A B|=|a b|$,

2. $|A C|=|a c|$,

3. $|B C| \leqslant|b c|$,

4. $H<h$, where $H$ is the distance from $A$ to the plane $\mathcal{E}$ and $h$ is the distance from a to the line $b c$,

then there exists a point $D$ on $\mathcal{E}$ such that the triangle pair $(A B D) \cup(A D C)$ is isometric (up to the fold) to abc. Furthermore, the number of such points is specified: if $|B C|=|b c|$, all points on the line $B C$ have this property. If $|B C|<|b c|$, then there exist exactly two such points.

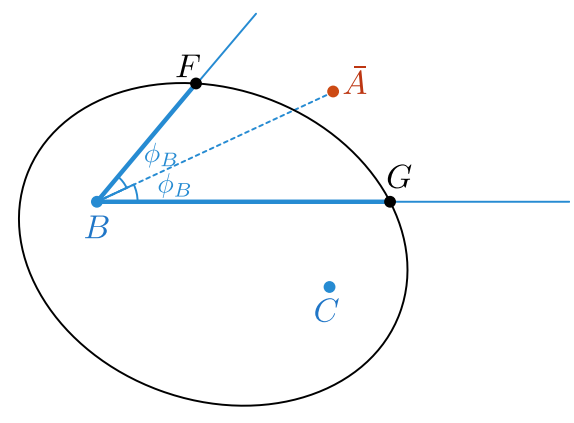

FIGURE 2.7: Illustration of the notation used in the proof of Theorem 2.4.2. Two rays from B intersect the ellipse with foci $B$ and $C$ in two points $F$ and $G$.

Proof of Theorem 2.4.2 Consider the set of all points $D \in \mathcal{E}$ with $|B D|+|D C|=|b c|$. This locus of points forms an ellipse $\eta$ in the plane $\mathcal{E}$ with foci $B$ and $C$. To each such $D$ corresponds exactly one point $d$ on the side $b c$ such that $|b d|=|B D|$ and $|d c|=|D C|$. It suffices to show that for some $D$ on this ellipse, the additional condition $|D A|=|d a|$ holds. 

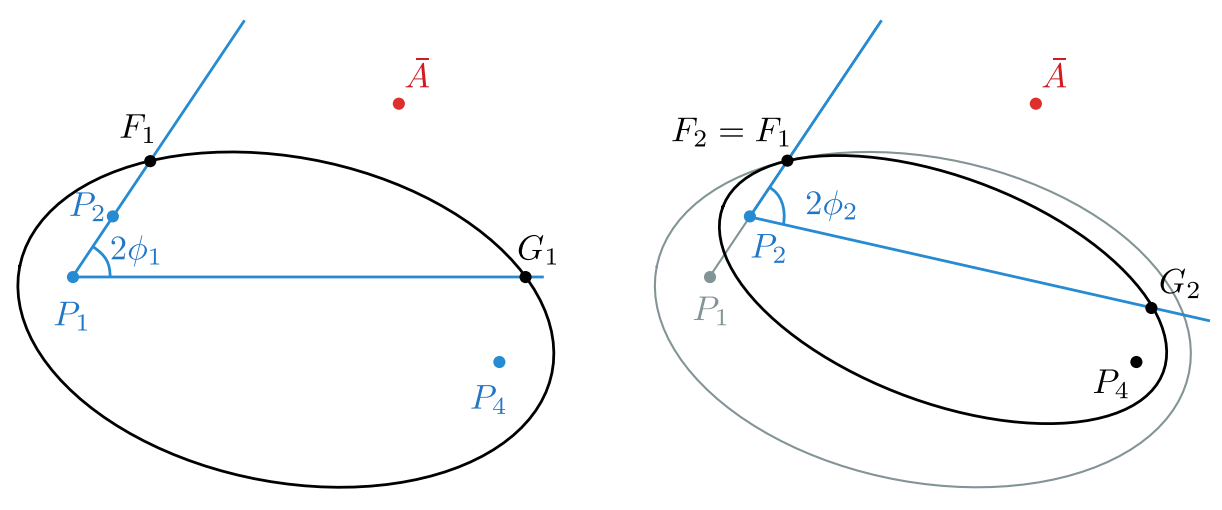

FiguRE 2.8: Two steps of the fan construction process for $n=4$. Left: $P_{2}$ is placed inside the $P_{1}-P_{4}$ ellipse. Right: $P_{2}$ and $P_{4}$ define a new ellipse and two new rays. Since $P_{3}$ is the last point, it must be at either intersection $F_{2}$ or $G_{2}$.

Let $\bar{A}$ be the orthogonal projection of $A$ onto $\mathcal{E}$. Lemma 2.3.5 implies that $|B D|=$ $|b d|$ and $|D A|=|d a|$ is only given if $D$ lies on one of two rays emanating from $B$ under an angle of $\phi_{B}$ (defined by Equation (2.3.1) with the line $B \bar{A}$, see Figure 2.7. However, since $B$ lies inside the ellipse $\eta$, the rays intersect the ellipse in precisely one point each. At these two points, all conditions are satisfied- $|B D|=|b d|$ and $|D A|=|d a|$ because all points on the ray satisfy this and $|D C|=|d c|$ because $D$ lies on the ellipse.

The above theorem is turned into a construction process as follows: first, if only a single interior fold line is to be added, such that $n=3$ and $P_{1}$ and $P_{3}$ are placed with $\left|P_{1} P_{3}\right|<$ $\left|p_{1} p_{3}\right|$, the position of $P_{2}$ is defined according to the above theorem. If more fold lines are desired, that is $n>3$ and $P_{1}, P_{n}$ have been placed such that $\left|P_{1} P_{n}\right|<\left|p_{1} p_{n}\right|$, then all but one of the interior vertices can be placed at will as long as the length constraints of a short embedding are satisfied, as the following corollary shows:

Corollary 1. Let $A, B, C, F, G$ be as in Figure 2.7 and let $P$ be any point on either line segment $B F$ or $B G$. Let $p$ be the corresponding point on the side $b c$ such that $|B P|=|b p|$. Then $|P C| \leqslant|p c|$.

Proof. As $P$ is in or on the ellipse, $|P C| \leqslant|B C|-|B P| \leqslant|b c|-|b p|=|p c|$.

The first interior point $P_{2}$ must be placed on one of the line segments $P_{1} F$ or $P_{1} G$. The corollary implies that the conditions of Theorem 2.4.2 are still satisfied, regardless of where $P_{2}$ is placed: $P_{2}$ and $P_{n}$ define a new ellipse and two new rays originating from $P_{2}$, so that $P_{3}$ can be placed etc. Thus, all but one of the points $P_{2}, \ldots, P_{n-2}$ can be chosen freely according to the above construction method, and $P_{n-1}$ must lie exactly on the $P_{n-2}-P_{n}$ ellipse. Figure 2.8 illustrates this construction process.

A skeleton embedding satisfying the premises of Theorem 2.4.2 is easily found: the top vertices are already embedded as a regular $k$-gon with side lengths exactly equal to their 
material counterpart. Thus, no vertices need to be added to the top boundary.
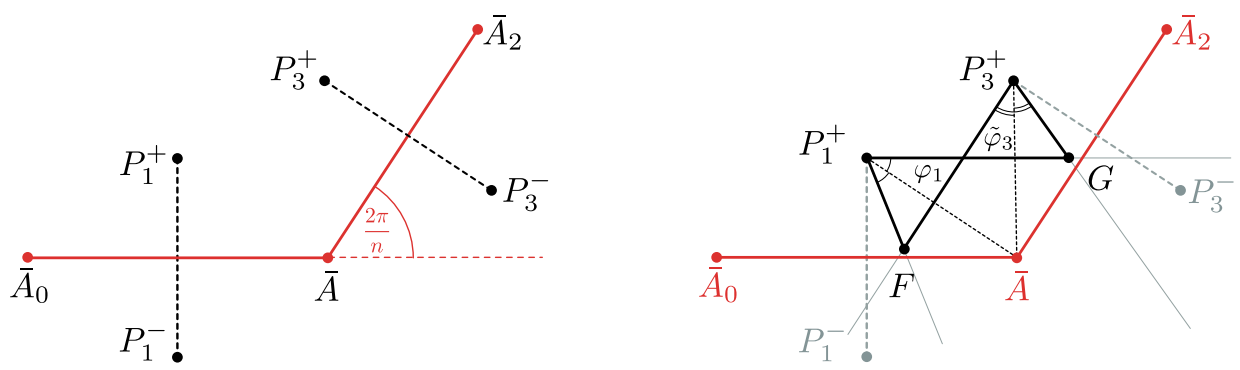

FigURE 2.9: Left: The four different end points of the short fan. Objects in red belong to the upper boundary. Right: From each $P_{i}^{+}$originate two rays at a specified angle. These intersect each other (and the ellipse with foci $P_{1}^{+}$and $P_{3}^{+}$) in exactly two points $F, G$, the possible locations of $P_{2}$.

Because the cylinder height is fixed, the triangles incident to each of the upper boundary's edges can embed in two ways: they either tilt towards the inside of the $k$-gon or towards its outside, fixing the vertices on the bottom boundary to one of two positions each. Furthermore, one of the two possibilities (all triangles tilt to the inside or all to the outside) results in a short embedding, as an easy computation shows. Choose coordinates such that three successive vertices on the top boundary embed as $(-1,0, H),(0,0, H)$, and $(\cos 2 \pi / k, \sin 2 \pi / k, H)$, see Figure 2.9. Depending on $u_{1}$, the two possible locations for $P_{1}$ are given by $P_{1}^{ \pm}=\left(u_{1}, \pm \sqrt{h^{2}-H^{2}}, 0\right)$ and, likewise, the two possibilities for $P_{3}$ are $P_{3}^{ \pm}=R^{2 \pi / k}\left(u_{1}+1, \pm \sqrt{h^{2}-H^{2}}, 0\right)$, where $R^{\alpha}$ indicates the matrix rotating by $\alpha$ around the vertical axis. The inwards tilting solutions are each given by taking the positive square root.

One of the two embeddings is short if at least one of the squared distances $d^{ \pm}=$ $\left|P_{1}^{ \pm} P_{3}^{ \pm}\right|^{2}$ is less than 1 . Both $d^{ \pm}$are minimal at $u_{1}=-1 / 2$ and their minima are less than 1 if and only if $\sqrt{h^{2}-H^{2}} \leqslant(\tan \pi /(2 k))^{\mp 1}$. However, since $k \geqslant 3$, we have $\frac{1}{\sqrt{3}} \geqslant \tan \pi /(2 k)>0$ and therefore it is enough to consider the less strict inequality

$$
\sqrt{h^{2}-H^{2}} \leqslant \frac{1}{\tan \frac{\pi}{2 k}}=: d_{k}
$$

As $H<h$ must still hold, this translates into $H \in\left[h-d_{k}, h\right)$ or, conversely, $h \in(H, H+$ $\left.d_{k}\right]$. Thus, for all values of $h$, there exists $H$ and for all $H$ there exists $h$ such that at least one of these inequalities is satisfied. This proves the existence of at least one short embedding of the truss with $u_{1}=-1 / 2$ that can be completed to an isometric embedding. Note, however, that even though not all pairs $(H, h)$ satisfy the inequality (2.4.1), if $h$ is chosen less than $d_{k}$, then every value of $H<h$ satisfies the inequality.

Summarily, the full procedure to generate a fold pattern that embeds to a given height $H$ is the following:

1. Pick $k \geqslant 3$ arbitrarily. 
2. Find $h$ such that the above inequality (2.4.1) is satisfied.

3. On the strip of dimensions $w \times h$, mark off $k$ equidistant vertices on each boundary, aligned such that to $u=-1 / 2$. Add fold lines in a zig-zag pattern, see Figure 2.9.

4. Given $H$, embed the vertices on the top boundary as a regular $k$-gon. Embed the vertices on the bottom boundary such that their distances are short.

5. For each neighboring pair of vertices $P, Q$ on the bottom boundary with apex $A$, construct additional folds into the fan. To this end, obtain the projection $\bar{A}$ of $A$ onto the boundary plane containing $P$ and $Q$ and repeat the following steps:

(a) Draw the ellipse with foci $P$ and $Q$.

(b) Draw two rays from $P$ with angle to the line $P \bar{A}$ given by Equation 2.3.1. Pick any new point $R$ on the ray inside the ellipse, mark $r$ in the fold pattern such that $|P R|=|p r|$.

(c) Set $P \leftarrow R$ and begin anew. Stop when $R$ lies exactly on the ellipse, as the fan is isometrically embeddable.

This construction process generates strips where one boundary embeds as a regular $k$ gon and the other may be irregular, depending on the different fan embeddings. However, the only condition on the correctness of this method is that every individual fan has an initial short embedding. Investigating which polygonal lines are valid shapes for the upper boundary in that the bottom vertices can be embedded short is an open question.

\subsection{Construction of multistable origami}

Another application of the rigid origami cylinder model is to analyze whether given fold patterns are multistable, that is, whether they can be folded to different cylinders of more than one height. Instead of applying the gap function approach used in the general rigidity analysis, this section provides an alternative approach to find embeddings at different heights with the additional restriction that one boundary of the strip remains unchanged. In this case, bistability reduces to the level of individual fans. The reason for this restriction is the following: a bistable strip can be glued to its mirror image to obtain a multistable twostrip origami cylinder whose boundaries are constant across all different configurations. If this boundary happens to be a regular $k$-gon, copies of this two-strip cylinder can again be stacked to structures with an even greater number of different embeddings.

Similar to the previous section, the construction process will first build individual fans, which are then assembled into a full strip fold pattern. Thus, consider a single fan with only a single interior vertex and given values of $u_{1}, u_{3}$, and $h$. In order to preserve the ability to 
stack strips as described before, we additionally impose that the fan's apex turning angle $\alpha$ must remain equal to a chosen value for all heights. For which value of $u_{2}$ (depending on $u_{1}$ and $\left.u_{3}\right)$ is this fan multistable?

The preceding section and Lemma 2.3.5 as well as Theorem 2.4.2 imply that, given locations for $A, P_{1}$ and $P_{3}$, the interior vertex $P_{2}$ lies at one of two possible locations $F$ and $G$, which can be obtained by intersecting two pairs of rays originating from $P_{1}$ and $P_{3}$, respectively.

Now instead of using Equation (2.3.1) to determine the turning angle $\phi_{2}$, this angle $\phi_{2}$ is expressed as a sum of the apex turning angle $\alpha$ and the boundary angles $\phi_{1}$ and $\psi_{3}$ (similar to the proof of Lemma 2.3.7). The resulting equation is then solved for $u_{2}$ instead. By rearranging Equation (2.3.1), this results in two separate equations, depending on which ray contains $P_{2}$ :

$$
\begin{aligned}
& u_{2}(H)=-\sqrt{h^{2}-H^{2}} \cot \left(\phi_{1}(H)+\frac{|\alpha|}{2}\right), \\
& u_{2}(H)=\sqrt{h^{2}-H^{2}} \cot \left(\psi_{3}(H)+\frac{|\alpha|}{2}\right) .
\end{aligned}
$$

A fan is bistable if for its value of $u_{2}$, multiple values of $H$ solve any one of these equations or, equivalently, the set of stable heights for a given value $u_{2}$ is exactly the union of the level sets for the value $u_{2}$ of these two functions. If we consider the right-hand sides as functions in $H$, we find that depending on the value of $u_{2}$, the fan can have up to three stable heights:

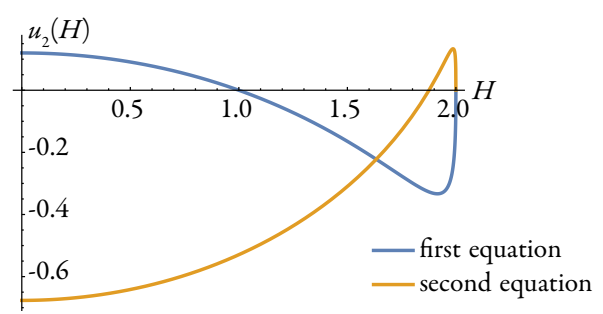

FIGURE 2.10: Plot of the two right-hand sides of Equation (2.5.2) for specific parameters $u_{1}=$ $-1, u_{3}=0.4, h=2$.

This analysis shows two things: there exist strips which are multistable (with up to three different heights) and for a simple class of fold patterns-periodic repetitions of a 3-vertex fan-these heights that do not change the strip's upper boundary can be computed directly from the fold pattern.

Unfortunately, the inverse problem - to find, given a set of heights, a fold pattern that embeds to precisely these heights-is more challenging and an open question. But a slightly weaker problem has an easy solution: if the fold pattern is only required to embed to at least the given heights, there is a general solution available.

Assume that a set of heights $\left\{H_{1}, H_{2}, \ldots, H_{n}\right\}$ is given. Then a fold pattern that em- 
beds to at least these heights can be constructed as follows: begin with a $k$-fold periodic repetition of a single fan. Embed the top boundary as a regular $k$-gon and the bottom vertices such that the lower boundary is embedded short for all $H_{i}$. Then use Theorem 2.4.2 to find for each height $H_{i}$ a separate interior point $P_{2, i}$. The union of all these folds is a fan with $n+2$ fold lines. It embeds to all heights $H_{i}$ : all except the $i$-th fold are straight folds whereas the $i$-th fold has the appropriate dihedral angle. The result of this process is illustrated in Figure 2.11 with two prescribed heights.

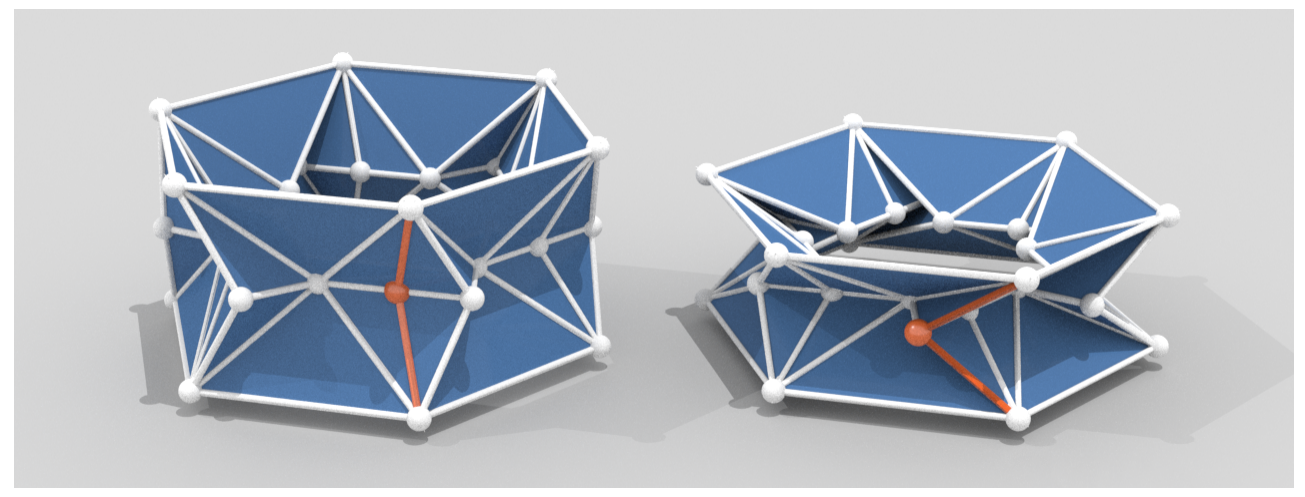

FIGURE 2.11: A bistable origami cylinder where a bistable strip has been glued to its mirror image. The two orange folds joined to the orange vertex are completely flat in the left embedding. Theorem 2.3.2 show that there exists no continuous isometric deformation between the two states. 
Rigid ORIgAMI 


\section{Nearly rigid origami}

The rigidity theorem 2.3.2 establishes that most origami cylinders are inflexible, contrary to the observation that various paper origami seem to compress without putting strain on the material. This evidence suggests that the deformations occurring in these instances are elastic, but not plastic, and thus that even though the material must accumulate some stretching energy, the maximal amount of that stretching remains small enough to prevent permanent damage to the material. To model these "invisibly elastic" deformations, we therefore introduce and analyze an extension to the rigid origami model.

The key feature of this nearly rigid origami model is that the compressive stress is localized at the prescribed folds. This localization property is motivated by two different arguments. On one hand, it has been observed in numerical simulations and experiments by Witten and Lobkovsky [39, 59]. They found that if the boundaries of a sheet of paper are confined into a frame bent at two opposite corners, then only the material near the ridge line experiences stress. On the other hand, this fact is supported by a careful analysis of the elastic equations. This was first done again by Lobkovsky [38], who used an approximate elastic shell model, and again by Conti and Maggi [17] for the fully general three-dimensional elastic energy. In both cases, the specific constraints on the material boundary allow to establish that the width of the strained region scales with $h^{1 / 3}$. This means that in the limit of zero thickness, the strained region consists of just the fold itself.

The nearly rigid model mimics this localization in the following way: while the faces of a fold pattern are rigid objects, the folds themselves may now deform and carry strain. This strain is encoded by a simple elastic model: imagine that the cylinder is cut open along all folds and that the faces' corners previously joined in a single vertex are now connected by linear springs, as illustrated in Figure 3.1. This network of rigid faces connected by elastic springs is completely flexible, but accumulates elastic energy. In order to determine the actual response of this network to axial compressions, we minimize the total elastic energy over the space of all possible configurations, subject to different constraints that clamp the cylinder's boundaries to specific heights.

In computational geometry, minimizing an elastic shell energy subject to handle con-
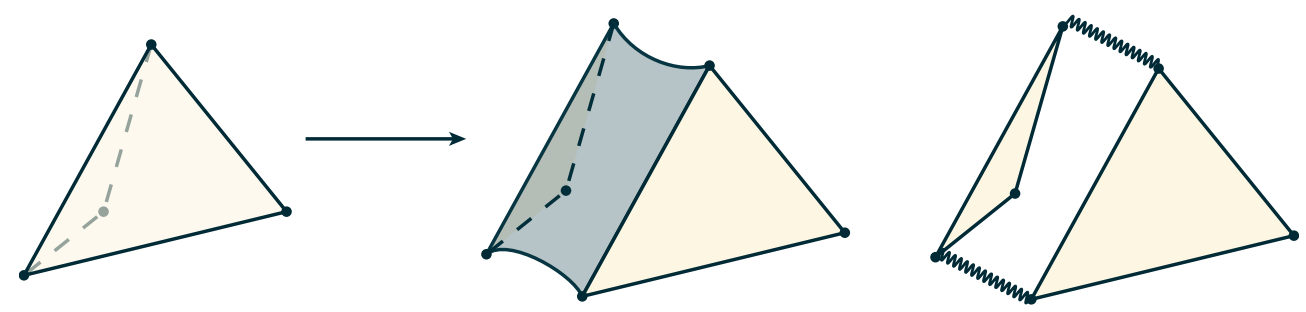

FIGURE 3.1: A single fold is cut and rejoined by an elastic rubber band of zero rest width. We consider the equivalent formulation of adding springs between the vertices. 
straints is a problem well known under the name of suface modeling. In fact, a variation of our nearly rigid origami model has also been proposed under the name of PriMo as an especially numerically stable and robust method by Botsch et al [9]. Seen from this perspective, our nearly rigid origami model is a special case of this PriMo model for infinitely thin shells. This restriction has a number of consequences, most prominently the fact bending the shell is a zero-energy transformation.

\subsection{Setup}

We begin by making precise the notion of a nearly rigid origami.

Definition 3.1.1. 1. A nearly rigid origami cylinder is a triangulated fold pattern together with an affine isometric embedding of each of its faces into $\mathbb{R}^{3}$. Note that faces which share a common fold line in the fold pattern need not map to joined flat faces, so that a single vertex might map to several different corners of flat triangles. Additionally, we require that two additional planarity constraints are satisfied: (a) all copies of each vertex in the fold pattern must lie in the same horizontal plane and (b) all vertices that lie on the same horizontal line in the fold pattern must embed to the same horizontal plane. Such groups of vertices will be called vertex layers.

2. A deformation of a nearly rigid origami cylinder is a one-parameter family of nearly rigid origami cylinders with the same triangular fold pattern such that the cylinder's height changes continuously.

Remark 3.1.2. The two additional planarity assumptions on nearly rigid fold patterns correspond to the assumption of horizontality on the old model of perfectly rigid origami cylinders. Their purpose is to make the nearly rigid origami model comparable to the perfectly rigid origami model, but they are not essential to the nearly rigid model itself. Removing them will affect the choice of parameterization as described in Section 3.3, which in turn possibly affects the nature of the minimizers. The effects on the minimizers will be analyzed in Section 5.2 .2 .

Note also that the planarity assumption allows nearly rigid cylinders to be decomposed into strips in the same way as perfectly rigid cylinders.

Remark 3.1.3. It is not strictly necessary to require that all faces are triangles. Rather, the subsequent choice of parametrization (in Section 3.3) remains exactly the same for arbitrary flat polygonal faces and the only adjustment to the actual implementation concerns computing the vertex coordinates from the parametrization. However, for the sake of simplicity, we only present the case of triangular faces.

To each nearly rigid origami we associate a model elastic energy in the following way: 
Definition 3.1.4. 1. A cluster of vertices is the collection of images of a single vertex in the fold pattern under the embeddings of all its incident faces and thus a set of vertices in $\mathbb{R}^{3}$. The set of all vertices of the nearly rigid origami can be partitioned into disjoint clusters.

2. The elastic strain of a nearly rigid origami cylinder is defined as follows. Partition the embedded vertices of the cylinder into clusters $\mathcal{C}_{i}:=\left\{v_{i, 1}, \ldots, v_{i, n_{i}}\right\}$. For each cluster, compute the average $m_{i}:=\frac{1}{n_{i}} \sum_{j} v_{i, j}$. The elastic energy is then defined by

$$
E\left(v_{i}\right):=\sum_{i} \sum_{j=1}^{n_{i}}\left|v_{i, j}-m_{i}\right|^{2} .
$$

The aim of this model remains to describe actual deformations of elastic thin-walled cylindrical shells. To this end, we begin with a reference embedding: a closed nearly rigid cylinder and fold pattern that - if the faces are rejoined into a single polyhedral cylinder-satisfy the Definition 2.2.2 of a perfectly rigid origami cylinder.

We then represent axial compressions of this reference cylinder by a family of nearly rigid cylinders of its underlying fold pattern whose cylinder heights match the prescribed compression. Moreover, for a given point in time, we require that the nearly rigid cylinder is a local minimum of the energy 3.1.1 in the space of all nearly rigid cylinders with the given fold pattern and cylinder height.

In practice, we perform a time-discrete compression with uniform compression. For each time step, we find a local minimum by solving a constrained minimization problem: we characterize valid embeddings of the underlying fold pattern as tuples of vertex coordinates satisfying constraints enforcing isometry of faces, planarity of vertex layers, and the correct cylinder height. Furthermore, we add certain inequality constraints that need to be satisfied in order to avoid unphysical effects such as eversion of the cylinder walls. We then minimize the energy as function of the vertex coordinates over this feasible set. More details on the nature of the constraints can be found in Section 3.3.

Although certain simple fold patterns such as the Guest and Pellegrino type [26] admit an exact solution to the minimization problem (presented in Section 3.2), this minimization is generally only approachable by numerical methods. In this thesis, we use an iterative descent method, which will be described in more detail in Section 3.3, while details of the implementation are presented in Chapter 8 .

\subsection{Analytic solution in special cases}

The class of fold patterns introduced by Guest and Pellegrino [26] allows finding an exact solution to the energy minimization problem if one makes a simplifying assumption on the 

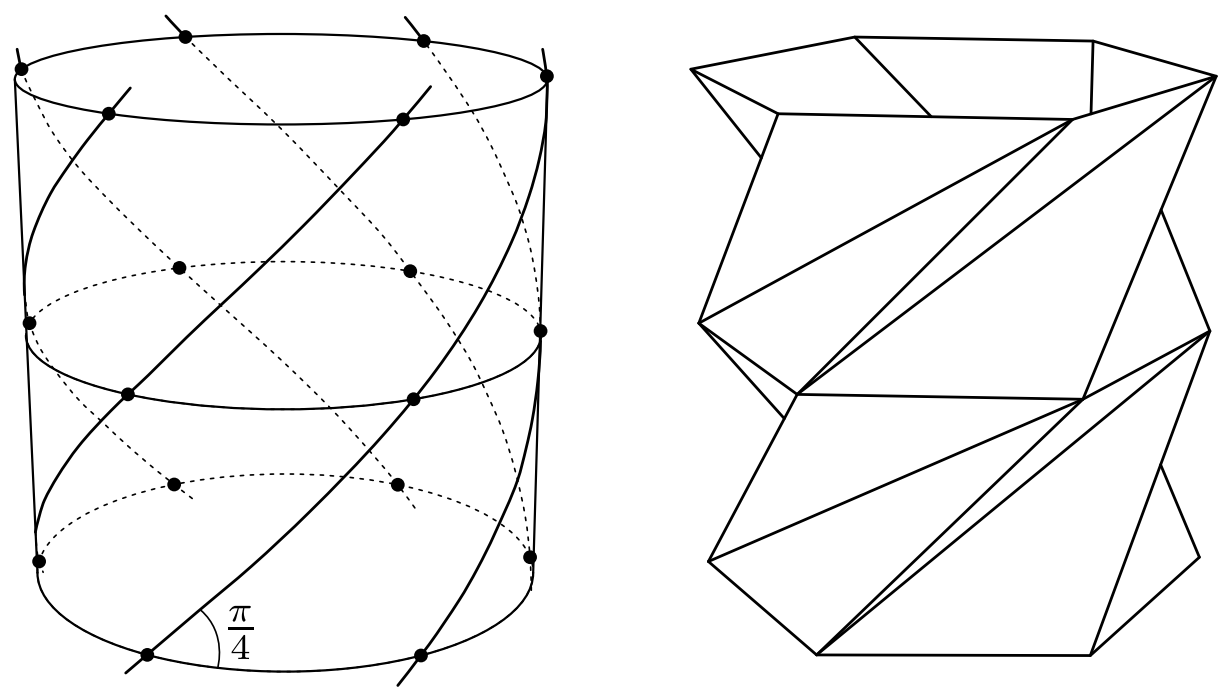

FIGURE 3.2: Illustration of the construction process for rotationally symmetric fold patterns, $n=$ $6, m=2$. Left: a cylinder is covered with helices of inclination $\pi / 4$; vertices are helical points of integer height. Right: the vertices are connected via straight lines and planar faces are filled in.

nature of the compression, which is that each strip and the cylinder as a whole maintains its original $n$-fold rotational symmetry throughout the compression. This assumption is motivated by the fact that the fold pattern itself is periodic, and its validity is tested in Chapter 5 by comparing the results obtained in this section with the unrestricted minimizers found numerically.

This class of fold patterns and their initial closed embedding is constructed from three parameters $r, n, m$ as follows. The positions of each vertex in the initial closed embedding are determined as depicted in Figure 3.2: on a cylinder of radius $r$ and vertical axis of symmetry, mark $n$ vertices in the $z=0$ plane that form a regular $n$-gon. Through these vertices draw $n$ helices of arbitrarily chosen inclination $\pi / 4$, parametrized by $t \mapsto$ $(\cos t+2 \pi i / n, \sin t+2 \pi i / n, t)$. All vertex positions are then given by the intersection of these helices with the planes $z=0,1,2, \ldots, m$, to the effect that each strip has embedded height 1 . Finally, connect vertices by straight edges, whose lengths then determine the fold pattern's final form. As Guest and Pellegrino have shown, this procedure generates a rigid origami cylinder in the sense of Chapter 2 with a fold pattern consisting of $2 \mathrm{~nm}$ congruent triangular faces that are arranged in a periodic truss-like configuration, see Figure 3.3. The corresponding nearly rigid origami (with separated faces) can be analyzed directly.

\subsubsection{Single strips}

For single strips, the assumption of rotational symmetry and the fact that all vertices have a fixed prescribed height allows for a reparametrization of the set of feasible embeddings to a given height $H$. 


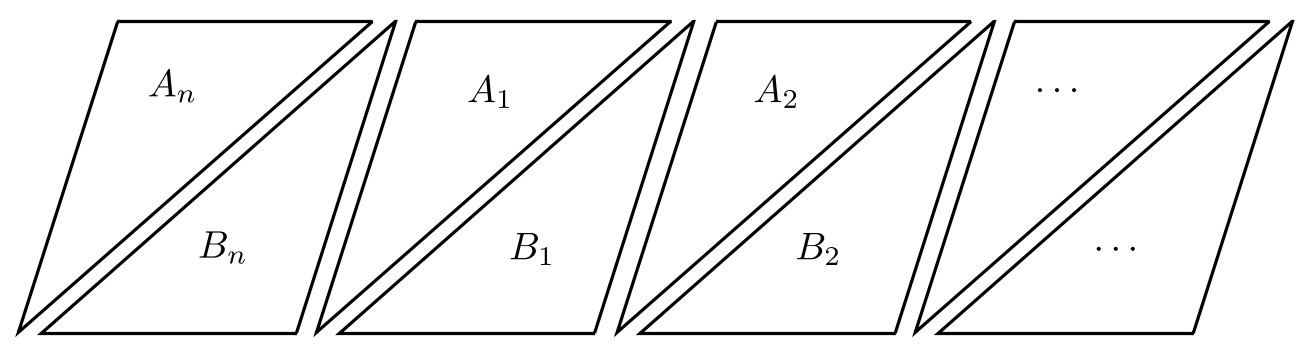

FIGURE 3.3: Exploded fold pattern of a single Guest and Pellegrino strip.

Because of rotational symmetry, the entire embedding is defined by the embedding of only two triangles, labelled as $A_{1}$ and $B_{1}$ in Figure 3.3, from which all other triangles are obtained as rotated and translated copies.

Any embedding of the first two triangles $A_{1}, B_{1}$ is characterized by three parameters in the following way. Let $H$ be the strip's embedded height. Up to rigid body motions, $A_{1}$ can only be embedded in two different ways, depending on the direction of the triangle's tilt. Since the direction of tilt cannot change during the compression procedure, we pick the one that tilts in the same direction as the initial cylinder's face.

Starting from the embedding of $A_{1}$, we construct, using techniques from the rigid origami model, a reference embedding for $B_{1}$ that is connected to $A_{1}$ at the joint edge. Again there are two such embeddings and we pick the one that coincides with the initial cylinder. The actual embedding of $B_{1}$ is then characterized by a rotation about the vertical axis by an angle $\alpha$ and a horizontal translation by $(x, y, 0)$ of this reference embedding.

Because of rotational symmetry, the next pair of triangles $A_{2}, B_{2}$ is obtained by rotating a copy of $A_{1}, B_{1}$ by $2 \pi / n$ about the vertical axis through $A_{1}$ and translating horizontally by $\left(d_{x}, d_{y}, 0\right)$. Likewise, all subsequent pairs $A_{k}, B_{k}$ are obtained iteratively by rotating a copy of $A_{k-1}, B_{k-1}$ by $2 \pi / n$ about $A_{k-1}$ and translating by $R^{2 \pi(k-1) / n}\left(d_{x}, d_{y}, 0\right)$. Altogether, the four positional parameters $p=\left(x, y, d_{x}, d_{y}\right)$ and the angular parameter $\alpha$ define the entire embedding of the single strip.

The elastic energy expressed in these five parameters can be written as a positive definite quadratic form

$$
E(p, \alpha)=\frac{1}{2} p^{T} A p+p^{T} b(\alpha)+c(\alpha)
$$

where the entries of $A \in \mathbb{R}^{4 \times 4}$ do not depend on $\alpha$ and $b, c \in \mathbb{R}^{4}$ depend linearly on $\cos \alpha$ and $\sin \alpha$. Its minimum can thus be computed variable-wise.

For fixed $\alpha$, the energy is a quadratic function in $p$, whose unique minimizer $p$ is given by $p_{\min }(\alpha)=-A^{-1} b(\alpha)$.

To conduct the final minimization with respect to the remaining variable $\alpha$, note that the univariate function $\tilde{E}(\alpha)=E\left(p_{\min }(\alpha), \alpha\right)$ is of the form $\tilde{E}(\alpha)=k_{0}+k_{c} \cos \alpha+$ $k_{s} \sin \alpha$. An easy computation shows that the global minimum of this function is $E_{\min }=$ $k_{0}-\sqrt{k_{c}^{2}+k_{s}^{2}}$ at $\alpha$ given by $\tan \alpha=k_{c} / k_{s}$ : up to the constant term $k_{0}, \tilde{E}(\alpha)$ is the dot product $\left\langle\left(k_{c}, k_{s}\right),(\cos \alpha, \sin \alpha)\right\rangle$, which is minimal if the two vectors are antiparallel. 
The minimal energy $E_{\min }$ is now purely a function of $H$ and the fold pattern. Figure 3.4 shows two examples of such functions for different values of $r$ and $n$.
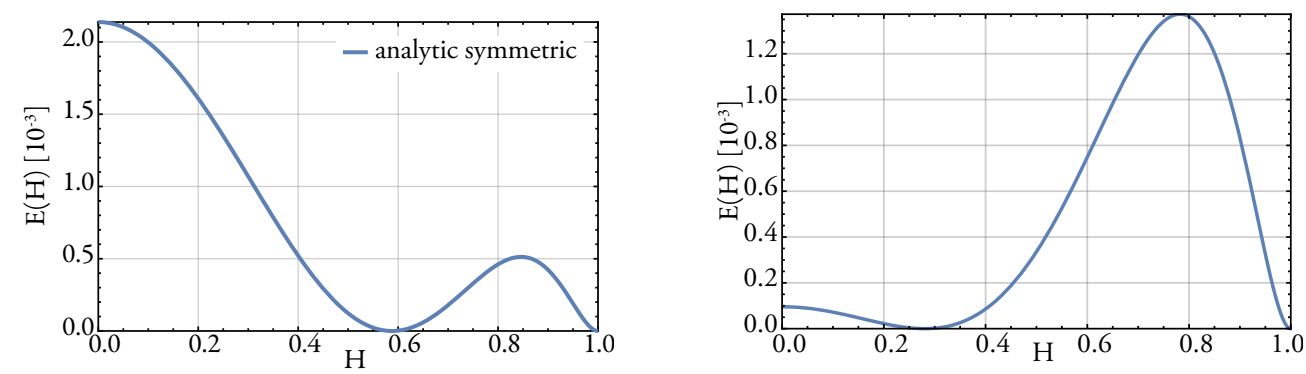

FIGURE 3.4: Two energy functions for different Guest and Pellegrino strips. Left: $r=1, n=8$. Right: $r=1.1, n=7$. In both cases, the initial height is $H=1$.

\subsubsection{Multiple strips}

A stack of $m$ strips can be parametrized in a similar fashion. The assumption of planar vertex layers allows to parametrize each strip individually, resulting in five parameters per strip, plus $m-1$ independent parameters $t_{i}$ that describe each strip's height (the $m$ strip heights are coupled by the condition that their total height equals $H$ ) and another $m-1$ parameters $\beta_{i}$ describing the relative rotation of adjacent strips.

In principle, minimization can again be split into separate steps: for each set of strip heights $t_{i}$ and angles $\beta_{i}$, minimize the energy with respect to each individual strip's embedding, and then minimize with respect to the remaining parameters.

An important special subset of embeddings are the ones where all strips have the same height and embedding, which we denote to be the symmetric state. In that case, the specific nature of the Guest and Pellegrino fold pattern allows the strips to be rotated such that their boundaries align perfectly, to the effect that the cylinder's energy is $m$ times the single strip energy. This implies that the number of degrees of freedom is the same as in the single strip case, and finding the exact minimizer is then simply a repetition of the steps done in the preceding section: first minimize with respect to $x, y, d_{x}, d_{y}$ and then minimize the resulting expression with respect to $\alpha$.

However, the solution strategy used for the single strip case is not applicable for multiple strips not in the symmetric state. While it is still possible to eliminate the positional variables $\left(x_{i}, y_{i}, d_{x, i}, d_{y, i}\right)$ as before, the resulting function is a multilinear trigonometric polynomial of degree 3 in the remaining variables $\alpha_{i}, \beta_{j}$ and does not admit an easy solution as in the single strip case. Nevertheless, numerical minimization of this function will provide reference results for the evaluation of the exponential descent method in Section 5.1.1.

Remark 3.2.1. If the elastic energy did not couple the different strips, the multi-strip case would be simpler: for each individual strip, find the optimal embedding as in the preceding section, depending on the strip's height. Then if $E_{\min }\left(t_{i}\right)$ is the energy of the $i$-th strip, 
the total energy is given by $\sum_{i=1}^{m} E_{\min }\left(t_{i}\right)$, where $H=\sum_{i=1}^{m} t_{i}$, and only needs to be minimized over the $t_{i}$. This model can be used to describe qualitatively the compressive behaviour of multi-layered strips while remaining accessible to analytic treatment. A more detailed discussion of this model of separate strips will be done in Section 3.4.

\subsection{Parametrizing the constraint manifold}

We now return to general fold patterns and embeddings, for which we solve the attendant minimization problem numerically. As the first step, we analyze the problem's feasible set. Recall that minimization takes place subject to equality constraints enforcing isometry of faces and planarity of vertex layers, and inequality constraints ensuring that no part of the cylinder "flips," i.e., everts, by requiring that each strip sub-cylinder has nonnegative height. These constraints will now be laid out in detail.

The degrees of freedom of a nearly rigid origami pattern are its 3 degrees of freedom per vertex. In order to establish a concise notation, we order vertices by layers as follows: if the fold pattern consists of $m$ strips, then there are $m+1$ vertex layers, with layer $i$ consisting of $n_{i}$ vertices, labelled $v_{i, 1}, \ldots$ (with coordinates $\left(x_{i, j}, y_{i, j}, z_{i, j}\right)$ ).

The constraints then take on the following form: isometry constraints are of the form $m_{k}(p)=\left|v_{i_{1, k}, j_{1, k}}-v_{i_{2, k}, j_{2, k}}\right|^{2}-l_{k}^{2}$ for every edge between two vertices $v_{i_{1, k}, j_{1, k}}$ and $v_{i_{2, k}, j_{2, k}}$. The planarity constraints are such that for each $i, z_{i, 1}=z_{i, 2}=\cdots=: z_{i}$. Finally, the boundary constraints set $z_{0}=c_{l o}, z_{m}=c_{h i}$ to given values $c_{l o / h i}$, and the inequality constraints are $b_{i}(p)=z_{i}-z_{i-1}=: t_{i} \geqslant 0$ for $i=1, \ldots, m$. Note that the two boundary heights $c_{l o}, c_{h i}$ change with time, such that each time step requires minimization of the same target function over a slightly different feasible set.

Altogether, the set of all feasible points forms a bounded embedded submanifold with boundary of the ambient space $\mathbb{R}^{9 N}$ :

Theorem 3.3.1. The set

$\mathcal{F}:=\left\{p \in \mathbb{R}^{9 N} \mid m_{k}(p)=0 \forall k=1, \ldots, E:=\#\right.$ edges $\left., b_{i}(p) \geqslant 0 \forall i=1, \ldots, m\right\}$

forms an embedded submanifold of $\mathbb{R}^{9 N}$ as long as the cylinder height is positive.

Proof. Let $p \in \mathcal{F}$. Let $g_{k}=\nabla m_{k}(p)$ be the equality constraint gradients and $h_{i}=$ $\nabla b_{i}(p)$ be the gradients of the active inequality constraints, that is, those where $b_{i}(p)=0$. By the implicit function theorem, it is sufficient to show that the set of vectors $\left\{g_{k}\right\} \cup\left\{h_{i}\right\}$ is linearly independent, since in that case 0 is a regular value of the differential of the constraints.

Suppose for contradiction that the vectors are linearly dependent, so that there exist $\lambda_{k}, \mu_{i} \in \mathbb{R}$ with $\sum_{k} \lambda_{k} g_{k}+\sum_{i} \mu_{i} h_{i}=0$. Out of these linear equations, consider first 
those corresponding to partial derivatives along $x_{i, j}$ and $y_{i, j}$ for any $i, j$. It is clear that for each vertex $v_{i, j}$, the only constraints affecting its horizontal coordinates are the three isometry conditions of the face containing that vertex. A simple calculation then shows that the only solution to this subsystem of linear equations is that the three $\lambda_{k}$ corresponding to the face's edges are zero unless the triangle is degenerate. By repeating this argument for every $i, j$, it follows that all $\lambda_{k}$ must be zero.

On the other hand, since $h_{i}=e_{z_{i}}-e_{z_{i-1}}$, the $(m+1) \times m$ matrix $H=\left(h_{i}\right)$ of all inequality constraint gradients is a band matrix and thus has full rank $m$. In other words, the set $\left\{h_{i}\right\}$ is linearly independent as long as not all inequality constraints are active at the same time, that is, not all strips are compressed flat.

\subsubsection{Planar vertex layers}

We now describe a global parametrization of this manifold. Given a reference cylinder $p_{0} \in$ $\mathcal{F}$, each point in $\mathcal{F}$ consists of a collection of triangles, each of which is congruent to a triangle of $p_{0}$. Since two congruent triangles differ by a three-dimensional rigid body motion, all of $\mathcal{F}$ can be described as (a subset of) the orbit of $p_{0}$ under a left action of the Lie group of rigid body motions. The details of this will be presented in the following section. For the purpose of simplicity, we will first disregard the inequality conditions and present a parametrization of points satisfying only the equality constraints, which is the rigid body motions' entire orbit. In a second step, we will then detail how to incorporate the inequality constraints.

The reference point While $p_{0}$ can in principle be chosen arbitrarily, we choose it in such a way that it is heuristically close to the previous time step's minimum (or the reference embedding for the first time step) $p_{\text {prev }}$. As $p_{\text {prev }}$ lies on the previous time step's constraint manifold, we project it onto the new $\mathcal{F}$ by reducing the value of $c_{h i}$ (and thus $z_{m}$ ) by the amount of compression $\Delta H$, followed by rotating each triangle in the topmost strip about its horizontal edge such that the value of $z_{m-1}$ does not change. Should the topmost strip be already compressed such that $z_{m}-z_{m-1}<\Delta_{H}$, $z_{m}$ is set to $z_{m-1}$ and the excess height change is applied to the next strip. This adjustment is repeated until the full change of height has been distributed. The effect of this method is that, expecting a time-continuous family of minimizers, the reference embedding, which will also be used as the initial guess for the subsequent minimization, is already close to a local minimum of the new constraint manifold.

Parametrizing rigid body motions The planarity assumption suggests to parametrize first the different vertex layers' $z$ coordinates. Let $m$ be the number of strips, such that there are $m+1$ vertex layers. For a given point $p \in \mathcal{F}$, we set for each of the $m-1$ non-boundary 
layers their common $z$ coordinate $z_{i}, i=1, \ldots, m-1$ as one of the parameters; the two boundary layer coordinates $z_{0}, z_{m}$ are fixed. Altogether, we obtain as the set of possible values $T:=\left\{z \in \mathbb{R}^{m-1} \mid z_{i}-z_{i-1} \in\left[0, h_{i}\right], i=1,2, \ldots, m\right\}$, where $h_{i}$ is the material height of the $i$-th strip.

Then, since the remaining equality constraints do not couple vertices of different triangles, each triangle of $p$ can be parametrized individually as a rigid body motion acting on the given reference embedding.

Parametrizing the group of rigid body motions of a single triangle is done using the following observation: for a given $z$ vector $\left(z_{1}, \ldots, z_{m-1}\right)$, each triangle has all its vertices' $z$ coordinates fixed, so that it can only be moved horizontally. Accordingly, the valid rigid body motions consist of a rotation of the reference triangle about the vertical axis and a horizontal translation, parametrized by an element of $\mathbb{R}^{2} \times S^{1}$. This product manifold can be endowed with a group structure in two different ways:

In the usual extrinsic viewpoint, an element $(d, \phi)$ would act as an affine transformation $x \mapsto R^{\phi} x+d$ (where $R^{\phi} \in S O(3)$ is the matrix rotating by $\phi$ about the vertical axis) of the ambient space $\mathbb{R}^{3}$ on each vertex of a triangle, with the resulting group structure being that of the semidirect product $\mathbb{R}^{2} \rtimes S^{1}$.

The other intrinsic viewpoint exchanges simplicity of the group action for simplicity of the group structure. Here, a pair $(d, \phi)$ acts on triples of vertices $\left(v_{1}, v_{2}, v_{3}\right)$ (i.e., on entire faces) by rotating about a body-fixed anchor. Although any anchor will result in the direct product group structure, we will adopt the convention of rotating about the vertical axis centered at the triangle's single horizontal edge. Thus, if the vertices are labelled such that the edge $v_{1} v_{2}$ is horizontal,

$$
\begin{aligned}
(d, \phi)\left(v_{1}, v_{2}, v_{3}\right) & :=\left(R^{\phi}\left(v_{1}-m\right)+m+d, \ldots\right), \\
m & :=\frac{v_{1}+v_{2}}{2} .
\end{aligned}
$$

Lemma 3.3.2. Concatenating two such rigid body motions results in the product group structure on $\mathbb{R}^{2} \times S^{1}$.

Proof. The claim can be verified using direct computation.

The parametrizing groups have even more structure: they are also smooth manifolds and thus Lie groups. Therefore, the following holds:

Theorem 3.3.3. The global constraint manifold $\mathcal{F}$ can be parametrized as a subset of the orbit of a Lie group action by the group

$$
G=\mathbb{R}^{m-1} \times \underset{i=1}{X}\left(\mathbb{R}^{2} \times S^{1}\right),
$$

where $N$ is the number of triangles and $m+1$ is the number of vertex layers. Elements of $G$ 
given by $\left(t, d_{1}, \phi_{1}, d_{2}, \phi_{2}, \ldots, d_{N}, \phi_{N}\right)$ act on elements of $\mathcal{F}$ by to the following procedure:

1. Vertically translate and rotate about the horizontal edge all triangles until the vertex layers' $z$ coordinates have changed by $t$.

2. Rotate each triangle by $\phi_{i}$ about the vertical axis through the horizontal edge midpoint and translate horizontally by $d_{i}$.

Note that this definition of the group action relies on the fact that every triangular face has precisely one horizontal edge - a consequence of the requirement in Definition 3.1.1 that the fold pattern is split into strips.

If one discards the planarity of vertex layers, the parametrization of feasible points changes. While it is still true that the new feasible set can be parametrized by one rigid body motion per face, this parametrization will depend on the number of boundary vertices in this face. The details of this parametrization will be laid out in the next section.

\subsubsection{Nonplanar layers}

We now briefly describe the minimization if the assumption of planarity of vertex layers is dropped. In this case, a nonplanar nearly rigid origami cylinder is simply facewise affine isometric embedding of a given fold pattern such that the boundary vertices lie in two horizontal planes. As before, compression is simulated via numerical minimization of the elastic energy (3.1.1) subject to isometry and boundary constraints and a number of inequality constraints preventing inversion. To achieve this, we need two inequality constraints per face, defined as follows: recall that, since the fold pattern is split into strips, every face has an edge that is horizontal in the fold pattern and in the reference embedding. Let $v_{1}, v_{2}$ be this edge's vertices and $v_{3}$ the face's third vertex, and let $z_{i}^{(0)}$ be the corresponding $z$ coordinates in the initial embedding. We then use the two constraints $\left(z_{3}-z_{1}\right)\left(z_{3}^{(0)}-z_{1}^{(0)}\right) \geqslant 0$ and $\left(z_{3}-z_{2}\right)\left(z_{3}^{(0)}-z_{2}^{(0)}\right) \geqslant 0$, meaning that $v_{1}$ and $v_{2}$ can never cross the horizontal plane containing $v_{3}$. Additionally, we also require for all vertices $v_{i}$ that $c_{l o} \leqslant z_{i} \leqslant c_{h i}$.

As before, one can show that the feasible set is an embedded manifold, and it can again be parametrized by a rigid body motion per triangle.

However, parametrizing each triangle's set of rigid body motions now depends on the type of triangle. Because of the absence of the planarity assumption, only the boundary vertices have a fixed $z$ coordinate. Therefore, we distinguish triangles according to the number of boundary elements:

Triangles without boundary vertices The easiest case is that of triangles without additional constraints. In this case, the admissible rigid body motions are composed of a rotation $A \in S O(3)$ and a translation $b \in \mathbb{R}^{3}$, i.e., as an element of $S O(3) \times \mathbb{R}^{3}$. By using again the intrinsic group action, the correct group structure is indeed the direct product structure 
as opposed to the semidirect product. An element $(A, b)$ acts via translating by $b$ and then applying the rotation $A$, anchored at the triangle's center of mass.

Triangles with a single vertex on the boundary Triangles with a single vertex on the cylinder boundary are parametrized by the group $S O(3) \times \mathbb{R}^{2}$ : the triangle can have arbitrary orientation, but its single boundary vertex can only move horizontally. We therefore let an element $(A, b) \in S O(3) \times \mathbb{R}^{2}$ act on a triangle $\left(v_{1}, v_{2}, v_{3}\right)$ (where $v_{1}$ is the boundary vertex) via rotating with $A$ about $v_{1}$ and then translating horizontally by $\left(b_{1}, b_{2}, 0\right) \in \mathbb{R}^{3}$.

Triangles with one edge on the boundary Triangles with one edge on the cylinder boundary can be parametrized by the group $\mathbb{R}^{2} \times S^{1} \times S^{1}$. Here, the element $(d, \phi, \psi)$ acts on a triangle $\left(v_{1}, v_{2}, v_{3}\right)$ as follows: if $v_{1}, v_{2}$ are the two boundary vertices, then first rotate $v_{3}$ by $\psi$ about the boundary edge $v_{1}-v_{2}$ and then rotate by $\phi$ about the vertical axis through the boundary edge midpoint $\left(v_{1}+v_{2}\right) / 2$. Finally, translate horizontally by $d$. The group structure is the simple product and the different rotations by $\phi$ and $\psi$ are commutative.

Triangles with two vertices on different boundaries If two vertices $v_{1}, v_{2}$ of a triangle $\left(v_{1}, v_{2}, v_{3}\right)$ are on different boundaries, the parameter Lie group is again $\mathbb{R}^{2} \times S^{1} \times S^{1}$. If $v_{1}$ and $v_{2}$ are the boundary vertices, then an element $(d, \phi, \psi)$ acts by translating by $d$, rotating by $\phi$ about the vertical axis through $\left(v_{1}+v_{2}\right) / 2$, and then rotating $v_{3}$ by $\psi$ about the new axis $v_{1}-v_{2}$.

Triangles with all three vertices on both boundaries Finally, it could also be the case that all three vertices are height-constrained, but that the vertices belong to different boundaries. This is the same situation as in the planar case, so that the rigid body motions are parametrized by $\mathbb{R}^{2} \times S^{1}$.

Triangles where every vertex is on the same boundary The last remaining possibility is that all vertices of a triangle are on the same boundary. Although such triangles do not appear in practice, this case is mentioned here for the sake of completeness. Flat triangles can be parametrized by $\mathbb{R}^{2} \times S^{1}$ as they can only be moved horizontally and rotated about the vertical axis.

All of these group actions on $\mathbb{R}^{9}$ have very nice properties. In particular:

Lemma 3.3.4. All actions of the groups defined above are transitive, free, and smooth.

Proof. Transitivity is obvious since any two congruent triangles that satisfy the additional conditions are related via a rigid body motion represented by an element in the Lie group. Freeness is obvious since no two rigid body motions act alike. Smoothness of the action is a consequence of the fact that all actions are polynomial in its arguments. 
Theorem 3.3.5. Without the assumption of planarvertex layers, the global constraint manifold $\mathcal{F}$ can be parametrized as a subset of the product of Lie groups

$$
G=\underset{i=1}{\chi_{i}^{N}} G_{i},
$$

where each $G_{i}$ is either $S O(3) \times \mathbb{R}^{3}$ or $\mathbb{R}^{2} \times S^{1} \times S^{1}$ or $\mathbb{R}^{2} \times S^{1}$, depending on the constraints on the $i$-th triangle.

The nonplanar initial guess It remains to find a base point that will generate the group action orbit. Analogous to the planar case, we generate this point from the previous time step's minimum by performing the following steps:

1. All faces in the topmost strip are vertically shifted by the compression $-\Delta H$.

2. Then perform the following adjustments for each face to guarantee that the inequality constraints are all satisfied:

(a) faces that still satisfy all inequality constraints are unchanged.

(b) Faces where all vertices violate the inequality constraints are completely outside of the two boundary planes. Vertically translate these faces such that they touch the boundary plane, then rotate about the unique axis that is both horizontal and in the triangle's plane, anchored at the vertex touching the boundary plane, until all three vertices satisfy the inequality constraints.

(c) For triangles where only one or two vertices lie outside, we perform the same rotation about the horizontal axis formed by intersecting the triangle with the boundary plane. (If a triangle intersects both boundary planes, we pick one at random.)

\subsubsection{Basics of Lie groups}

The great advantage of parametrizing $\mathcal{F}_{1}$ via a Lie group is the availability of the Lie group's exponential map. Loosely speaking, it allows to map tangent vectors to the underlying manifold in a way that connects the group structures of tangent space and Lie group itself. In this section we will briefly introduce the notion of Lie groups and the exponential map and only refer to the literature for a more thorough introduction to the subject, for instance to [29].

Definition 3.3.6. 1. A Lie group is a group $G$ which is also a smooth manifold such that both group multiplication $m: G \times G \rightarrow G$ and inversion $i: G \rightarrow G$ are smooth maps.

2. For $g \in G$, the maps $R_{g}: G \rightarrow G, h \mapsto h g$ and $L_{g}: G \rightarrow G, h \mapsto g h$ are called right and left multiplication maps, respectively, and are all diffeomorphisms. 
A central object in the study of Lie groups are its tangent spaces. Because of the presence of the left and right multiplication maps, all tangent spaces can be canonically identified, to the effect that it suffices to consider the group's Lie algebra:

Definition 3.3.7. Let $G$ be a Lie group and $e \in G$ be the neutral element. The tangent space $T_{e} G$ is called the Lie algebra $\mathfrak{g}$ of $G$.

Remark 3.3.8. We defined the Lie algebra as a mere vector space. Textbooks on Lie groups and algebras also define a Lie bracket on this vector space, turning it into a proper Lie algebra (hence the name), but this extra structure is irrelevant to the purpose at hand.

Definition 3.3.9. Let $X$ be a smooth vector field on $G$. $X$ is said to be right-invariant if it commutes with the right multiplication differential: for all $g, h \in G$, it holds that

$$
X_{g h}=\left(d_{g} R_{h}\right)\left(X_{g}\right)
$$

Left-invariant vector fields are defined analogously.

We use the notion of invariant vector fields to define the exponential map. First, they are in one-to-one correspondence with $\mathfrak{g}$ :

Theorem 3.3.10. The Lie algebra $\mathfrak{g}$ is isomorphic to the set of right-invariant vector fields. It is furthermore isomorphic to the set of one-parameter subgroups, that is, subgroups $H$ of $G$ for which there exists a continuous group homomorphism from $(\mathbb{R},+)$ into $H$.

Proof. The first isomorphism is trivial: invariance uniquely gives $X_{g}=d_{e} R_{g}\left(X_{e}\right)$ for all $g \in G$. The second isomorphism is given by the flows of invariant vector fields, see e.g. [47, Proposition 5.3.1] and the subsequent remarks.

Corollary 2. For all $v \in \mathfrak{g}$, there exists a unique homomorphism of groups $\theta_{v}$ of $\mathbb{R}$ into $G$ such that $\theta_{v}^{\prime}(0)=v$.

The exponential map is the map identifying the one-parameter subgroups of $\mathfrak{g}$, which are given by one-dimensional subspaces, with the one-parameter subgroups of $G$.

Definition 3.3.11. The exponential map of $G$ is the map

$$
\exp : \mathfrak{g} \rightarrow G, v \mapsto \theta_{v}(1),
$$

where $\theta_{v}$ is the unique group homomorphism defined in Corollary 2. It is smooth and satisfies the addition theorem

$$
\exp (s+t) v=\exp (s v) \exp (t v)
$$

for all $v \in \mathfrak{g}$ and $s, t \in \mathbb{R}$. 
More general, the exponential map can be defined at arbitrary base points $g \in G$.

$$
\exp _{g}: \mathfrak{g} \rightarrow G, \exp _{g}(v):=\exp (v) g
$$

It can also be understood as a map from $T_{g} G$ to $G$ by precomposing it with the explicit isomorphism of $T_{g} G$ and $T_{e} G=\mathfrak{g}$ defined by $d_{g} R_{g^{-1}}$ :

$$
\begin{aligned}
\exp _{g} & =R_{g} \circ \exp \circ d_{g} R_{g^{-1}}, \\
\exp _{g}(v) & =\exp \left(d_{g} R_{g^{-1}} v\right) g .
\end{aligned}
$$

Theorem 3.3.12. The tangent vectors to the curves $t \mapsto \exp _{g}(t v)$ are given by

$$
\left.\frac{\mathrm{d}}{\mathrm{d} t}\left(\exp _{g}(t v)\right)\right|_{t=t_{0}}=d_{g} R_{\exp _{g}\left(t_{0} v\right)}(v)
$$

Proof. The claim follows from the fact that the curve $t \mapsto \exp (t v)$ is the integral curve to the right-invariant vector field $X$ with $X_{e}=v$.

Using the exponential map in an iterative descent method allows to map directly from the tangent space back to the constraint manifold instead of taking a linear step and then projecting back, see Figure 3.5. One obvious advantage of this method is that the next iteration is always located "close" to the base point, which is not guaranteed if the step-andproject method is applied carelessly.
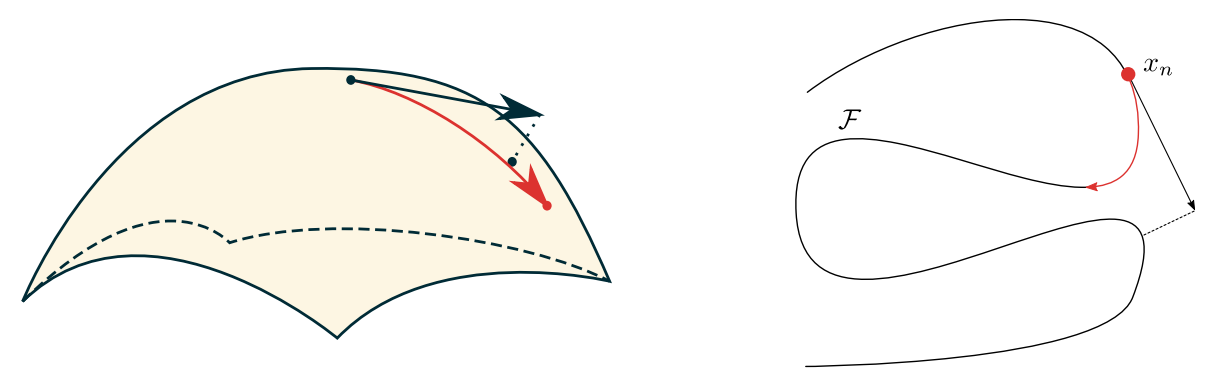

FIGURE 3.5: Left: taking exponential steps never leaves the constraint manifold. Right: step-andproject can make nonlocal steps for large stepsizes.

\section{The exponential map of $\mathcal{F}$}

In order to obtain the exponential map of the Lie group containing $\mathcal{F}$, we employ the following theorem about product Lie groups:

Theorem 3.3.13. Let $G, H$ be two Lie groups with Lie algebras $\mathfrak{g}, \mathfrak{h}$ and exponential maps $\exp _{G}, \exp _{H}$, respectively. Then the Cartesian product $G \times H$ is a Lie group whose exponential map and Lie algebra are given by the product structure: the algebra of $G \times H$ is $\mathfrak{g} \times \mathfrak{h}$ and 
the exponential map is given by $\exp _{G \times H}=\exp _{G} \times \exp _{H}$, such that for tangent vectors $\xi \in T_{g} G, \nu \in T_{h} H$

$$
\left(\exp _{G \times H}\right)_{(g, h)}(\xi, \nu)=\left(\left(\exp _{G}\right)_{g}(\xi),\left(\exp _{H}\right)_{h}(\nu)\right) .
$$

Therefore, the exponential map of the Lie group $\mathcal{F}_{1}$ is the product map of the exponential maps of $\mathbb{R}^{k}, S O(3)$, and $S^{1}$.

Proof. See e.g. [47, Example (5.3.16)].

The exponential maps of the constituent Lie groups are well-known and only stated here for completeness:

Theorem 3.3.14. $\quad$ 1. The Lie algebra of $\mathbb{R}^{k}$ is $\mathbb{R}^{k}$ itself and the exponential map of $\mathbb{R}^{k}$ is $\exp _{p}(v)=p+v$.

2. The Lie algebra of $S O(3)$ is the space $\mathfrak{s o}(3)$ of $3 \times 3$ skew-symmetric matrices. For $A \in S O(3)$, any $v \in T_{A} S O(3)$ is of the form $\eta A, \eta \in \mathfrak{s o}(3)$ and the exponential map is given by $\exp _{A}(\eta A)=e^{\eta} A$, where $e^{\eta}$ is the usual matrix exponential, realized here via Rodrigues' rotation formula

$$
e^{\eta}=\operatorname{Id}_{3}+\sin (\theta) \cdot \eta+(1-\cos (\theta)) \cdot \eta^{2},
$$

where $2 \theta=\sum_{i, j=1}^{3} \eta_{i j}^{2}$.

3. The Lie algebra of $S^{1}$ is $\mathbb{R}$ and the exponential map is given by $\exp _{\phi}(t)=\phi+t$ $\bmod 2 \pi$.

Remark 3.3.15. In general, the exponential map could be defined using either left-invariant or right-invariant vector fields, which results in different maps for noncommutative Lie groups. Since the Lie group in question acts via a left action on the set of feasible points, we choose to define the exponential map via right-invariant vector fields to obtain the proper group action.

\subsubsection{Incorporating the inequality constraints}

Having parametrized the entire space of rigid body motions by a suitable Lie group, adding the inequality constraints means restricting the parameter space to a submanifold (with boundary) of the Lie group, which does not need to be a subgroup. This fact brings two complications: first, on the boundary, the tangent directions no longer form a linear tangent space, but rather a tangent cone, requiring more effort to produce a feasible descent direction. A method for efficiently finding such a suitable direction will be presented in Section 4.1 .3 . 
Second, the exponential map is no longer defined for tangent vectors of arbitrary length, putting another limit on the maximal step size. The line search method detailed in Section 4.2 thus not only checks if the candidate step length is suitable for the purpose of the minimization, but also if the step's result is located in the feasible set.

Finally, note that cutting away a part of the Lie group means potentially creating boundary minima: if the current iterate lies on the boundary and the chosen descent direction is tangent to the boundary, it might be that no positive feasible step size exists. While these boundary minima are a natural part of the chosen model, they can possibly impact the numerical efficiency: sliding along the boundary towards a minimum will require very small step sizes and thus many iterations of the descent process.

\subsection{The simple energy model}

As established in section 3.2, the nearly rigid origami model is only accessible numerically even for simple and regular fold patterns. To complement the numerical computations detailed in Chapter 4 , we implement in this section a slight modification that greatly reduces the model's complexity: instead of considering the full elastic energy as introduced above, treat the cylinder as a stack of independent strips.

This directly gives an expression for the energy function of several strips: let $f(t)$ be the energy of a single strip of height $t$. Then a stack of identical strips of heights $t_{i}$ has a cumulative energy of $g\left(t_{1}, \ldots, t_{m}\right)=\sum_{i} f\left(t_{i}\right)$. Fixing the cylinder's height to a value $x$ corresponds to restricting the vector $\left(t_{1}, \ldots, t_{m}\right)$ to the set

$$
D_{m}(x):=\left\{\left(t_{1}, \ldots, t_{m}\right) \mid 0 \leqslant t_{i} \leqslant h, \sum t_{i}=x\right\},
$$

reflecting the constraint that strips cannot turn inside out $\left(t_{i} \geqslant 0\right)$ or extend beyond their material height $\left(t_{i} \leqslant h\right)$. Note that in contrast to the full model, it is not necessary to exclude the extremal case $t_{i}=h$.

Analogously to the nearly rigid origami model, the cylinder of fixed height $x$ will adjust the values of $t_{i}$ to a (local) minimum of $g$.

To be precise, we say that the strip heights form a trace:

Definition 3.4.1. A trace is a function $t(x)=\left(t_{1}(x), \ldots, t_{m}(x)\right)$ of the cylinder height $x$ such that the following conditions are satisfied:

1. For $x$ near the initial height $m, t_{i}(x)=x / m$ for all $i$ : compression starts symmetric.

2. For all $x, t(x)$ is a local minimum of $g$, either in the interior or on the boundary.

3. $t$ is lower semicontinuous with finitely many points of discontinuity. At these points, the lower limits satisfy the following property: if $x_{0}$ is a point of discontinuity and 


$$
\begin{aligned}
t_{0}=t\left(x_{0}\right), t_{1} & =\lim _{x \searrow x_{0}} t(x), \text { then } \\
& \partial_{t} g\left(t, x_{0}\right) \cdot\left(t_{1}-t_{0}\right)<0 \quad \forall t \text { between } t_{0} \text { and } t_{1} .
\end{aligned}
$$

The intuition behind this definition is the following: at height $x_{0}$, the cylinder state $t\left(x_{0}\right)$ is a local minimum of the function $g$. If $x_{0}$ is decreased by a small amount $\varepsilon$, the energy landscape changes slightly. If the local minimum persists, the cylinder (and the function $t$ ) will follow that minimum. If, however, the local minimum disappears, for instance because it turns into a saddle point, the cylinder will immediately descend to the nearest minimum. This behaviour mimics that of the minimization by iterative descent method employed for the full energy minimization.

Finding traces is equivalent to characterizing the set of local minima of the function $g$ on the set $D_{m}(x)$ with varying $x$. Local minima can arise in two different ways: they are either critical points or-if $m=2$, such that $D_{m}(x)$ is a line segment-they can be boundary minima.

At least one critical point must always exist:

Lemma 3.4.2. The symmetric point $t_{s}(x)=(x / m, \ldots, x / m)$ is a critical point of $g$ for all values of $x$.

Proof. At $t_{s}$, all entries of the gradient $\nabla g$ are identical. But the vector $(1, \ldots, 1)$ is perpendicular to the set $D_{m}(x)$, so that $\left.g\right|_{D_{m}(x)}$ is minimal at $t_{s}$.

Furthermore, there exists a simple condition for the symmetric configuration being a local minimum:

Lemma 3.4.3. The point $t_{s}$ is a local minimum of $g$ if $f^{\prime \prime}(x / m)>0$. It is a local maximum if $f^{\prime \prime}(x / m)<0$.

Proof. Fix $x$. In order to obtain the intrinsic constrained Hessian, reparametrize $g$ by replacing $t_{m}=x-\sum_{i=1}^{m-1} t_{i}$. Then the function $g$ depends only on $m-1$ variables which are locally unconstrained near $t_{s}$. In these modified coordinates, the Hessian is the usual matrix of second partial derivatives and reads

$$
H_{m}:=\operatorname{Hess} g=f^{\prime \prime}\left(\frac{x}{m}\right) \cdot\left(C_{m-1}+\operatorname{Id}_{m-1}\right),
$$

where $\operatorname{Id}_{m-1}$ is the $(m-1) \times(m-1)$ identity matrix and $C_{m-1}$ is the $(m-1) \times(m-1)$ matrix whose entries are all equal to 1 . The eigenvalues of $H_{m}$, as computed in the following Lemma 3.4.4, show the claim.

Lemma 3.4.4. The eigenvalues of $H_{m}$ are given by $f^{\prime \prime}(x / m)$ with multiplicity $m-2$ and $m f^{\prime \prime}(x / m)$ with multiplicity 1 . 
Proof. An eigenbasis is given by the vectors $(1, \ldots, 1)$ to the eigenvalue $m f^{\prime \prime}(x / m)$ and by the vectors $\{(1,-1,0, \ldots, 0),(0,1,-1,0, \ldots, 0), \ldots,(0, \ldots, 0,1,-1)\}$ to the eigenvalue $f^{\prime \prime}(x / m)$.

The other minima need to be found via a more general different method: the set of critical points is exactly the zero set of the gradient function $\nabla g$ and thus an algebraic variety. As such, it is locally a submanifold, with isolated points at which more complicated phenomena such as branching out into different directions occur. In order to describe the procedure for analyzing the set of minima in detail, we consider the simplest nontrivial case of $m=2$ strips.

\subsubsection{The case $m=2$}

For only two strips, reparametrize $g$ to a function $g(t, x)=f(t)+f(x-t)$. For a fixed value of $x$, critical points of $g$ are given by zeros of the partial derivative $\partial_{t} g(t, x)=f^{\prime}(t)-$ $f^{\prime}(x-t)$ and minima are the subset of critical points where $\partial_{t}^{2} g(t, x)=f^{\prime \prime}(t)+f^{\prime \prime}(x-t)$ is positive.

In order to determine the shape of the set of critical points, we employ the following observation. Let $(t, x)$ be a critical point of $g$. First, as long as the gradient $\nabla\left(\partial_{t} g\right)$ is nonzero, the set of critical points is locally a manifold by the implicit function theorem. Such a manifold may "end" in two different ways: it can reach a point where the gradient becomes zero (which we call branch points), or it may end on the domain boundary. Furthermore, since we are mainly interested in slices $x=$ const, there exists an additional type of termination: minima and maxima can join and transform into saddle points which then disappear, which occurs when $\nabla\left(\partial_{t} g\right) \|(0,1)$ (which is equivalent to the point being a saddle point of the function $t \mapsto g(t, x))$. We will now discuss all three types of events.

\section{Branch points}

We begin with branch points. Let $\left(t_{0}, x_{0}\right)$ be a zero of $\nabla \partial_{t} g$ and let $s \mapsto(t(s), x(s))$ be a smooth parametrization of a curve of critical points starting at $(t(0), x(0))=\left(t_{0}, x_{0}\right)$. We first establish the possible tangent directions this curve can have.

Expand the function $p: s \mapsto \partial_{t} g(t(s), x(s))$ into a Taylor series around $s=0$ :

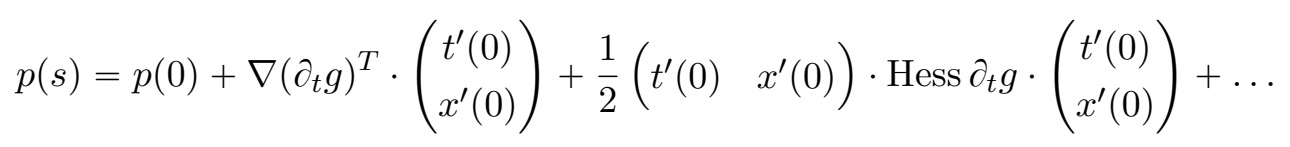

Since $\nabla \partial_{t} g\left(t_{0}, x_{0}\right)=0$, the first-order change is zero. Thus, possible tangent directions for the set of critical points are those directions where the next-higher order change, given by the Hessian if it is nonzero, vanishes. A necessary and sufficient condition for the existence 
of these directions is that the Hessian is neither positive definite nor negative definite, i.e., the determinant is negative.

Explicitly, the Hessian is given by

$$
\operatorname{Hess} \partial_{t} g=\left(\begin{array}{cc}
f^{\prime \prime \prime}(t)-f^{\prime \prime \prime}(x-t) & f^{\prime \prime \prime}(x-t) \\
f^{\prime \prime \prime}(x-t) & -f^{\prime \prime \prime}(x-t)
\end{array}\right)=\left(\begin{array}{cc}
a-b & b \\
b & -b
\end{array}\right),
$$

so its determinant is $-a b$. Let $(r, s)$ be a tangent vector such that $(a-b) r^{2}+2 b r s-b s^{2}=$ 0 . If $a=b$, then $s=0$ or $r=s / 2$, providing four different possible tangent directions; if $a \neq b$, then solving the quadratic equation gives two distinct real solutions for $r$, depending linearly on $s$, if and only if $a b>0$.

In both cases, there are two pairs of opposing possible tangent directions, meaning that in general, the set of critical points branches into four arms.

If, on the other hand, $a b<0$, then no possible tangent directions exist as the critical point is an isolated local minimum of $\partial_{t} g$. Finally, if $a=0, r=s$ follows and if $b=0$, $r=0$ follows, leaving only two possible tangent directions. In these cases, no branching occurs.

Remark 3.4.5. It is still possible to find tangent directions in the special case that the Hessian is the zero matrix by considering third (or even higher) order changes. This special case does not appear in practice, though, and will thus remain unaddressed at this point.

Remark 3.4.6. For the symmetric configuration $t=x / 2$, the Hessian satisfies $a=b$. The four tangent directions are therefore always given by $\pm(1,0)$ and $\pm(1,2)$ and in particular always constitute a branch point.

How are the branch points found? As $\nabla \partial_{t} g=\left(f^{\prime \prime}(t)+f^{\prime \prime}(x-t),-f^{\prime \prime}(x-t)\right) \stackrel{!}{=}$ $(0,0)$, both $f^{\prime \prime}(t)=0$ and $f^{\prime \prime}(x-t)=0$, as well as $\partial_{t} g(t, x)=0$, which implies $f^{\prime}(t)=f^{\prime}(x-t)$. Thus, in order to list all branching points, first find all zeros $z_{1}, \ldots, z_{k}$ of $f^{\prime \prime}$. These form all possible values for $t$. All possible values of $x$ are formed by sums $z_{i}+z_{j}$ with $f^{\prime}\left(z_{i}\right)=f^{\prime}\left(z_{j}\right)$. Then discard from this set of branch candidates those where the Hessian is not indefinite to obtain the set of proper branching points.

Remark 3.4.7. The conditions for branch points are such that in the general case, the only branch points are of the form $\left(z_{i}, 2 z_{i}\right)$.

Finally, we analyze which parts of the set of critical points can be minima. Since the second derivative $\partial_{t}^{2} g$ which determines the type of critical point is at the same time the first component of $\nabla \partial_{t} g$, it is clear that points on the same branch are either all maxima or all minima and that at a branching point, $\partial_{t}^{2} g$ is zero. (Recall that $\partial_{t}^{2} g=0$ indicates either a branch point if $\partial_{t} \partial_{t} g=0$ or a pair event if $\partial_{t} \partial_{t} g \neq 0$.)

This means that the type of critical point is determined by the lowest-order change of $\partial_{t}^{2} g$ along the four tangent directions originating at a branch point.

Let $(t, x)$ be a branch point. We consider first the case $t=x / 2$. Here, the first-order change of $\partial_{t}^{2} g$ along $\pm(1,0)$ is zero since $\nabla \partial_{t}^{2} g(x / 2, x)=\left(0, f^{\prime \prime \prime}(x / 2)\right)$. Similarly con- 


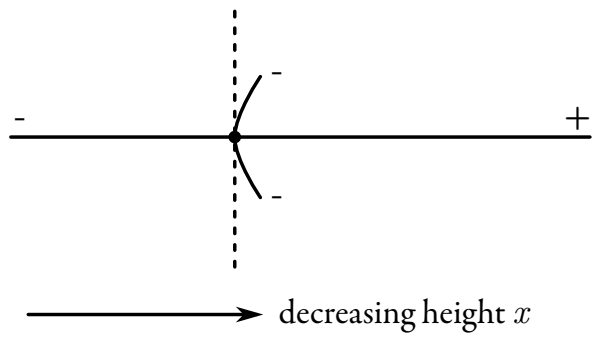

FIgURE 3.6: As $x$ decreases during the compression, the critical point changes its type. The label "-" indicates that the critical point is a minimum, the label "+" indicates a maximum. Horizontal lines correspond to constant ratio $t / x$.

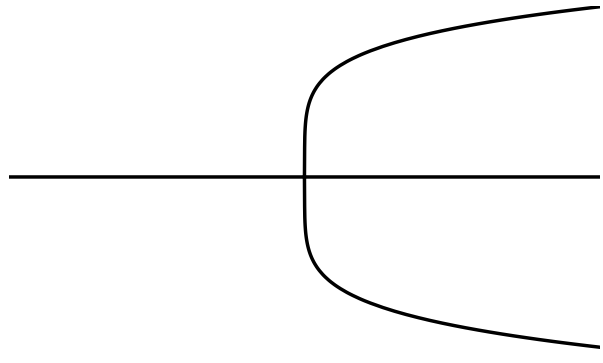

FIgURE 3.7: A local minimum splits into two local minima enclosing a local maximum. Horizontal lines correspond to constant ratio $t / x$

sidering the Hessian of $\partial_{t}^{2} g$ shows that the second-order changes all both positive multiples of $f^{(4)}(x / 2)$, to the effect that both $\pm(1,0)$ are of the same type of critical point.

The first-order changes along the other two directions $\pm(1,2)$ are opposite in sign, to the effect that one of the directions contains maxima and one contains minima.

Unless gradient and Hessian are zero, this leaves four general cases according to the signs of $f^{\prime \prime \prime}(x / 2)$ and $f^{(4)}(x / 2)$. For the purpose of illustration, consider first the case that both are positive. Then $\pm(1,0)$ and $(1,2)$ are directions of minima and $(-1,-2)$ is a direction of maxima. In other words, decreasing $x$ (corresponding to going forwards in time) will change the critical point at $t=x / 2$ from a local minimum to a local maximum, as illustrated in Figure 3.6.

It is clear that the branches with tangent $\pm(1,2)$ are branches of symmetric critical points with $t=x / 2$. What is the shape of the other branches with tangent $\pm(1,0)$ ? At first glance, it is even possible that each tangent direction spawns more than one branch, as multiple branches might share the same tangent at the branch point. However, all these branches must be minima and no branch can stay on the line $x=$ const, since that would correspond to a continuous set of local minima of the nonconstant univariate function $g(\cdot, x)$.

Additionally, in Figure 3.6, no branch can turn left towards the minima branch: since two local minima enclose a local maximum, there would have to be a branch of local maxima also approaching the branching point from the left. But that has already been ruled out as the only tangent of maxima exits the branching point towards the right. Therefore, all branches with initial tangent $\pm(1,0)$ are minima and must curve towards the right branch of maxima.

Finally, there is exactly one branch of minima per tangent direction: if there were more branches, they would have to enclose an additional branch of local maxima (which would have to merge with the minima at the branching point and, furthermore, have the same tangent as the two branches of minima), which leads to the same contradiction as before. This means that the end result must be a splitting event as depicted in Figure 3.7.

Repeating this line of argument for all other combinations of signs of $f^{\prime \prime \prime}(x / 2)$ and 


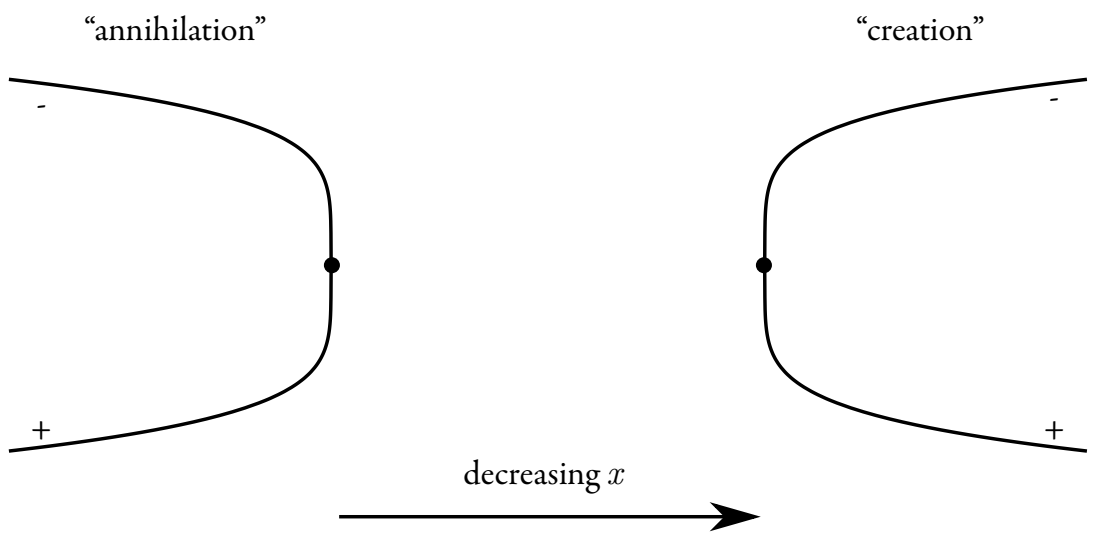

FIGURE 3.8: Two different pair events may occur. Left: a minimum and a maximum annibilate into a saddle which then disappears. Right: a pair of minimum and maximum is created from a saddle point.

$f^{(4)}(x / 2)$ yields the following table:

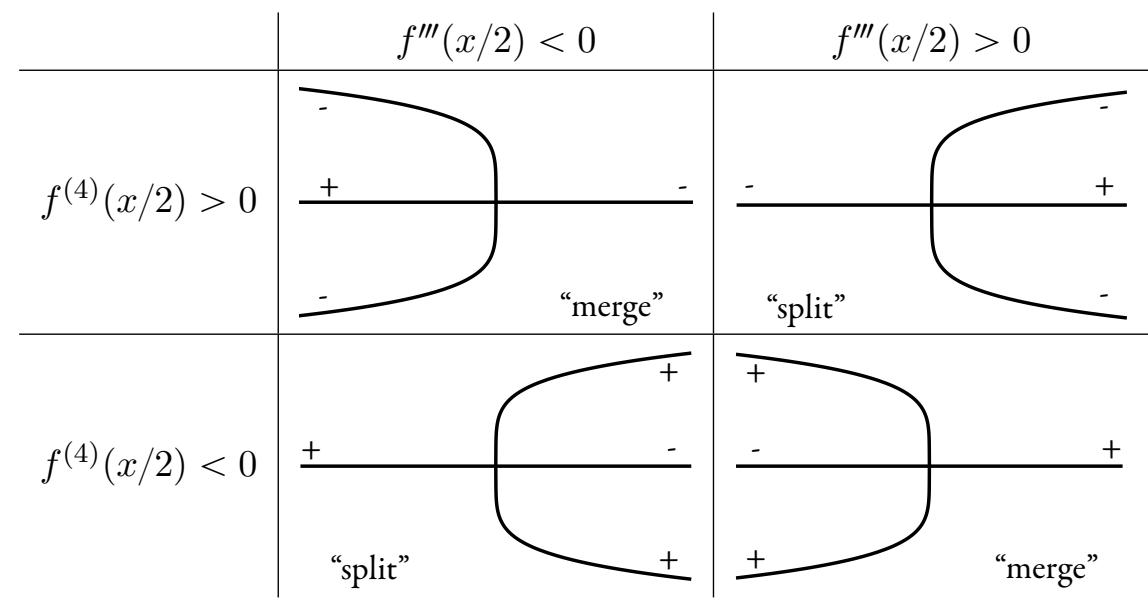

TABLE 3.1: The four general cases of branching events with $t=x / 2$.

\section{Pairing events}

The second type of changes to a branch of minima consists of the transformations of a local maximum and a local minimum into a saddle point when $\nabla \partial_{t} g \|(0,1)$ or, equivalently, $\partial_{t}^{2} g=0$ and $f^{\prime \prime}(x-t) \neq 0$. In this case, the two valid tangent directions are $\pm(1,0)$, with associated first-order change of $\partial_{t}^{2} g$ given by $\partial_{t}^{3} g(t, x)=f^{\prime \prime \prime}(t)-f^{\prime \prime \prime}(x-t)$. Since this quantity is in general nonzero, the tangent directions lead to branches of different types of critical point, so that either of the two scenarios depicted in Figure 3.8 occurs. We note again that more complicated pairing events may occur if the first-order change of $\partial_{t}^{2} g$ is zero, but that this situation does not appear in practice.

But which type of pair event occurs? As with the other types of events, the answer is determined by the signs of derivatives of $g$, in this case of $\partial_{t}^{3} g$ and $\partial_{x} \partial_{t} g$. To see this, consider the case that $\left(\nabla \partial_{t} g\right)_{2}=\partial_{x} \partial_{t}>0$ and $\partial_{t}^{3} g>0$. As previously established, the 


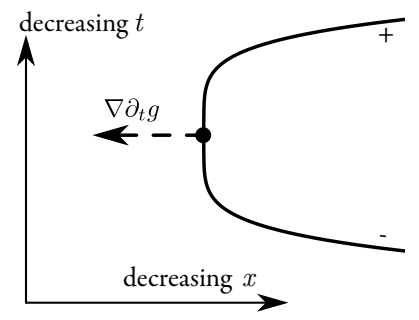

FIGURE 3.9: The sign of $\partial_{t}^{3} g$ determines both the type of branch and whether they curve towards or away from $\nabla \partial_{t} g$.

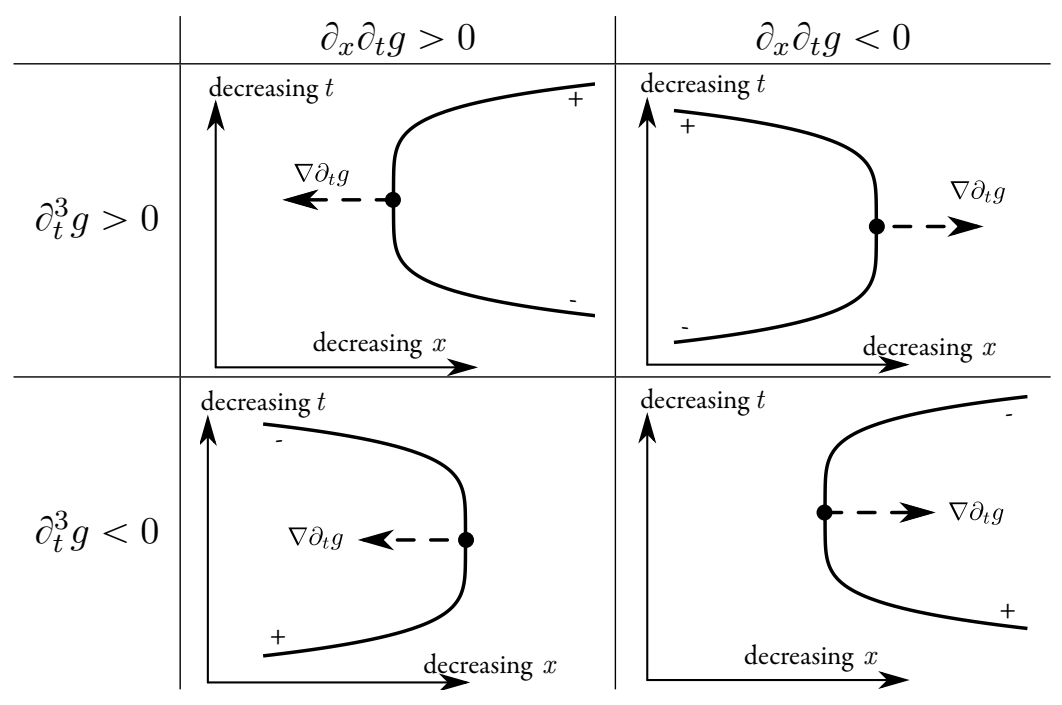

TABLE 3.2: The four general cases of pair events.

third derivative determines that the branch of critical points with tangent $(1,0)$ is a branch of maxima and the opposite branch consists of minima.

Now consider the gradient's first component $\left(\nabla \partial_{t} g\right)_{1}$. Its first-order change along the tangent direction $(1,0)$ is given by $\partial_{t}^{3} g$ as well, indicating that (together with the assumption that the second component $\left(\nabla \partial_{t} g\right)_{2}=\partial_{x} \partial_{t} g$ is positive) both components of $\nabla \partial_{t} g$ are positive on the $(1,0)$ branch near the saddle point. As a consequence, the branch's tangent vector, being perpendicular to the gradient, must have alternating signs and thus the branch curves towards the $-x$ direction. A similar argument shows that the branch of minima also curves towards the $-x$ direction. In this case, the event is a creation event, see Figure 3.9. An analogous discussion of the other cases gives Table 3.2.

Finding the saddle points of $g$ requires finding common zeroes to the two functions $\partial_{t} g(t, x)$ and $\partial_{t}^{2} g(t, x)$. This is in general a hard problem, and we only sketch a possible solution that guarantees completeness of the output. First, note that the explicit form of the single strip energy $f$ is in general rather complex, to the effect that solution by direct algebraic manipulation is infeasible. We therefore approximate $f$ by piecewise polynomial spline functions $f_{i}$. Using the approximation $f_{i}$ instead of $f$ for the construction of the function $g$, this turns the search for simultaneous zeroes into a bivariate polynomial system. 
This broad class of problems appears on the fundamental level in many different areas of mathematics and so it is no surprise that considerable effort has been undertaken to develop efficient numerical algorithms for its solution. As one of the more recent results, we point to work by Berberich, Emeliyanenko, and Sagraloff [7].

For the purposes of this analysis, it is enough to require fourth-order derivatives (to detect second-order change of the second derivative) of $f$, to the effect that the required degree of the interpolating polynomials is 9 . For simplicity, we use $k$ equidistant data points $t_{i}=i /(k-1)$ and require for additional precision that the interpolating polynomials agree with $f$ at the data points up to fifth order. Replacing $f$ by a piecewise polynomial function converts $g$ into a piecewise polynomial as well, defined on two-dimensional patches $t_{i} \leqslant$ $t \leqslant t_{i+1}, t_{j} \leqslant x-t \leqslant t_{j+1}$. On each piece, the bivariate polynomial system is solved using Mathematica's builtin REDUCE[] function. A final filtering step discards all solutions outside of the current patch.

Remark 3.4.8. While conceptually simple, interpolation by polynomial splines suffers from rapidly degenerating quality of approximation of higher-order derivatives but have the benefit that finding saddle points of polynomials is an explicitly solvable problem. We only note here that there exist more advanced approximation methods grouped under the name of quasi-interpolation that guarantee better approximation of derivatives (see exemplarily the work by Ma and Wu [40] or by Schaback [42]), but for the purposes of this thesis, the simple interpolation is sufficient.

\section{Events on the boundary}

The final type of events may happen when a branch of critical points meets the domain boundary. For $m=2$ strips, $t$ must lie in the interval $[x-h, h]$ if $x>h$ and in the interval $[0, x]$ if $x \leqslant h$ (because none of the two strips can extend beyond the material height of $h$ or invert to negative heights). Because $g(\cdot, x)$ is symmetric about $t=x / 2$ for all $x$, it is sufficient to check the lower boundary $t_{\text {min }}=\max \{0, x-h\}$ for the presence of a minimum.

Suppose that $\left(t_{0}, x_{0}\right)=\left(t_{\min }\left(x_{0}\right), x_{0}\right)$ is a critical point of $g$ that lies exactly on the boundary, such that $\partial_{t} g\left(t_{0}, x_{0}\right)=0$. Two pieces of information are available: first, the second partial derivative $\partial_{t}^{2} g\left(t_{0}, x_{0}\right)$ determines the type of critical point and second, the function $s: x \mapsto \partial_{t} g\left(t_{\min }(x), x\right)$ determines whether $g$ has a boundary minimum or a boundary maximum in the vicinity of $x_{0}$. The important aspect about this second function is whether its zero at $x_{0}$ constitutes a zero with or without sign change. To denote the different kinds of sign changes, we adopt the following notation: if $s$ changes sign from positive to negative with increasing $x$, we write $s \succ 0$; if $s$ changes from negative to positive with increasing $x$, we write $s f 0$. To determine in practical applications which of the two cases applies, it is usually enough to check the sign of the first derivative $s^{\prime}\left(x_{0}\right)$.

With these two pieces of information, there are again four different cases. Consider first the case that $\partial_{t}^{2} g$ is positive and $s \neq 0$ at $x_{0}$. Then as $x$ decreases, the boundary changes 
from maximum to minimum. Since $\partial_{t}^{2} g \neq 0$, so is $\nabla\left(\partial_{t} g\right)$, to the effect that the set of critical points has exactly one tangent direction at $\left(t_{\min }, x_{0}\right)$, which must be a branch of minima. Arguing analogously to the branching point case, there can only be a single branch of minima which furthermore must turn towards the left:

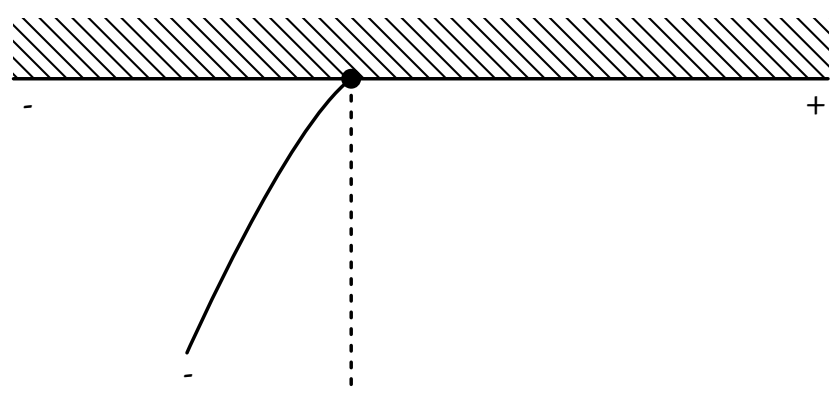

Repeating this argument for all four cases leads to the following table:

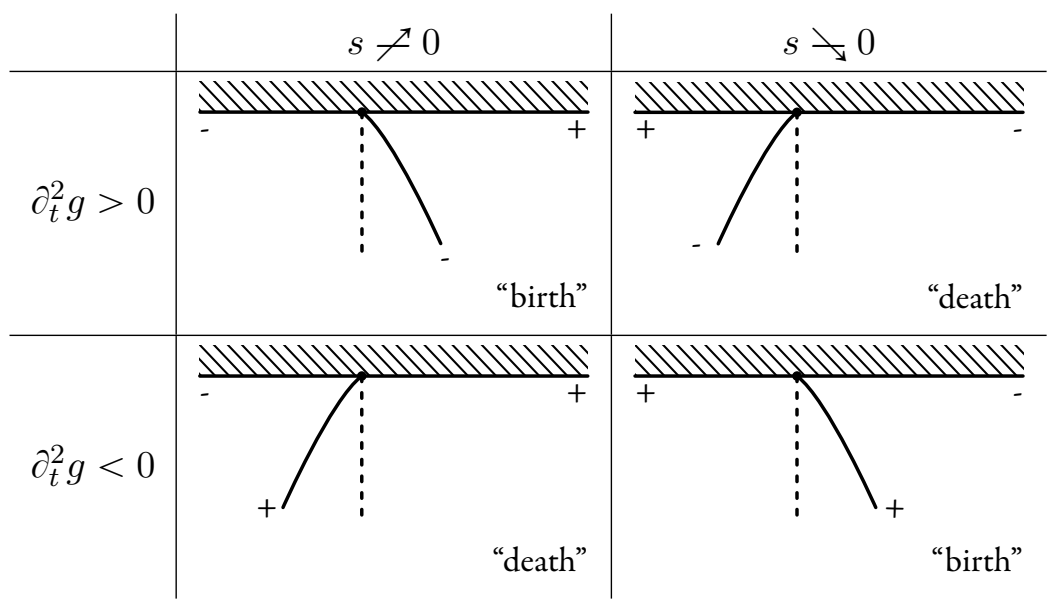

TABLE 3.3: The four different cases for boundary events.

Behaviour near $x=2$

Finally, we identify the initial branch for small amounts of compressions. To this end, note that the initial configuration $t=1$ is a stable embedding of the single strip and therefore a minimum of the single strip energy $f$. This implies that for $t$ near $1, f^{\prime \prime}$ is negative, so that $\partial_{t}^{2} g(x / 2, x)=2 f^{\prime \prime}(x / 2)$ is negative for $x$ near 2 . This means that for $x$ near 2 , the symmetric branch is a branch of minima. In particular, all traces start on this branch:

Lemma 3.4.9. Let $t$ be a trace. Then for $x$ near $2, t(x)=x / 2$.

Proof. There are only finitely many events on the $t=x / 2$ branch (pair and boundary events cannot occur, and branching events are defined by the finitely many zeroes of $f^{\prime \prime}$ ) and the 
initial configuration is given by $t=1, x=2$. Departure from the symmetric branch can thus only occur at branching events.

A similar argument can be made for $x$ near 0 . Depending on the sign of $f^{\prime \prime}(t)$ for small $t, g(\cdot, x)$ is either concave or convex, to the effect that its only minimum is on the boundary (if concave) or at $t=x / 2$ (if convex).

\section{Tracing out minima}

This information is enough to determine traces uniquely. To this end, perform the following algorithm:

1. First, find all zeroes $z_{1}>\cdots>z_{k}$ of $f^{\prime \prime}$. Partition the $z_{i}$ into sets with equal $f^{\prime}\left(z_{i}\right)$. Then all branching points are in the set $\left\{\left(z_{i}, z_{i}+z_{j}\right) \mid f^{\prime}\left(z_{i}\right)=f^{\prime}\left(z_{j}\right)\right\}$. Determine the nature of the branching points.

2. Next, find all boundary events by determining the zeroes of the function $s(x)=$ $\partial_{t} g\left(t_{\min }(x), x\right)$. Determine the type of event.

3. Then find all pair events by finding all joint zeroes of $\partial_{t} g$ and $\partial_{t}^{2} g$.

4. From this information, assemble an ordered list of local minima and maxima for each $x$. Starting at $t=1, x=2$ with a single minimum, decrease $x$ and adjust the list at each branch event accordingly.

5. Then, starting at $t=1, x=2$ in the uncompressed state, find the values of the trace $t(x)$ as follows:

(a) As long as no branching occurs, the evolution of $t$ is uniquely prescribed.

(b) Upon reaching a splitting event, pick one of the new branches of minima at random.

(c) If the current branch of minima merges into a saddle point $\left(t_{s}, x_{s}\right)$, descend to the nearest minimum. To be precise, find the nearest minimum in the current connected component of the sublevel set of $g$, that is, find the nearest minimum $\left(t_{m}, x_{s}\right)$ such that for each $t \in\left(t_{s}, t_{m}\right),\left(t_{m}-t_{s}\right) \cdot \partial_{t} g\left(t, x_{s}\right) \geqslant 0$.

(d) Likewise, if the current branch of minima merges with the boundary, remain on the boundary until $g$ no longer has a boundary minimum. As soon as that happens, find the nearest local minimum.

This procedure allows a qualitative prediction of the cylinder configuration at each intermediate point in time during the compression. The cylinder can be in either of three states: it can be in the symmetric configuration where $t=x / 2$, it can be in the asymmetric configuration $\left(t_{\min }(x), x\right)$ where one strip is maximally extended, or it can be in a transitional state where $t$ is neither on the boundary nor equal to $x / 2$. The preceding procedure 
of tracing out allows to determine with certainty for each $x$ the state of the cylinder. Furthermore, only in the third case the exact value of $t$ is unknown.

The predictions obtained by counting branching points is tested in Section 5.2 against the actual numerical results. 


\section{Implementation details}

Having laid out the basic theory behind the parameterization in the previous chapter, this chapter turns to describing the intricacies of the actual implementation. All code was implemented in the Wolfram Mathematica language and does not use high-level Mathematica functions unless explicitly stated.

Reiterating the task at hand, the problem of finding the actual configuration of a nearly rigid origami cylinder is essentially a minimization of the energy function (as function of the vertex positions) on some embedded submanifold with boundary $\mathcal{F} \subset \mathbb{R}^{9 N}=: \mathbb{R}^{n}$ defined by equality constraints enforcing isometry (three constraints per triangle, realized via edge length equality constraints), planarity (one constraint per vertex) or clamping the boundary vertex to the current height (one constraint per boundary). Additional inequality constraints prevent the "flipping" of strips.

In this section, we present the main tool to perform this numerical minimization, an exponential gradient descent method. This iterative method consists of the following basic steps to proceed from the current iteration $x_{b} \in \mathcal{F}$ to the next one:

1. Compute the gradient of the energy in ambient coordinates at $x_{b}$. This will be some vector of $\mathbb{R}^{n}$.

2. Project the gradient onto the tangent space $T_{x_{b}} \mathcal{F}$. Note that if $x_{b} \in \partial \mathcal{F}$, the tangent space is not a linear space but only a tangent cone.

3. Take an exponential step in that tangent direction. Find a step length that leads to a feasible point and is suitable from a numerical point of view, see Section 4.2 .

4. Terminate if the length of the projected gradient is less than some threshold.

Step 1 is easily done: since the energy function expressed in vertex coordinates is a quadratic polynomial, computation of the gradient vector is performed using Mathematica's builtin automatic differentiation capacity.

\subsection{Projection onto tangent cones}

More involved is the second step of projecting the gradient onto the set of tangent vectors. Let $x_{b} \in \mathcal{F}$ be the current iteration and thus the base point of the tangent space, and let $y \in \mathbb{R}^{n}$ be the energy gradient. Depending on the presence of active inequality constraints (that is, whether some $b_{j}\left(x_{b}\right)$ are exactly zero), $y$ is projected on either the whole tangent linear space $T_{x_{b}} \mathcal{F}_{1}$ or a subset thereof, which we will label the tangent cone. While projection 
onto a linear subspace is a standard task, projection onto cones is more involved and we present two different methods in Sections 4.1.2 and 4.1.3.

\subsubsection{Projection onto tangent spaces}

Assume first that no inequality constraint is active. In this case, the tangent set $T_{x_{b}} \mathcal{F}=$ $T_{x_{b}} \mathcal{F}_{1}$ is a full linear space which is the orthogonal complement of the span of all gradients of the equality constraints $g_{i}, i=1, \ldots, k$. Equivalently, the tangent space is the kernel of the matrix $G^{T}=\left(g_{i}\right)^{T} \in \mathbb{R}^{k \times n}$.

If the projection is rephrased as a separate distance minimization problem

$$
\begin{array}{r}
\min _{x \in \mathbb{R}^{n}} \frac{1}{2}\|y-x\|^{2} \\
\text { subject to } G^{T} x=0,
\end{array}
$$

it can be solved using Lagrange multipliers. The Lagrangian is

$$
L(x, \lambda)=\frac{1}{2}\|y-x\|^{2}+\lambda^{T} G^{T} x
$$

whose critical points are given by the solutions to

$$
\begin{cases}(x-y)+G \lambda & =0 \\ G^{T} x & =0\end{cases}
$$

which in turn translates into a linear system in $x$ :

$$
\underbrace{\left(\begin{array}{c|c}
\operatorname{Id}(n) & G \\
\hline G^{T} & 0
\end{array}\right)}_{=: A}\left(\begin{array}{l}
x \\
\lambda
\end{array}\right)=\left(\begin{array}{l}
y \\
0
\end{array}\right) .
$$

As $G$ has full rank (see the proof of Theorem 3.3.1), so does $A \in \mathbb{R}^{(n+k) \times(n+k)}$. We find the solution to this equation by solving the corresponding least squares problem given by $\min _{x, \lambda}\left\|A(x, \lambda)^{T}-(y, 0)^{T}\right\|^{2}$ using Mathematica's builtin LEASTSQUAREs[] function.

\subsubsection{Tangent cone projection: straightforward method}

We now deal with the case of active inequality constraints. Let $h_{i} \in \mathbb{R}^{n}, i=1, \ldots, r$ be the gradients of the active inequality constraints. Let $H=\left(h_{i}\right)$. The corresponding 
minimization problem is thus

$$
\begin{array}{r}
\min _{x \in \mathbb{R}^{n}} \frac{1}{2}\|y-x\|^{2} \\
\text { subject to } G^{T} x=0, \\
H^{T} x \geqslant 0 .
\end{array}
$$

(For vectors $x \in \mathbb{R}^{m}$, the notation " $x \geqslant 0$ " indicates that all components of $x$ are positive.)

We first show that it is sufficient to solve this minimization problem if both initial point $y$ and constraint vectors $h_{j}$ are projected onto the full linear space $T_{x_{n}} \mathcal{F}_{1}$.

Lemma 4.1.1. Let $y \in \mathbb{R}^{n}$ and let $y=y_{G}+y^{\perp}$ be the decomposition of $y$ into components in $\operatorname{ker} G^{T}$ and $\left(\operatorname{ker} G^{T}\right)^{\perp}$. Likewise, let $H=H_{G}+H^{\perp}$ be the columnwise decomposition of the inequality constraint gradients. Then the projection problem $\min _{x}\|x-y\|^{2}$ s.t. $G^{T} x=$ $0, H^{T} x \geqslant 0$ has the same unique solution as the problem $\min _{x \in \operatorname{ker} G^{T}}\left\|x-y_{G}\right\|^{2}$ s.t. $H_{G}^{T} x \geqslant$ 0 .

Proof. Observe that both problems have the same set of feasible points. By the Pythagorean theorem, $\|x-y\|^{2}=\left\|x_{G}-y_{G}\right\|^{2}+\left\|y^{\perp}\right\|^{2}$ for any feasible $x$, such that the two target functions only differ by a constant offset.

We will thus work only within the linear space $\operatorname{ker} G^{T}$ and omit the indices indicating the decomposition. Then the feasible set of this second minimization problem is an intersection of half-spaces and called a polyhedral cone. This notion, together with that of a finitely generated cone, will be important in formulating the actual projection method.

Definition 4.1.2. 1. A polyhedral cone is defined to be the intersection of finitely many closed half spaces. For any polyhedral cone $K$, there exist finitely many vectors $h_{i}$ such that

$$
K=\left\{x \in \mathbb{R}^{n} \mid\left\langle h_{i}, x\right\rangle \geqslant 0 \text { for all } i\right\} .
$$

If $H \in \mathbb{R}^{k \times n}$ is the matrix with rows $h_{i}$, we also use the notation $K=: \operatorname{ker}_{+}(H)$.

2. A finitely generated cone is defined to be the positive-span of a number of vectors: if $M \in \mathbb{R}^{n \times m}$, the set

$$
\operatorname{Im}_{+}(M):=\left\{M x \mid x \in \mathbb{R}^{m}, x \geqslant 0\right\}
$$

is a finitely generated cone.

3. If $K \subset \mathbb{R}^{n}$ is a cone of either type, the dual cone $K^{*}$ is defined to be the set

$$
K^{*}:=\left\{x \in \mathbb{R}^{n} \mid\langle x, r\rangle \geqslant 0 \forall r \in K\right\}
$$

The most straightforward method to project $y$ onto this polyhedral cone is to reduce the problem to ordinary orthogonal projection onto subspaces. To this end, note that the 
cone's faces (including lower-dimensional boundary components) are characterized by subsets of the set of active inequality constraint gradients $\left\{h_{i}, i=1, \ldots, k\right\}$ : each subset $I \subset\{1, \ldots, k\}$ defines a corresponding face by $\left\{x \mid\left\langle h_{j}, x\right\rangle=0 \forall j \in I,\left\langle h_{j}, x\right\rangle>\right.$ $0 \forall j \notin I\}$.

This cone projection algorithm proceeds as follows: project $y$ onto the span of each boundary component $\left\{x \mid\left\langle h_{j}, x\right\rangle=0 \forall j \in I\right\}$. From these intermediate projections $y_{I}, I \subset\{1, \ldots, k\}$, discard those that do not lie on the face (if $\left\langle h_{j}, y_{I}\right\rangle<0$ for some $j \notin I)$ and finally select the one that is closest to the original $y$.

The major disadvantage of this approach is that it scales exponentially with the number of active inequality constraints: if there are $k$ active constraints, one cone projection requires $2^{k}$ subspace projections.

\subsubsection{Tangent cone projection: Lemke's algorithm}

The speed of the projection method can be improved greatly following [55].

The first step is to consider the well-known fact that every cone projection problem can be written as an orthogonality problem [31, Proposition 3.2.3]

Lemma 4.1.3. Let $K$ be a polyhedral cone in $\mathbb{R}^{n}$, and let $K^{*}$ be its dual cone. Then for every vector $y \in \mathbb{R}^{n}$ there exists a unique vector $r \in \mathbb{R}^{n}$ such that

$$
\begin{aligned}
r & \in K, \\
r-y & \in K^{*}, \\
\langle r, r-y\rangle & =0 .
\end{aligned}
$$

This vector $r$ is the projection of $y$ onto $K$.

We apply this to our case. Here, we project $y$ onto the cone $K=\operatorname{ker}_{+}\left(H^{T}\right)$. The projection problem reads:

$$
\text { Find } r \in \operatorname{ker}_{+}\left(H^{T}\right) \text { such that } r-y \in \operatorname{ker}_{+}\left(H^{T}\right)^{*} \text { and }\langle r-y, r\rangle=0 .
$$

Furthermore, the dual cone of $K$ can be expressed as a finitely generated cone using a reformulation of Farkas' lemma [5, Theorem 2.3.5]:

Lemma 4.1.4 (Farkas' lemma). Let $A \in \mathbb{R}^{n \times k}$ and $b \in \mathbb{R}^{n}$. Then exactly one of the following is true:

1. There exists $x \in \mathbb{R}^{k}$ such that $A x=b$ and $x \geqslant 0$.

2. There exists $y \in \mathbb{R}^{n}$ such that $A^{T} y \geqslant 0$ and $\langle y, b\rangle<0$.

Lemma 4.1.5. Let $H \in \mathbb{R}^{n \times k}$. Then

$$
\operatorname{ker}_{+}(H)^{*}=\operatorname{Im}_{+}\left(H^{T}\right),
$$




$$
\operatorname{Im}_{+}(H)^{*}=\operatorname{ker}_{+}\left(H^{T}\right) .
$$

Proof. Straightforward consequence of Farkas' Lemma.

This allows a reformulation of the projection problem using Lemmas 4.1.3 and 4.1.5:

$$
\text { Find } r \in \operatorname{ker}_{+}\left(H^{T}\right) \text { such that } r-y \in \operatorname{Im}_{+}(H) \text { and }\langle r-y, r\rangle=0 \text {, }
$$

or, equivalently by substituting $z=r-y$,

$$
\text { Find } z \in \operatorname{Im}_{+}(H) \text { such that } r=y+z \in \operatorname{ker}_{+}\left(H^{T}\right) \text { and }\langle z, y+z\rangle=0 .
$$

Using the definition of $\operatorname{Im}_{+}(H)$ and $\operatorname{ker}_{+}(H)$, this problem turns into a linear complementary problem: write $z=H v$ for $0 \leqslant v \in \mathbb{R}^{k}$ and set $w=H^{T}(y+z)$. Then the problem reads:

Find vectors $v, w \in \mathbb{R}^{k}$ such that $w-H^{T} H v=H^{T} y$ and $v, w \geqslant 0$ and $\langle v, w\rangle=0$.

This is now a problem in only $k$ dimensions and there exists a tableau pivoting algorithm known as Lemke's algorithm to solve it.

\section{Linear complementarity problems and Lemke's method}

Formally, the linear complementarity problem is defined as follows:

Definition 4.1.6. Let $M \in \mathbb{R}^{k \times k}$ and $y \in \mathbb{R}^{k}$. The linear complementarity problem (LCP) is to find vectors $v, w \in \mathbb{R}^{k}$ such that

$$
\begin{aligned}
v-M w & =y, \\
v, w & \geqslant 0, \\
\langle v, w\rangle & =0 .
\end{aligned}
$$

Note that if $(v, w)$ is a solution to this problem, then at most one of $v_{i}, w_{i}$ is positive for all $i$. Out of the $2 k$ variables, the $k$ variables that may be positive are called base variables.

In order to solve the problem, note that if $y \geqslant 0$, then $v=y, w=0$ is a solution. If $y \neq 0$, an artificial variable $\lambda \in \mathbb{R}$ is introduced which is added to every linear equation of $v-M w=y$, leading to a modified problem

$$
\begin{aligned}
v-M w-\lambda e & =y, \\
v, w, \lambda & \geqslant 0, \\
\langle v, w\rangle & =0 .
\end{aligned}
$$

Here, $e \in \mathbb{R}^{k}$ is the vector containing 1 in every component. If $\lambda$ is chosen large enough (and positive) that $y+\lambda e$ is positive, then $w=0$ and $v=y+\lambda e$ is a valid solution to this 
modified system. Applying successive pivot operations to the tableau (i.e., changing which variables belong to the basis) will result in eliminating the artificial variable $\lambda$ from the basis, thereby finally providing a solution to the original problem.

This procedure is called Lemke's algorithm and is formulated in terms of table pivots: given a table $T$ and a pivot element $t_{i j}$,

1. divide the $i$-th row by $t_{i j}$,

2. for all $s \neq i$, add to the $s$-th row $\left(-t_{s j} / t_{i j}\right)$ times the original $i$-th row.

Lemke's algorithm then proceeds as follows:

1. Assemble the initial tableau as

$$
\left[\begin{array}{lll}
\operatorname{Id}_{k} & -M & -e \mid y
\end{array}\right]
$$

Each row of the tableau corresponds to one of the basis variables (initially the $v_{i}$ ), and each column of the tableau corresponds to one of the problem variables (the first $k$ columns correspond to the $v_{i}$, the next $k$ columns correspond to the $w_{i}$ and the last two columns correspond to $\lambda$ and the right-hand side, respectively). Furthermore, it is important to keep track of which variables are on which position in the basis: let $b_{1}, \ldots, b_{k}$ be the $k$ basis variables. Each $b_{i}$ is initially set to $v_{i}$.

2. Perform the initial pivot: , let $i \in\{1, \ldots, k\}$ be such that $y_{i}=\min \left\{y_{j}, 1 \leqslant j \leqslant\right.$ $k\}$.

(a) Pivot the table at row $i$ and column $2 k+1$ (corresponding to the auxiliary variable $\lambda$ ).

(b) Update the list of base variables: set $b_{i} \leftarrow \lambda$.

(c) Finally, store which variable is to enter the base in the next pivot: set $j \leftarrow k+i$ (corresponding to the index of the $w_{i}$ column).

Now perform repeated table pivots. Generally speaking, each pivot operation will introduce the complementary variable to the one that left the base in the previous step. For instance, since the initial pivot eliminated the variable $v_{i}$ from the basis, the first pivot will add $w_{i}$ to the basis. Likewise, if a previous pivot operation removed the variable $w_{\ell}$, the subsequent pivot will add the variable $v_{\ell}$. Each individual iteration proceeds as follows:

3. Let $d$ be the $j$-th tableau column and let $\bar{y}$ be the last column of the tableau.

3.1 If $d \leqslant 0$, the problem is unbounded. Abort.

3.2 Otherwise, determine the pivot row $i$ via

$$
i \leftarrow \operatorname{argmin}\left\{\frac{\bar{y}_{i}}{d_{i}} \mid d_{i}>0\right\} .
$$


3.3 If $b_{i}=\lambda$, the algorithm has found a solution to the original LCP. Perform the pivot and terminate.

3.4 If $b_{i} \neq \lambda$,

a) perform the pivot,

b) set $b_{i} \leftarrow$ (variable corresponding to column $j$ ),

c) set $j \leftarrow s$ if $b_{i}$ was $w_{s}$ before the pivot or $j \leftarrow s+k$ if $b_{i}$ was $v_{s}$ before the pivot.

One can show that this algorithm terminates after finitely many pivots with a solution to the LCP if the problem is consistent:

Theorem 4.1.7. Let $M$ be copositive-plus, that is, $x^{T} M x \geqslant 0$ for all $x \geqslant 0$ and if $x \geqslant 0$ satisfies $x^{T} M x=0$, then $\left(M+M^{T}\right) x=0$. Then Lemke's algorithm, applied to the LCP $v-M w=y, v, w \geqslant 0,\langle v, w\rangle=0$ terminates in finitely many steps with a solution to the $L C P$.

Proof. See Theorem 11.3 .8 of [5].

The conditions of this theorem are satisfied in the cone projection case: the matrix $M$ is given by $H^{T} H$. Thus, for all vectors $x \in \mathbb{R}^{k}, x^{T} M x=\|H x\|^{2} \geqslant 0$ and if $H x=0$ for some $x \geqslant 0$, then $\left(M+M^{T}\right) x=2 M x=0$ as $M^{T}=M$. Thus $M$ is copositive-plus.

Using Lemke's method for projection scales far better with the number of active inequality constraints $k$. Although the convergence theorem does not provide bounds on the number of pivot operations, we have observed that that number is usually comparable to $k$ itself. Unsurprisingly, in the examples presented in Chapter 5 (where $k \lesssim 10$ ), projection using Lemke's method is consistently faster by at least two orders of magnitude than the brute force approach.

\subsection{The line search method}

Once a descent direction has been identified, we need to find a suitable step size for the exponential gradient descent. This is done via a simple line search method. But what constitutes a suitable step size?

Mimicking the discussion by Nocedal and Wright [46, Chapter 3], let $x_{b} \in \mathcal{F}$ be the current base point, and let $v$ be the chosen tangent descent direction. Then consider the univariate function $g(t):=E\left(\exp _{x_{b}}(t v)\right)$. A suitable step size $t$ should satisfy two constraints: first, the target function should decrease by a sufficient amount (that is, faster than the tangent of $g$ at 0 ), and second, the new base point $x(t)=\exp _{x_{b}}(t v)$ should lie closer to 
a local minimizer, indicated by the fact that the derivative $g^{\prime}(t)$ is small in magnitude compared to $g^{\prime}(0)$. In the case of regular straight descent, these two conditions are equivalent to the strong Wolfe conditions.

In our case, the derivative $g^{\prime}(t)$ is given by

$$
\begin{aligned}
g^{\prime}(t) & =\left\langle\nabla_{\exp _{x_{b}}(t v)} E,\left(\left.\frac{\mathrm{d}}{\mathrm{d} s} \exp _{x_{b}}(s v)\right|_{s=t}\right)\right\rangle \\
& =\left\langle\nabla_{\exp _{x_{b}}(t v)} E, d R_{\exp _{x_{b}}(t v)} v\right\rangle
\end{aligned}
$$

since the tangent vectors of the curve $s \mapsto \exp (s v)$ are given by the differential of the right-multiplication map, see Theorem 3.3.12. This leads to the exponential Wolfe conditions

$$
\begin{aligned}
E\left(\exp _{x_{b}}(t v)\right) & \leqslant E\left(x_{b}\right)+c_{1} t\left\langle\nabla_{x_{b}} E, v\right\rangle \\
\left|\left\langle\nabla_{\exp _{x_{b}}(t v)} E, d R_{\exp _{x_{b}}(t v)} v\right\rangle\right| & \leqslant c_{2}\left|\left\langle\nabla_{x_{b}} E, v_{k}\right\rangle\right| .
\end{aligned}
$$

The two constants $0<c_{2}<c_{1}$ determine the "aggressiveness" of the conditions. For this thesis, they are chosen to be $c_{1}=10^{-1}, c_{2}=10^{-4}$ according to best practices [46, Chapter 3].

Remark 4.2.1. Similar to the usual Wolfe conditions, a weak version of the exponential Wolfe conditions can be obtained by not taking absolute values in the curvature condition (4.2.2).

In order to find a step size $t$ that satisfies both conditions, we implement the following bisecting line search method taken again from Nocedal and Wright [46, Algorithm 3.6]:

1. Start with a lower bound of $\alpha=0$, an upper bound $\beta=\infty$, and a candidate value $t=1$.

2. Repeat the following steps until both Wolfe conditions are satisfied:

(a) If condition (4.2.1) is violated, the step is so large that it left the region where $g$ decreases. Thus set $\beta \leftarrow t$.

(b) If (4.2.1) is satisfied but condition (4.2.2) is violated, the step length can either be increased to step even closer to a local minimizer, or the minimum has already been overstepped. An easy computation shows that the second case is equivalent to $g^{\prime}(t) \cdot(\beta-\alpha) \geqslant 0$ if $\beta$ is finite or simply $g^{\prime}(t) \geqslant 0$ if $\beta$ is infinite. If that is the case, set $\beta \leftarrow \alpha$. Furthermore, in both cases, set $\alpha \leftarrow t$.

(c) Find a new candidate value. If $\beta<\infty$, set $t \leftarrow(\alpha+\beta) / 2$; otherwise set $t \leftarrow 2 \alpha$.

The step length determined by this method is guaranteed to satisfy the exponential Wolfe conditions, but does not necessarily result in a point that is still contained in the feasible set $\mathcal{F}$. Therefore, the step length is halved until either it becomes too small (in which 
case the gradient descent algorithm terminates) or a feasible point is reached. Note though that this new feasible step length is no longer guaranteed to satisfy the exponential Wolfe conditions.

\subsection{Coordinate conversion}

The final building block in the individual steps is switching between points in $\mathcal{F}$, given as vertex coordinates in $\mathbb{R}^{9 N}$, and elements of the parametrizing Lie group. Translating a Lie group element $T$ into vertex coordinates is straightforward: simply apply the rigid body motions of each triangle jointly encoded by $T$ to the given reference embedding. Solving the inverse problem - finding the explicit rigid body motion from vertex coordinates-amounts primarily to extracting translations as well as axes and angles of rotations. Similar conversions also need to be done for tangent vectors: the descent direction is given as a vector in $\mathbb{R}^{9 N}$ and needs to be converted to an element of the Lie algebra in order to perform the exponential step.

A common task during the conversions is to compute the signed angle $\theta$ between two horizontal vectors $v, w \in \mathbb{R}^{3}$. The naive approach via $\langle v, w\rangle=\|v\| \cdot\|w\| \cdot \cos \theta$ requires taking inverse cosines, which has two main drawbacks: first, the argument is confined to the interval $[-1,1]$, which leads to problems when numerical imprecisions when $v$ and $w$ are almost parallel, and second, the derivative(s) are very large near \pm 1 and thus ill-conditioned.

In order to mitigate these problems, the standard approach is to use instead the tangent half-angle formula

$$
\theta=2 \arctan \frac{\sin \theta}{1+\cos \theta}=2 \arctan \frac{(v \times w)_{z}}{\|v\| \cdot\|w\|+\langle v, w\rangle}
$$

where $(u)_{z}$ means taking the $z$ coordinate of the vector $u$. Computing the inverse tangents is done using Mathematica's implementation of the ATAN2 function, which incorporates program logic to return the correct sign and deal with the case of small denominators.

More generally, if $v$ and $w$ are arbitrary vectors in $\mathbb{R}^{3}$, their enclosed angle can be computed with similar robustness via the similar formula

$$
\theta=2 \arctan \frac{\|v \times w\|}{\|v\| \cdot\|w\|+\langle v, w\rangle}
$$

\section{Feasible points}

We first deal with the problem of finding the Lie group element mapping the reference point $x_{0} \in \mathcal{F}$ to a given point $x \in \mathcal{F}$. Because each triangle is parametrized separately, this 
conversion is done per triangle.

Let $\Delta_{0}=\left(b_{1}, b_{2}, b_{3}\right)$ be the reference triangle, where the vertices have been labelled such that the edge $b_{1} b_{2}$ is horizontal, and let $\Delta=\left(v_{1}, v_{2}, v_{3}\right)$ be the new triangle (recall that because of the planarity assumption and the decomposition into strips, every triangle has precisely one horizontal edge). We now compute the rotation angle $\phi \in S^{1}$, the translation $d \in \mathbb{R}^{2}$, and the relevant two components of the change in vertex layer heights $t \in \mathbb{R}^{m-1}$.

Tilt angles In preparation for subsequent computations, we first examine the triangle's tilt angle $\psi \in(-\pi, \pi]$, which is defined as follows: let $\left(b_{1}, b_{2}, b_{3}\right)$ be the vertices of a triangle such that $b_{1} b_{2}$ is horizontal. Let $f_{3}$ be the orthogonal projection of $b_{3}$ onto the line $b_{1} b_{2}$. The tilt $\psi$ is defined as the signed angle between $b_{3}-f_{3}$ and the vertical direction, such that $R_{b_{2}-b_{1}}^{\psi}\left(0,0,\left\|b_{3}-f_{3}\right\|\right)=b_{3}-f_{3}$. See Figure 4.1 for an illustration.
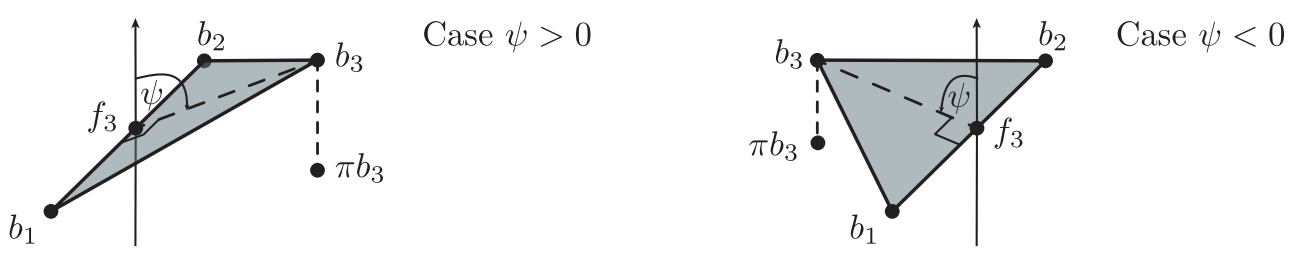

FIGURE 4.1: Illustration of the tilt angle $\psi$.

Tilt angles are computed from vertex coordinates by the following procedure: let $\pi b_{3}$ be the vertical projection of $b_{3}$ onto the horizontal plane containing the edge $b_{1} b_{2}$ and let $\Delta z=\left(b_{3}\right)_{z}-\left(b_{1}\right)_{z}$. Then with $h_{3}=\left\|b_{3}-f_{3}\right\|, h_{3} \cos \psi=\Delta z$ and $h_{3} \sin \psi=$ $\left\|\pi b_{3}-f_{3}\right\|$. Using Equation 4.3.1,

$$
\psi=2 \arctan \frac{\left\|\pi b_{3}-f_{3}\right\|}{h_{3}+\Delta z} .
$$

Vertex layers Assume that the horizontal edge $b_{1} b_{2}\left(\right.$ and $\left.v_{1} v_{2}\right)$ lies in the $i$-th vertex layer and that the respective third vertices are in the $j$-th layer. Then $t_{i}=v_{1}-b_{1}=v_{2}-b_{2}$ and $t_{j}=v_{3}-b_{3}$.

Once $t_{i}$ and $t_{j}$ have been determined, transform $\Delta_{0}$ first to an intermediate triangle $\left(\tilde{b}_{1}, \tilde{b}_{2}, \tilde{b}_{3}\right)$ whose vertices have the same $z$ coordinate as the $\Delta$ vertices: vertically translate by $t_{i}$, then rotate $\tilde{b}_{3}$ about the axis $\tilde{b}_{2}-\tilde{b}_{1}$ by the difference in tilt angles $\psi_{v}-\psi_{b}$. This will result in $\left(\tilde{b}_{k}\right)_{z}=\left(v_{k}\right)_{z}$ for $k=1,2,3$.

Translations In general, the translation $d$ is simply the difference between the two triangle's anchor points of the body-fixed frame. Depending on the type of constraints posed on the triangle, this anchor point is one of several different locations. To be precise, with the planarity assumptions, it is the midpoint of the horizontal edge. If planarity is not assumed, 
the anchor of e.g. unconstrained triangles is the triangle's center of mass. See Section 3.3.1 for the comprehensive list.

Rotations We determine the correct rotation element by finding both angle and axis of rotation. Under the planarity assumptions, the axis is always given by the vertical line through the horizontal edge midpoint and the angle $\phi$ is simply the signed angle between e.g. $v_{1}-$ $m_{v}$ and $b_{1}-m_{b}$, obtained again via (4.3.1).

Without the planarity assumption, the axis of rotation $n$ is normal to the plane spanned by the vectors $\left(v_{k}-m_{v}\right)-\left(b_{k}-m_{b}\right), k=1,2,3$, and thus obtained via a simple cross product. Once the axis is known, the angle of rotation $\phi$ is given similar to Equation (4.3.1) as

$$
\phi=2 \arctan \frac{\left\langle\left(v_{1}-m_{v}\right) \times\left(b_{1}-m_{b}\right), n\right\rangle}{\left\|v_{1}-m_{v}\right\| \cdot\left\|b_{1}-m_{b}\right\|+\left\langle v_{1}-m_{v}, b_{1}-m_{b}\right\rangle} .
$$

\section{Tangent vectors}

Conversion between tangent vectors $t \in T_{x} \mathcal{F}$ and elements $v \in \mathfrak{g}$ is similarly done on a per-triangle basis. The direction $v \rightarrow t$ is straightforward addition (for infinitesimal translations) and matrix multiplications (for infinitesimal rotations).

The reverse direction corresponds to finding infinitesimal translations and rotations. For a triangle with vertices $v_{1}, v_{2}, v_{3}$ and corresponding tangents $v_{1}, v_{2}, v_{3}$, the infinitesimal translation is simply the infinitesimal movement of the body frame anchor: $d=\left(y_{1}+\right.$ $\left.y_{2}\right) / 2$ with planarity assumptions and analogous expressions without planarity assumption.

Infinitesimal rotations General infinitesimal rotations can be described in two equivalent ways, either as a cross product or via an element of $\mathfrak{s o}(3)$. If $A(t)$ is a continuous rotation by an angle $\alpha(t)$ about a fixed axis, the infinitesimal rotation of a point $v$ is given by the tangent vector $r=(A v)^{\prime}(0)$. An easy calculation shows that $r=a \times v$, where $a$ is parallel to the axis of rotation of $A(t)$ and has length equal to $\alpha^{\prime}(0)$.

In the case at hand, the vectors $r_{k}=v_{k}-d$ ( $d$ again being the infinitesimal translation obtained in the previous paragraph) describe the vertices' infinitesimal rotation, and we need to determine the vector $a$ such that $a \times\left(v_{k}-m_{v}\right)=r_{k}$. The direction of $a$ is simply the normal to the plane spanned by $\left\{r_{k}, k=1,2,3\right\}$ and is obtained by e.g. $r_{1} \times r_{2}$, the length of $a$ is obtained by

$$
\left\|r_{k}\right\|=\|a\|\left\|v_{k}-m_{v}\right\| \sin \angle\left(a, v_{k}-m_{v}\right) .
$$

The angle $\angle\left(a, v_{k}-m_{v}\right)$ does not depend on the length of $a$. It is thus equal to $\angle\left(r_{1} \times\right.$ $r_{2}, v_{k}-m_{v}$ ), which is computed using Equation (4.3.1). 


\section{Results and discussion}

As a first step, the implementation detailed in the previous section was run with Guest and Pellegrino type input fold patterns of various different type. Described in more detail in Section 3.2, this class of input patterns essentially depends on three parameters: the realvalued parameter $r$ determines the radius of the initial cylinder, the integer $n$ determines the order of rotational symmetry, and the integer $m$ denotes the number of initially identical strips that constitute the cylinder. As each strip's initial embedded height is 1 independent of $n$ and $r$, the cylinder's initial height is also given by $m$.

In the subsequent numerical experiments, the ratio $r / m$ was varied between 0.2 and 1.5 , covering the range from long thin "pipes" to wide and flat "bands". An illustration of the cylinders for different values of $r$ and $n$ is given in Figure 5.1.

In this section, we will only exemplarily highlight the nature of the results by presenting two different selected examples. For a more comprehensive list, we refer to part $A$ of the Appendix.

Simulation details All results in this section have been obtained using the following procedure: from the initial embedding with height $H_{0}=2$, decrease $H$ in steps of $\delta H=0.02$. Adapt the old minimizer, which is now no longer on the constraint manifold, to the new boundary heights by changing only the top strip's height, as outlined in Section 3.3.1.

Before this initial guess is fed into the descent algorithm, it is further perturbed by picking at random a tangent vector of small magnitude and performing an exponential step in that direction. This is done in order to deal with situations where the initial configuration is already near to a local maximum: in those cases, our chosen termination criterion will instantly stop unless the starting point is moved towards one of the accompanying minima.
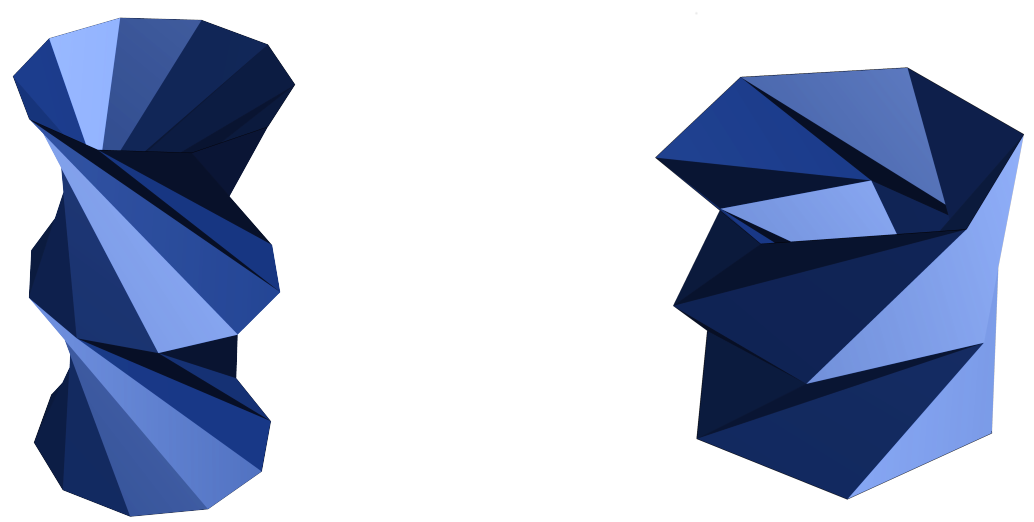

FIGURE 5.1: Two examples of input fold patterns. Left: $r=0.6, n=10$, right: $r=1.2, n=6$. 


\subsection{Code validation}

We first validate the correctness of the code, for which we consider two different checks. First, if the output turns out to be nearly rotationally symmetric (even though that is not explicitly enforced), it can be compared to the energy values obtained semi-analytically in Section 3.2. Second, the results of the exponential minimizer code can be compared against a minimization of the energy with soft constraints, that is, the constraints are added as additional energy penalty to the target function. This latter minimization is essentially unconstrained and was performed with Mathematica's builtin numerical minimization capabilities.

\subsubsection{Comparison to analytic results}

For the Guest and Pellegrino type fold patterns, Section 3.2 established a semi-exact solution to the minimization problem under the assumption of rotational symmetry. Comparing the minimizer obtained by the exponential descent method to the analytic solution shows two things: first, the numerically obtained minimizer is indeed rotationally symmetric and second, the energies match very well with their analytic predictions. As Figure 5.3 shows, the height of the middle vertex layer is almost always either equal to half the cylinder height or maximally asymmetric and the energy values agree with the corresponding analytic predictions. This behavior-the cylinder is either in its symmetric or in its maximally asymmetric state-has been consistently observed across the range of parameters $0.4 \leqslant r \leqslant 2.8$ and $4 \leqslant n \leqslant 10$.

\subsubsection{Comparison to soft constraint minimization}

If the hard constraints ensuring rigidity of each face are replaced by penalty terms added to the energy, the problem becomes essentially unconstrained: the remaining planarity conditions amount to simple variable substitution and the inequality conditions constrains only a single variable. This new problem can be approached using off-the-shelf methods, in particular Mathematica's FindMinimum[] function. This section shows the results for the set of parameters $r=1, n=8$.

Figure 5.2 shows that there is general qualitative agreement with the soft constrained solution: both solutions oscillate between totally asymmetric and symmetric states (visible from the left plot), and the respective energies coincide. The only visible difference is that the soft constrained solution transitions from asymmetric to symmetric configuration at an earlier time. Similar behavior - agreement between soft and hard constrained minimization except for the location of transitions - is observed across the entire range of parameters.

Summarily, the two comparison experiments show that the numerical minimization process performs as expected over the entire range of parameters considered in this thesis. 

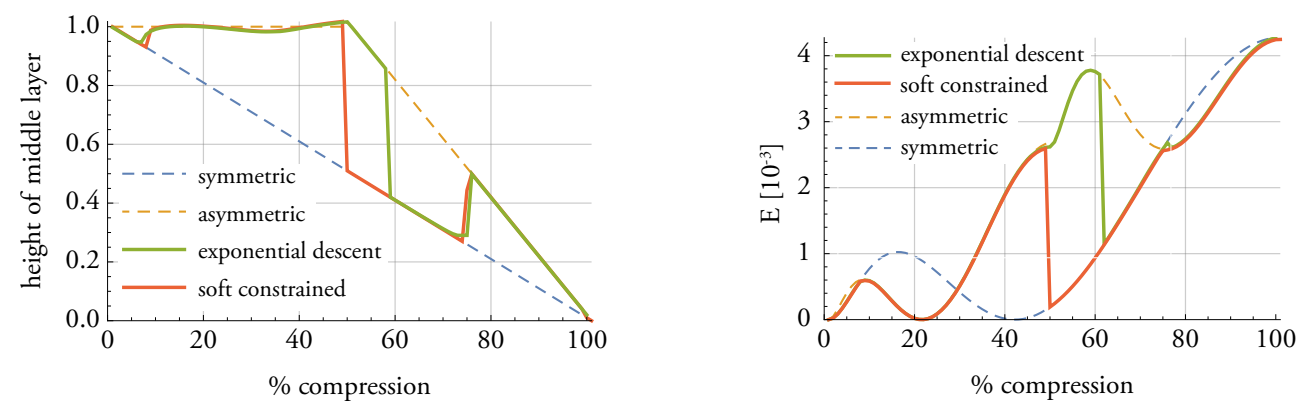

FIGURE 5.2: Comparison between minimization by exponential descent and soft-constrained minimization for $r=1, n=8$.

Even though there is evidently a discrepancy to the soft constrained results, a closer examination of the associated energy landscape functions (see also next section) shows that the discrepancy must be an artifact of the soft constraints, likely due to the change in energy landscape. For instance, Figure 5.4 shows that at $H=1.2$, the energy landscape has three local minima. Since the minimization result of the previous time step is in the asymmetric configuration and cannot cross to the much lower local minimum of the symmetric configuration because of a small energy barrier, so that even a small alteration of the landscape induced by the soft constraints might be sufficient to eliminate this barrier.

\subsection{Results}

The main output of the numerical minimization process is threefold. On the one hand, the minimization process delivers the compressive strain (the value of the target function) and the minimizer as functions of the cylinder height $x$, directly giving the kinematic response to and required loads for compression the input folded cylinders. On the other hand, under the assumption of planar vertex layers, a similar minimization process with fixed vertex layer heights (that is, minimizing only with respect to horizontal motions of faces) generates for each value of $x$ an energy landscape function of the middle vertex layer position $t$, similar to the simple energy function $g(t, x)$. This landscape function, whose local minima represent the possible outputs of the full numerical minimization, will provide useful information for explaining the minimizers' behavior.

We will highlight the results and the dependence of the energy landscape families on the parameters $r, n, m$ by selected examples. For an overview over the entire parameter range, we refer to part $A$ of the appendix. 

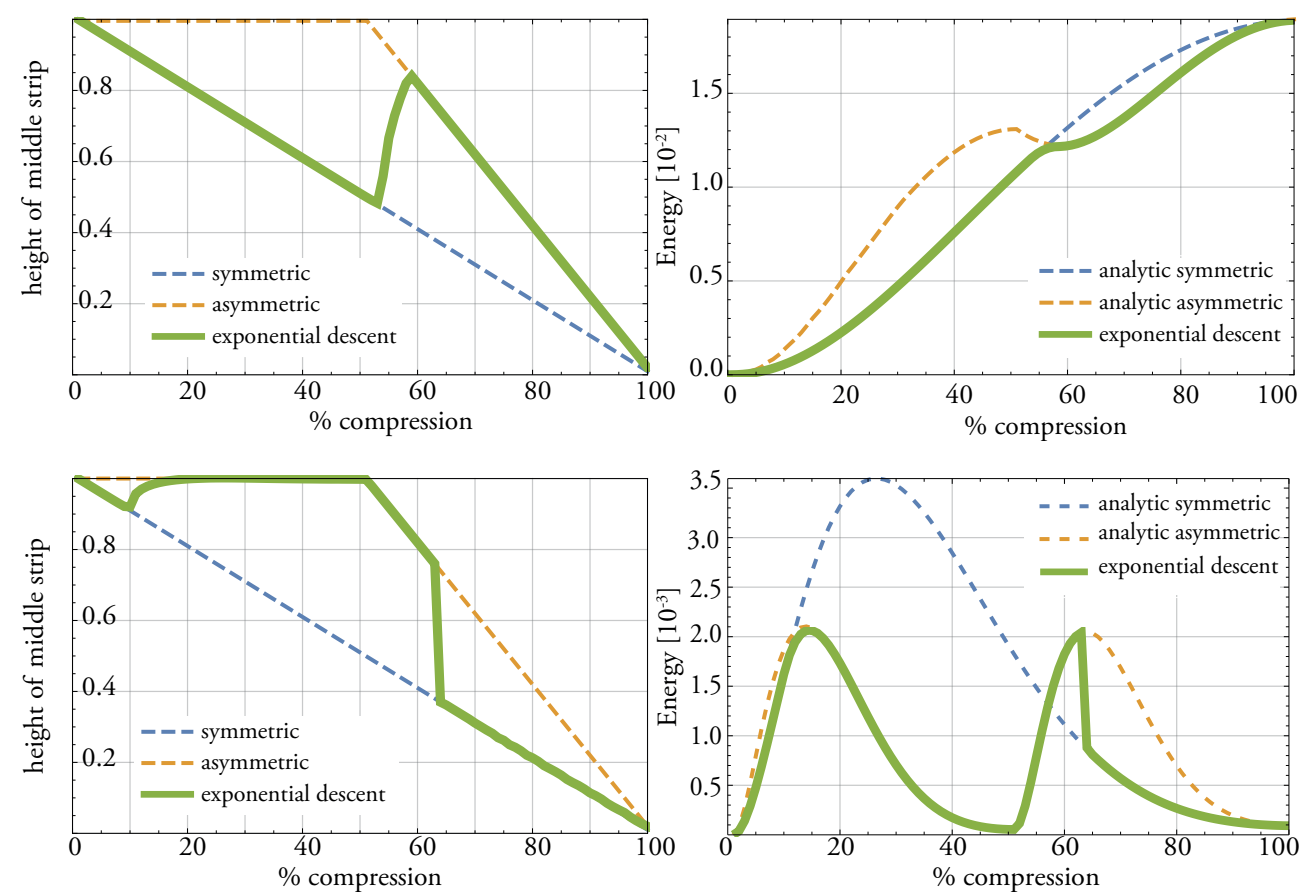

FIGURE 5.3: Plot of the middle layer's heights (right) and elastic energies (left) for two sets of parameters (top: $r=0.6, n=10$, bottom: $r=1.2, n=6$ ). Marked in green is the numerical minimizer, marked in blue and orange are the exact minimizers for symmetric and asymmetric special case.

\section{Example 1}

The first example with $r=0.6, n=10, m=2$ showcases the most common qualitative behavior: The energy, depicted in the top right part of Figure 5.3, increases monotonically and exhibits a single saddle point at about $50 \%$ compression, where it transitions from following the exact energy of the symmetric state to following the exact solution of the asymmetric state. The minimizer itself reflects this observation in that at first, both strips compress equally, until the middle vertex layer snaps to either top or bottom boundary, as depicted in the top left of Figure 5.3.

\section{Example 2}

If the ratio $r / H_{0}$ is chosen around 0.5 to 0.6 , the minimizers exhibit a different behavior. While the energy function still follows closely either the symmetric or the asymmetric exact solution, there exist multiple jumps in both the height and the energy function, as seen in the bottom row of Figure 5.3, where $r / H_{0}=0.6$.

In this example, three things stand out: first, the energy function may change discontinuously between different heights (as evidenced at around 65\% compression in the energy plot). Second, the local minimum found by the exponential descent method is not necessarily the global minimum (see the discrepancy between green and blue curve around 60\%). Finally, the cylinder generally seems to be in either the symmetric and totally asymmetric 
state with only short transitory intervals.
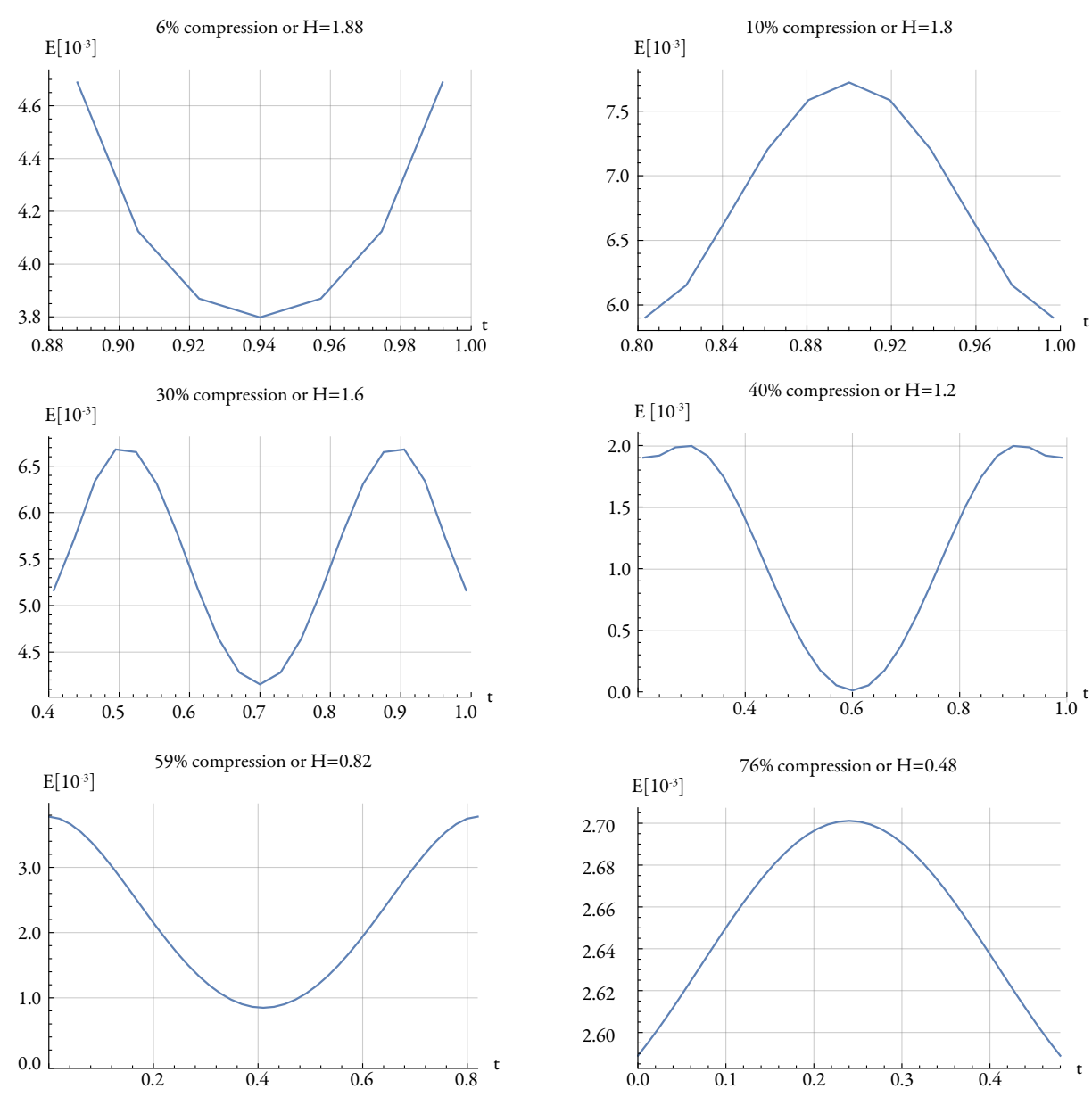

FIGURE 5.4: The energy landscape as a function of the middle vertex layer position t for $r=1, n=8$ and different values of $H$. Total height of the cylinder decreases from left to right and top to bottom.

Over the entire range of parameters, some common features are observed. First, the solution transitions at least once from symmetric to asymmetric configuration. This transition occurs continuously as opposed to abruptly. There are no other stable configurations, such as for instance a compression ratio of $2: 1$. Moreover, if the input fold pattern is rotationally symmetric, this symmetry is preserved throughout the compression, even if the initial guess is randomly perturbed by a small amount before beginning the minimization process.

Furthermore, some choices of parameters exhibit more than one transition. In contrast to the first transition from symmetric to asymmetric, these other transitions can be discontinuous. Within the parameter range discussed in this chapter, the maximal number of transitions is 3 .

Moreover, even if the minimizer undergoes a rapid (but continuous) transition, the energy might not change as drastically. For instance, the top right of Figure 5.3 shows a transition from symmetric to asymmetric configuration at 55\%, drastically altering the visual appearance of the solution but barely affecting the energy. 

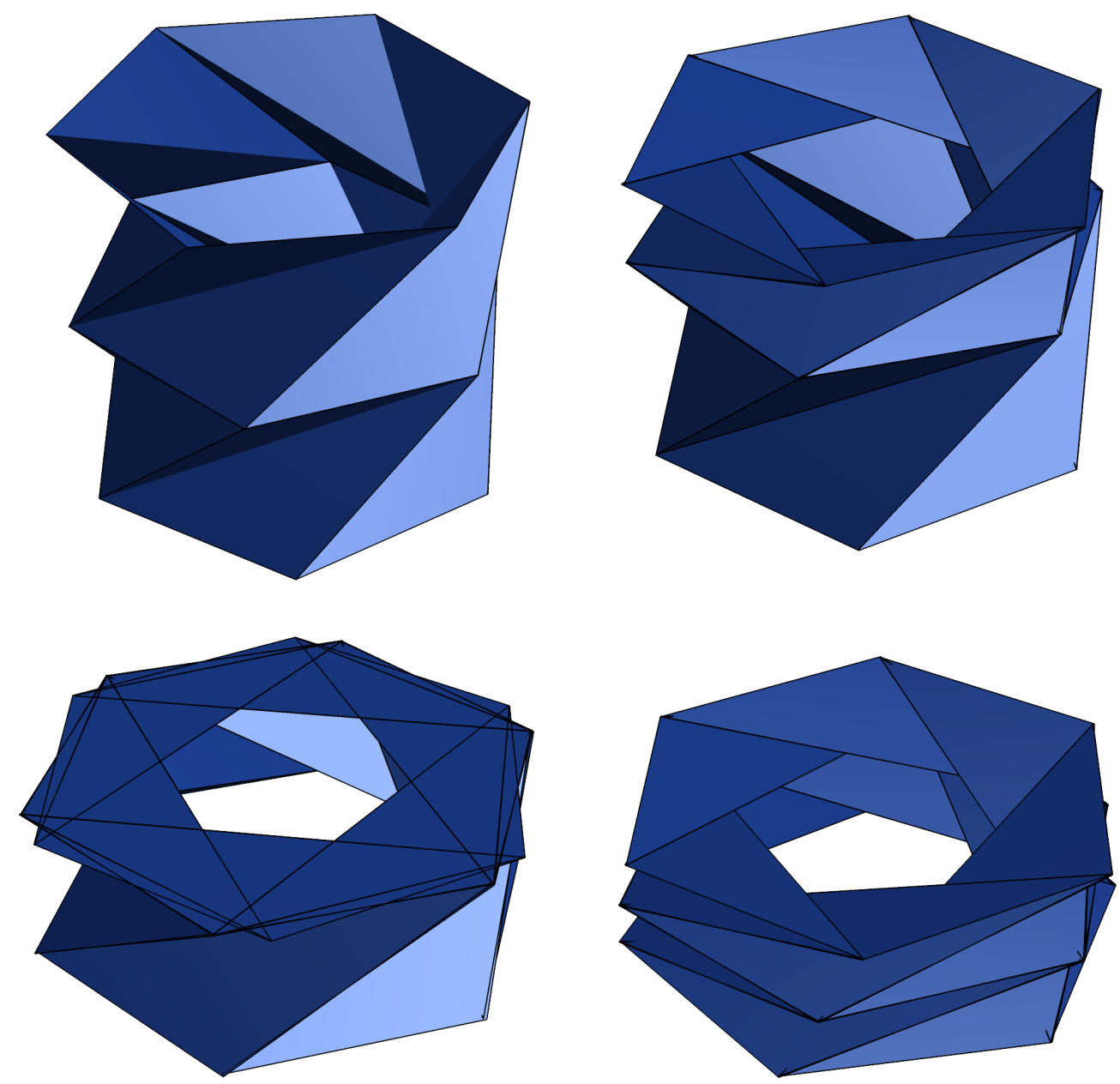

FIGURE 5.5: Snapshots of the compression process for $r=1.2, n=6$. Top left: the initial cylinder in its rest state. Top right: the initial phase sees only the top strip compressed and the lower strip unchanged. Bottom row: at about $65 \%$ compression, the cylinder suddenly snaps from asymmetric configuration with one completely flat strip (left) to the symmetric configuration where both strips are compressed by the same amount (right).

Finally, some energy landscapes exhibit minima of zero energy, implying that the respective fold pattern is bistable. This can be seen for instance for the set of parameters $r=$ $1, n=8$, depicted in Figure 5.2.

All these observations can be seen as consequences of the shape of the energy landscape functions obtained by a separate minimization. It is observed that the energy landscape changes continuously with the cylinder height $H$ and that the numerically found minimizer follows along a given local minimum as the landscape changes. As the critical points of the landscape merge or split, a continuous tracking of minima is not always possible. In cases such as this, the numerical minimizer code instantaneously "rolls downhill", causing both the minimizer and the resultant energy to undergo a discontinuous jump. In particular, we find that these observed discontinuities are not artificially introduced by e.g. the time-discretization.

Knowing the energy landscape in advance is thus a valuable tool in predicting the qual- 
itative behavior of the family of minima. While the landscapes cannot be found exactly or a priori, they can at least be estimated by the simple energy model. The next section will therefore showcase how to obtain such a prediction following the theoretical discussion in Section 3.4 .1 and then compare these predictions against the actual minima.

\subsubsection{The simple energy model}

In practice, the predictions of the simple model were obtained by the following process: starting from the initial configuration $t=1, x=2=H_{0}, x$ was iteratively decreased in steps of $\Delta x=H_{0} / 100$ to match with the time step of the numerical minimization. In the $k$-th step, the predicted position $t_{k}$ of the middle vertex layer was chosen out of all local or boundary minima of $g(\cdot, x)$ such that the distance $\left|t_{k}-t_{k-1}\right|$ was minimal and $\partial_{t} g(t, x)$. $\left(t_{k}-t_{k-1}\right)<0$ for all $t \in\left(t_{k}, t_{k-1}\right)$ (to the effect that $g$ is monotonically decreasing from $t_{k-1}$ to $\left.t_{k}\right)$. Accordingly, the predicted energy value is given by $g_{k}=g\left(t_{k}, x_{k}\right)$.

For the first of the examples presented in Section 5.2, the top row of Figure 5.6 compares the predicted vertex layer height against the numerically obtained minimizer of the true energy. Furthermore, both the simple energy value and the true energy value of this prediction are compared against the true energy value of the numerically obtained minimizer.
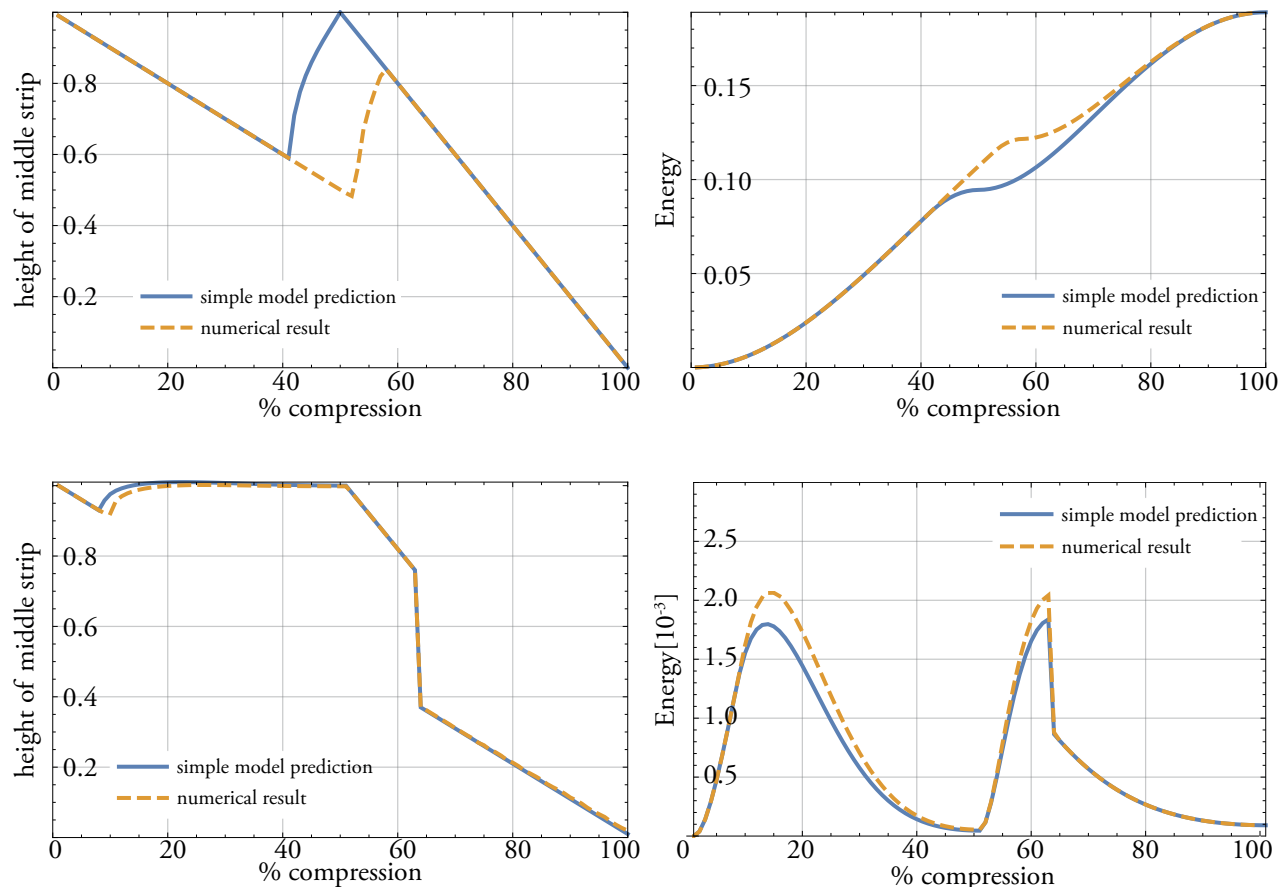

FIGURE 5.6: Comparison of simple energy prediction versus numerically obtained minimizer of the true energy, both in middle vertex layer height (left) and respective energy values (right). Top row shows the parameters $r=0.6$ and $n=10$-fold rotational symmetry; bottom row shows $r=1.2, n=6$.

Similarly, the second example compares very well to the minimizer of the "true" elastic 


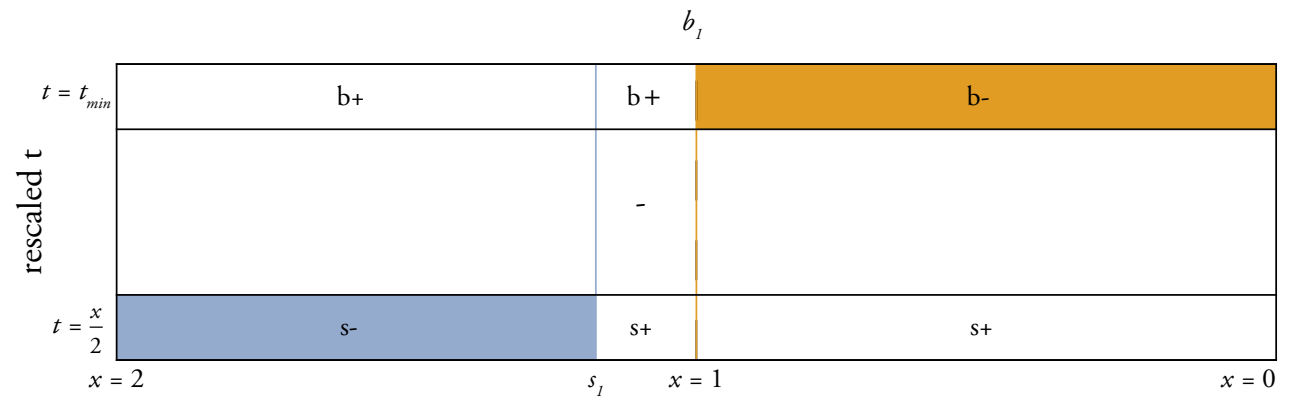

FIGURE 5.7: Prediction plot for the first example. The $t$ interval has been rescaled to constant width; only one half is shown. " $b \pm$ " signifies that $g$ has a boundary maximum ("+") or minimum ("-"), "s士" signifies that the symmetric configuration is a maximum or a minimum. Additional signs indicate other local extrema and their order.

energy. The top row of Figure 5.6 shows that, even though there are quantitative differences, the general shape of the curves, in particular of the vertex heights, are very similar.

We observe this general agreements over the entire range of parameters: while it may be that some transitions are shifted (that is, occur at slightly different values of $x$, as in the top row of Figure 5.6), the general behavior of the minimizer's middle layer height and in particular the energy are very well predicted.

\section{Qualitative prediction of minima}

Finally, we verify the quality of the prediction of the actual configuration using only information about the different types of branching and merging events of the set of minima of $g$.

Example 1 The first example shows only two different events: at $x=1.17=: s_{1}$, the local minimum at $t=x / 2$ splits into two minima flanking a maximum. At $x=1=: b_{1}$, a local minimum is absorbed by the boundary, which turns from a maximum into a minimum. No pair events occur. Thus, the local minimum that meets the boundary at $b_{1}$ must be the one that split off at $x=s_{1}$. We can thus assemble Figure 5.7 and predict the behavior of this cylinder under compression. Since there exists only one local minimum for all $x$, the prediction is straightforward: for $x \geqslant s_{1}$, the cylinder must be in the symmetric state; it continuously transitions for $s_{1}<x<b_{1}$ towards the boundary and remains in the asymmetric state until the end. The validity of this prediction is confirmed by the left column of Figure 5.3. Even though the transition period occurs later than predicted, the sequence of states (first symmetric, then transitory, then asymmetric) is correctly captured.

Example 2 In the second example, an analysis of the single strip energy indicates that there exist only two branching events at $x=1.85=: s_{1}$ and $x=1.12=: s_{2}$ with $t=x / 2$ each. At $s_{1}$, a minimum splits into two minima enclosing a maximum and at $s_{2}$, a maximum splits into two maxima enclosing a minimum. Furthermore, there exist only two 


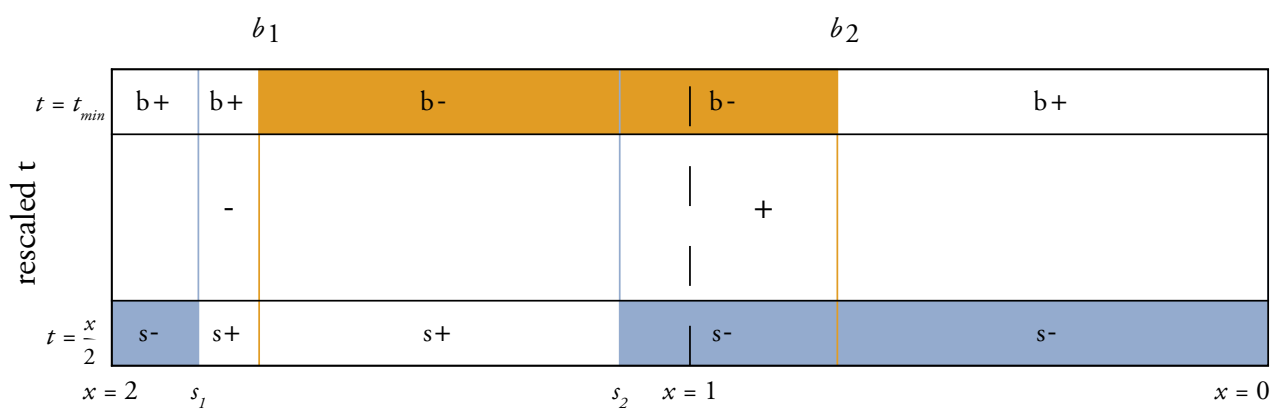

FIGURE 5.8: Prediction plot for the second example. The variable thas been rescaled to constant width; only one half is shown. "b士" signifies that $g$ has a boundary maximum ("+") or minimum ("-"), "s." signifies that the symmetric configuration is a maximum or a minimum. Additional signs indicate other local extrema.

different points where the type of boundary extremum changes: at $x=1.75=: b_{1}$ and $x=0.75=: b_{2}$. At $b_{1}$, a minimum merges with the boundary and at $b_{2}$, the boundary absorbs a maximum. Since no other local extrema are generated or removed by pair events, we know that the minimum spawned at $s_{1}$ must be the one reaching the boundary in $b_{1}$ and that likewise the maximum spawned in $s_{2}$ must reach the boundary in $b_{2}$. We can thus assemble the complete list of extrema as shown in Figure 5.8 .

It is then straightforward to obtain a prediction for the cylinder's behavior under compression: for $x \geqslant s_{1}$, both strips must compress symmetrically. For $s_{1} \geqslant x \geqslant b_{1}$, the cylinder is in transition and reaches the asymmetric state at $x=b_{1}$. It remains asymmetric up until $x=b_{2}$, at which point there is only a single local minimum left. Thus, at $x=b_{2}$, the cylinder instantaneously reverts back to the symmetric configuration and remains there for all remaining $x<b_{2}$. As evidenced by Figure 5.6, this prediction coincides very well with the actual result, with the only difference being that the event $s_{1}$ occurs a little later than predicted.

Limits of the simple model For most of the examples, the simple model can accurately predict the qualitative behavior of the numerical minimization result, indicating that the simplification - neglecting the coupling between different layers-has comparatively small influence on the energy landscape. But in some cases, where the single strip energy function $f$ and thus the simple model energy $g$ exhibit delicate features, the neglected terms become quantitatively important. As shown in Figure 5.9 for $r=1, n=8$, both jumps predicted by the simple model occur too soon compared to the actual numerical minimizer. Accordingly, the predicted vertex heights also differ.

Examining the simple energy landscape confirms this suspicion: as Figure 5.10 shows, the true energy barrier separating the boundary minimum from the symmetric configuration is very small, to the effect that the same place is a maximum of the simple energy. 


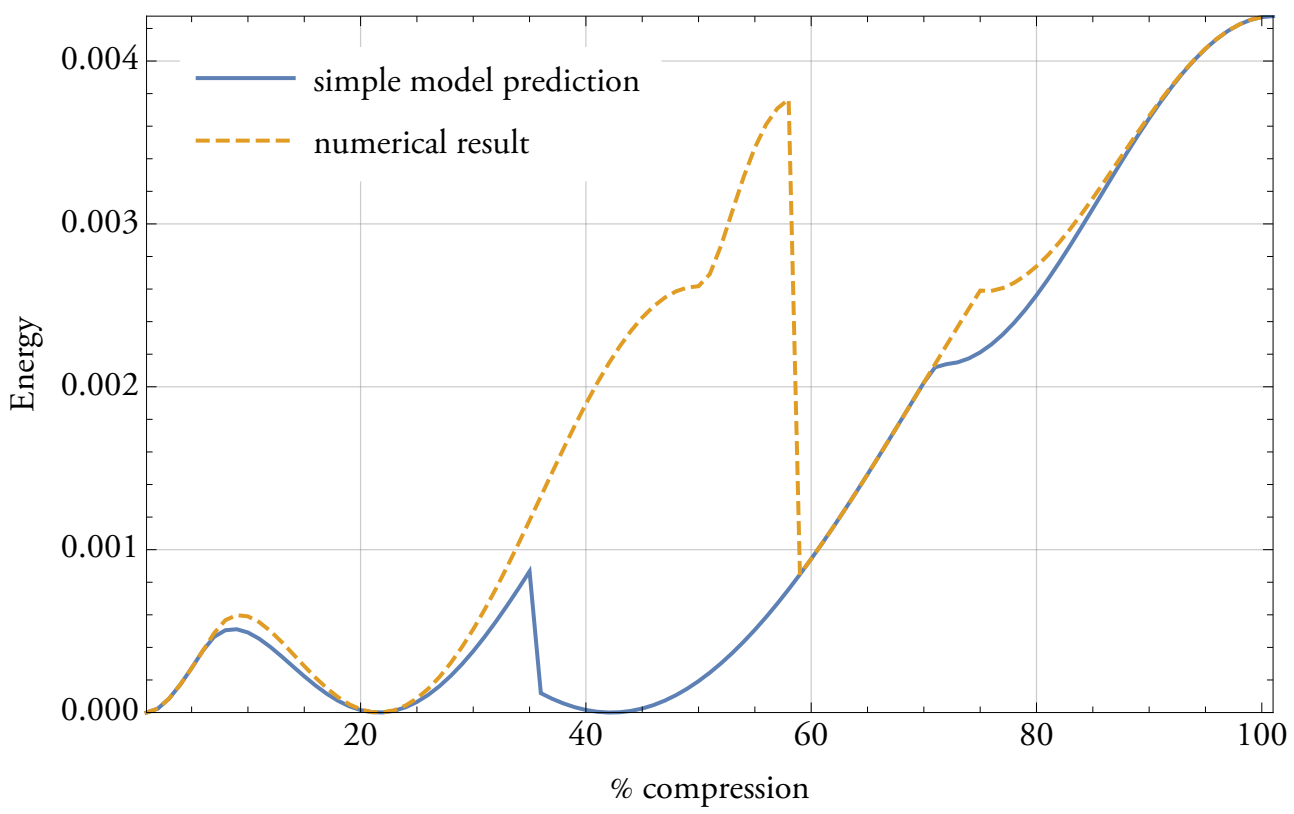

FIGURE 5.9: Energy predictions for $r=1, n=8$ deviate significantly in two places.

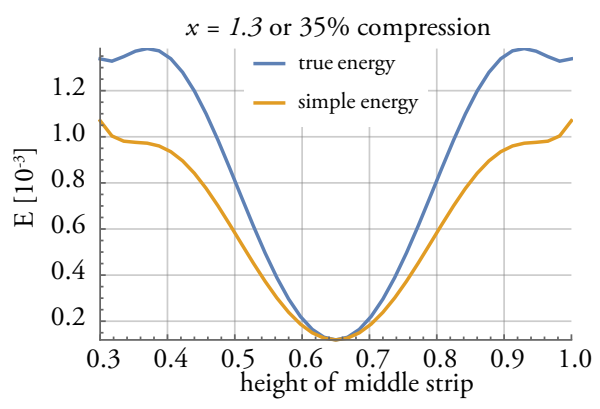

FIgURE 5.10: Two landscape functions showing different shape of simple and true energy. Note that the true energy has three minima while the simple energy has only one local minimum.

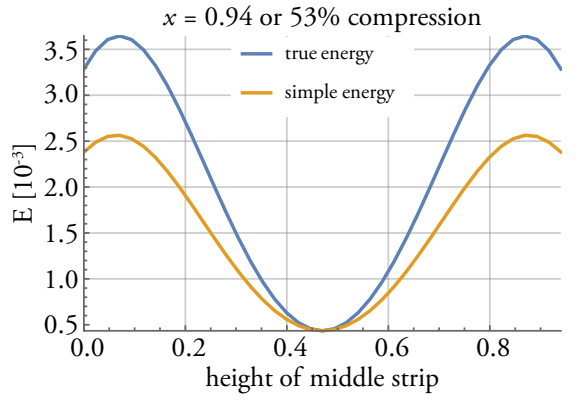

FIgure 5.11: At a later time, the landscape prediction is again correct. 

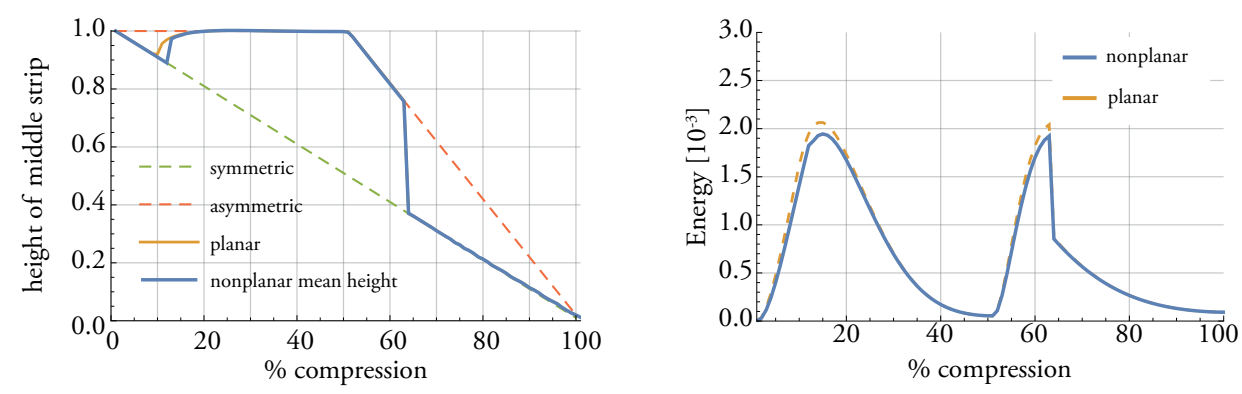

FIGURE 5.12: Comparing planar and nonplanar minimizers

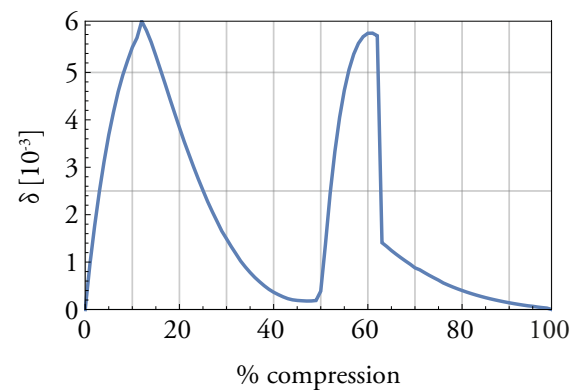

FIGURE 5.13: Plot of $\delta$ for $r=0.6, n=10$.

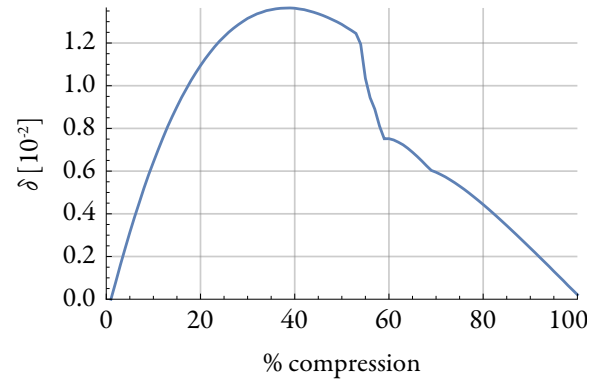

FiguRE 5.14: Plot of $\delta$ for $r=1.2, n=6$.

\subsubsection{Verifying the planarity assumption}

Finally, we verify the validity of the planarity assumption made for the nearly rigid model. To this end, we examine the span of $z$ coordinates of the non-boundary vertices: if $\mathfrak{z}:=$ $\left\{z_{i_{1}}, \ldots, z_{i_{n}}\right\}$ is the set of $z$ coordinates, $\delta:=\max \mathfrak{z}-\min \mathfrak{z}$ measures the deviation from planarity.

Furthermore, we have already seen that the energy landscapes of the planar minimization process may have more than one local minimum and that the exponential minimization cannot cross local maxima even if the local minimum on the other side is lower than that at the current location. Removing the planarity assumption opens up new degrees of freedom, to the effect that the nonplanar minimization process could hypothetically circumvent the energy barriers which appeared above. We therefore also compare the values in $\mathfrak{z}$ to the vertex layer height of the planar minimizer.

For the two examples presented in this section, the corresponding curves are shown in Figures 5.12 and 5.15, together with the deviation from planarity given by $\delta$ in Figures 5.13 and 5.14. Evidently, planarity is very well satisfied as $\delta$ is very small at all times. More importantly, even though removing the planarity assumption has some effect on both the energy and the heights of the nonboundary vertices, the qualitative behavior is exactly the same: both planar and nonplanar minimizers are mostly either in the symmetric or in the asymmetric state and transition at exactly the same points in time.

The aforementioned phenomenon of barrier circumnavigation is tested for by using the planar minimizers as inputs for a second round of minimization without the planarity 

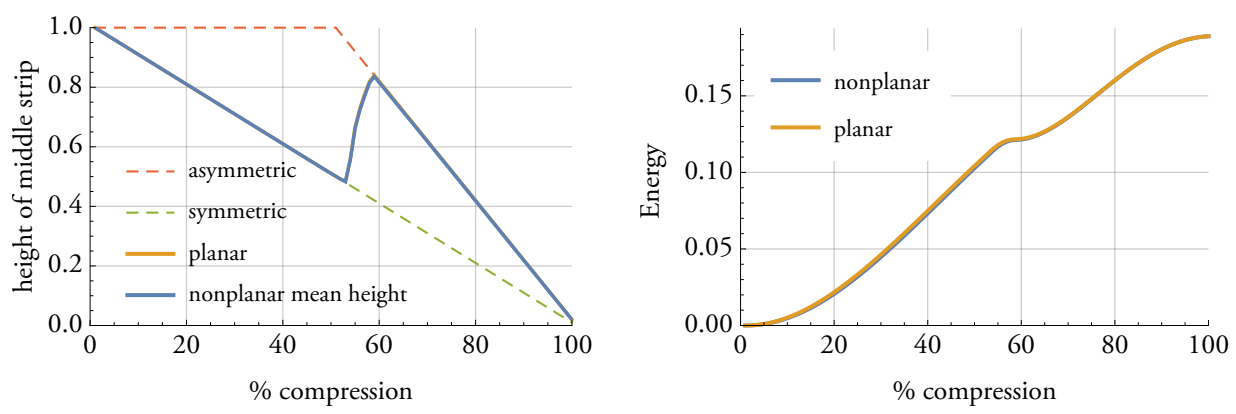

FIGURE 5.15: Comparison of nonplanar and planar minimizers.

assumption. The output of this second round is the same (up to numerical accuracy) as depicted in Figures 5.12 and 5.15 even if substantial random noise is added. We thus conclude that not only do planar and nonplanar minimizers coincide very well, but that in general no circumnavigation effects occur.

\subsubsection{Further observations}

\section{Estimating the minimal elastic yield length}

Another feature that can be extracted from the numerical minimization results is an estimate on the required elastic yield length. This material quantity denotes the maximum length a material can be forcibly extended before it enters the regime of plastic deformations and does not revert back to its original rest state when released, that is, when its deformation can no longer be modeled within the theory of elasticity. The yield length can be estimated from the numerical minimizers by the following procedure: recall that the elastic energy was obtained from computing first the centers of mass of each cluster of vertices and then the sum of squares of distances to the center of mass as in Equation 3.1.1. At each time step, the maximal extension is given by the maximum

$$
l(x)=\max _{i} \max _{j=1, \ldots, n_{i}}\left|v_{i, j}(x)-m_{i}(x)\right|,
$$

to the effect that the yield length must be at least $L=\max _{x} l(x)$. A cylinder supposed to deform elastically must therefore have a yield length of at least $L$.

Applied to the two examples, we obtain the diagrams given in Figures 5.16 and 5.17 .

\subsection{Conclusion}

The results obtained both by numerical minimization and analytically in the frame of the simplified model are very promising. For the type of regular fold pattern that has been the 


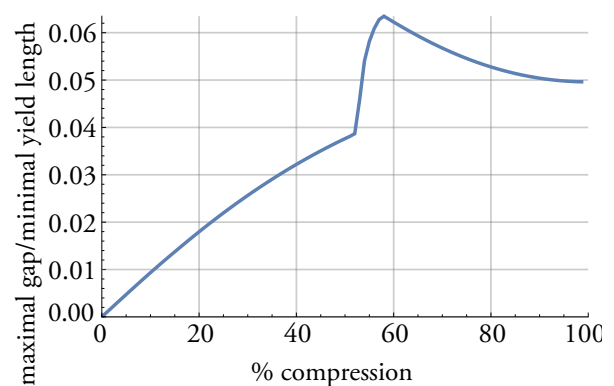

FIGURE 5.16: Plot of the elastic yield for $r=$ 0.6 and $n=10$.

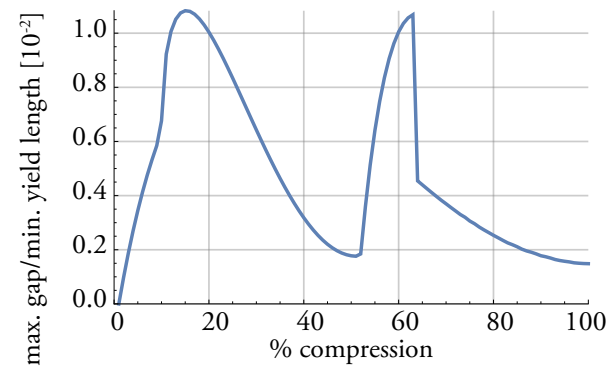

FIGURE 5.17: Plot of the elastic yield for $r=$ 1.2 and $n=10$.

focus of the current simulations, the qualitative behavior of the nearly rigid origami can be predicted very well in a short amount of time and can then be quantitatively fixed by a subsequent numerical minimization.

Furthermore, from a theoretic point of view, our model showed that discontinuous phenomena, manifesting in the physical world as buckling effects, can already arise at a very basic level. 


\section{Conclusion}

The kinematics of folded structures is a long standing subject in mathematics, physics, and the various mechanical engineering disciplines. In this work, we have approached the associated phenomenon of folded thin shells from both the mathematical perspective, using idealized, perfectly rigid objects, and from a physical perspective, using a very simple elastic model and numerical simulations.

Perfectly rigid origami This thesis has brought to light several interesting facts about the global rigidity of prefolded cylindrical structures. Assuming perfect rigidity of the constituent material, it has been shown that flexibility in the axial direction necessitates very strict conditions (Theorems 2.3.2 and 2.3.3), the most visible one being the existence of vertical folds which, furthermore, must remain sharp at all times. To prove these theorems, a model of rigid origami was introduced that, starting from the language of differential geometry of surfaces and very general assumptions on the shape of the folded cylinder, reduced the rigidity problem to the characterization of a univariate real analytic function by means of elementary trigonometry. As it turned out, the assumption of planar faces usually made for rigid origami can be relaxed: if the embedding map is piecewise $C^{4}$, all faces must necessarily be planar.

Within the context of this model, other applications related to the design of folded structures are easily tackled, such as finding folded structures that embed to specified heights (Section 2.4) and structures that possess multiple stable heights (Section 2.5). In particular, it has been shown that a "skeleton" fold pattern provided by the user can be refined to a fold pattern that embeds at a given height by the addition of isolated fold lines.

There is a class of cylindrical rigid origami not covered by the model. Most notably, cylinders may be non-developable or may compress such that the horizontality assumption is violated. Related work by other researchers (we exemplarily highlight recent work by Filipov and others [22]) has shown that non-developable rigid origami cylinders in particular seem to exhibit a much greater variety of flexibility. Extending the rigidity analysis to nondevelopable cylinders would achieve a much more general characterization of the preconditions of flexibility.

For "nonhorizontal" cylinders, that is, those where horizontal cross-sections of the fold pattern do not remain horizontal polygonal lines, a simple counterexample shows that flexibility is easy to obtain: consider a stack of identical regular tetrahedra, each connected to its neighbors along a joint edge, as depicted in Figure 6.1. This surface is developable: at each interior vertex, six equilateral triangles meet, so that the Gauß curvature is zero. Consequently, the stack of tetrahedra is almost a rigid origami cylinder in our sense, with the only fault being that the cylinder walls have been crushed to line segments in multiple different locations. However, even though the cylinder does not possess vertical fold lines, it is 

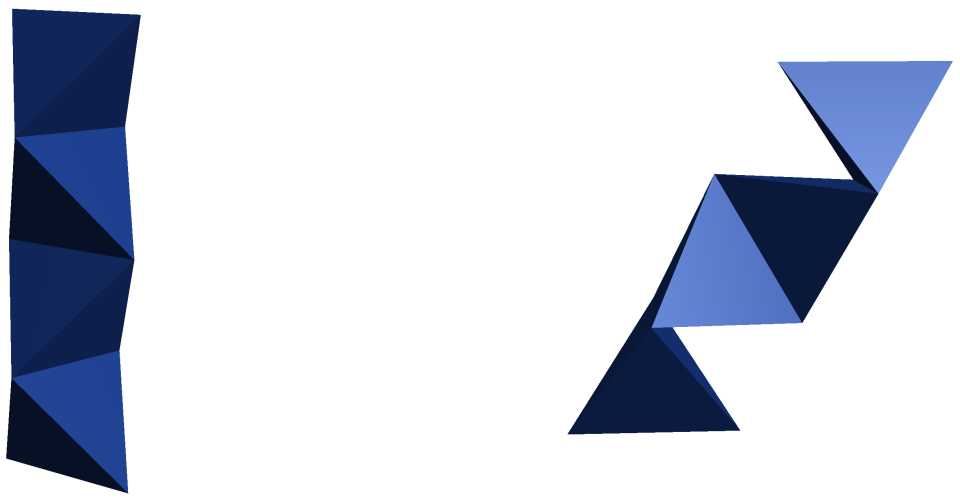

FIGURE 6.1: Another compressible origami cylinder. Left: uncompressed state. Right: compressed state.

clearly rigidly flexible: by tilting the middle pair of tetrahedra about the edge joints, one can change the height of the complete stack without violating isometry or even the planarity of the boundaries.

It is currently unknown whether there exist flexible origami cylinders that compress non-horizontal, but also do not degenerate, and even Theorem 2.3.3 directly implies a degeneracy of any compressible rigid origami cylinder in that vertical fold lines must exist and fold sharply, so that the two adjacent faces lie on top of each other. On the other hand, the results of our nearly rigid model seems to indicate that planarity is a natural state if the fold pattern is sufficiently regular. In light of this, it seems worthwhile to investigate the role of degeneracy of embeddings for the flexibility of rigid objects.

Pseudo-elastic models Relaxing the condition of perfect rigidity, a simple model has been formulated that mimics the key feature of the experimentally observed elastic behavior of creased thin sheets in that faces remain rigid and only creases may deform elastically. As an extension of the perfectly rigid model, this nearly rigid model is perfectly compatible with the previously obtained results, while at the same time allowing the formulation of a geometric method for finding numerically the kinematic response and requisite strain for axial compressions of cylindrical structures.

The results encourage and justify the inclusion of simplifying assumptions into the perfectly rigid model towards for instance rotational symmetry or horizontality of cross sections. Moreover, they exhibit buckling phenomena of different kinds, indicating that the existence of buckling is a direct consequence of the geometry of the problem and not necessarily predicated by complex and subtle interactions of the physical laws of elasticity. Further examination would shed more light on the complex nature of buckling phenomena in real-world folded structures.

Another natural extension of the model would see the current elastic energy replaced by a more complex one that reflects the energy scaling behavior of elastic ridges known from 
experiments and numerical simulations, such as the crease energy formulated by Witten and Lobkovsky [39]. Doing so would bridge the gap to physically motivated models of elasticity and thus provide another possible avenue for the high-level exploration of phenomena related to elasticity in general, but this scaling behavior has not yet been extended to multiple interacting creases. Finding the correct general crease energy would be or even determining whether it can be reduced to a simple per-crease expression is ongoing work.

Analytic predictions of buckling phenomena Finally, it has been empirically shown that a further simplification of decoupling the cylinder's strips allows to predict with great ease the qualitative behavior of multi-strip systems. Thus, it is certainly within the scope of this simple model to pose the inverse problem: instead of computing the elastic response of a given folded structure, the task now becomes to find a folded structure whose behavior is equal or at least close to a user-given target response. Although certainly more challenging than the forward problem treated in this thesis, solving the inverse problem would benefit various applications in engineering and architecture.

The current model already presents a crude solution: by providing an easier method to obtain the elastic response compared to finite element methods, the model allows to quickly narrow down the range of candidate parameter values. Finding a true solution to the inverse problem would alleviate the need for extensive cataloging and sampling operations, thus providing an efficient tool for designing folded cylinders with a prescribed elastic response. 
Conclusion 


\section{Bibliography}

[1] Abel, Z., Connelly, R., Demaine, E. D., Demaine, M. L., Hull, T. C., Lubiw, A., AND TACHI, T. Rigid Flattening of Polyhedra with Slits. Origami6: I. Mathematics (2015), 109-118.

[2] Alexandrov, A. D. Convex Polyhedra. Springer, Berlin, Heidelberg, 2005.

[3] Balkcom, D. J., Demaine, E. D., Demaine, M. L., Ochsendorf, J. A., And You, Z. Folding Paper Shopping Bags. In Origami 4, R. J. Lang, Ed., vol. 1. A K Peters/CRC Press, Boca Raton, 2009, ch. 28, pp. 315--333.

[4] BARDOs, L. C. Interesting things to make out of paper-from mathematical models to toys. How to make Steffen's flexible polyhedron.

[5] BazaraA, M. S., And Shetty, C. M. Nonlinear Programming, Theory and Applications. John Wiley \& Sons, New York, New York, USA, 1979.

[6] Belcastro, S.-M., and Hull, T. C. Modelling the folding of paper into three dimensions using affine transformations. Linear Algebra and its Applications 348, 1-3 (2002), 273-282.

[7] Berberich, E., Emeliyanenko, P., and Sagraloff, M. An Elimination Method for Solving Bivariate Polynomial Systems : Eliminating the Usual Drawbacks. In Proceedings of the Thirteenth Workshop on Algorithm Engineering and Experiments (ALENEX) (2011), pp. 35-47.

[8] Bös, F., Wardetzky, M., Vouga, E., and Gottesman, O. On the Incompressibility of Cylindrical Origami Patterns. Journal of Mechanical Design 139, 2 (dec 2016), 21404-21409.

[9] Botsch, M., Pauly, M., Gross, M., and Kobbelt, L. PriMo : Coupled Prisms for Intuitive Surface Modeling.

[10] BRICARD, R. Mémoire sur la théorie de l'octaèdre articulé. Journal de Mathématiques Pures et Appliquées 3 (1897), 113-148.

[11] BRICARD, R. Memoir on the Theory of the Articulated Octahedron. arXiv preprint (2010).

[12] Cauchy, A. L. Recherche sur les polyèdres-premier mémoire. Journal de l'Ecole Polytechnique 9 (1813), 68-86.

[13] Ciarlet, P. G. Mathematical Elasticity, vol. 1 of Three-dimensional elasticity. Elsevier, Amsterdam, 1993. 
[14] Connelly, R. A counterexample to the rigidity conjecture for polyhedra. Publications Mathématiques del I.H.E.S. 47 (1977), 333-338.

[15] Connelly, R. The Rigidity of Polyhedral Surfaces. Mathematics Magazine 52, 5 (1979), 275-283.

[16] Connelly, R., Sabitov, I., and Walz, A. The Bellows Conjecture. Beiträge zur Algebra und Geometrie 38, 1 (1997), 1-10.

[17] Conti, S., and Maggi, F. Confining Thin Elastic Sheets and Folding Paper. Arch. Rational Mech. Anal. 187 (2008), 1-48.

[18] Coppa, A. P. Inextensional Buckling Configurations of Conical Shells. AIAA Journal 5, 4 (1967), 750-754.

[19] do Carmo, M. P. Differential Geometry of Curves and Surfaces, 1 ed. Prentice Hall, Upper Saddle River, 1976.

[20] Dudte, L. H., Vouga, E., Tachi, T., And Mahadevan, L. Programming curvature using origami tessellations. Nature Materials 15, January (2016), 583-588.

[21] Euler, L. Opera Postuma Mathematica et Physica. St. Petersburg, 1862.

[22] Filipov, E. T., Paulino, G. H., and Tachi, T. Origami tubes with reconfigurable polygonal cross-sections. Proceedings of the Royal Society A: Mathematical, Physical and Engineering Science 472, 2185 (2016).

[23] Friesecke, G., James, R. D., and Müller, S. A theorem on geometric rigidity and the derivation of nonlinear plate theory from three dimensional elasticity. 2002.

[24] Friesecke, G., James, R. D., And Müller, S. A Hierarchy of Plate Models Derived from Nonlinear Elasticity by Gamma-Convergence. Arch. Rational Mech. Anal. 180 (2006), 183-236.

[25] GLuCK, H. Almost all simply connected closed surfaces are rigid. Springer Berlin Heidelberg, Berlin, Heidelberg, 1975, pp. 225-239.

[26] Guest, S. D., and Pellegrino, S. The Folding of Triangulated Cylinders, Part I: Geometric Considerations. Journal of Applied Mechanics 61, 4 (1994), 773-777.

[27] Guest, S. D., and Pellegrino, S. The Folding of Triangulated Cylinders, Part II: The Folding Process. Journal of Applied Mechanics 61, 4 (1994), 778-783.

[28] Guest, S. D., And Pellegrino, S. The Folding of Triangulated Cylinders, Part III: Experiments. Journal of Applied Mechanics 63, 1 (1996), 77-83. 
[29] Hall, B. C. Lie Groups, Lie Algebras, and Representations: An Elementary Introduction, 2 ed. Graduate Texts in Mathematics. Springer International Publishing, 2015.

[30] Hartman, P., And Wintner, A. On the Fundamental Equations of Differential Geometry. American Journal of Mathematics 72, 4 (1950), 757-774.

[31] Hiriart-Urruty, J.-B., And Lemaréchal, C. Convex analysis and minimization algorithms I: Fundamentals, 1 ed., vol. 305. Springer-Verlag, Berlin, Heidelberg, 1993.

[32] Huffman, D. A. Curvature and Creases: A Primer on Paper. IEEE Transactions on Computers C-25, 10 (1976), 1010-1019.

[33] Kilian, M., Flöry, S., Chen, Z., Mitra, N. J., Sheffer, A., and Pottmann, H. Curved Folding. In ACM SIGGRAPH 2008 Papers (New York, NY, USA, 2008), vol. 27, ACM.

[34] Kirchioff, G. Über das Gleichgewicht und die Bewegung einer elastischen Scheibe. Journal für die reine und angewandte Mathematik 40 (1850), 51-88.

[35] Kuribayashi, K., Tsuchiya, K., You, Z., Tomus, D., Umemoto, M., Ito, T., and SASAKI, M. Self-deployable origami stent grafts as a biomedical application of Ni-rich TiNi shape memory alloy foil. Materials Science and Engineering: A 419, 1-2 (2006), $131-137$.

[36] Le Dret, H., ANd Raoult, A. The nonlinear membrane model as a variational limit of nonlinear three-dimensional elasticity. Journal de Mathématiques Pures et Appliquées 74 (1995), 549-578.

[37] Loвкоvsкy, A. E. Boundary layer analysis of the ridge singularity in a thin plate. Physical Review E 53, 4 (1996), 3750-3760.

[38] Lobkovsky, A. E. Structure of Crumpled Thin Elastic Membranes. PhD thesis, University of Chicago, 1996.

[39] Lobkovsky, A. E., And Witten, T. A. Properties of ridges in elastic membranes. Physical Review E 55, 2 (1997), 1577-1589.

[40] Ma, L., AND Wu, Z. Approximation to the k-th derivatives by multiquadric quasiinterpolation method. Journal of Computational and Applied Mathematics 231, 2 (2009), 925-932.

[41] Massey, W. S. Surfaces of Gaussian Curvature Zero in Euclidean 3-Space. Tohoku Mathematical Journal, Second Series 14, 1 (1962), 73-79.

[42] Mirzaei, D., Schaback, R., and Dehghan, M. On Generalized Moving Least Squares and Diffuse Derivatives. IMA Journal of Numerical Analysis 32, 3 (2012), 983-1000. 
[43] Miura, K. Proposition of Pseudo-Cylindrical Concave Polyhedral Shells. ISAS Report 34, 9 (1969), 141-163.

[44] Mivra, K. Method of packaging and deployment of large membranes in space. In Proceedings of 31 st Congress International Astronautical Federation (1980), pp. 1-10.

[45] MiURA, K., AND TACHI, T. Synthesis of rigid-foldable cylindrical polyhedra. Journal of the International Society for the Interdisciplinary Study of Symmetry (aug 2010), 204213.

[46] Nocedal, J., And Wright, S. J. Numerical Optimization. Springer, New York, NY, USA, 2006.

[47] Rudolph, G., And Schmidt, M. Differential Geometry and Mathematical Physics Part I. Manifolds, Lie Groups and Hamiltonian Systems, 1 ed. Springer Science+Business Media, Dordrecht, 2013.

[48] Saito, K., Tsukahara, A., and Okabe, Y. Designing of self-deploying origami structures using geometrically misaligned crease patterns. Proceedings of the Royal Society A 472, 2185 (2016), 1-16.

[49] Silverberg, J. L., Evans, A. A., Mcleod, L., Hayward, R. C., Hull, T. C., SanTANGelo, C. D., AND Cohen, I. Using origami design principles to fold reprogrammable mechanical metamaterials. Science 345, 6197 (2014), 647-650.

[50] Silverberg, J. L., Na, J.-H., Evans, A. A., Liu, B., Hull, T. C., Santangelo, C. D., LANG, R. J., HaYward, R. C., AND Cohen, I. Origami structures with a critical transition to bistability arising from hidden degrees of freedom. Nat Mater 14, 4 (apr 2015), 389-393.

[51] Song, J., Chen, Y., ANd Lu, G. Axial crushing of thin-walled structures with origami patterns. Thin-Walled Structures 54 (may 2012), 65-71.

[52] Streinu, I., and Whiteley, W. Single-vertex origami and spherical expansive motions. In Discrete and Computational Geometry. Springer, Berlin, Heidelberg, oct 2004, pp. 161-173.

[53] TACHI, T. Generalization of Rigid Foldable Quadrilateral Mesh Origami. In Symposium of the International Association For Shell And Spatial Structures (2009) (Valencia, 2009), A. Domingo and C. Lazaro, Eds., pp. 2287-2294.

[54] TACHI, T. One-dof cylindrical deployable structures with rigid quadrilateral panels. Proceedings of the IASS Symposium October, October (2009), 2295-2305.

[55] UJVÁRI, M. On the projection onto a finitely generated cone. 2007. 
[56] Venkataramani, S. C. Lower bounds for the energy in a crumpled elastic sheet-a minimal ridge. Nonlinearity 17 (2004), 301-312.

[57] Venkataramani, S. C. The energy of crumpled sheets in Föppl-von Kármán plate theory. 2008.

[58] Wang, K., and Chen, Y. Folding a patterned cylinder by rigid origami. Origami 5: Fifth International Meeting of Origami Science, Mathematics, and Education 5 (2011), 265-276.

[59] Witten, T. A. Stress focusing in elastic sheets. Reviews of Modern Physics 79, 2 (apr 2007), 643-675.

[60] Wu, W., AND You, Z. Modelling rigid origami with quaternions and dual quaternions. Proceedings of the Royal Society A 466, 2119 (2010), 2155-2174.

[61] Wu, W., And You, Z. A solution for folding rigid tall shopping bags. Proceedings of the Royal Society A 467, 2133 (2011), 2561-2574.

[62] Yasuda, H., Yein, T., TAchi, T., Miura, K., and Taya, M. Folding behaviour of Tachi - Miura polyhedron bellows. Proceedings of the Royal Society A 469, 2159 (2013), 201303.

[63] Yoshimura, Y. On the Mechanism of Buckling of a Circular Cylindrical Shell Under Axial Compression. Tech. rep., National Advisory Committee for Aeronautics, Washington, 1955.

[64] Zhang, X., Cheng, G., You, Z., and Zhang, H. Energy absorption of axially compressed thin-walled square tubes with patterns. Thin-Walled Structures 45, 9 (2007), 737-746. 
A. Further results 


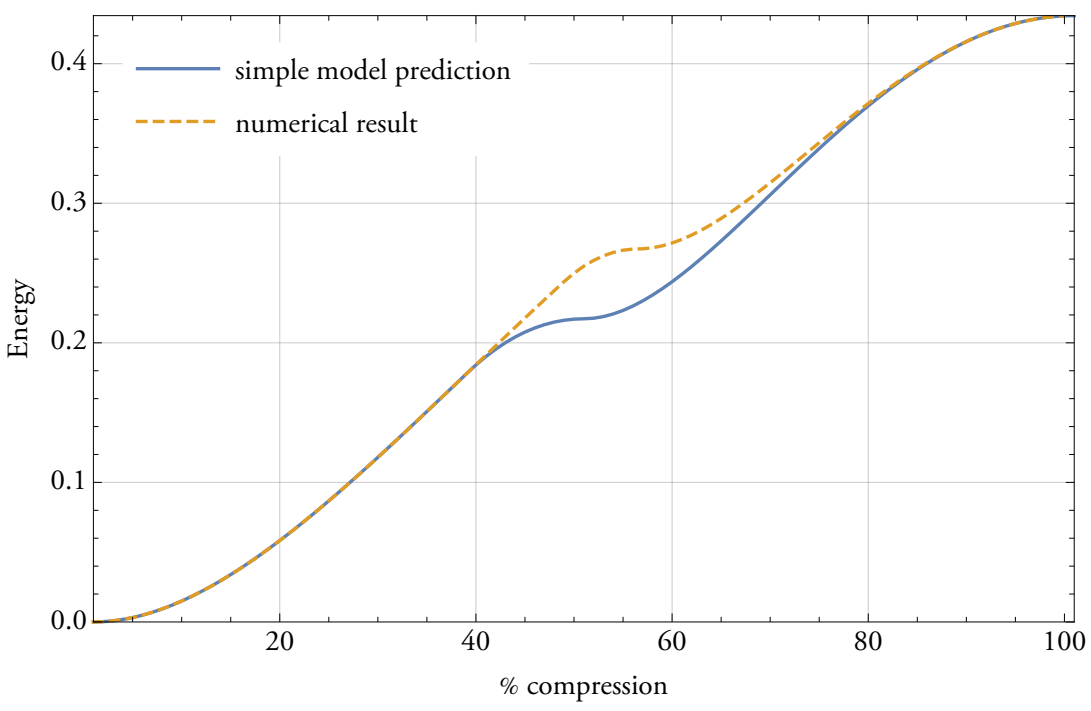

FIGURE A.1: simple energy for $r=0.6, n=5, m=2$

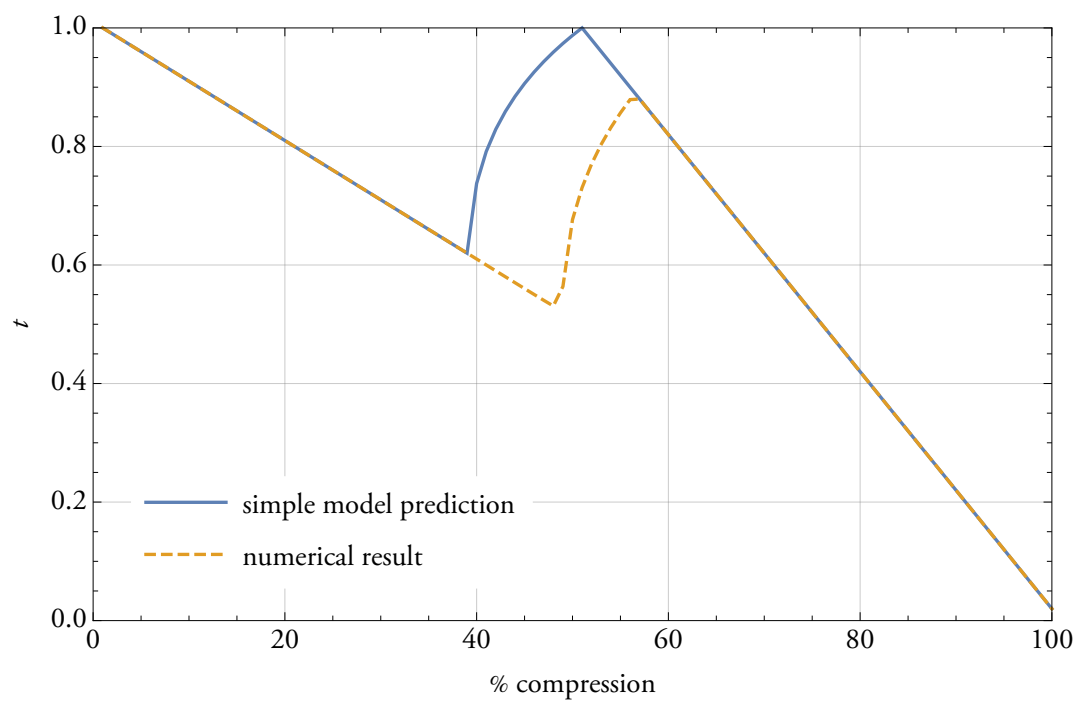

FIGURE A.2: intermediate layer height for $r=0.6, n=5, m=2$

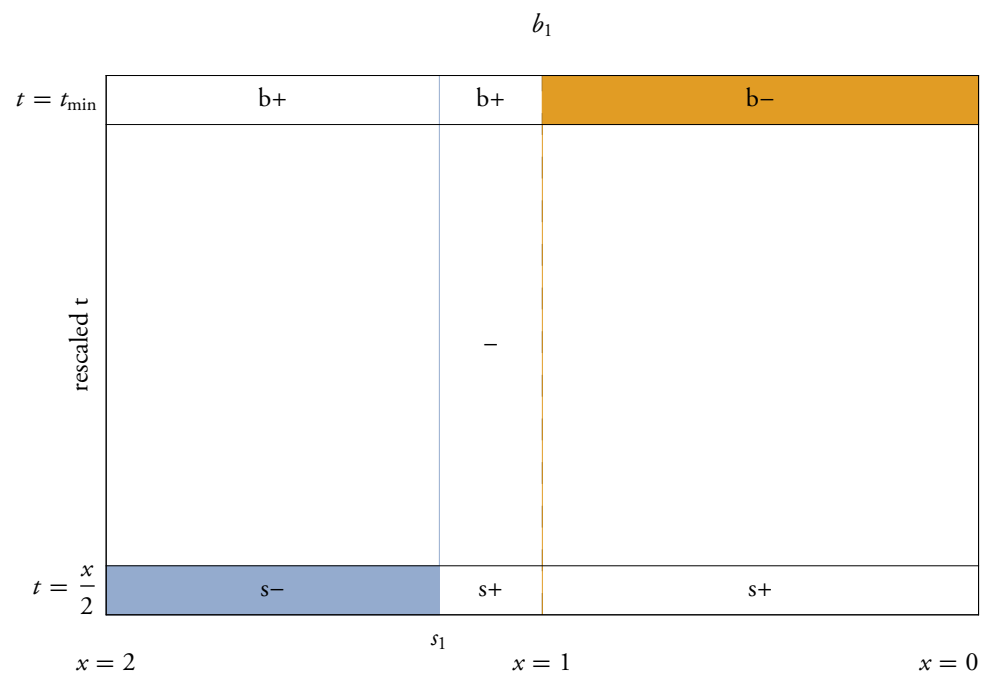

FIGURE A.3: prediction graphic for $r=0.6, n=5, m=2$ 


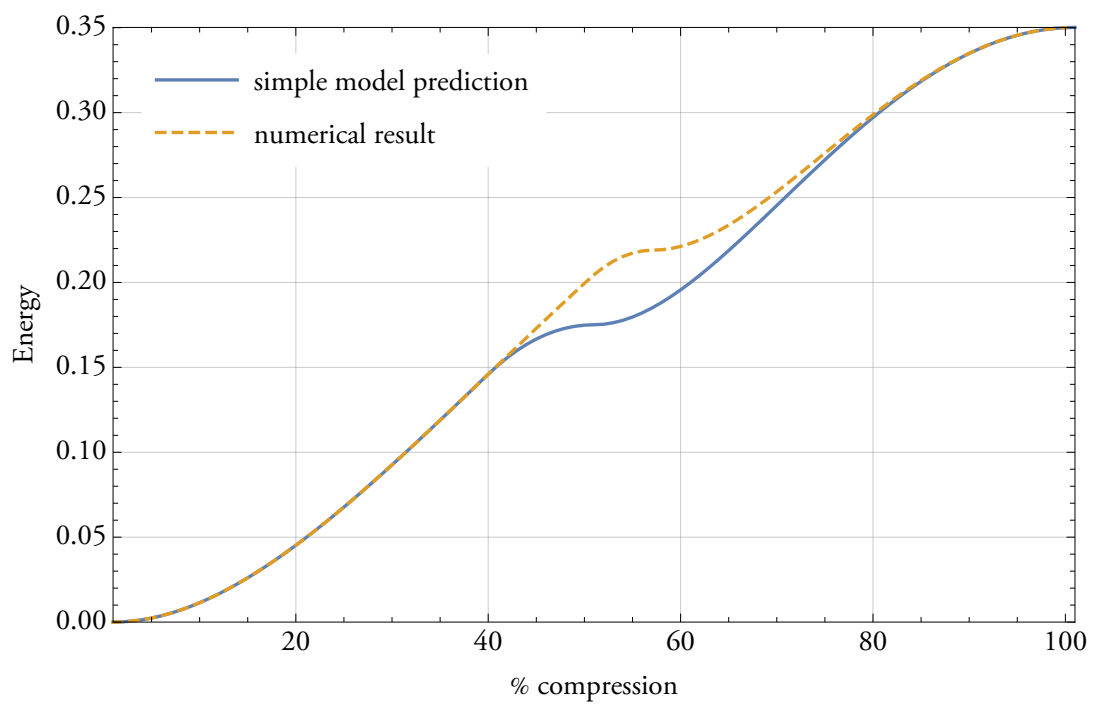

FIGURE A.4: simple energy for $r=0.6, n=6, m=2$

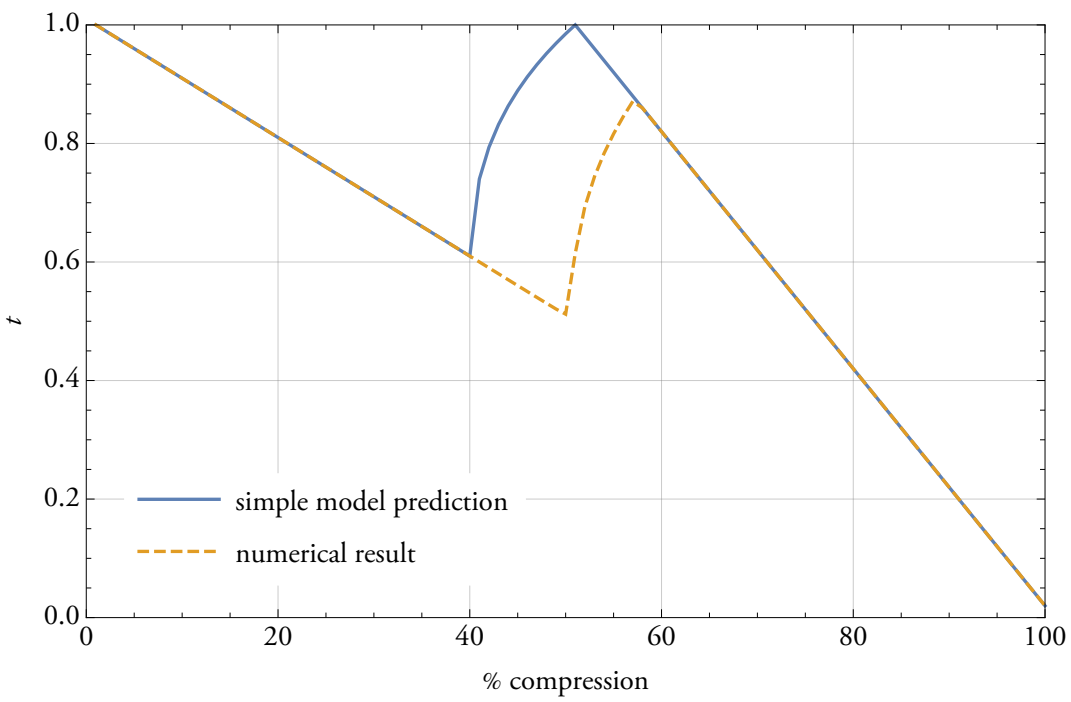

FIGURE A.5: intermediate layer height for $r=0.6, n=6, m=2$

$b_{1}$

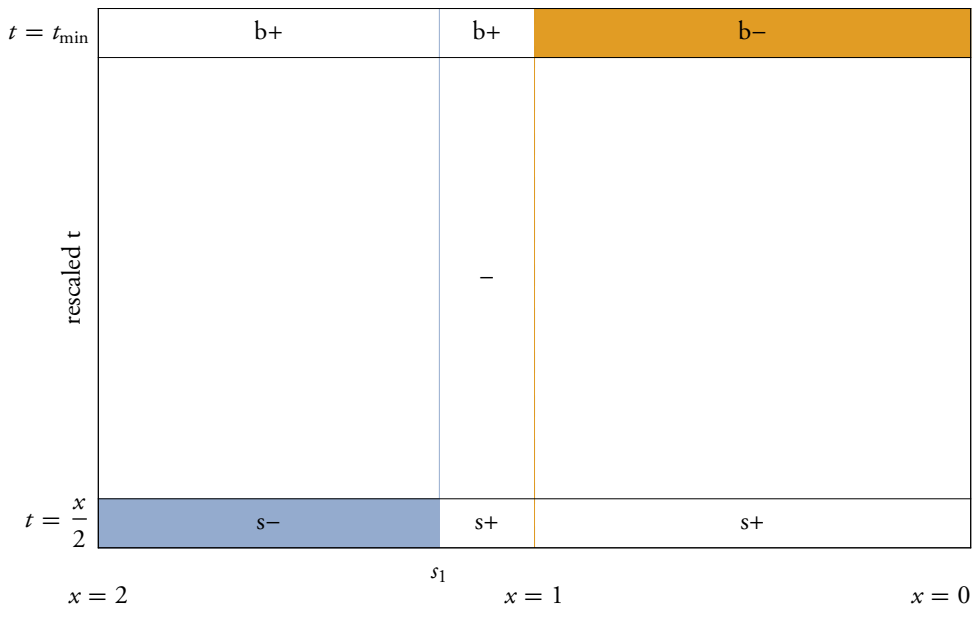

FIGURE A.6: prediction graphic for $r=0.6, n=6, m=2$ 


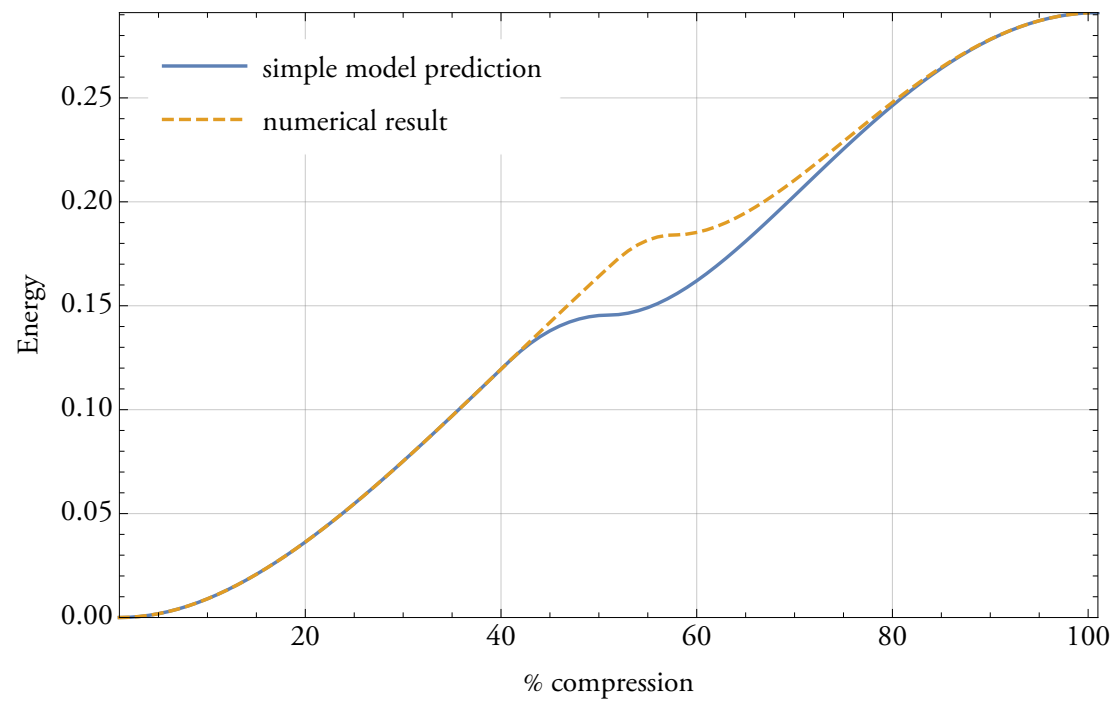

FIGURE A.7: simple energy for $r=0.6, n=7, m=2$

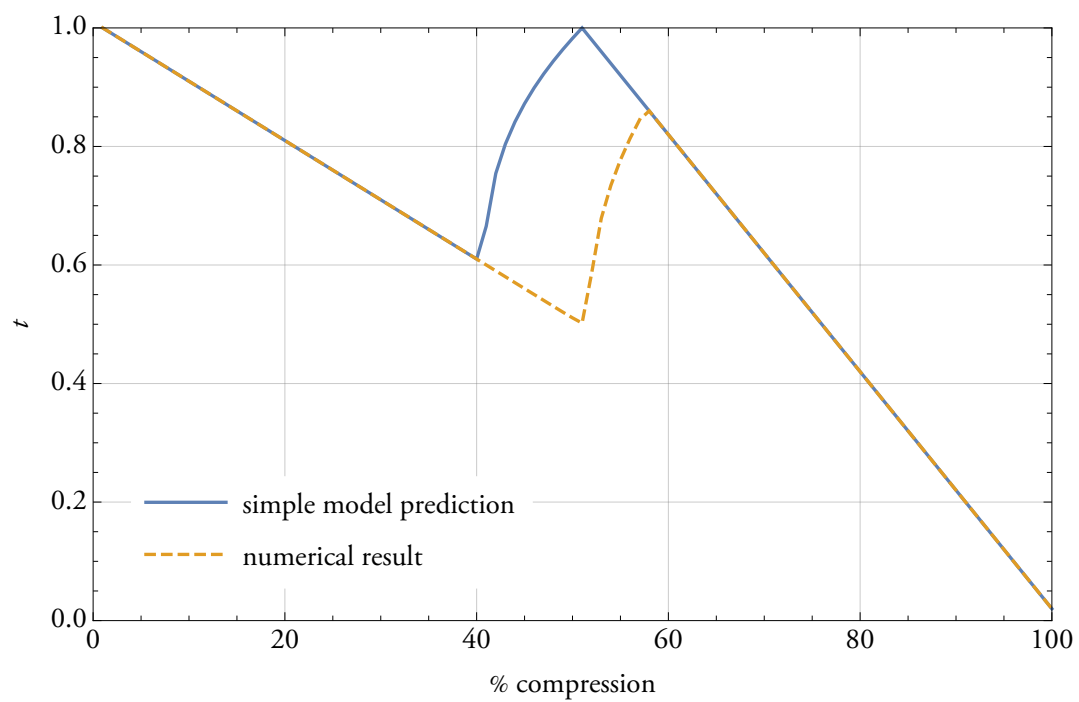

FIGURE A.8: intermediate layer height for $r=0.6, n=7, m=2$

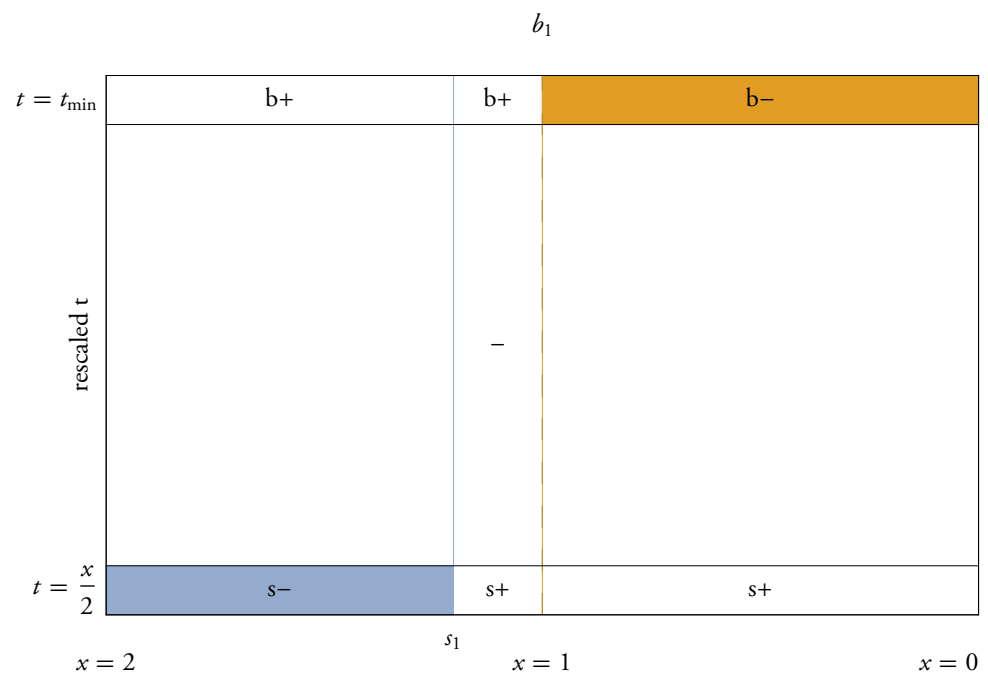

FIGURE A.9: prediction graphic for $r=0.6, n=7, m=2$ 


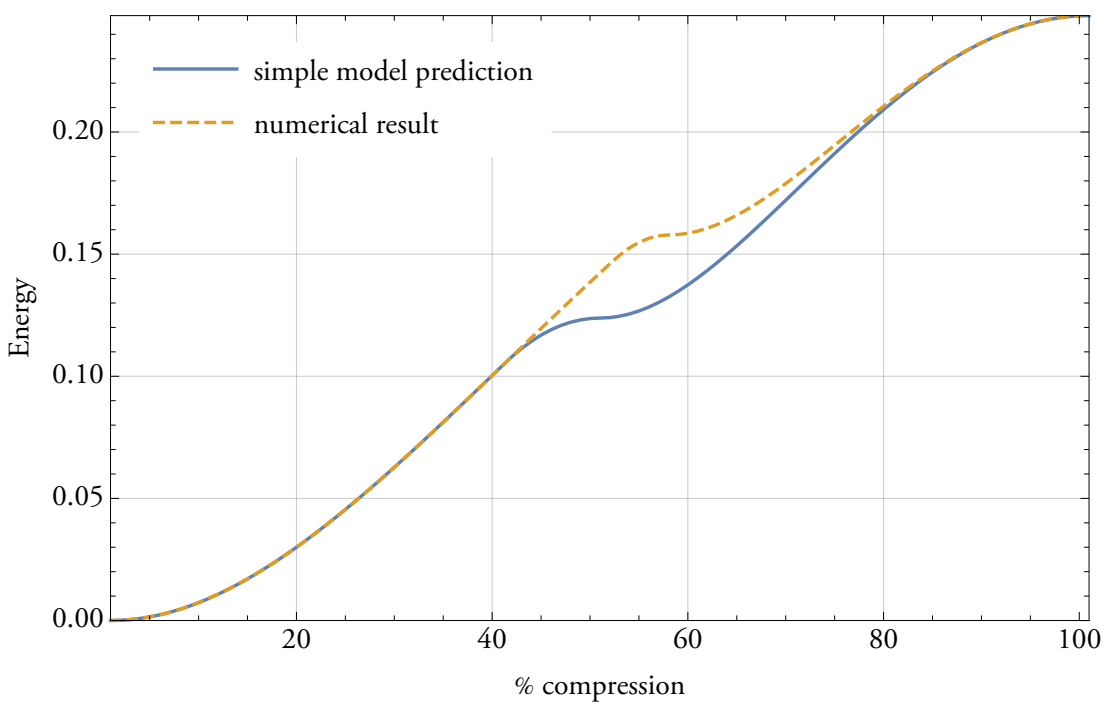

FIGURE A.10: simple energy for $r=0.6, n=8, m=2$

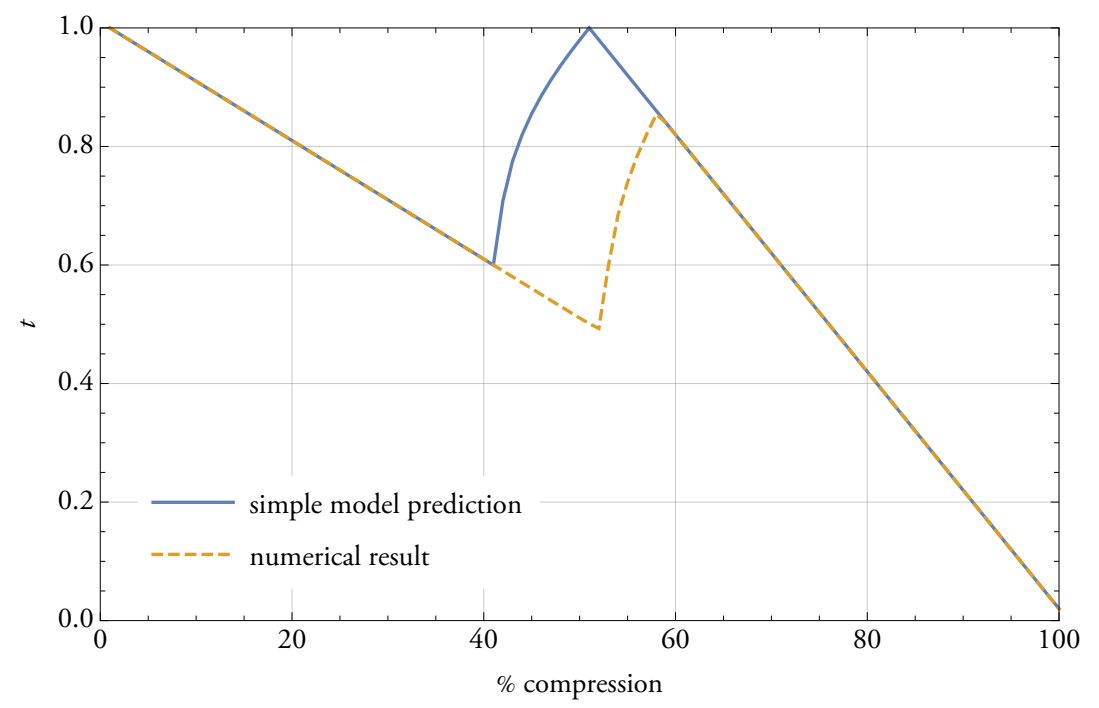

FIGURE A.11: intermediate layer height for $r=0.6, n=8, m=2$

$b_{1}$

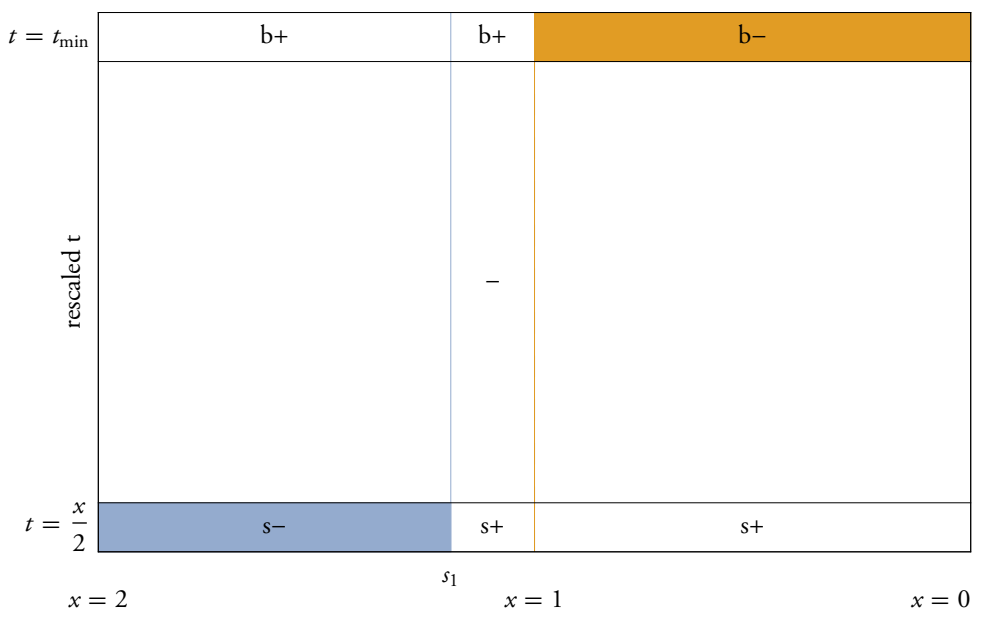

FIGURE A.12: prediction graphic for $r=0.6, n=8, m=2$ 


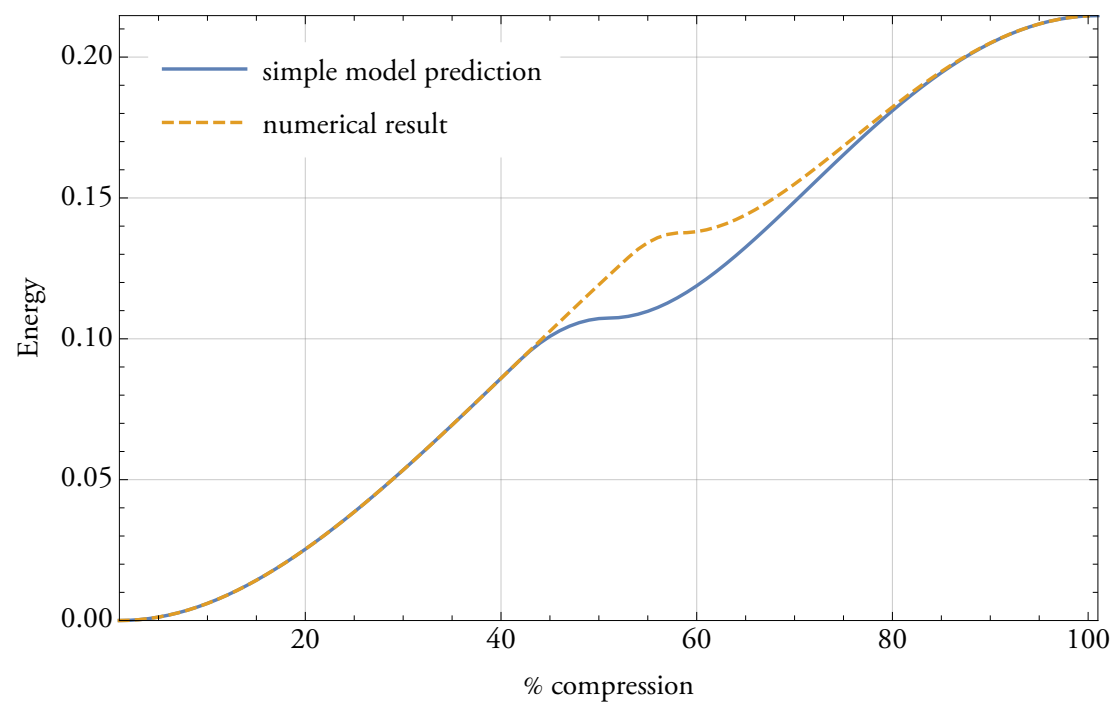

FIGURE A.13: simple energy for $r=0.6, n=9, m=2$

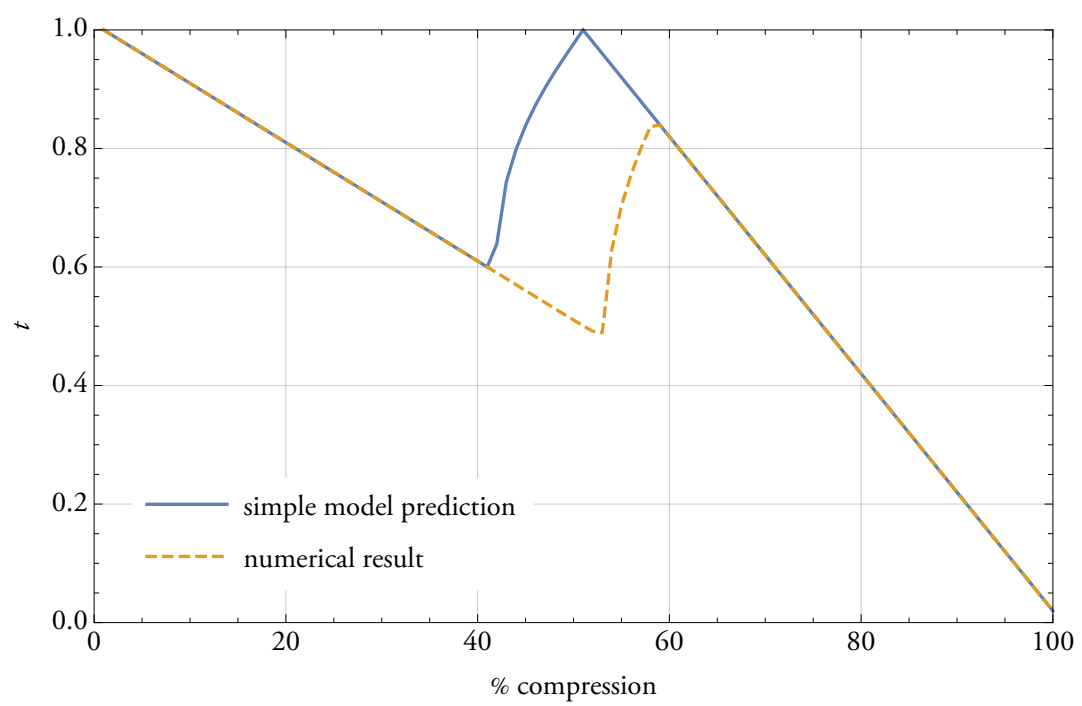

FIGURE A.14: intermediate layer height for $r=0.6, n=9, m=2$

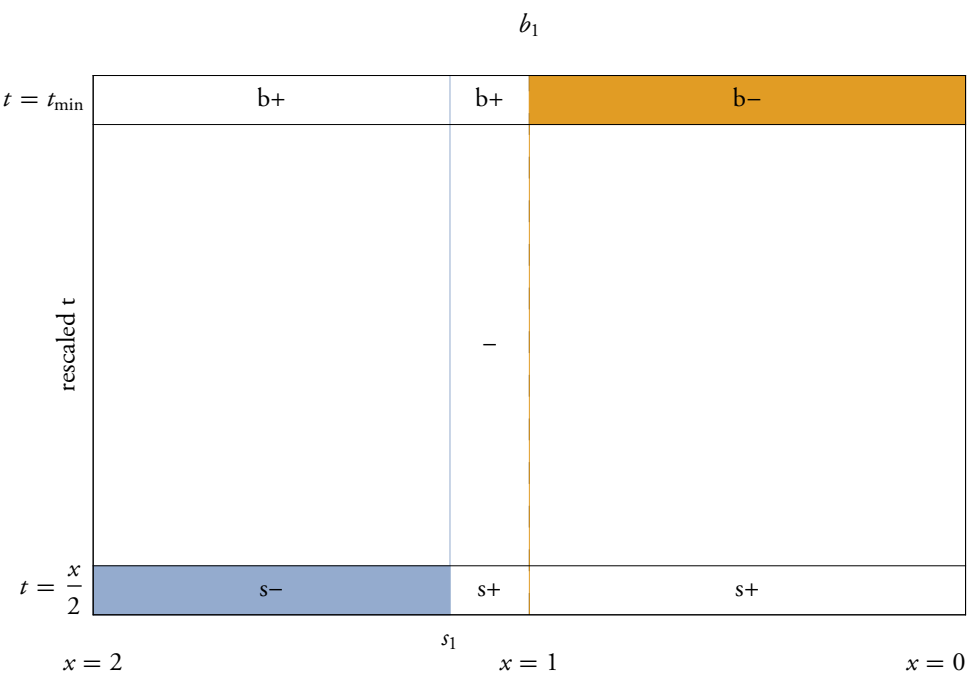

FIGURE A.15: prediction graphic for $r=0.6, n=9, m=2$ 


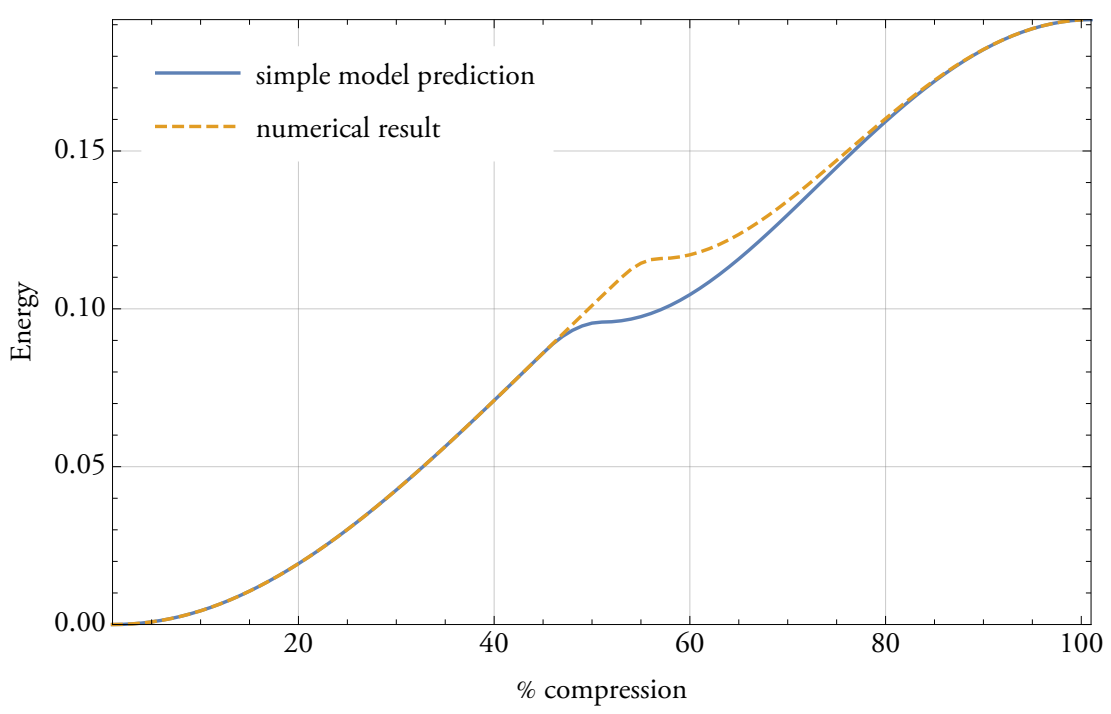

FIGURE A.16: simple energy for $r=0.8, n=5, m=2$

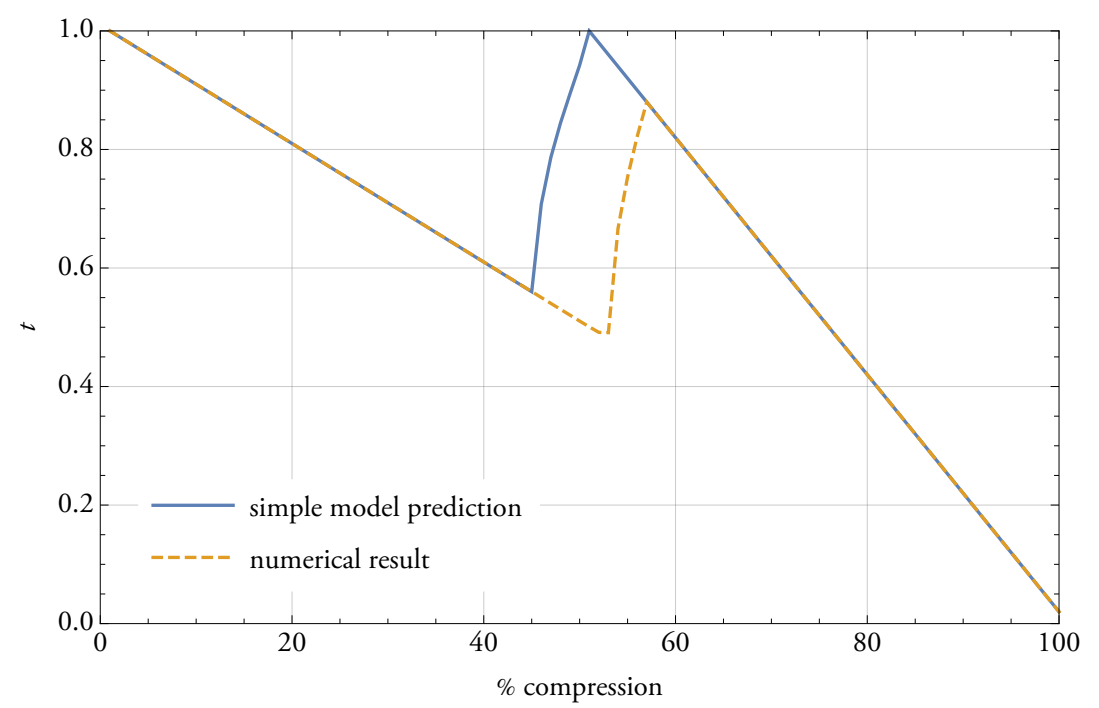

FIGURE A.17: intermediate layer height for $r=0.8, n=5, m=2$

$b_{1}$

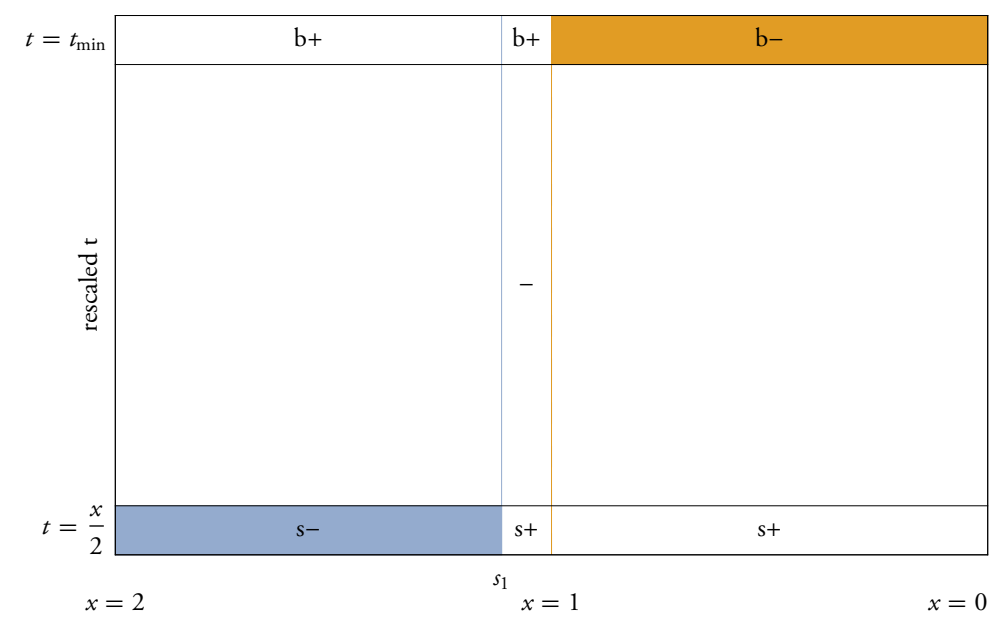

FIGURE A.18: prediction graphic for $r=0.8, n=5, m=2$ 


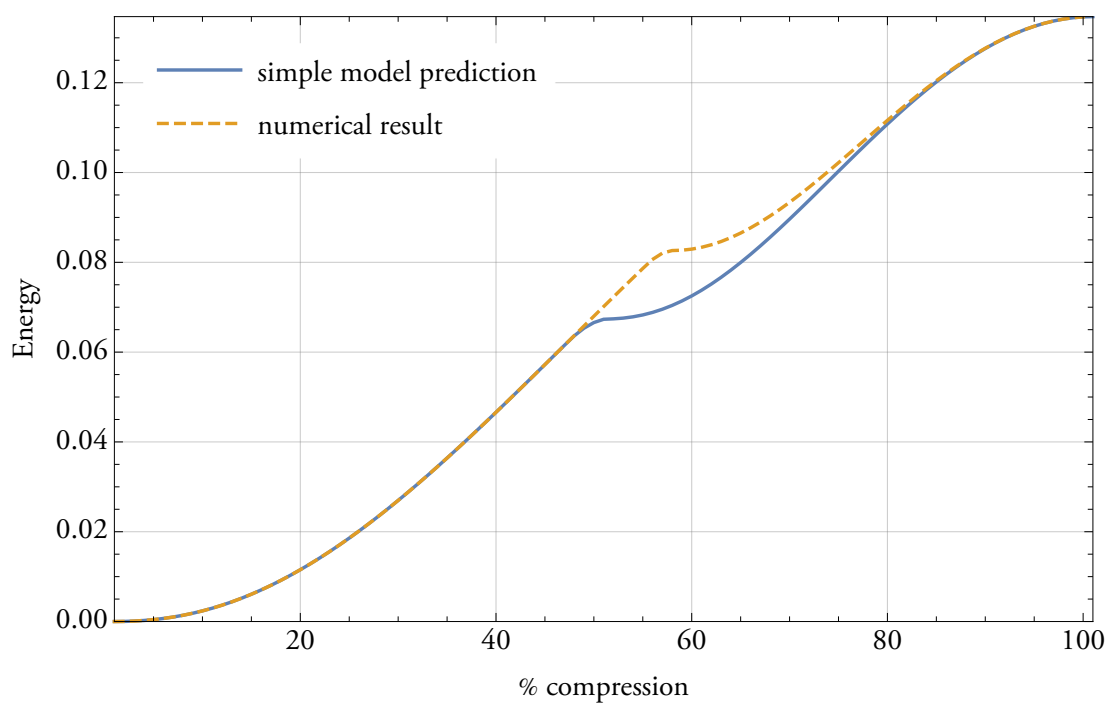

FIGURE A.19: simple energy for $r=0.8, n=6, m=2$

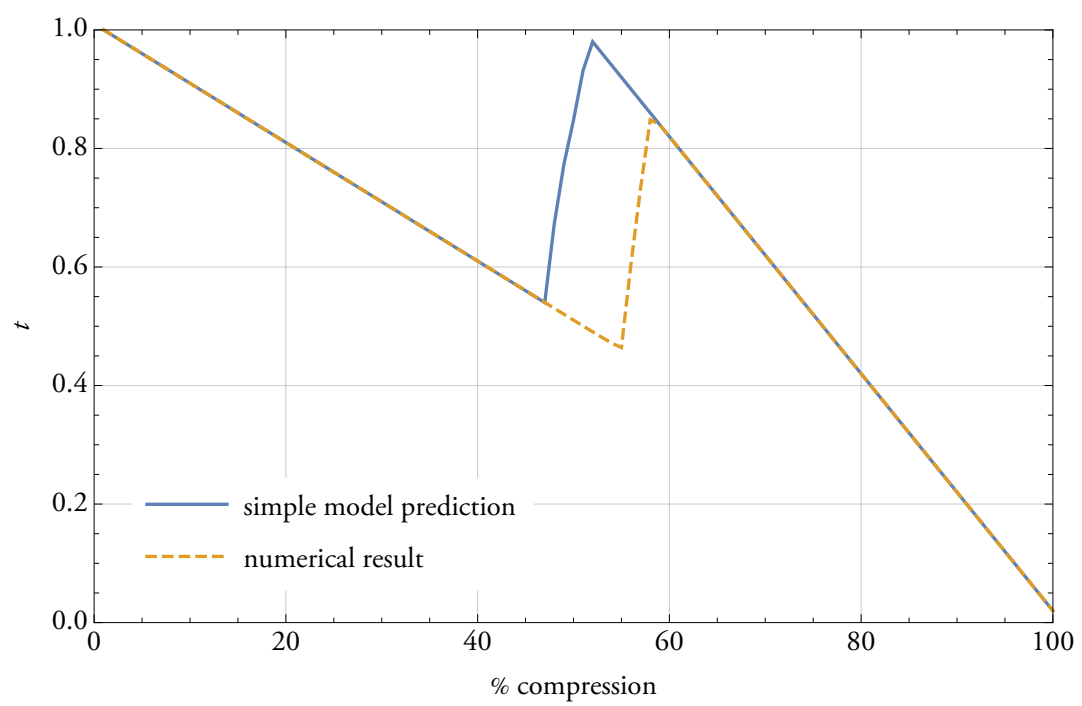

FIGURE A.20: intermediate layer height for $r=0.8, n=6, m=2$

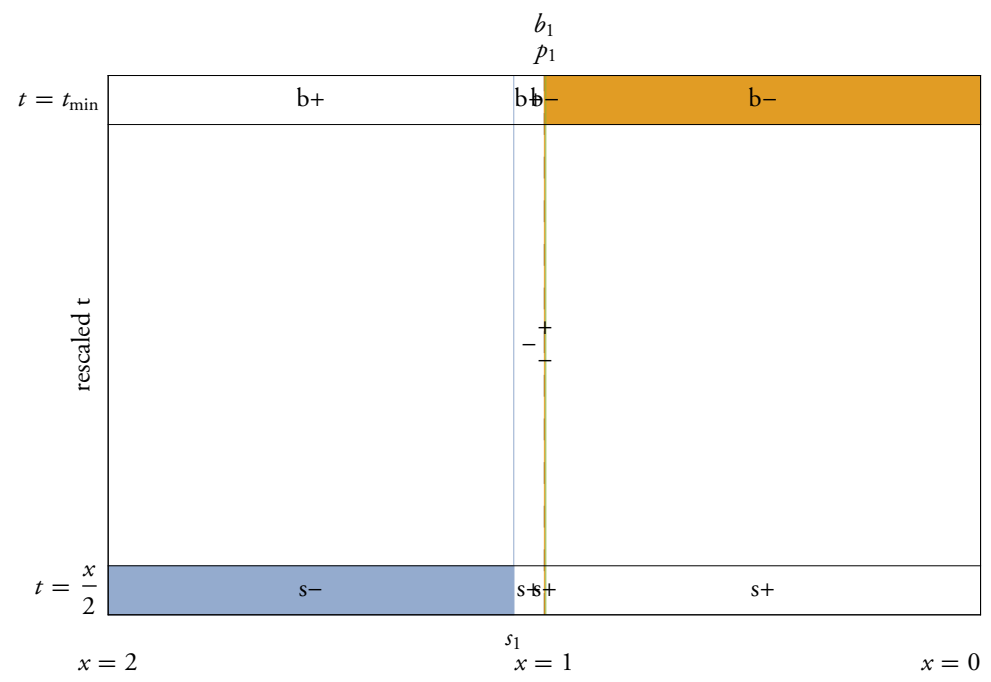

FIGURE A.21: prediction graphic for $r=0.8, n=6, m=2$ 


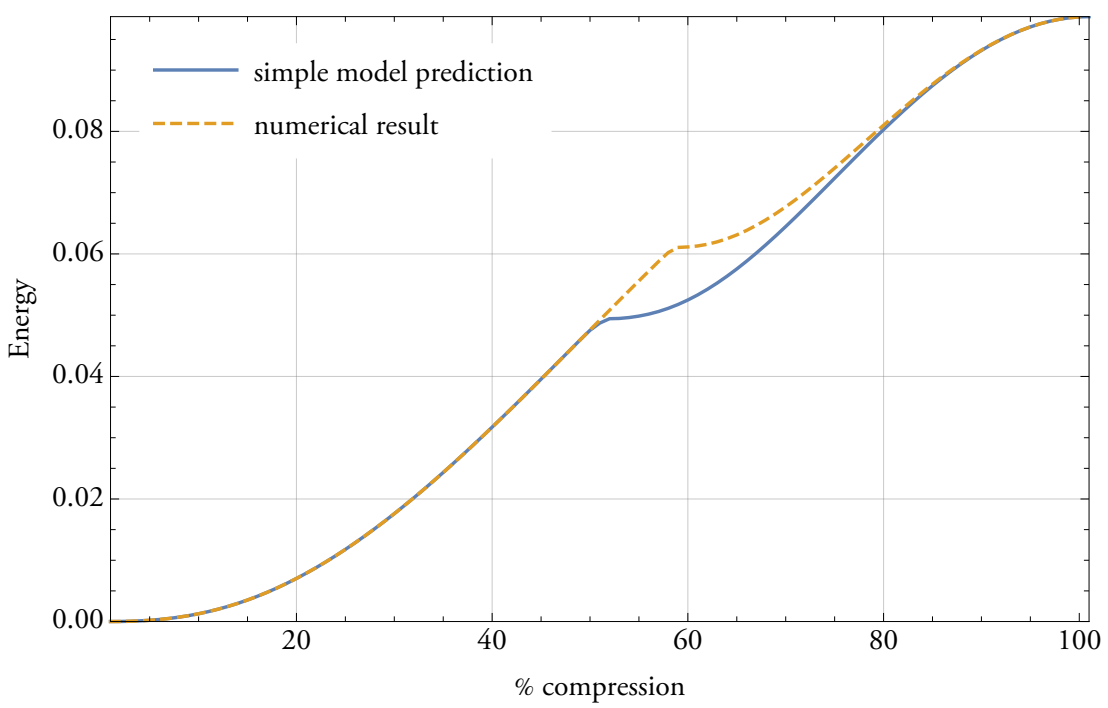

FIGURE A.22: simple energy for $r=0.8, n=7, m=2$

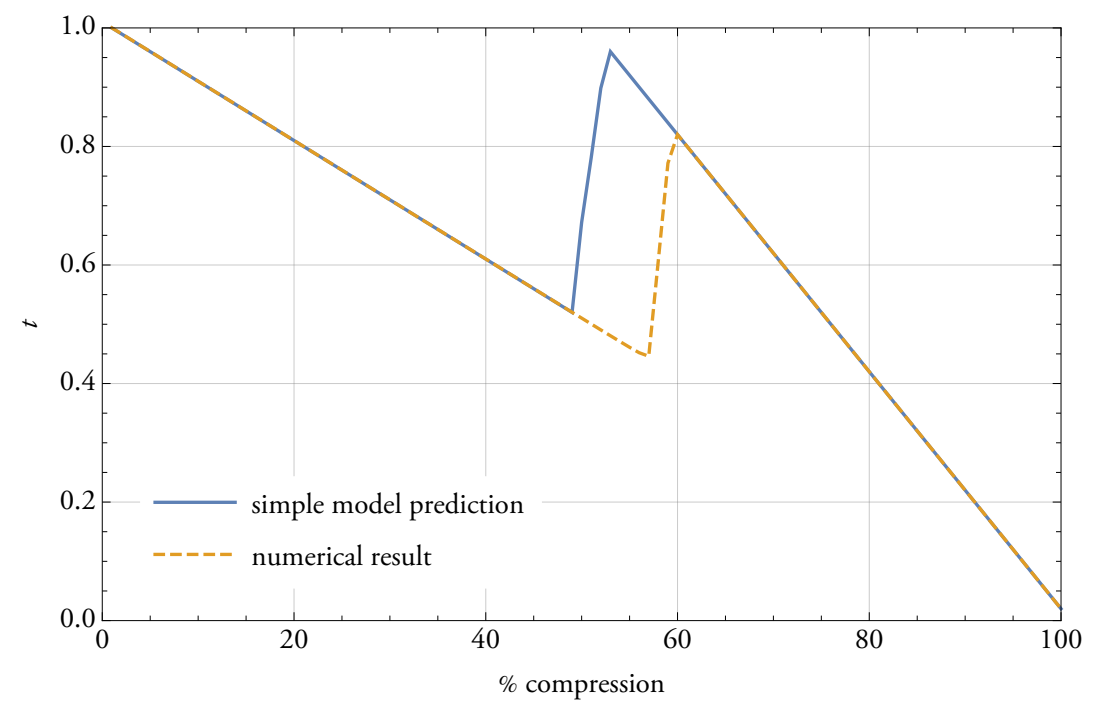

FIGURE A.23: intermediate layer height for $r=0.8, n=7, m=2$

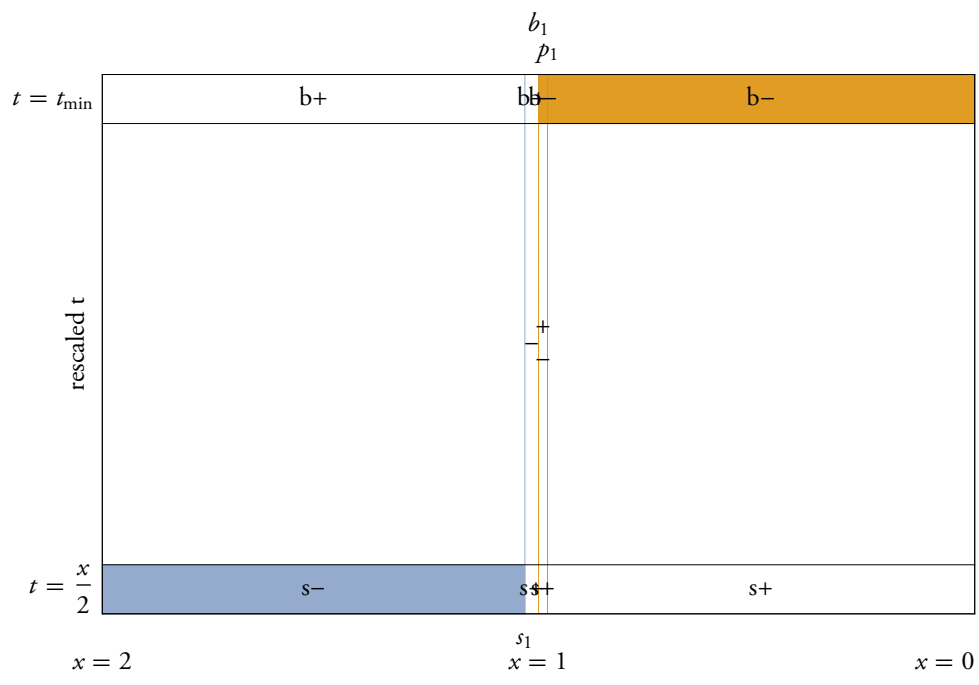

FIGURE A.24: prediction graphic for $r=0.8, n=7, m=2$ 


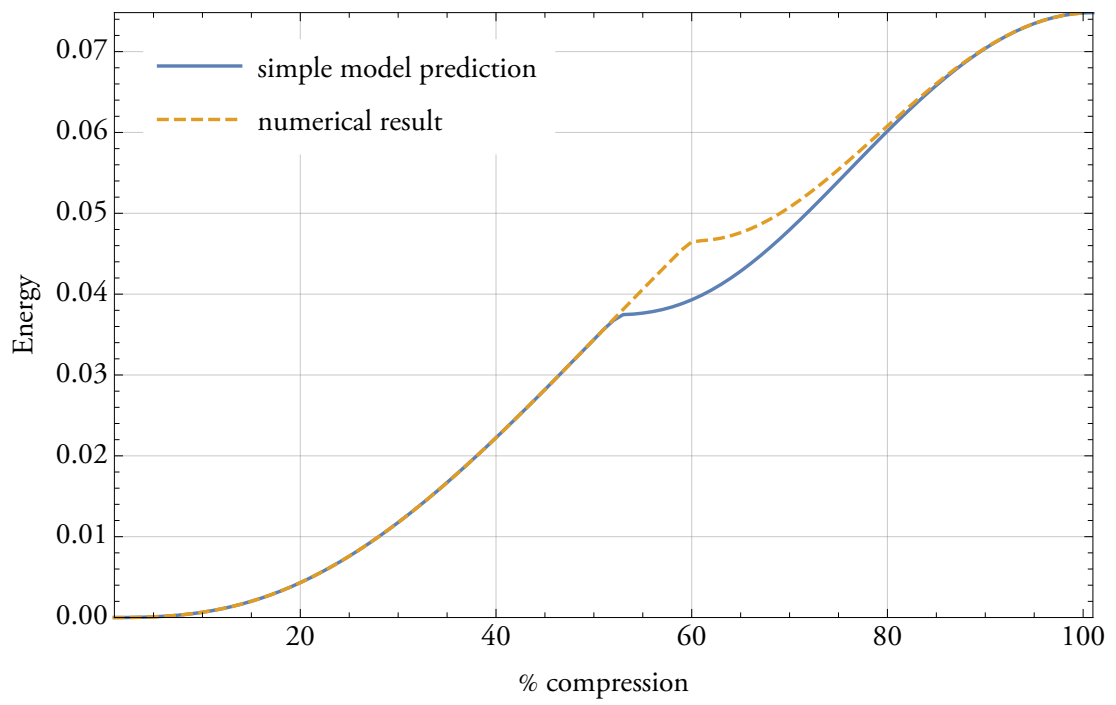

FIGURE A.25: simple energy for $r=0.8, n=8, m=2$

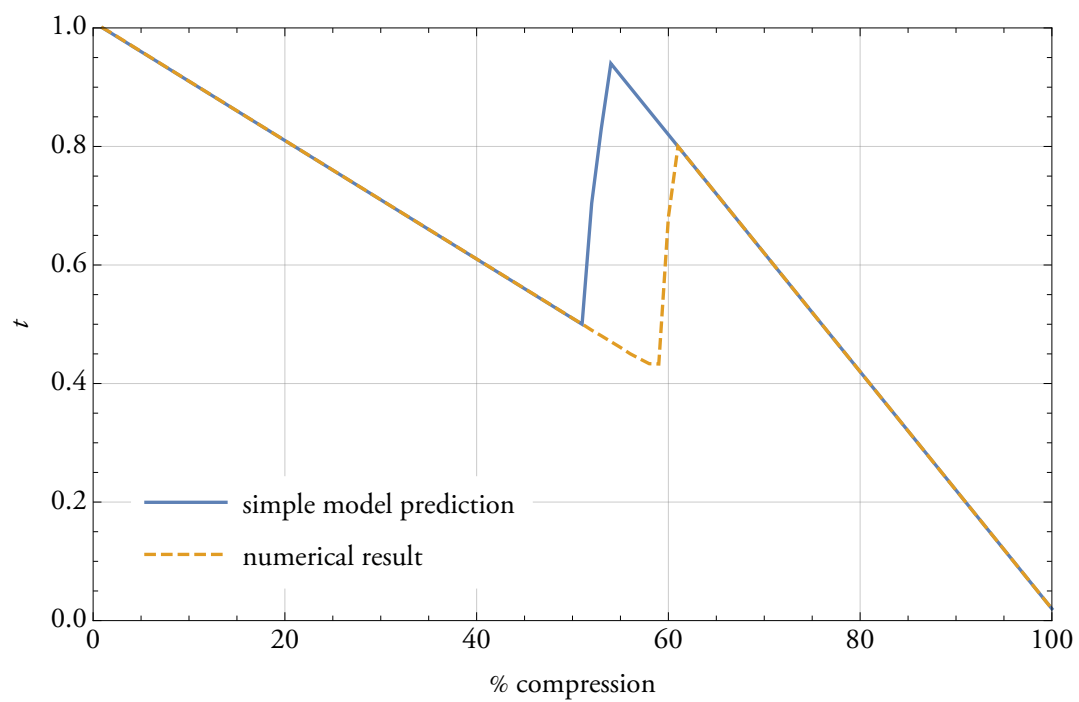

FIGURE A.26: intermediate layer height for $r=0.8, n=8, m=2$

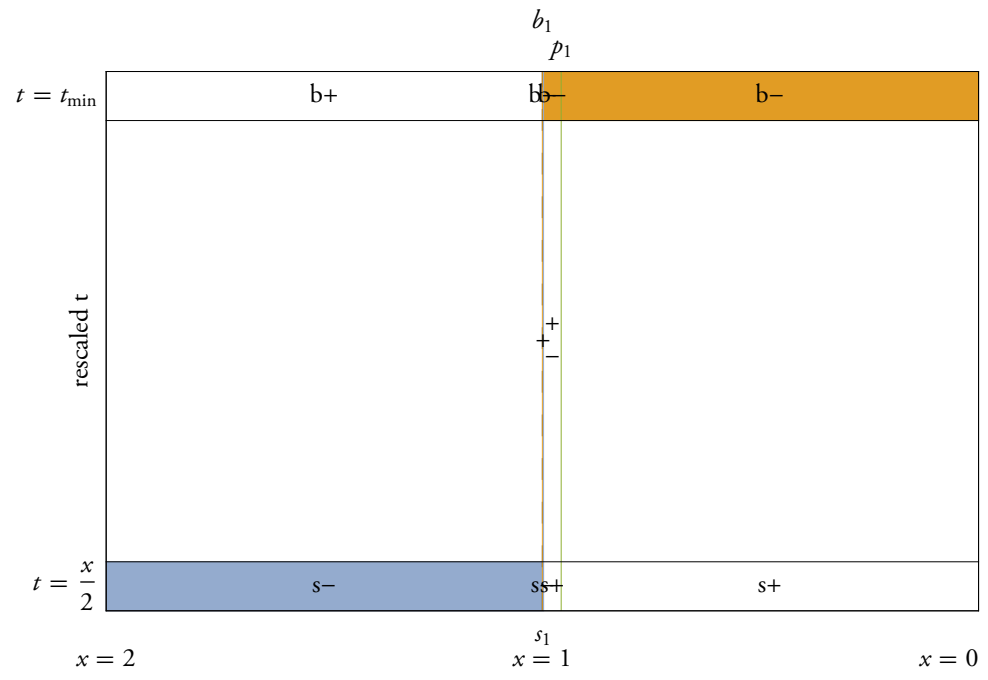

FIGURE A.27: prediction graphic for $r=0.8, n=8, m=2$ 


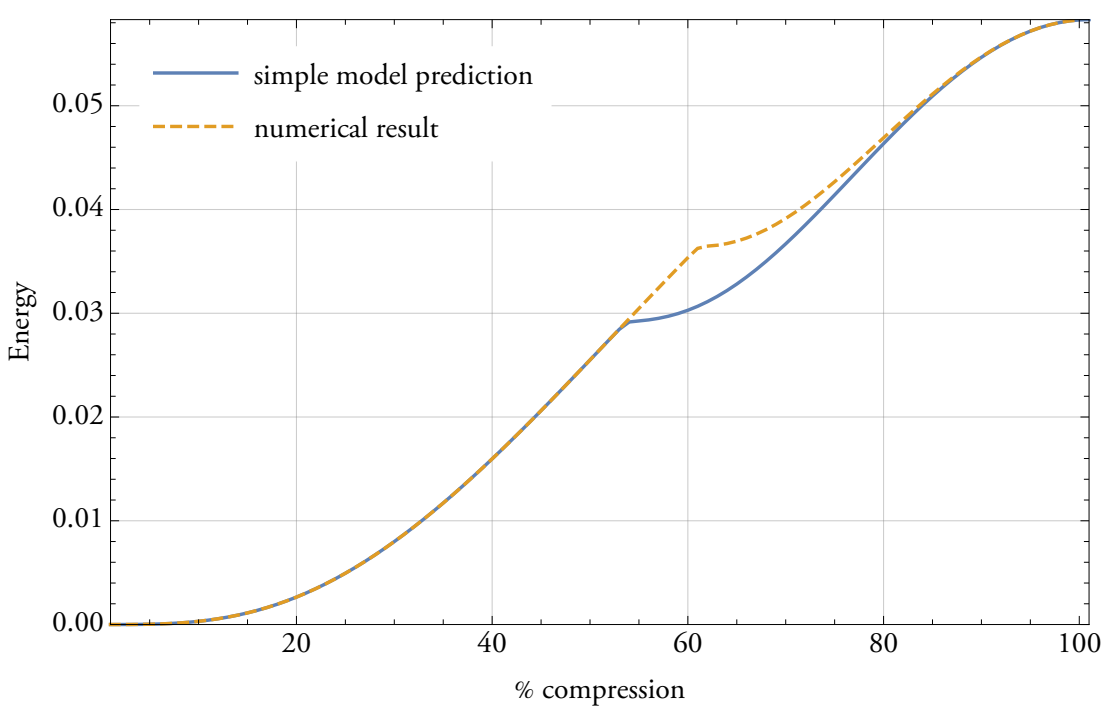

FIGURE A.28: simple energy for $r=0.8, n=9, m=2$

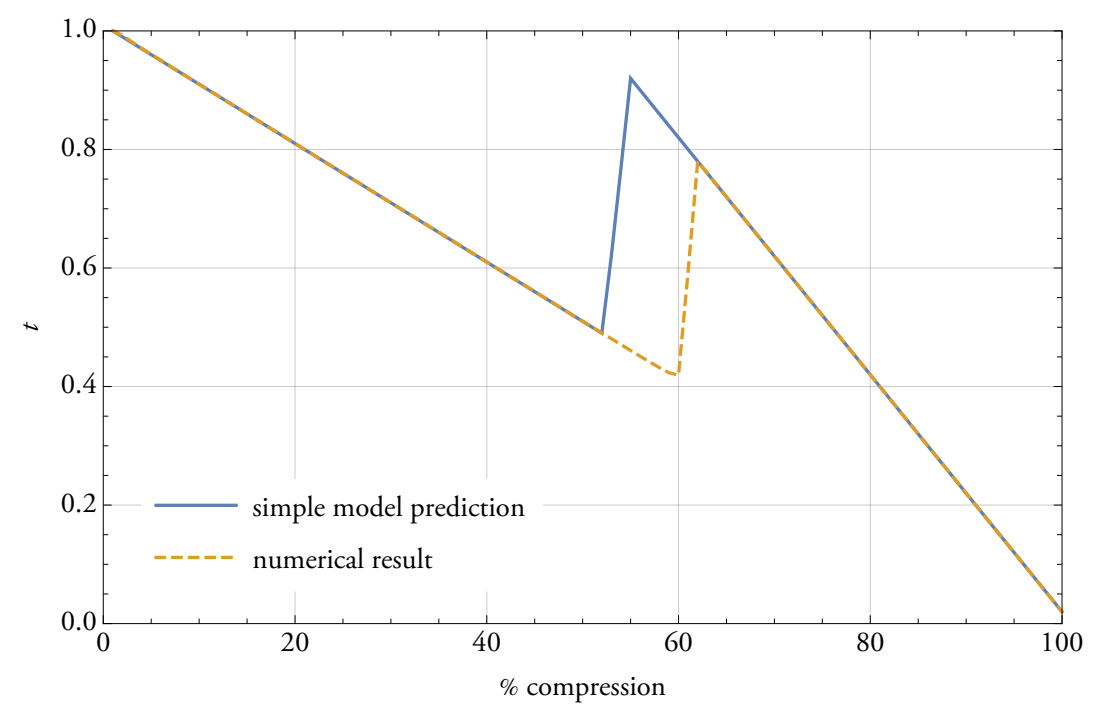

FIGURE A.29: intermediate layer height for $r=0.8, n=9, m=2$

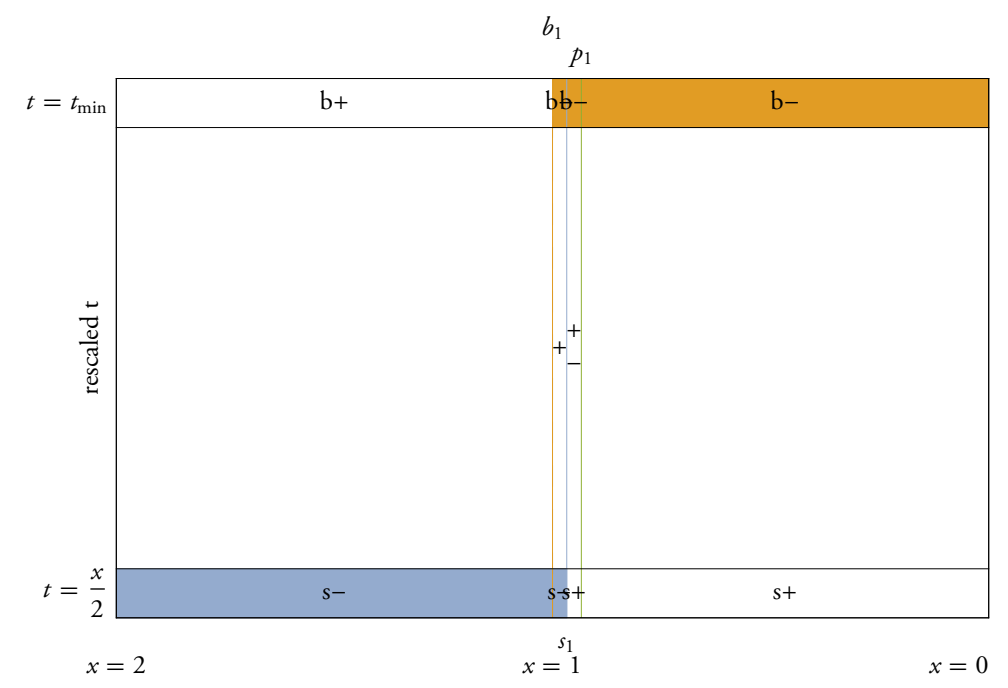

FIGURE A.30: prediction graphic for $r=0.8, n=9, m=2$ 


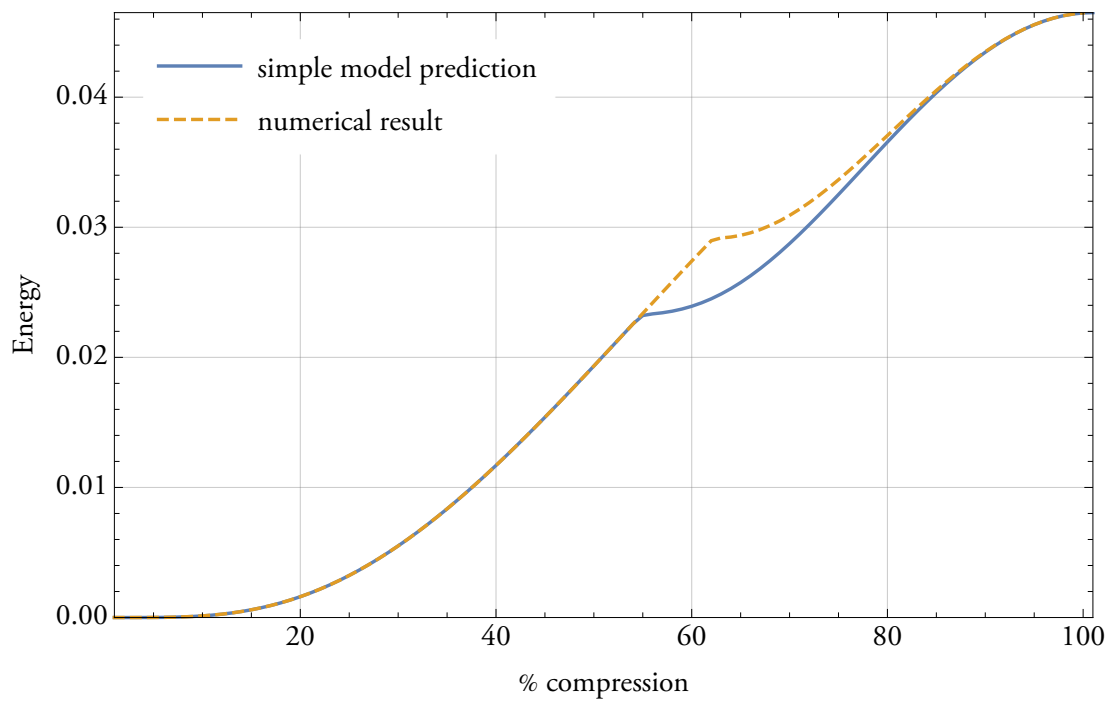

FIGURE A.31: simple energy for $r=0.8, n=10, m=2$

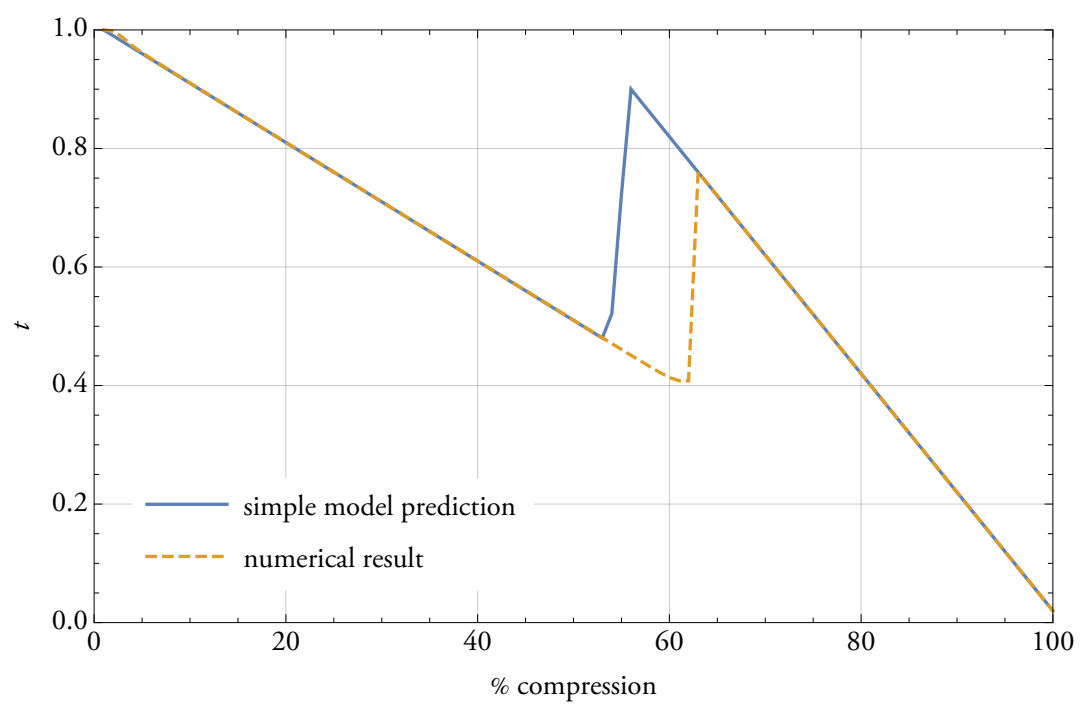

FIGURE A.32: intermediate layer height for $r=0.8, n=10, m=2$

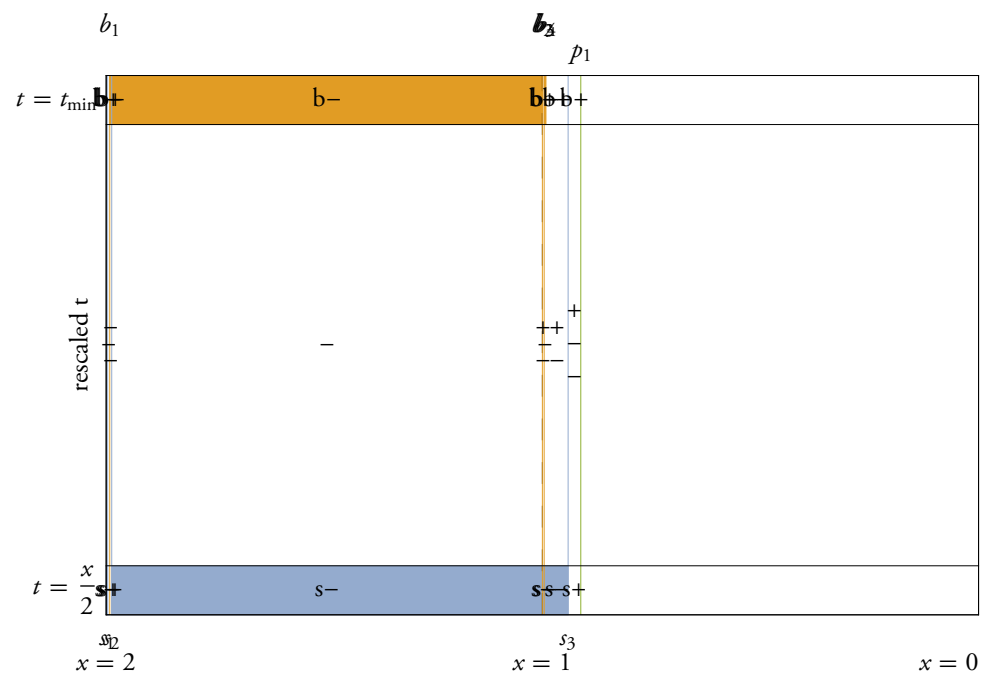

FIGURE A.33: prediction graphic for $r=0.8, n=10, m=2$ 


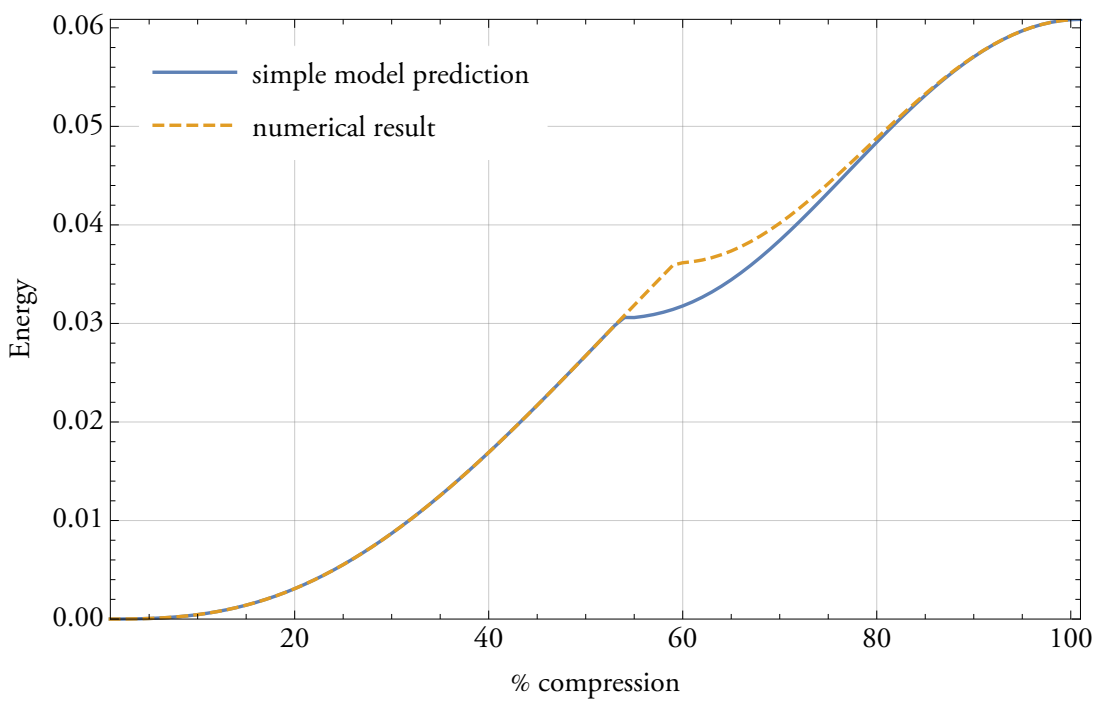

FIGURE A.34: simple energy for $r=1 ., n=5, m=2$

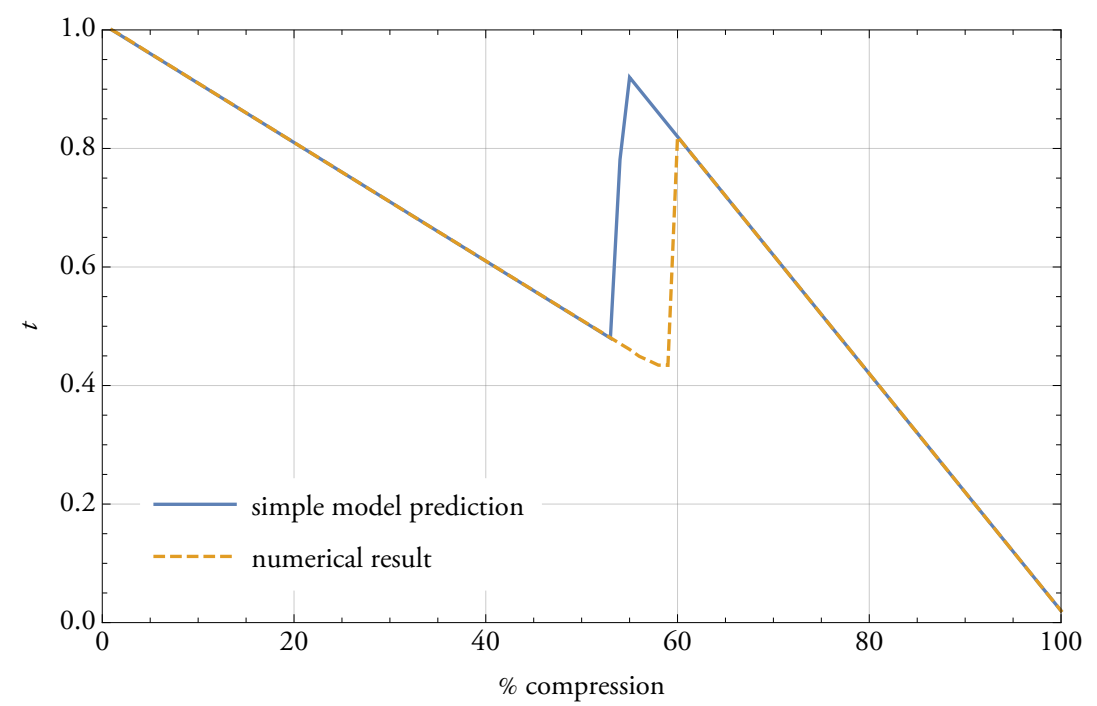

FIGURE A.35: intermediate layer height for $r=1 ., n=5, m=2$

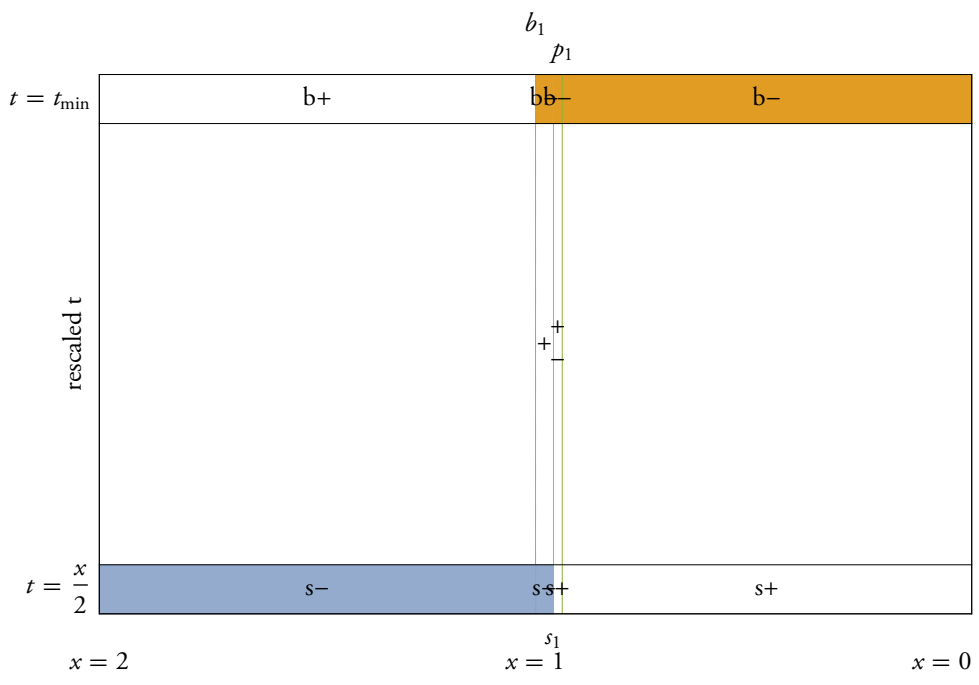

FIgURE A.36: prediction graphic for $r=1 ., n=5, m=2$ 


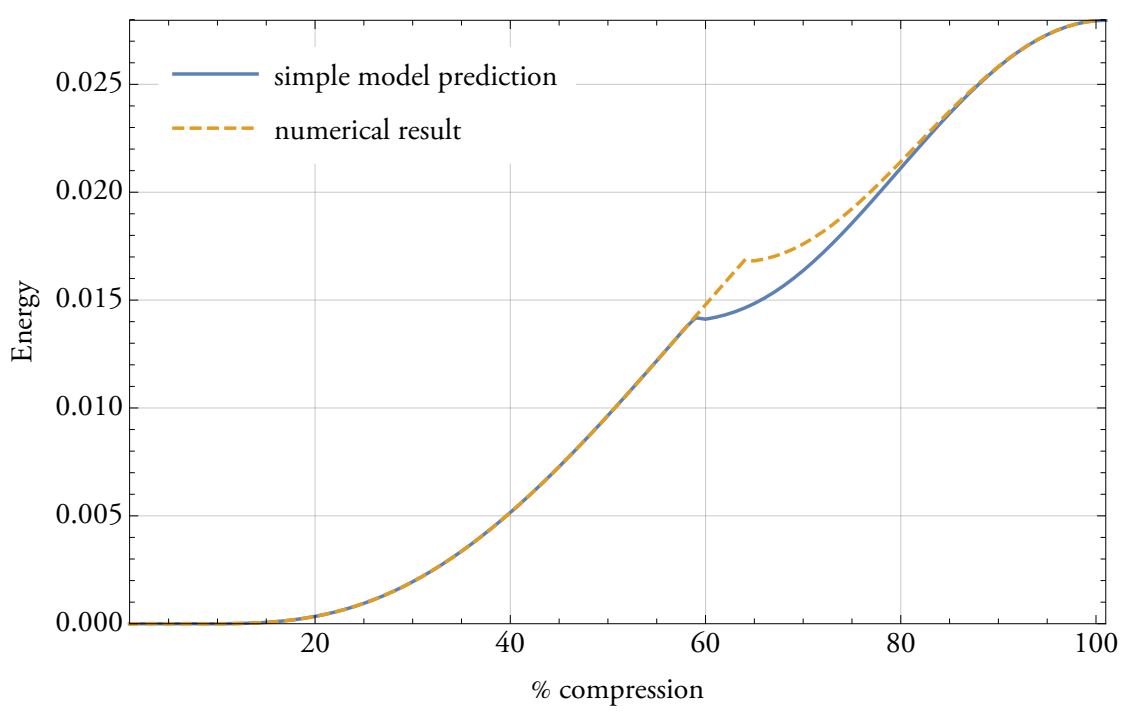

FIGURE A.37: simple energy for $r=1 ., n=6, m=2$

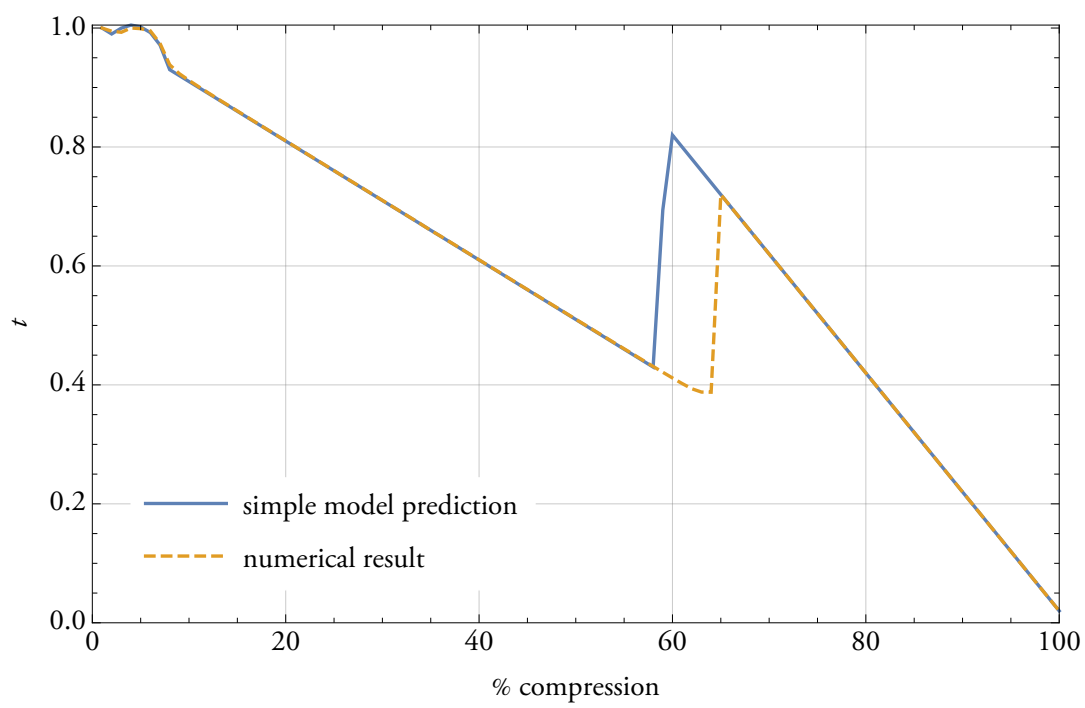

FIGURE A.38: intermediate layer beight for $r=1$., $n=6, m=2$

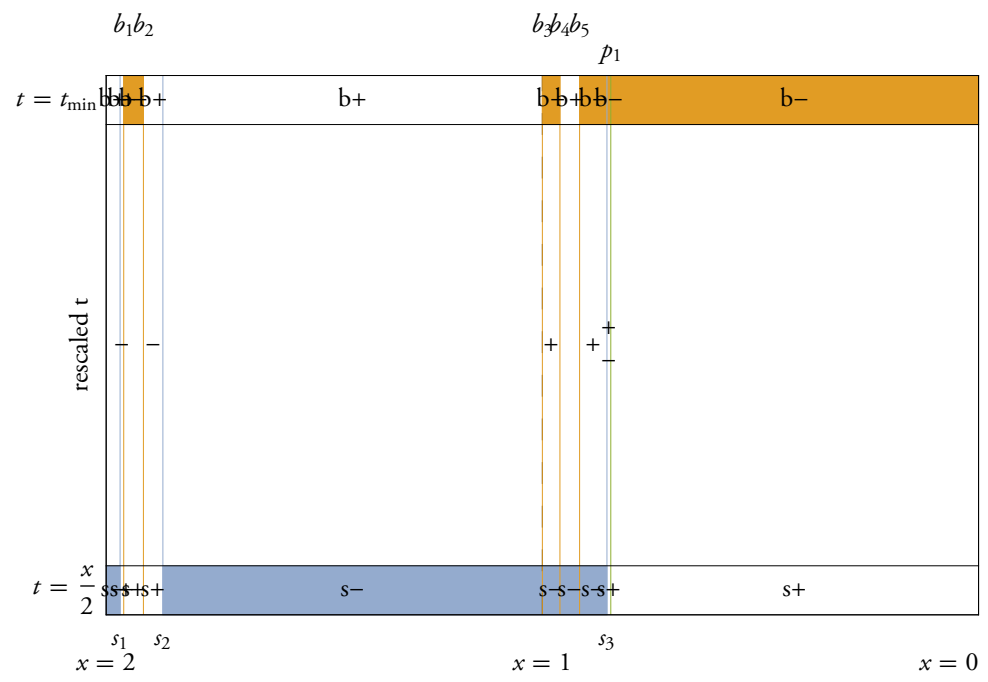

FIGURE A.39: prediction graphic for $r=1 ., n=6, m=2$ 


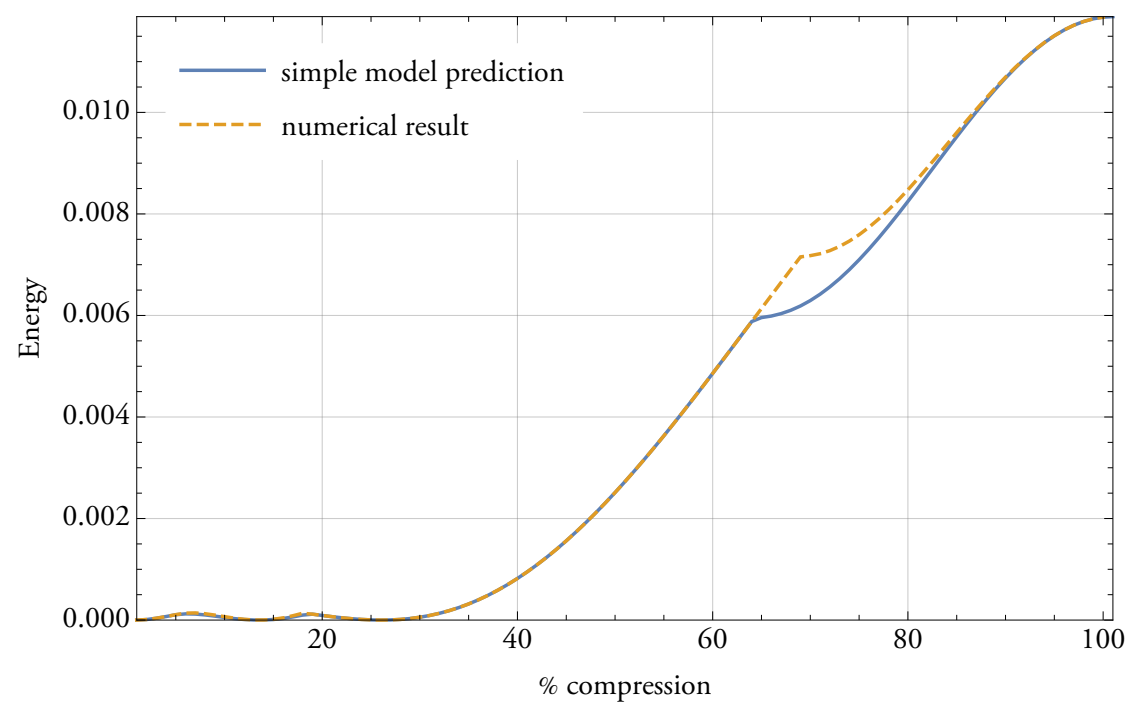

FIGURE A.40: simple energy for $r=1 ., n=7, m=2$

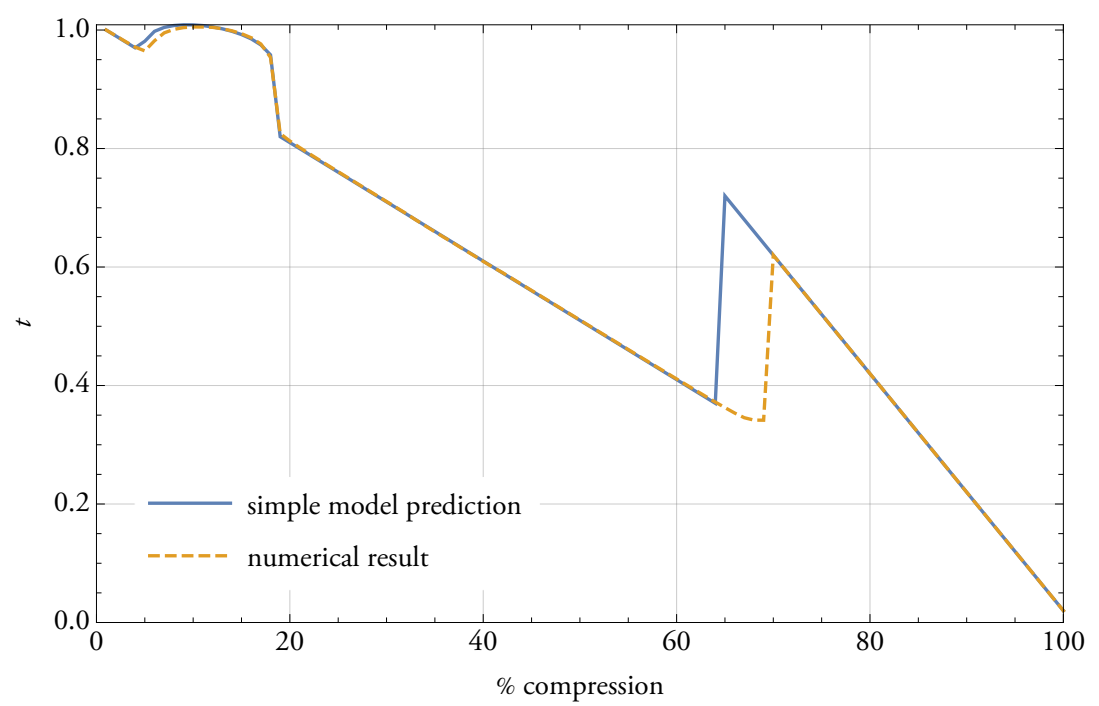

FIGURE A.41: intermediate layer height for $r=1 ., n=7, m=2$

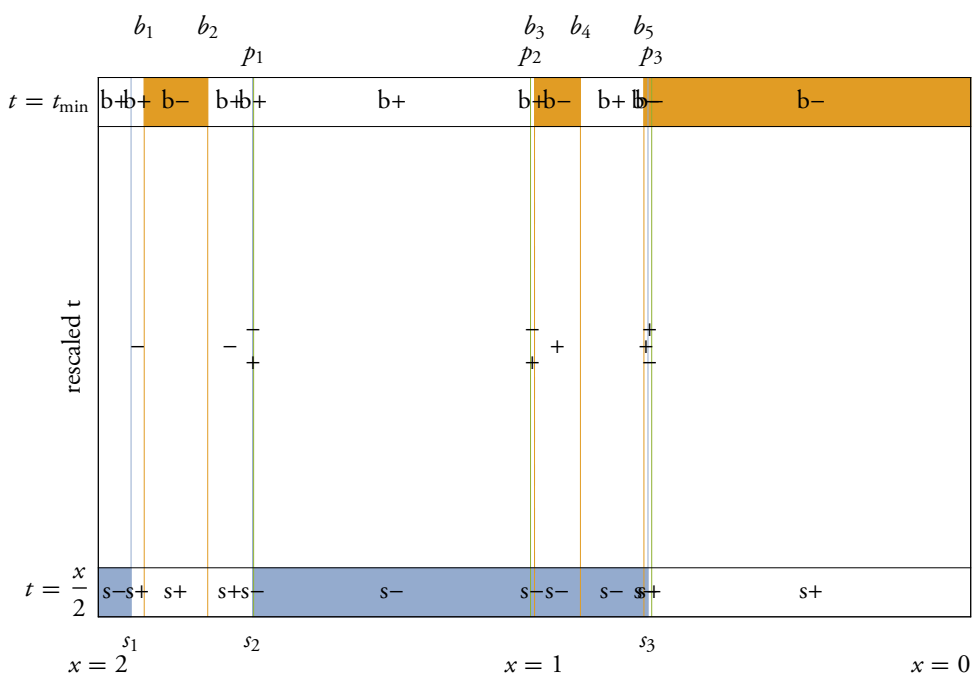

FIGURE A.42: prediction graphic for $r=1 ., n=7, m=2$ 


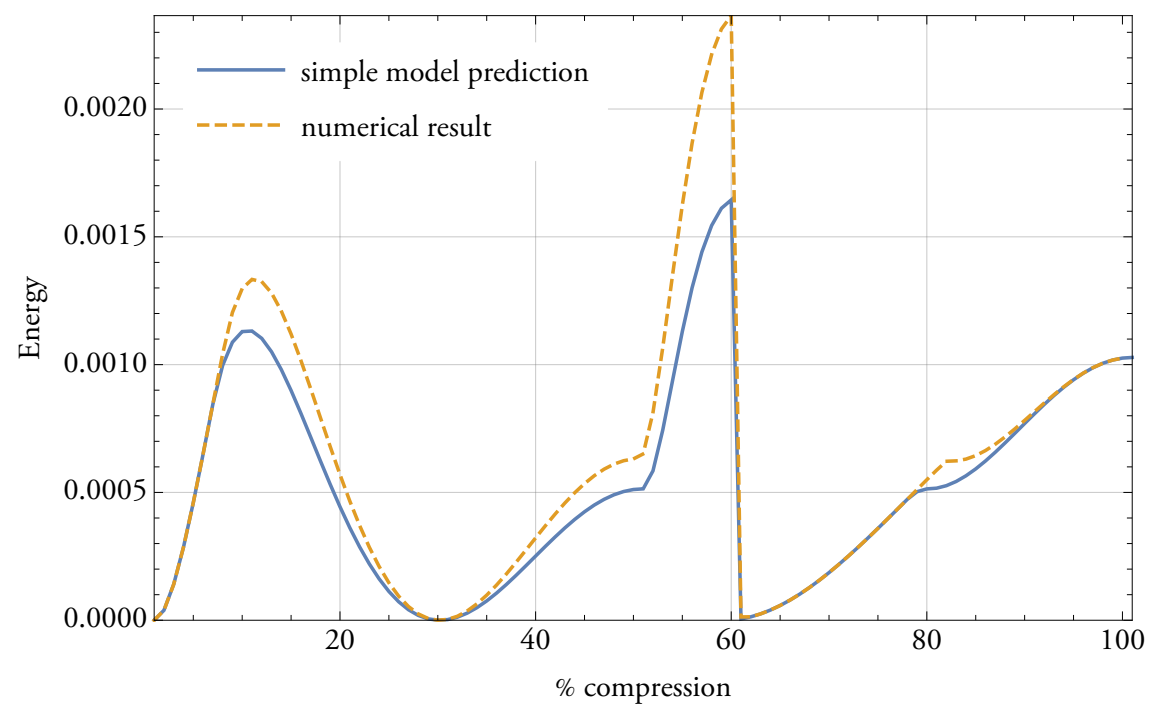

FIGURE A.43: simple energy for $r=1 ., n=9, m=2$

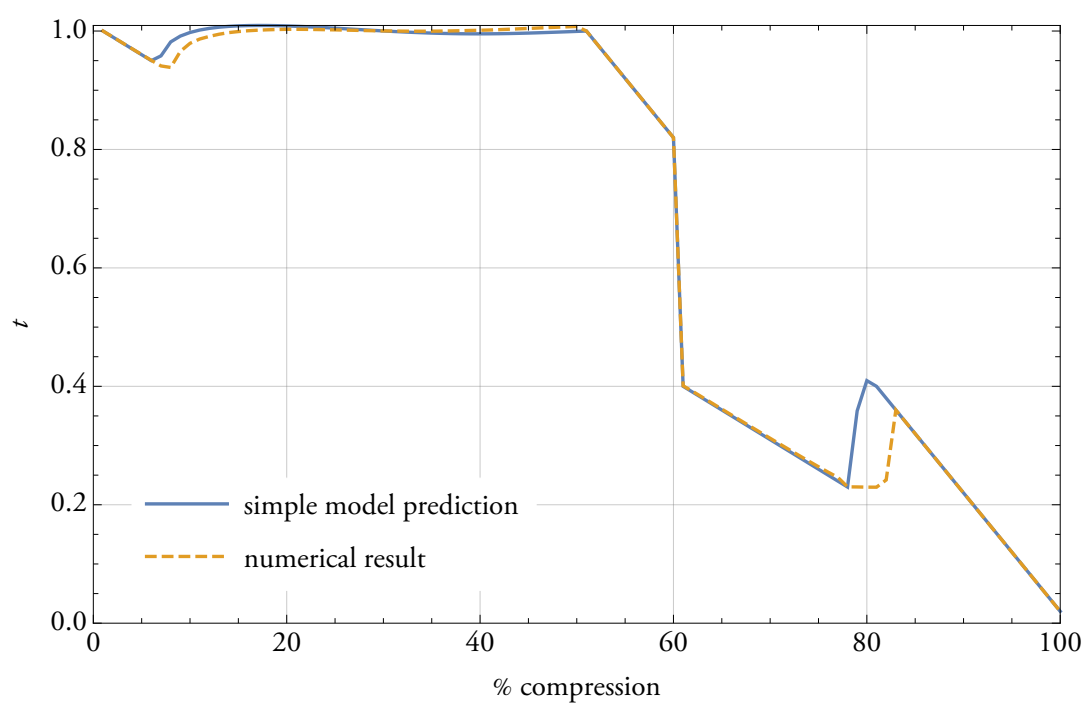

FIGURE A.44: intermediate layer height for $r=1 ., n=9, m=2$

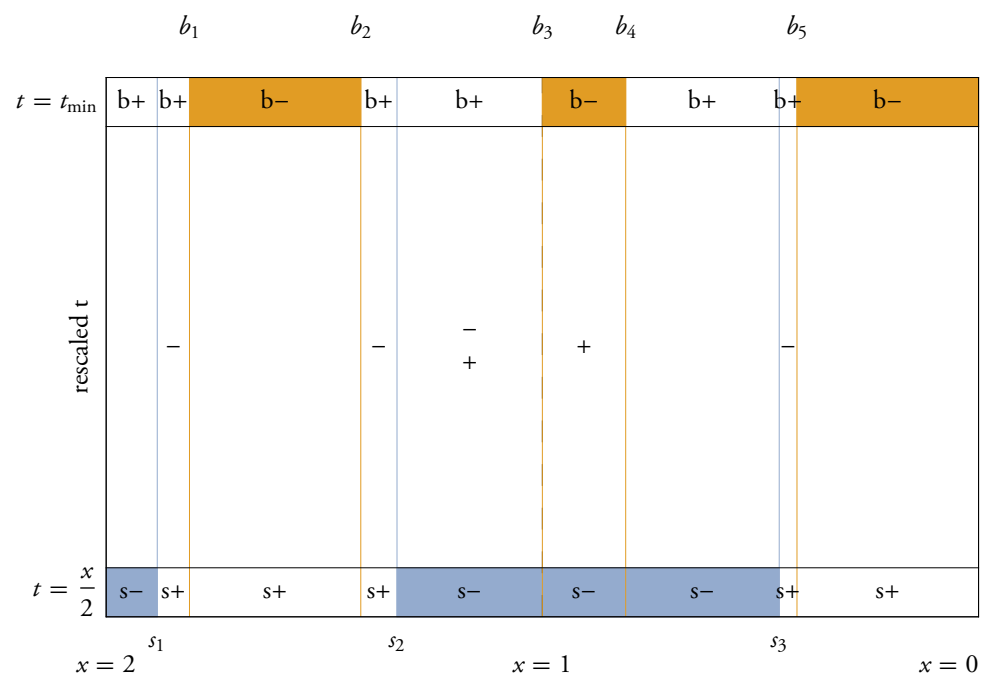

FIGURE A.45: prediction graphic for $r=1 ., n=9, m=2$ 


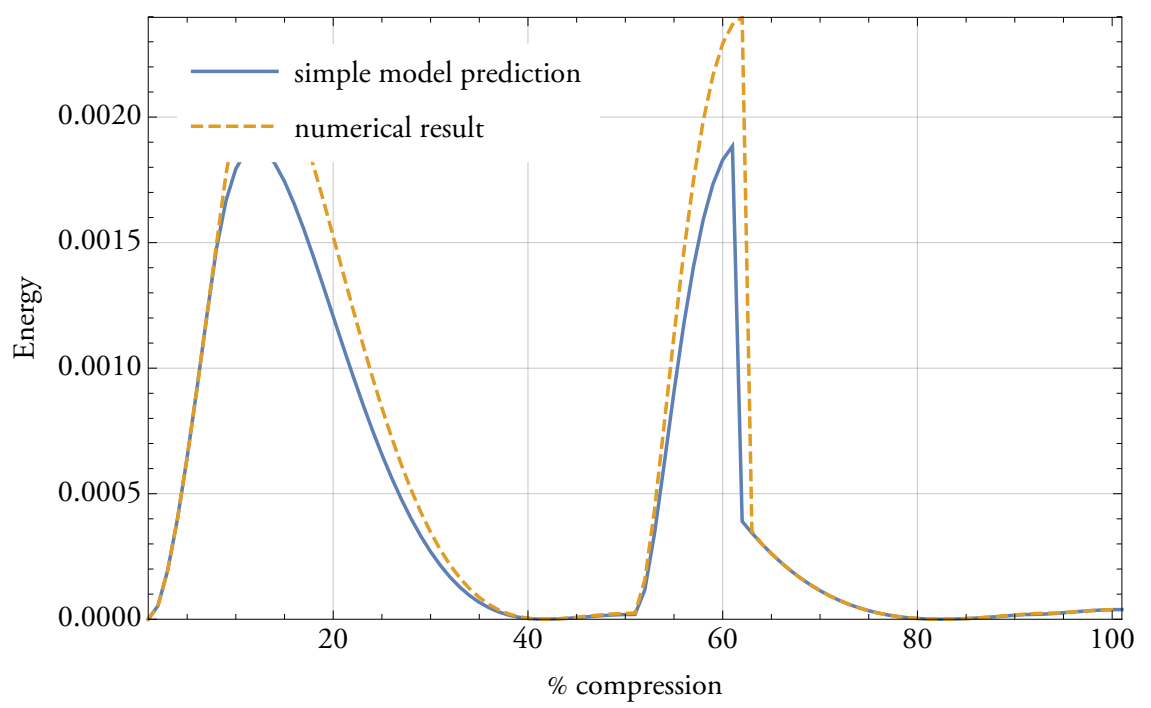

FIGURE A.46: simple energy for $r=1 ., n=10, m=2$

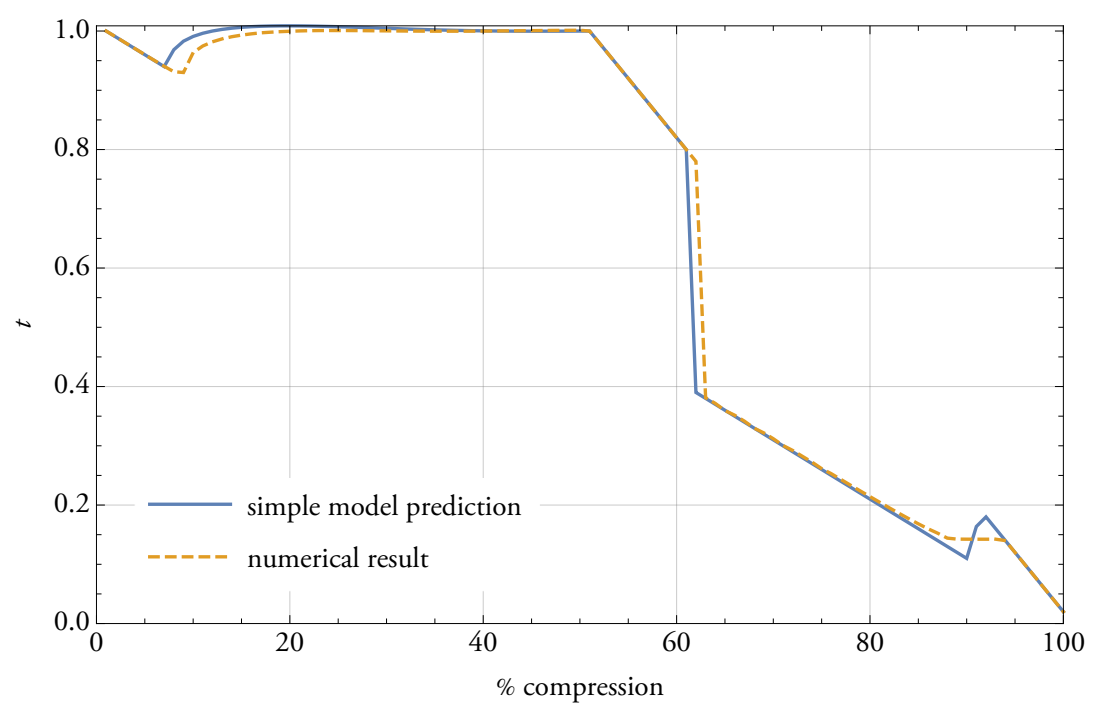

FIGURE A.47: intermediate layer height for $r=1 ., n=10, m=2$

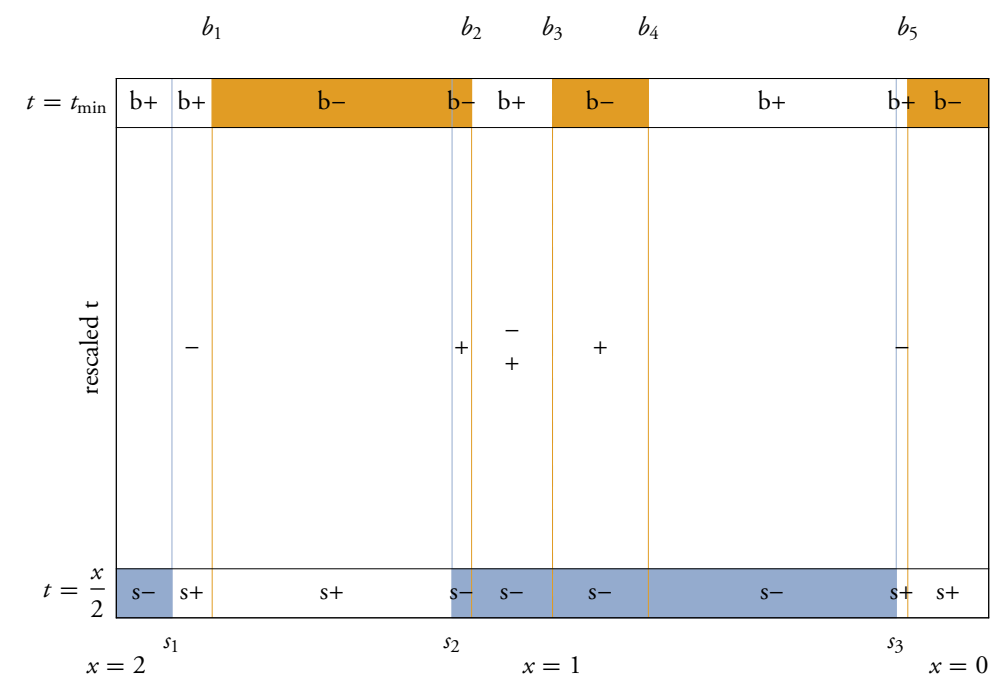

FIGURE A.48: prediction graphic for $r=1 ., n=10, m=2$ 


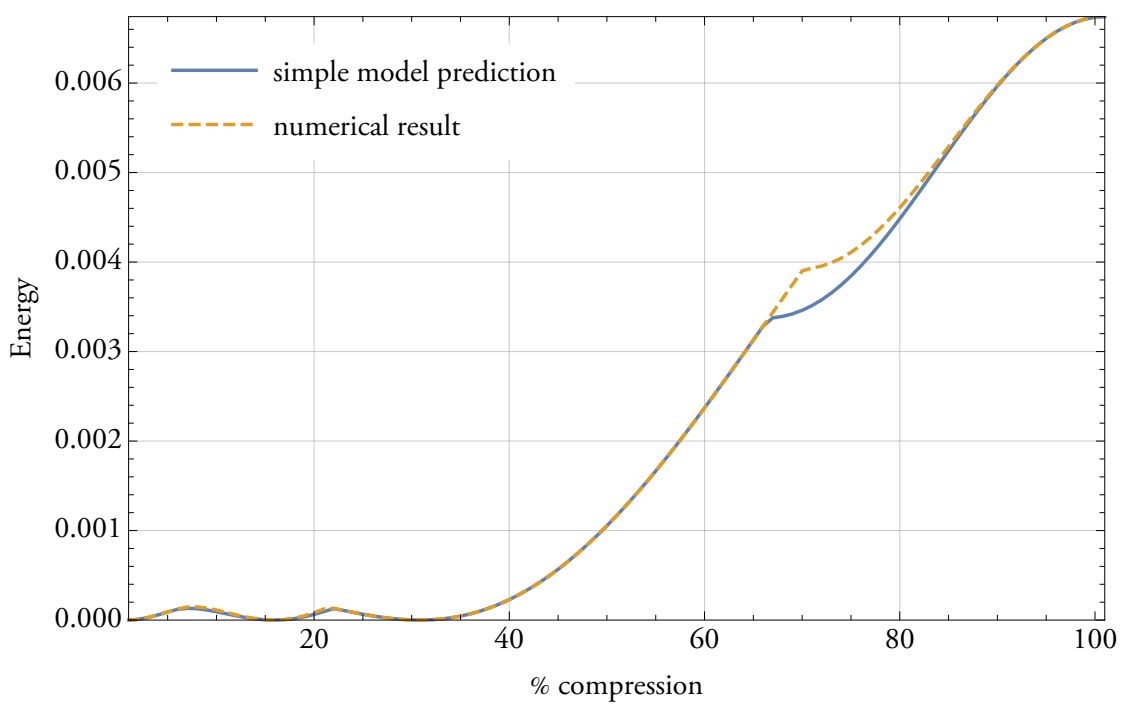

FIGURE A.49: simple energy for $r=1.2, n=5, m=2$

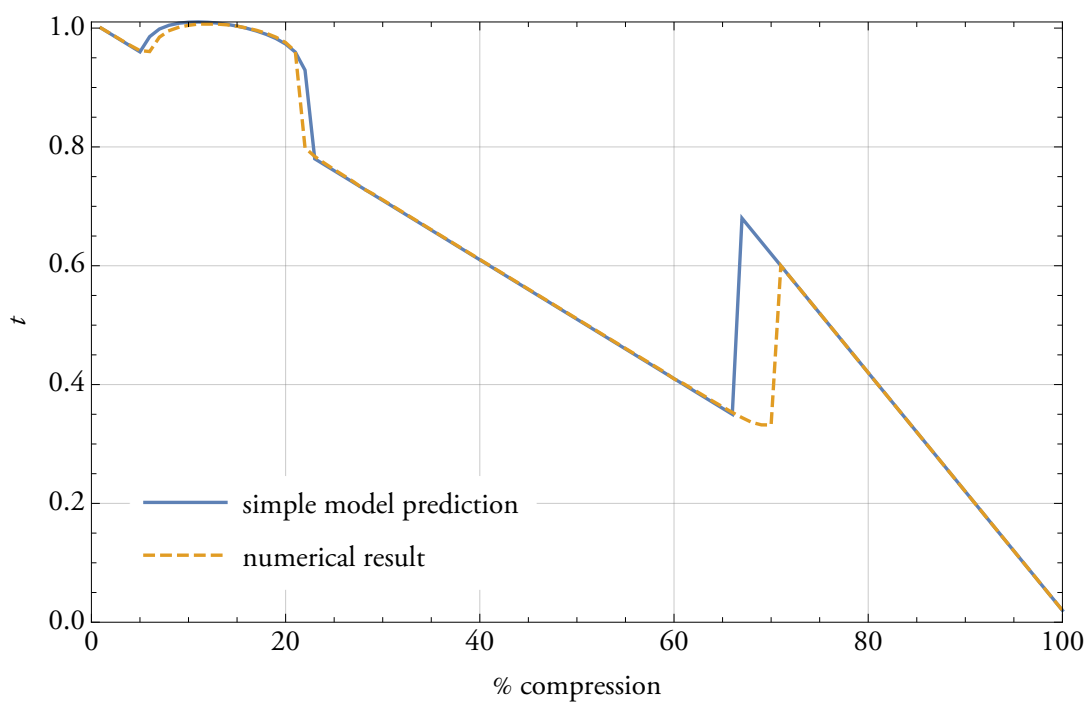

FIGURE A.50: intermediate layer height for $r=1.2, n=5, m=2$

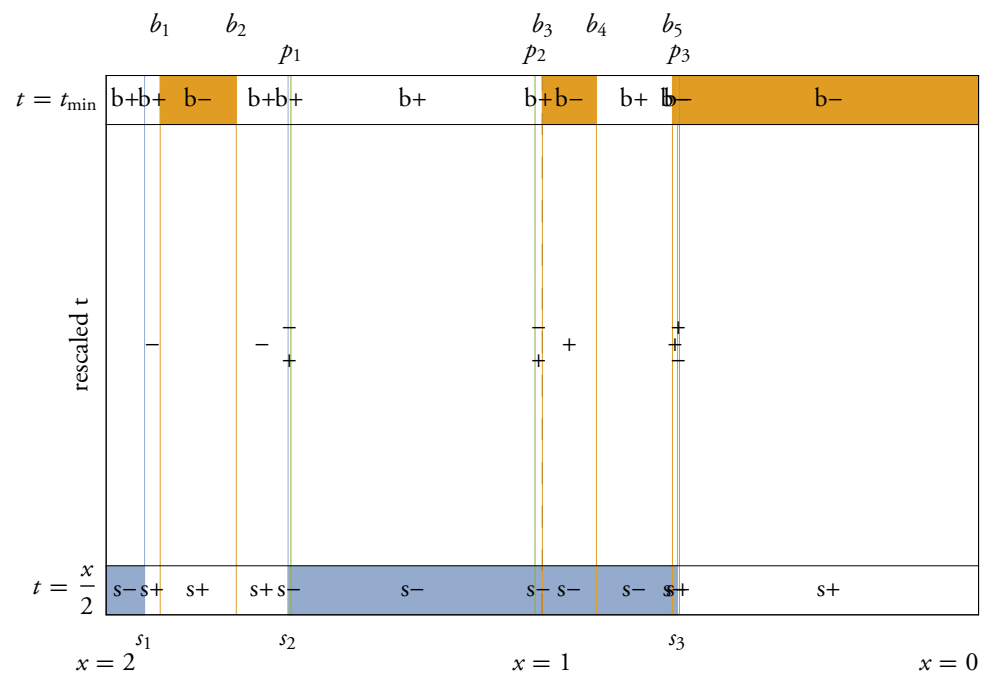

FIGURE A.51: prediction graphic for $r=1.2, n=5, m=2$ 


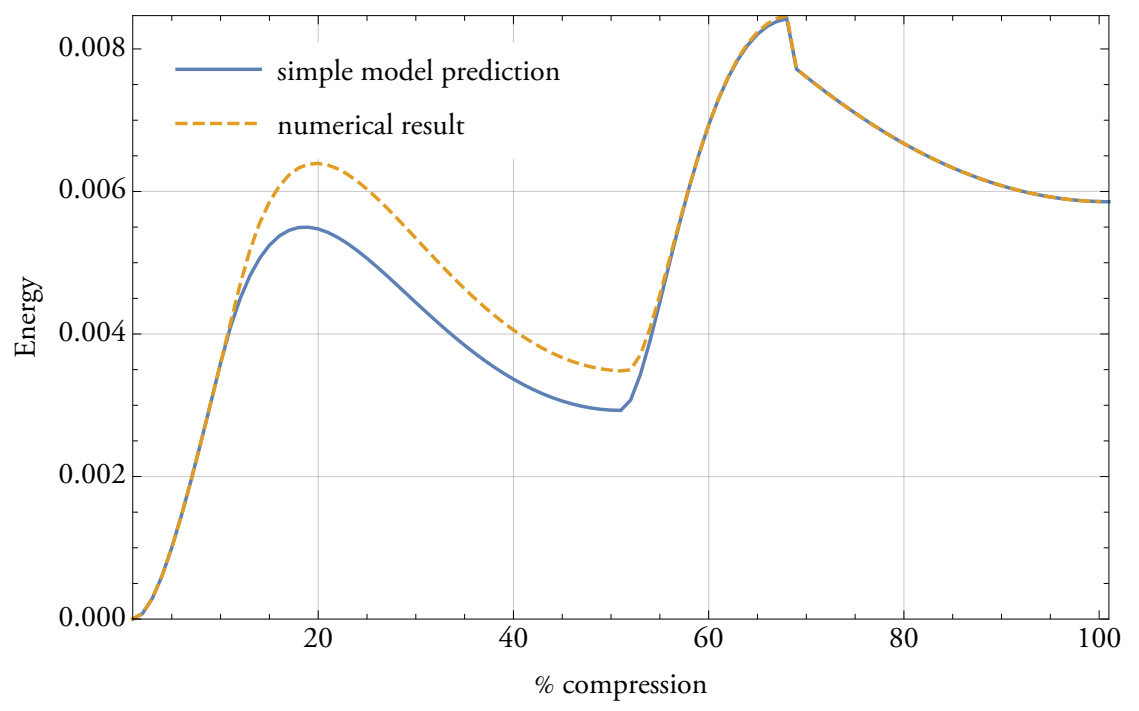

FIGURE A.52: simple energy for $r=1.2, n=7, m=2$

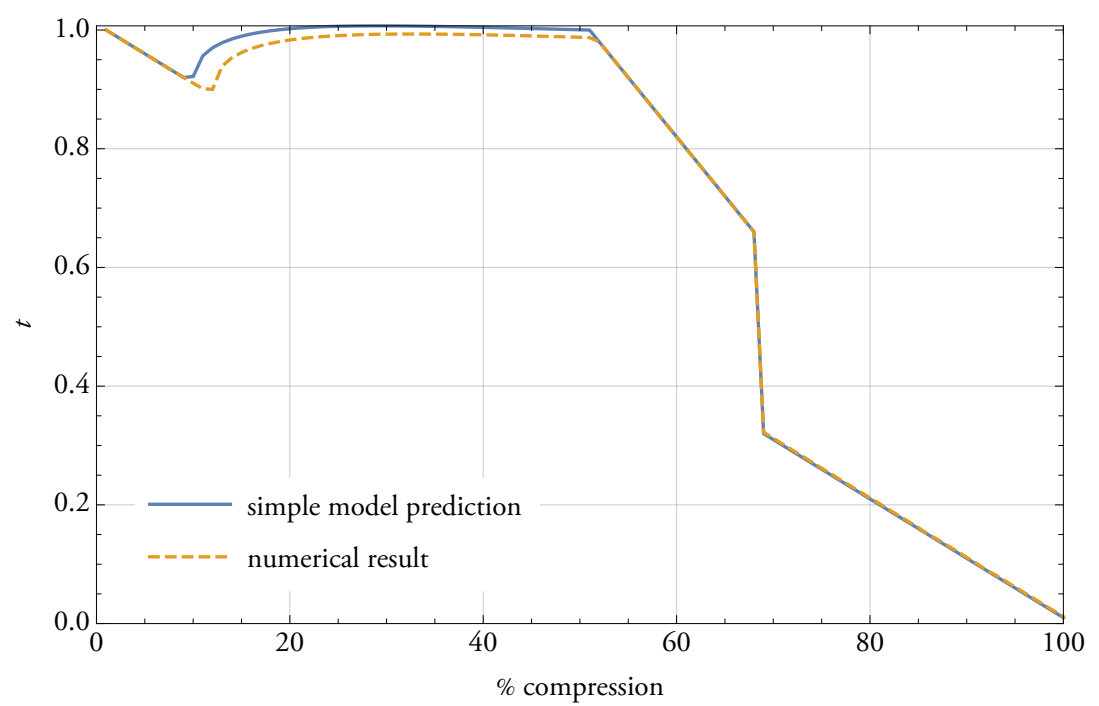

FIGURE A.53: intermediate layer height for $r=1.2, n=7, m=2$

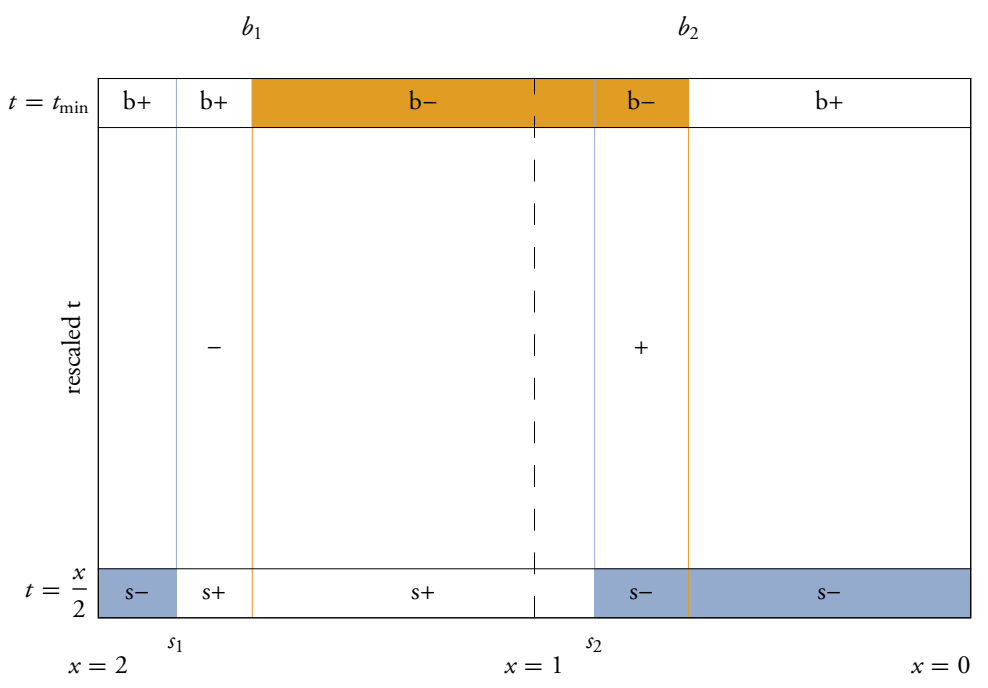

FIGURE A.54: prediction graphic for $r=1.2, n=7, m=2$ 


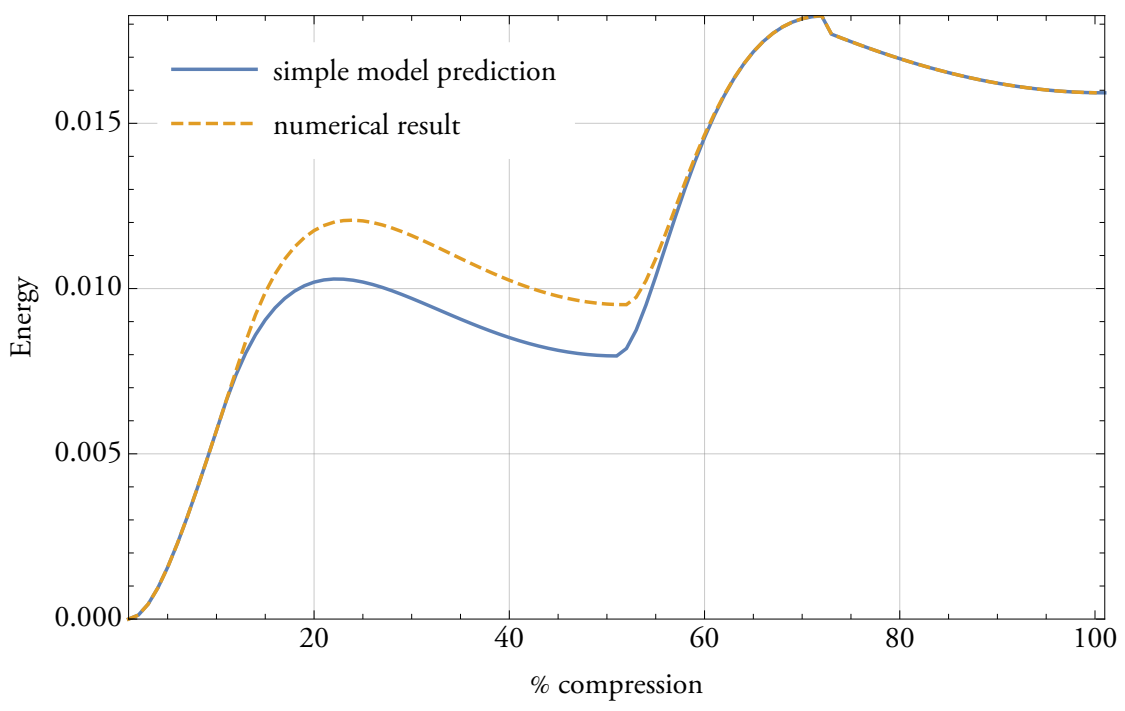

FIGURE A.55: simple energy for $r=1.2, n=8, m=2$

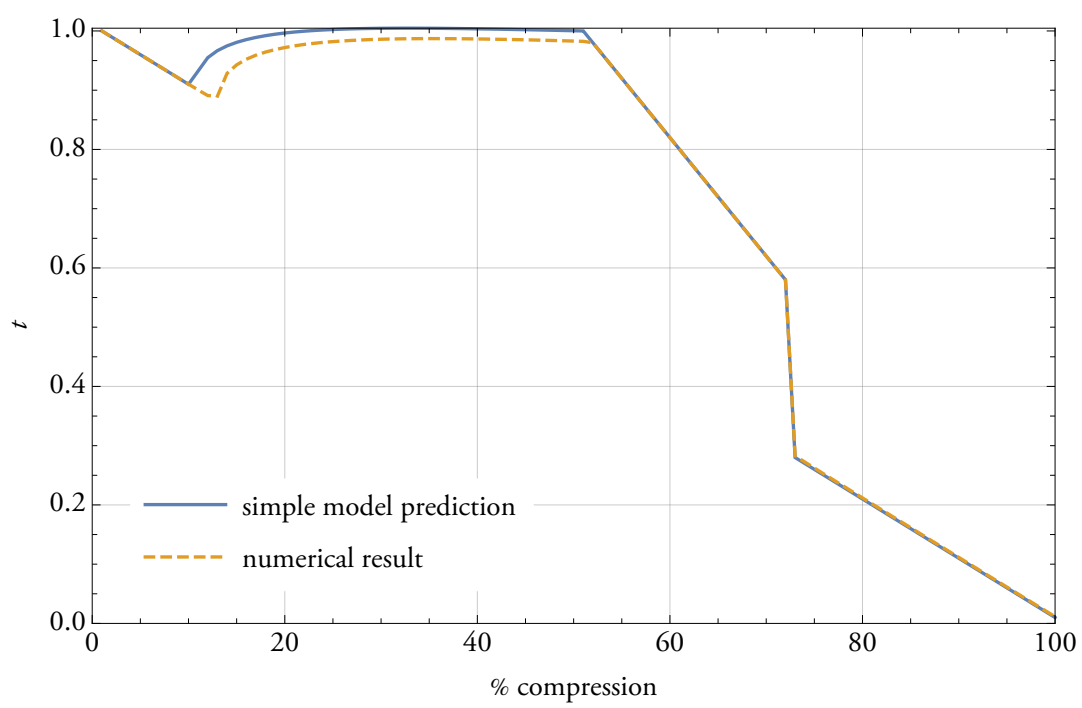

FIGURE A.56: intermediate layer height for $r=1.2, n=8, m=2$

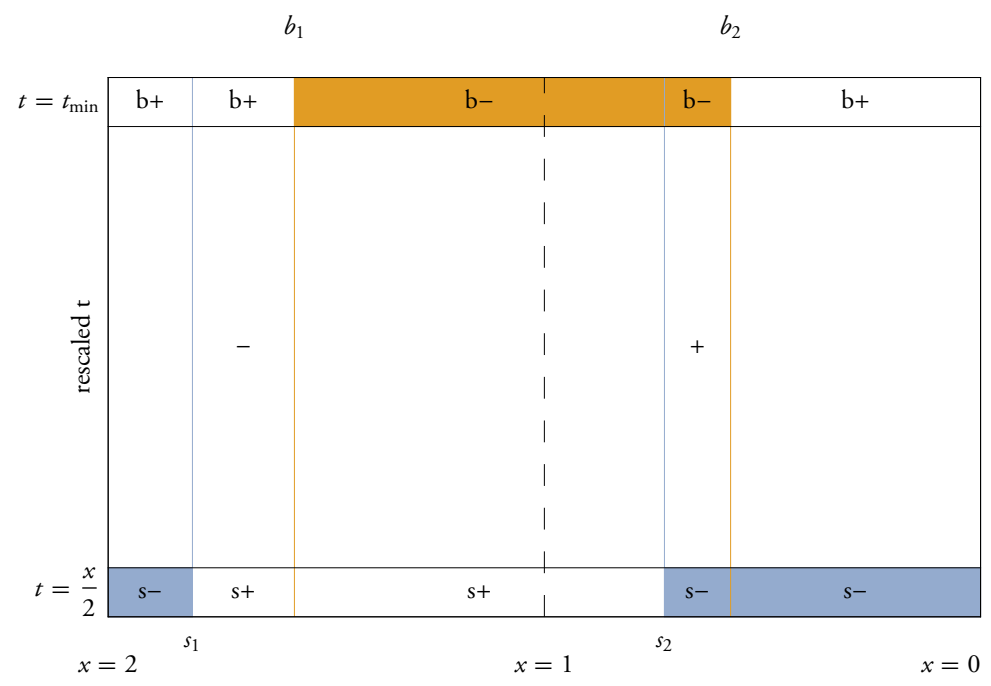

FIGURE A.57: prediction graphic for $r=1.2, n=8, m=2$ 


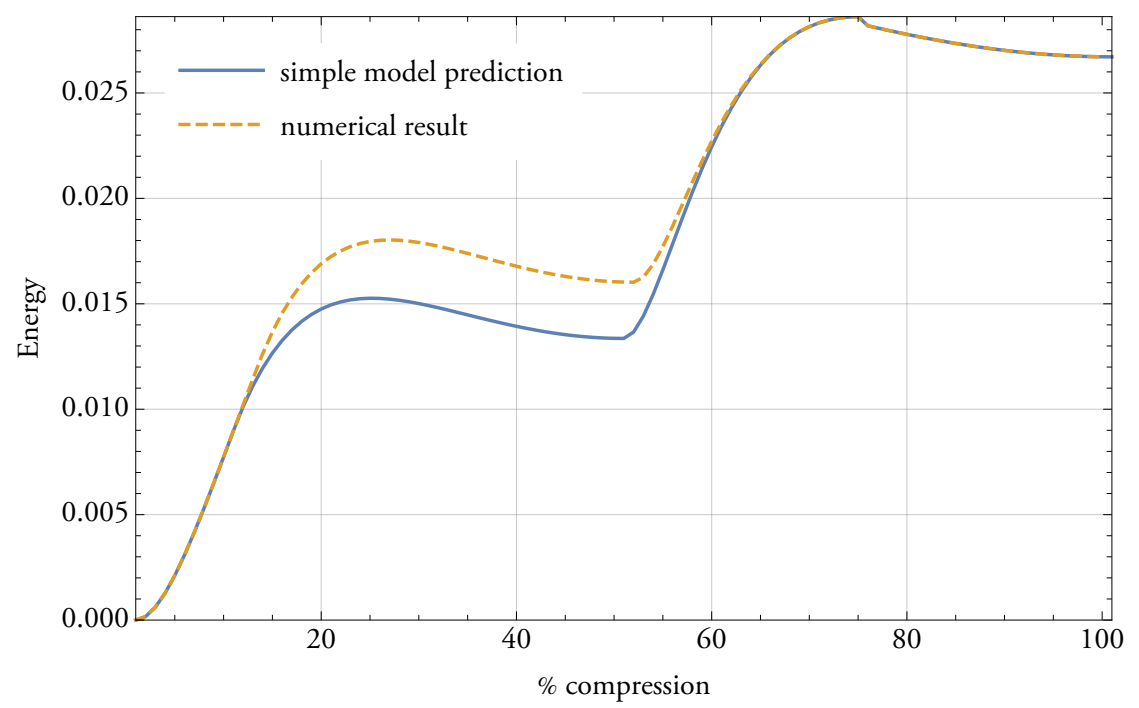

FIGURE A.58: simple energy for $r=1.2, n=9, m=2$

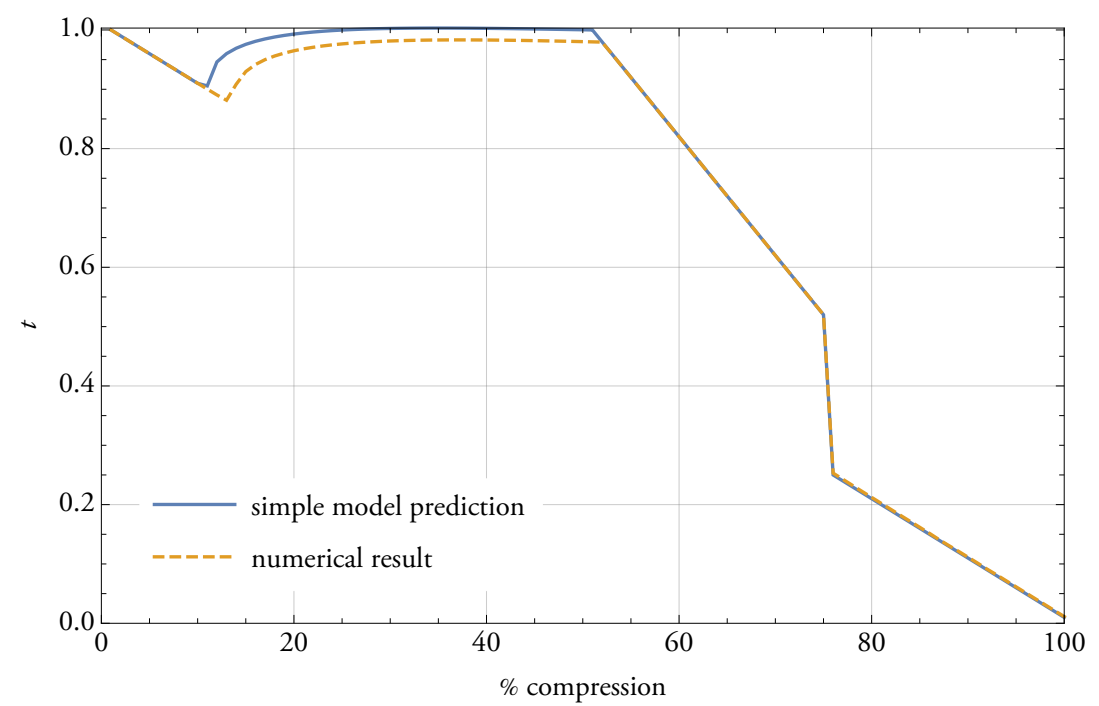

FIGURE A.59: intermediate layer height for $r=1.2, n=9, m=2$

$b_{1} \quad b_{2}$

\begin{tabular}{|c|c|c|c|c|c|}
\hline$t=t_{\min }$ & $\mathrm{b}+$ & $\mathrm{b}+$ & b- & $\mathrm{b}-$ & $\mathrm{b}+$ \\
\hline 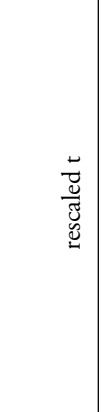 & & - & & + & \\
\hline$t=\frac{x}{2}$ & $s-$ & $s+$ & $s+$ & s- & $s-$ \\
\hline
\end{tabular}

FIGURE A.60: prediction graphic for $r=1.2, n=9, m=2$ 


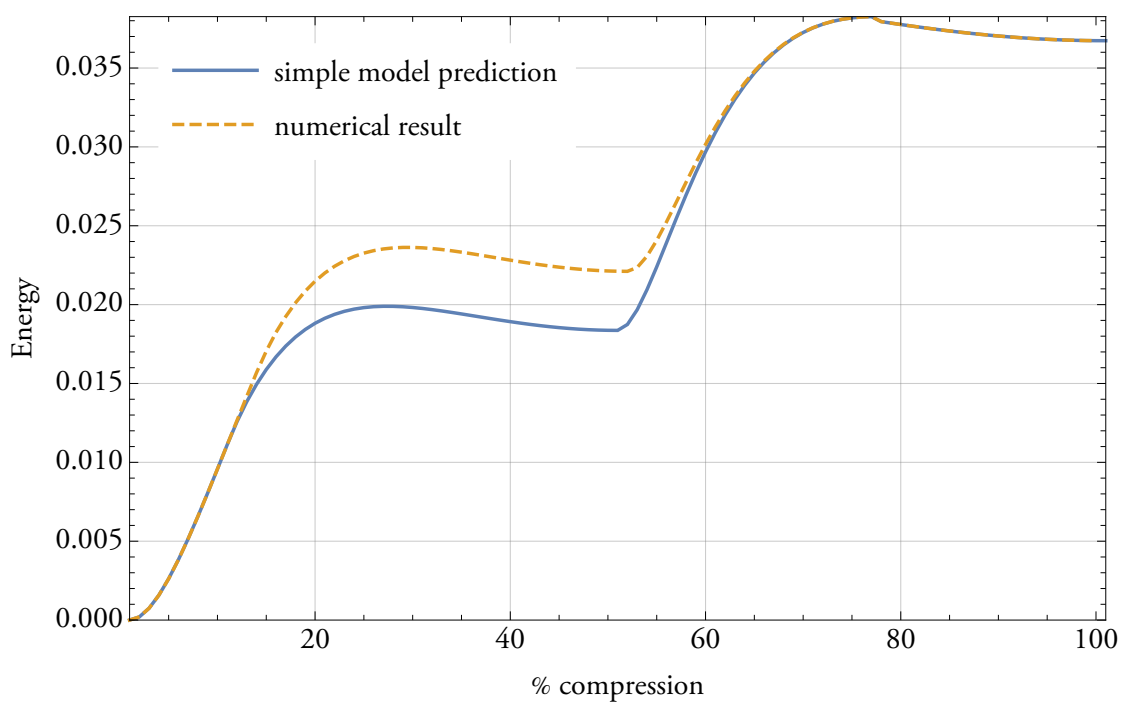

FIGURE A.61: simple energy for $r=1.2, n=10, m=2$

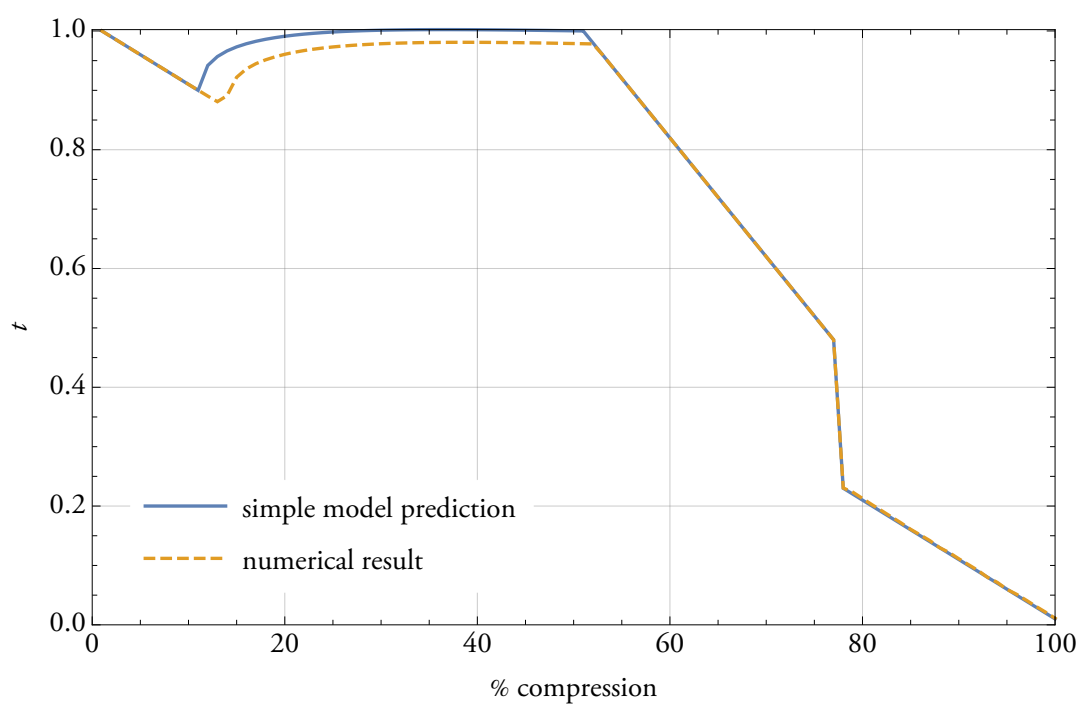

FIGURE A.62: intermediate layer height for $r=1.2, n=10, m=2$

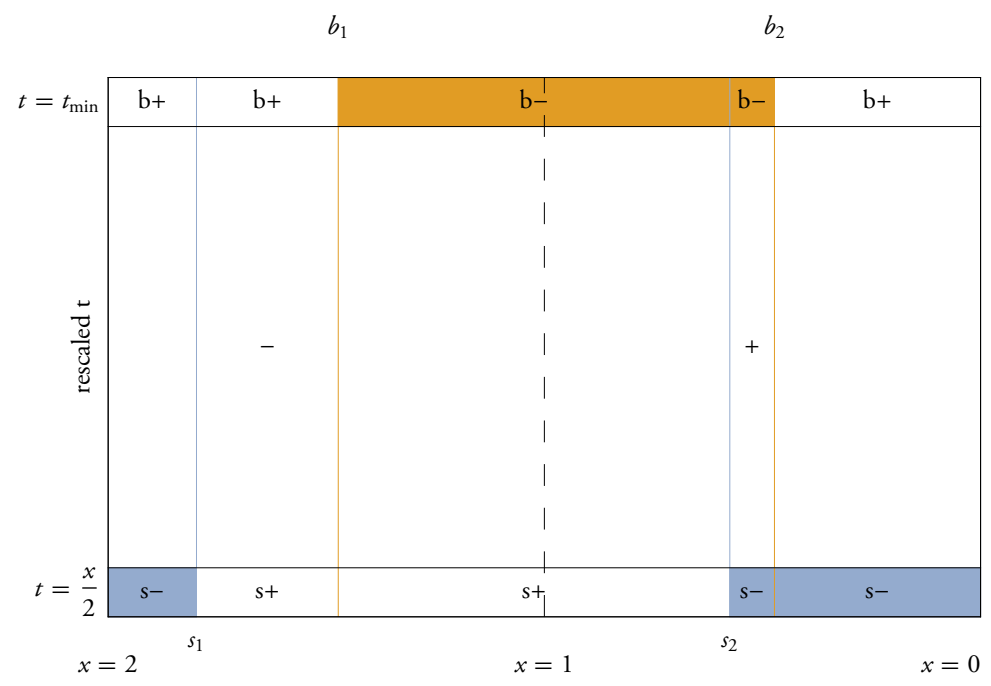

FIGURE A.63: prediction graphic for $r=1.2, n=10, m=2$ 


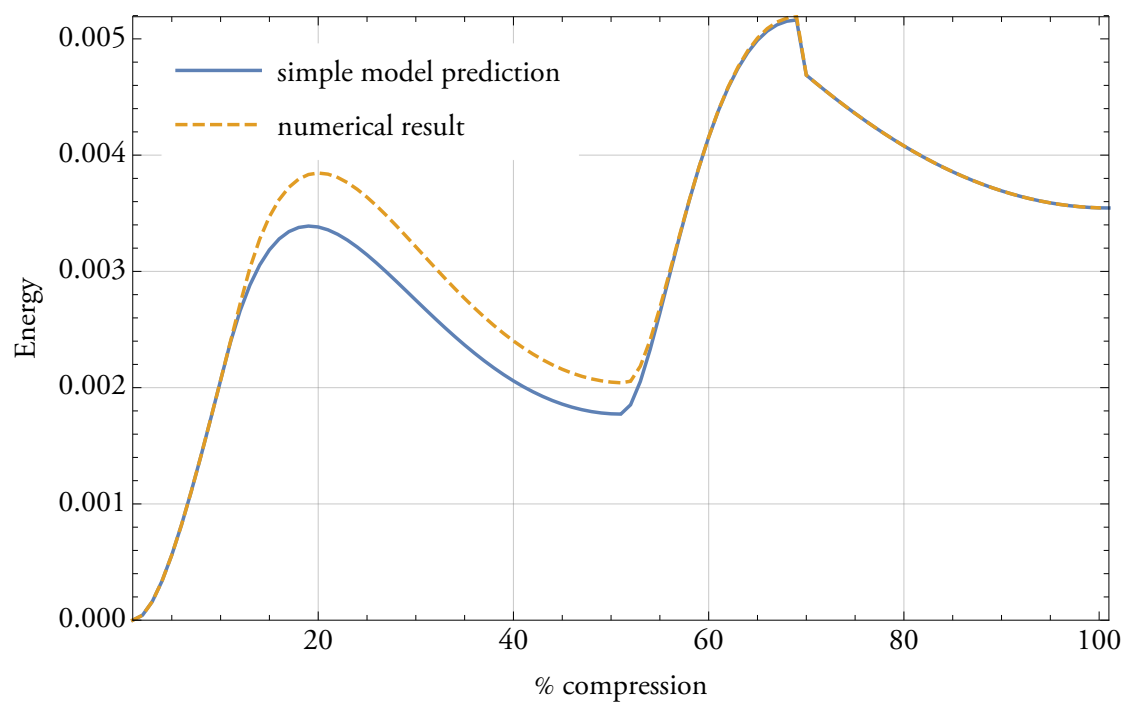

FIGURE A.64: simple energy for $r=1.4, n=5, m=2$

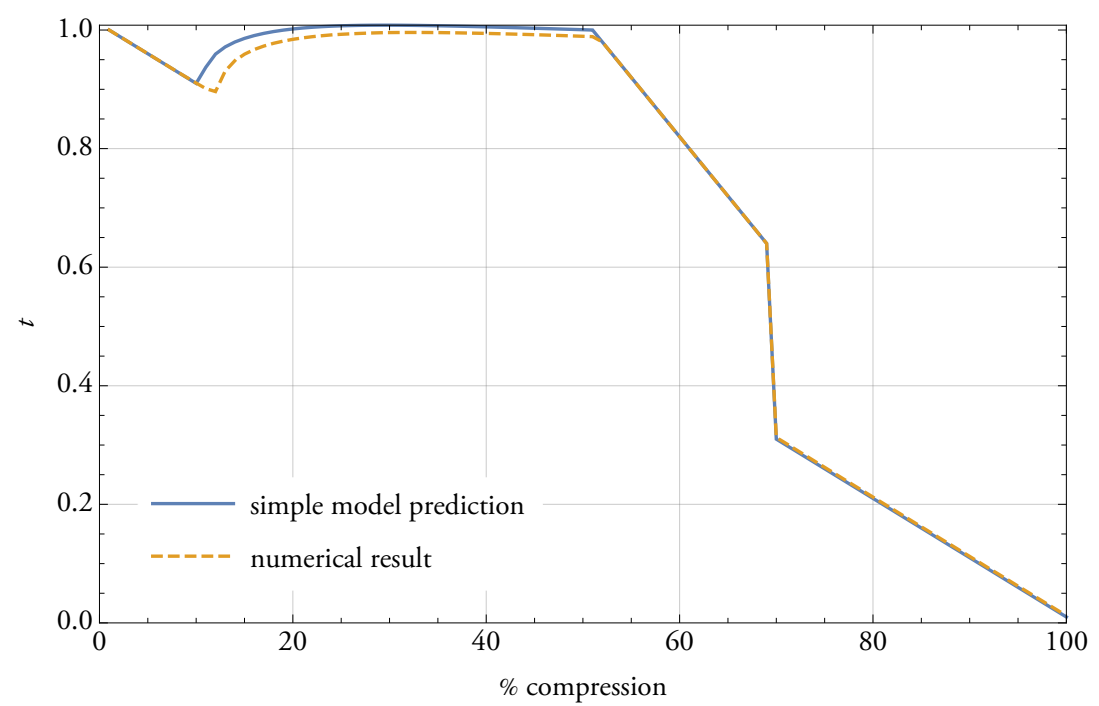

FIGURE A.65: intermediate layer height for $r=1.4, n=5, m=2$

$b_{1}$

$b_{2}$

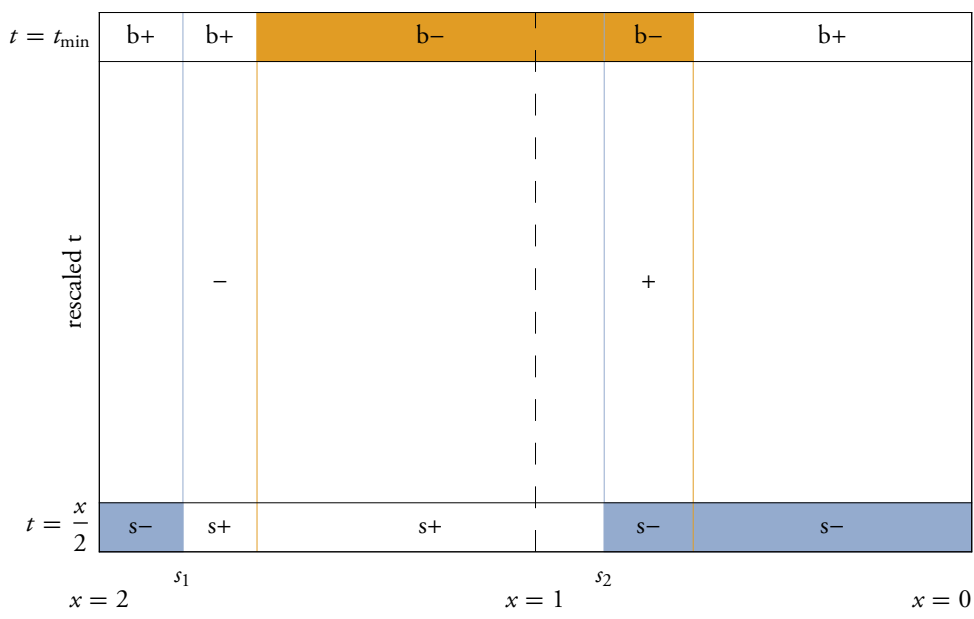

FIGURE A.66: prediction graphic for $r=1.4, n=5, m=2$ 


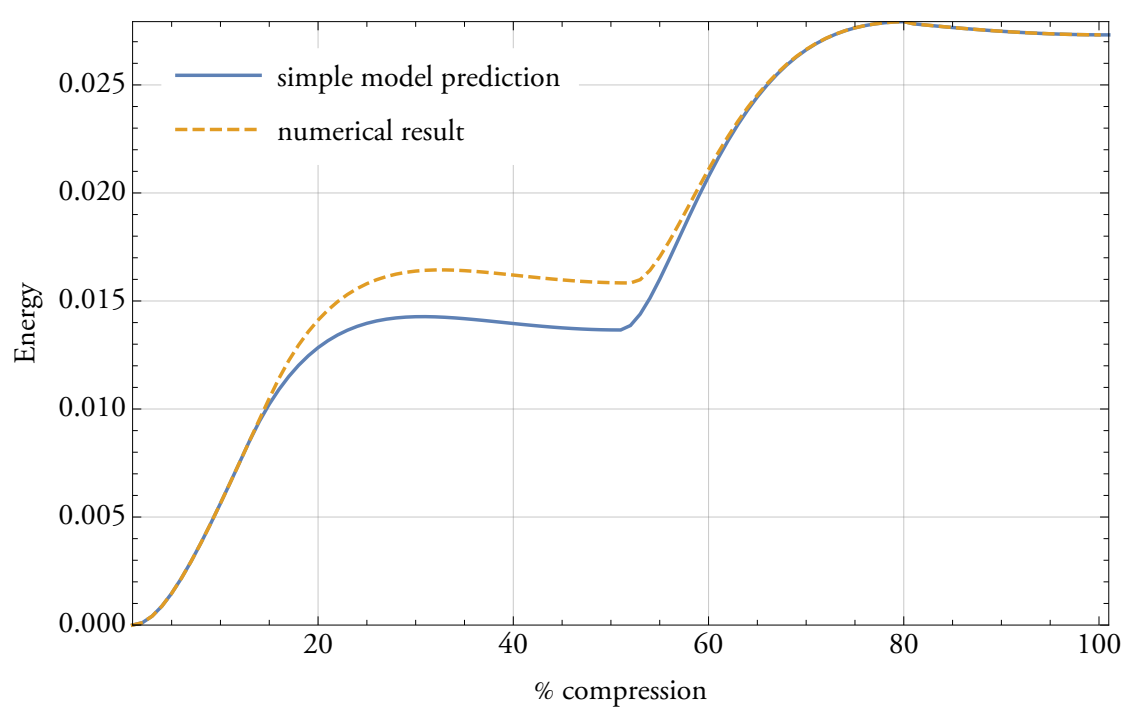

FIGURE A.67: simple energy for $r=1.4, n=6, m=2$

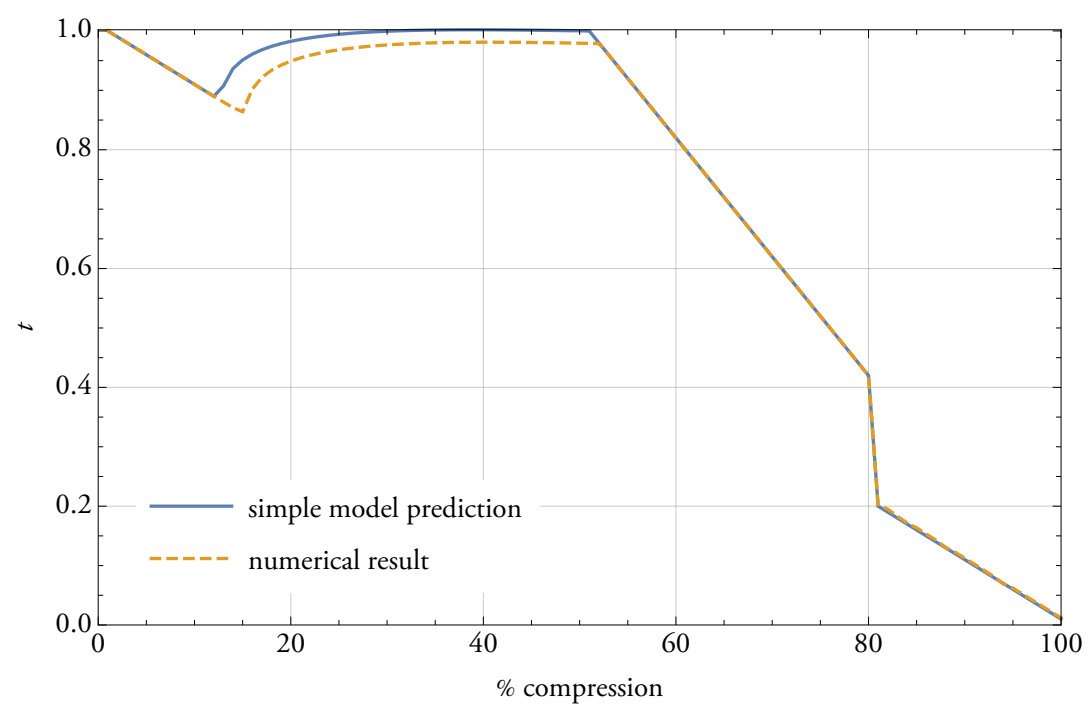

FIGURE A.68: intermediate layer height for $r=1.4, n=6, m=2$

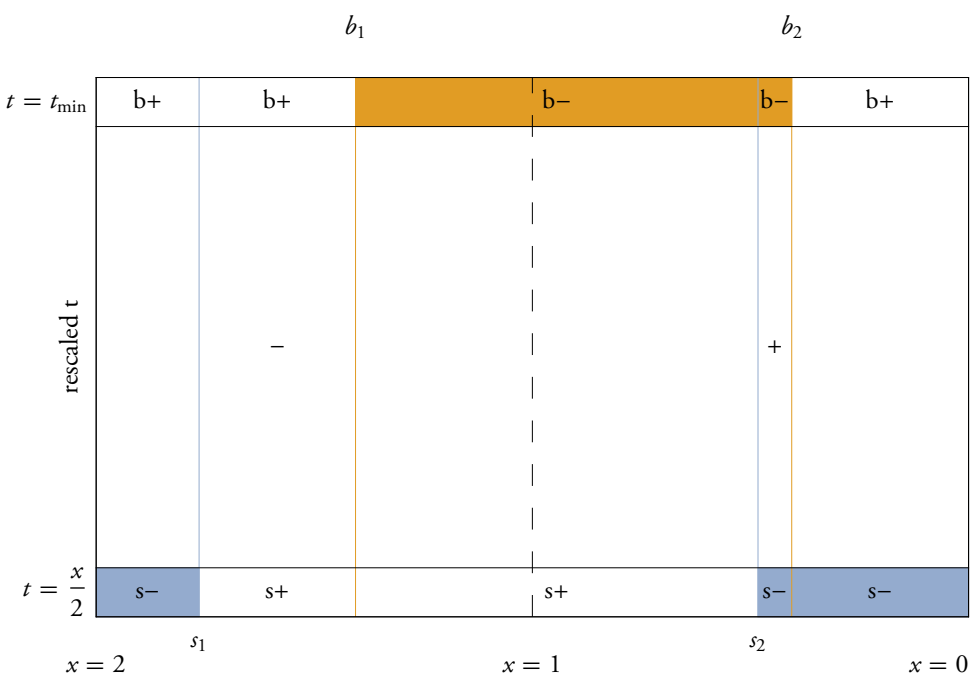

FIGURE A.69: prediction graphic for $r=1.4, n=6, m=2$ 


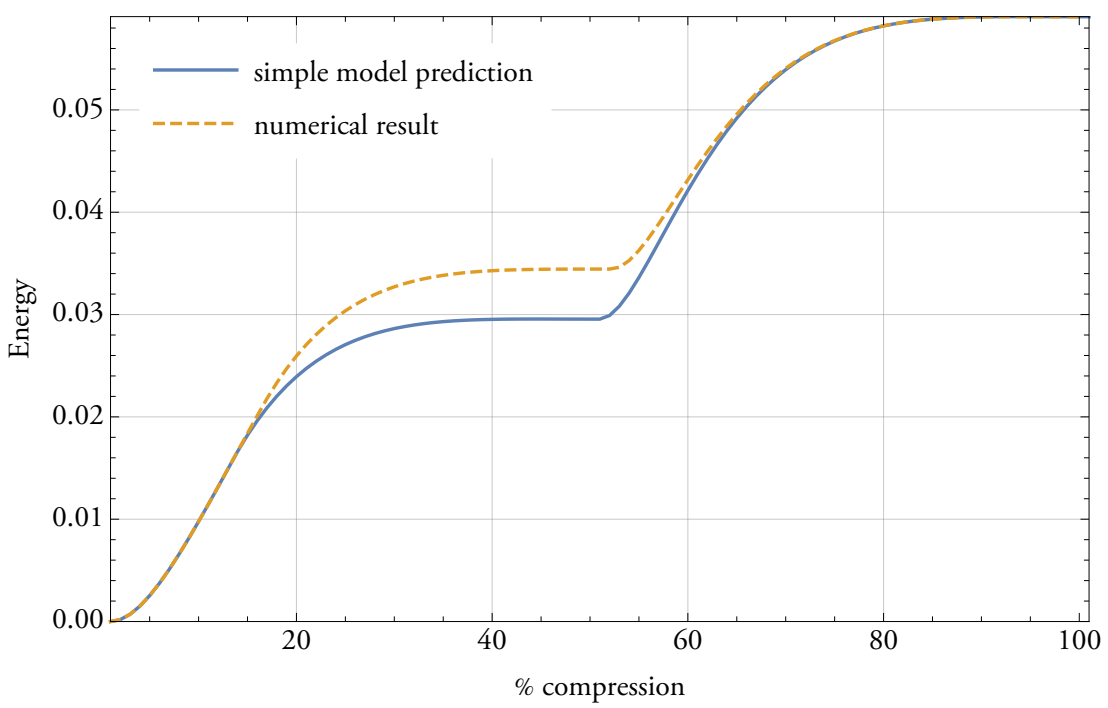

FIGURE A.70: simple energy for $r=1.4, n=7, m=2$

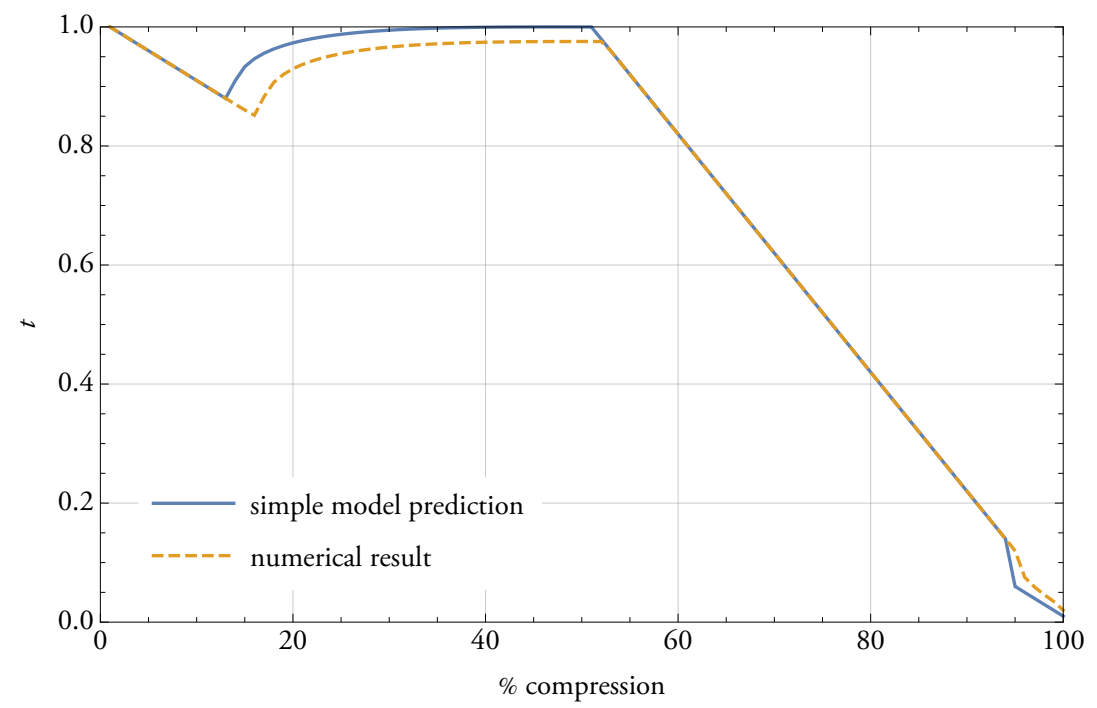

FIGURE A.71: intermediate layer height for $r=1.4, n=7, m=2$

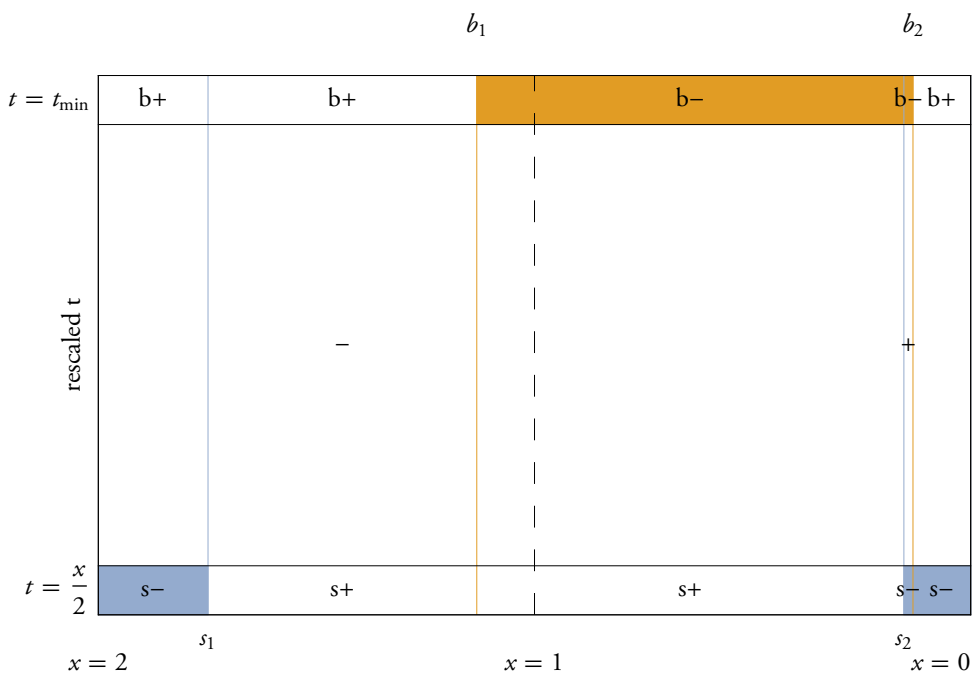

FIGURE A.72: prediction graphic for $r=1.4, n=7, m=2$ 


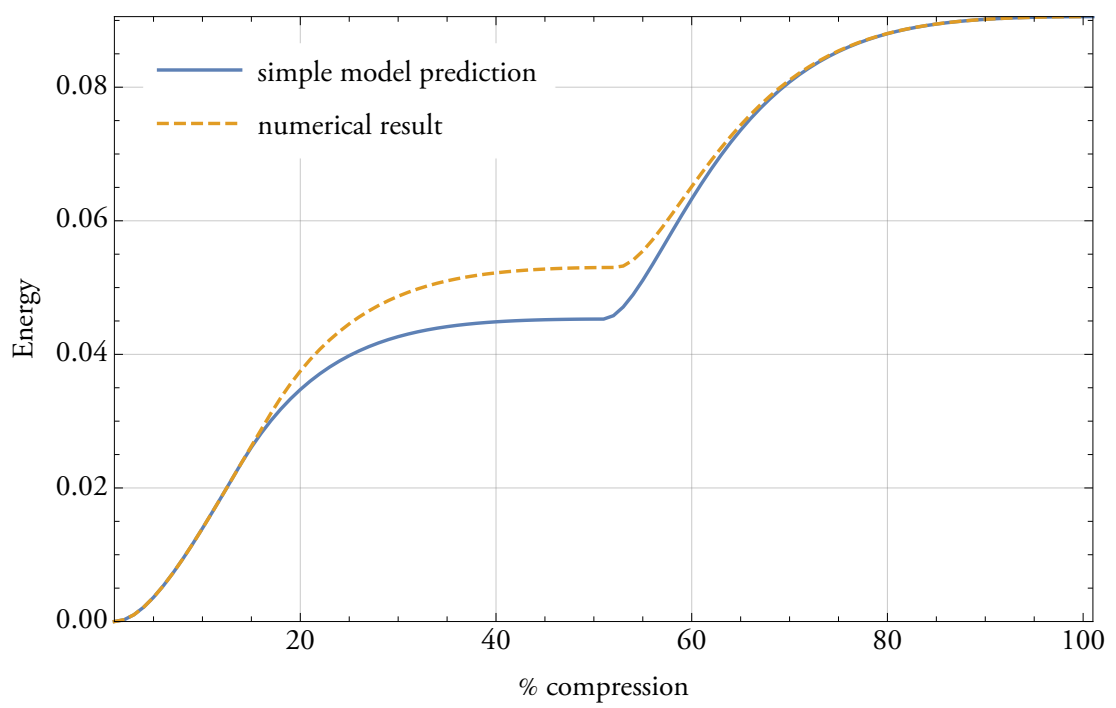

FIGURE A.73: simple energy for $r=1.4, n=8, m=2$

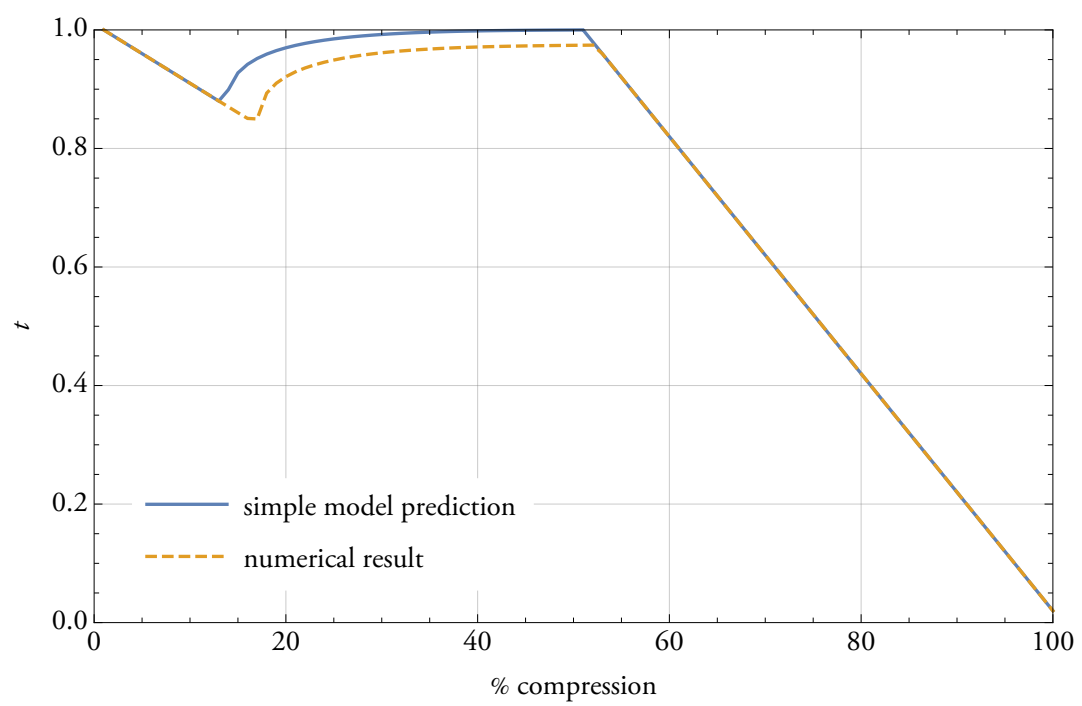

FIGURE A.74: intermediate layer height for $r=1.4, n=8, m=2$

$b_{1}$

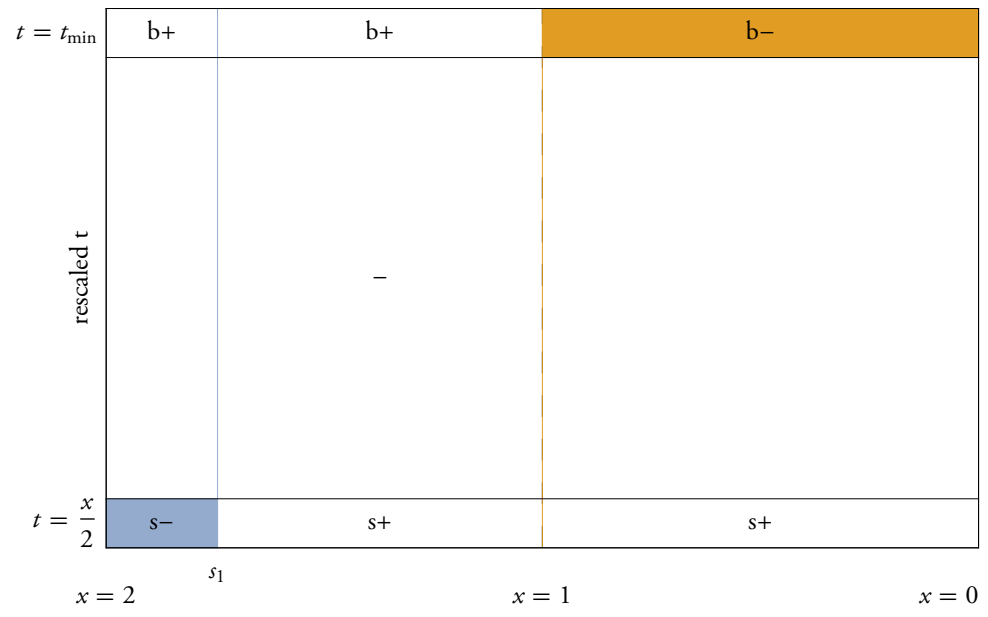

FIGURE A.75: prediction graphic for $r=1.4, n=8, m=2$ 


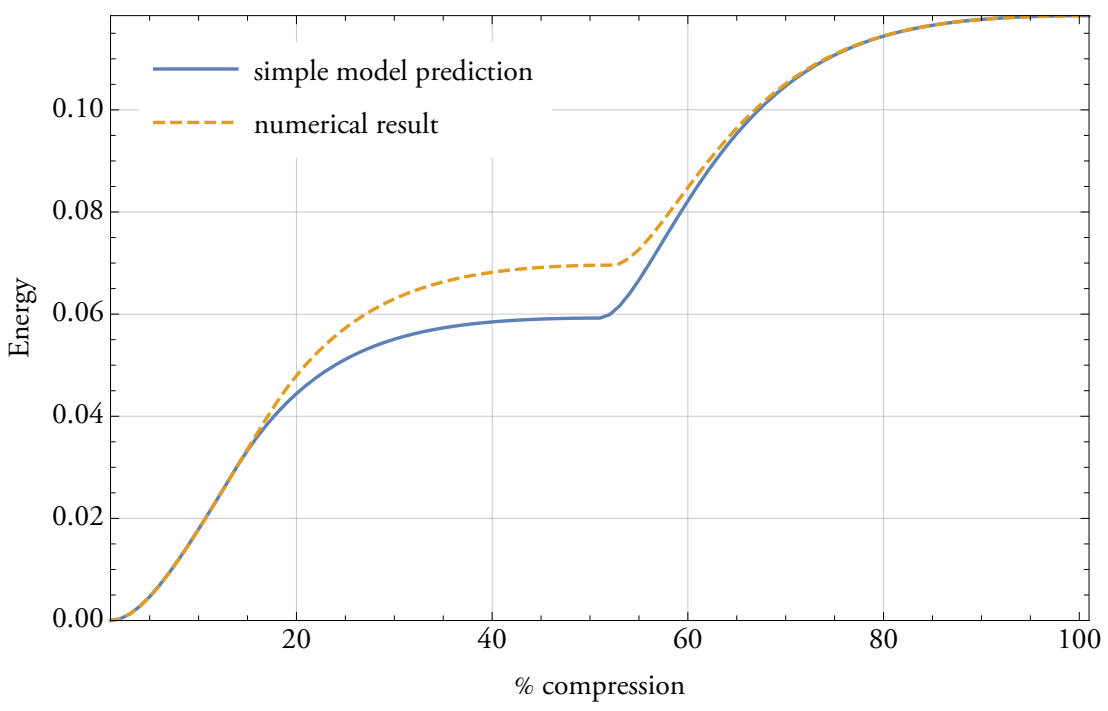

FIGURE A.76: simple energy for $r=1.4, n=9, m=2$

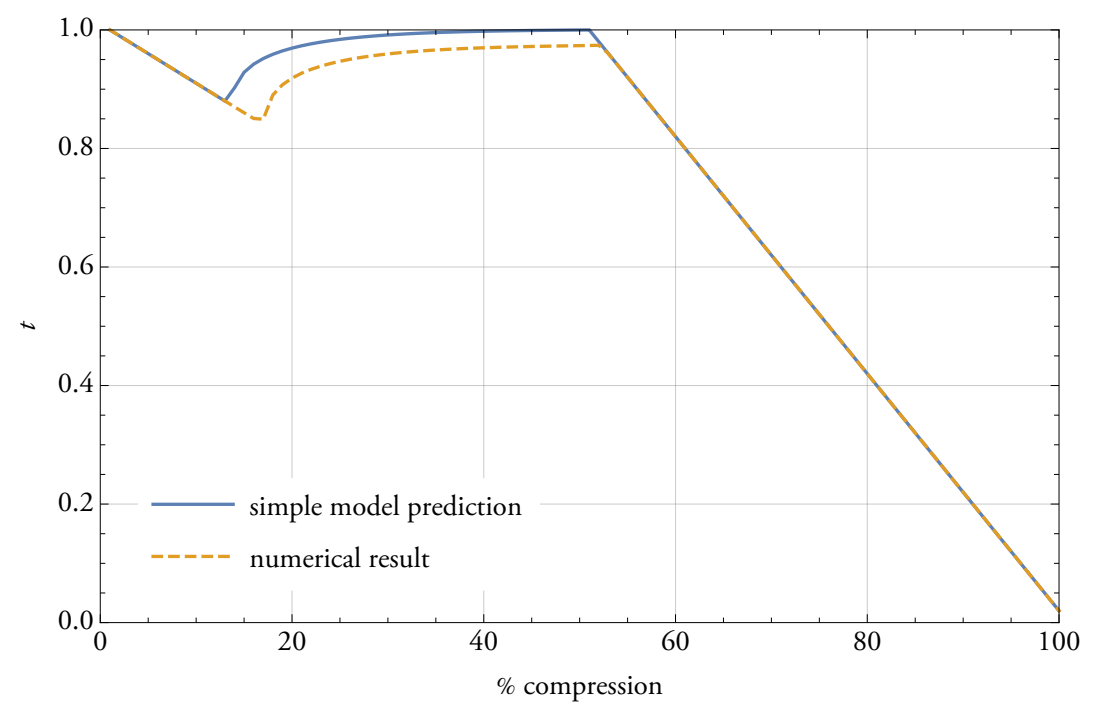

FIGURE A.77: intermediate layer height for $r=1.4, n=9, m=2$

$b_{1}$

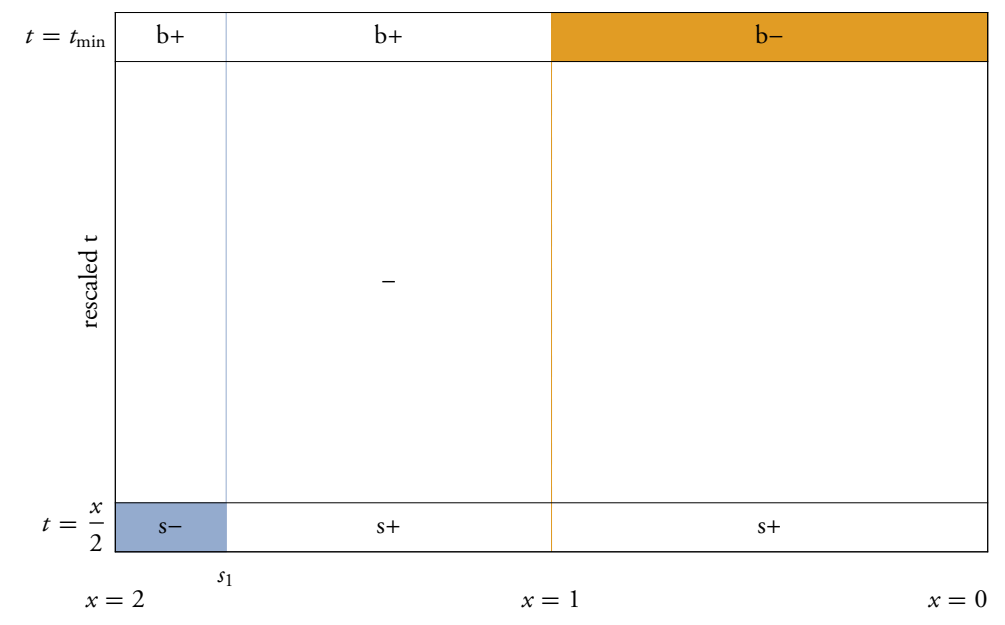

FIGURE A.78: prediction graphic for $r=1.4, n=9, m=2$ 


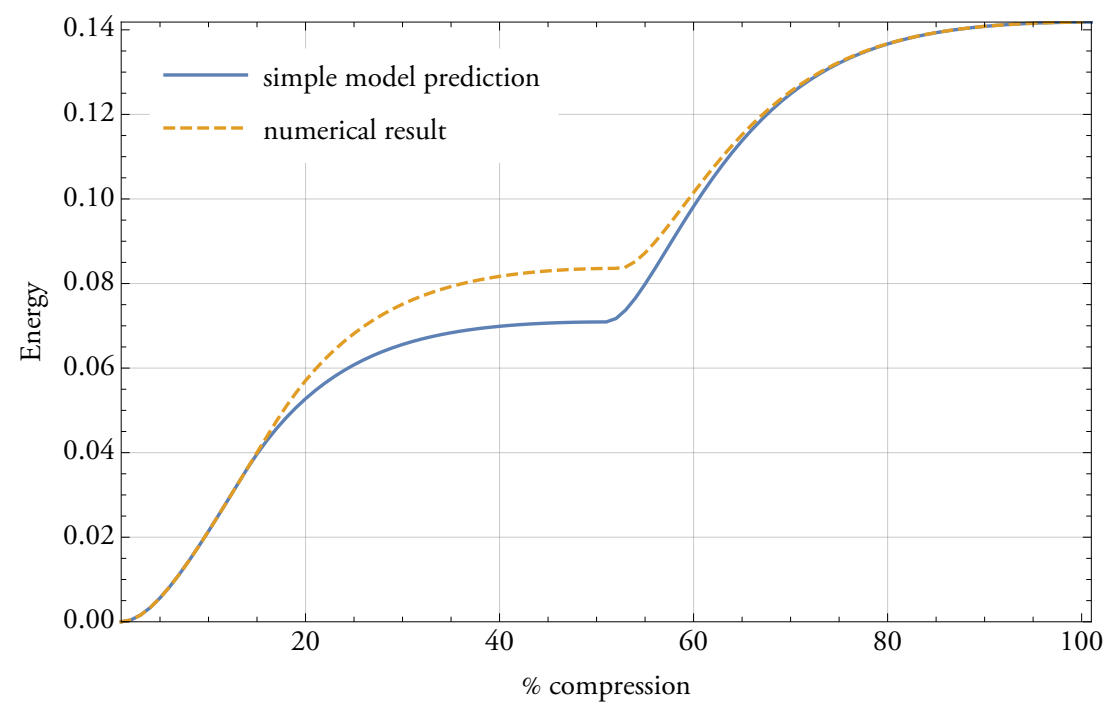

FIGURE A.79: simple energy for $r=1.4, n=10, m=2$

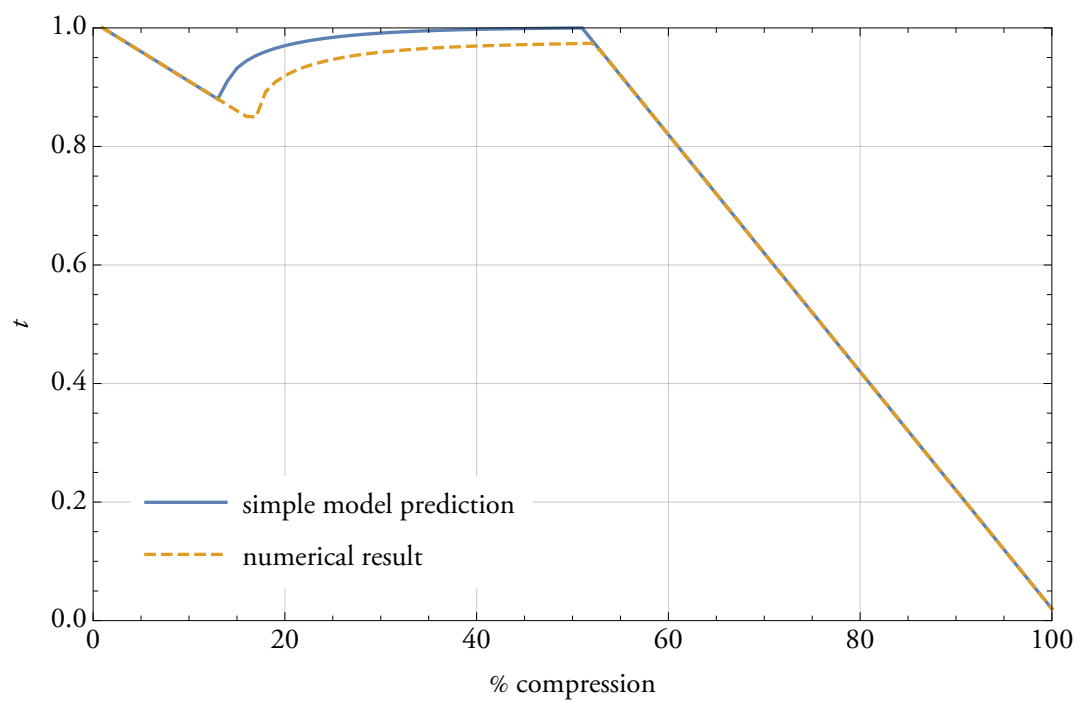

FIGURE A.80: intermediate layer height for $r=1.4, n=10, m=2$

$b_{1}$

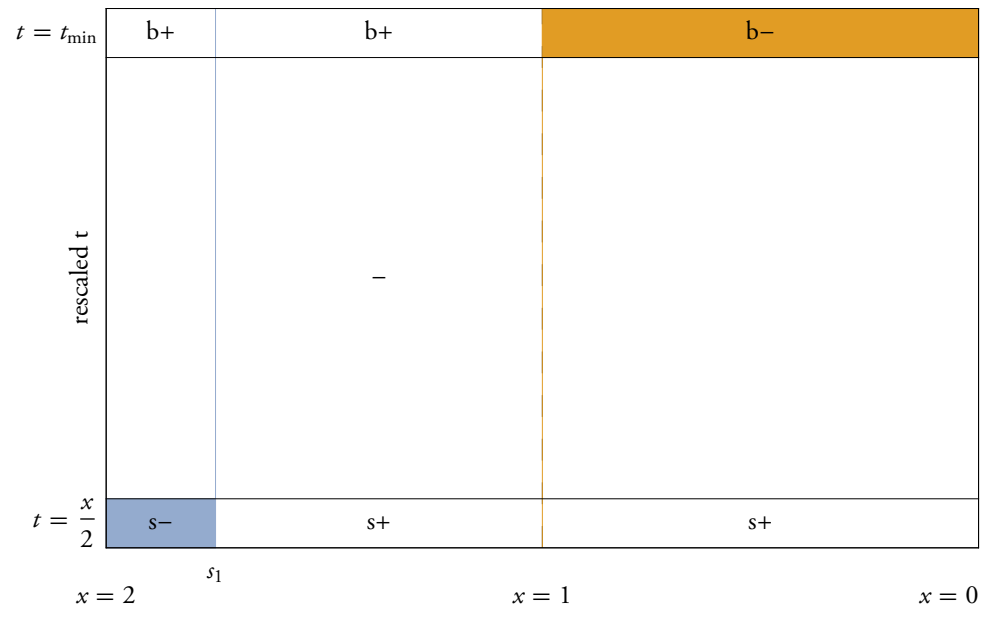

FIGURE A.81: prediction graphic for $r=1.4, n=10, m=2$ 


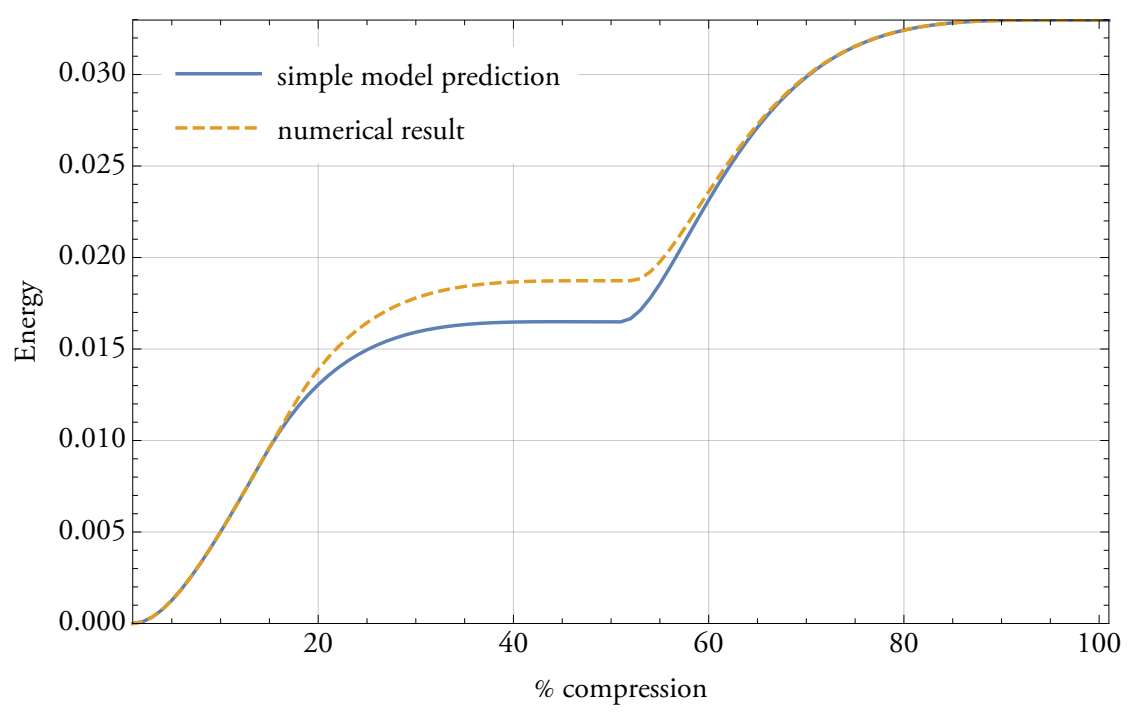

FIGURE A.82: simple energy for $r=1.6, n=5, m=2$

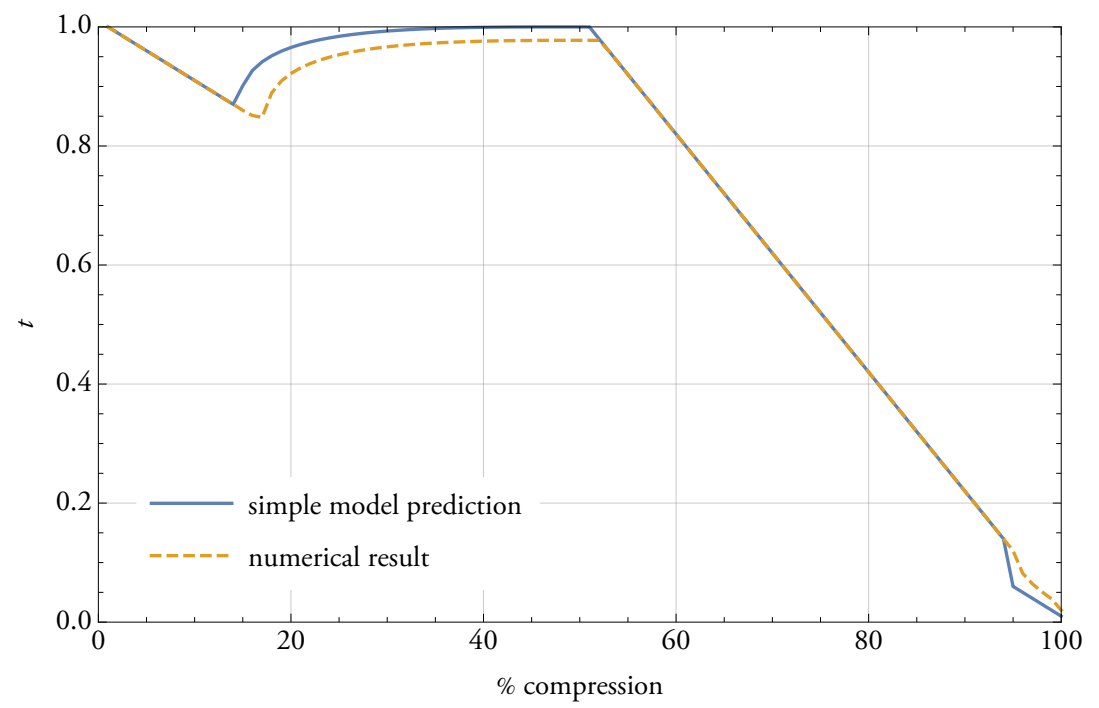

FIGURE A.83: intermediate layer height for $r=1.6, n=5, m=2$

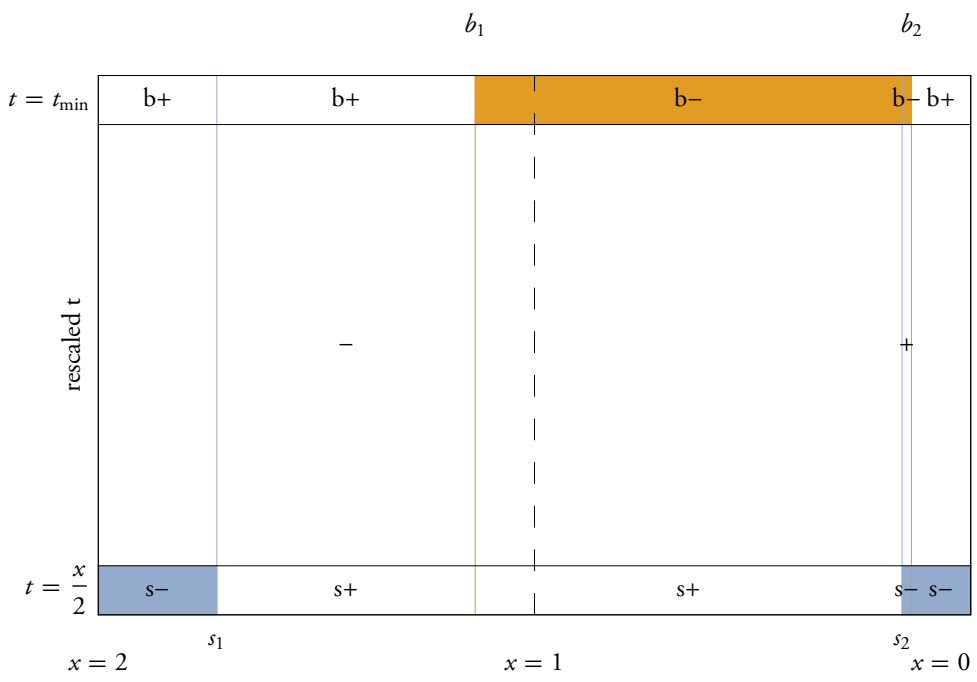

FIGURE A.84: prediction graphic for $r=1.6, n=5, m=2$ 


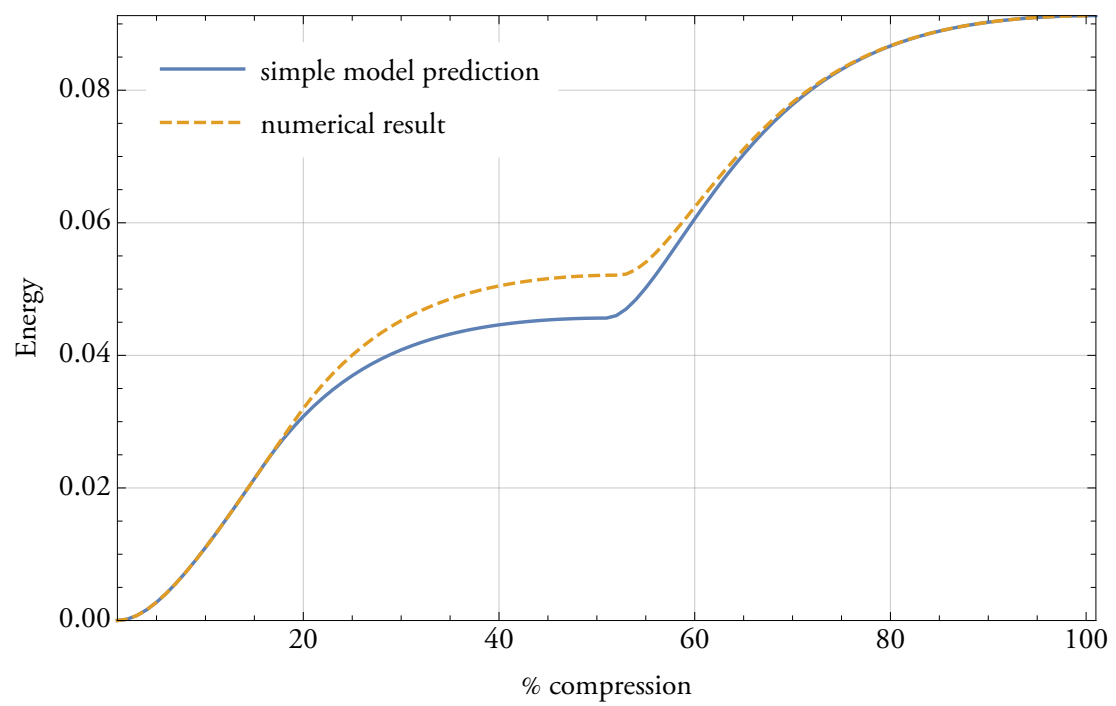

FIGURE A.85: simple energy for $r=1.6, n=6, m=2$

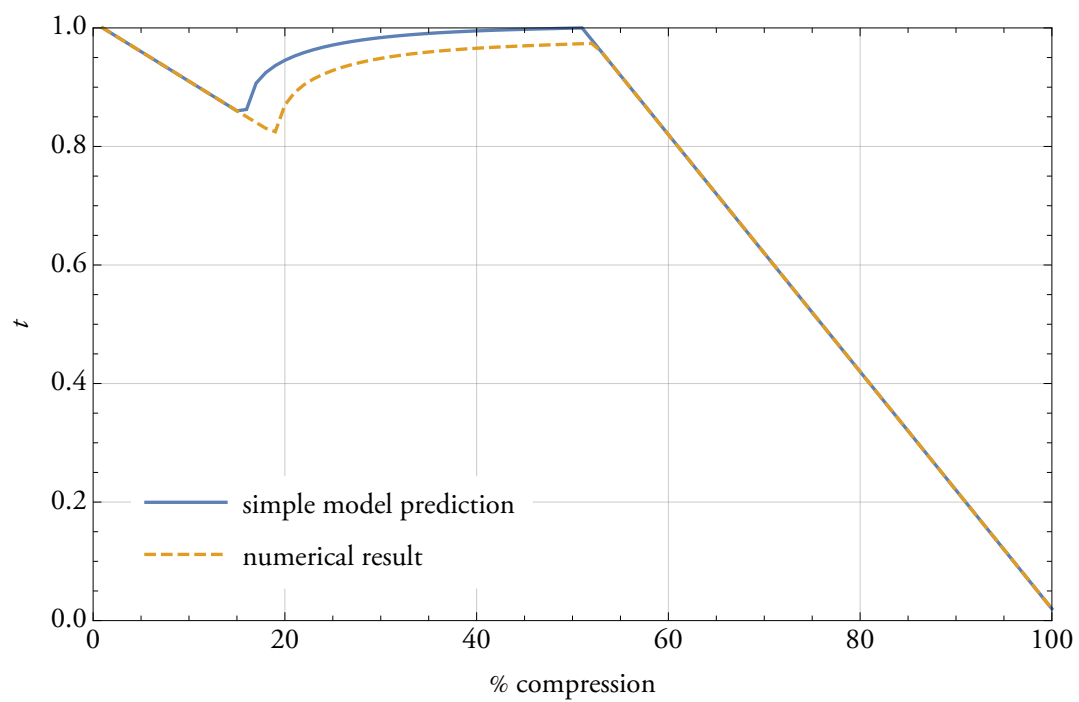

FIGURE A.86: intermediate layer height for $r=1.6, n=6, m=2$

$b_{1}$

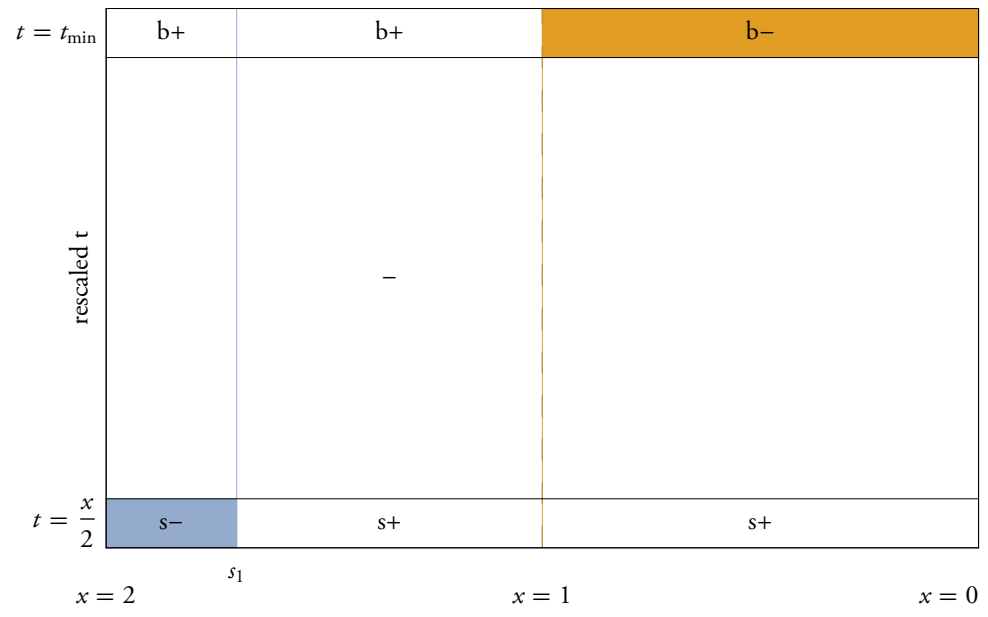

FIGURE A.87: prediction graphic for $r=1.6, n=6, m=2$ 


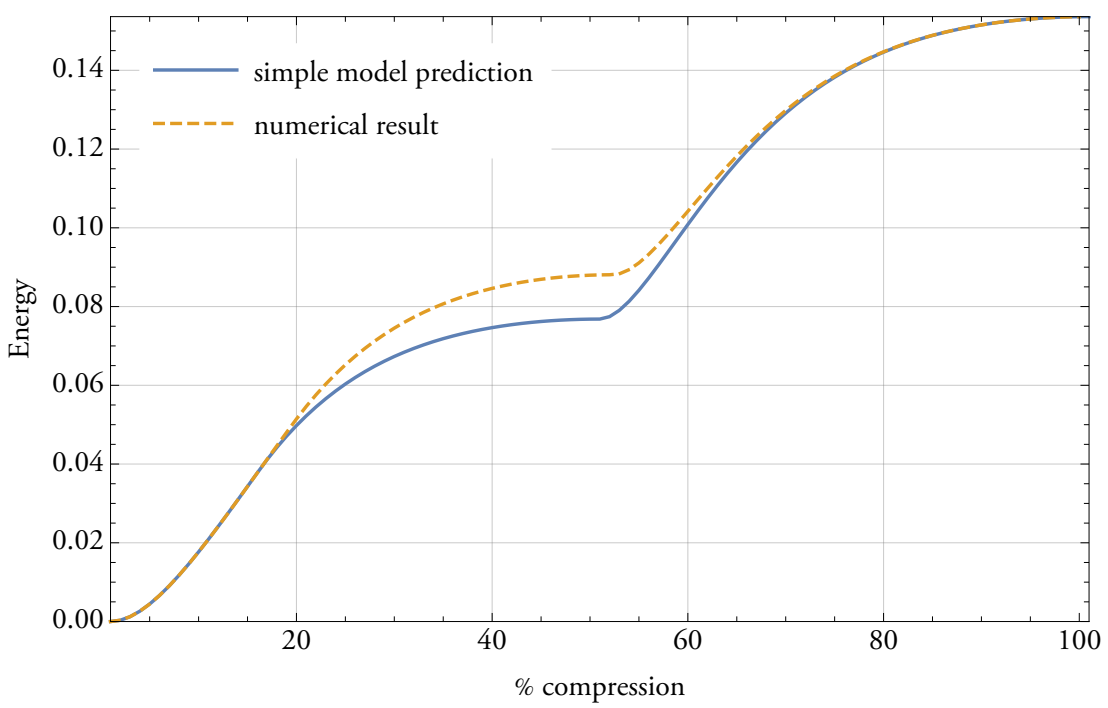

FIGURE A.88: simple energy for $r=1.6, n=7, m=2$

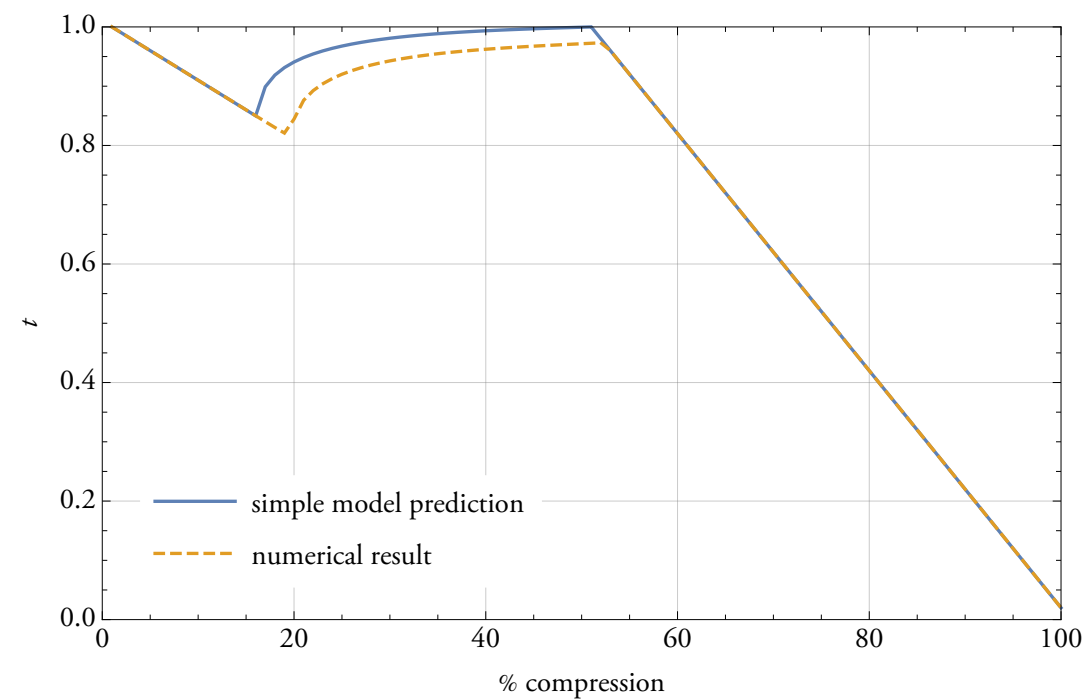

FIGURE A.89: intermediate layer height for $r=1.6, n=7, m=2$

$b_{1}$

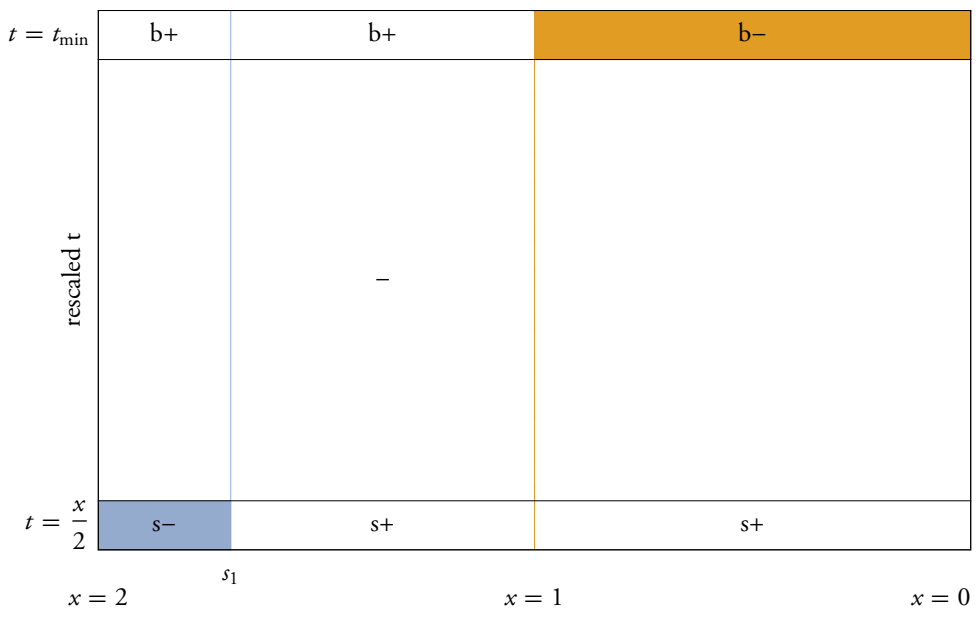

FIGURE A.90: prediction graphic for $r=1.6, n=7, m=2$ 


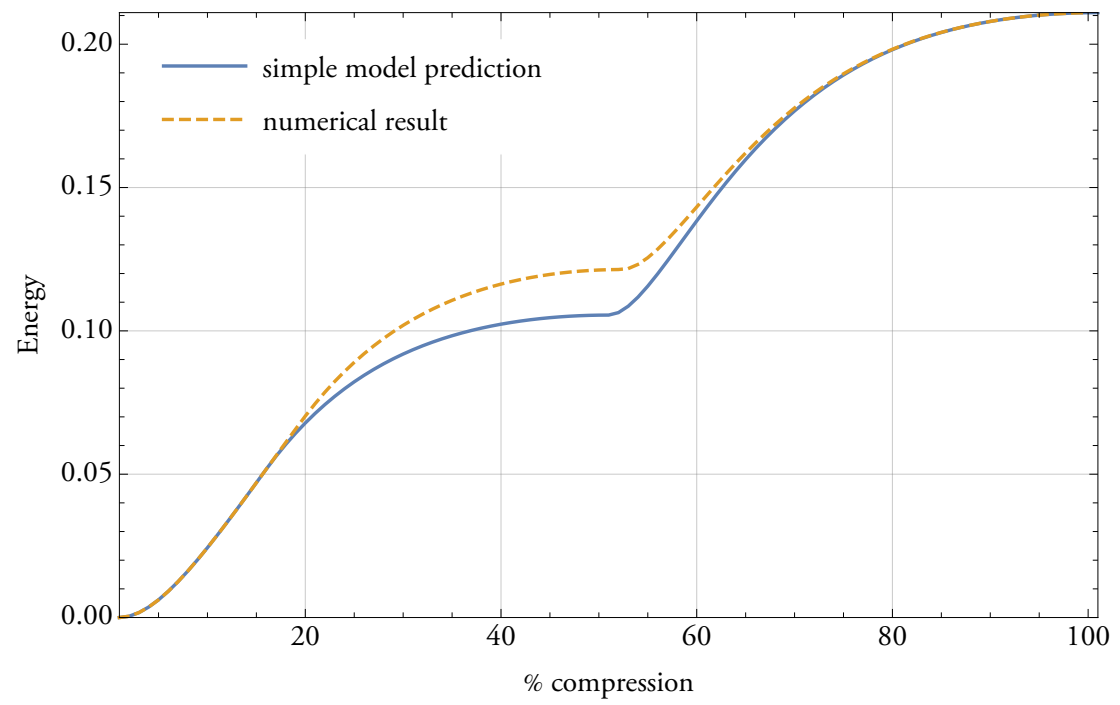

FIGURE A.91: simple energy for $r=1.6, n=8, m=2$

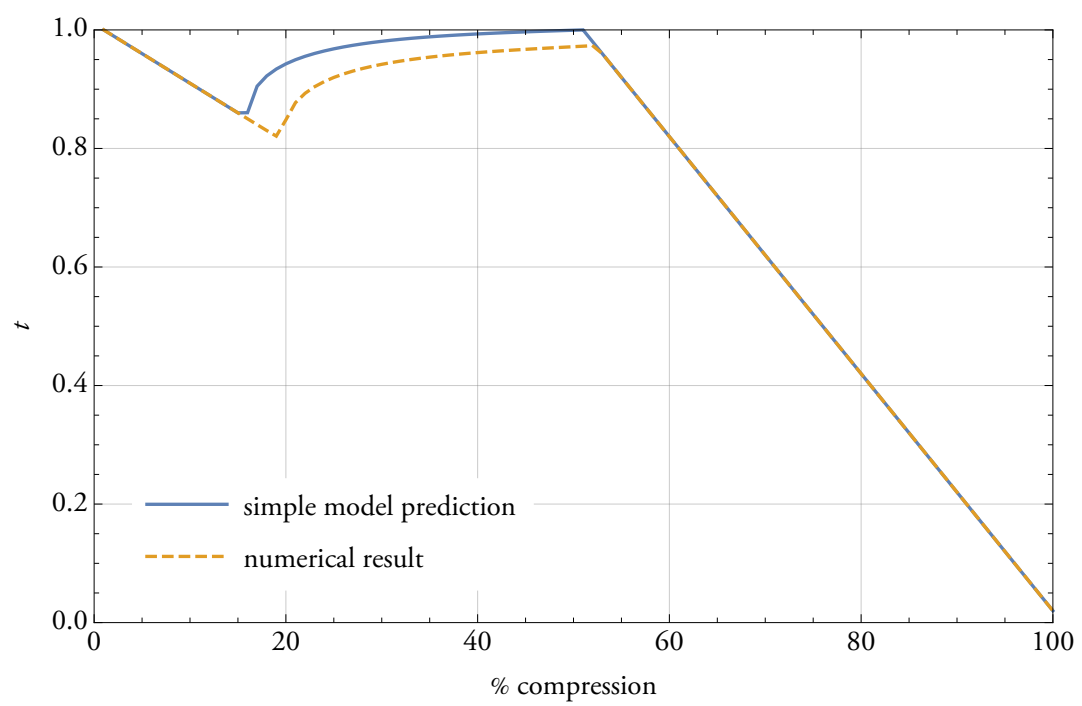

FIGURE A.92: intermediate layer height for $r=1.6, n=8, m=2$

$b_{1}$

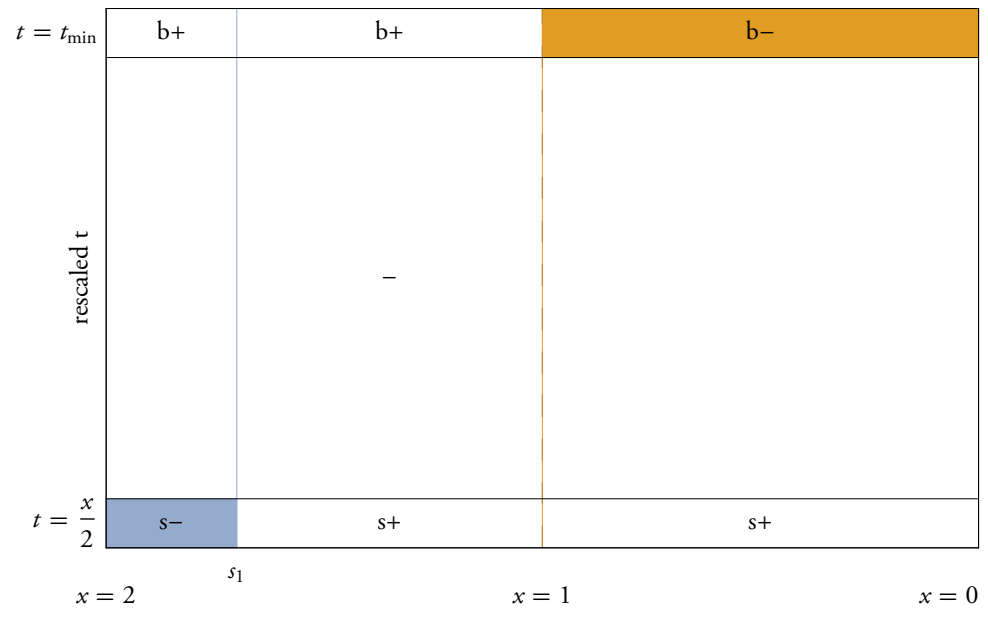

FIGURE A.93: prediction graphic for $r=1.6, n=8, m=2$ 


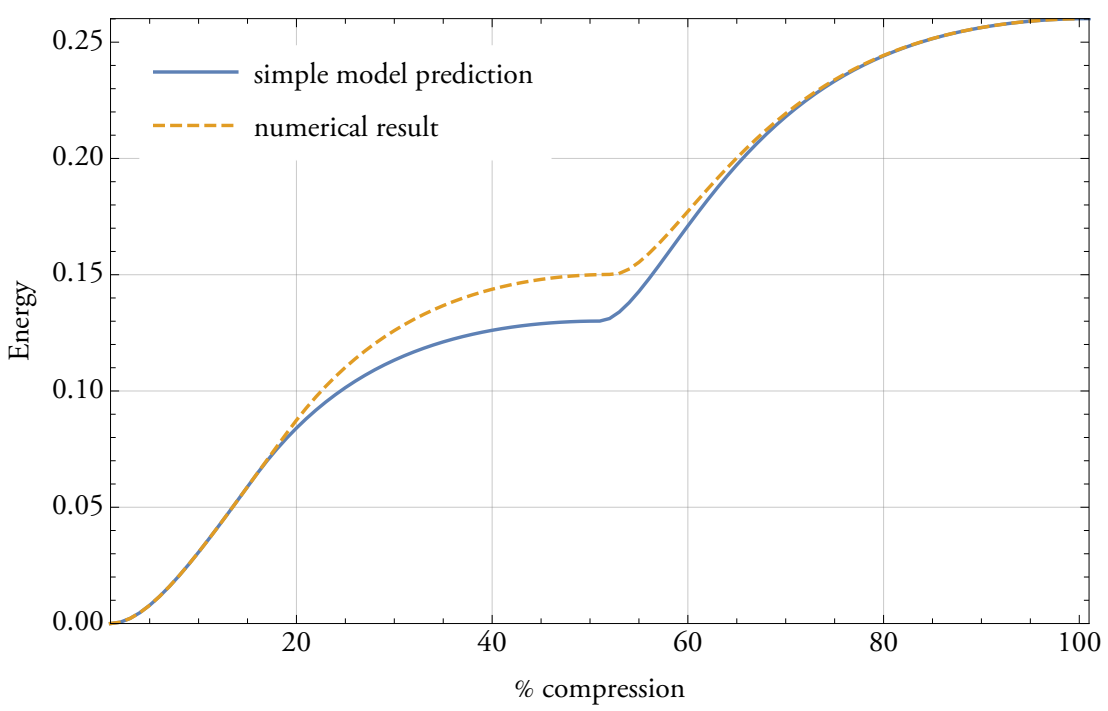

FIGURE A.94: simple energy for $r=1.6, n=9, m=2$

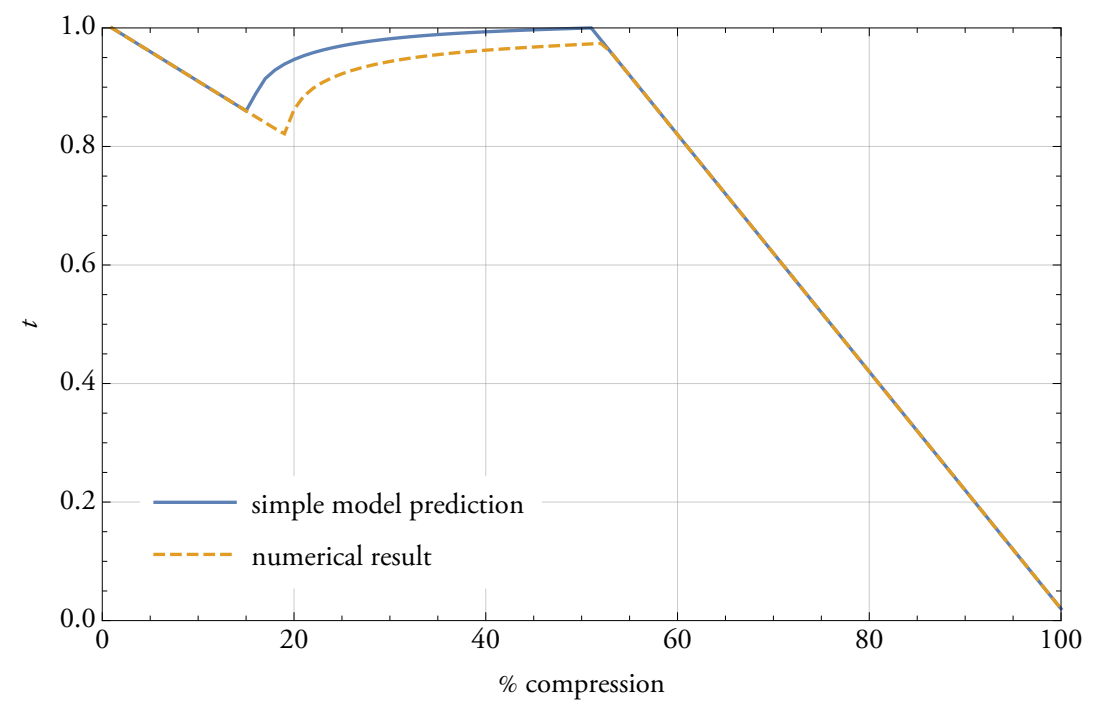

FIGURE A.95: intermediate layer height for $r=1.6, n=9, m=2$

$b_{1}$

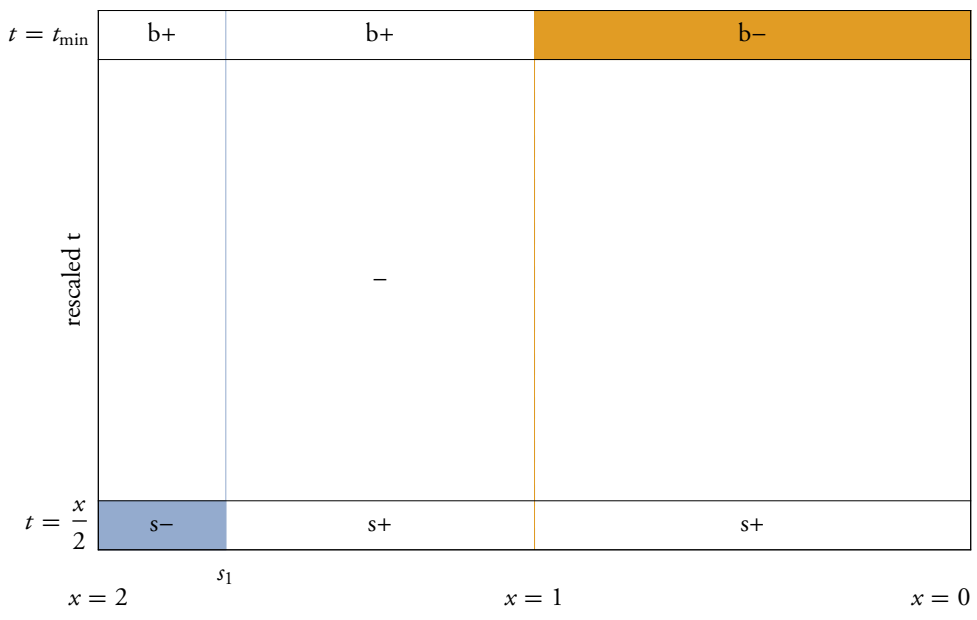

FIGURE A.96: prediction graphic for $r=1.6, n=9, m=2$ 


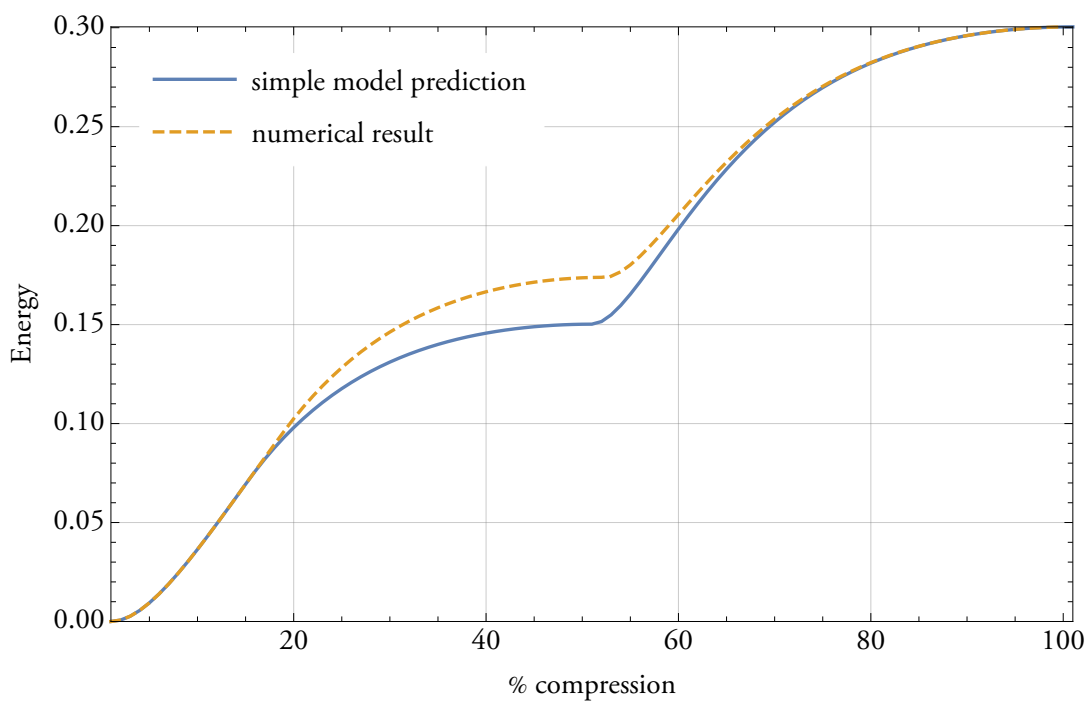

FIGURE A.97: simple energy for $r=1.6, n=10, m=2$

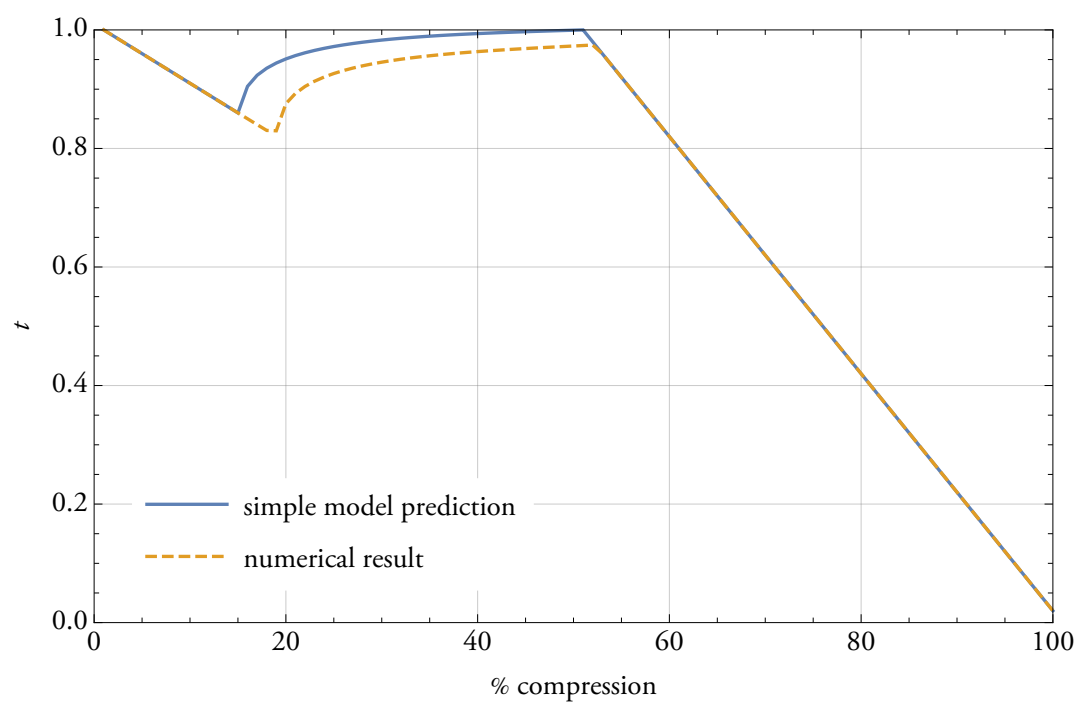

FIGURE A.98: intermediate layer height for $r=1.6, n=10, m=2$

$b_{1}$

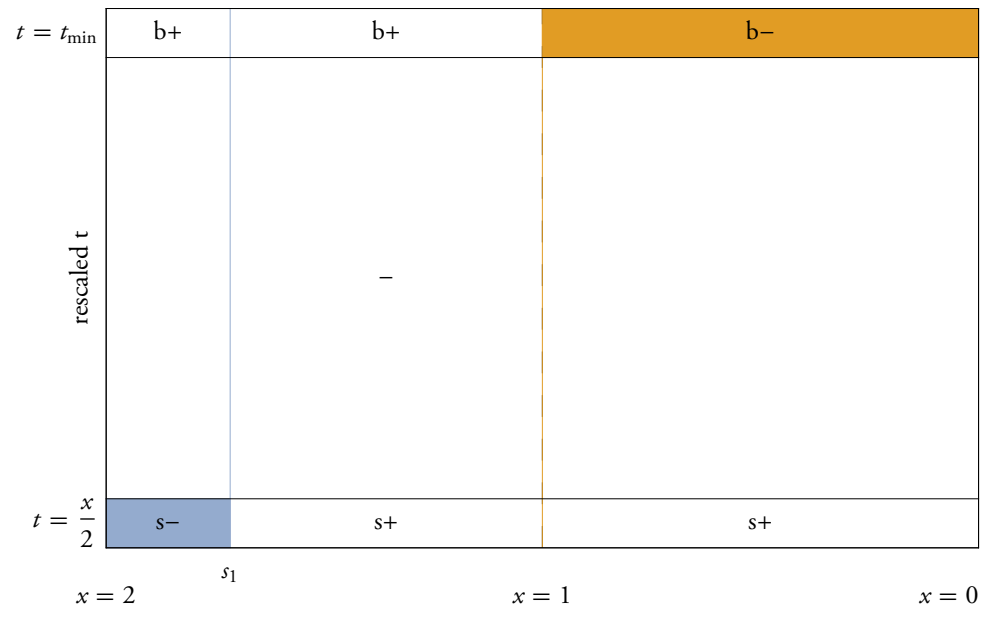

FIGURE A.99: prediction graphic for $r=1.6, n=10, m=2$ 


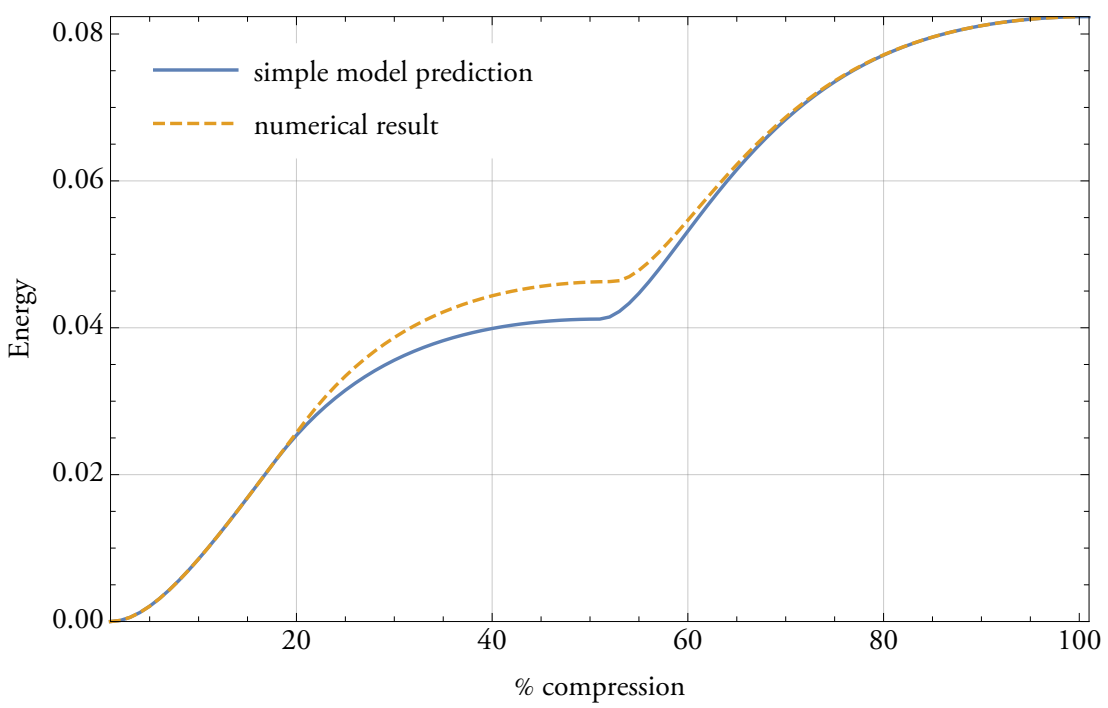

FIGURE A.100: simple energy for $r=1.8, n=5, m=2$

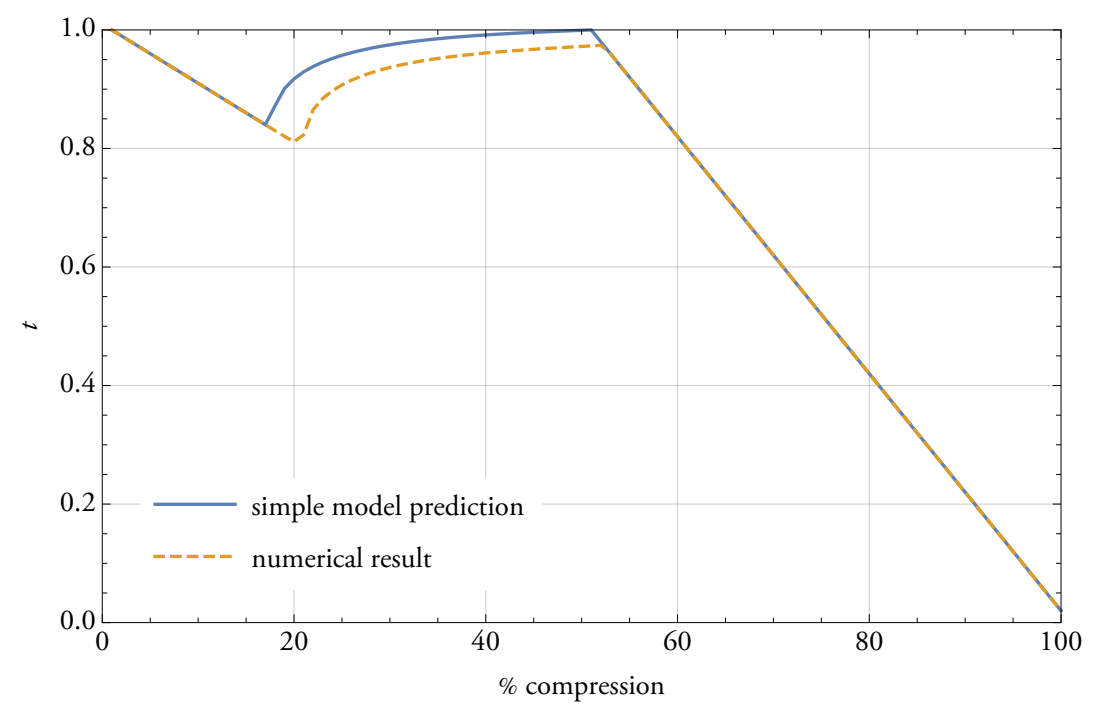

FIGURE A.101: intermediate layer height for $r=1.8, n=5, m=2$

$b_{1}$

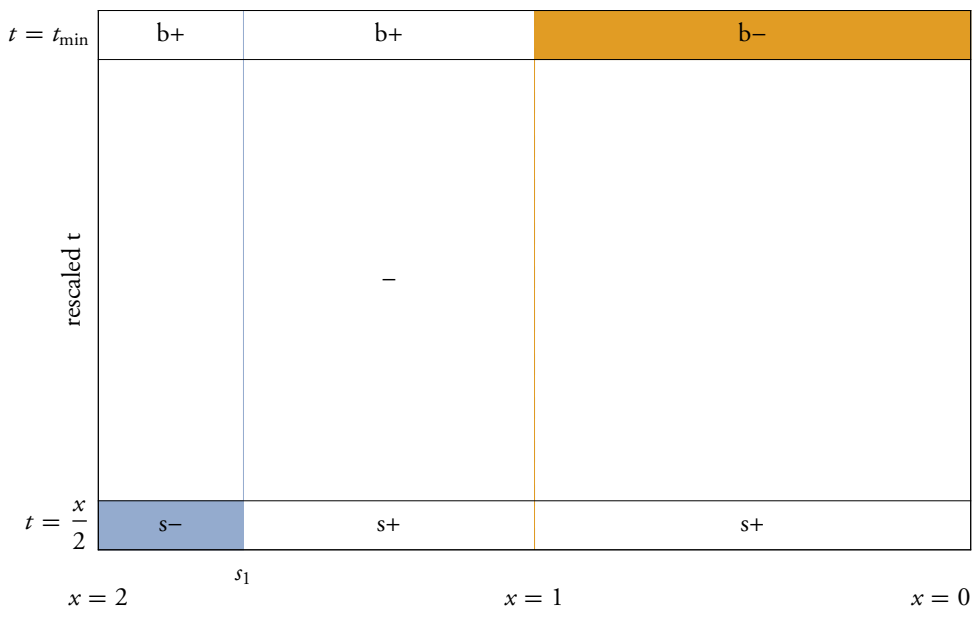

FIGURE A.102: prediction graphic for $r=1.8, n=5, m=2$ 


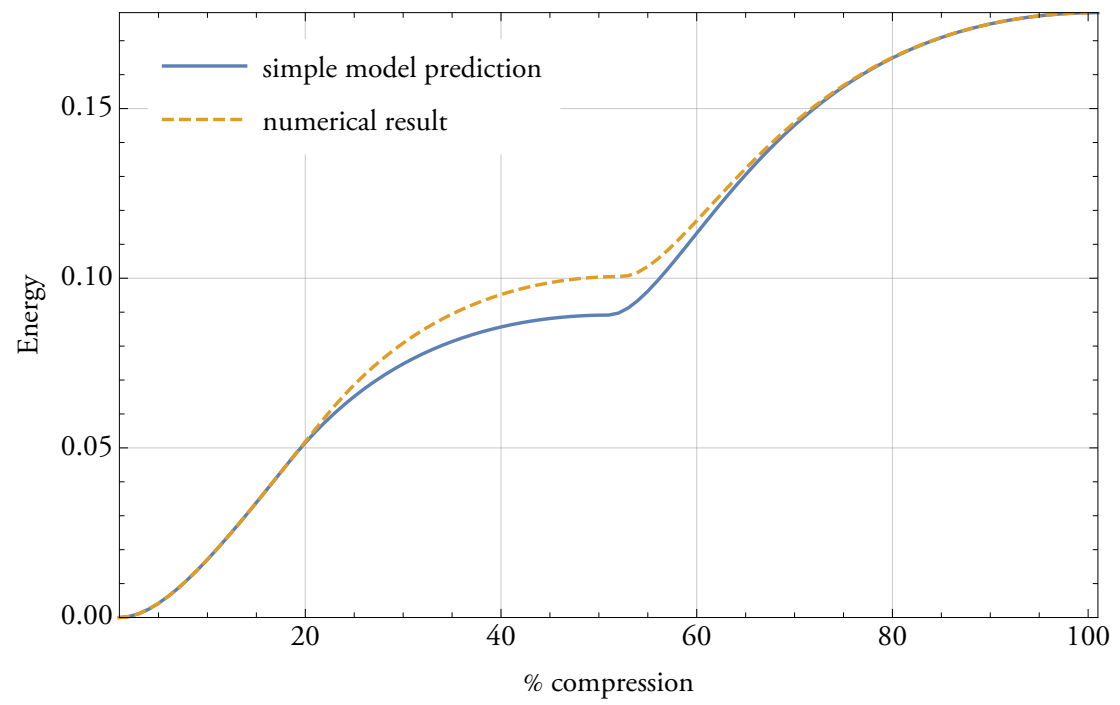

FIGURE A.103: simple energy for $r=1.8, n=6, m=2$

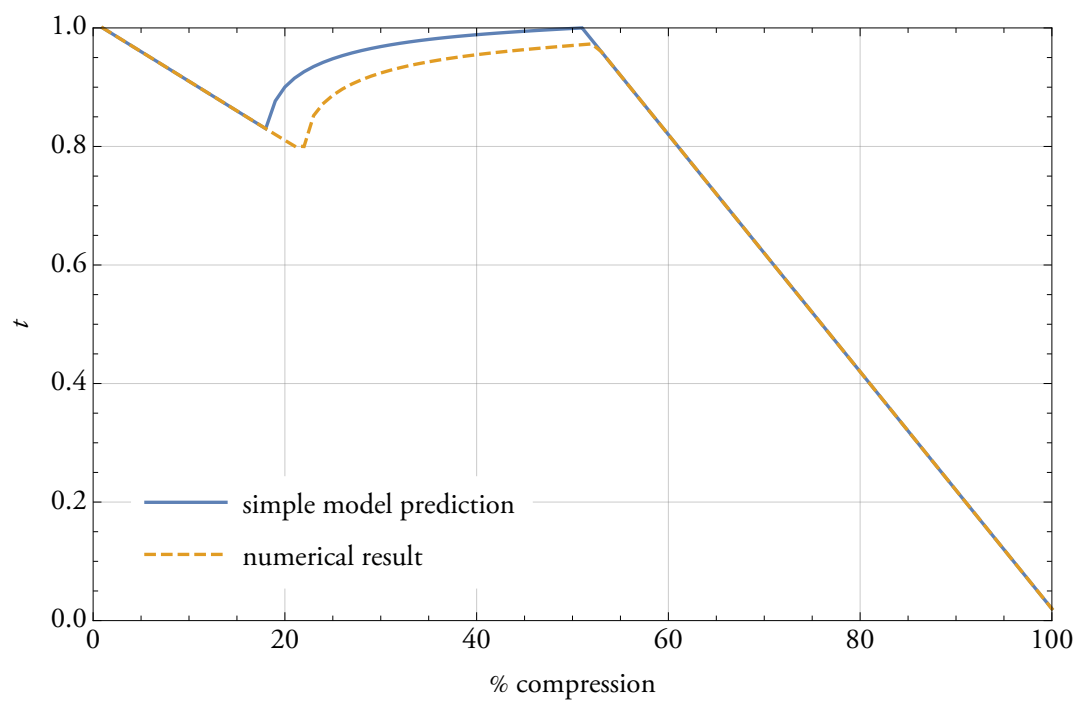

FIGURE A.104: intermediate layer height for $r=1.8, n=6, m=2$

$b_{1}$

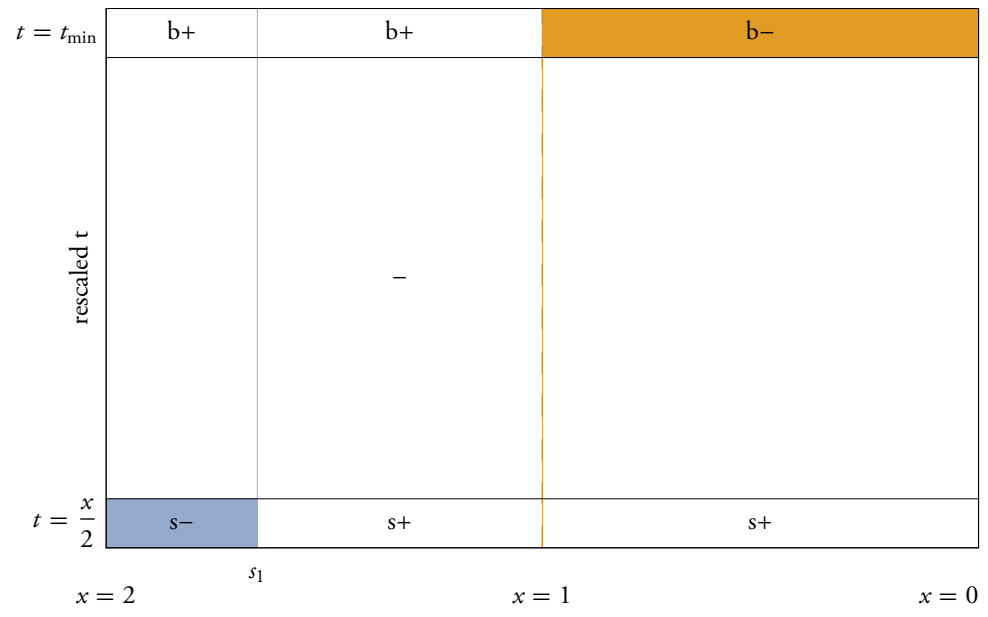

FIGURE A.105: prediction graphic for $r=1.8, n=6, m=2$ 


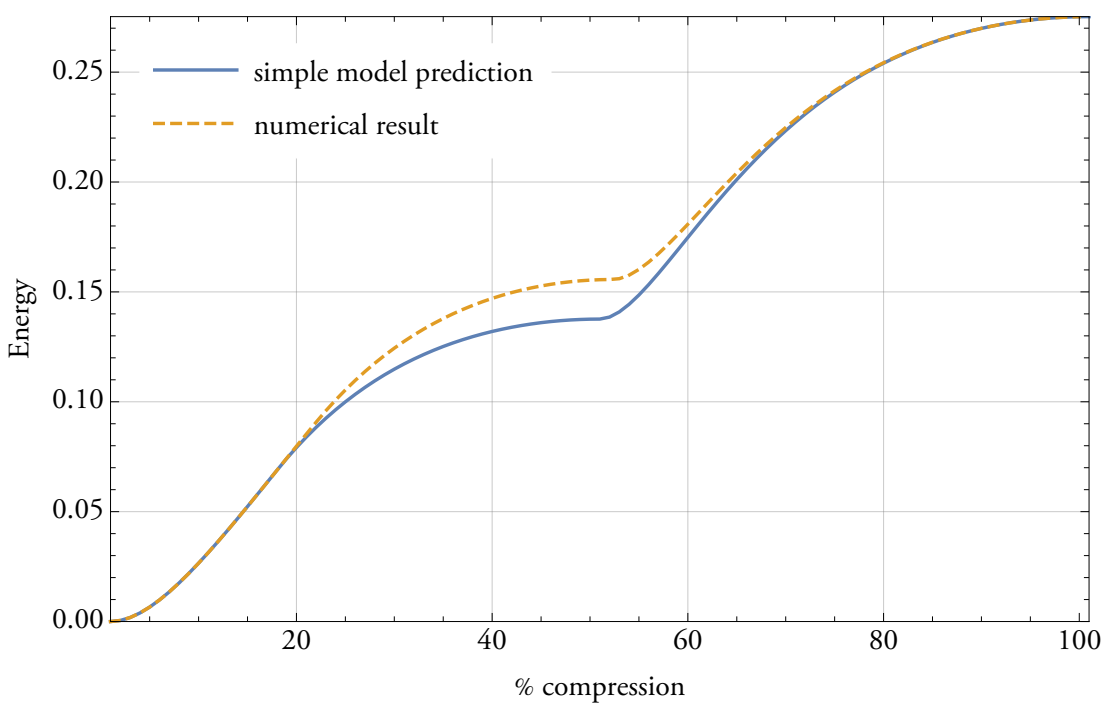

FIGURE A.106: simple energy for $r=1.8, n=7, m=2$

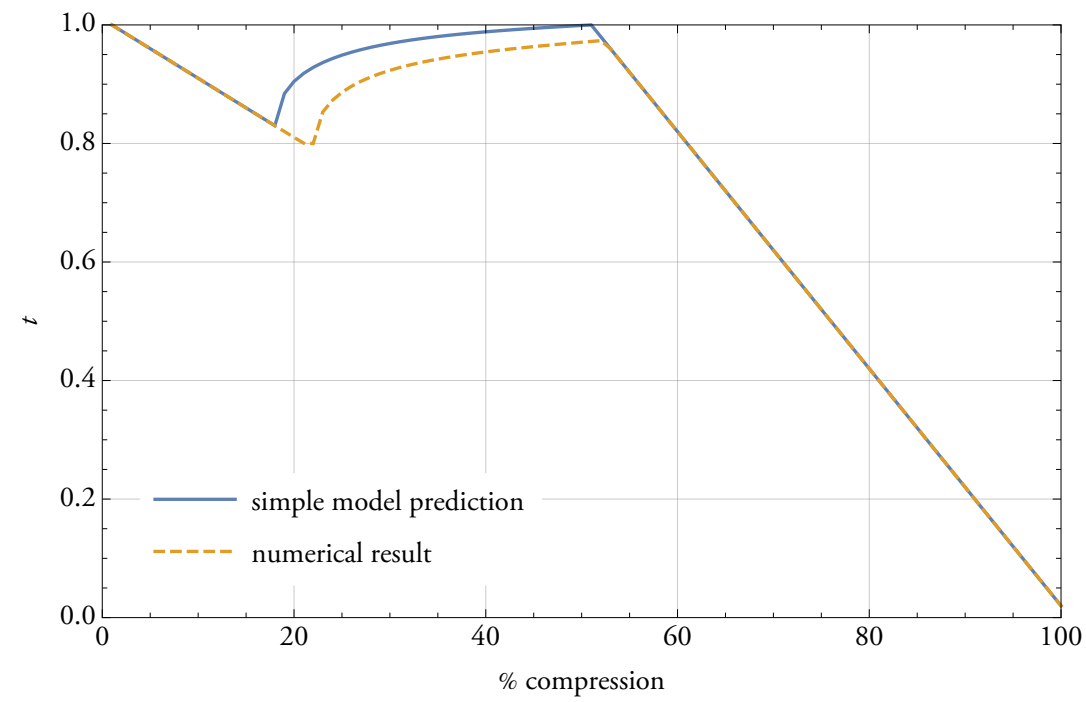

FIGURE A.107: intermediate layer height for $r=1.8, n=7, m=2$

$b_{1}$

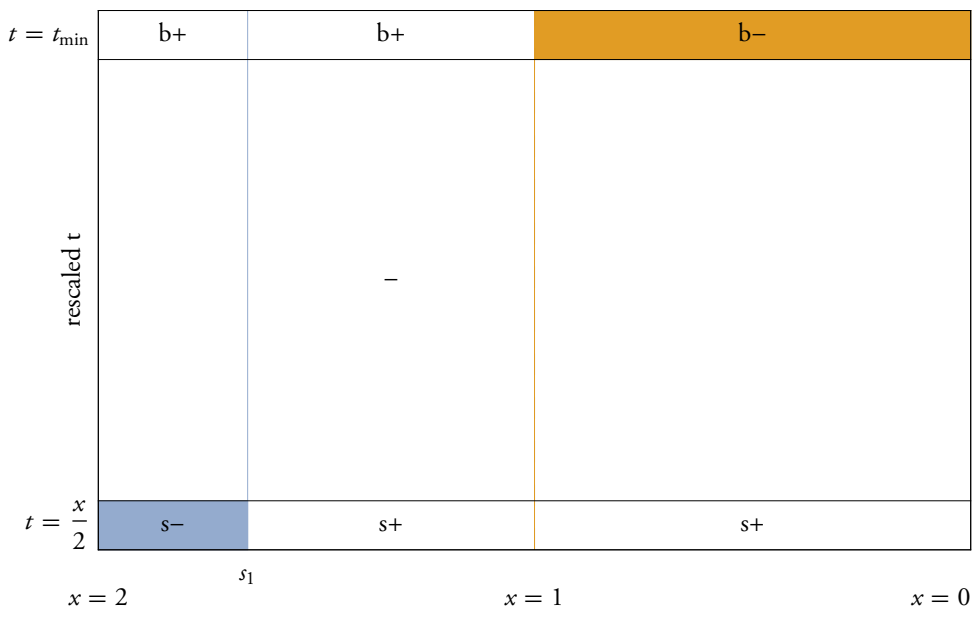

FIGURE A.108: prediction graphic for $r=1.8, n=7, m=2$ 


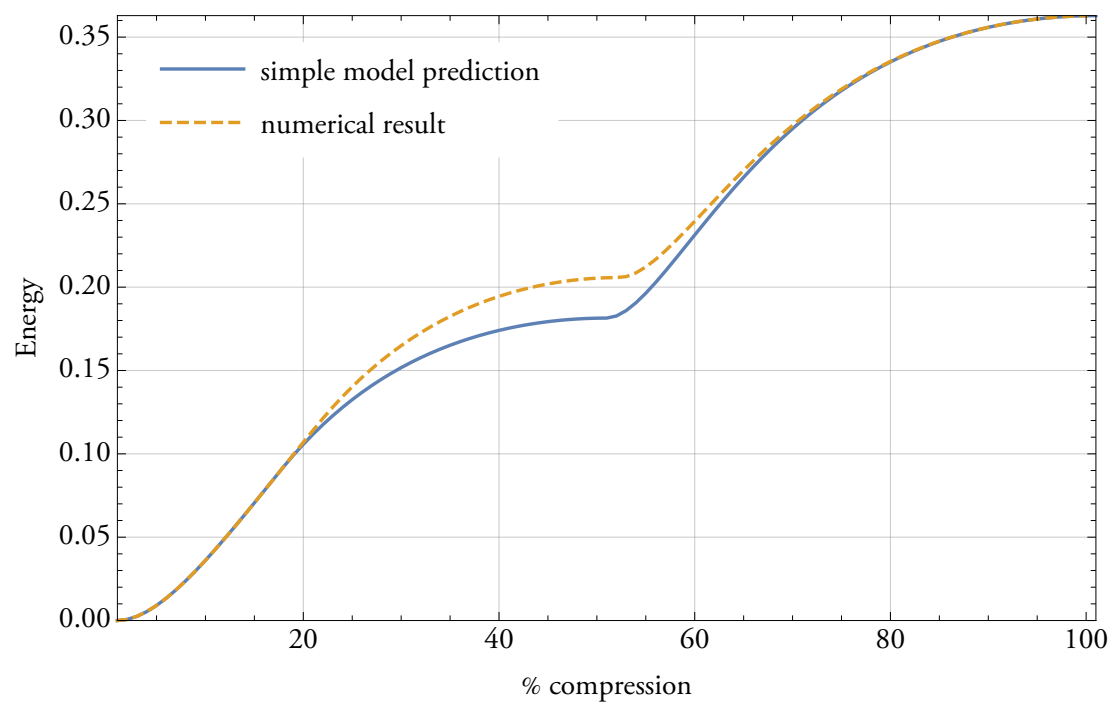

FIGURE A.109: simple energy for $r=1.8, n=8, m=2$

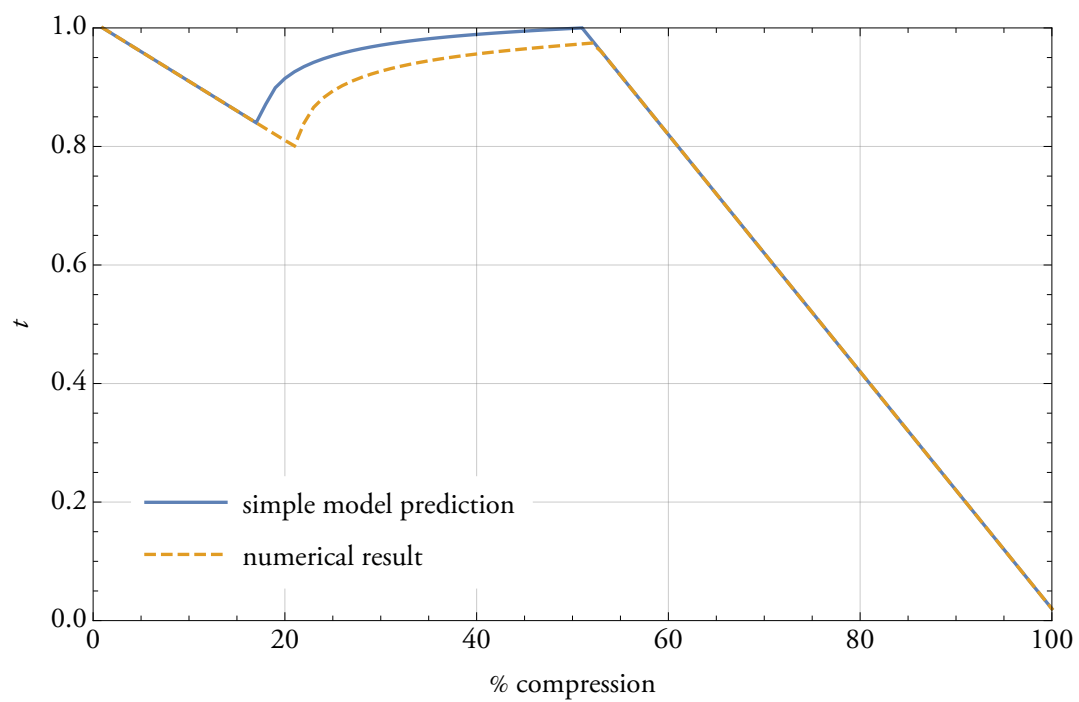

FIGURE A.110: intermediate layer height for $r=1.8, n=8, m=2$

$b_{1}$

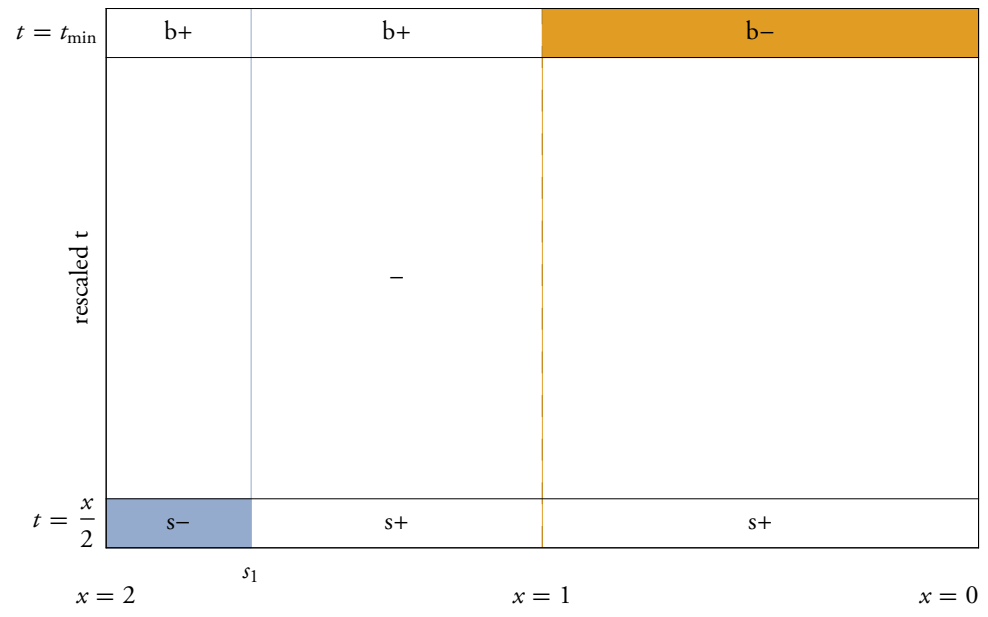

FIGURE A.111: prediction graphic for $r=1.8, n=8, m=2$ 


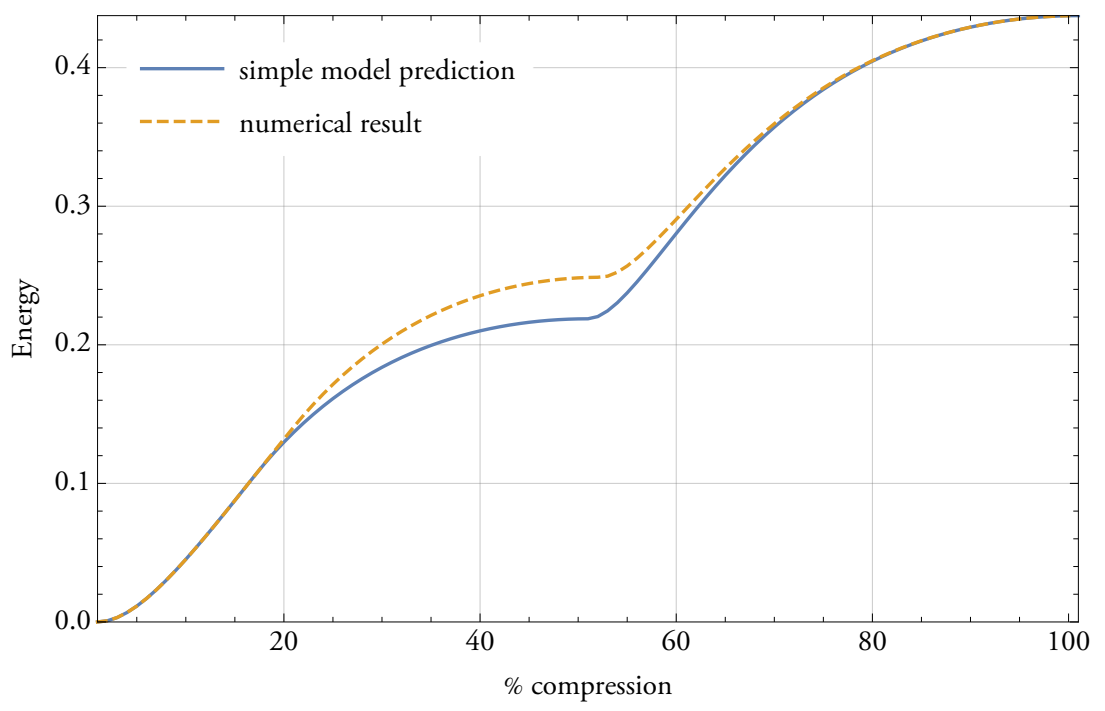

FIGURE A.112: simple energy for $r=1.8, n=9, m=2$

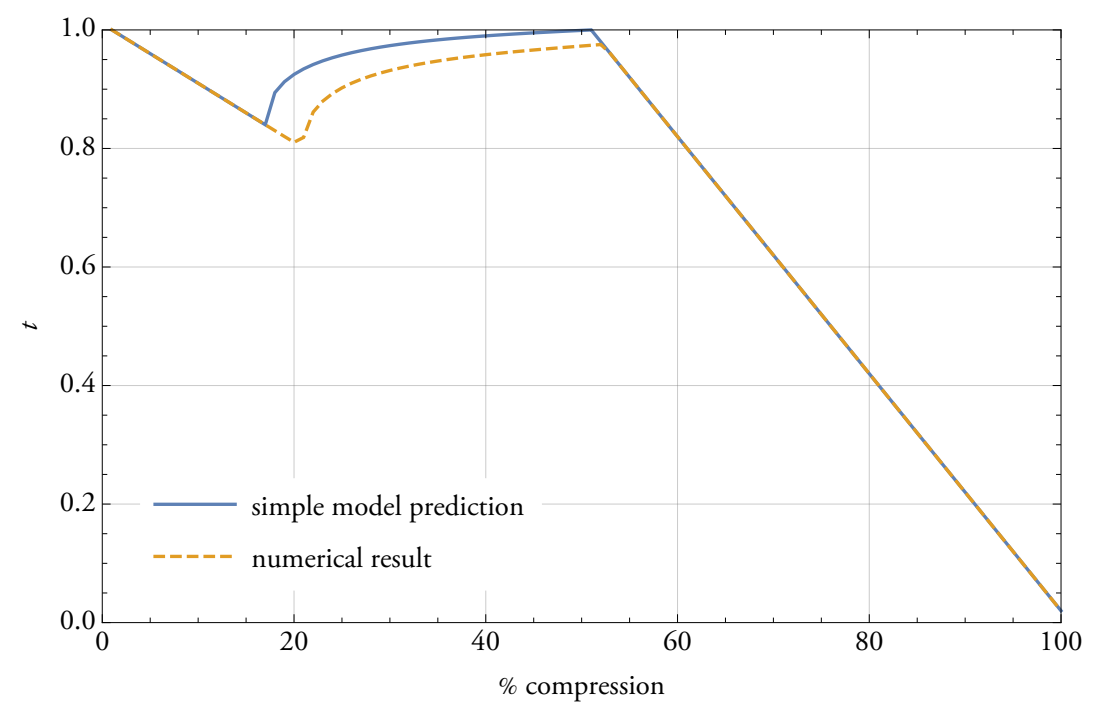

FIGURE A.113: intermediate layer height for $r=1.8, n=9, m=2$

$b_{1}$

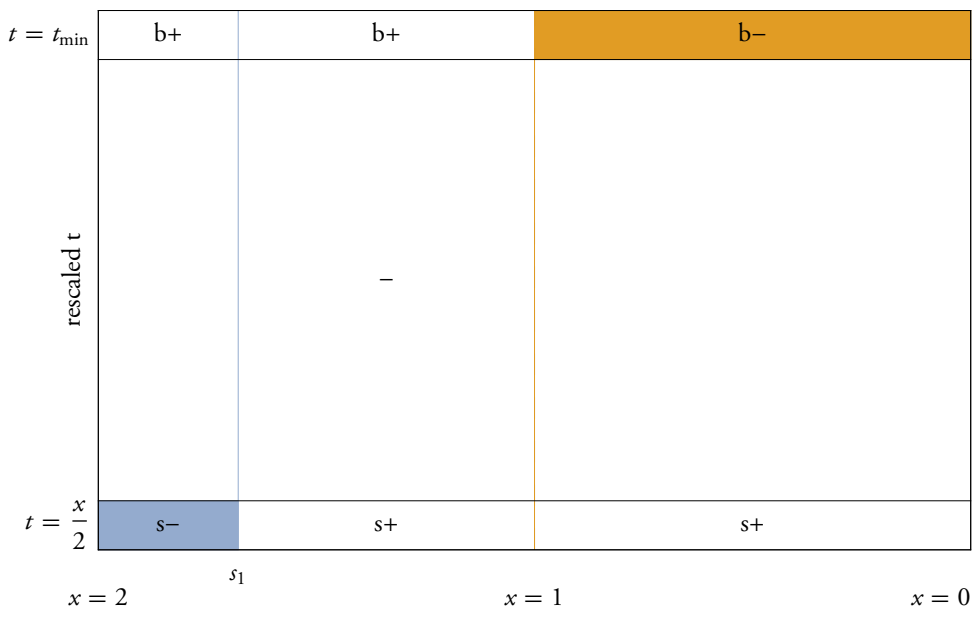

FIGURE A.114: prediction graphic for $r=1.8, n=9, m=2$ 


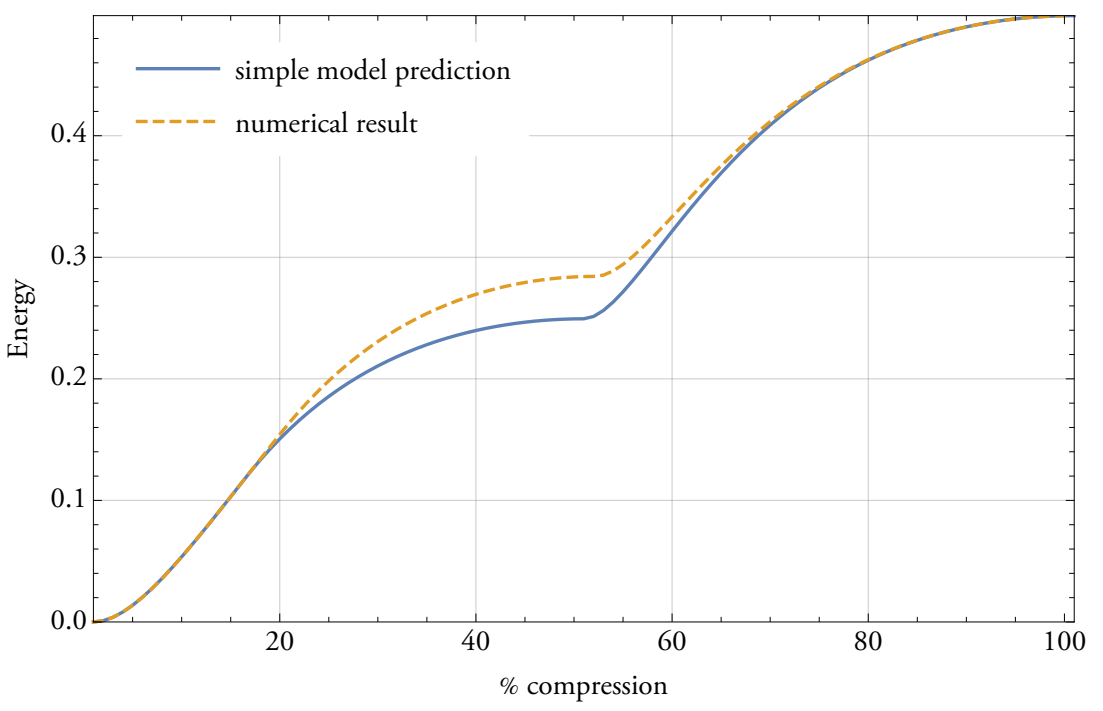

FIGURE A.115: simple energy for $r=1.8, n=10, m=2$

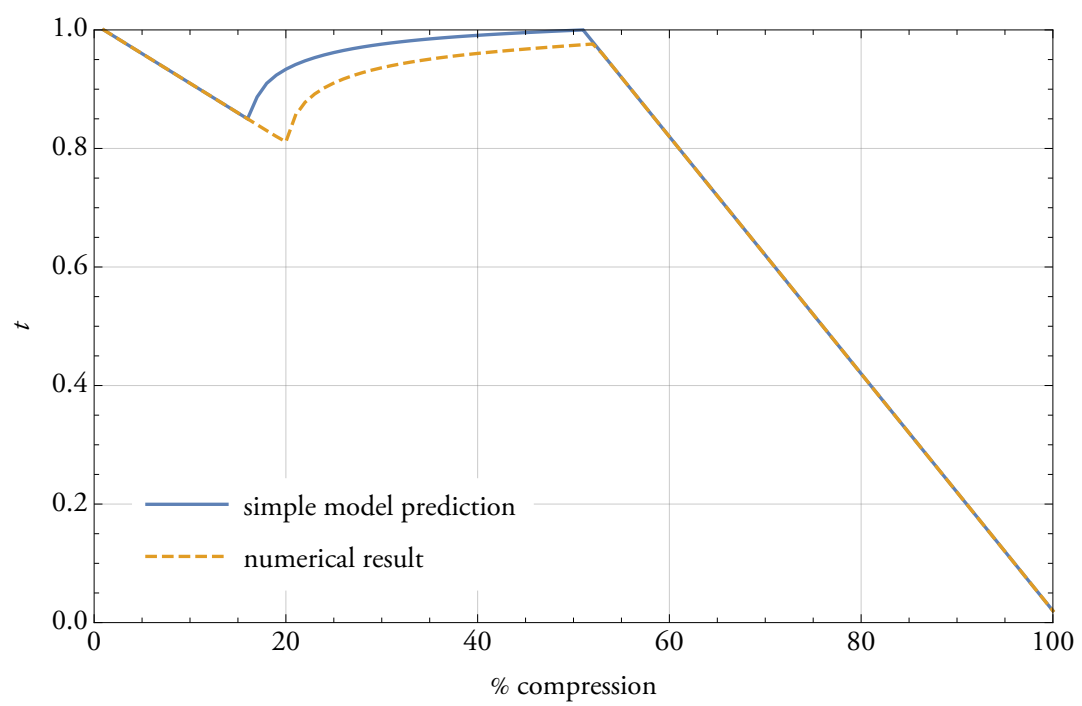

FIGURE A.116: intermediate layer height for $r=1.8, n=10, m=2$

$b_{1}$

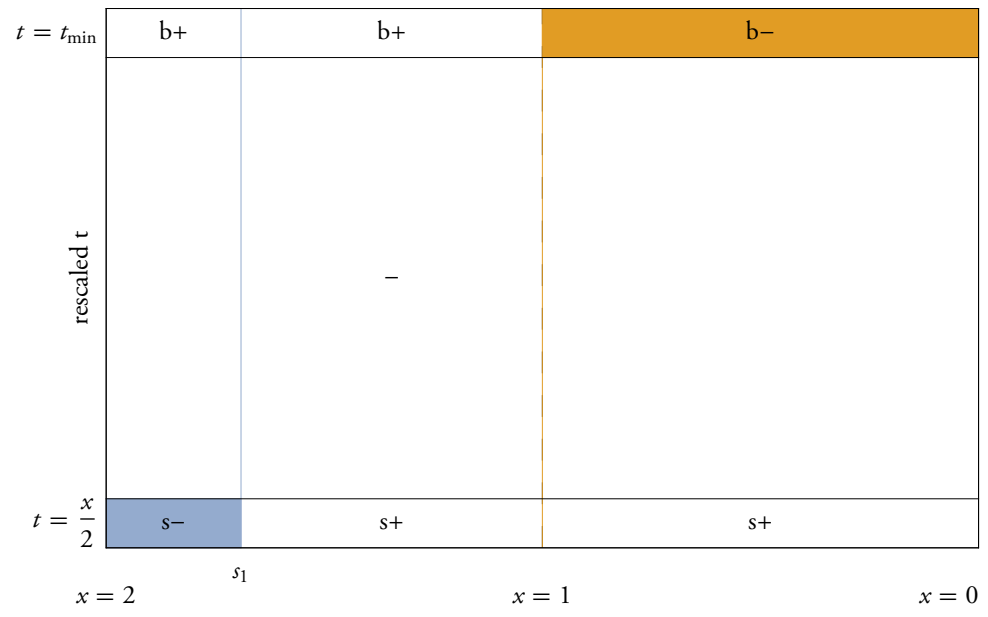

FIGURE A.117: prediction graphic for $r=1.8, n=10, m=2$ 


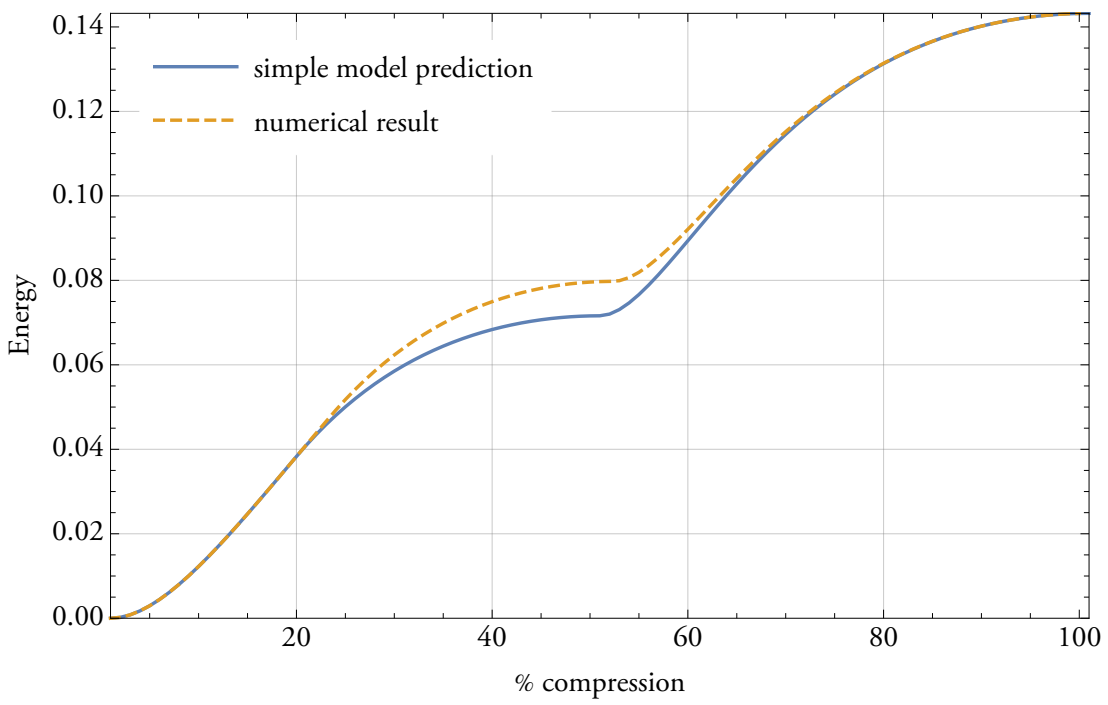

FIGURE A.118: simple energy for $r=2 ., n=5, m=2$

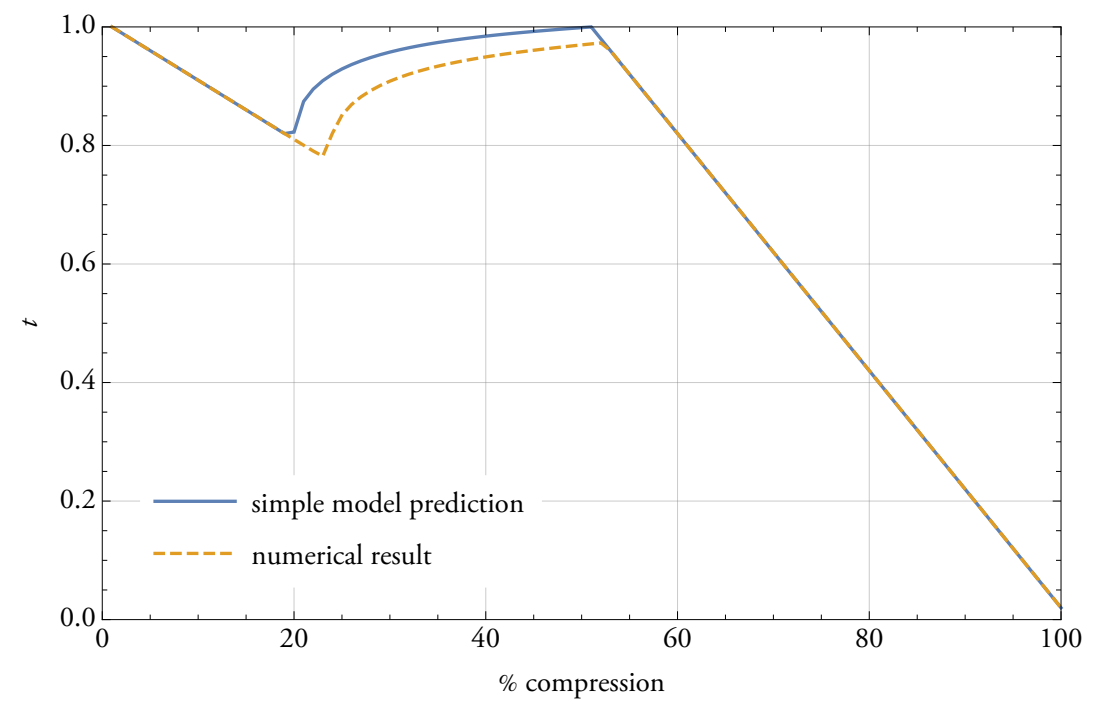

FIGURE A.119: intermediate layer height for $r=2 ., n=5, m=2$

$b_{1}$

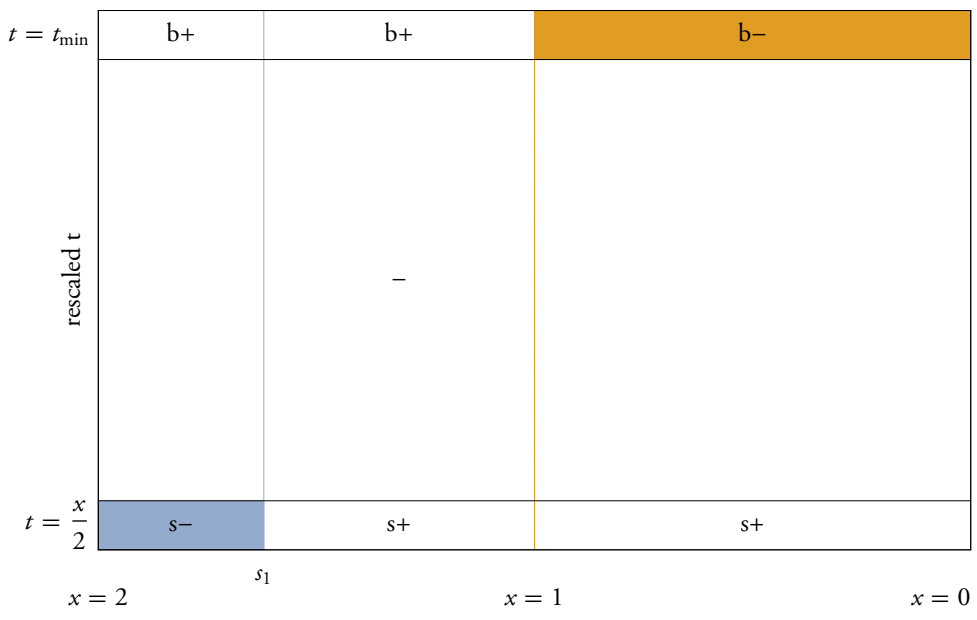

FIGURE A.120: prediction graphic for $r=2 ., n=5, m=2$ 


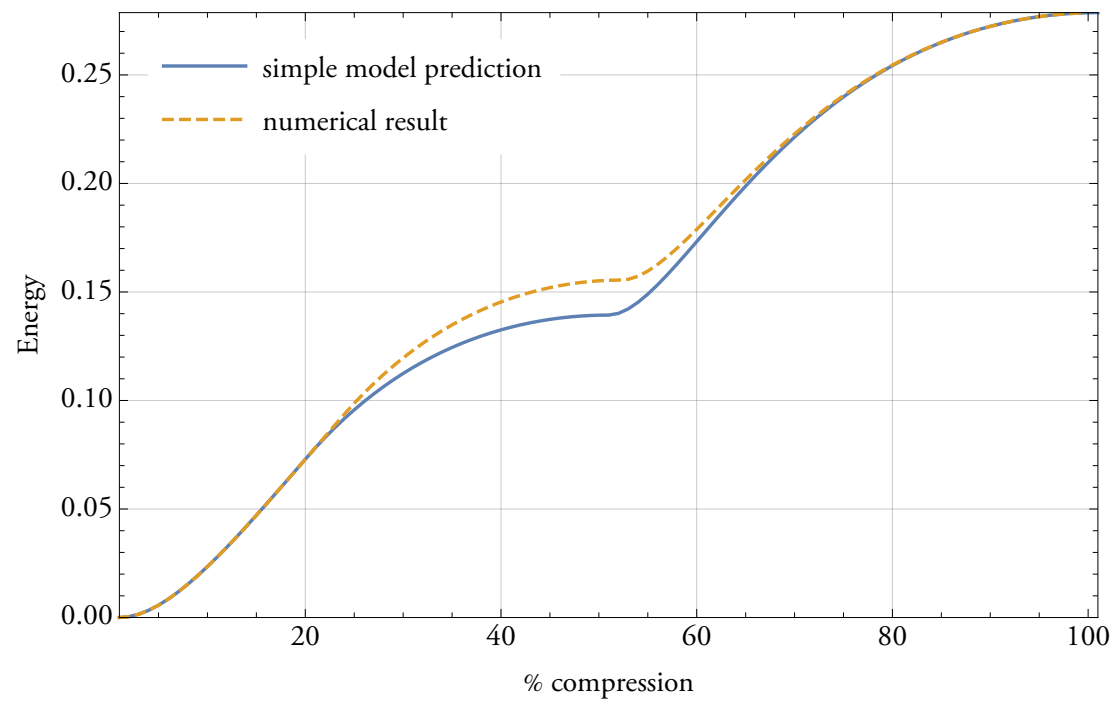

FIGURE A.121: simple energy for $r=2 ., n=6, m=2$

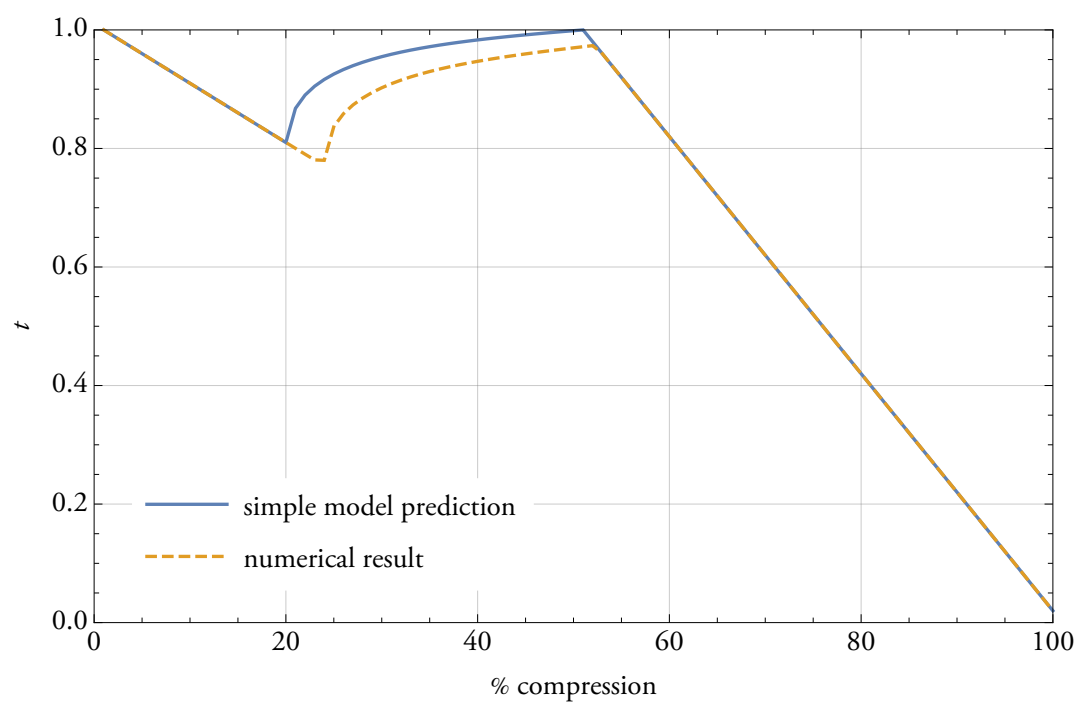

FIGURE A.122: intermediate layer height for $r=2 ., n=6, m=2$

$b_{1}$

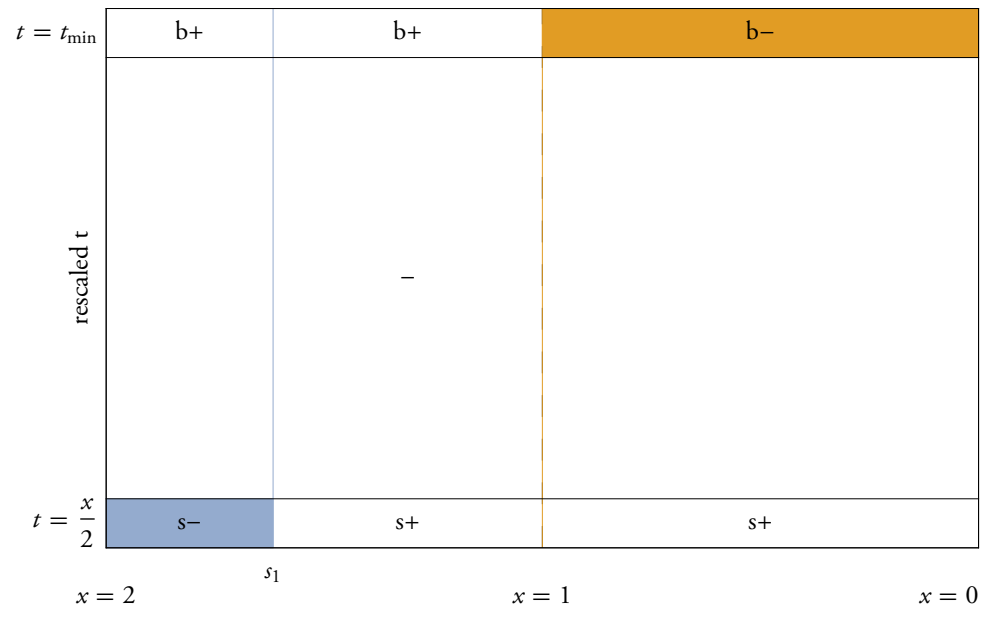

FIGURE A.123: prediction graphic for $r=2 ., n=6, m=2$ 


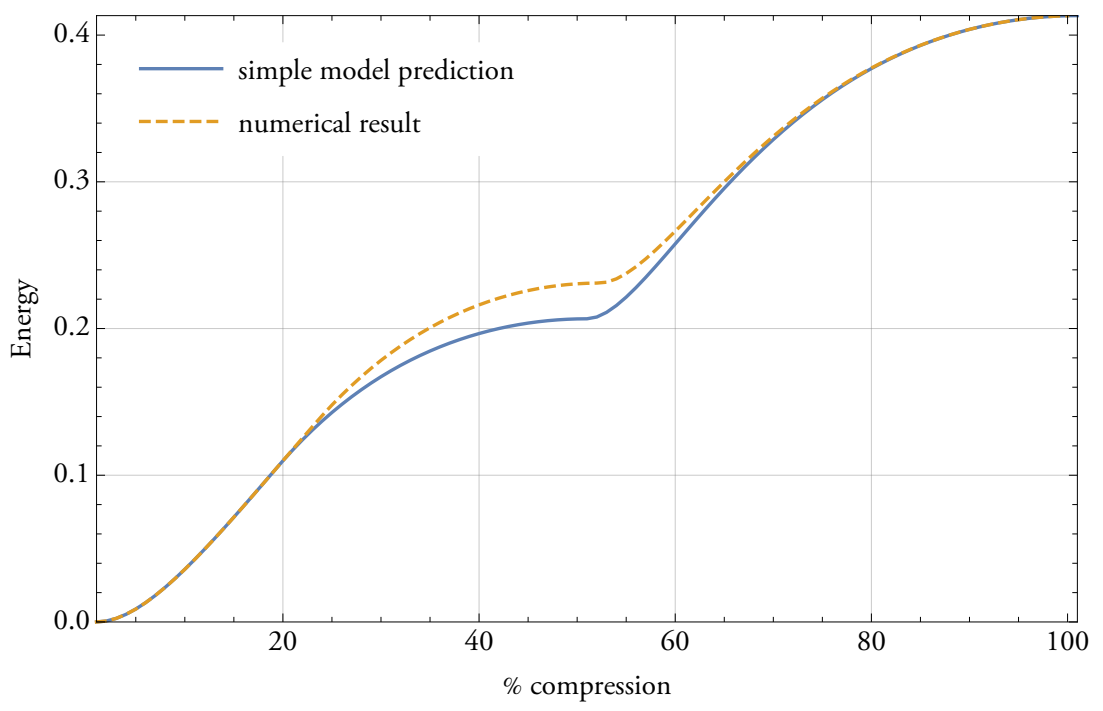

FIGURE A.124: simple energy for $r=2 ., n=7, m=2$

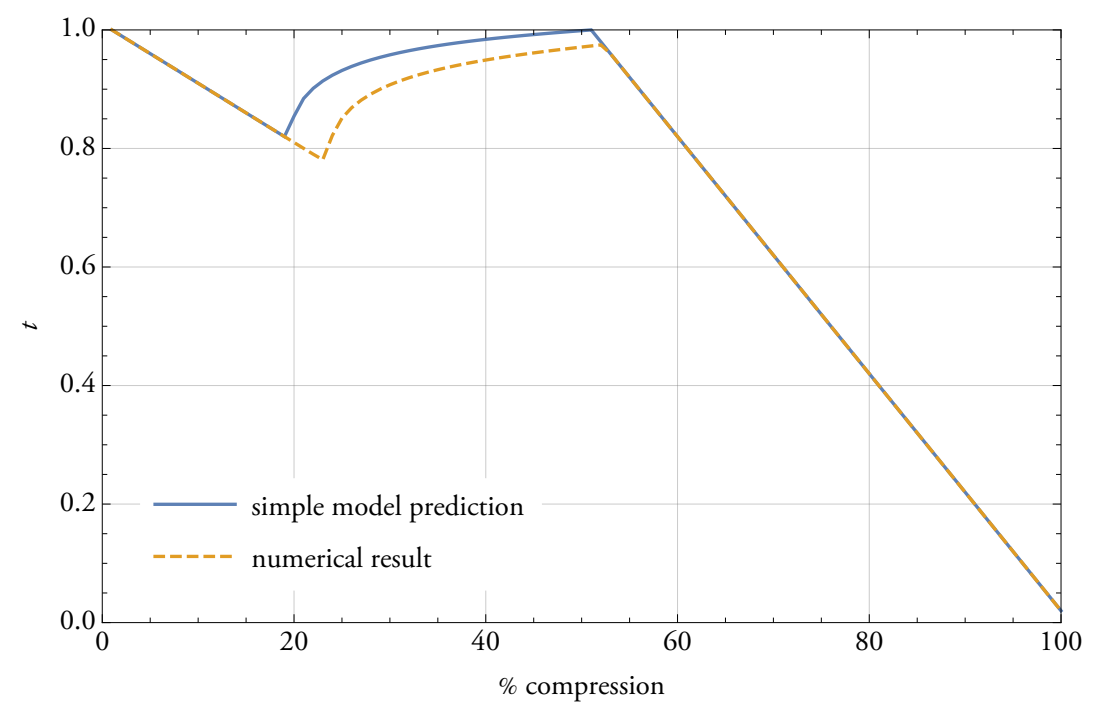

FIGURE A.125: intermediate layer height for $r=2 ., n=7, m=2$

$b_{1}$

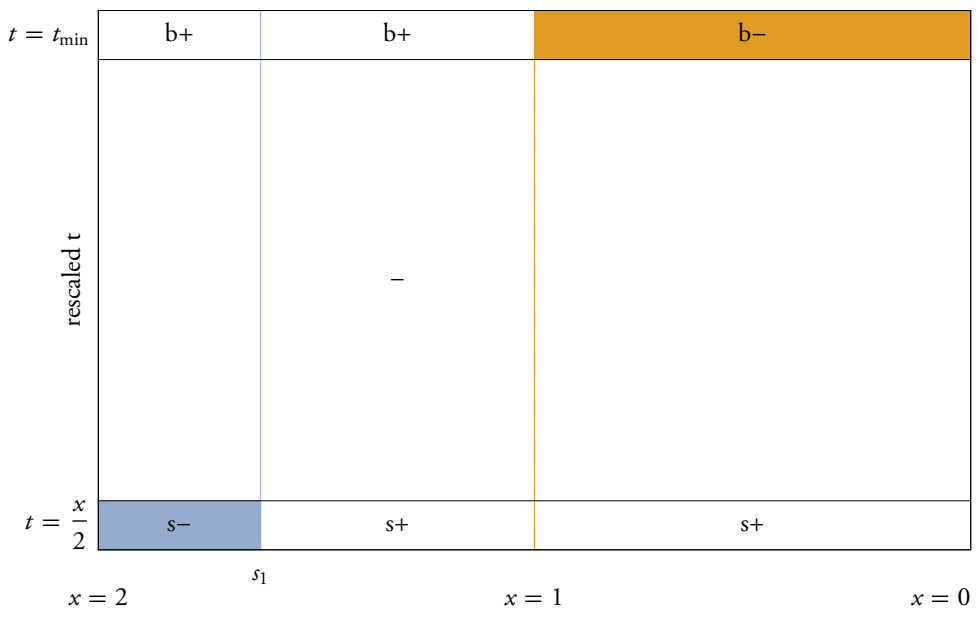

FIGURE A.126: prediction graphic for $r=2 ., n=7, m=2$ 


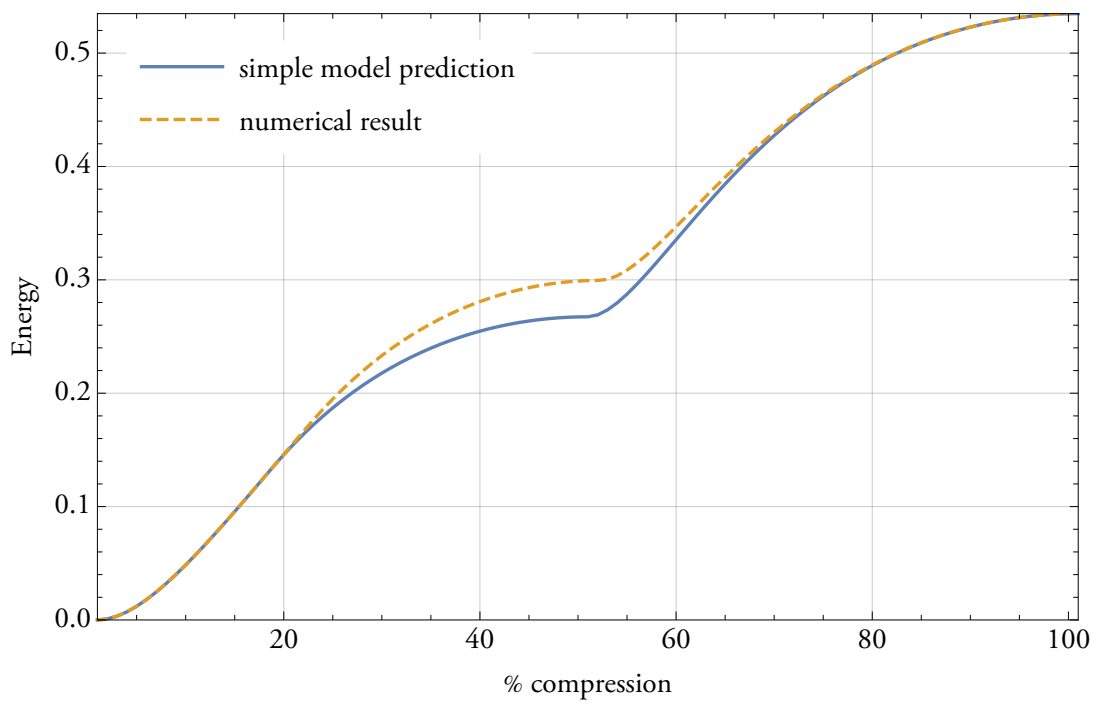

FIGURE A.127: simple energy for $r=2 ., n=8, m=2$

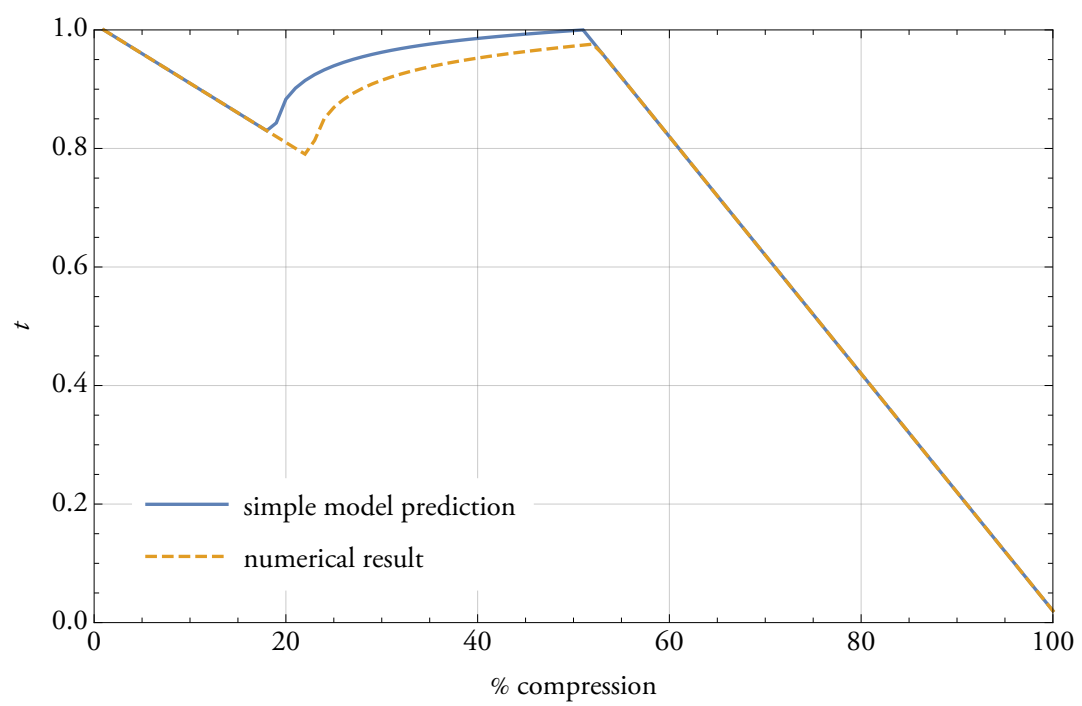

FIGURE A.128: intermediate layer height for $r=2 ., n=8, m=2$

$b_{1}$

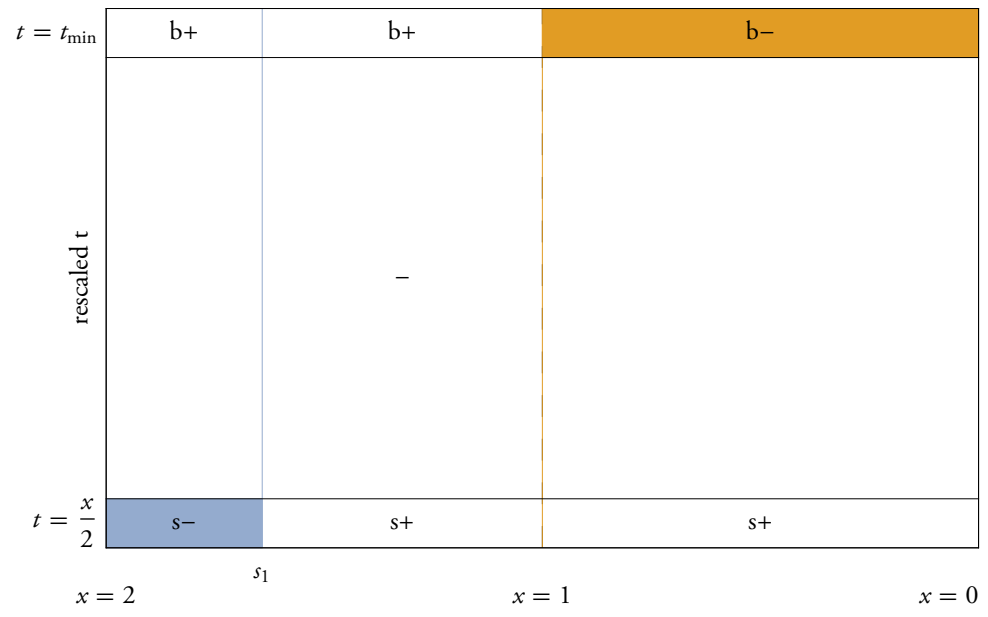

FIGURE A.129: prediction graphic for $r=2 ., n=8, m=2$ 


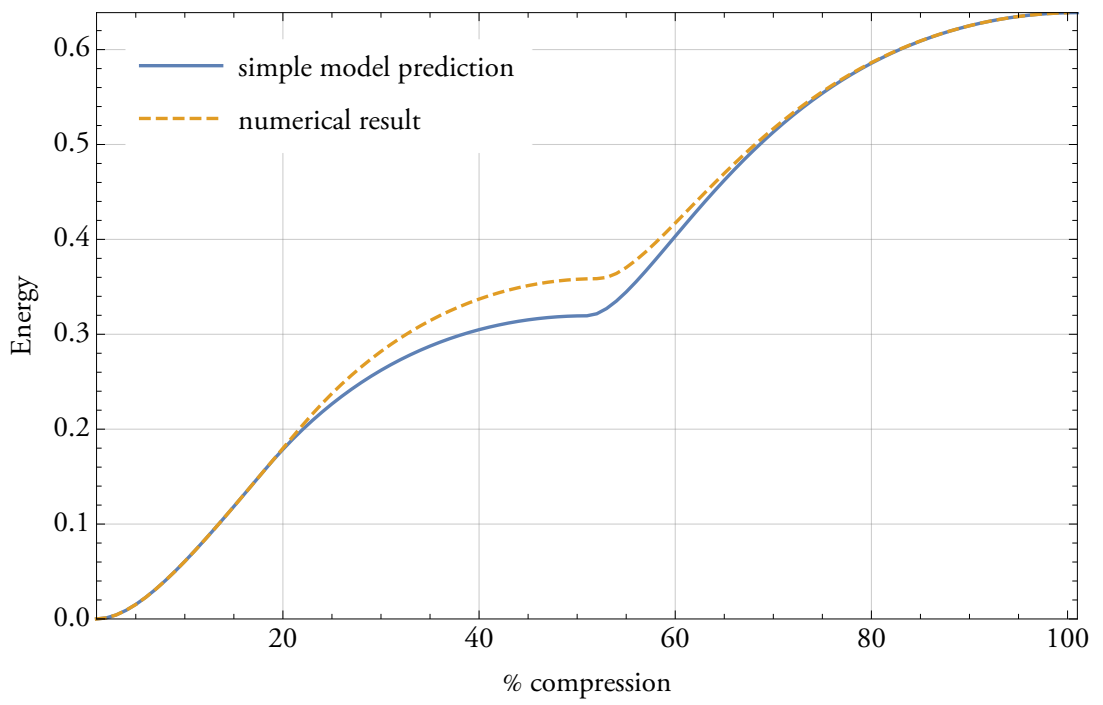

FIGURE A.130: simple energy for $r=2 ., n=9, m=2$

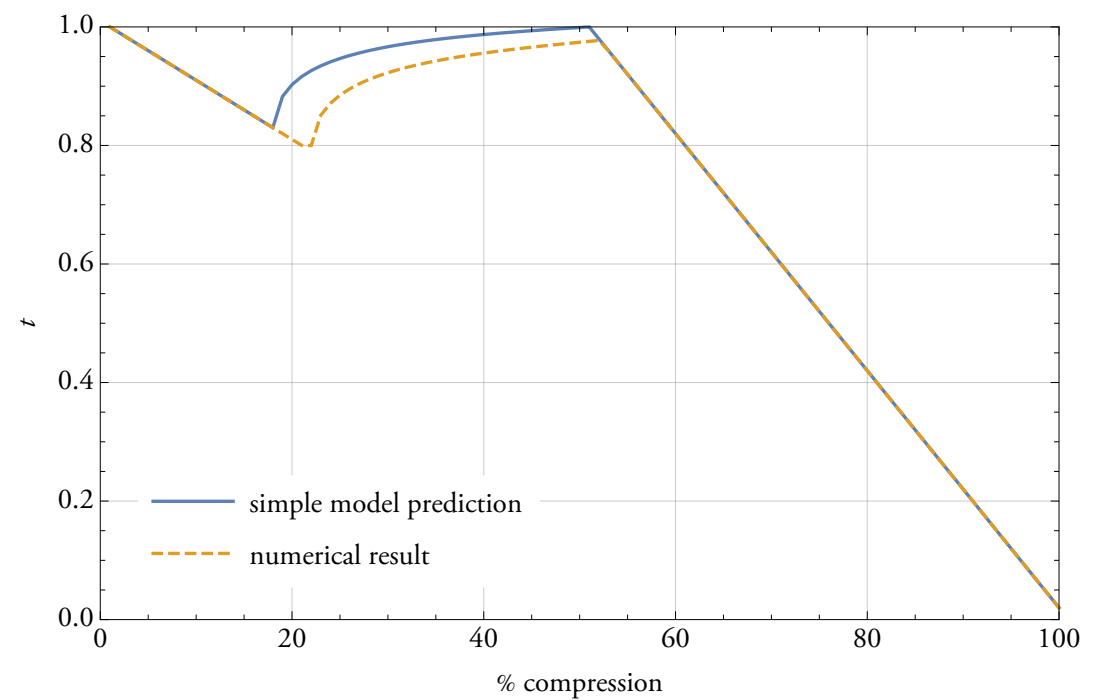

FIGURE A.131: intermediate layer height for $r=2 ., n=9, m=2$

$b_{1}$

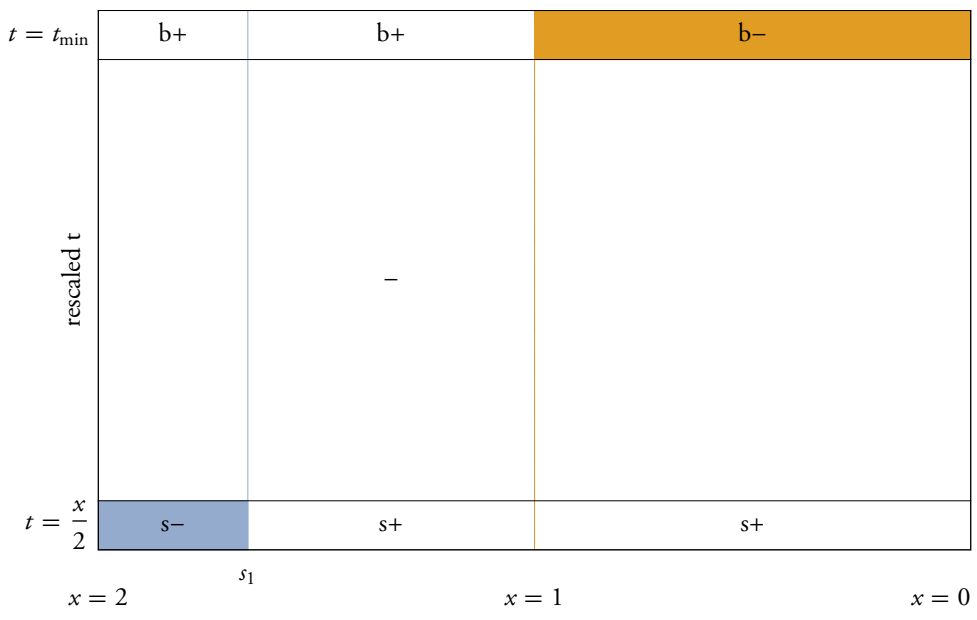

FIGURE A.132: prediction graphic for $r=2 ., n=9, m=2$ 


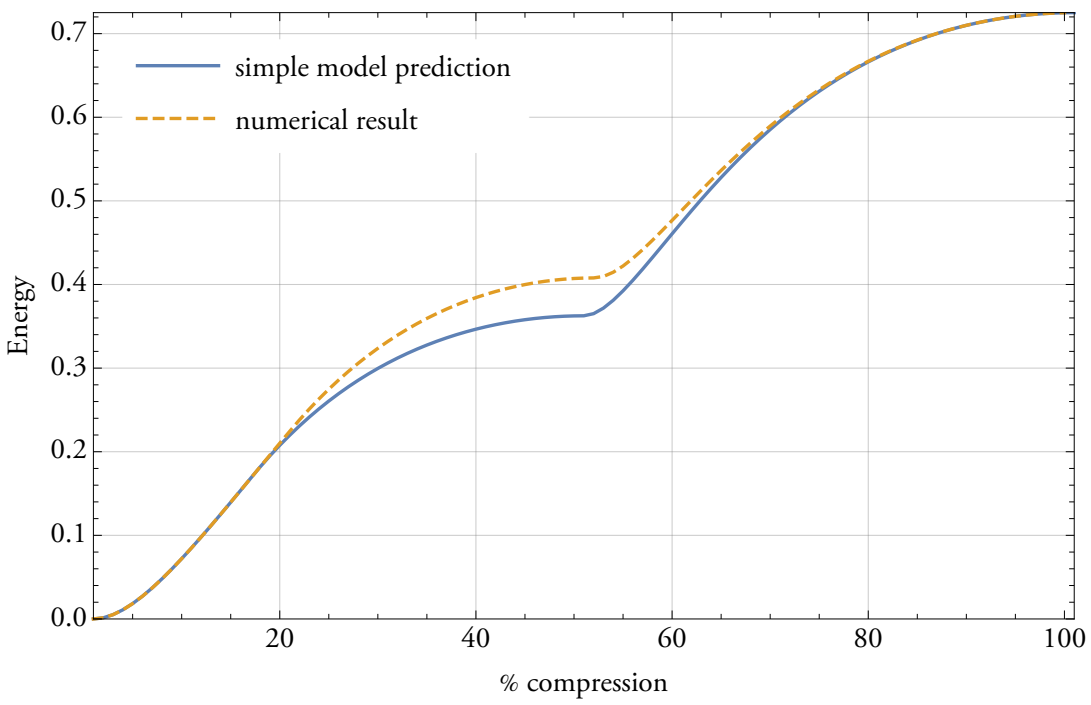

FIGURE A.133: simple energy for $r=2 ., n=10, m=2$

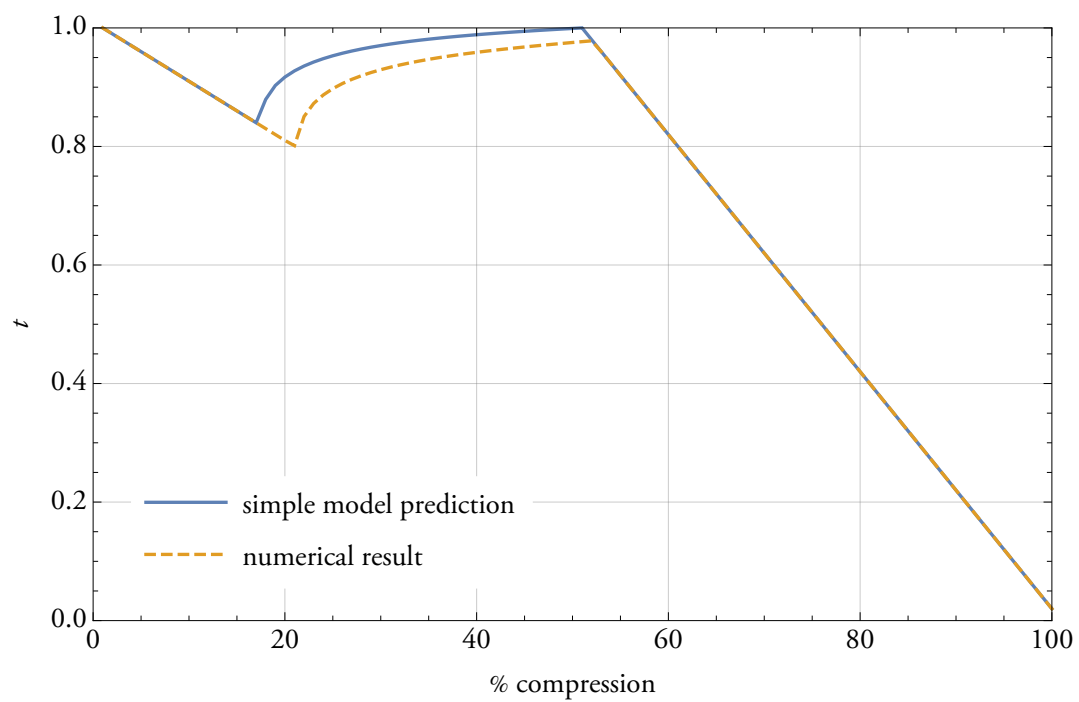

FIGURE A.134: intermediate layer height for $r=2 ., n=10, m=2$

$b_{1}$

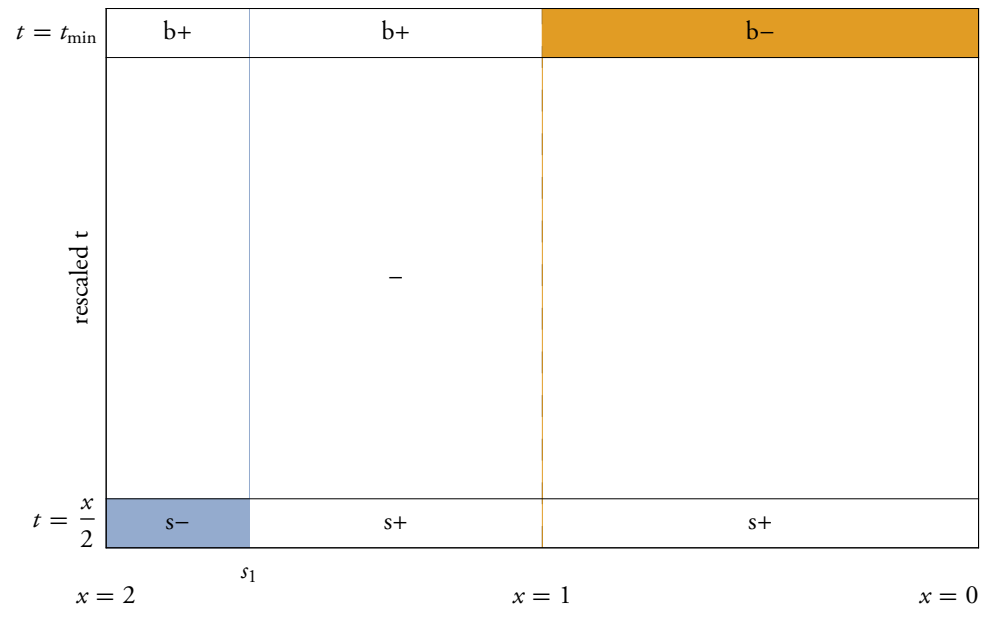

FIGURE A.135: prediction graphic for $r=2 ., n=10, m=2$ 


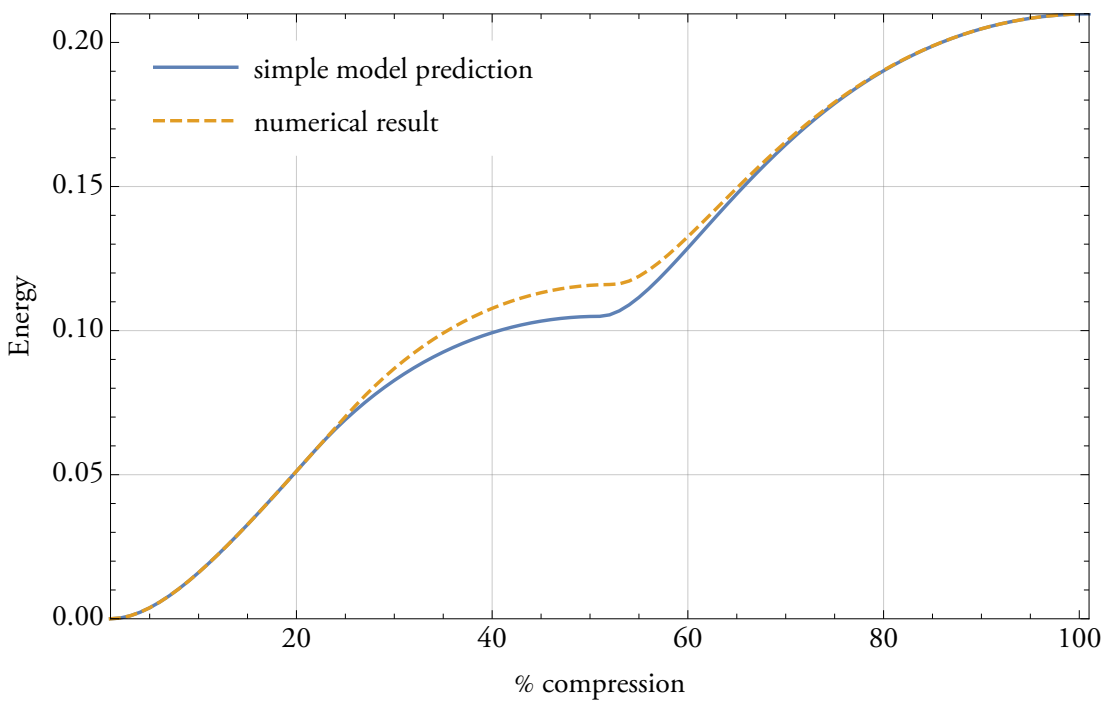

FIGURE A.136: simple energy for $r=2.2, n=5, m=2$

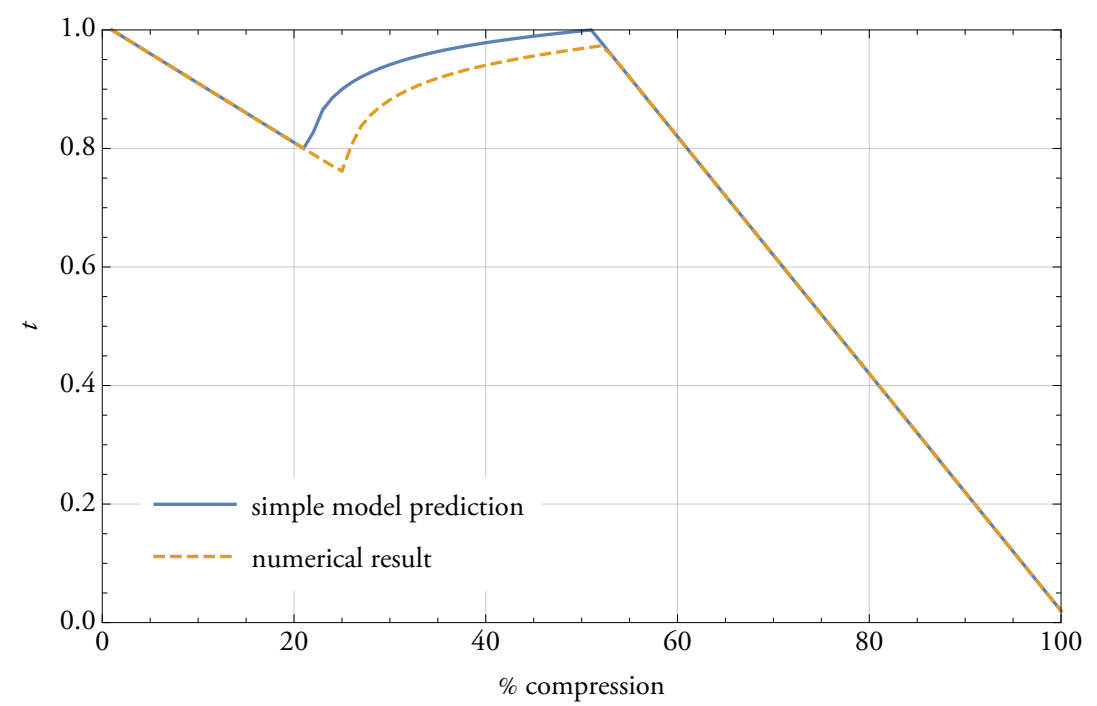

FIGURE A.137: intermediate layer height for $r=2.2, n=5, m=2$

$b_{1}$

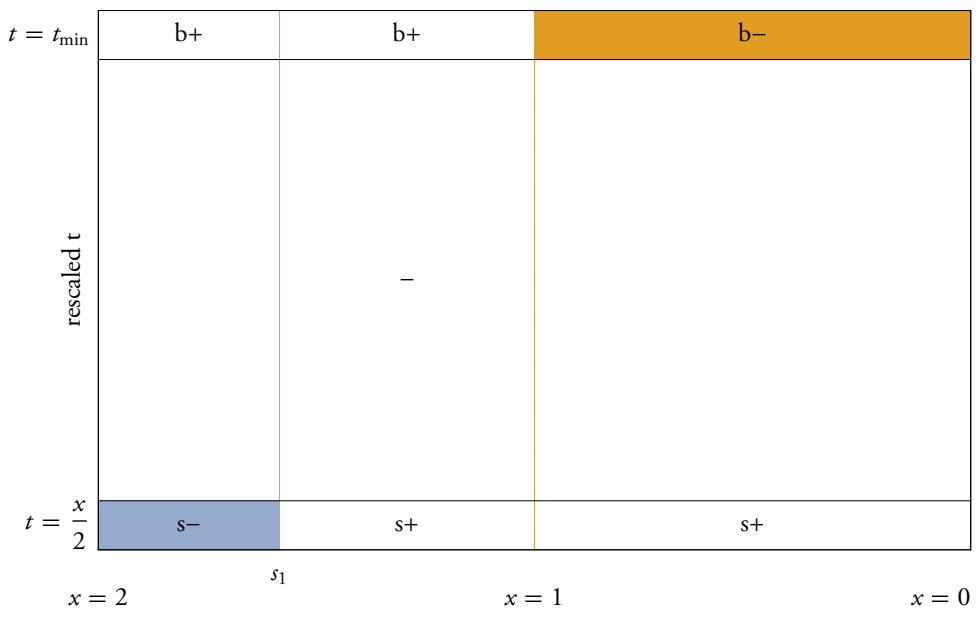

FIGURE A.138: prediction graphic for $r=2.2, n=5, m=2$ 


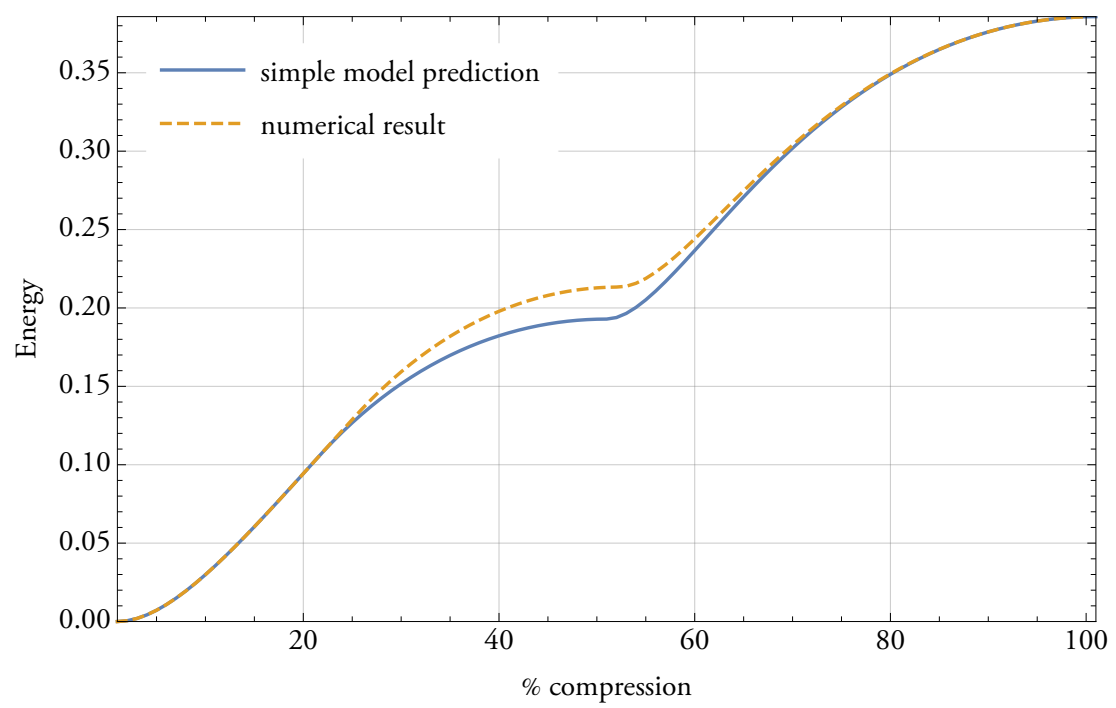

FIGURE A.139: simple energy for $r=2.2, n=6, m=2$

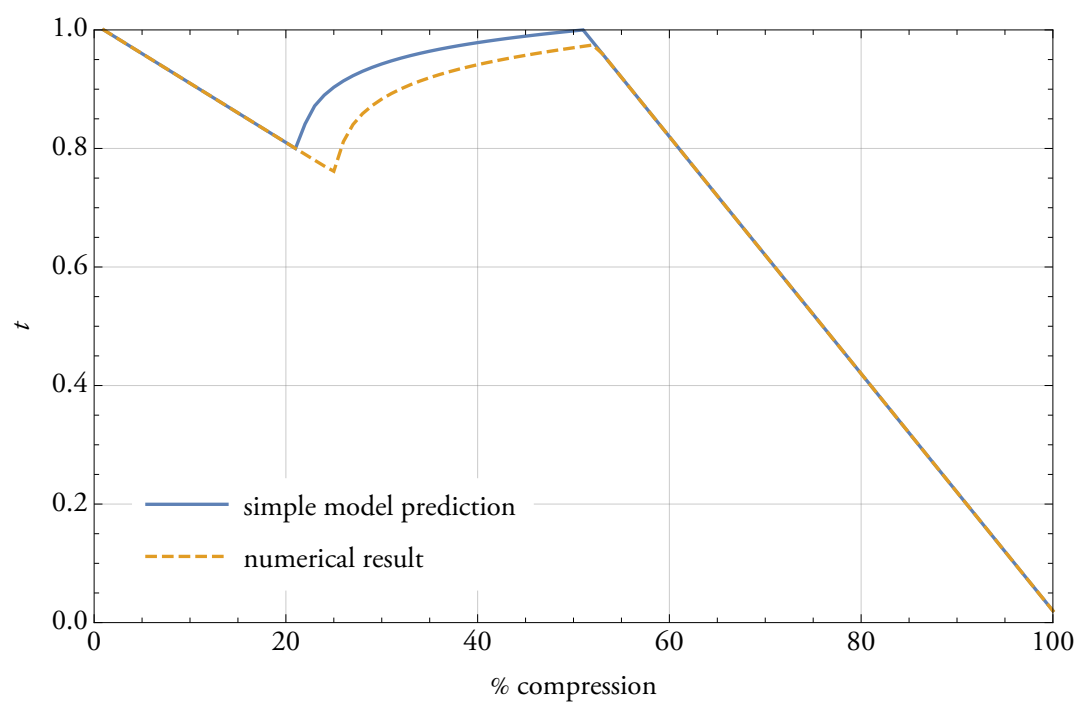

FIGURE A.140: intermediate layer height for $r=2.2, n=6, m=2$

$b_{1}$

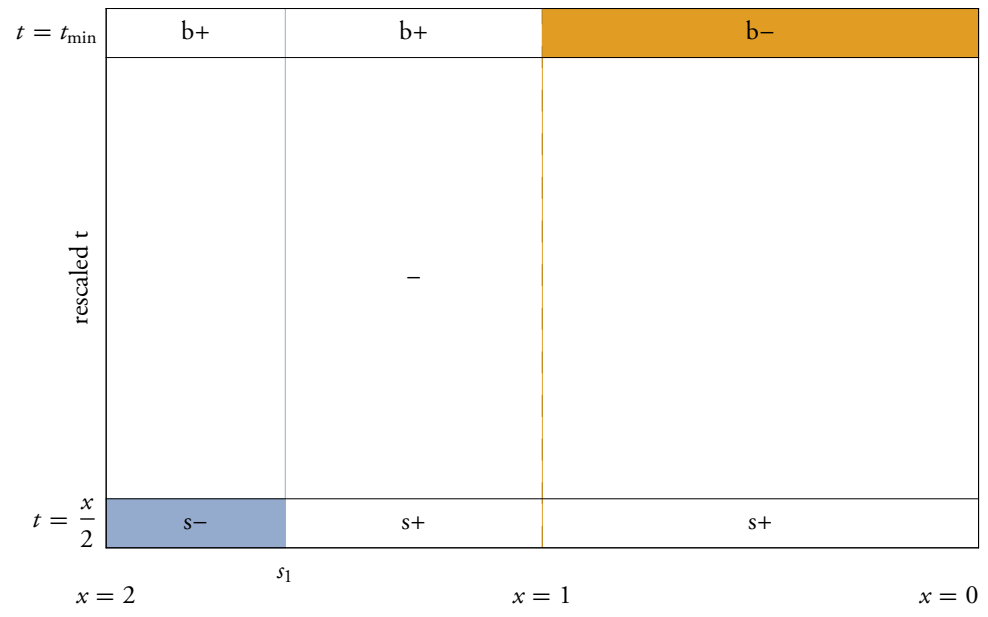

FIGURE A.141: prediction graphic for $r=2.2, n=6, m=2$ 


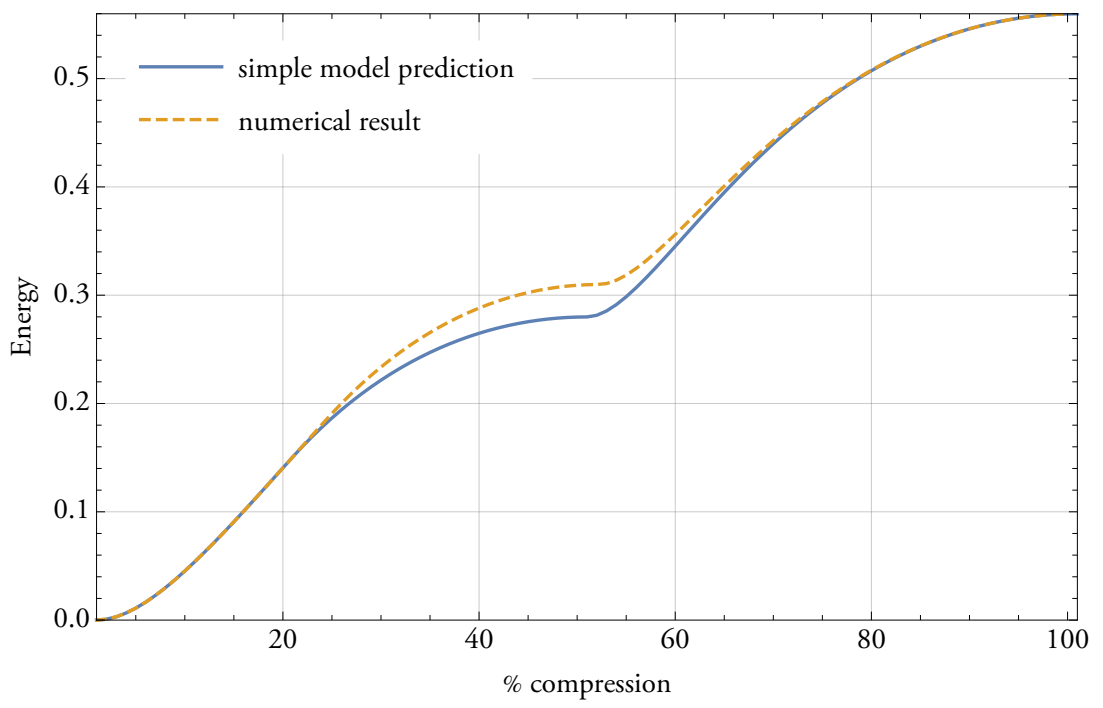

FIGURE A.142: simple energy for $r=2.2, n=7, m=2$

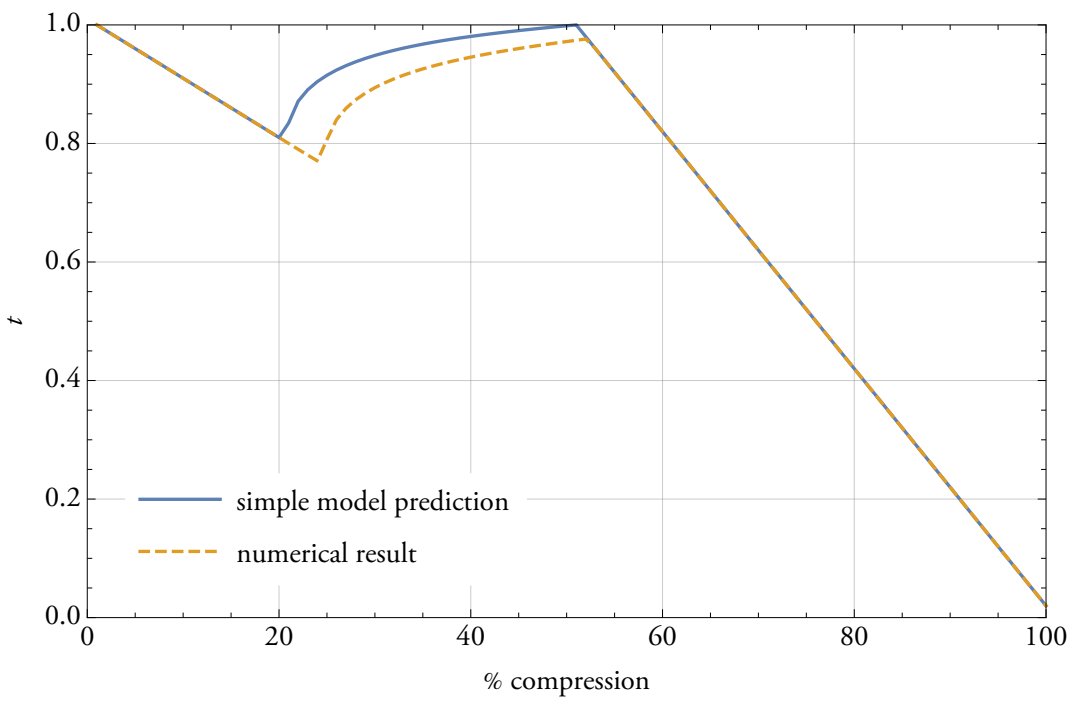

FIGURE A.143: intermediate layer height for $r=2.2, n=7, m=2$

$b_{1}$

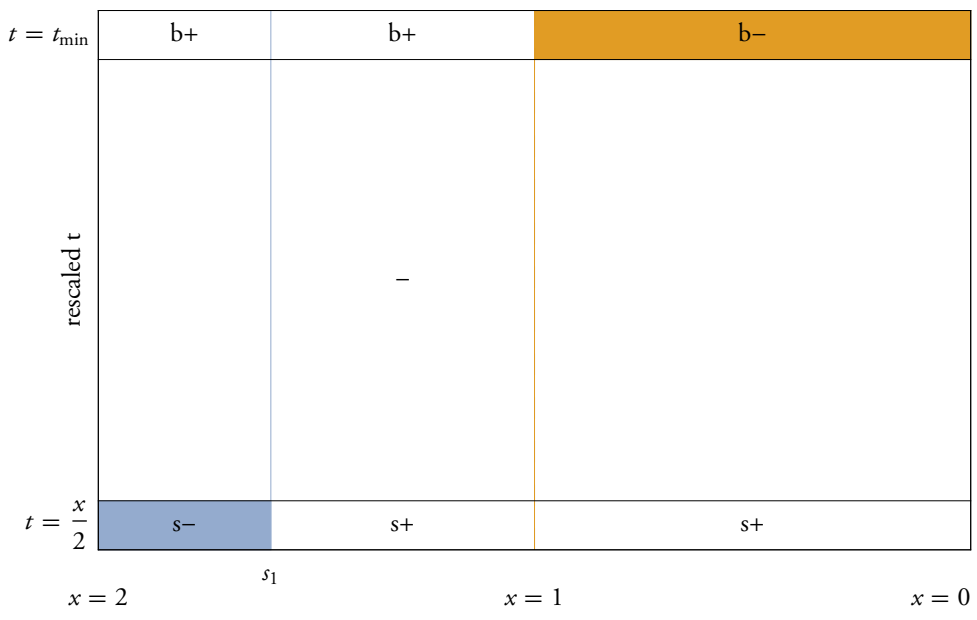

FIGURE A.144: prediction graphic for $r=2.2, n=7, m=2$ 


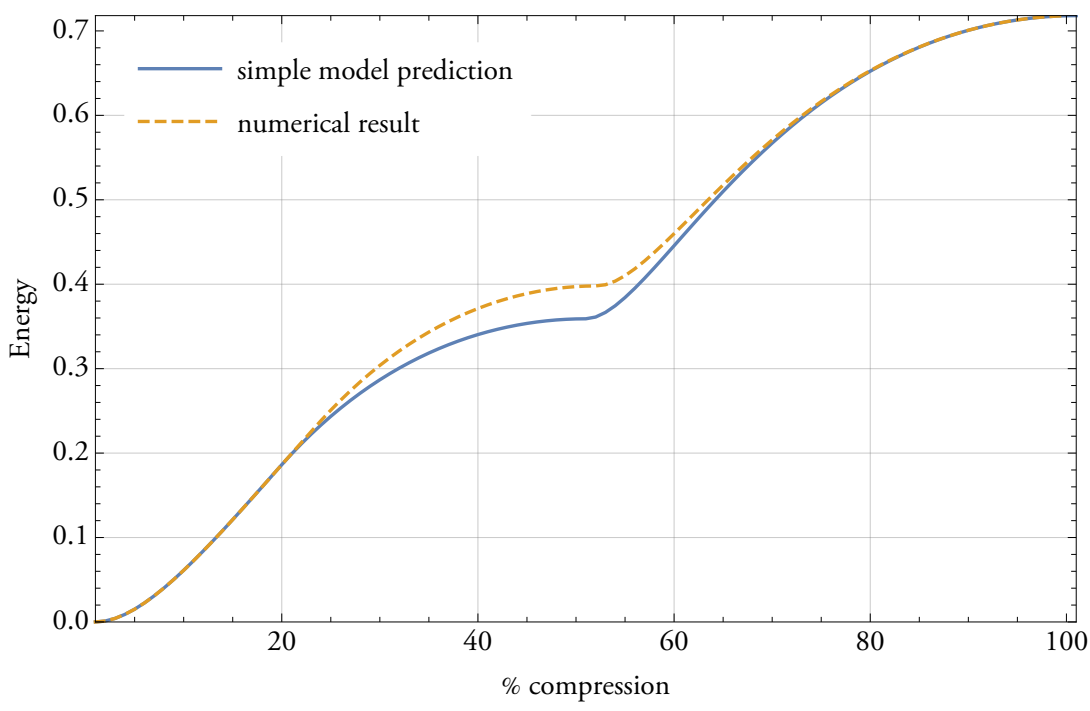

FIGURE A.145: simple energy for $r=2.2, n=8, m=2$

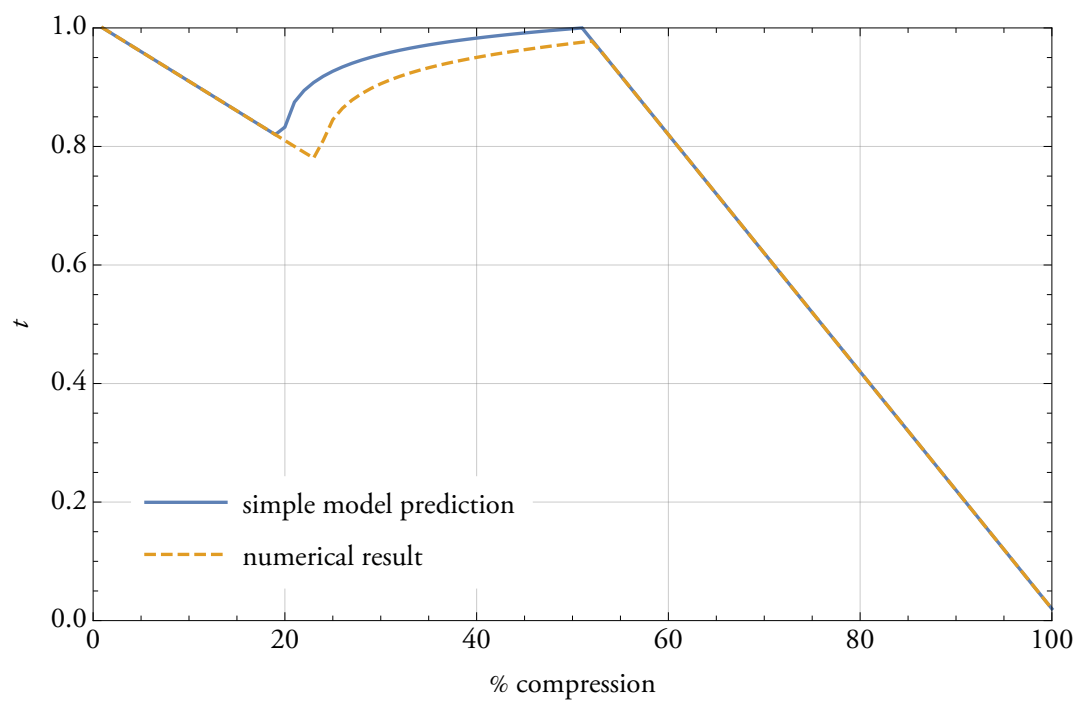

FIGURE A.146: intermediate layer height for $r=2.2, n=8, m=2$

$b_{1}$

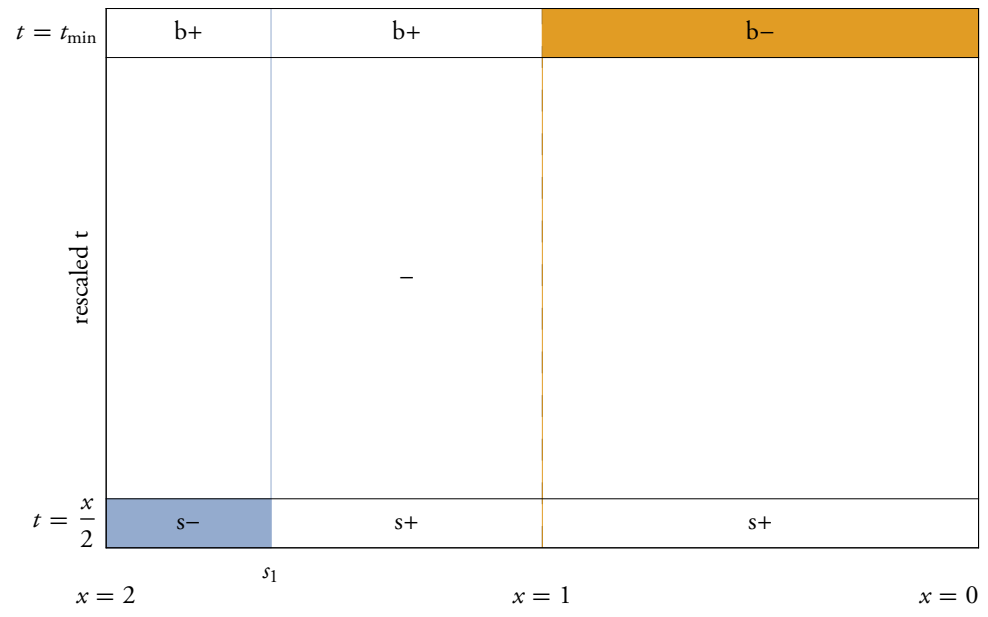

FIGURE A.147: prediction graphic for $r=2.2, n=8, m=2$ 


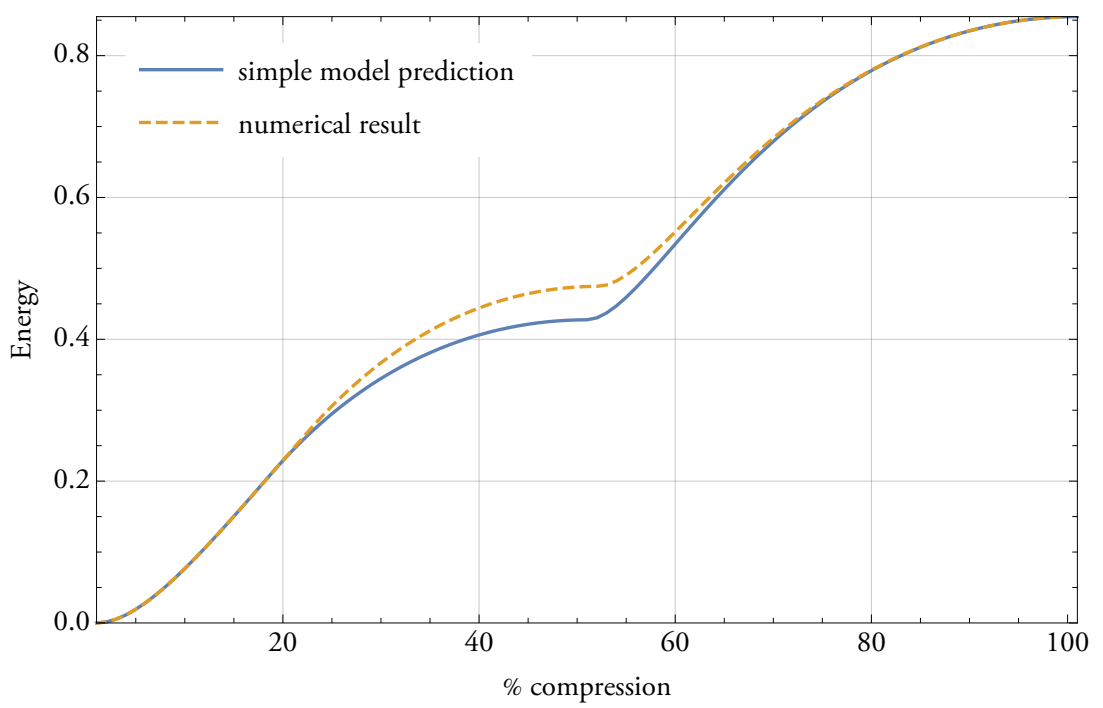

FIGURE A.148: simple energy for $r=2.2, n=9, m=2$

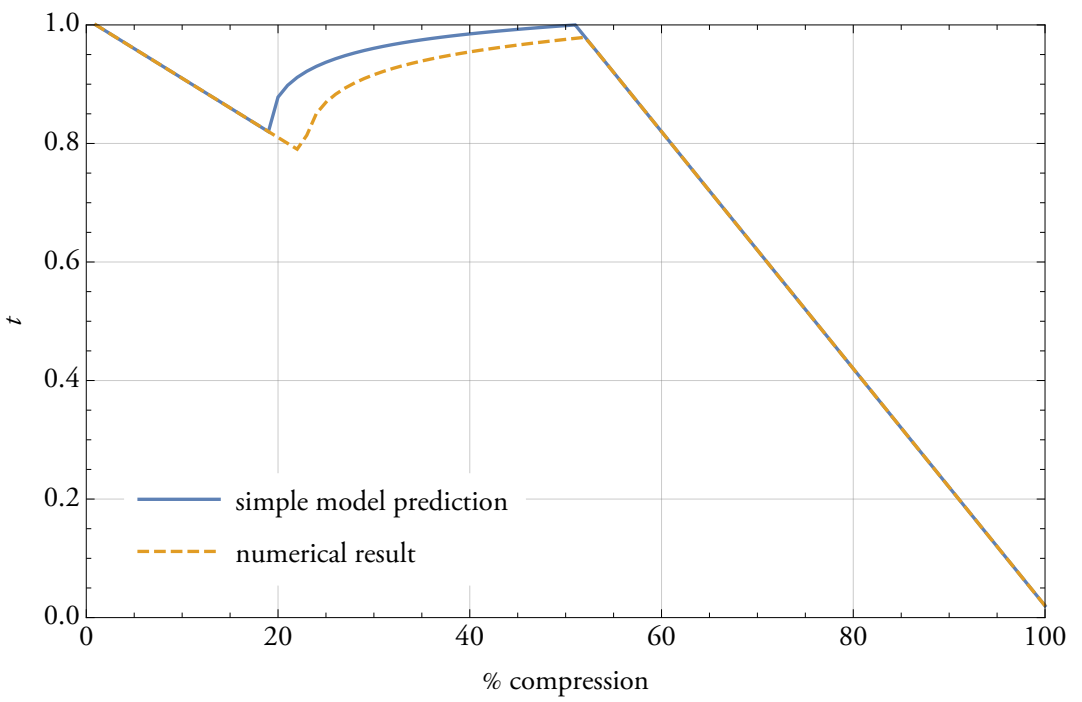

FIGURE A.149: intermediate layer height for $r=2.2, n=9, m=2$

$b_{1}$

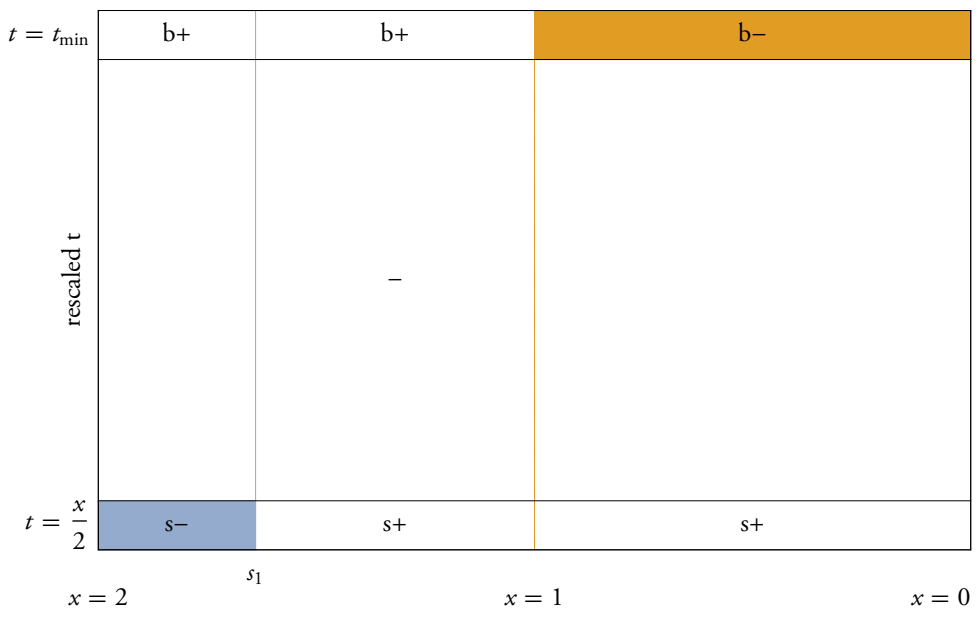

FIGURE A.150: prediction graphic for $r=2.2, n=9, m=2$ 


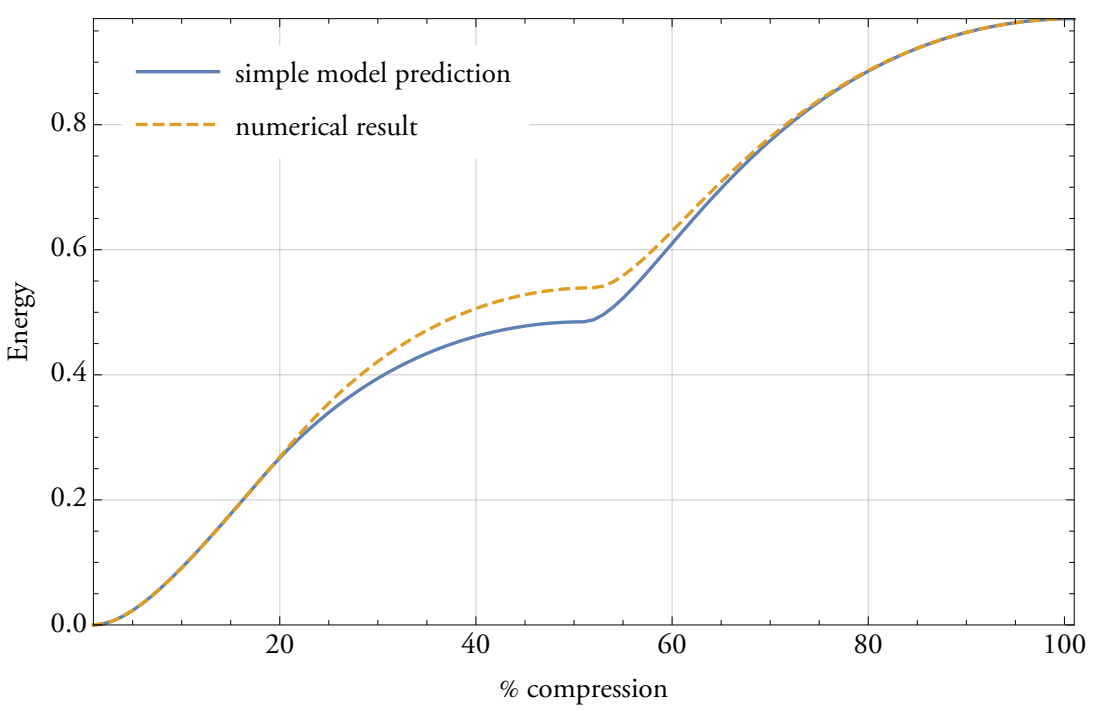

FIGURE A.151: simple energy for $r=2.2, n=10, m=2$

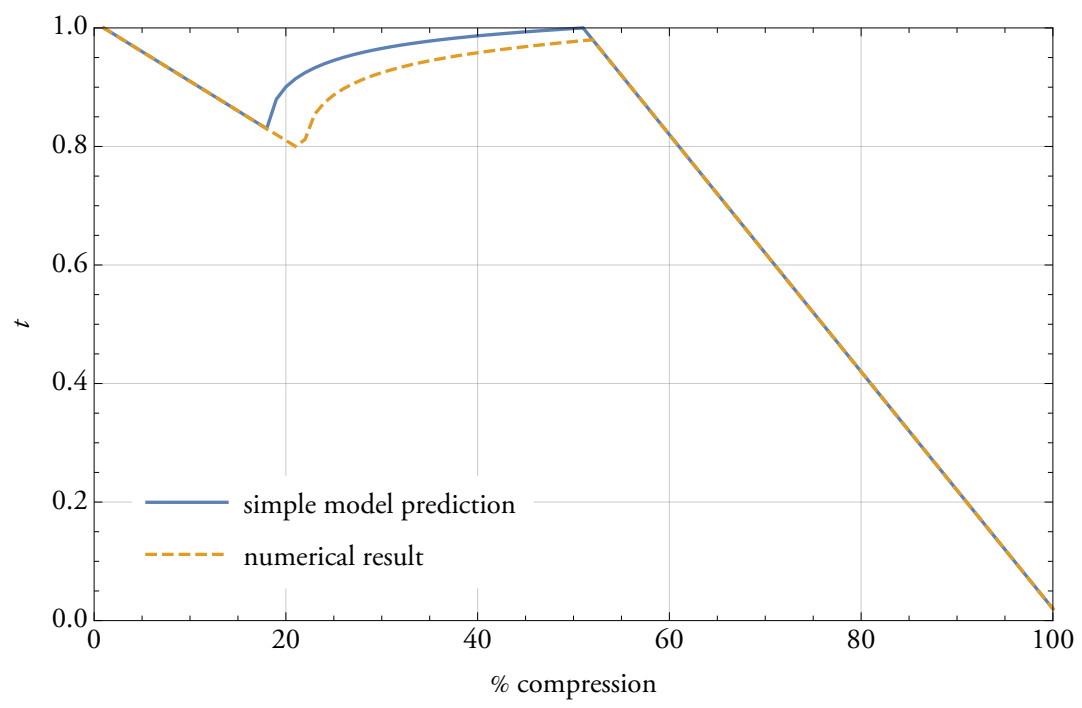

FIGURE A.152: intermediate layer height for $r=2.2, n=10, m=2$

$b_{1}$

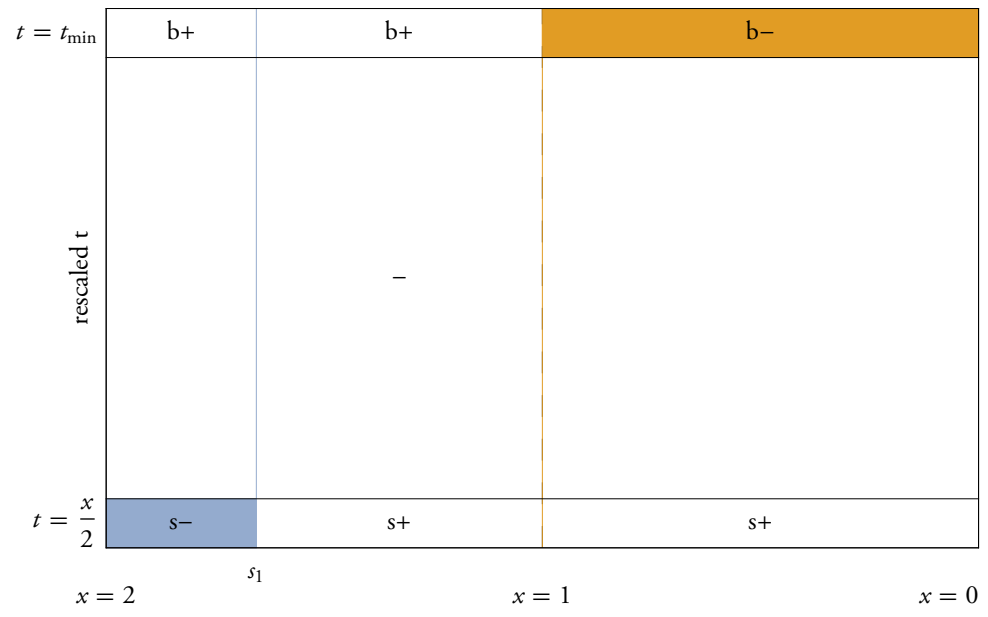

FIGURE A.153: prediction graphic for $r=2.2, n=10, m=2$ 


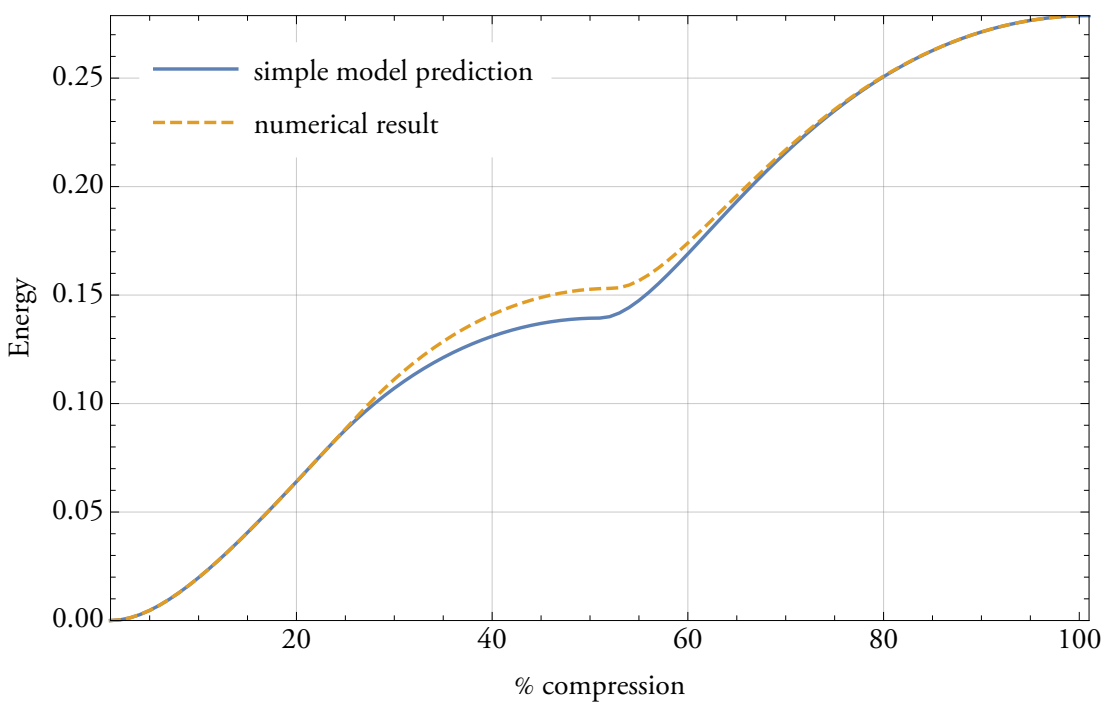

FIGURE A.154: simple energy for $r=2.4, n=5, m=2$

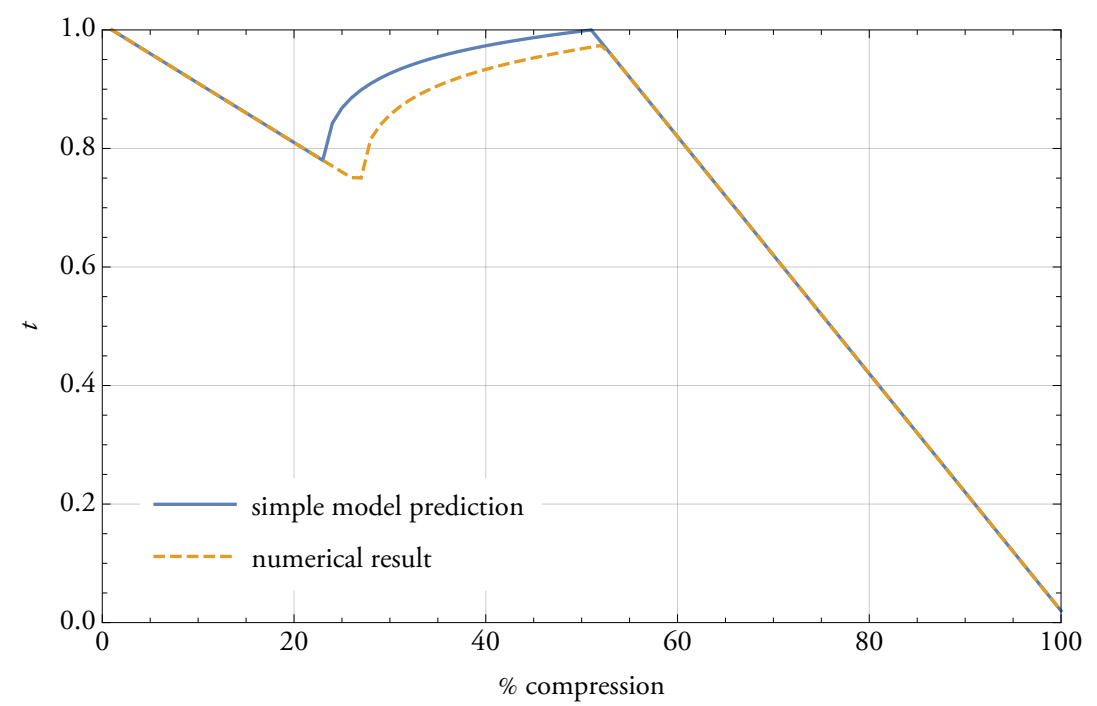

FIGURE A.155: intermediate layer height for $r=2.4, n=5, m=2$

$b_{1}$

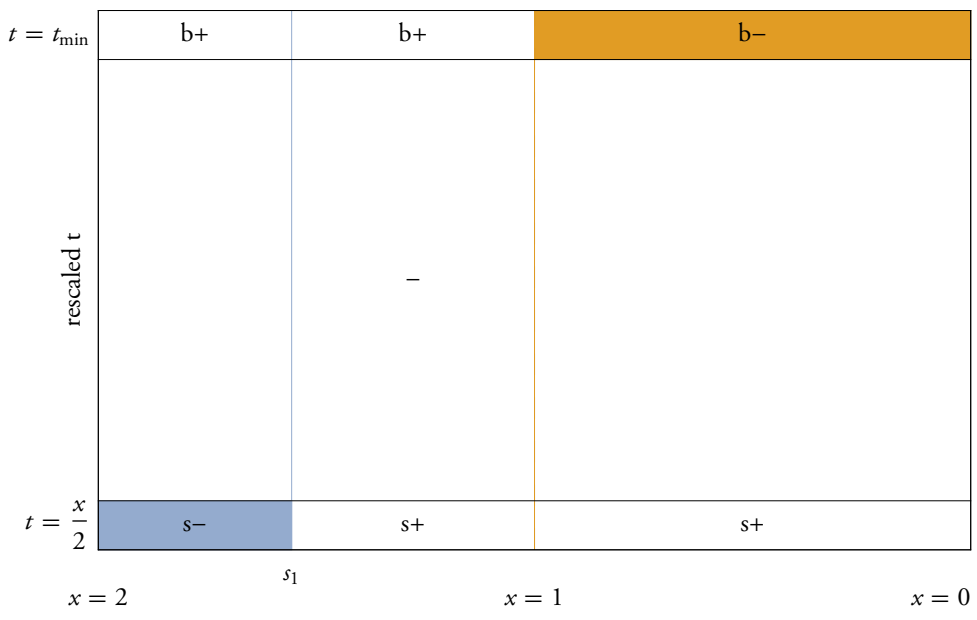

FIGURE A.156: prediction graphic for $r=2.4, n=5, m=2$ 


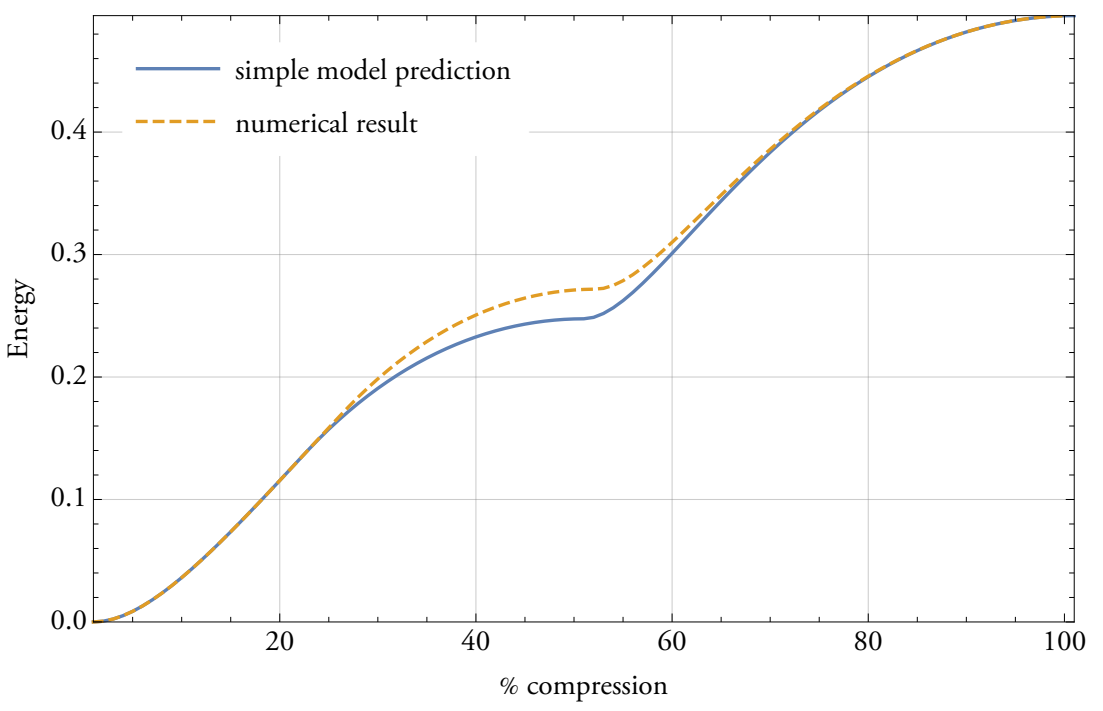

FIGURE A.157: simple energy for $r=2.4, n=6, m=2$

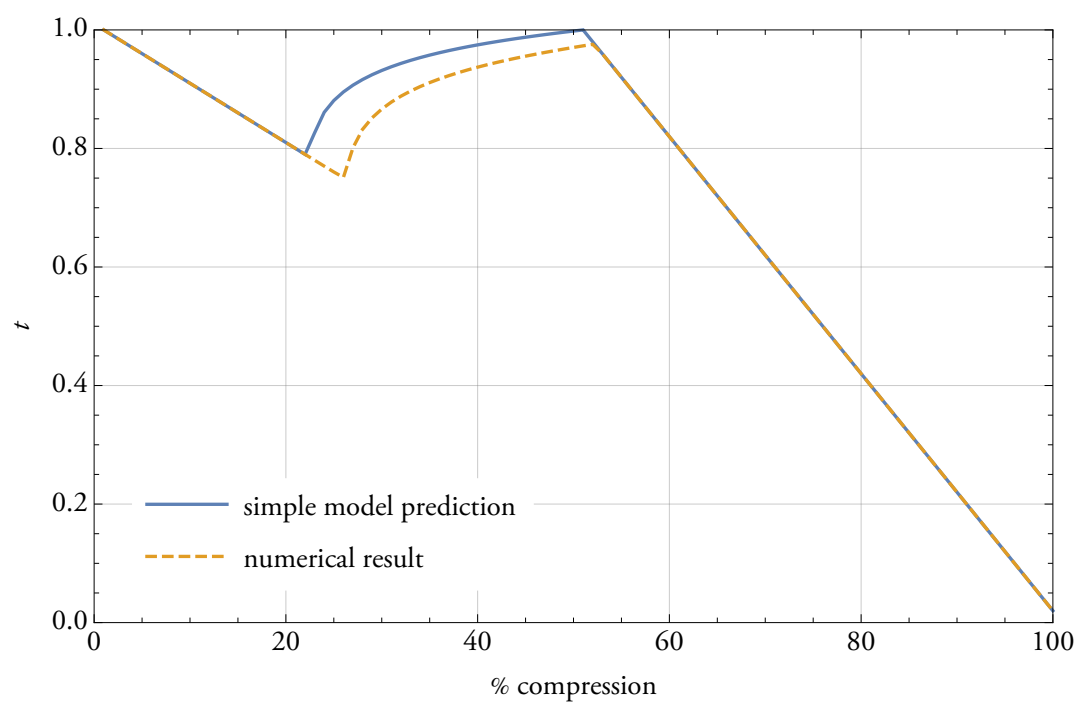

FIGURE A.158: intermediate layer height for $r=2.4, n=6, m=2$

$b_{1}$

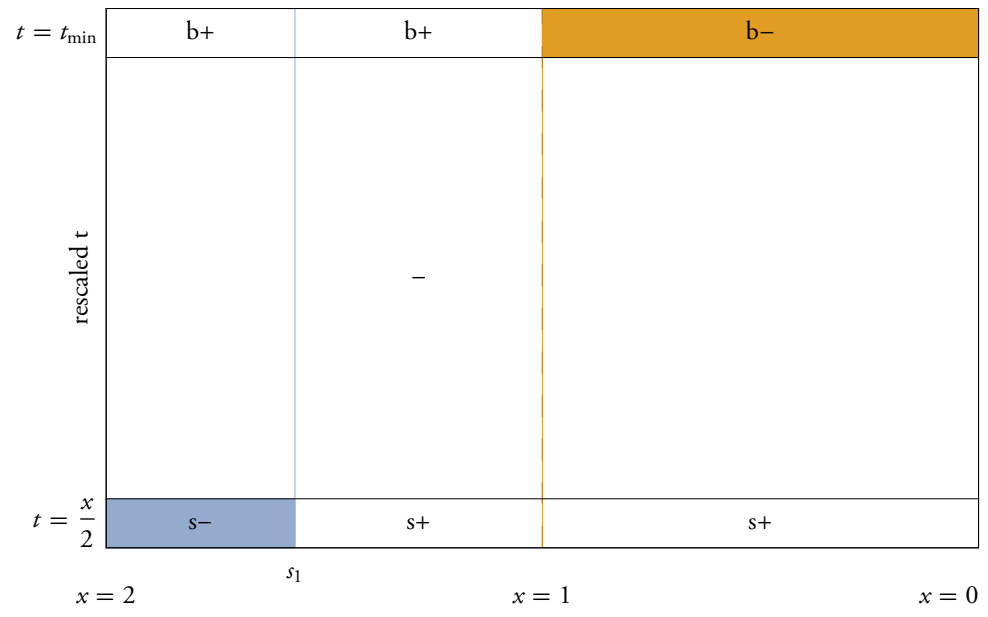

FIGURE A.159: prediction graphic for $r=2.4, n=6, m=2$ 


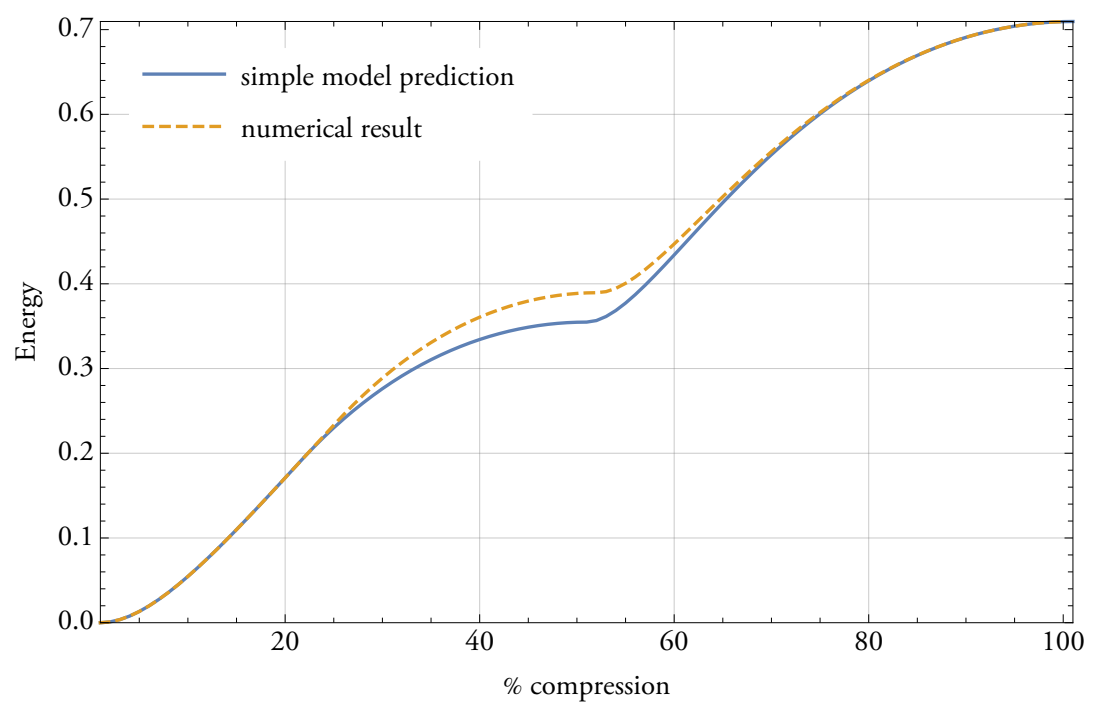

FIGURE A.160: simple energy for $r=2.4, n=7, m=2$

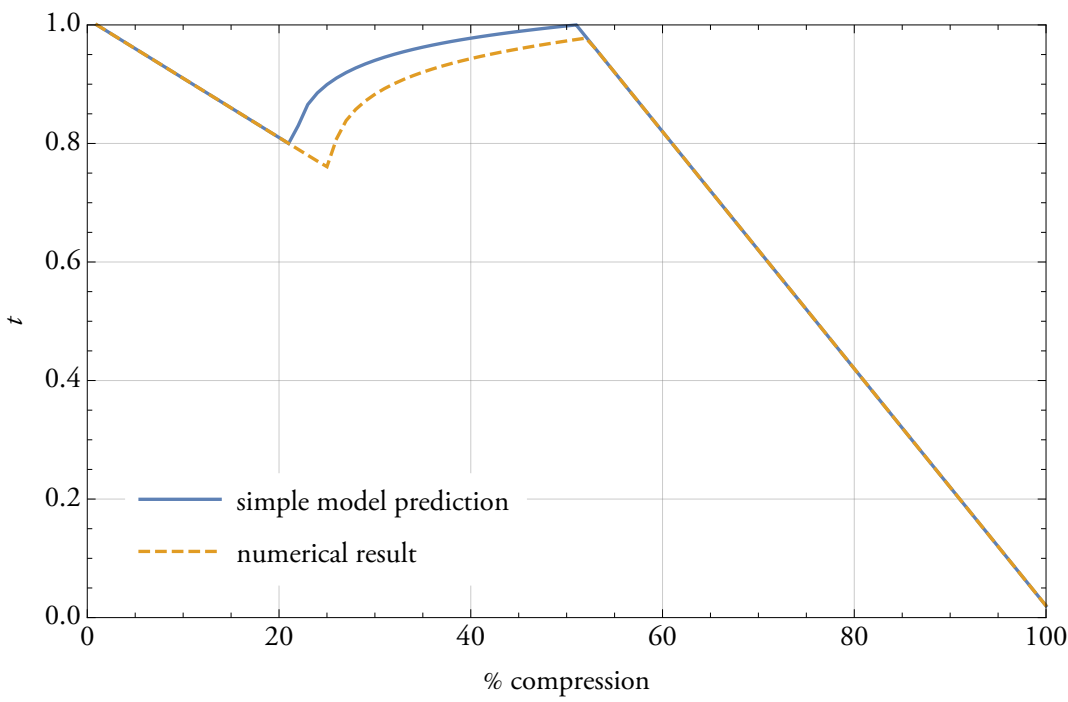

FIGURE A.161: intermediate layer height for $r=2.4, n=7, m=2$

$b_{1}$

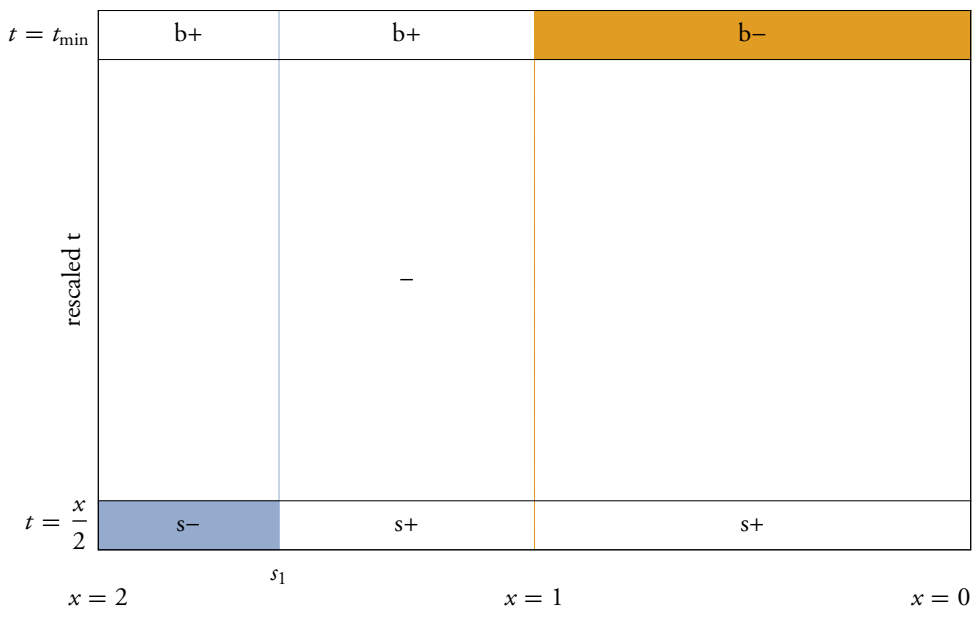

FIGURE A.162: prediction graphic for $r=2.4, n=7, m=2$ 


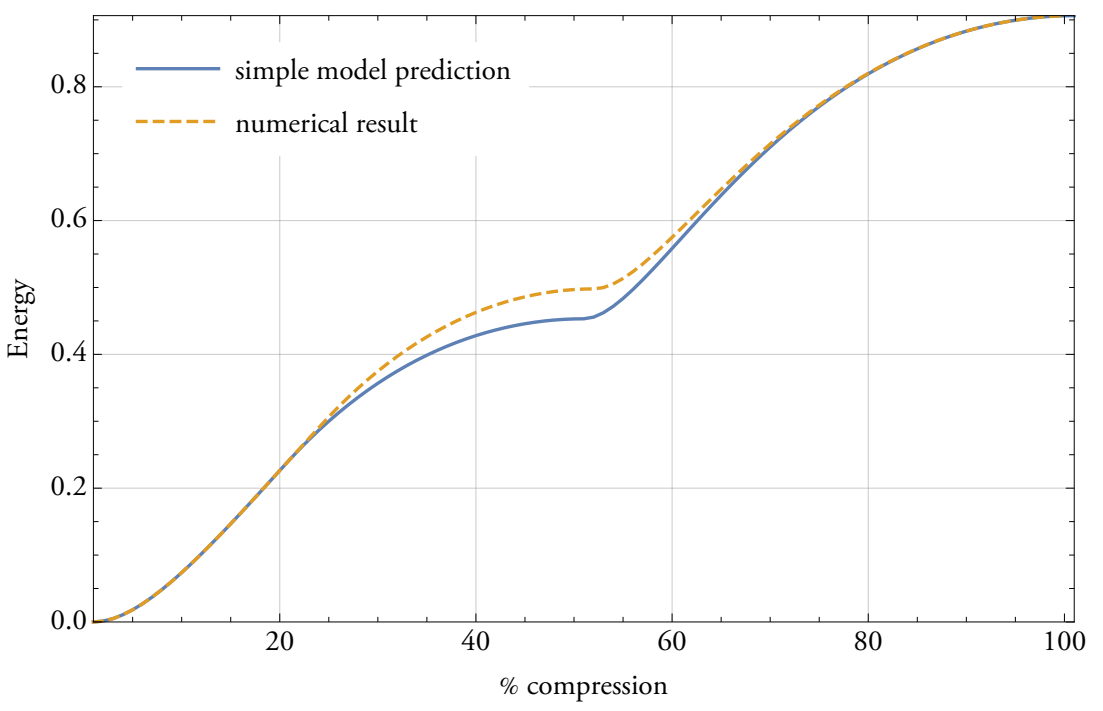

FIGURE A.163: simple energy for $r=2.4, n=8, m=2$

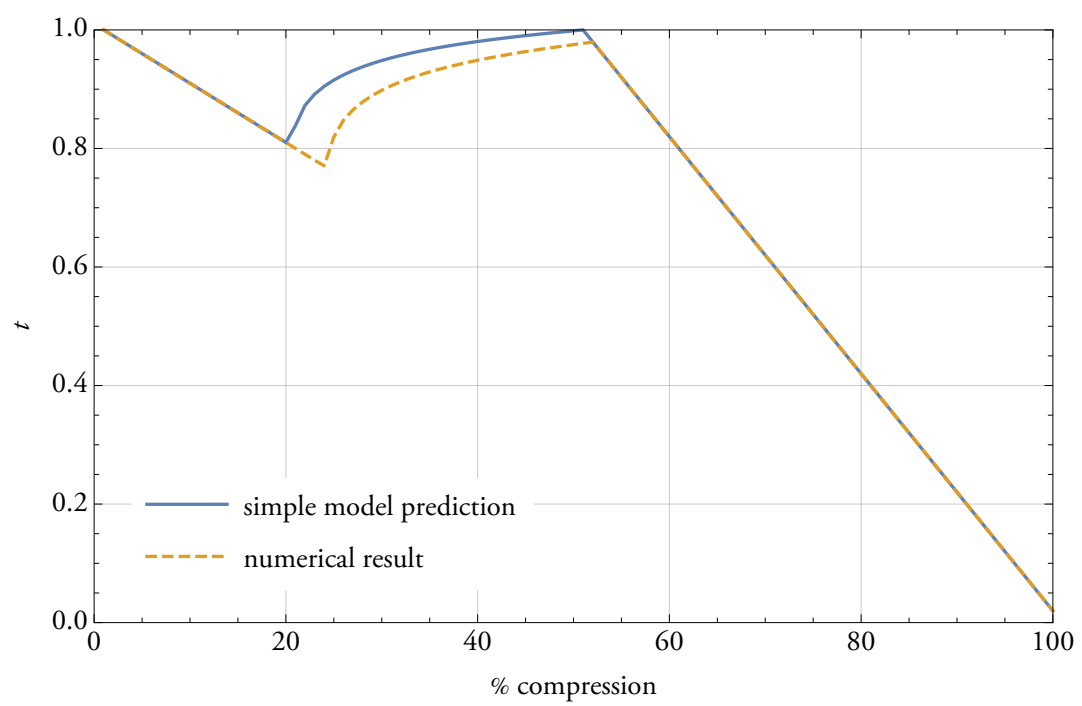

FIGURE A.164: intermediate layer height for $r=2.4, n=8, m=2$

$b_{1}$

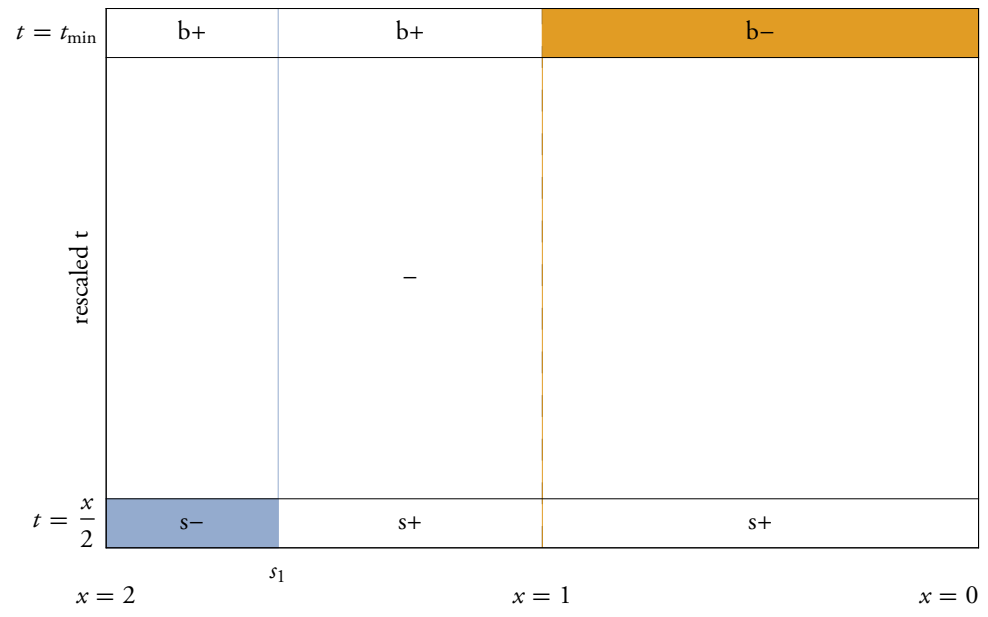

FIGURE A.165: prediction graphic for $r=2.4, n=8, m=2$ 


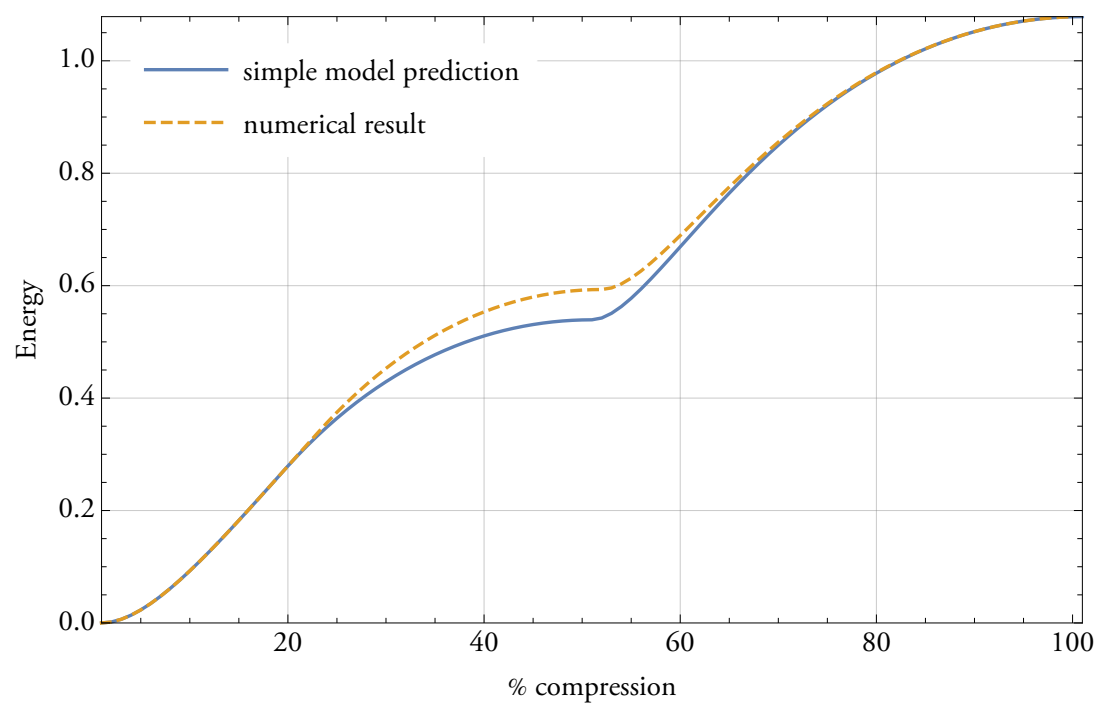

FIGURE A.166: simple energy for $r=2.4, n=9, m=2$

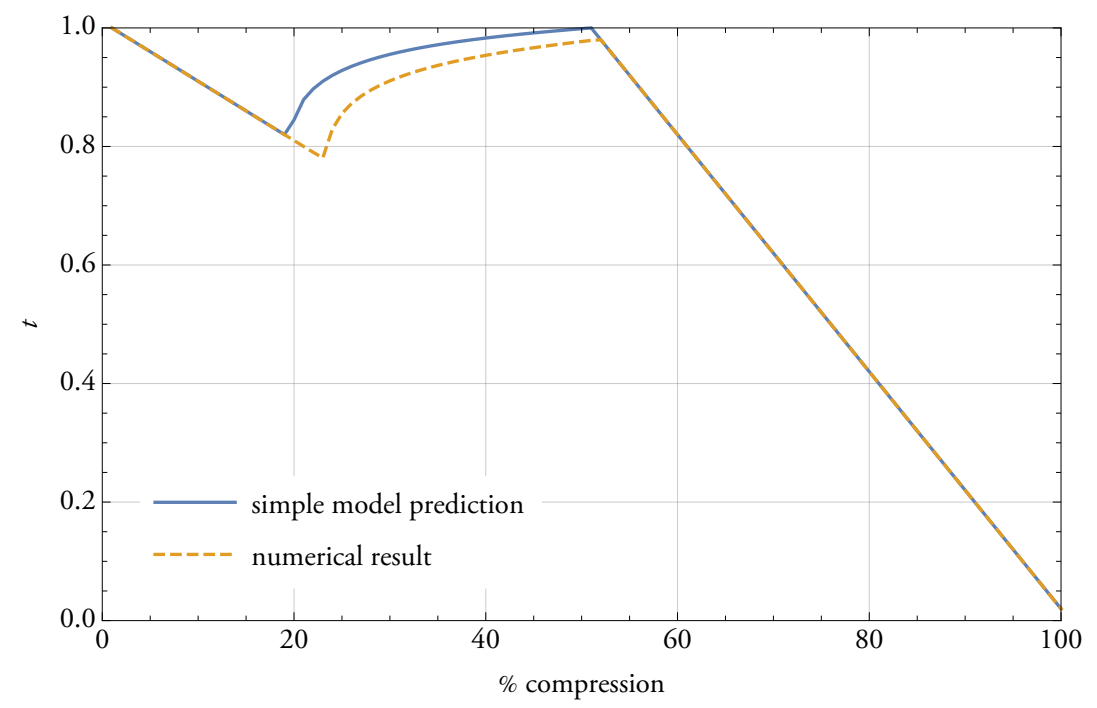

FIGURE A.167: intermediate layer height for $r=2.4, n=9, m=2$

$b_{1}$

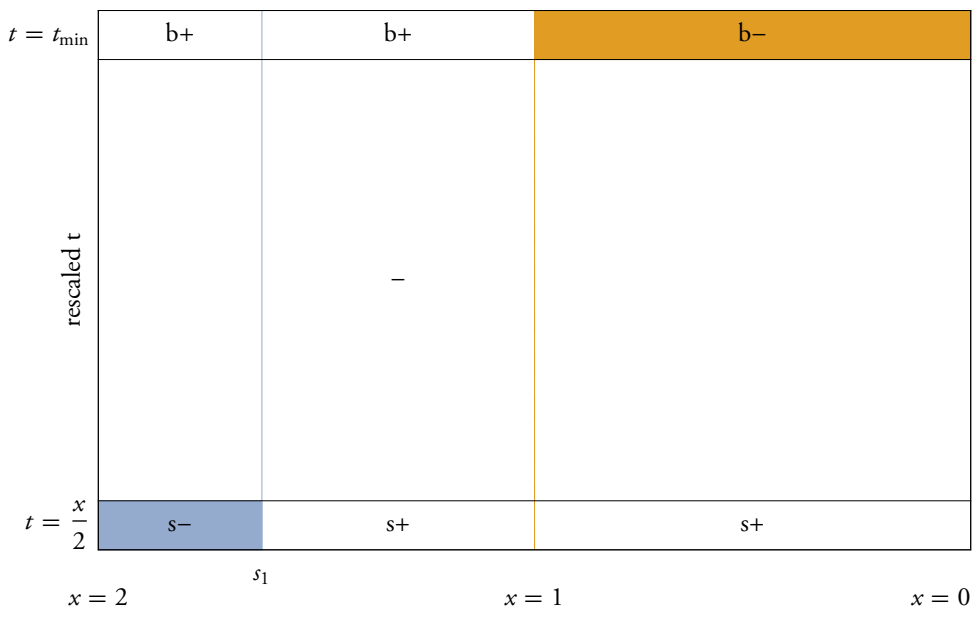

FIGURE A.168: prediction graphic for $r=2.4, n=9, m=2$ 


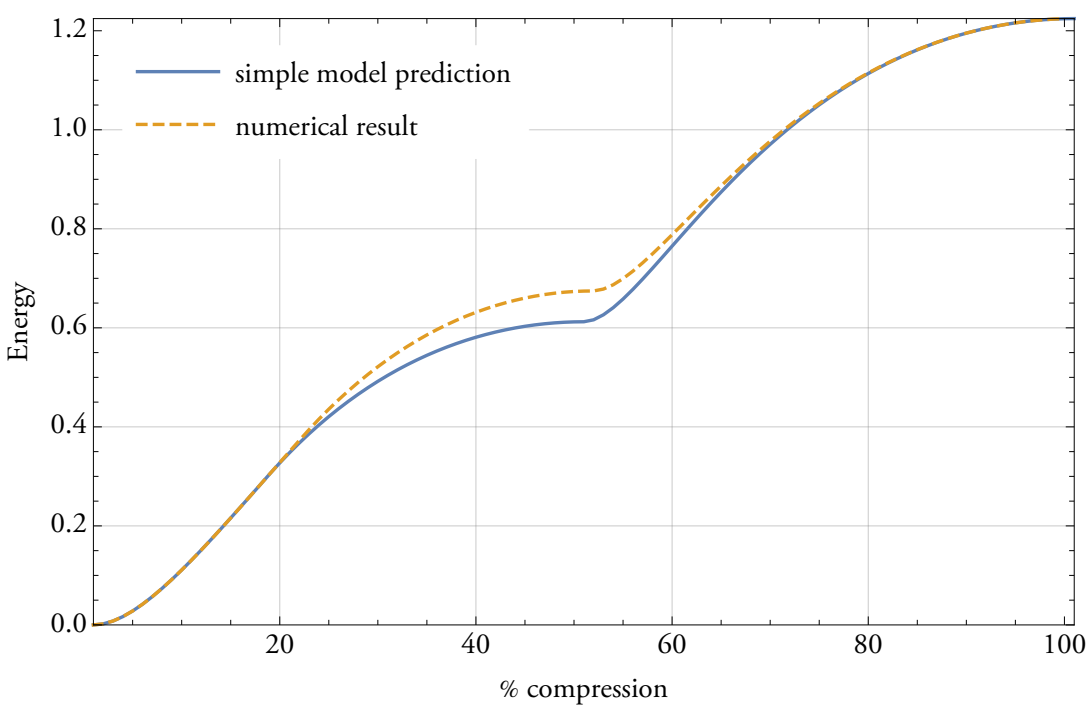

FIGURE A.169: simple energy for $r=2.4, n=10, m=2$

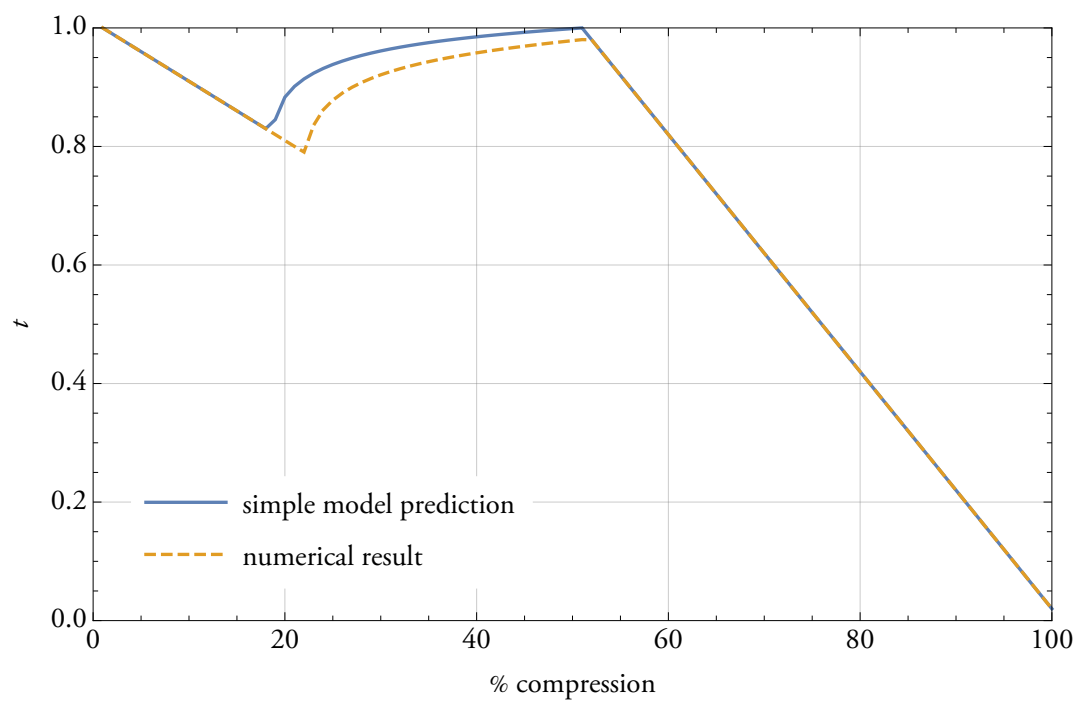

FIGURE A.170: intermediate layer height for $r=2.4, n=10, m=2$

$b_{1}$

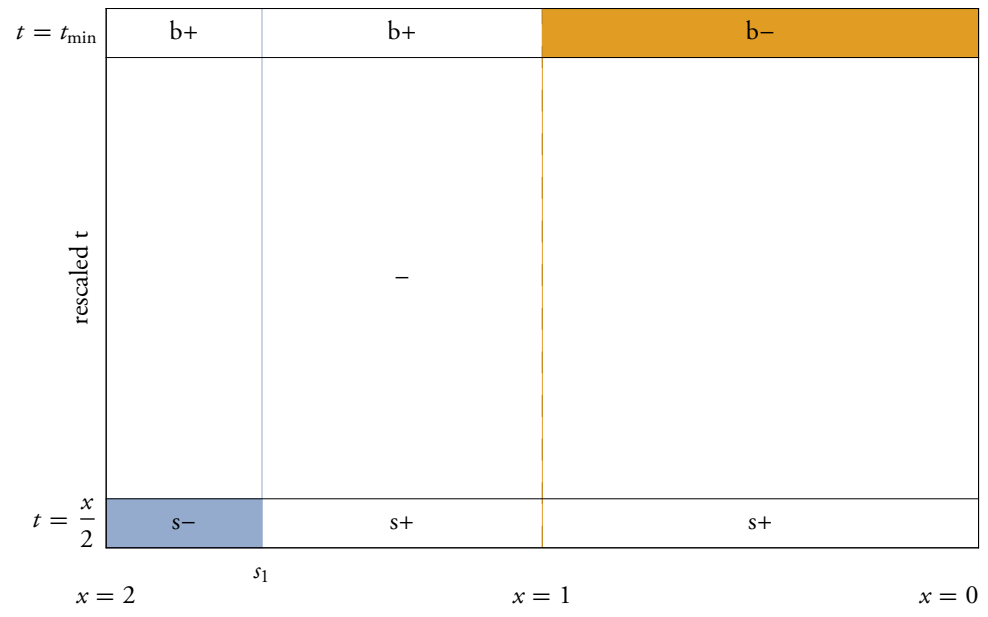

FIGURE A.171: prediction graphic for $r=2.4, n=10, m=2$ 


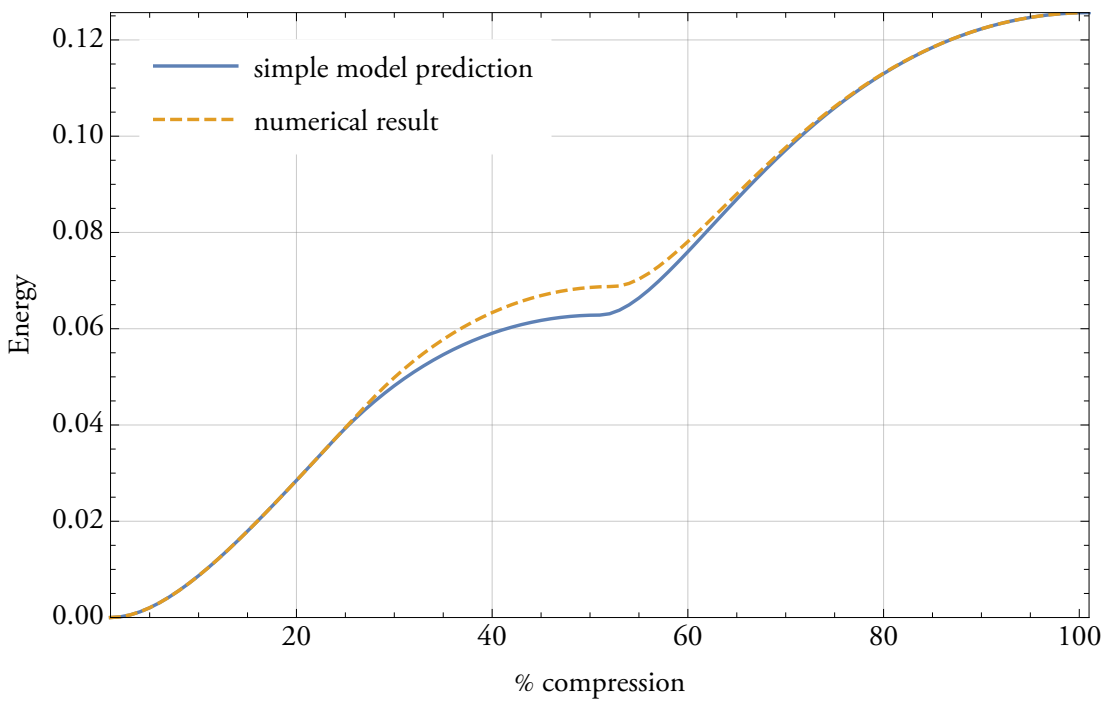

FIGURE A.172: simple energy for $r=2.6, n=4, m=2$

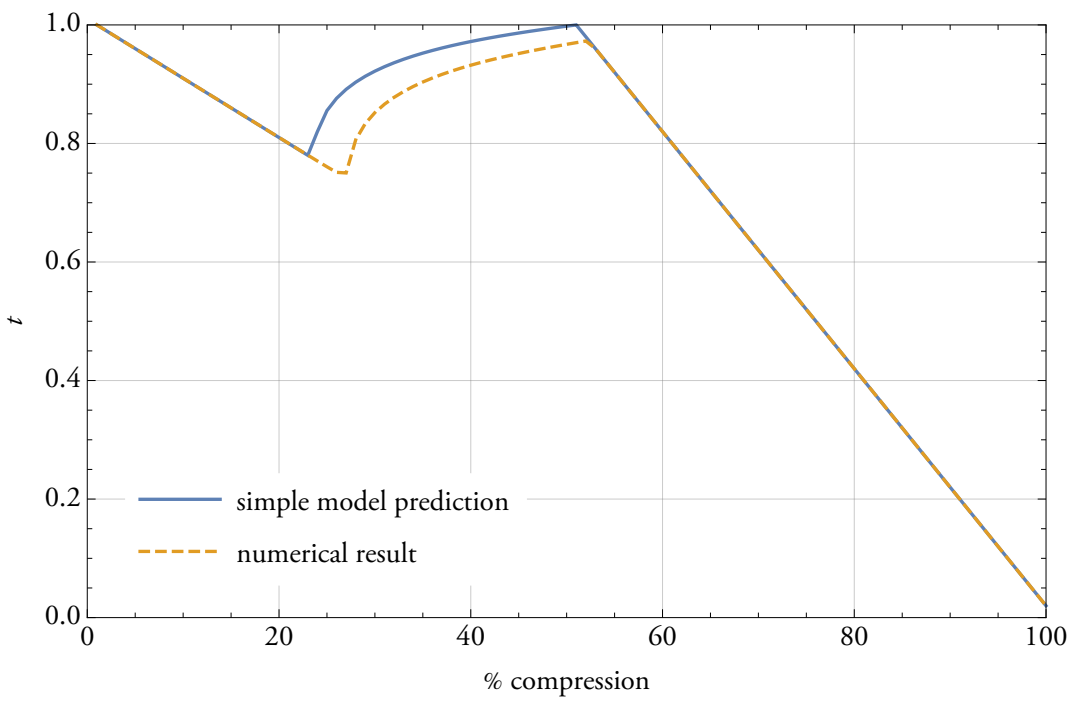

FIGURE A.173: intermediate layer height for $r=2.6, n=4, m=2$

$b_{1}$

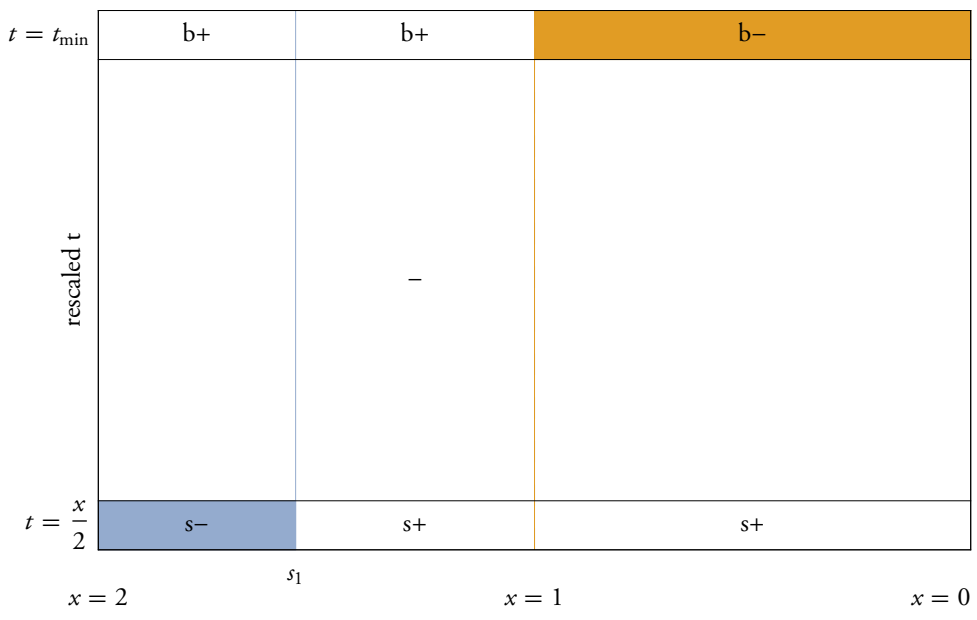

FIGURE A.174: prediction graphic for $r=2.6, n=4, m=2$ 


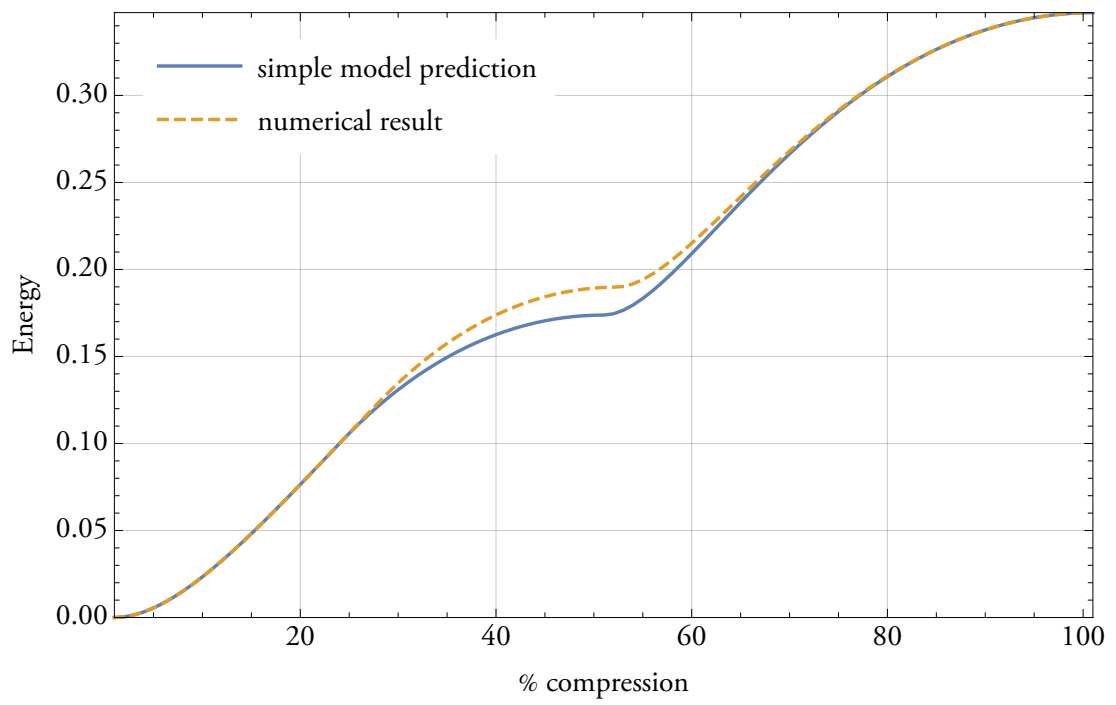

FIGURE A.175: simple energy for $r=2.6, n=5, m=2$

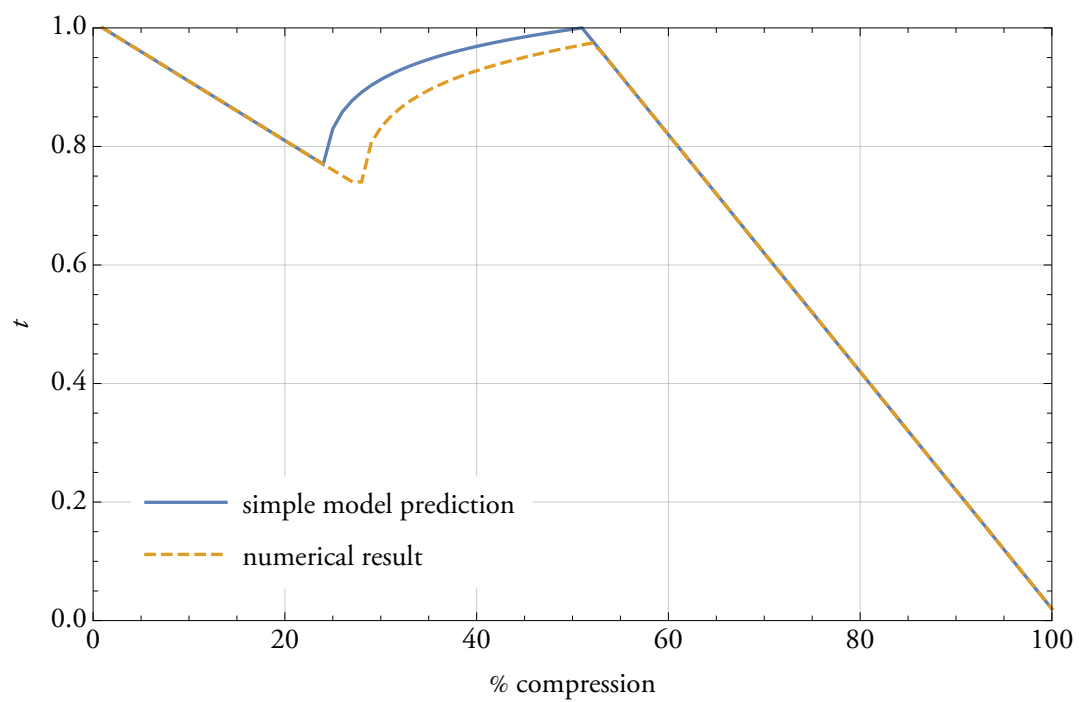

FIGURE A.176: intermediate layer height for $r=2.6, n=5, m=2$

$b_{1}$

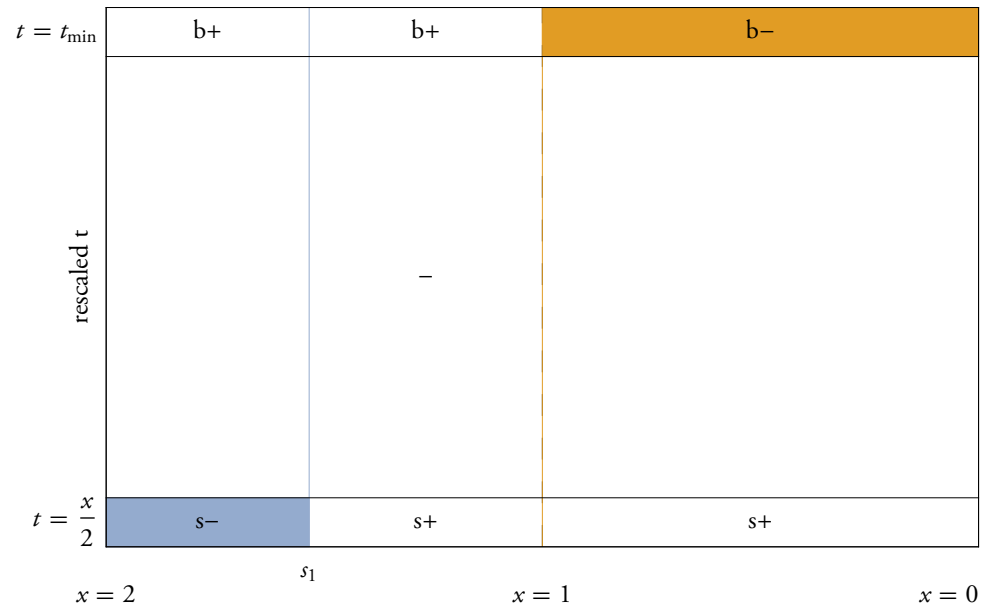

FIGURE A.177: prediction graphic for $r=2.6, n=5, m=2$ 


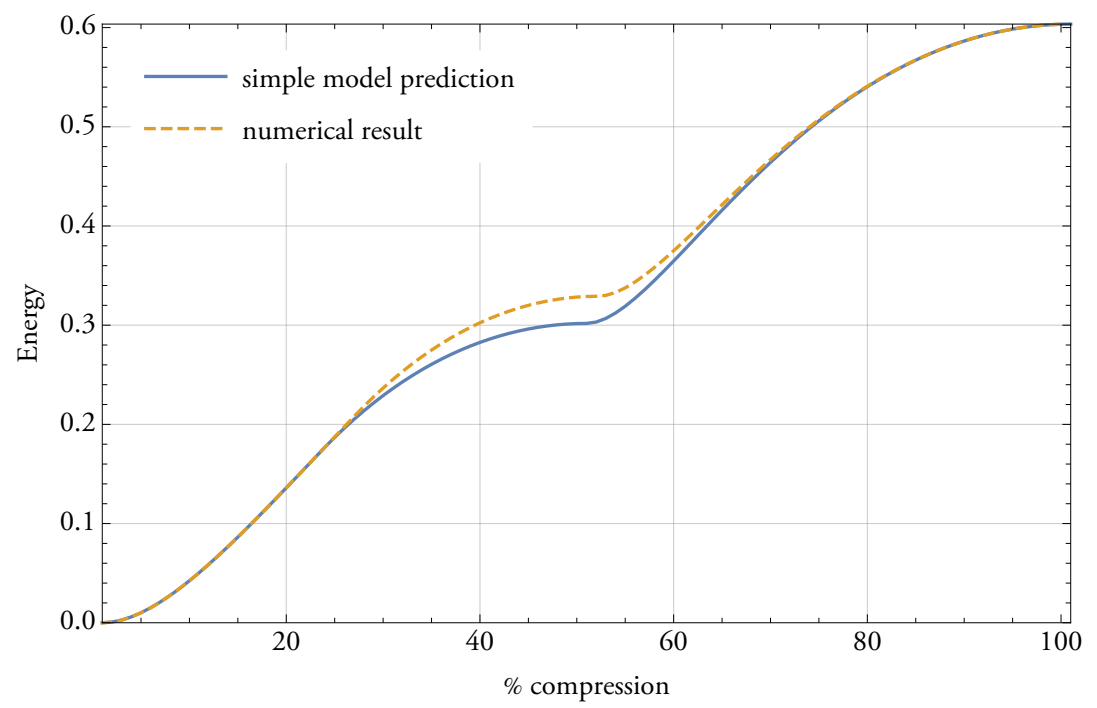

FIGURE A.178: simple energy for $r=2.6, n=6, m=2$

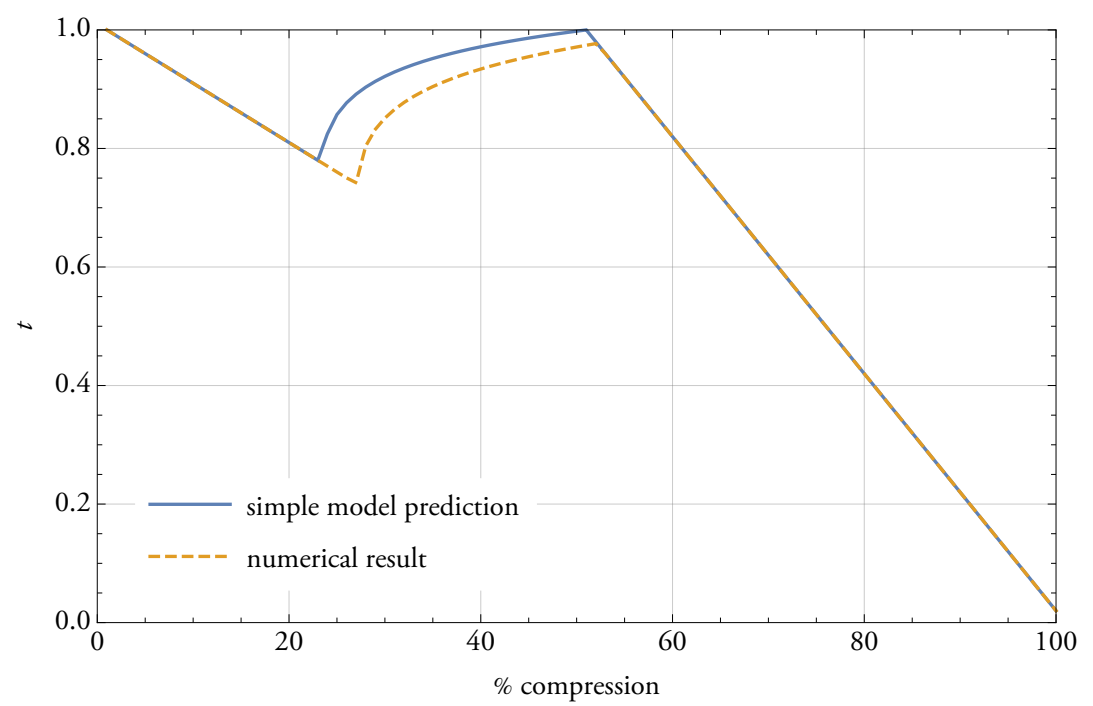

FIGURE A.179: intermediate layer height for $r=2.6, n=6, m=2$

$b_{1}$

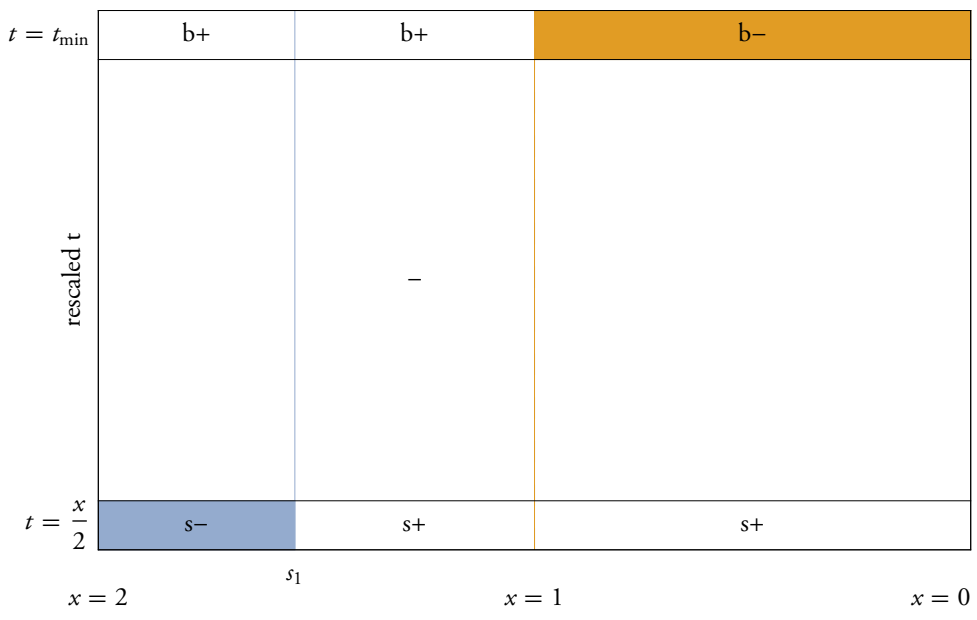

FIGURE A.180: prediction graphic for $r=2.6, n=6, m=2$ 


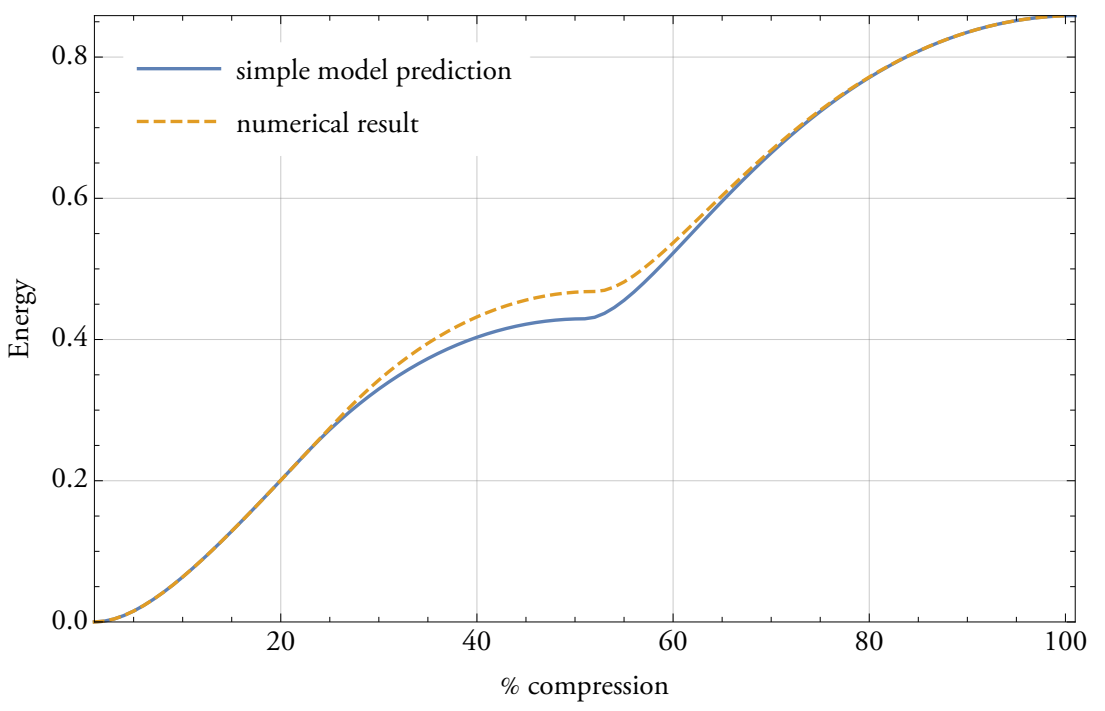

FIGURE A.181: simple energy for $r=2.6, n=7, m=2$

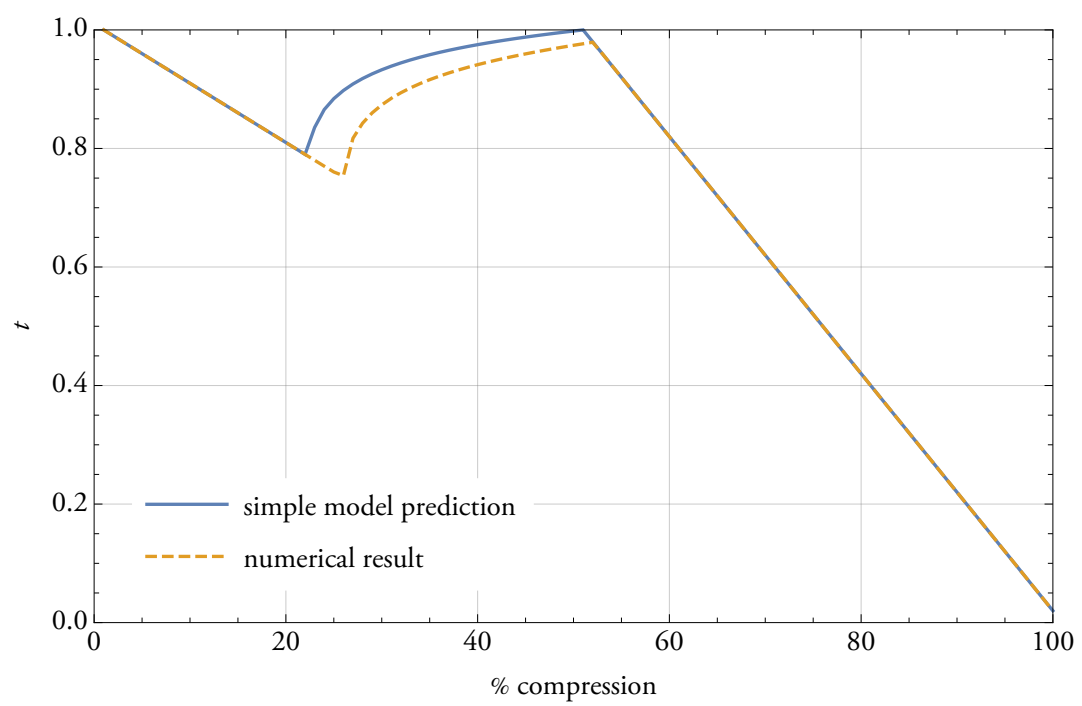

FIGURE A.182: intermediate layer height for $r=2.6, n=7, m=2$

$b_{1}$

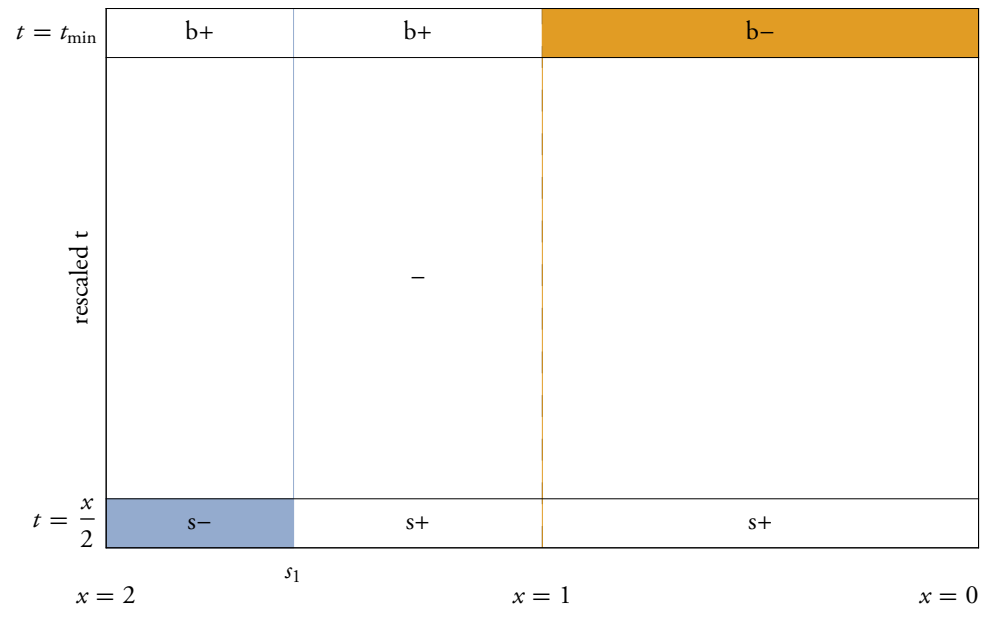

FIGURE A.183: prediction graphic for $r=2.6, n=7, m=2$ 


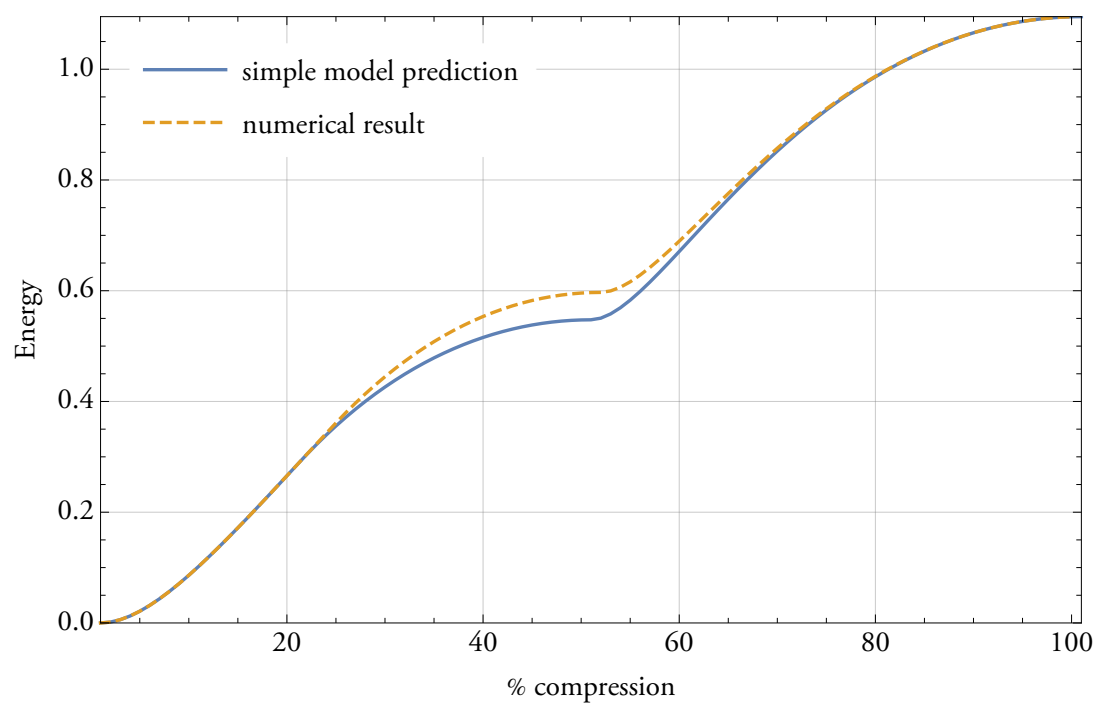

FIGURE A.184: simple energy for $r=2.6, n=8, m=2$

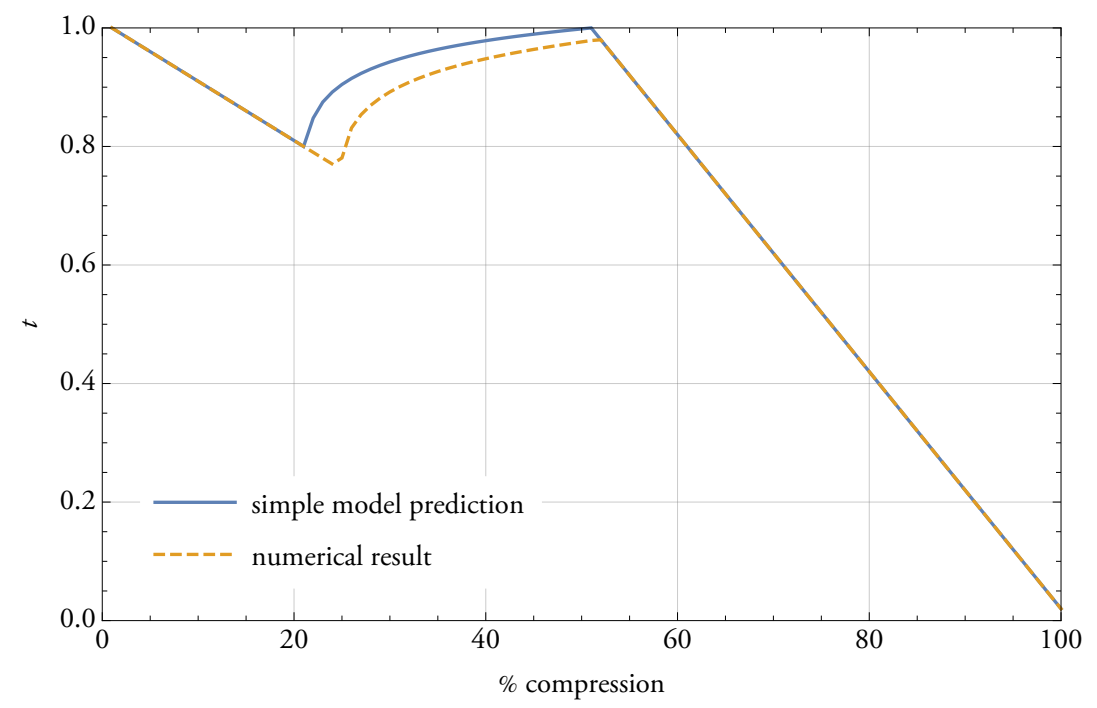

FIGURE A.185: intermediate layer height for $r=2.6, n=8, m=2$

$b_{1}$

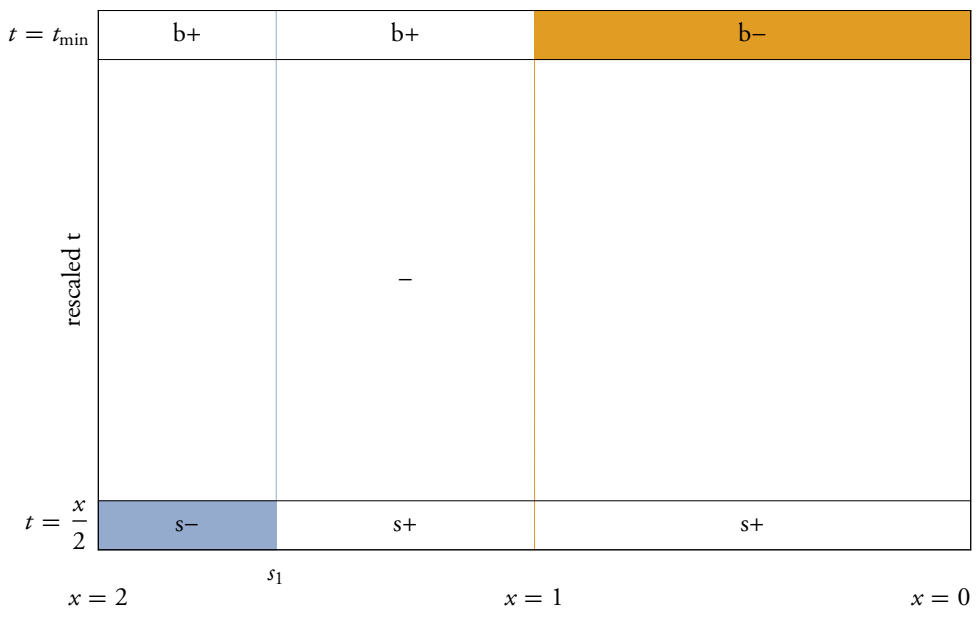

FIGURE A.186: prediction graphic for $r=2.6, n=8, m=2$ 


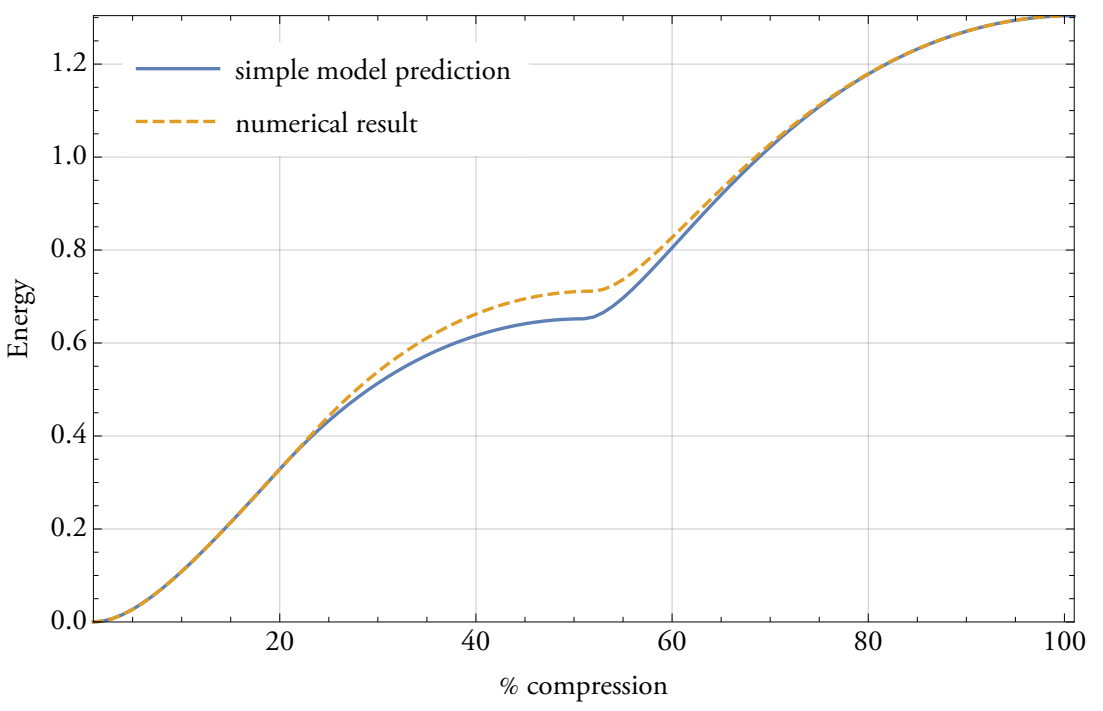

FIGURE A.187: simple energy for $r=2.6, n=9, m=2$

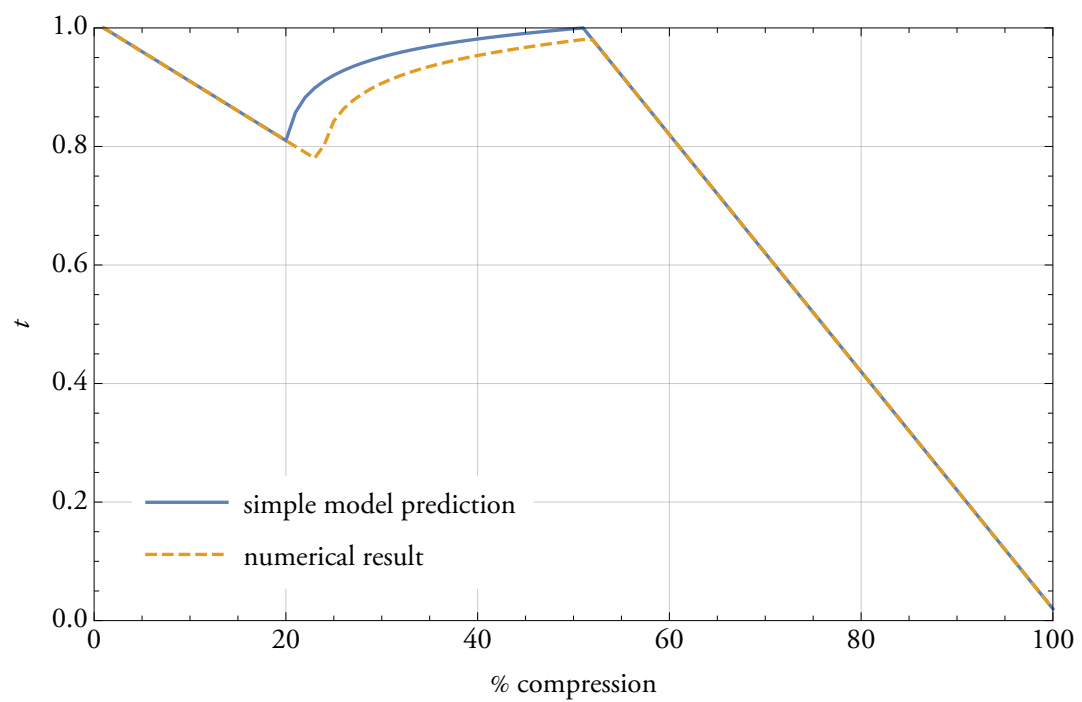

FIGURE A.188: intermediate layer height for $r=2.6, n=9, m=2$

$b_{1}$

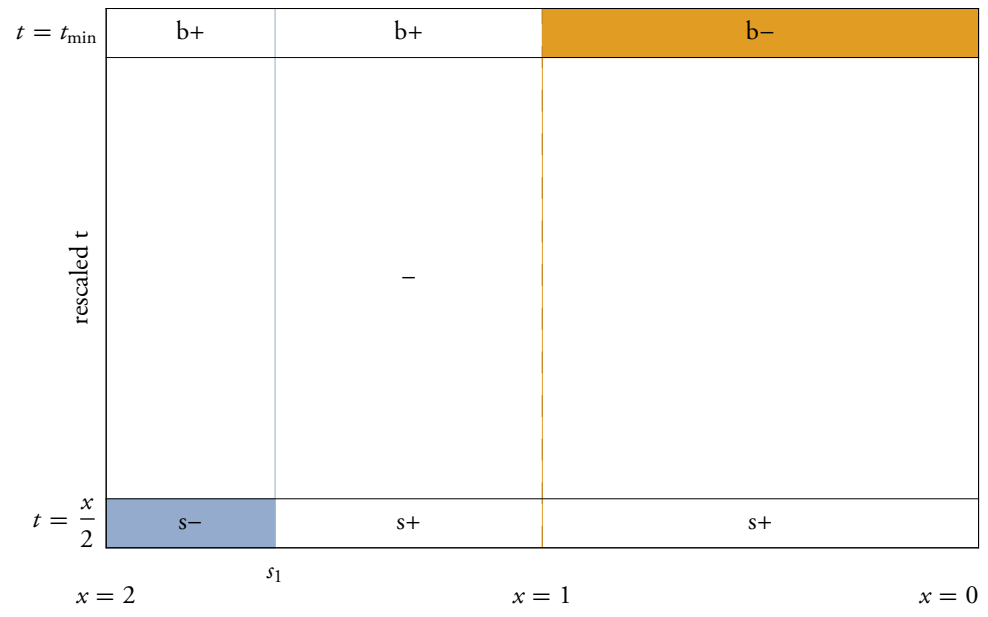

FIGURE A.189: prediction graphic for $r=2.6, n=9, m=2$ 


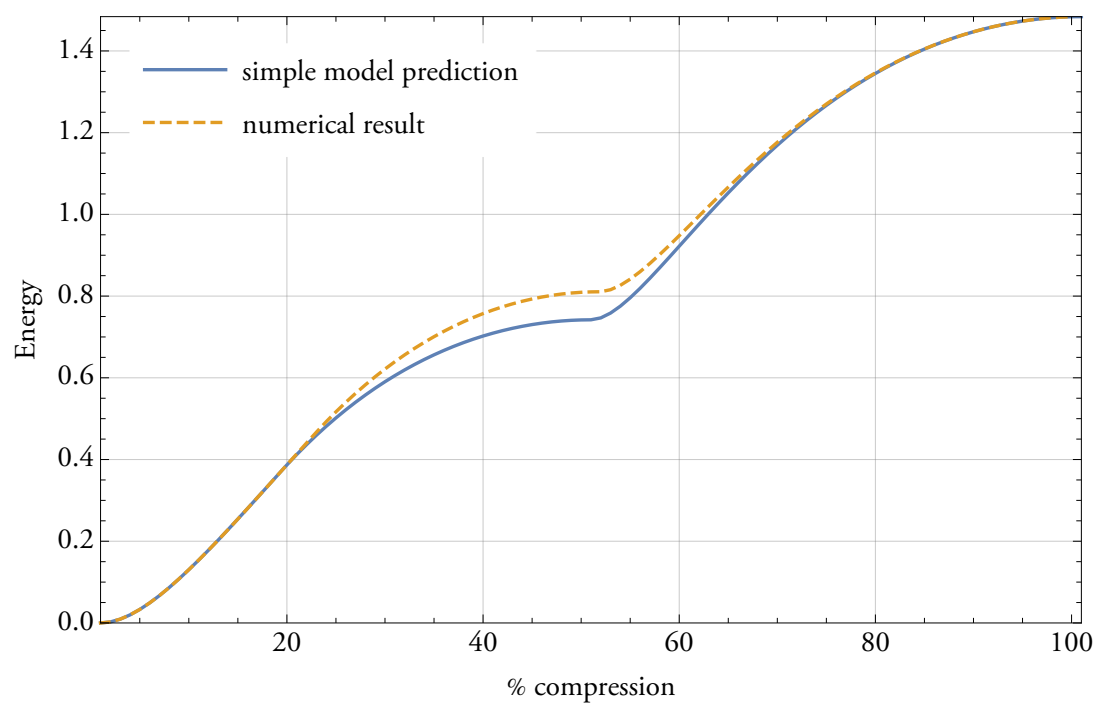

FIGURE A.190: simple energy for $r=2.6, n=10, m=2$

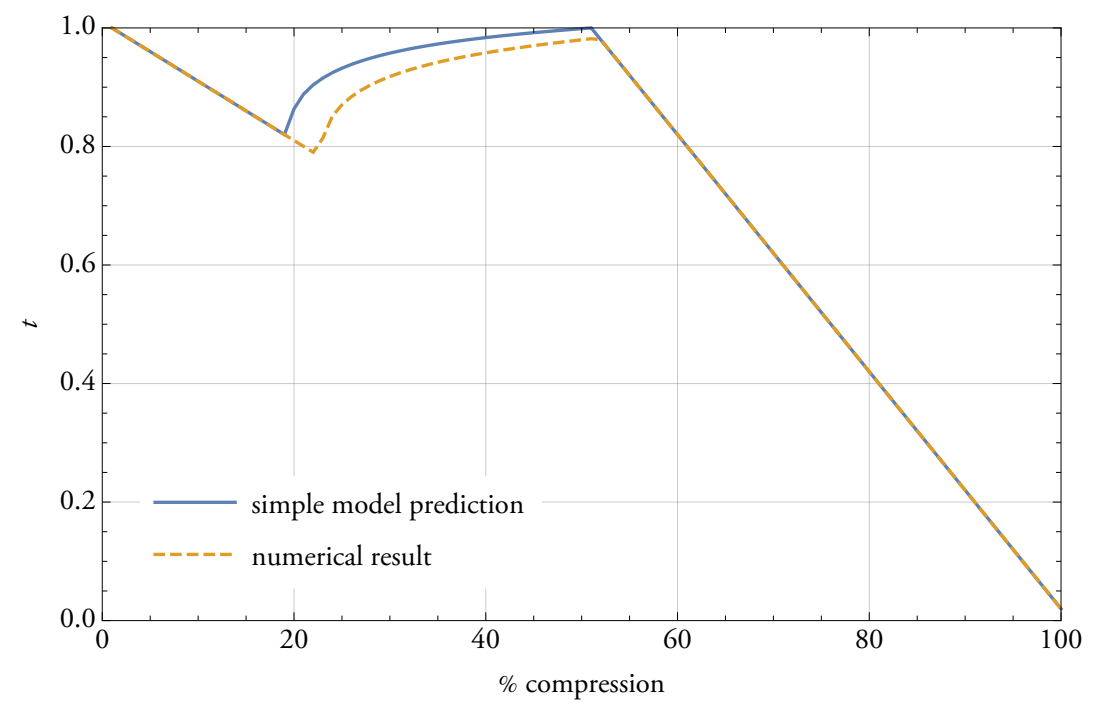

FIGURE A.191: intermediate layer height for $r=2.6, n=10, m=2$

$b_{1}$

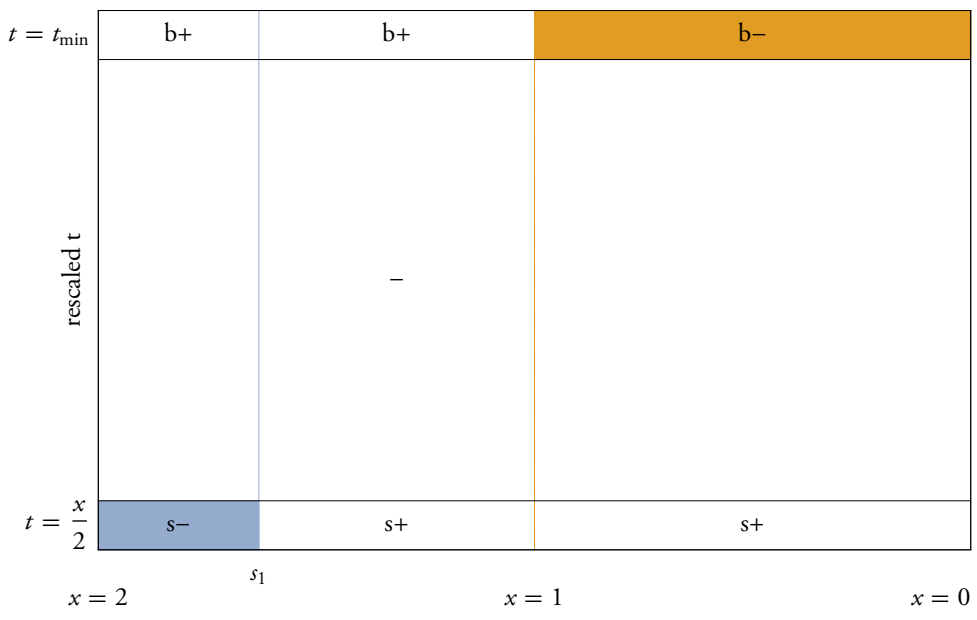

FIGURE A.192: prediction graphic for $r=2.6, n=10, m=2$ 


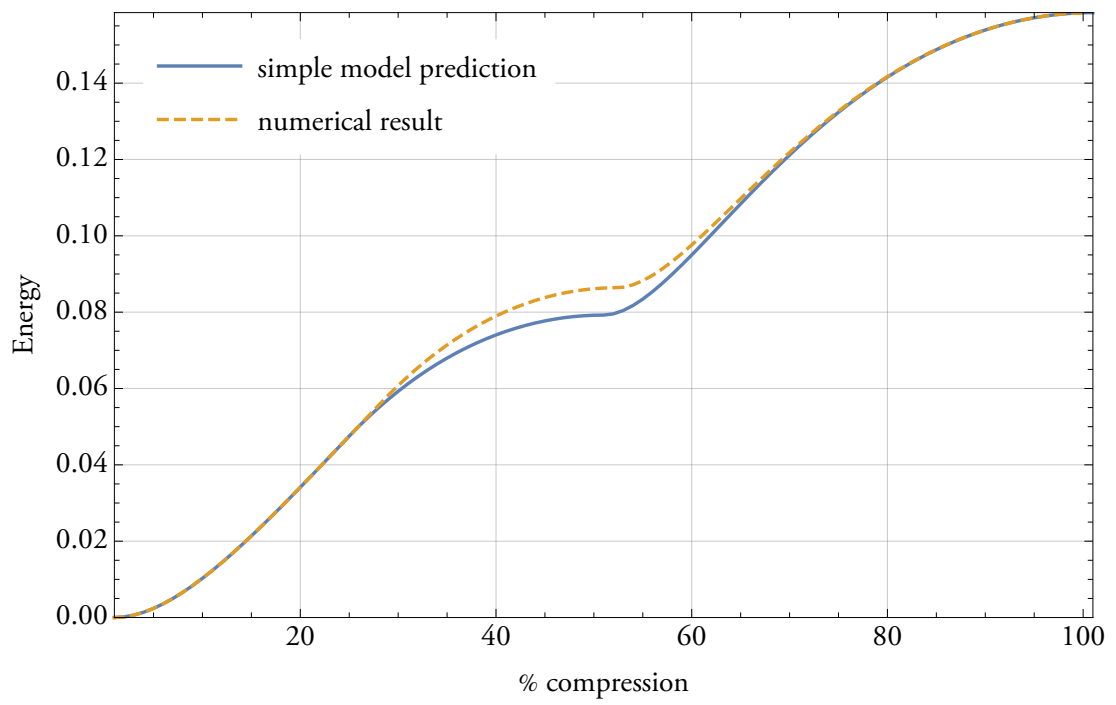

FIGURE A.193: simple energy for $r=2.8, n=4, m=2$

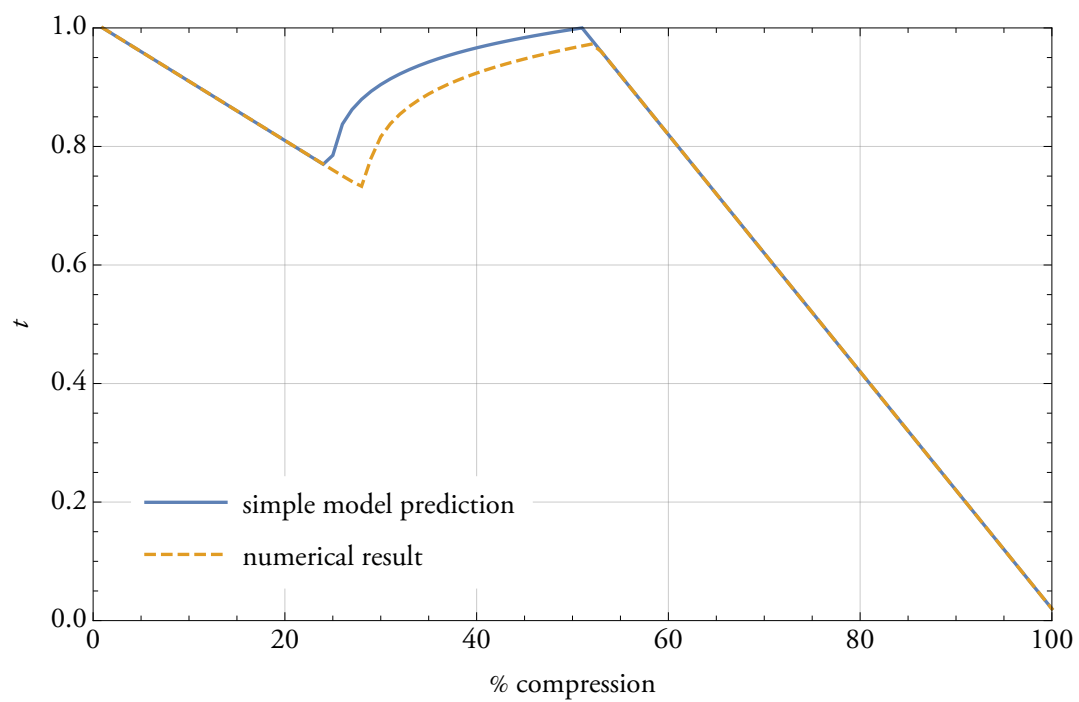

FIGURE A.194: intermediate layer height for $r=2.8, n=4, m=2$

$b_{1}$

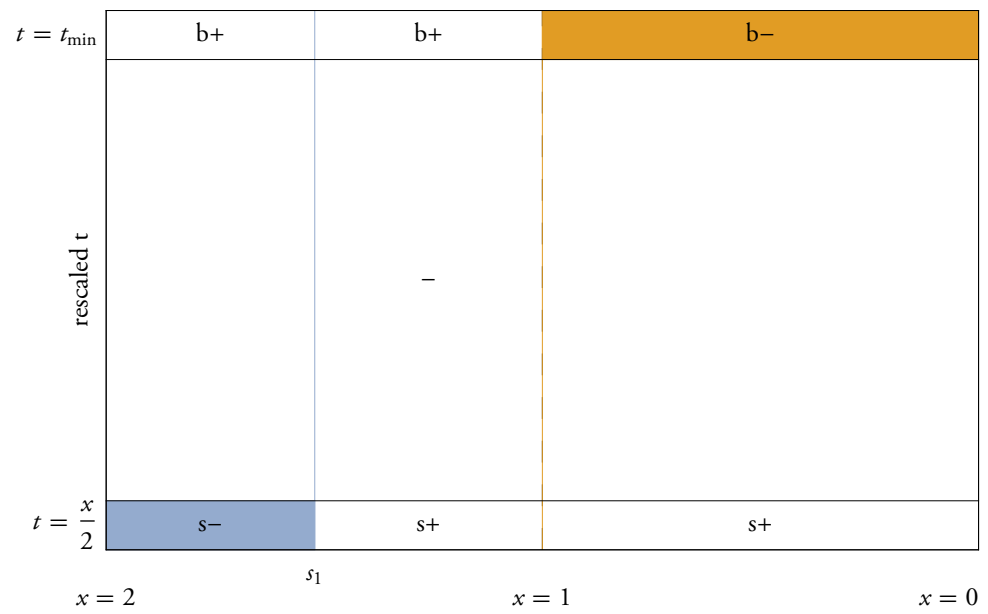

FIGURE A.195: prediction graphic for $r=2.8, n=4, m=2$ 


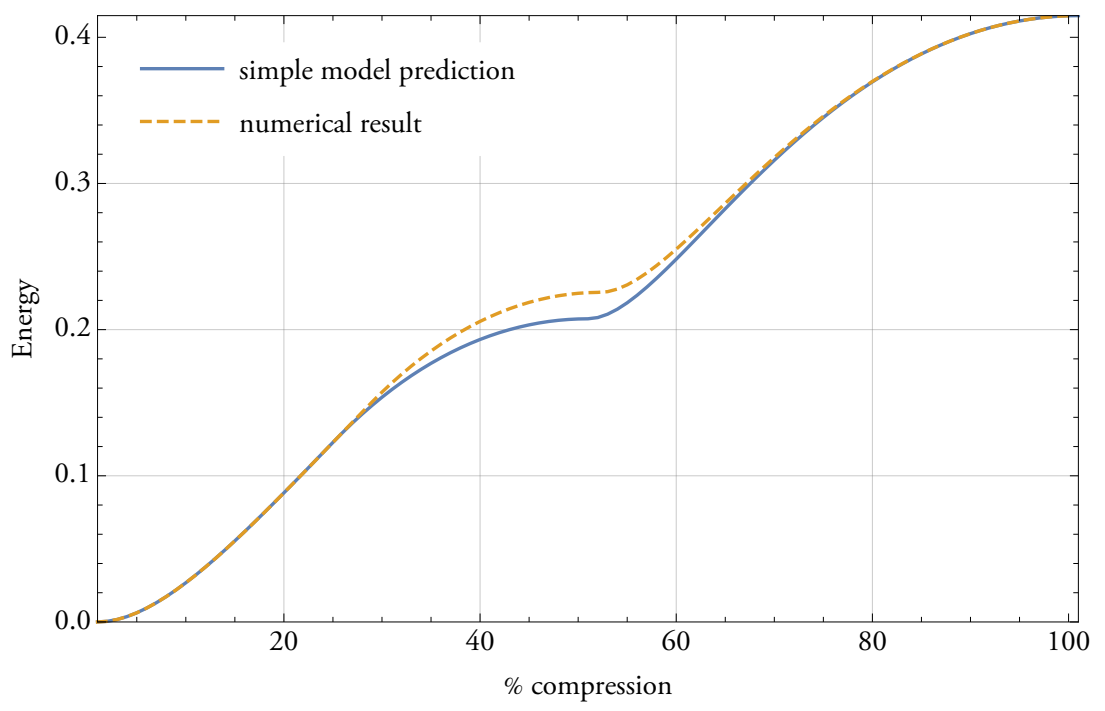

FIGURE A.196: simple energy for $r=2.8, n=5, m=2$

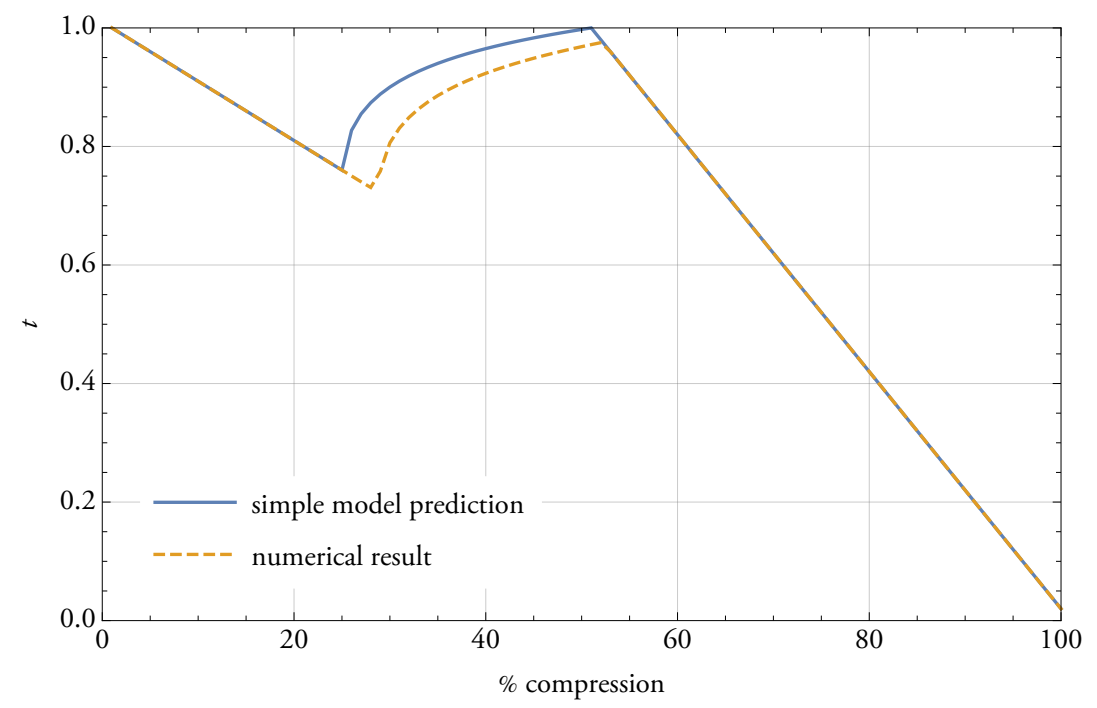

FIGURE A.197: intermediate layer height for $r=2.8, n=5, m=2$

$b_{1}$

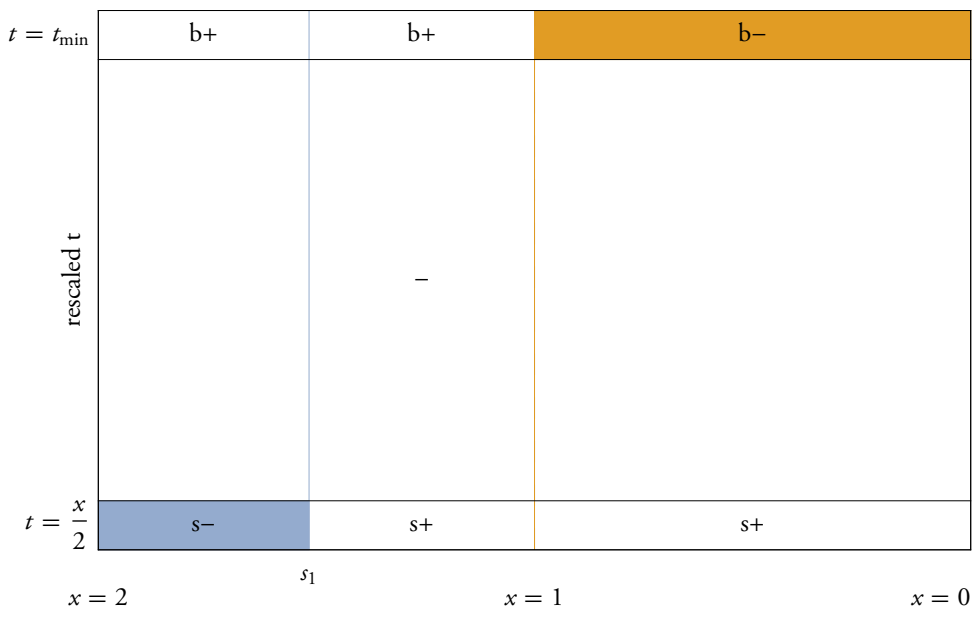

FIGURE A.198: prediction graphic for $r=2.8, n=5, m=2$ 


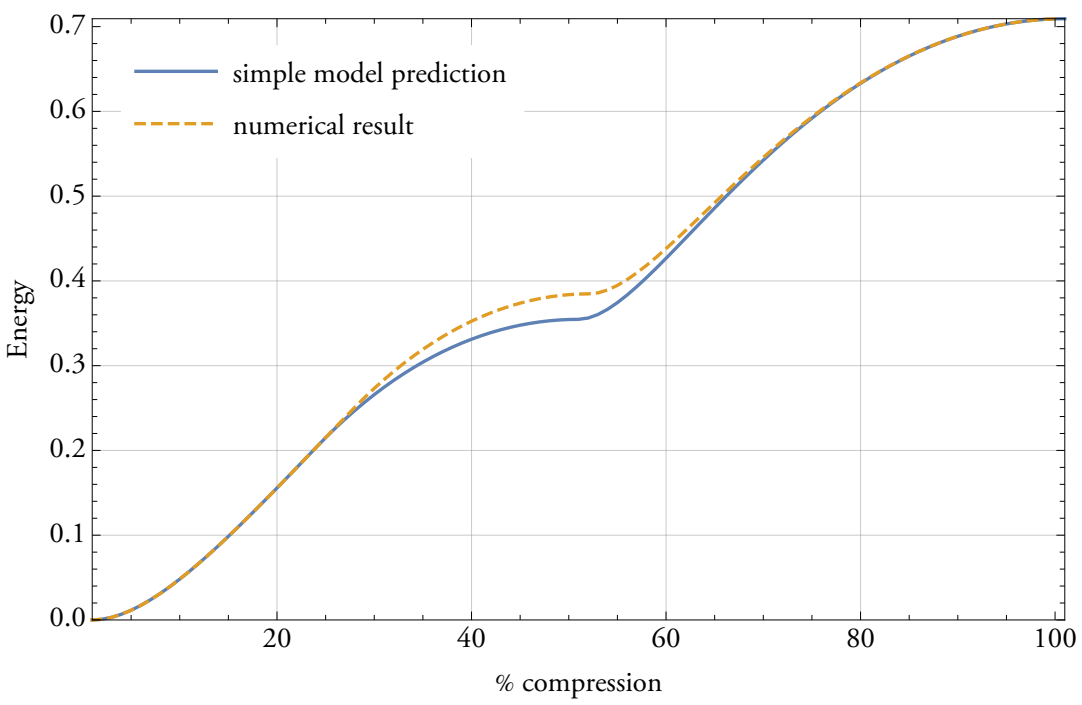

FIGURE A.199: simple energy for $r=2.8, n=6, m=2$

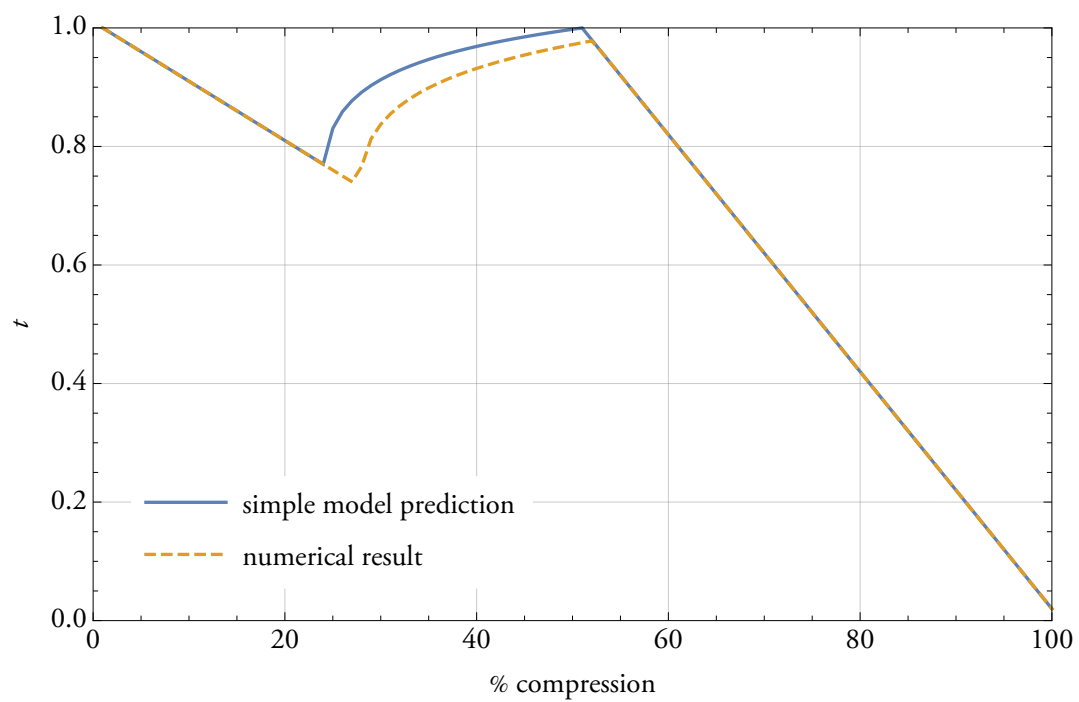

FIGURE A.200: intermediate layer height for $r=2.8, n=6, m=2$

$b_{1}$

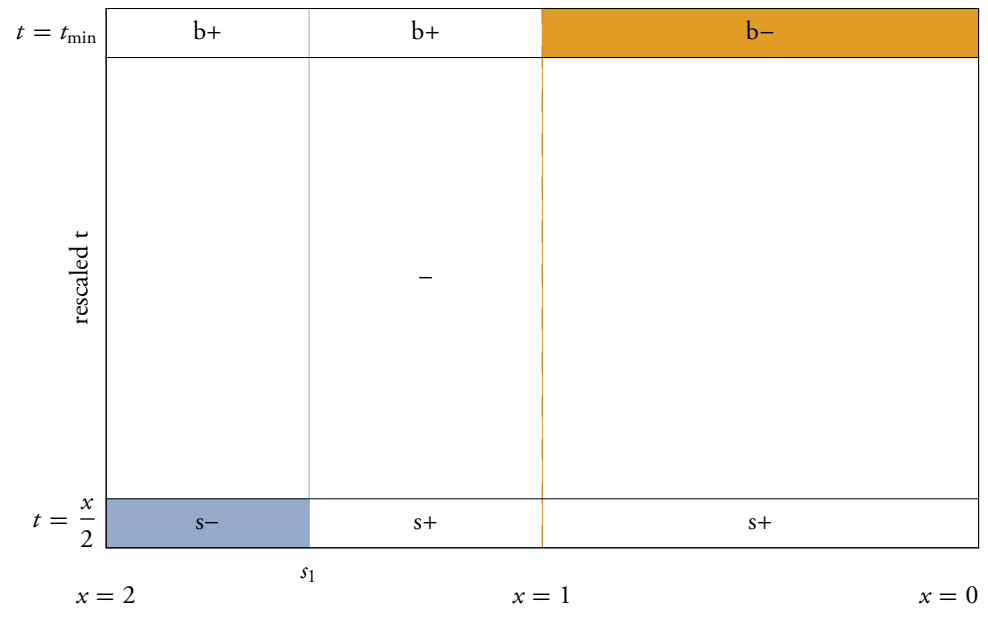

FIGURE A.201: prediction graphic for $r=2.8, n=6, m=2$ 


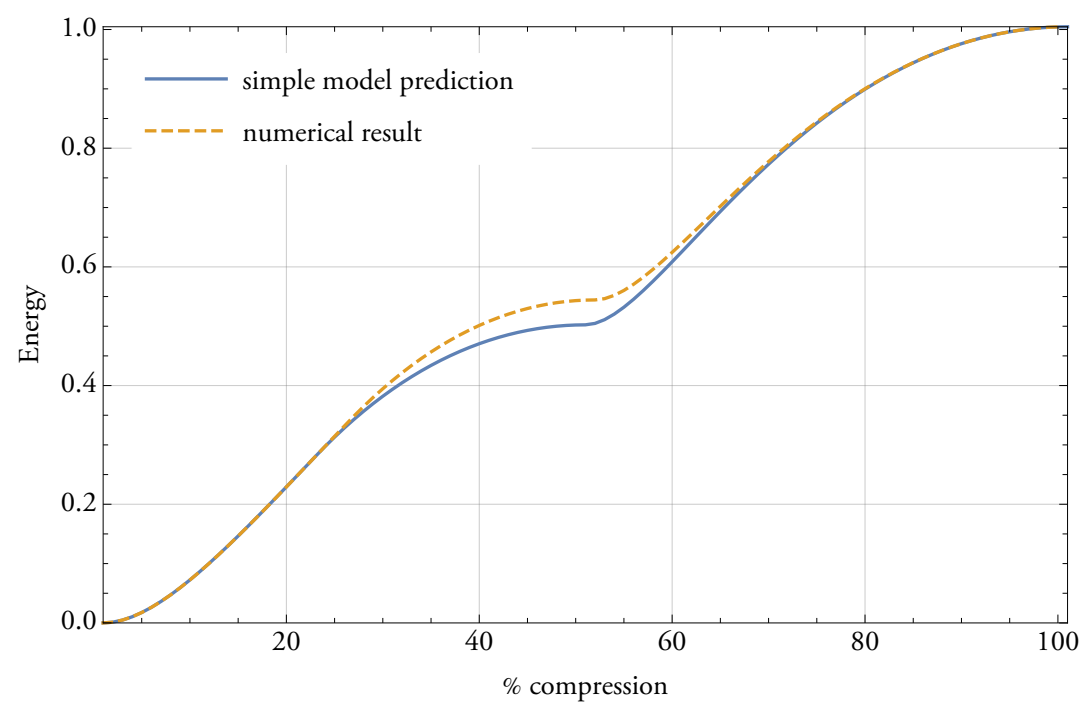

FIGURE A.202: simple energy for $r=2.8, n=7, m=2$

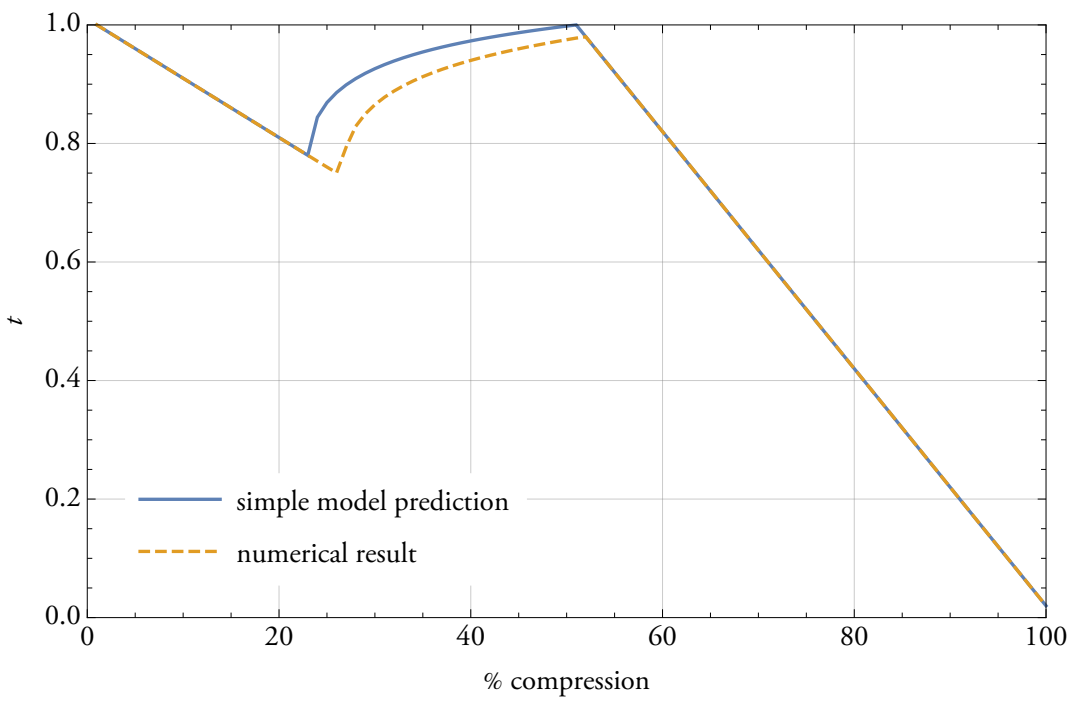

FIGURE A.203: intermediate layer height for $r=2.8, n=7, m=2$

$b_{1}$

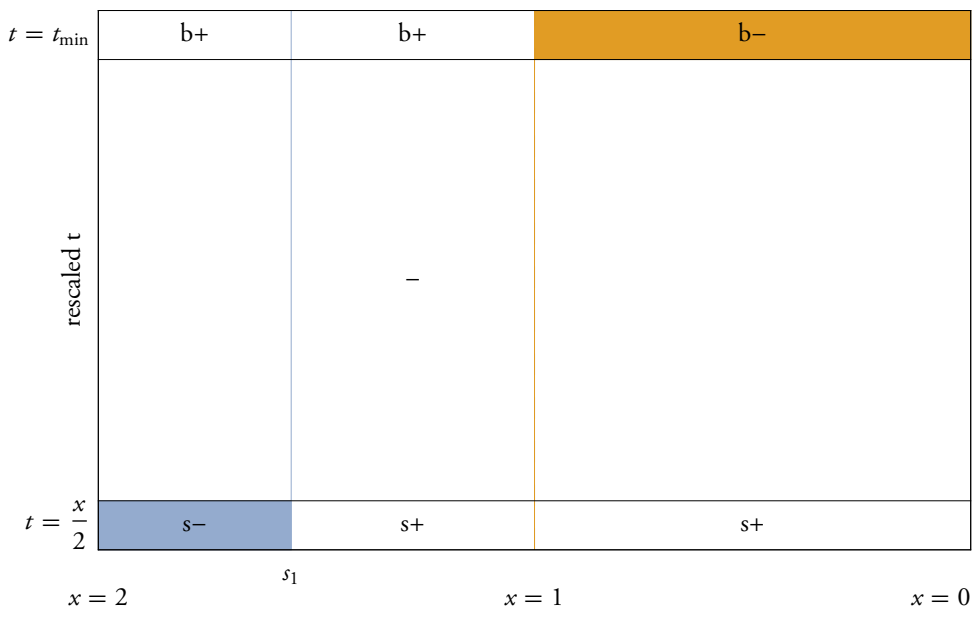

FIGURE A.204: prediction graphic for $r=2.8, n=7, m=2$ 


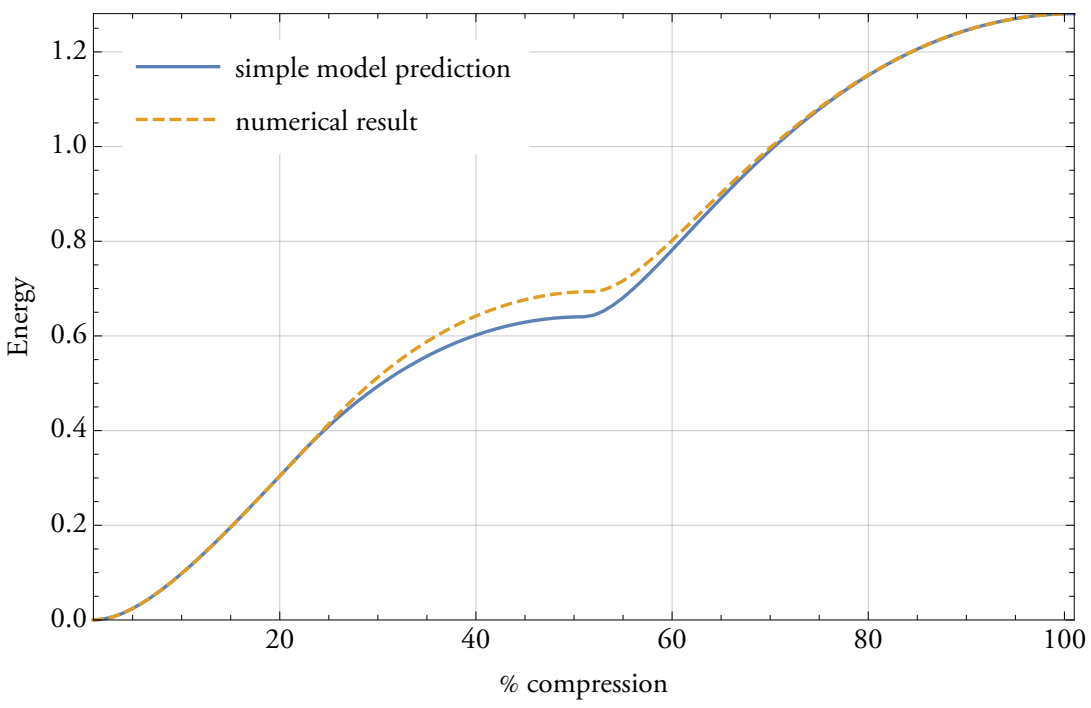

FIGURE A.205: simple energy for $r=2.8, n=8, m=2$

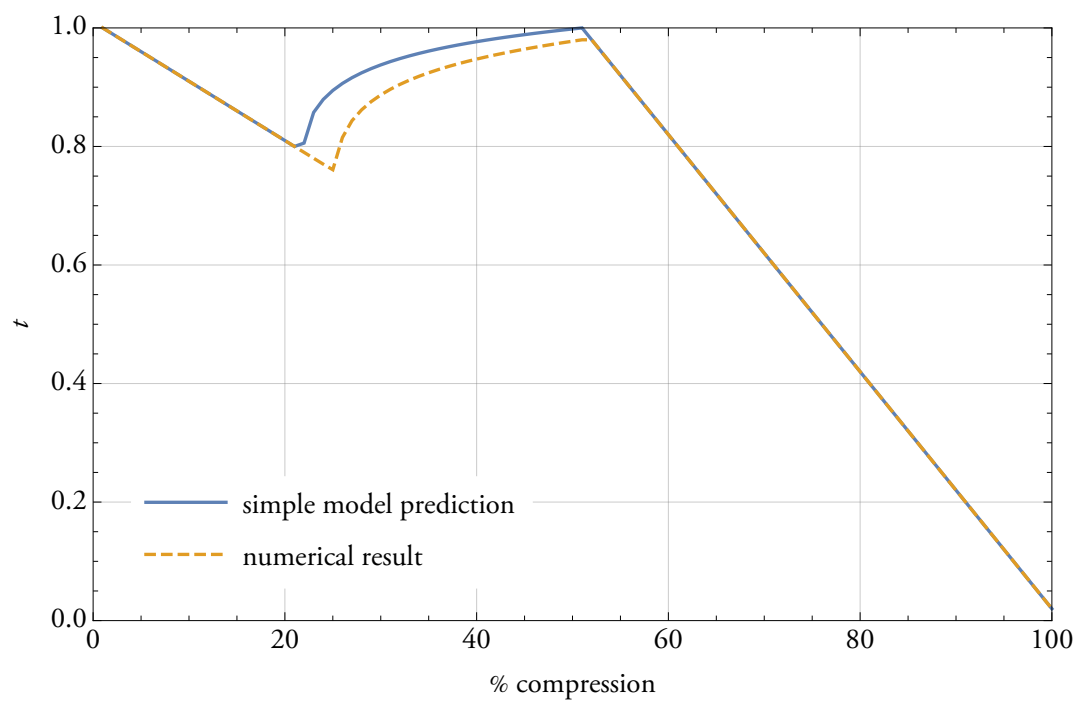

FIGURE A.206: intermediate layer height for $r=2.8, n=8, m=2$

$b_{1}$

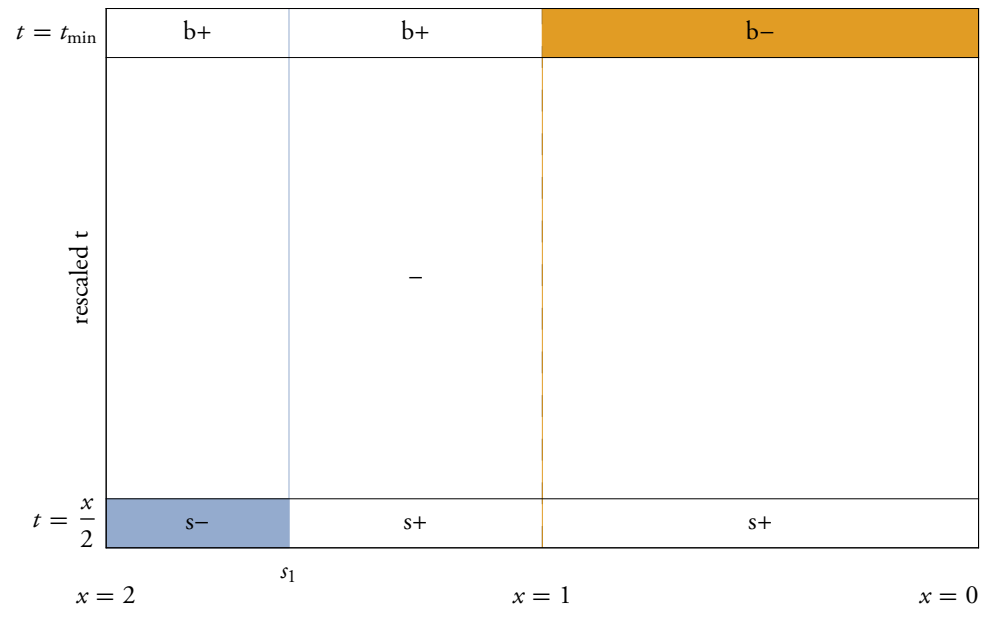

FIGURE A.207: prediction graphic for $r=2.8, n=8, m=2$ 


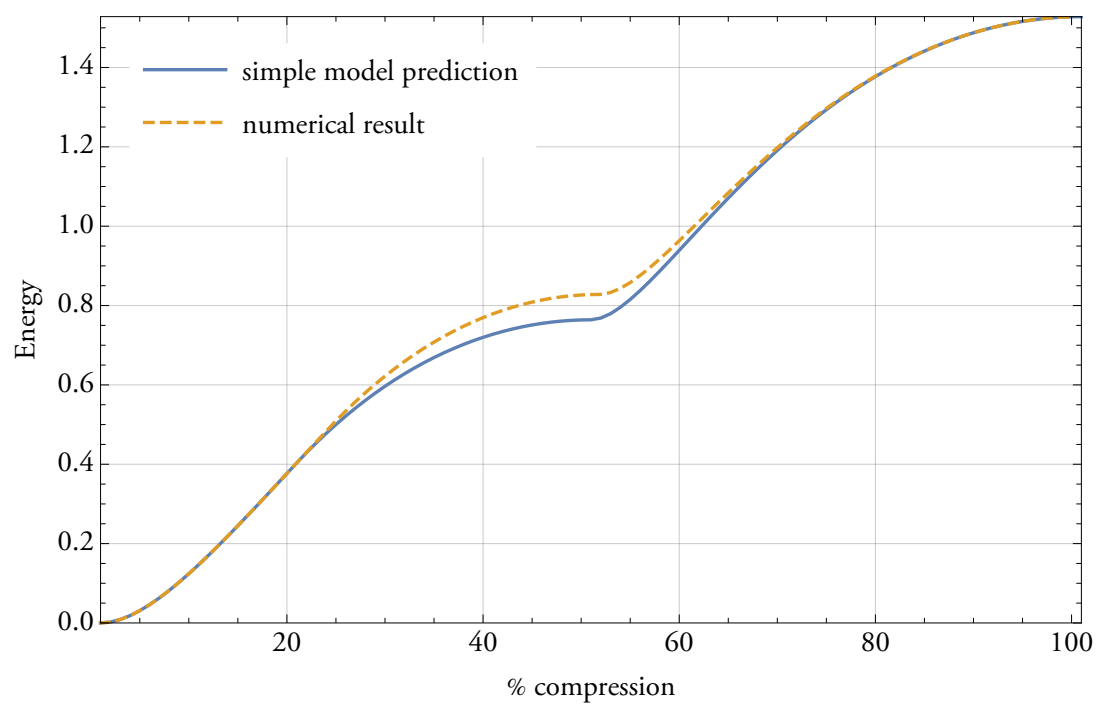

FIGURE A.208: simple energy for $r=2.8, n=9, m=2$

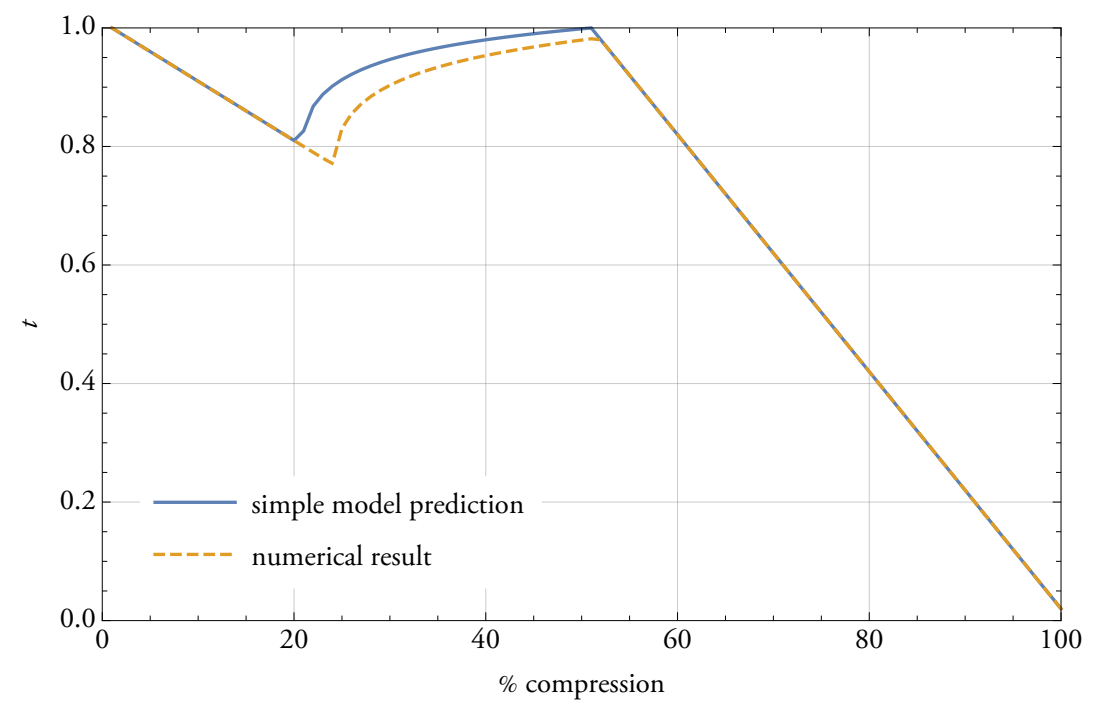

FIGURE A.209: intermediate layer height for $r=2.8, n=9, m=2$

$b_{1}$

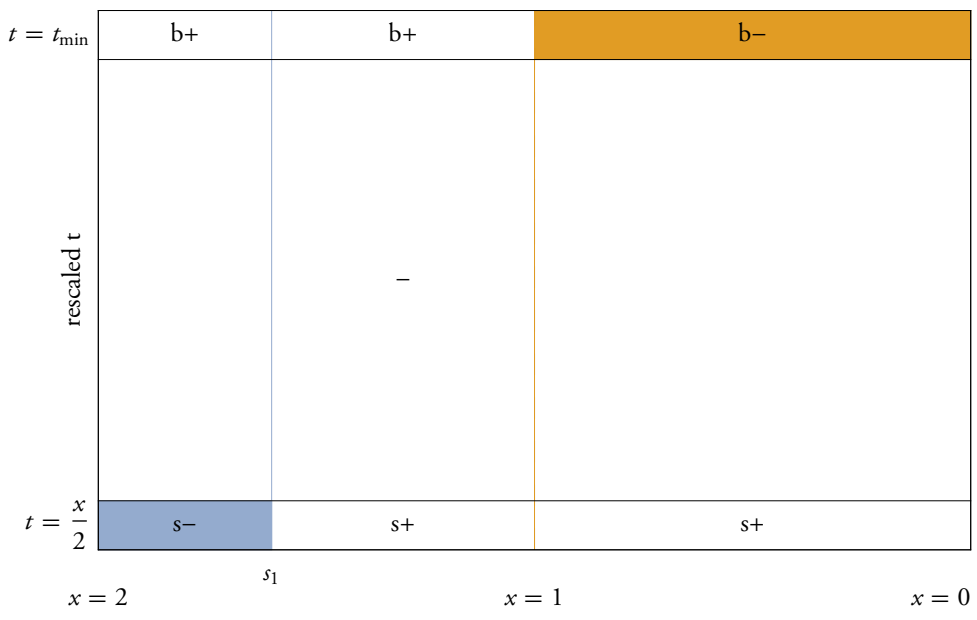

FIGURE A.210: prediction graphic for $r=2.8, n=9, m=2$ 


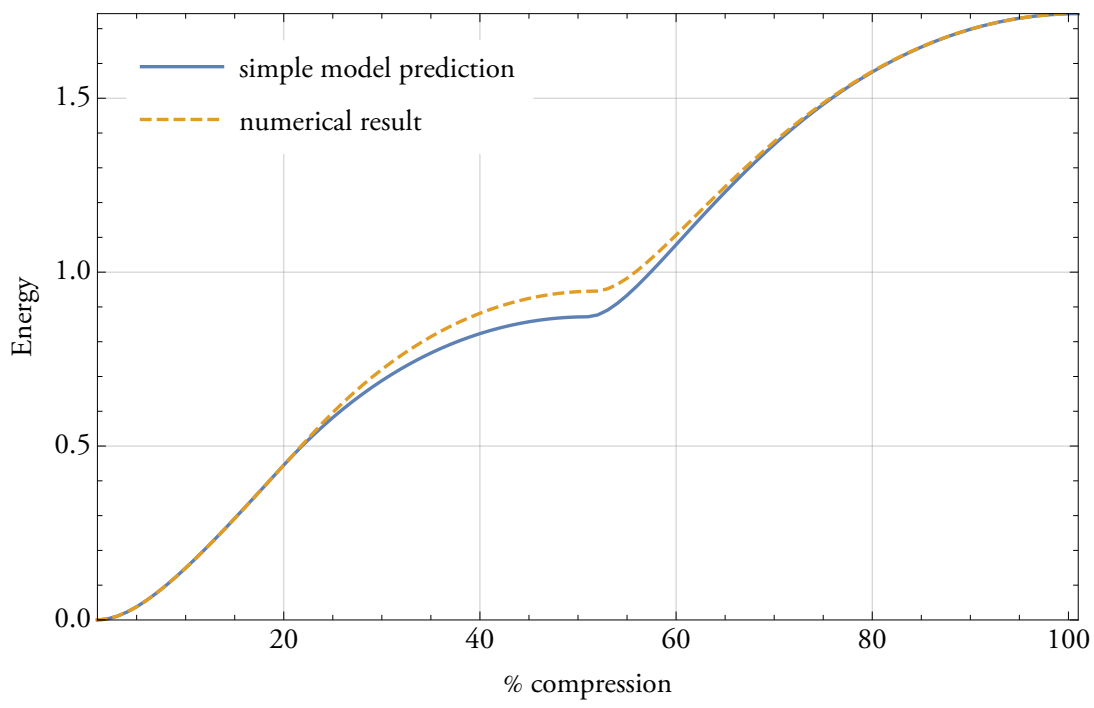

FIGURE A.211: simple energy for $r=2.8, n=10, m=2$

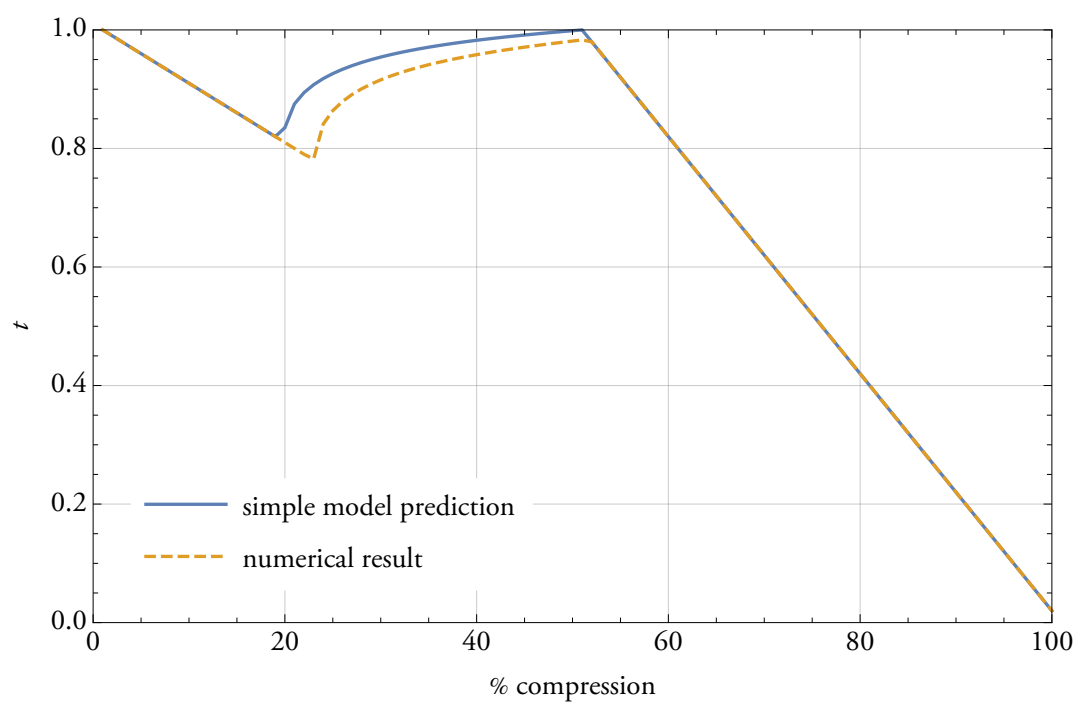

FIGURE A.212: intermediate layer height for $r=2.8, n=10, m=2$

$b_{1}$

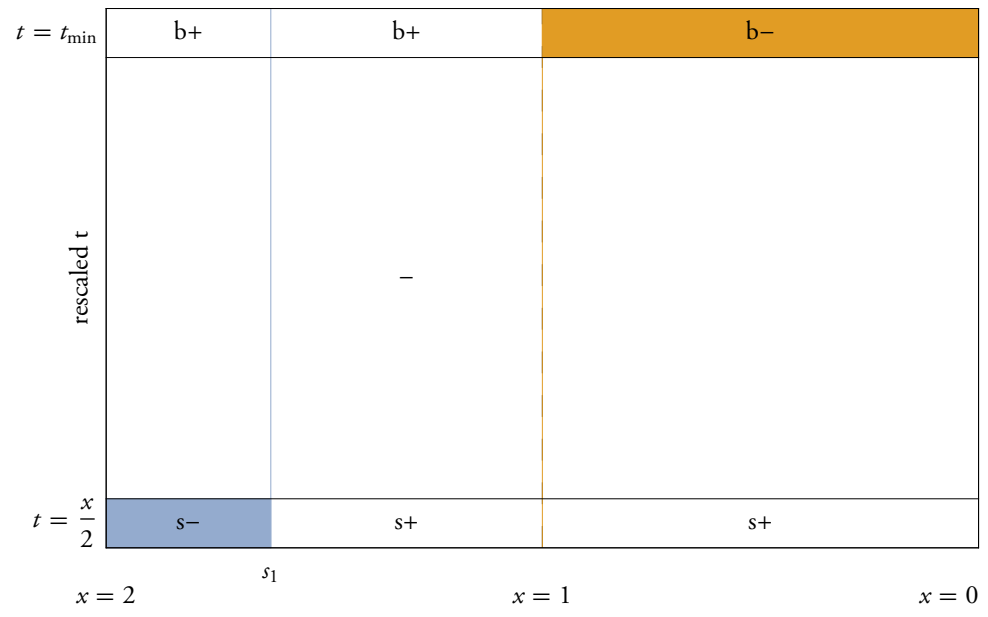

FIGURE A.213: prediction graphic for $r=2.8, n=10, m=2$ 


\section{B. Fold patterns}

This section includes fold patterns for the origami cylinders discussed in this thesis. 


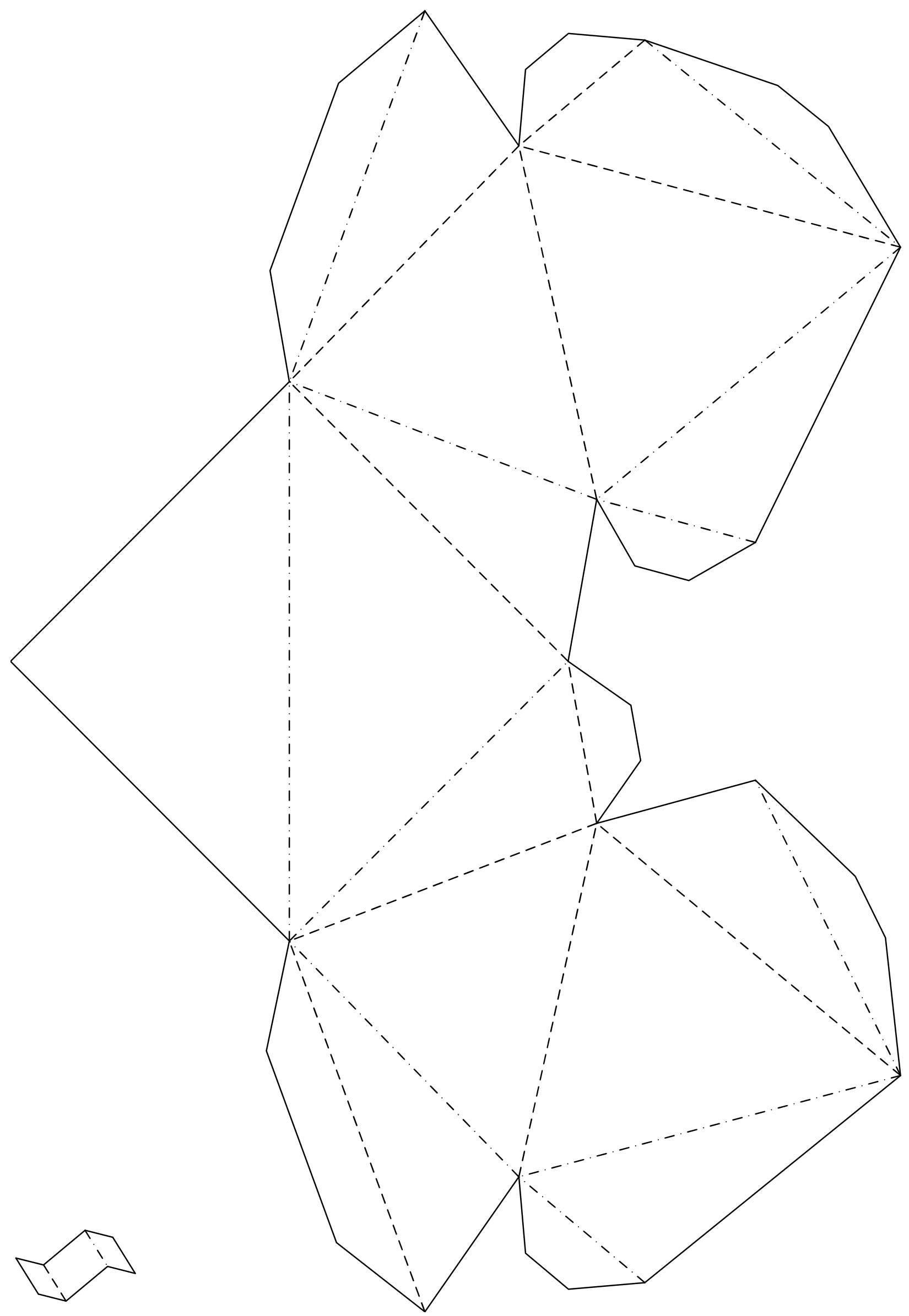

FIGURE B.1: Folding pattern for Steffen's polyhedron, the simplest continuously flexible rigid polyhedron. Obtained from [4] 
The Tachi-Miura

pattern

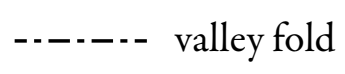

------ mountain fold

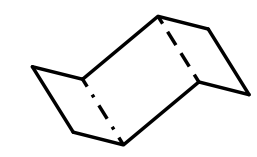

The slope $\alpha$ of the nonvertical lines can be chosen arbitrarily. This fold pattern shows a slope of $45^{\circ}$.

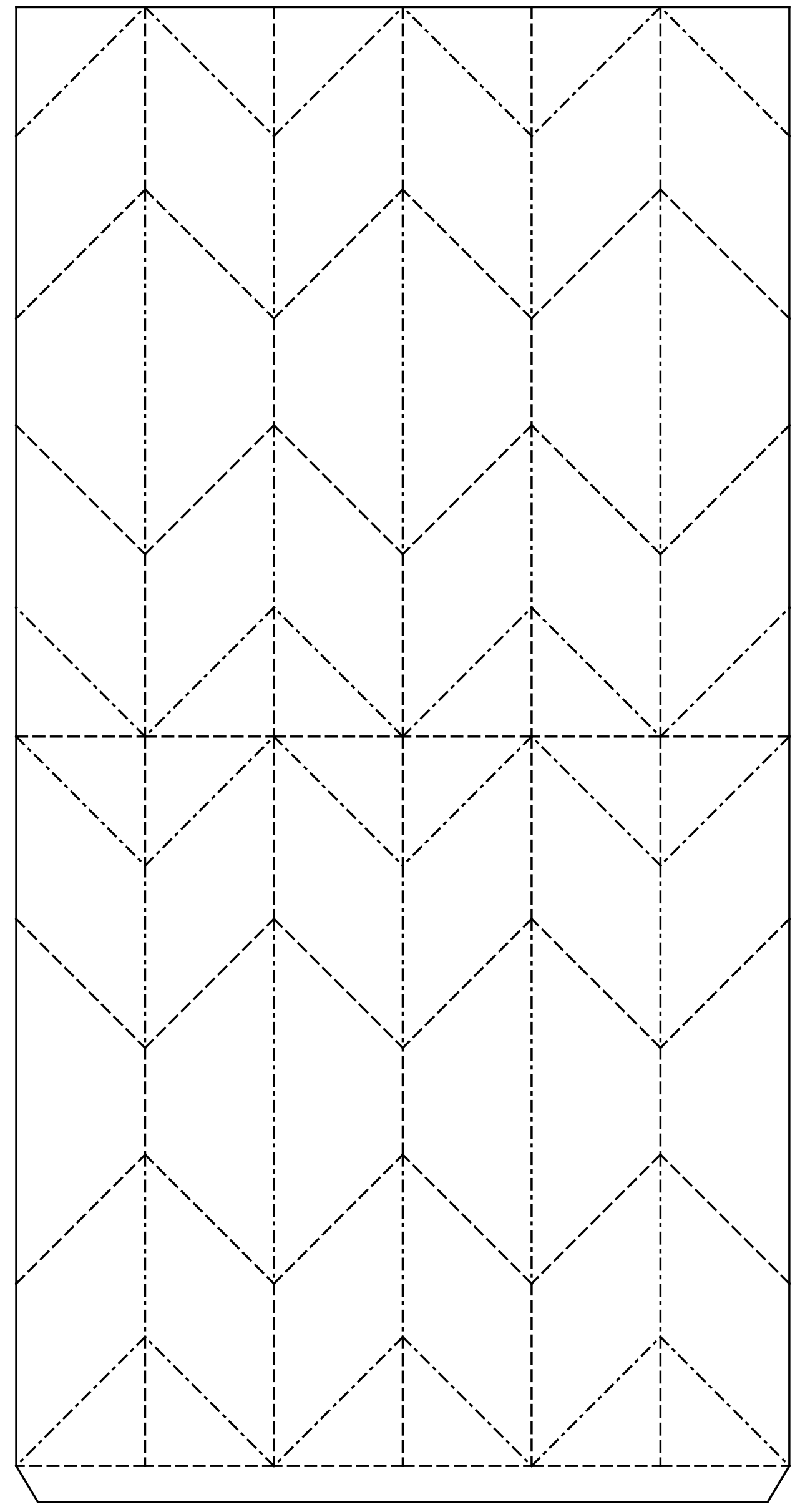

FIGURE B.2: Folding pattern for the Tachi-Miura pattern. More strips can be added by periodic horizontal repetition. 


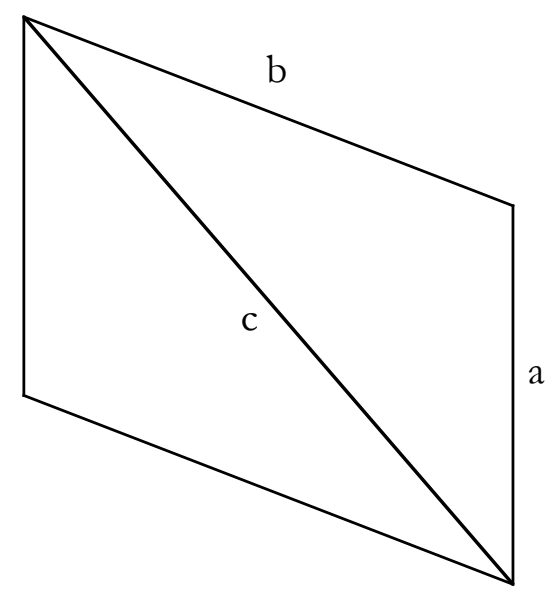

Find $a, b, c$ from $r / H, n, m$ via

$$
\begin{aligned}
a & =2 r \sin \frac{\pi}{n} \\
b & =\sqrt{4 r^{2} \sin \left(\frac{H}{2 m}\right)^{2}+\frac{H^{2}}{m^{2}}} \\
c & =\sqrt{4 r^{2} \sin \left(\frac{\pi}{n} \frac{H}{2 m}\right)^{2}+\frac{H^{2}}{m^{2}}}
\end{aligned}
$$

valley fold

mountain fold

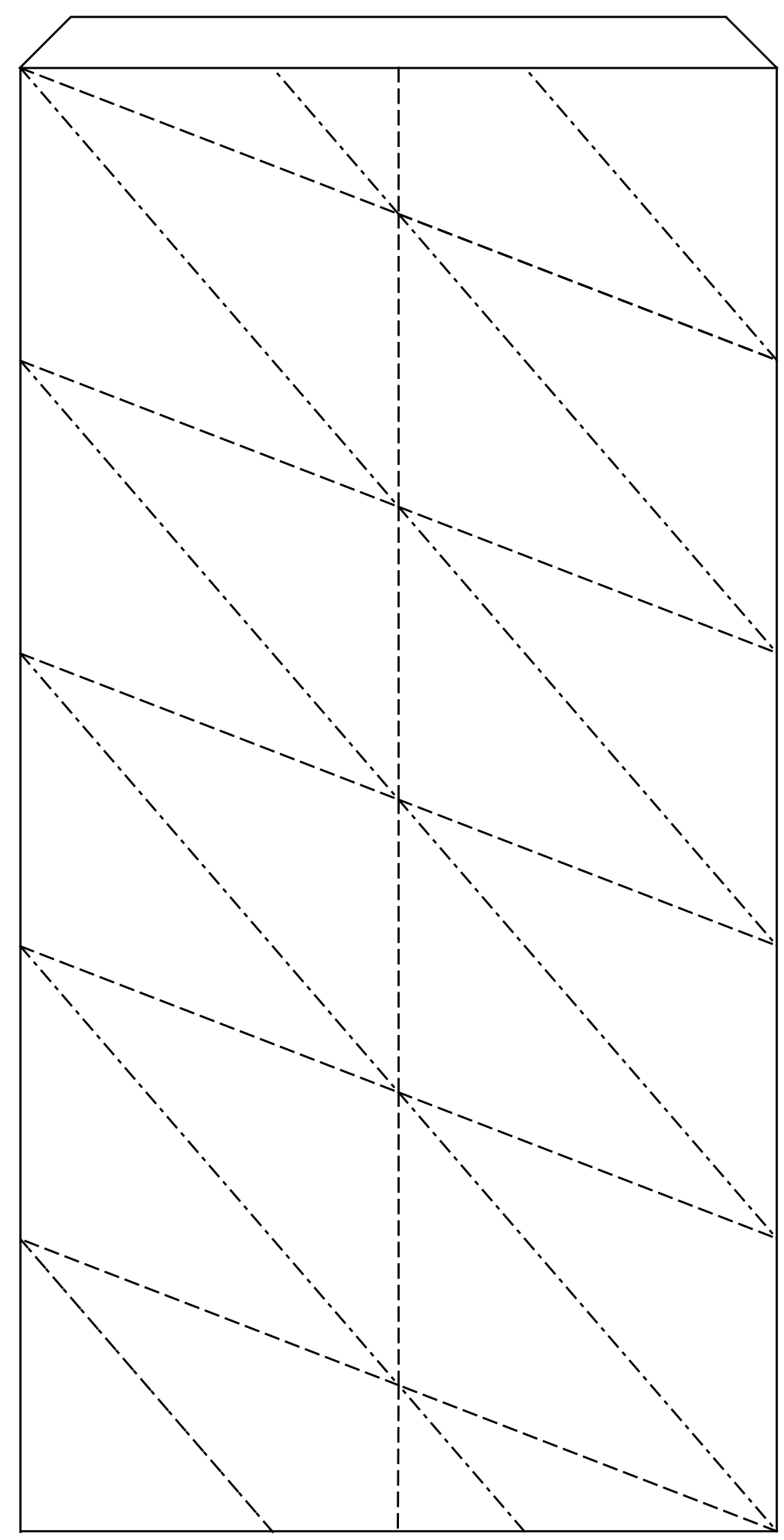

FigURE B.3: Folding pattern for the Guest and Pellgrino type cylinders, here depicted with $r=1.0, n=6, m=2$. 


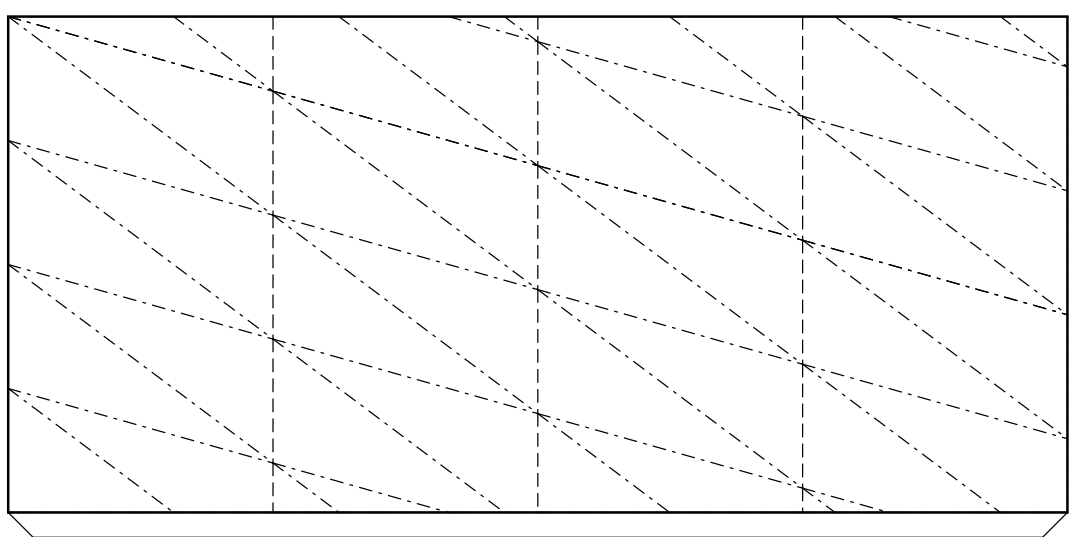

FIGURE B.4: Fold pattern for the $r=0.4, n=4$ Guest and Pellegrino cylinder

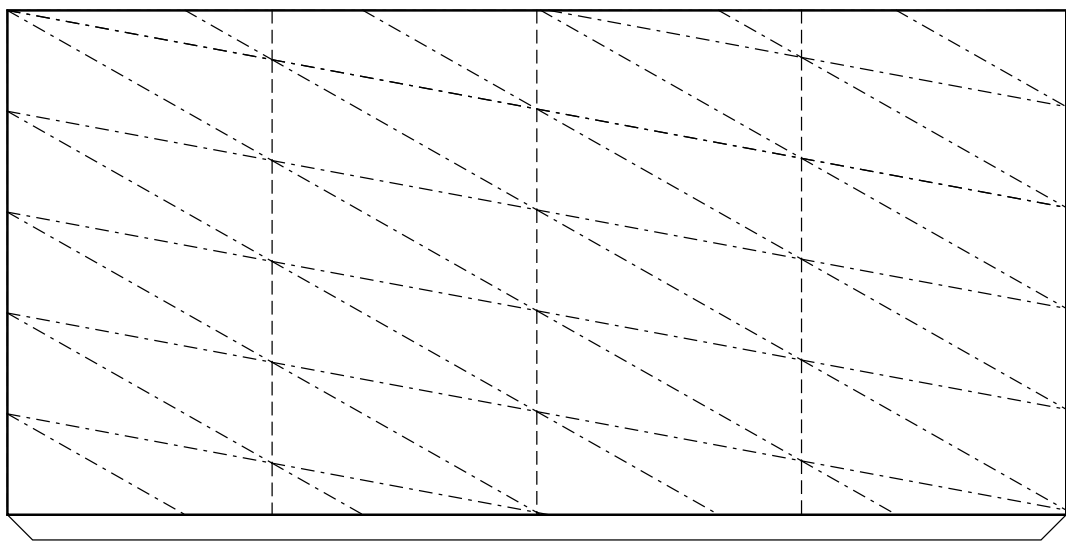

FIGURE B.5: Fold pattern for the $r=0.4, n=5$ Guest and Pellegrino cylinder

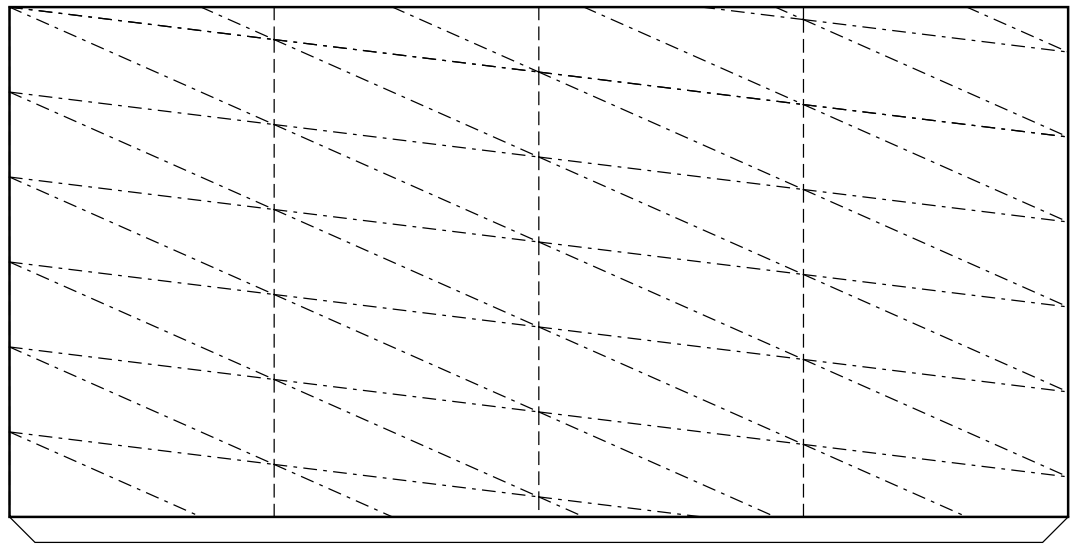

FIGURE B.6: Fold pattern for the $r=0.4, n=6$ Guest and Pellegrino cylinder 


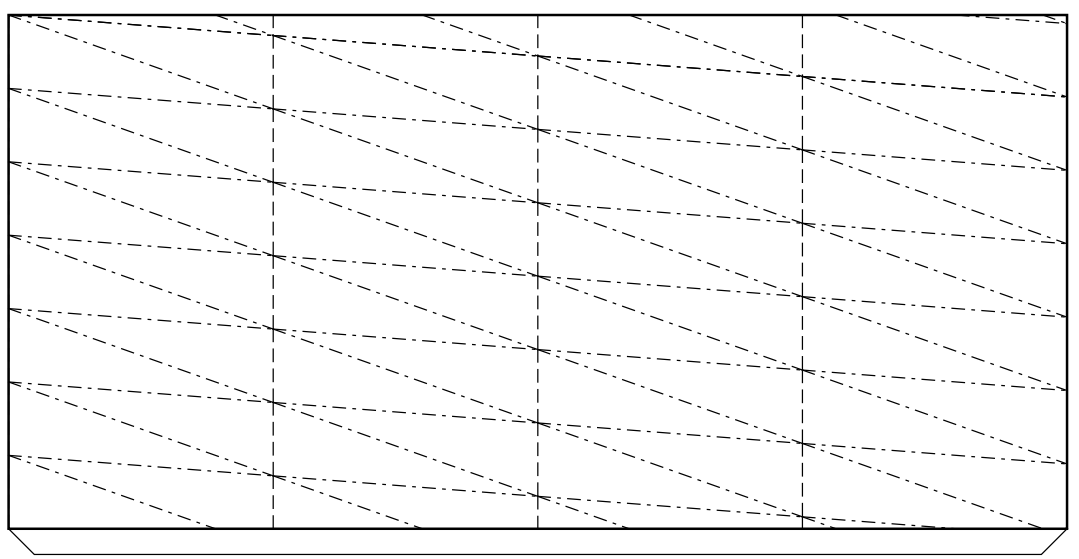

FiguRE B.7: Fold pattern for the $r=0.4, n=7$ Guest and Pellegrino cylinder

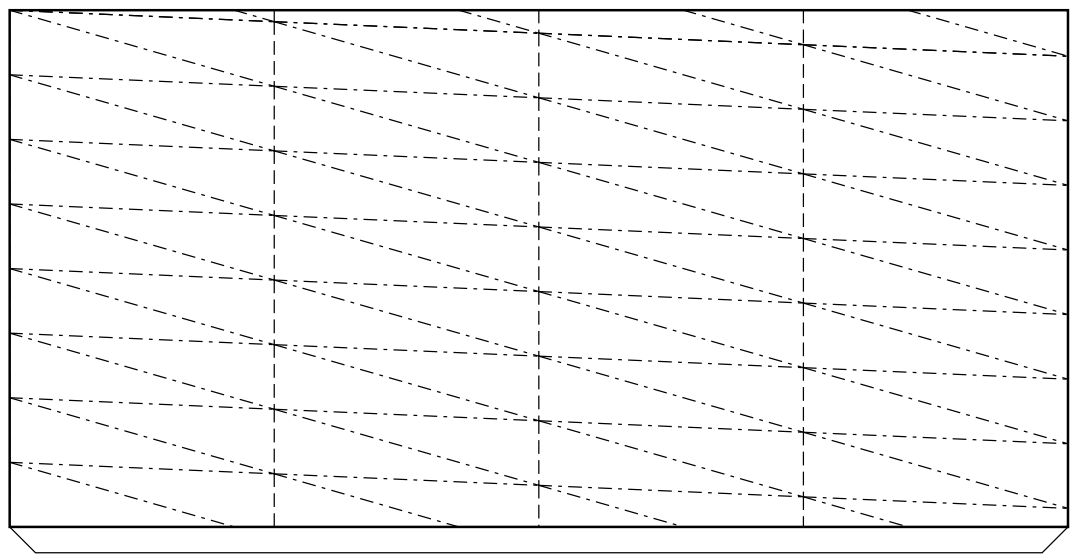

FigURE B.8: Fold pattern for the $r=0.4, n=8$ Guest and Pellegrino cylinder

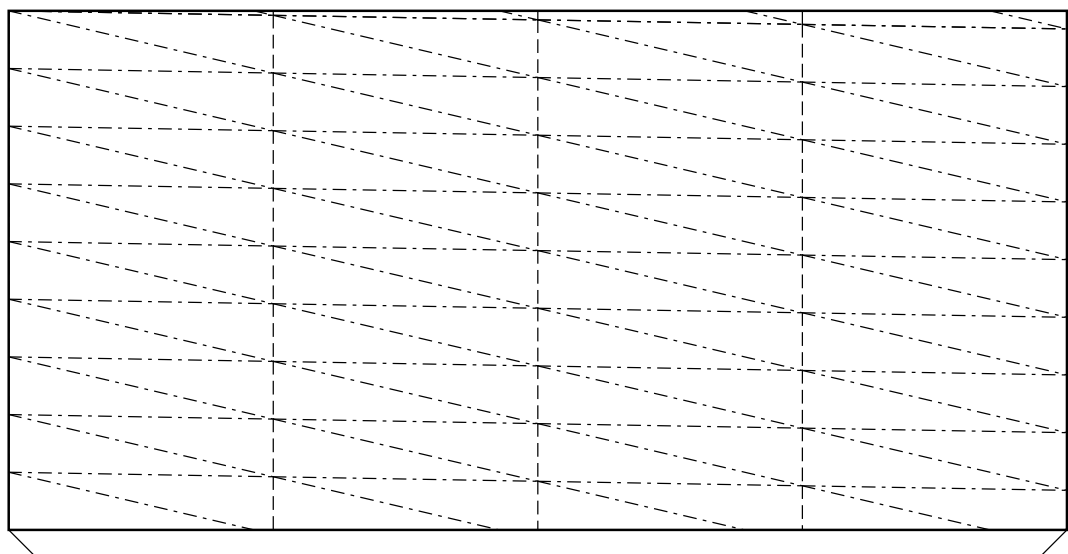

FigURE B.9: Fold pattern for the $r=0.4, n=9$ Guest and Pellegrino cylinder 


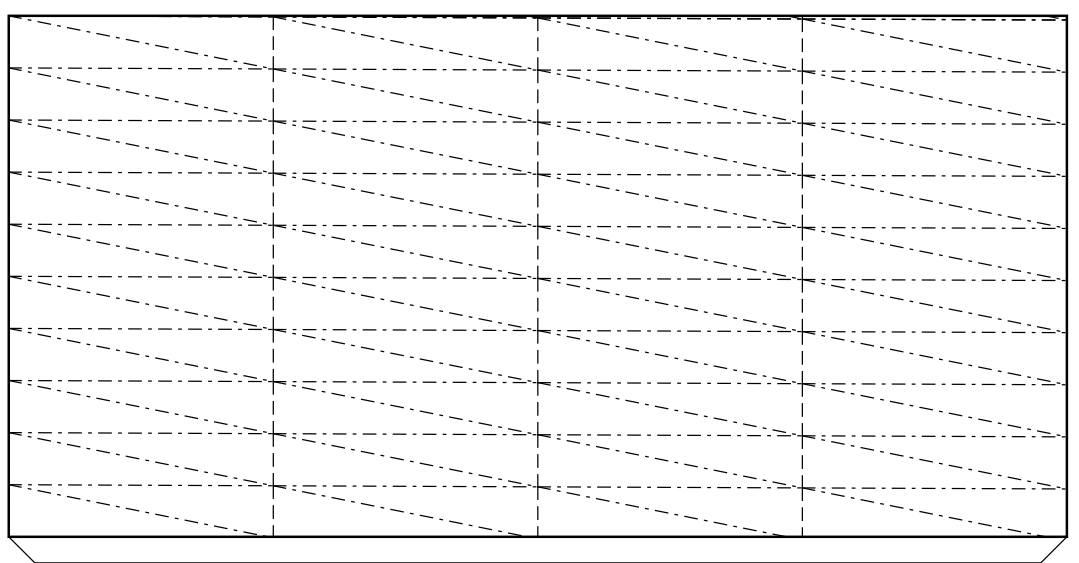

FIGURE B.10: Fold pattern for the $r=0.4, n=10$ Guest and Pellegrino cylinder

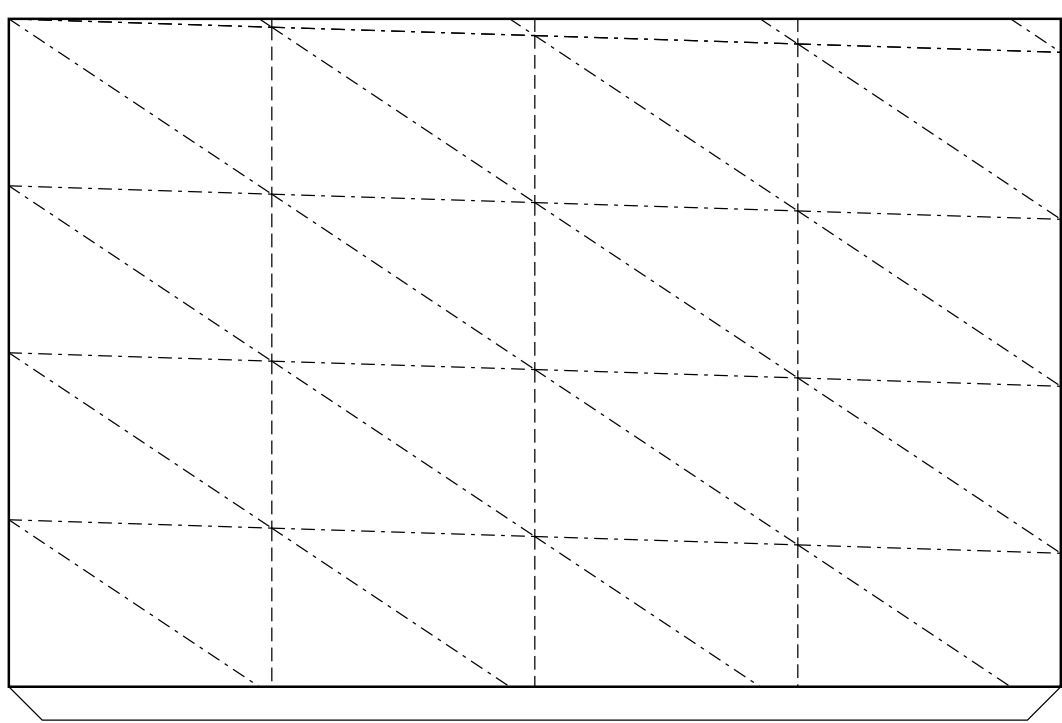

FIGURE B.11: Fold pattern for the $r=0.6, n=4$ Guest and Pellegrino cylinder

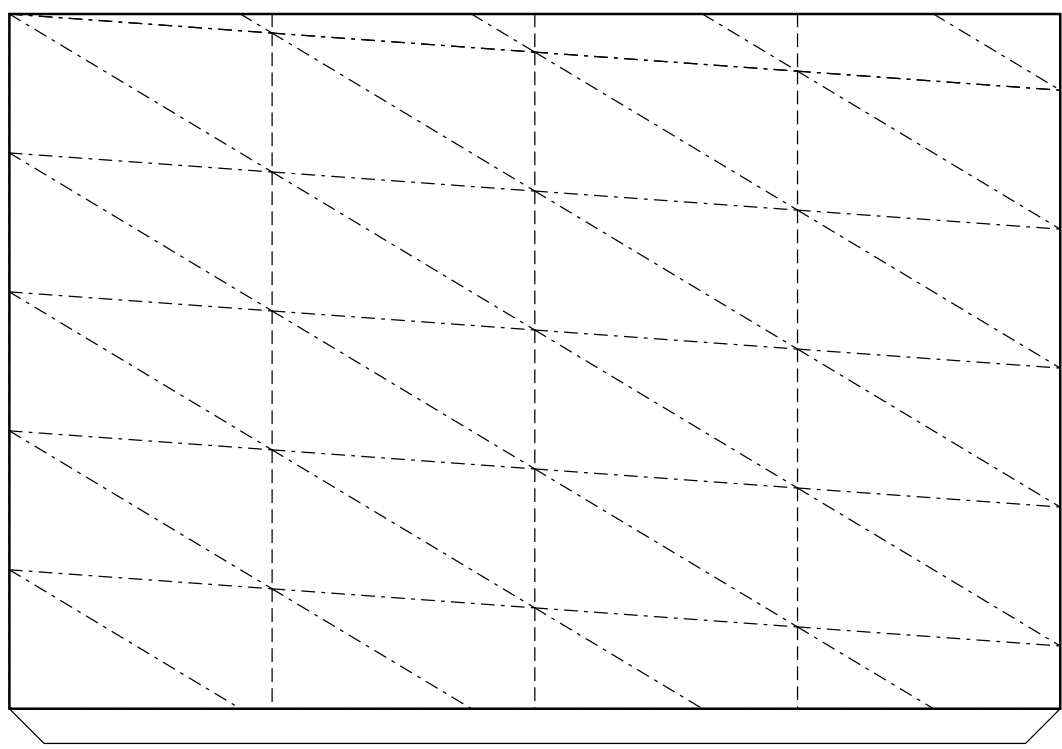

FIGURE B.12: Fold pattern for the $r=0.6, n=5$ Guest and Pellegrino cylinder 


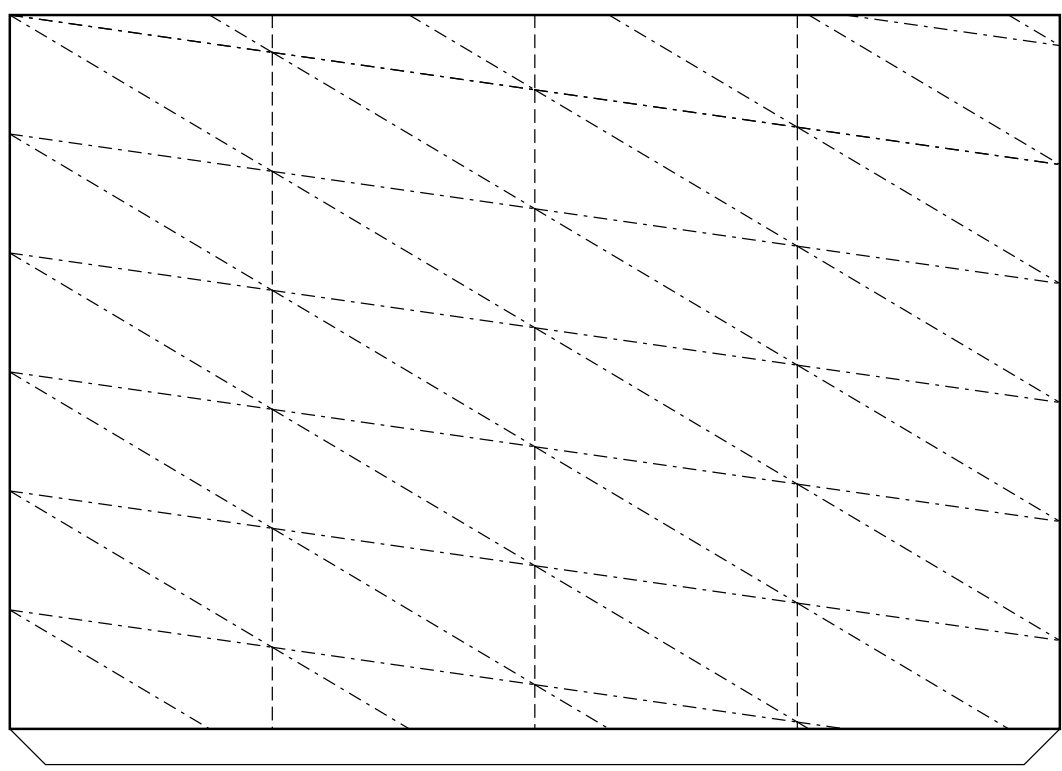

FIGURE B.13: Fold pattern for the $r=0.6, n=6$ Guest and Pellegrino cylinder

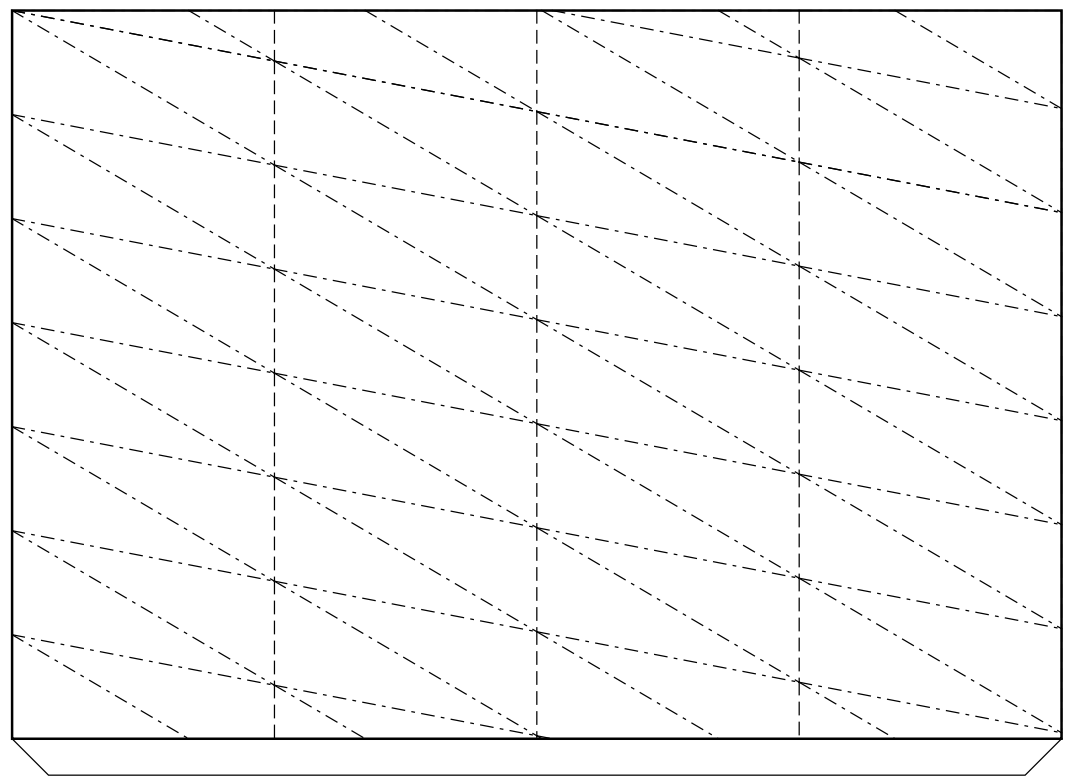

FIGURE B.14: Fold pattern for the $r=0.6, n=7$ Guest and Pellegrino cylinder 


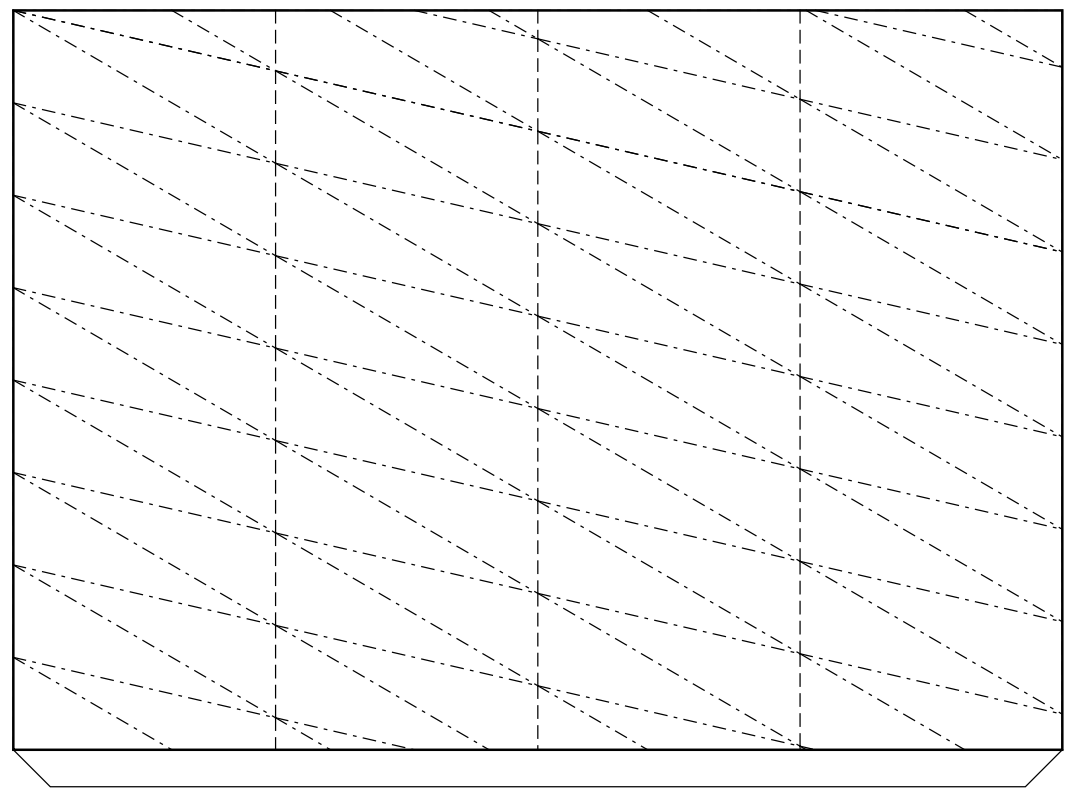

FIGURE B.15: Fold pattern for the $r=0.6, n=8$ Guest and Pellegrino cylinder

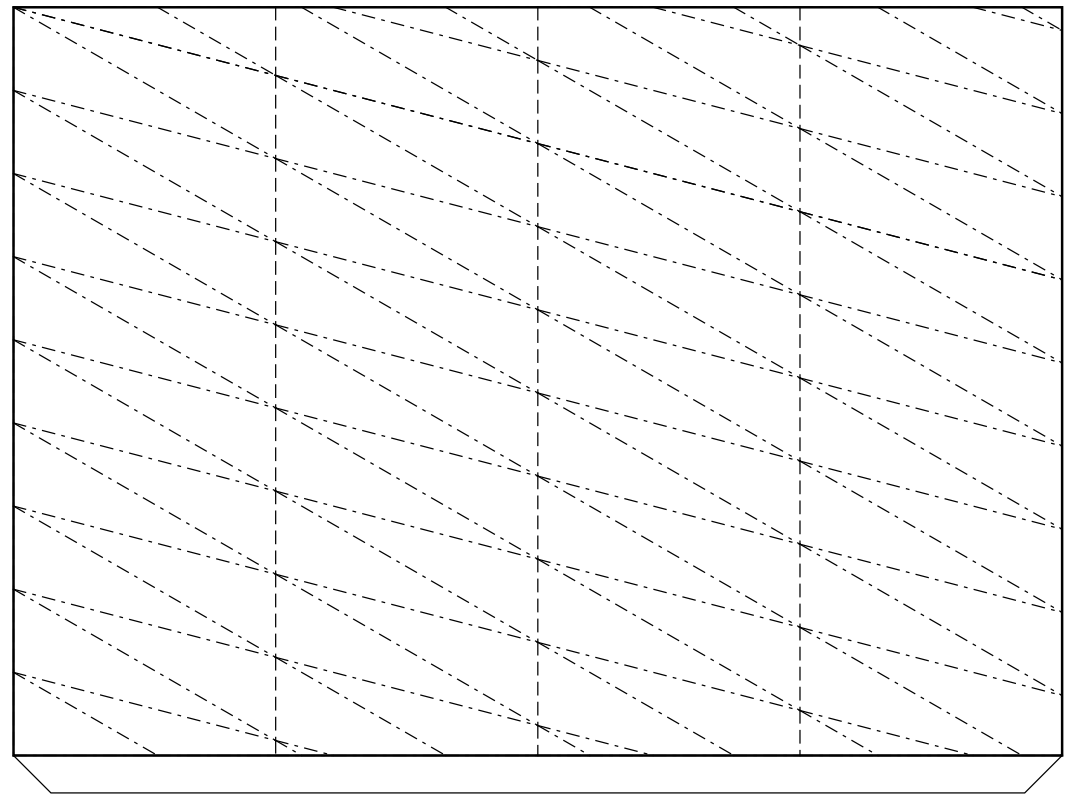

FIGURE B.16: Fold pattern for the $r=0.6, n=9$ Guest and Pellegrino cylinder 


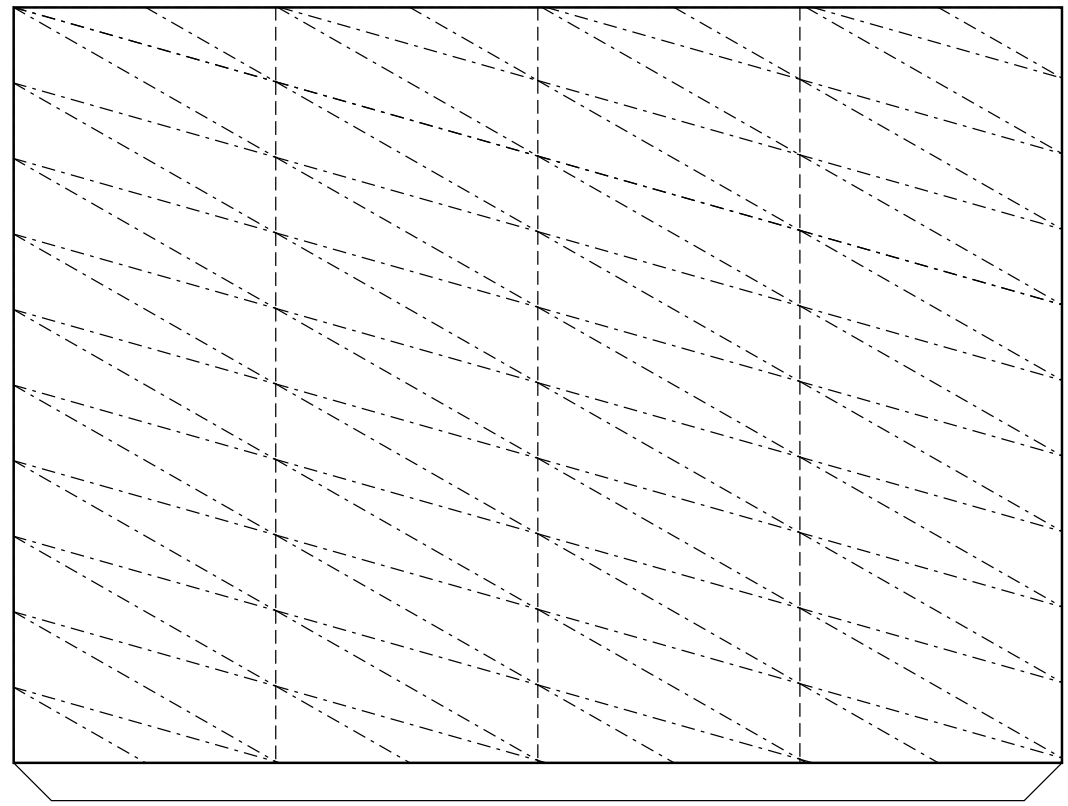

FIGURE B.17: Fold pattern for the $r=0.6, n=10$ Guest and Pellegrino cylinder

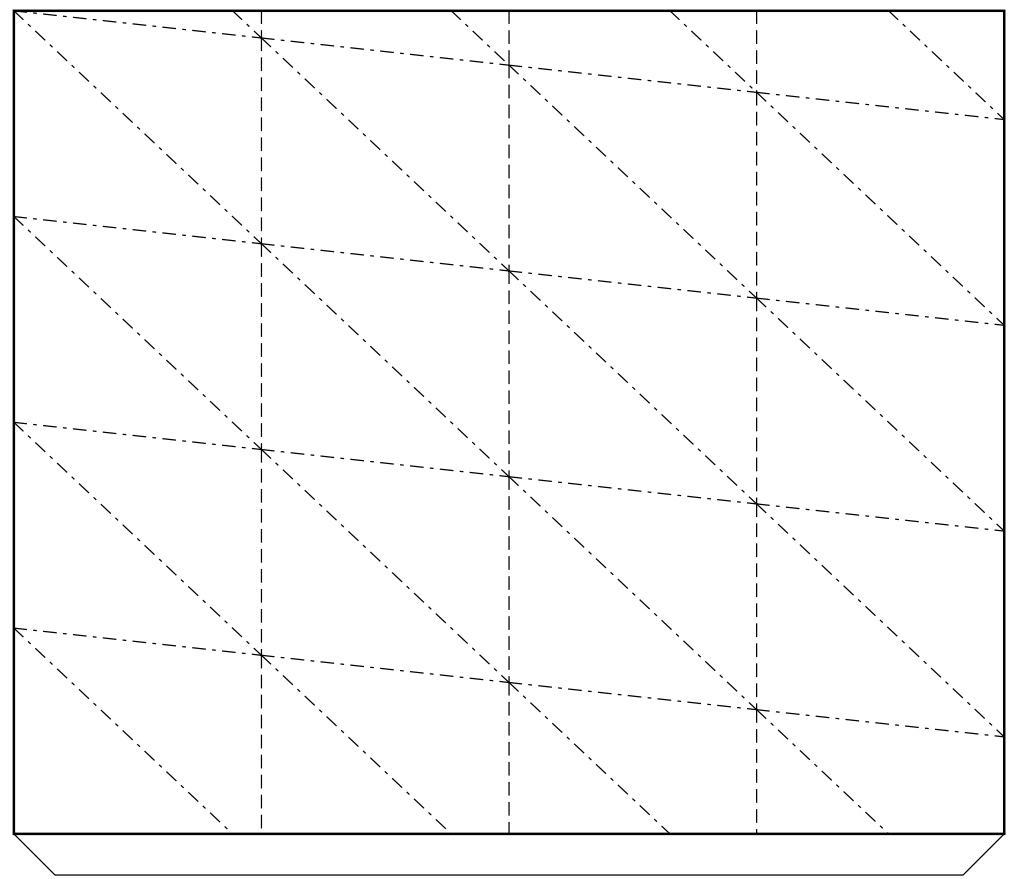

FIGURE B.18: Fold pattern for the $r=0.8, n=4$ Guest and Pellegrino cylinder 


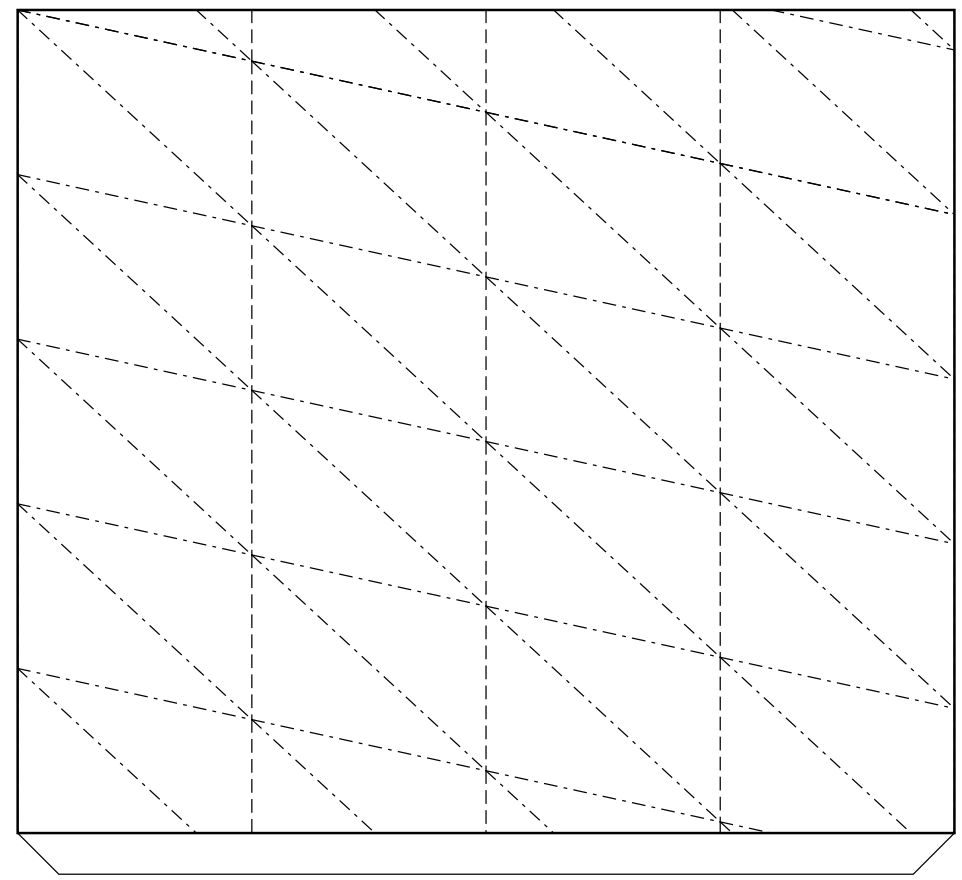

FIGURE B.19: Fold pattern for the $r=0.8, n=5$ Guest and Pellegrino cylinder

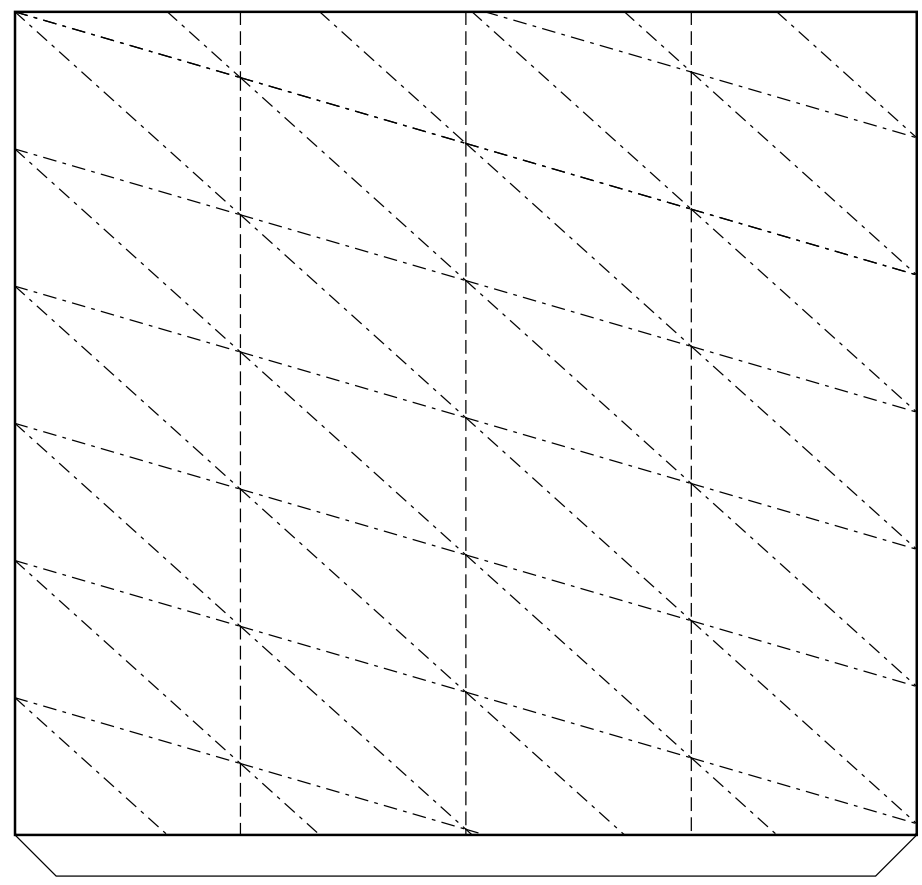

FIGURE B.20: Fold pattern for the $r=0.8, n=6$ Guest and Pellegrino cylinder 


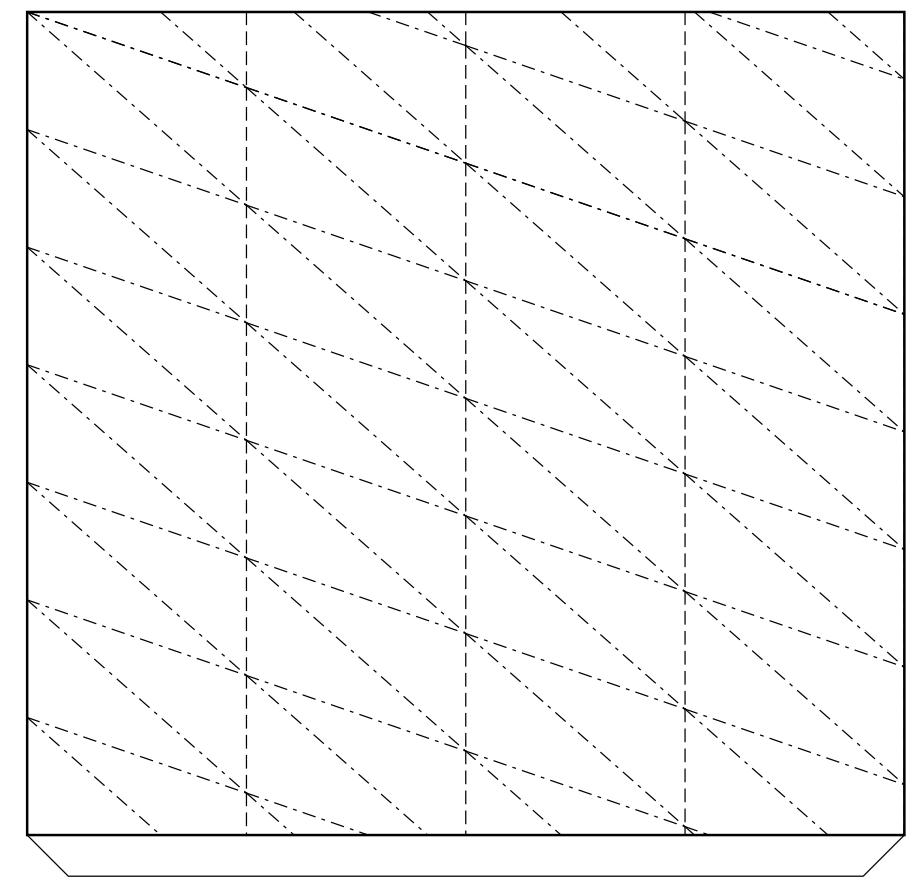

FIGURE B.21: Fold pattern for the $r=0.8, n=7$ Guest and Pellegrino cylinder

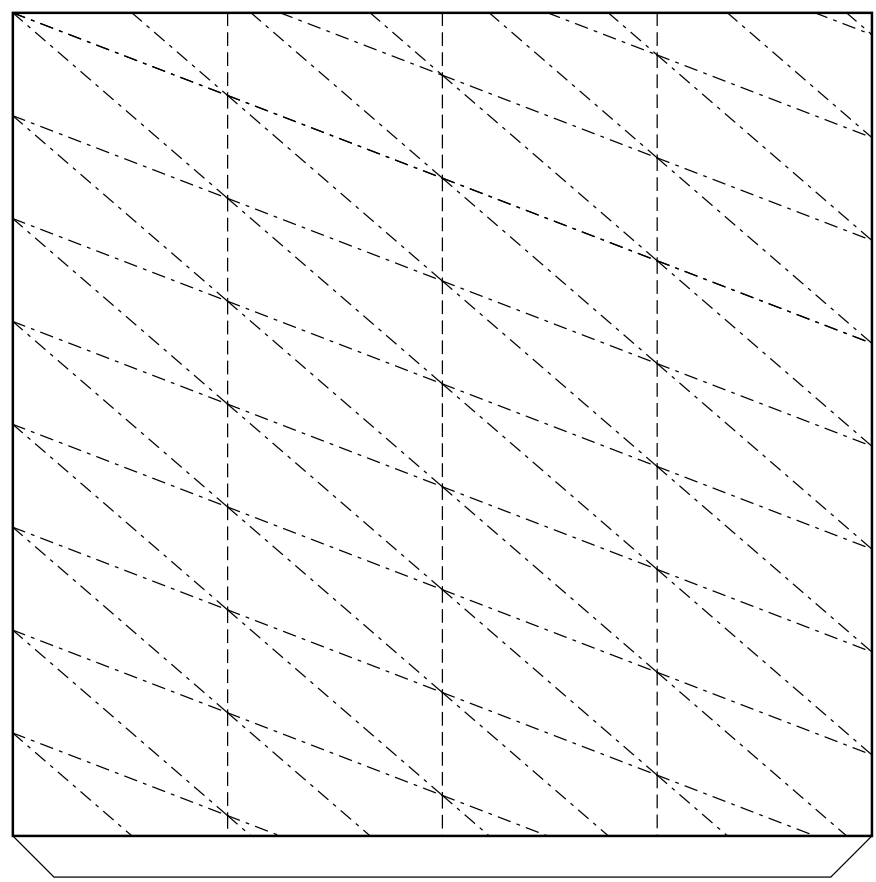

FIGURE B.22: Fold pattern for the $r=0.8, n=8$ Guest and Pellegrino cylinder 


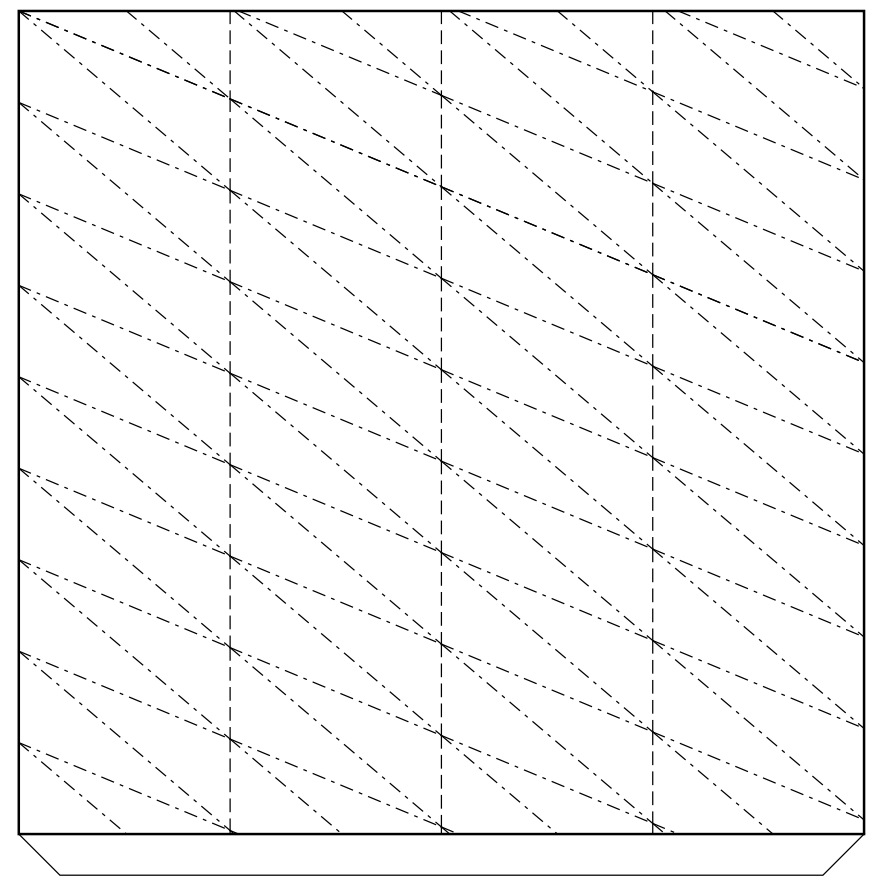

FIGURE B.23: Fold pattern for the $r=0.8, n=9$ Guest and Pellegrino cylinder

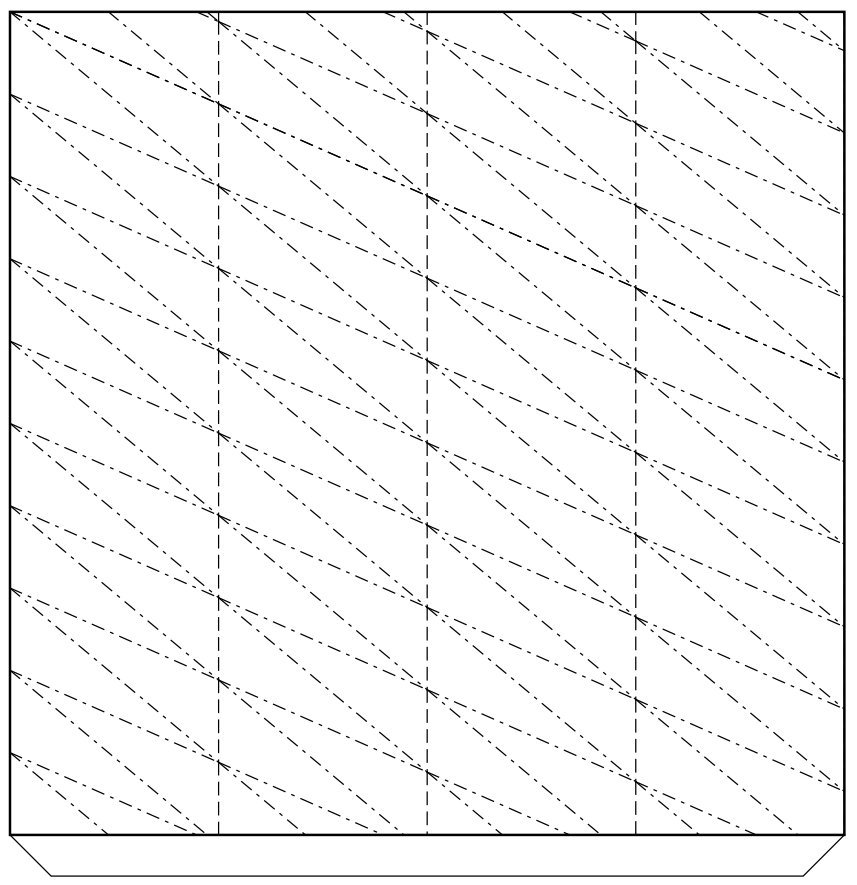

FIGURE B.24: Fold pattern for the $r=0.8, n=10$ Guest and Pellegrino cylinder 


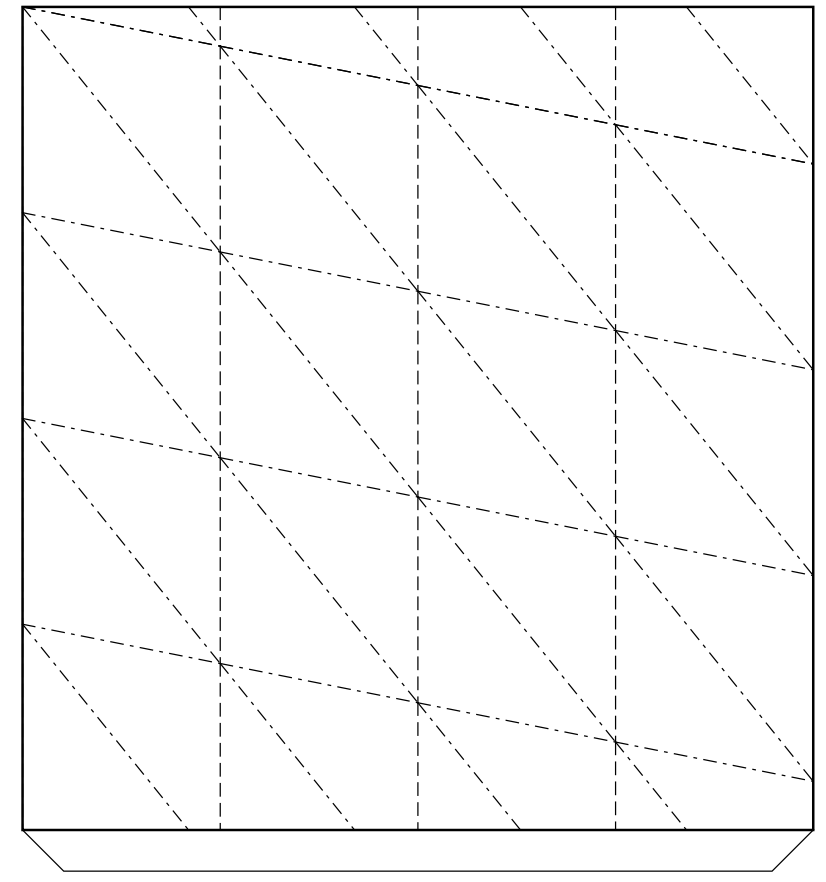

FIGURE B.25: Fold pattern for the $r=1 ., n=4$ Guest and Pellegrino cylinder

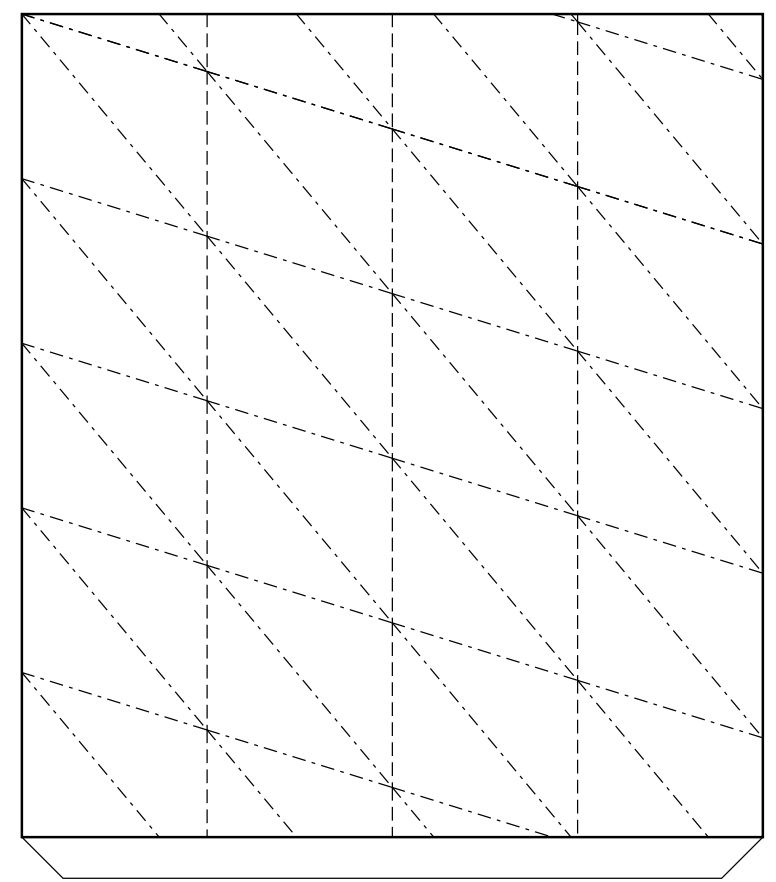

FiguRE B.26: Fold pattern for the $r=1 ., n=5$ Guest and Pellegrino cylinder 


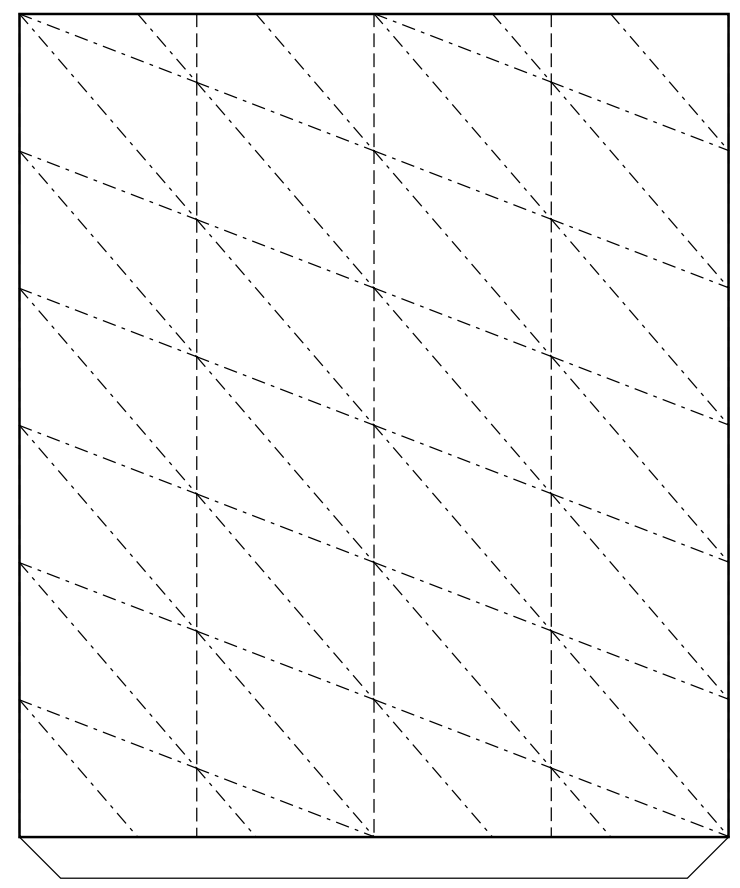

FIGURE B.27: Fold pattern for the $r=1 ., n=6$ Guest and Pellegrino cylinder

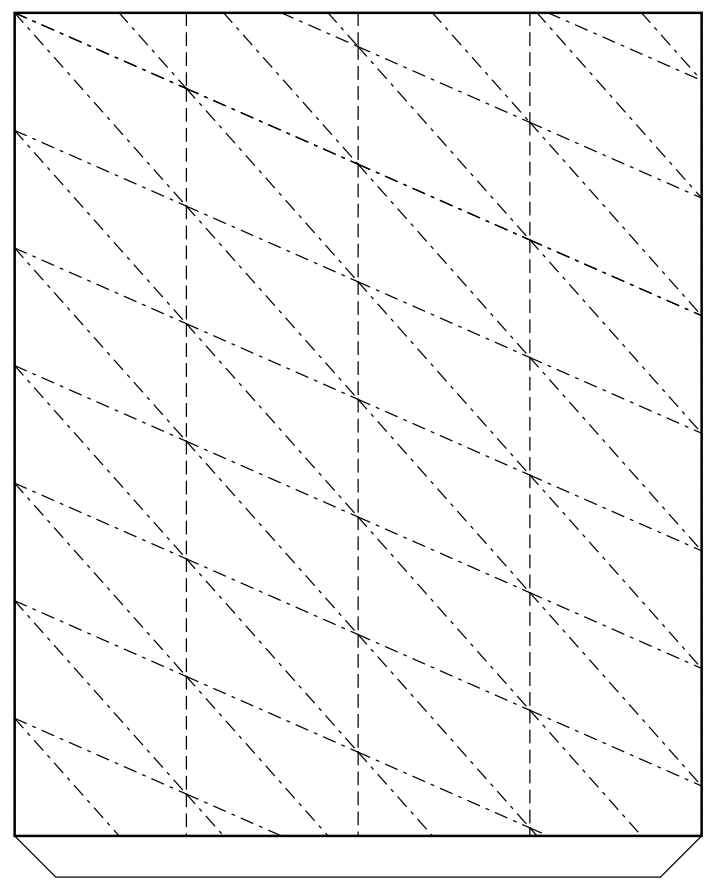

FIGURE B.28: Fold pattern for the $r=1 ., n=7$ Guest and Pellegrino cylinder 


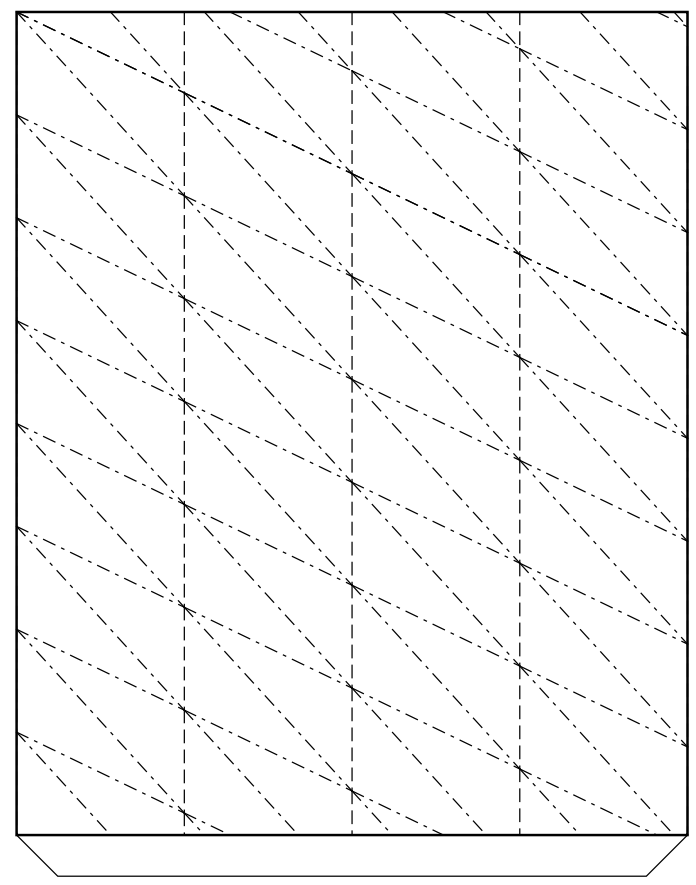

FIGURE B.29: Fold pattern for the $r=1 ., n=8$ Guest and Pellegrino cylinder

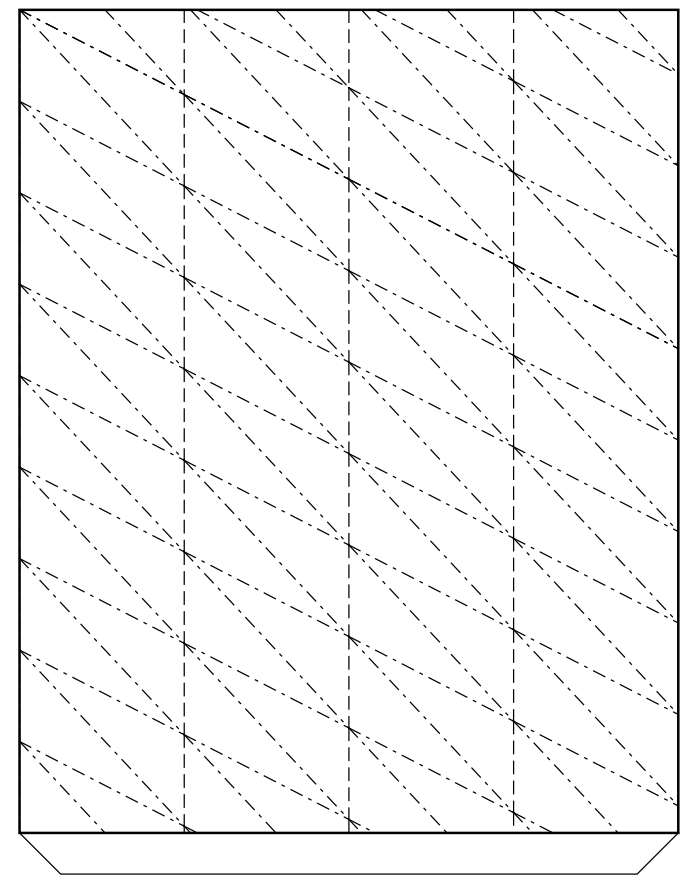

FiguRE B.30: Fold pattern for the $r=1 ., n=9$ Guest and Pellegrino cylinder 


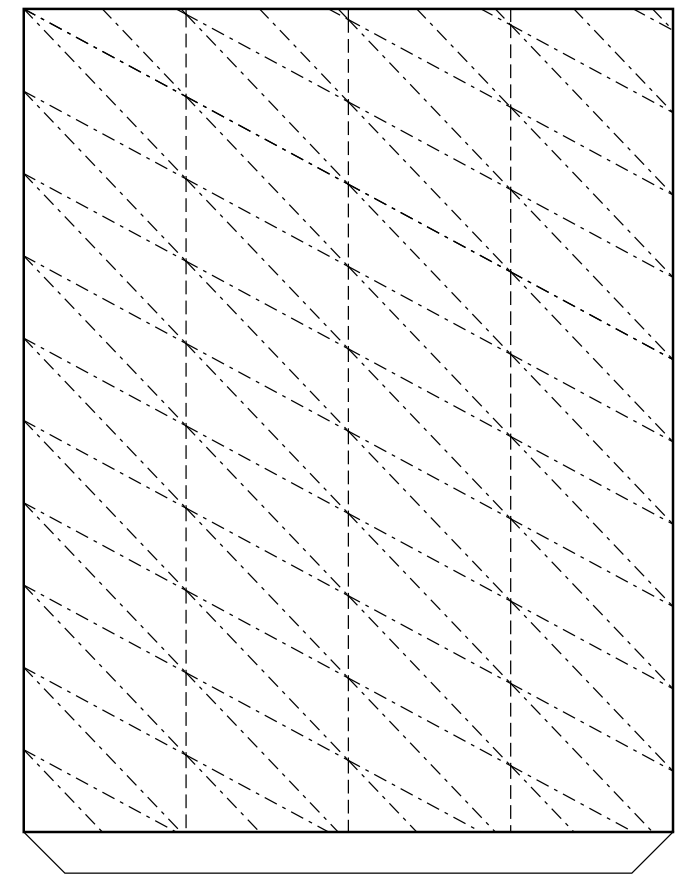

FIGURE B.31: Fold pattern for the $r=1 ., n=10$ Guest and Pellegrino cylinder

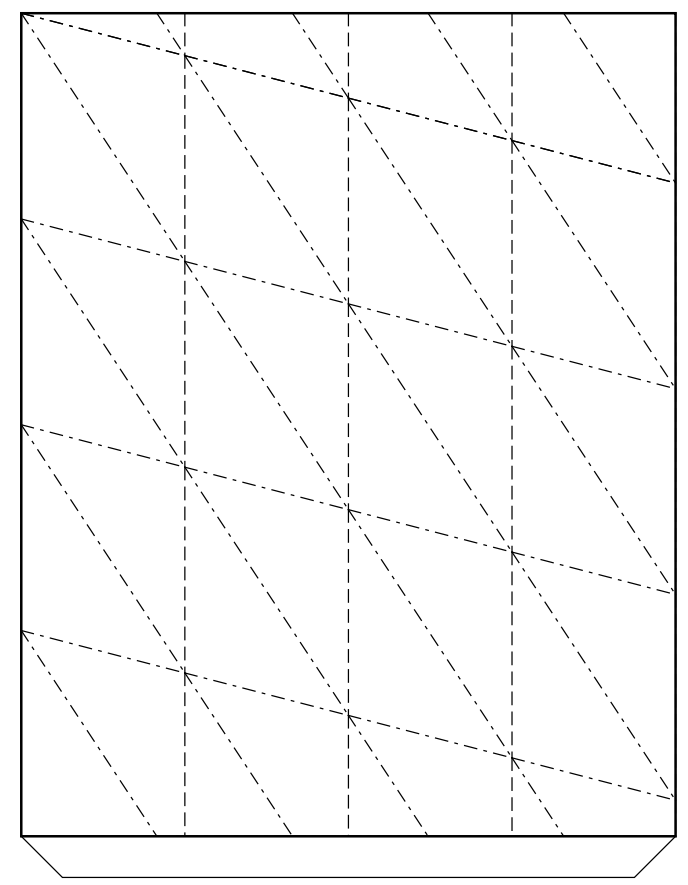

FIGURE B.32: Fold pattern for the $r=1.2, n=4$ Guest and Pellegrino cylinder 


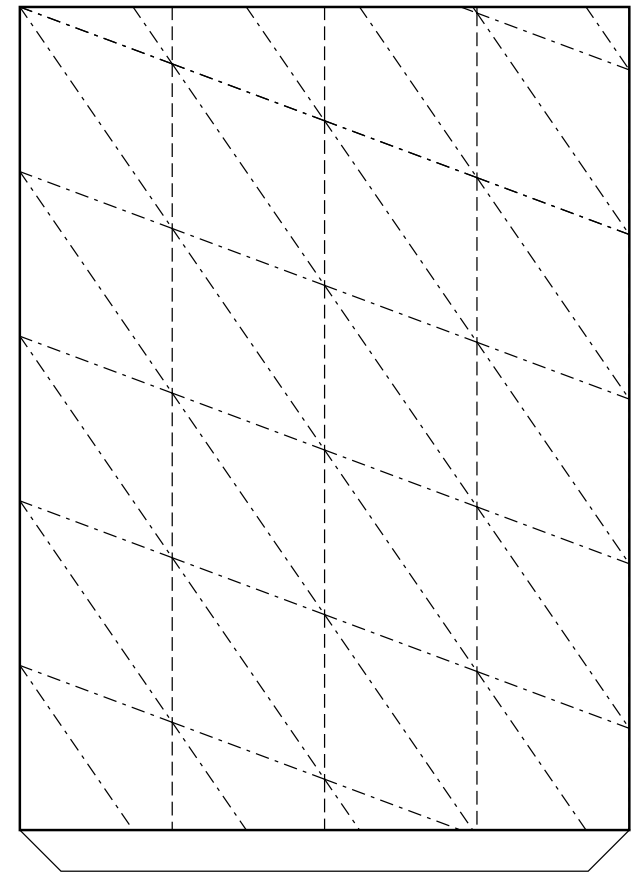

FIGURE B.33: Fold pattern for the $r=1.2, n=5$ Guest and Pellegrino cylinder

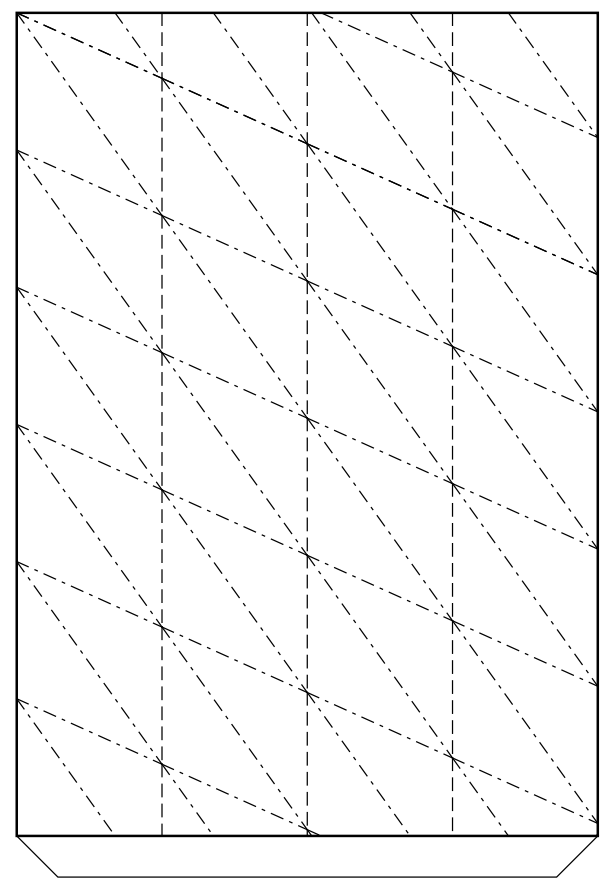

FIGURE B.34: Fold pattern for the $r=1.2, n=6$ Guest and Pellegrino cylinder 


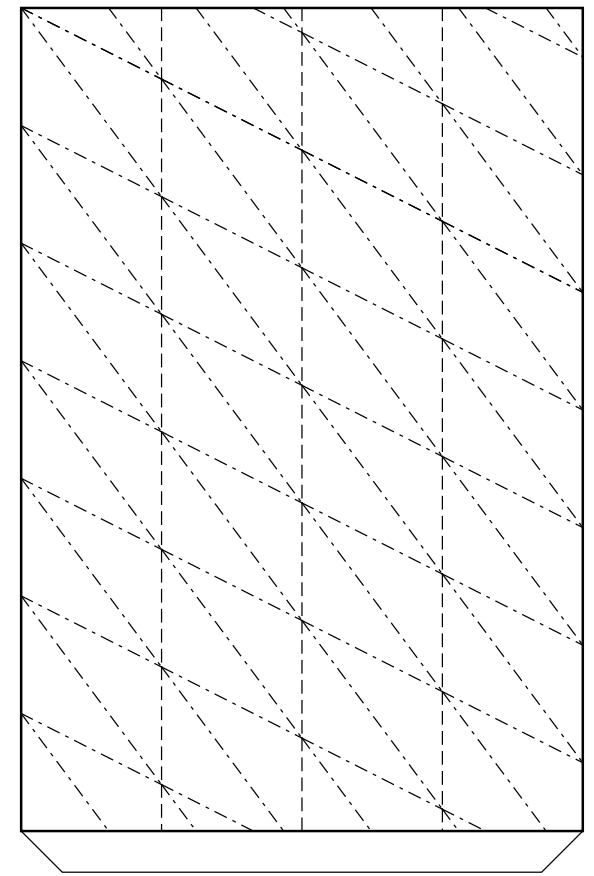

FIGURE B.35: Fold pattern for the $r=1.2, n=7$ Guest and Pellegrino cylinder

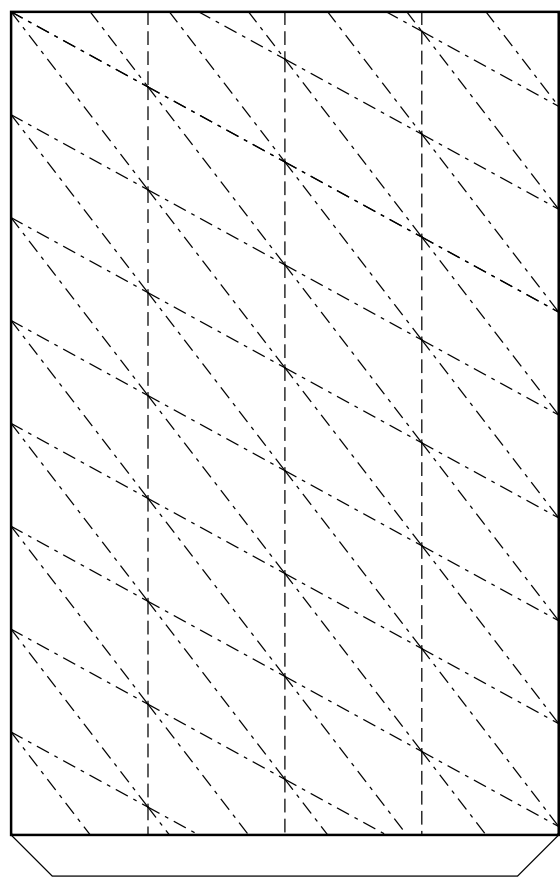

FIGURE B.36: Fold pattern for the $r=1.2, n=8$ Guest and Pellegrino cylinder 


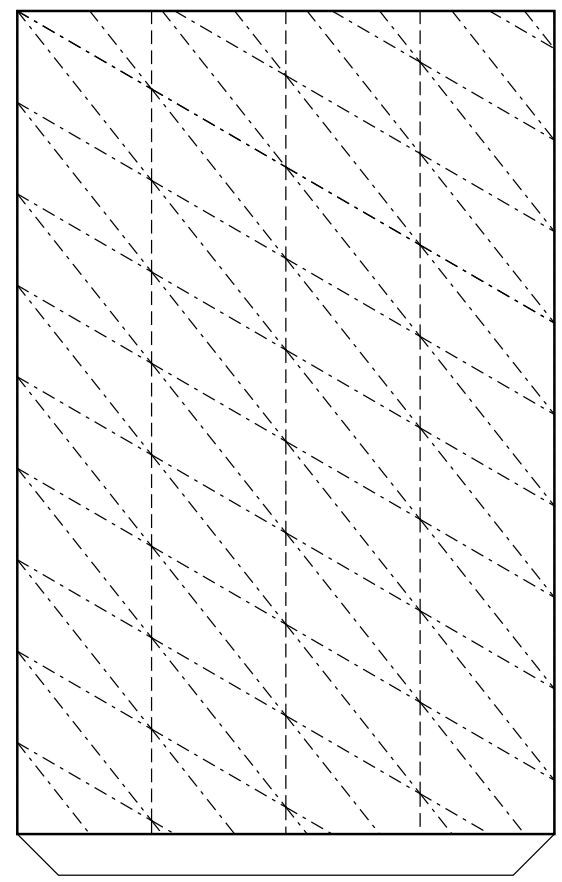

FIGURE B.37: Fold pattern for the $r=1.2, n=9$ Guest and Pellegrino cylinder

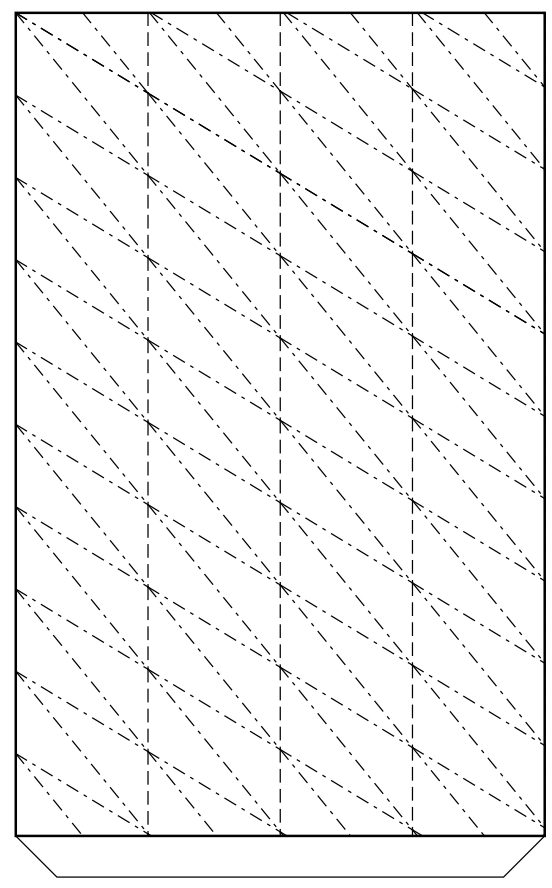

FIGURE B.38: Fold pattern for the $r=1.2, n=10$ Guest and Pellegrino cylinder 


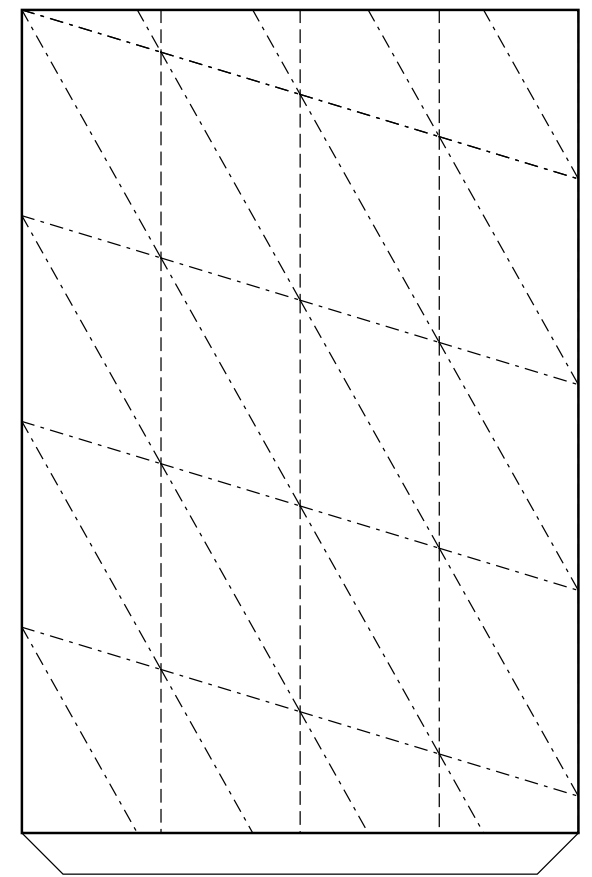

FIGURE B.39: Fold pattern for the $r=1.4, n=4$ Guest and Pellegrino cylinder

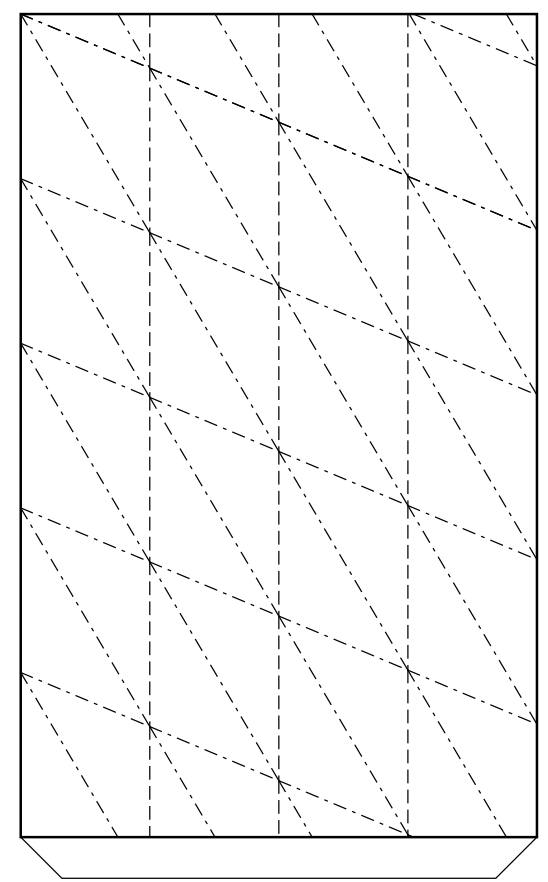

FIGURE B.40: Fold pattern for the $r=1.4, n=5$ Guest and Pellegrino cylinder 


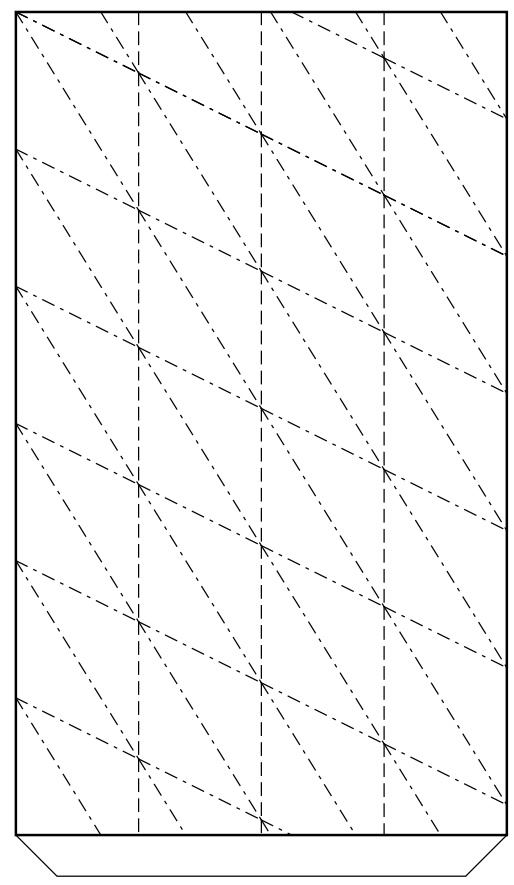

FIGURE B.41: Fold pattern for the $r=1.4, n=6$ Guest and Pellegrino cylinder

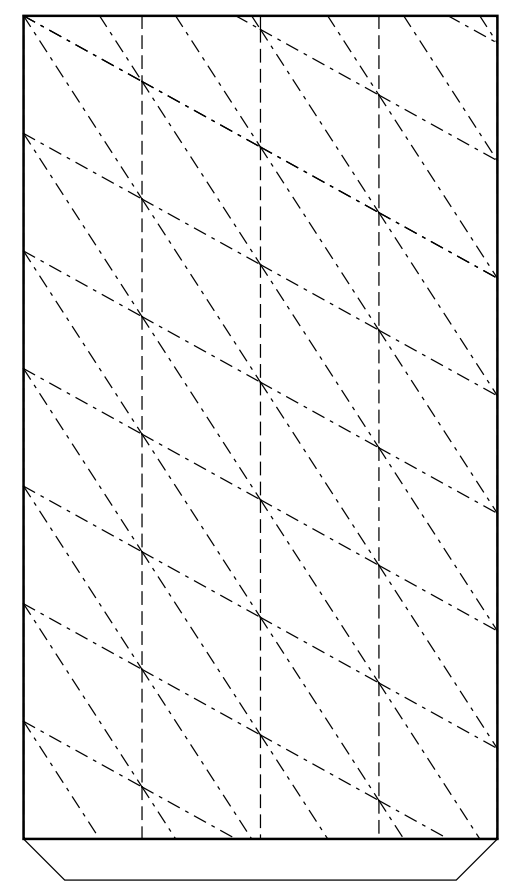

FIGURE B.42: Fold pattern for the $r=1.4, n=7$ Guest and Pellegrino cylinder 


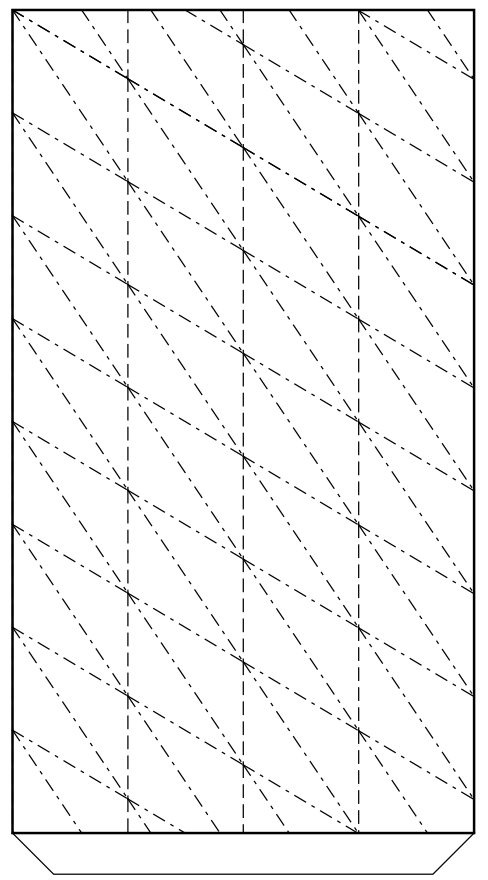

FiguRE B.43: Fold pattern for the $r=1.4, n=8$ Guest and Pellegrino cylinder

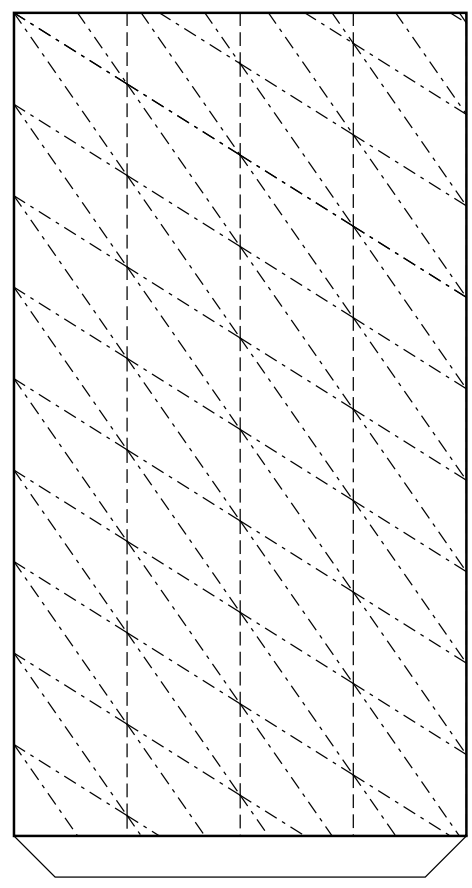

FIGURE B.44: Fold pattern for the $r=1.4, n=9$ Guest and Pellegrino cylinder 


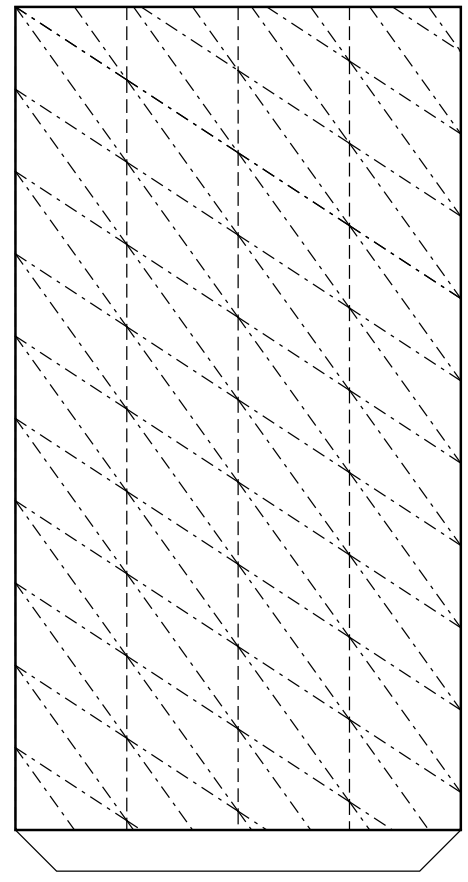

FIGURE B.45: Fold pattern for the $r=1.4, n=10$ Guest and Pellegrino cylinder

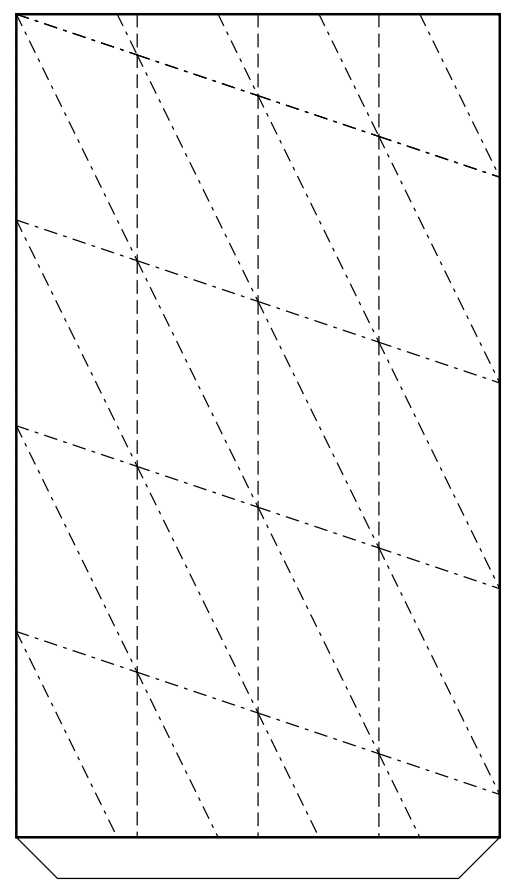

FIGURE B.46: Fold pattern for the $r=1.6, n=4$ Guest and Pellegrino cylinder 


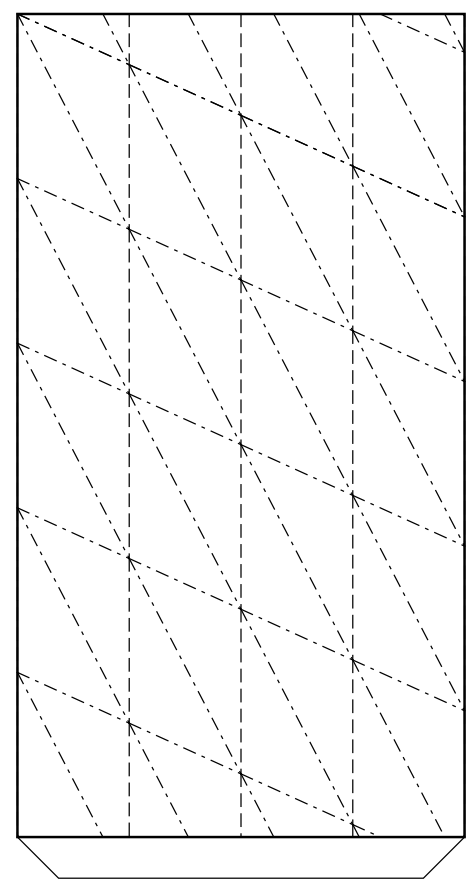

FIGURE B.47: Fold pattern for the $r=1.6, n=5$ Guest and Pellegrino cylinder

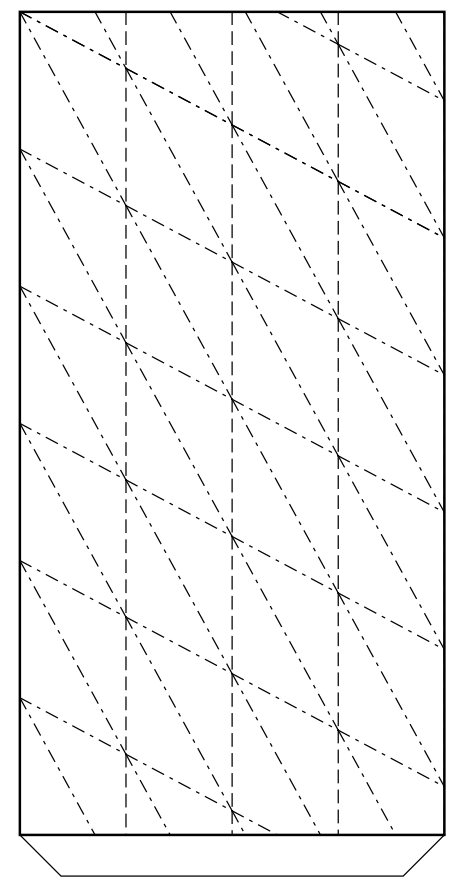

FIGURE B.48: Fold pattern for the $r=1.6, n=6$ Guest and Pellegrino cylinder 


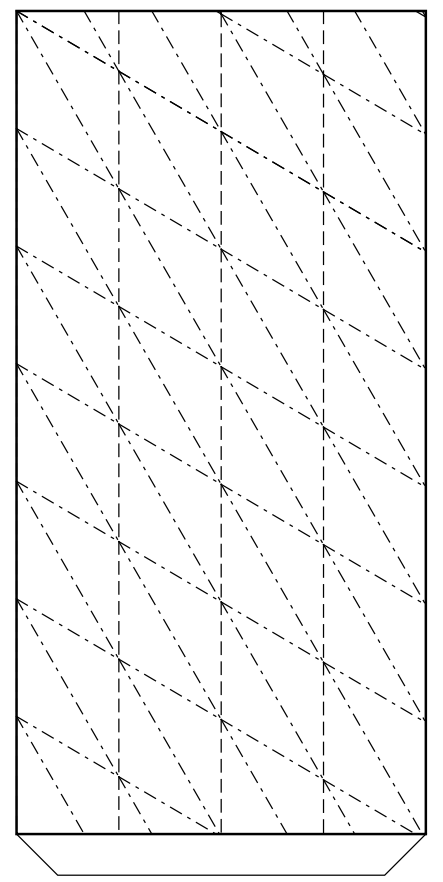

FIGURE B.49: Fold pattern for the $r=1.6, n=7$ Guest and Pellegrino cylinder

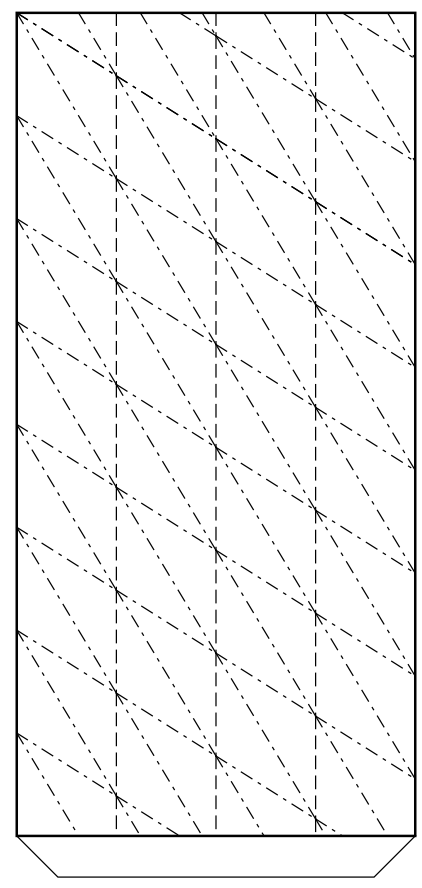

FIGURE B.50: Fold pattern for the $r=1.6, n=8$ Guest and Pellegrino cylinder 


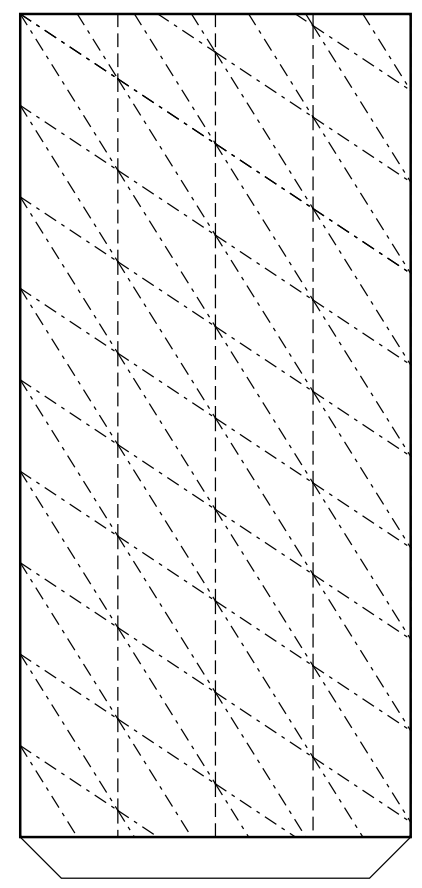

FIGURE B.51: Fold pattern for the $r=1.6, n=9$ Guest and Pellegrino cylinder

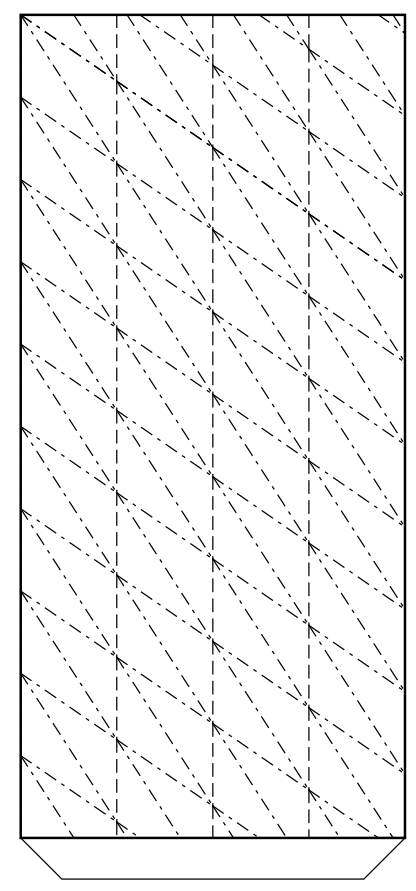

FIGURE B.52: Fold pattern for the $r=1.6, n=10$ Guest and Pellegrino cylinder 


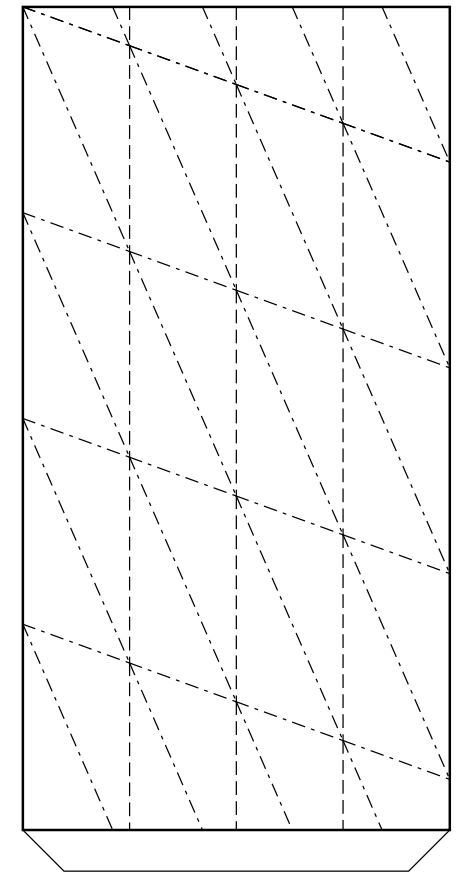

FIGURE B.53: Fold pattern for the $r=1.8, n=4$ Guest and Pellegrino cylinder

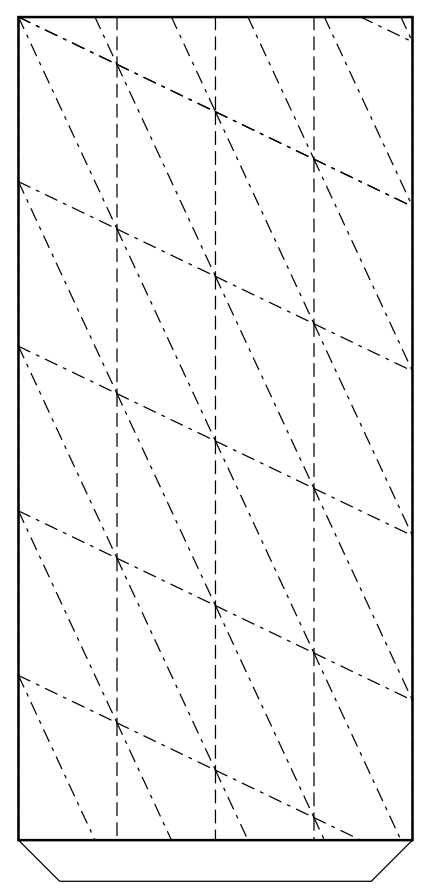

FIGURE B.54: Fold pattern for the $r=1.8, n=5$ Guest and Pellegrino cylinder 


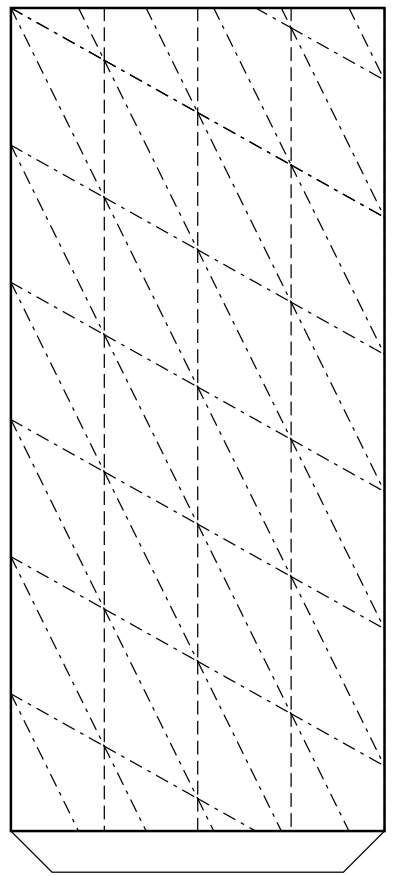

FIGURE B.55: Fold pattern for the $r=1.8, n=6$ Guest and Pellegrino cylinder

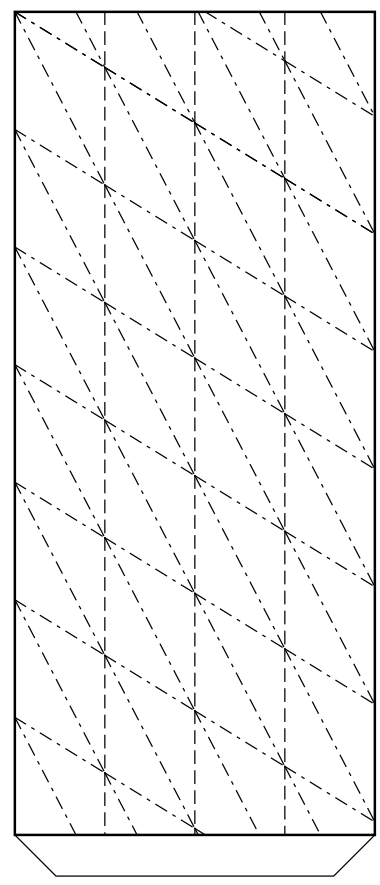

FIGURE B.56: Fold pattern for the $r=1.8, n=7$ Guest and Pellegrino cylinder 


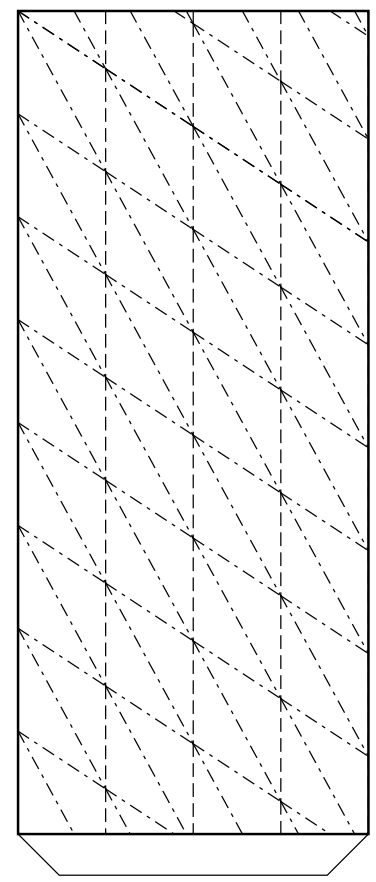

FIGURE B.57: Fold pattern for the $r=1.8, n=8$ Guest and Pellegrino cylinder

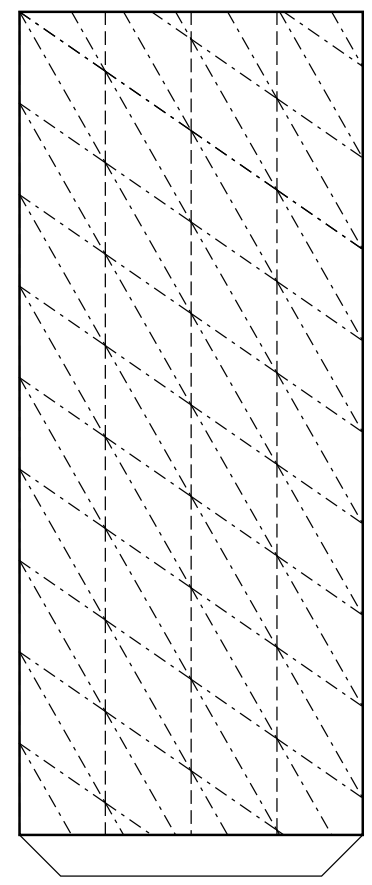

FIGURE B.58: Fold pattern for the $r=1.8, n=9$ Guest and Pellegrino cylinder 


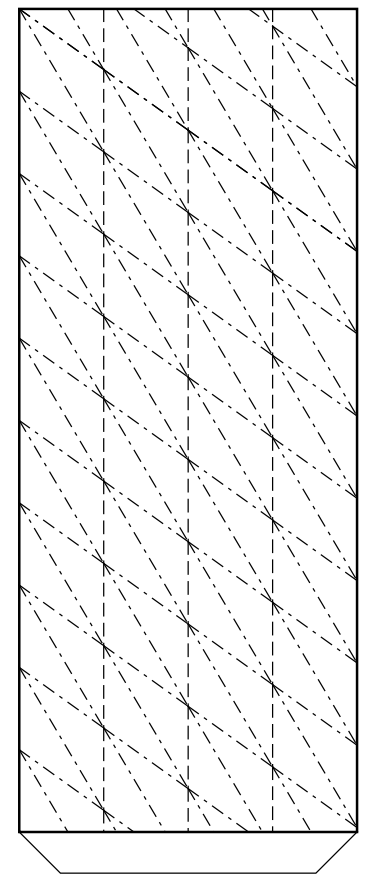

FIGURE B.59: Fold pattern for the $r=1.8, n=10$ Guest and Pellegrino cylinder

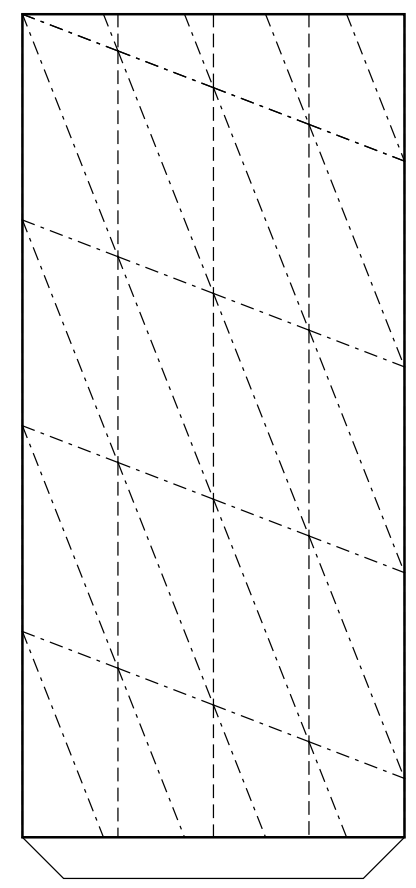

FiguRE B.60: Fold pattern for the $r=2 ., n=4$ Guest and Pellegrino cylinder 


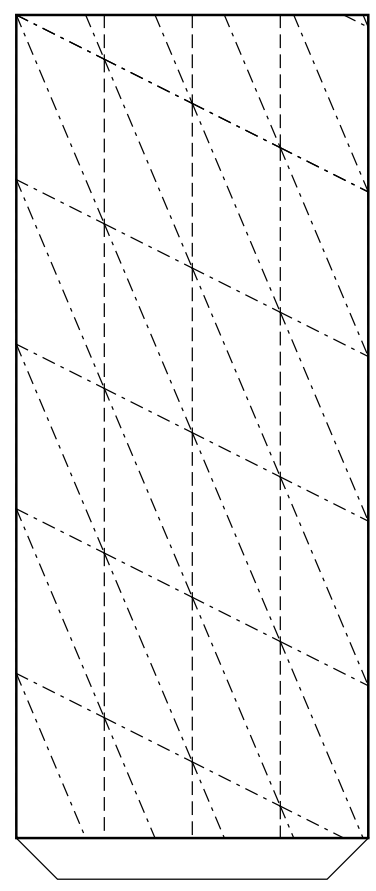

FIgURE B.61: Fold pattern for the $r=2 ., n=5$ Guest and Pellegrino cylinder

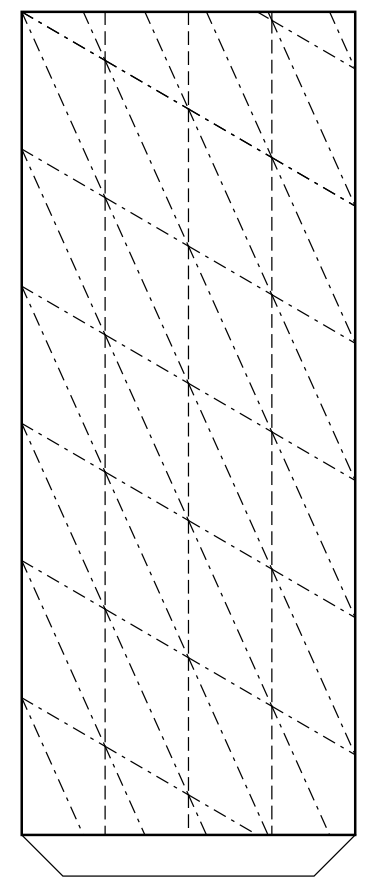

FIGURE B.62: Fold pattern for the $r=2 ., n=6$ Guest and Pellegrino cylinder 


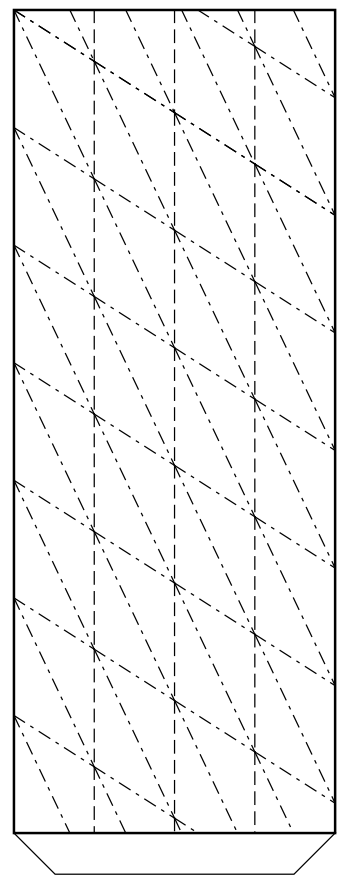

FIgURE B.63: Fold pattern for the $r=2 ., n=7$ Guest and Pellegrino cylinder

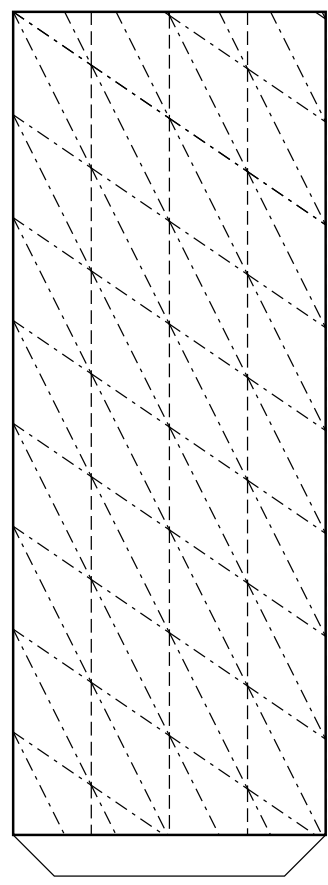

FIGURE B.64: Fold pattern for the $r=2 ., n=8$ Guest and Pellegrino cylinder 


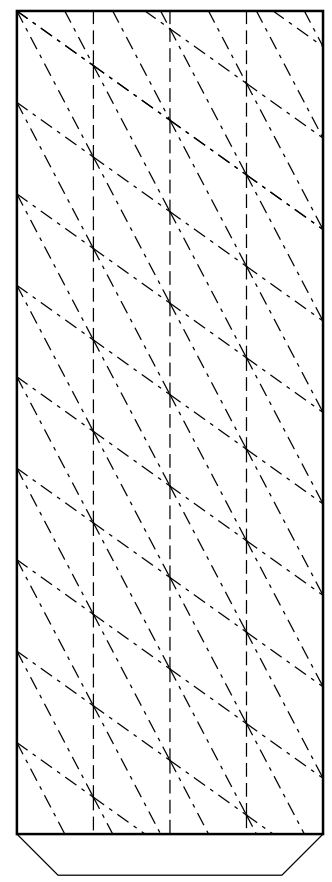

FIGURE B.65: Fold pattern for the $r=2 ., n=9$ Guest and Pellegrino cylinder

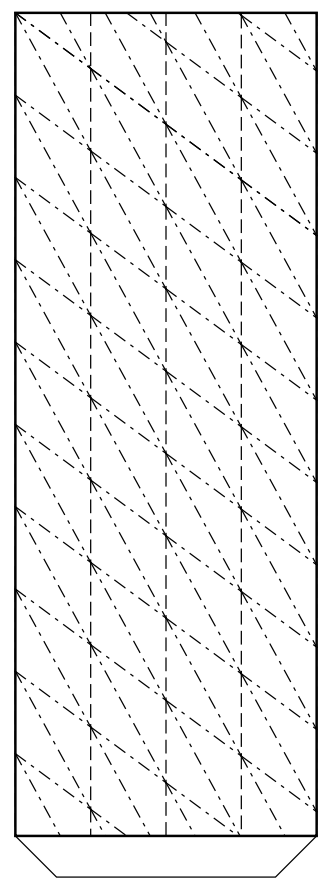

FIGURE B.66: Fold pattern for the $r=2 ., n=10$ Guest and Pellegrino cylinder 


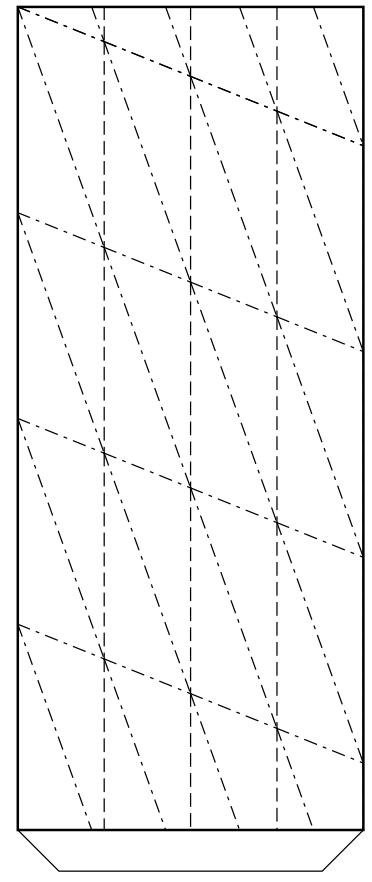

FIGURE B.67: Fold pattern for the $r=2.2, n=4$ Guest and Pellegrino cylinder

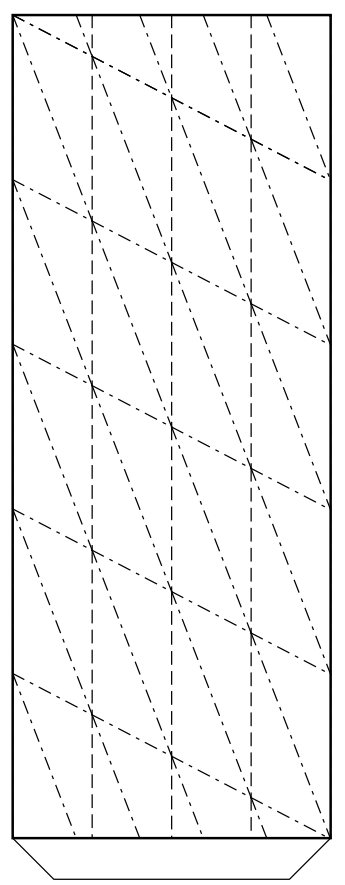

FIGURE B.68: Fold pattern for the $r=2.2, n=5$ Guest and Pellegrino cylinder 


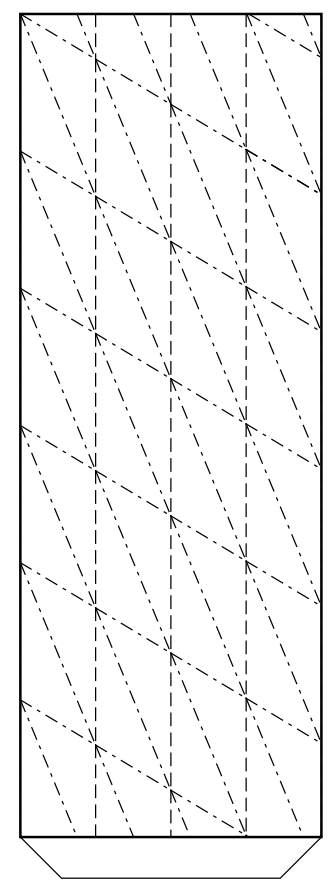

FIGURE B.69: Fold pattern for the $r=2.2, n=6$ Guest and Pellegrino cylinder

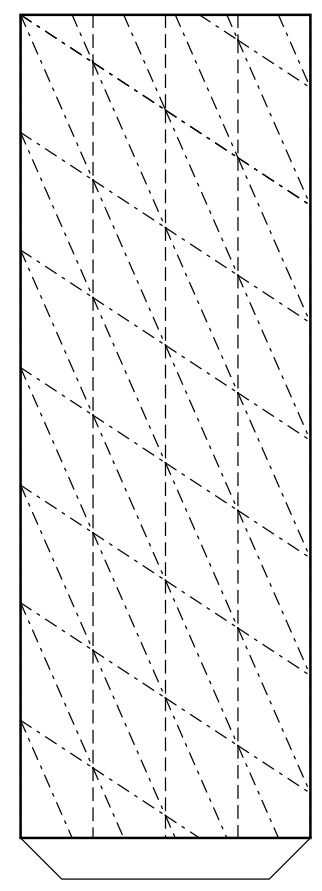

FIGURE B.70: Fold pattern for the $r=2.2, n=7$ Guest and Pellegrino cylinder 


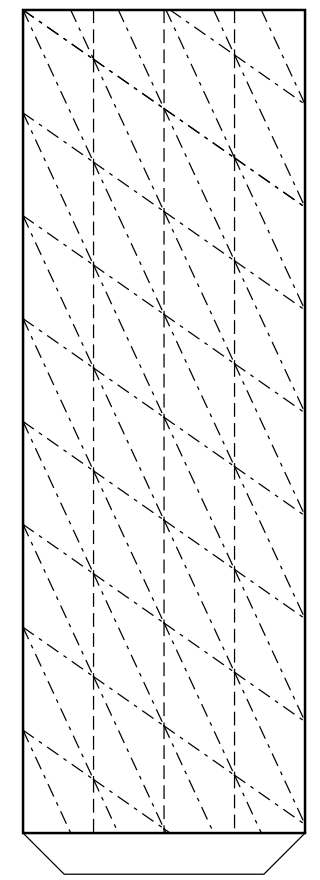

FIGURE B.71: Fold pattern for the $r=2.2, n=8$ Guest and Pellegrino cylinder

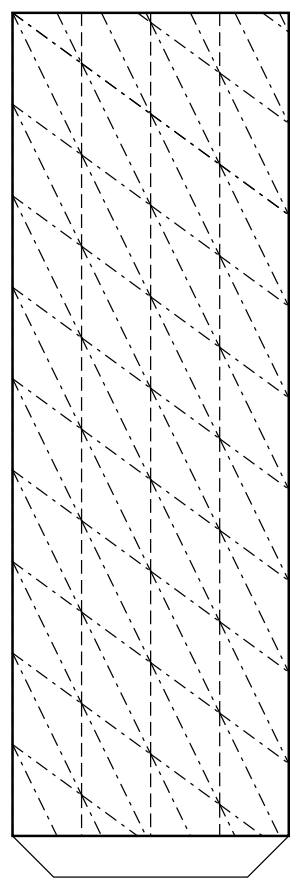

FIGURE B.72: Fold pattern for the $r=2.2, n=9$ Guest and Pellegrino cylinder 


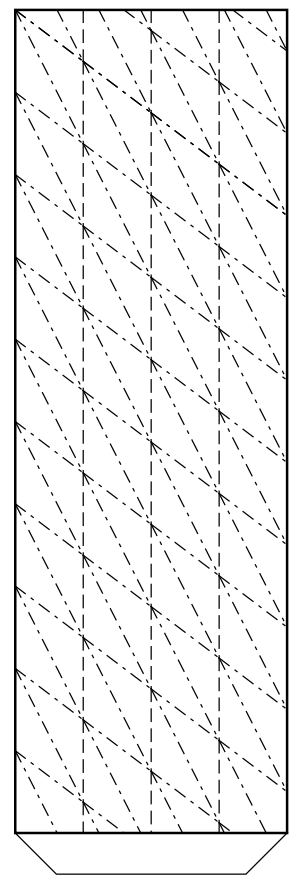

FIGURE B.73: Fold pattern for the $r=2.2, n=10$ Guest and Pellegrino cylinder

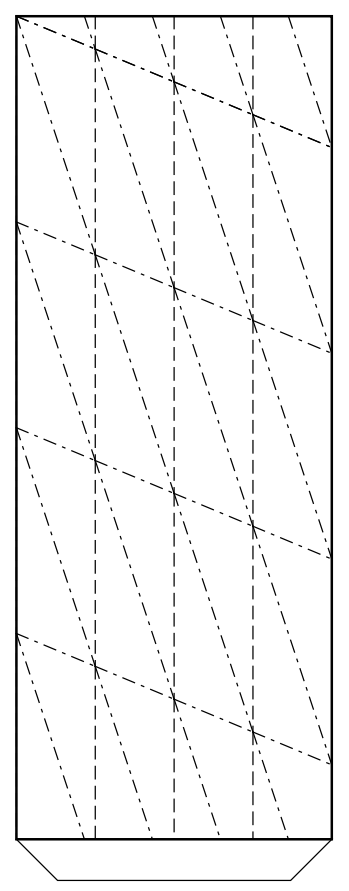

FIGURE B.74: Fold pattern for the $r=2.4, n=4$ Guest and Pellegrino cylinder 


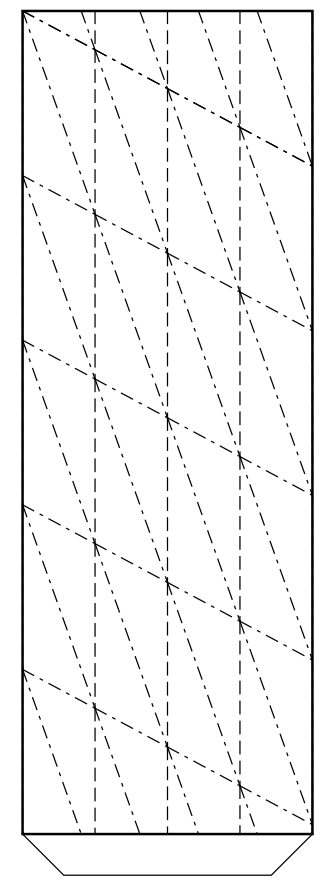

FIGURE B.75: Fold pattern for the $r=2.4, n=5$ Guest and Pellegrino cylinder

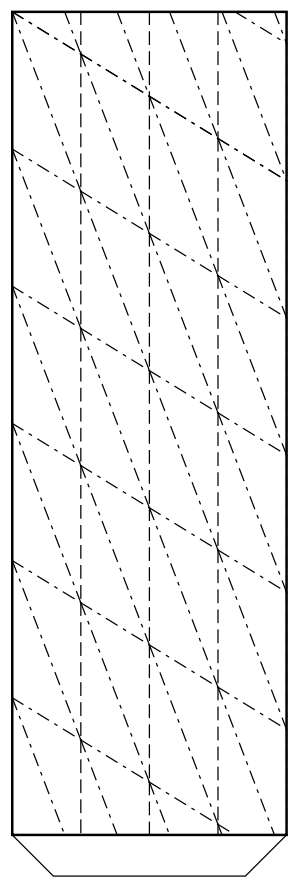

FIGURE B.76: Fold pattern for the $r=2.4, n=6$ Guest and Pellegrino cylinder 


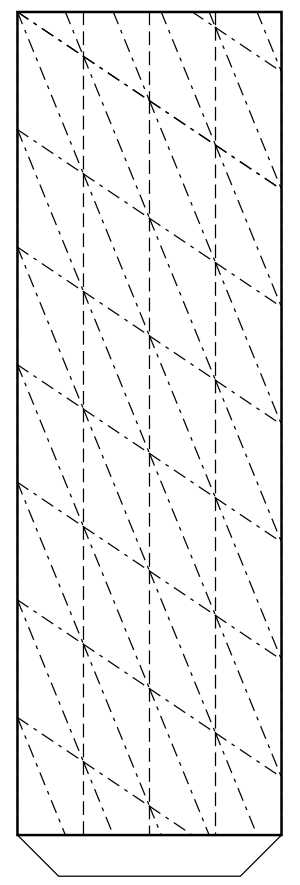

FIGURE B.77: Fold pattern for the $r=2.4, n=7$ Guest and Pellegrino cylinder

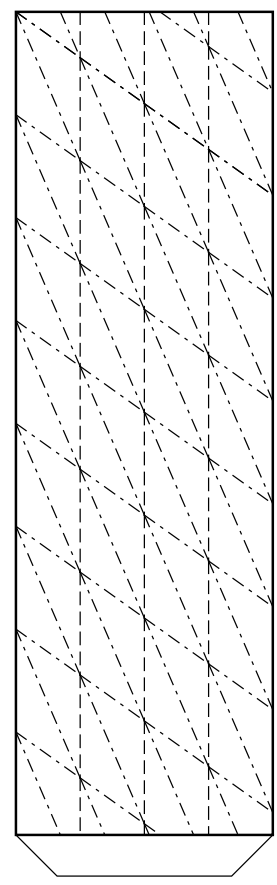

FIGURE B.78: Fold pattern for the $r=2.4, n=8$ Guest and Pellegrino cylinder 


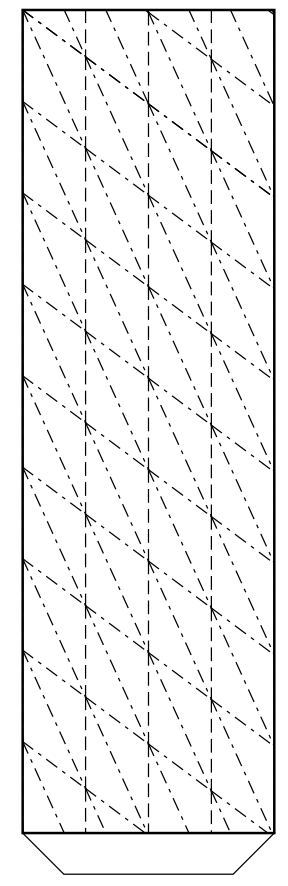

FIGURE B.79: Fold pattern for the $r=2.4, n=9$ Guest and Pellegrino cylinder

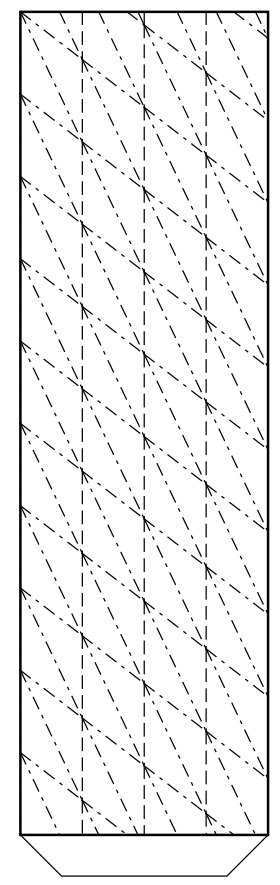

FIGURE B.80: Fold pattern for the $r=2.4, n=10$ Guest and Pellegrino cylinder 


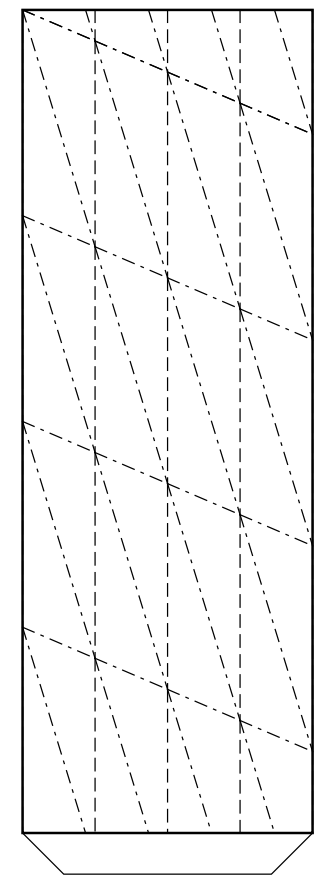

FIGURE B.81: Fold pattern for the $r=2.6, n=4$ Guest and Pellegrino cylinder

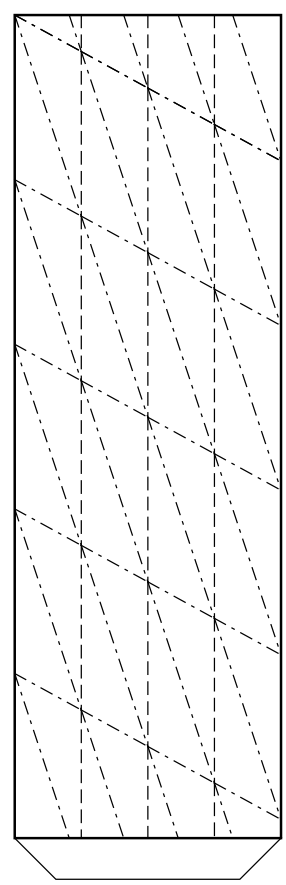

FIGURE B.82: Fold pattern for the $r=2.6, n=5$ Guest and Pellegrino cylinder 


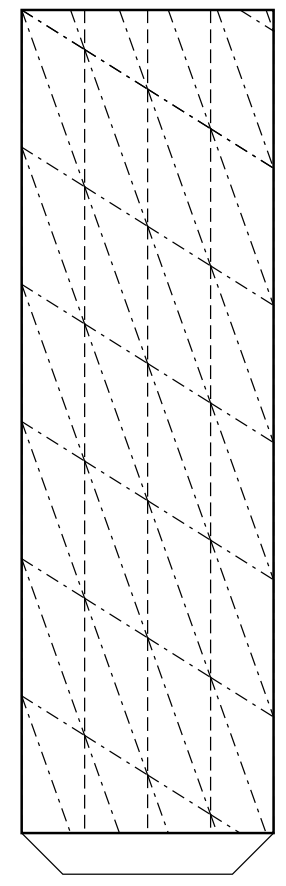

FIGURE B.83: Fold pattern for the $r=2.6, n=6$ Guest and Pellegrino cylinder

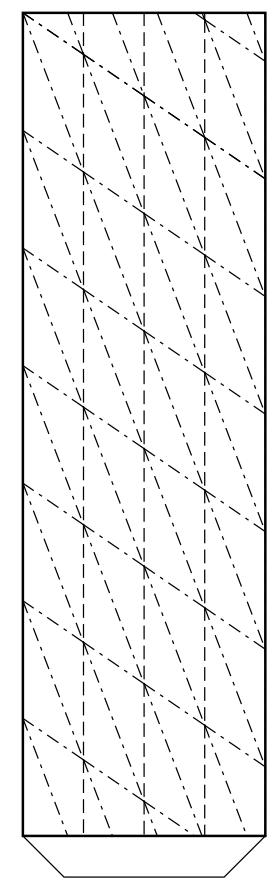

FIGURE B.84: Fold pattern for the $r=2.6, n=7$ Guest and Pellegrino cylinder 


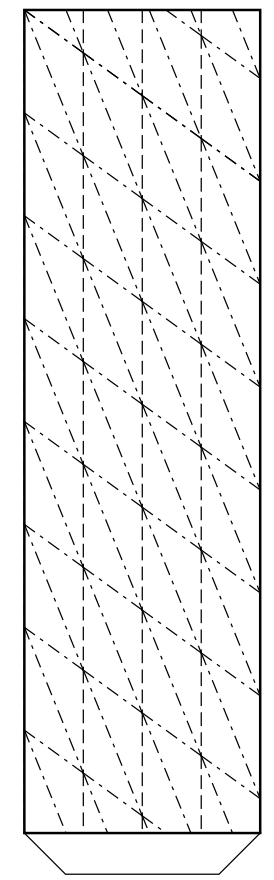

FIGURE B.85: Fold pattern for the $r=2.6, n=8$ Guest and Pellegrino cylinder

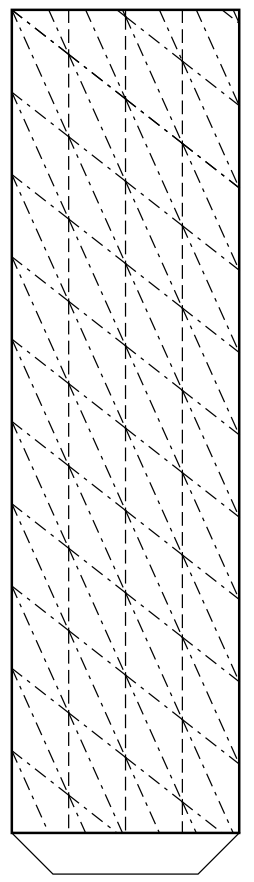

FIGURE B.86: Fold pattern for the $r=2.6, n=10$ Guest and Pellegrino cylinder 


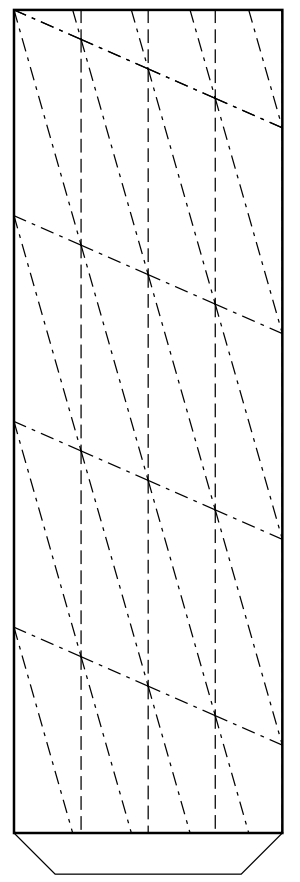

FIGURE B.87: Fold pattern for the $r=2.8, n=4$ Guest and Pellegrino cylinder

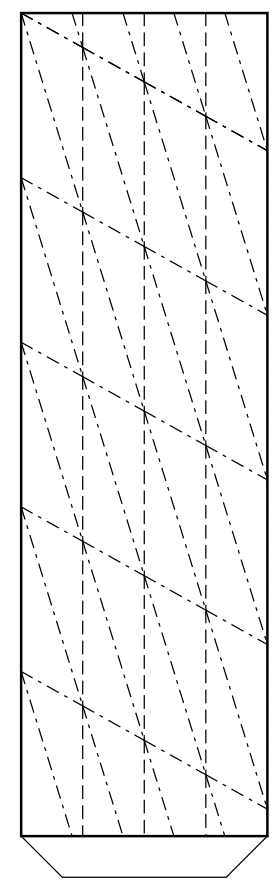

FIGURE B.88: Fold pattern for the $r=2.8, n=5$ Guest and Pellegrino cylinder 


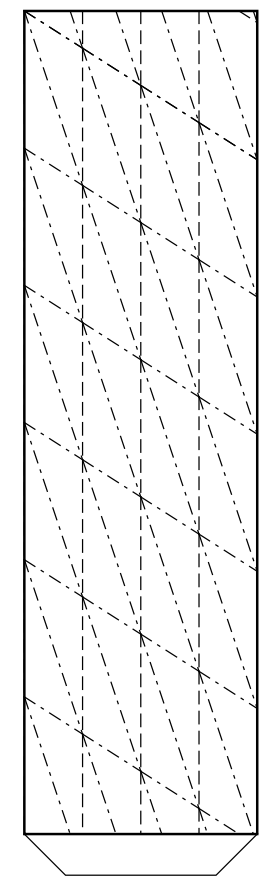

FIGURE B.89: Fold pattern for the $r=2.8, n=6$ Guest and Pellegrino cylinder

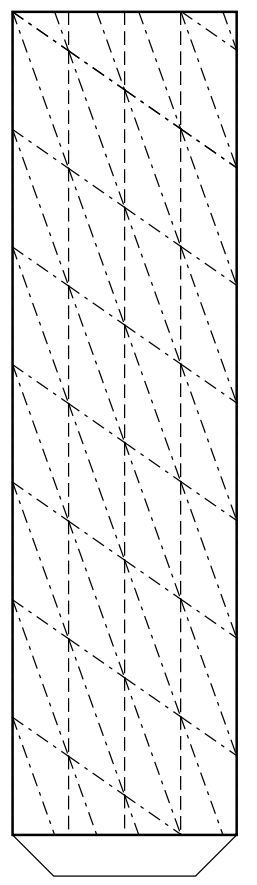

FIGURE B.90: Fold pattern for the $r=2.8, n=7$ Guest and Pellegrino cylinder 


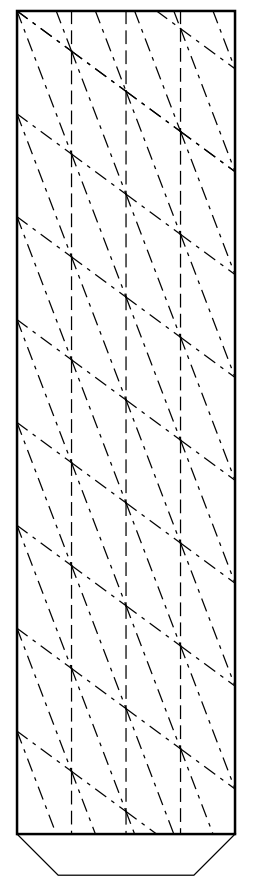

FIGURE B.91: Fold pattern for the $r=2.8, n=8$ Guest and Pellegrino cylinder

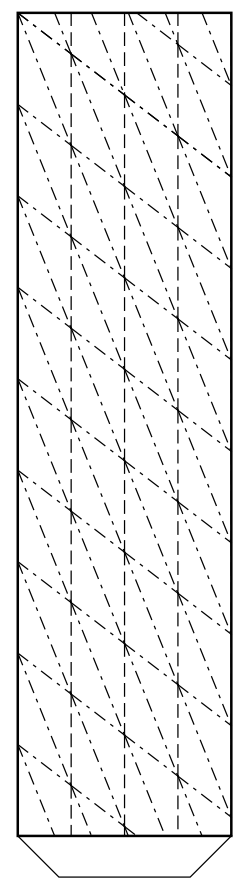

FIGURE B.92: Fold pattern for the $r=2.8, n=9$ Guest and Pellegrino cylinder 


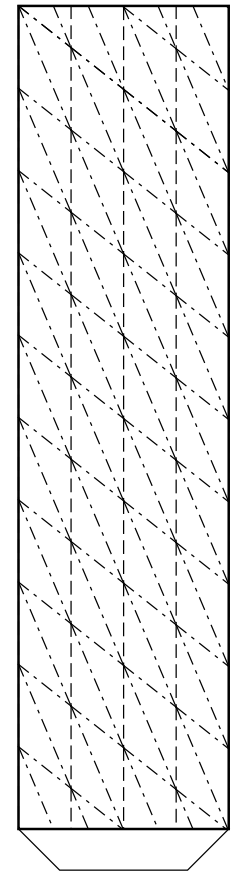

FIGURE B.93: Fold pattern for the $r=2.8, n=10$ Guest and Pellegrino cylinder

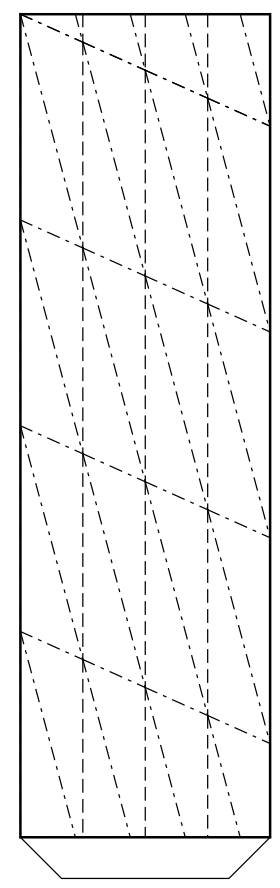

FiguRE B.94: Fold pattern for the $r=3 ., n=4$ Guest and Pellegrino cylinder 


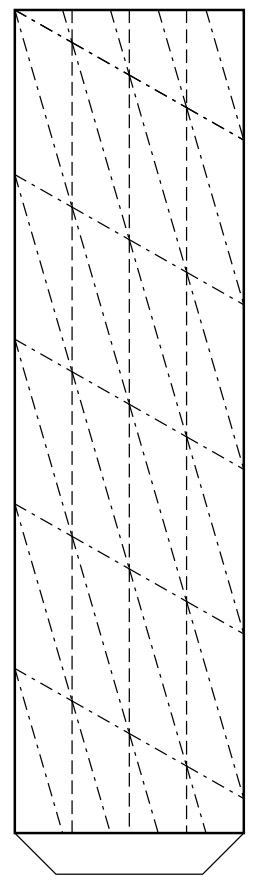

FIgURE B.95: Fold pattern for the $r=3 ., n=5$ Guest and Pellegrino cylinder

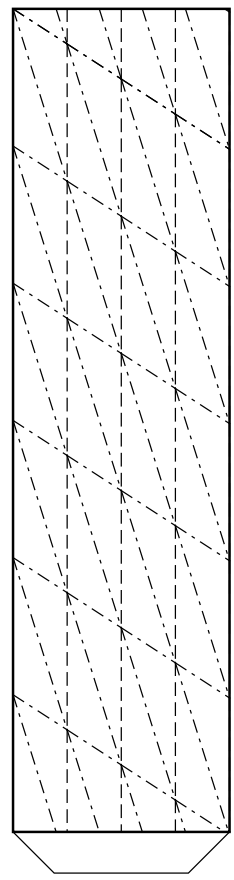

FIGURE B.96: Fold pattern for the $r=3 ., n=6$ Guest and Pellegrino cylinder 


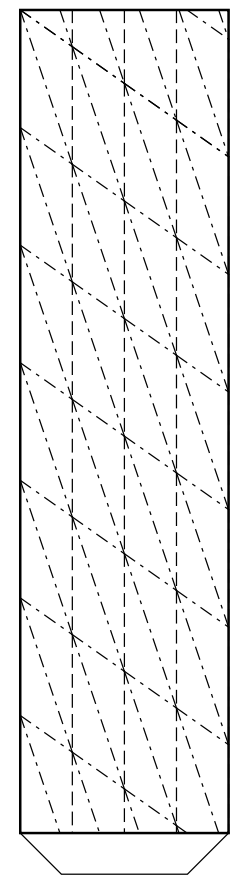

FIGURE B.97: Fold pattern for the $r=3 ., n=7$ Guest and Pellegrino cylinder

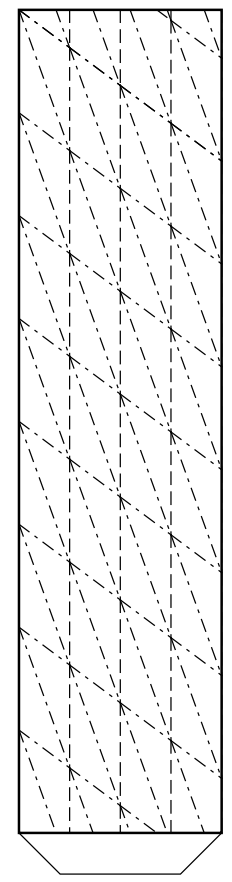

FIGURE B.98: Fold pattern for the $r=3 ., n=8$ Guest and Pellegrino cylinder 


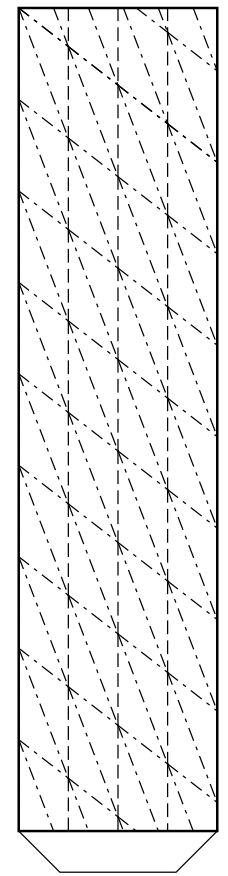

FIGURE B.99: Fold pattern for the $r=3 ., n=9$ Guest and Pellegrino cylinder

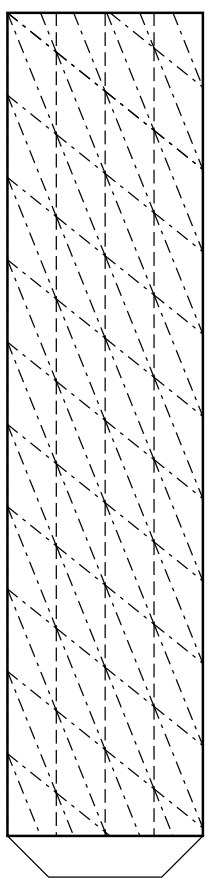

FIGURE B.100: Fold pattern for the $r=3 ., n=10$ Guest and Pellegrino cylinder 


\section{B.1 Acknowledgments}

Just as any other work of a certain size, the creation of this thesis would not have been possible without the help of a number of people.

First of all, I would like to thank my advisor Max Wardetzky, as this work could not have happened without his support. I would also like to thank Etienne Vouga, and Omer Gottesman, with whom I had the great pleasure of collaborating closely over the course of my PhD. I am also no less grateful towards my colleagues of the DDG lab, Knut Heidemann, Andrew O'Shea Sageman-Furnas, and Henrik Schumacher, who not only provided the most pleasant atmosphere one could hope for but who also readily let themselves be drawn into helpful discussions, towards Georg Jahn, Peter Hintz, and Benjamin Willenberg for smoothing out the rough edges of this thesis; and my family, Jule, and all other friends with whom I had the pleasure of sharing the past three and a half years.

This work was supported by the BMBF project MusiKa. 